\title{
Information Processing Speed in Ageing: Is it task dependent?
}

\author{
Torrens-Burton, Anna
}

How to cite:

Torrens-Burton, Anna (2018) Information Processing Speed in Ageing: Is it task dependent?. Doctoral thesis, Swansea University.

http://cronfa.swan.ac.uk/Record/cronfa40835

Use policy:

This item is brought to you by Swansea University. Any person downloading material is agreeing to abide by the terms of the repository licence: copies of full text items may be used or reproduced in any format or medium, without prior permission for personal research or study, educational or non-commercial purposes only. The copyright for any work remains with the original author unless otherwise specified. The full-text must not be sold in any format or medium without the formal permission of the copyright holder. Permission for multiple reproductions should be obtained from the original author.

Authors are personally responsible for adhering to copyright and publisher restrictions when uploading content to the repository.

Please link to the metadata record in the Swansea University repository, Cronfa (link given in the citation reference above.)

http://www.swansea.ac.uk/library/researchsupport/ris-support/ 
Information Processing Speed in Ageing: Is it task dependent?

\author{
Anna Torrens-Burton
}

Submitted to Swansea University in fulfilment of the requirements for the Degree of Doctor of Philosophy

Swansea University

2018 


\section{ABSTRACT}

Evidence indicates that information processing speed slows as age increases and disproportionately so with impaired cognition and various neurodegenerative diseases such as Alzheimer's disease. The new DSM-5 criteria for neuro-cognitive disorders state that measuring information processing speed associated with attentional function should be included within dementia diagnosis. However, what is not clarified is that outcome variability can occur, in part, as a result of methodological factors i.e. type of attention-related test/ attentional function and by person-related factors such as sex and education. In addition, there appears to be a dichotomy between the types of tests used within research studies and clinical settings which should be addressed [Haworth et al, 2016].

The aim of the research presented in this thesis was to investigate how using different tests of attentional function in similar groups of young and older adults may affect the outcome measure of information processing speed (RT) and its variability (IIV). Part of this aim was to determine whether the number of trials may influence performance i.e. RT, IIV and accuracy (number of errors). Another aim was to determine whether result outcome is affected similarly across tests by a variety of person-related factors i.e. sex, education, objective cognitive measures and particularly previously un-tested factors of subjective memory function and perceived test difficulty which may help determine whether subjective feelings are associated with slower and more variable information processing speed and may influence study outcome.

The first study [Chapter 2] comprised of a visual search test commonly used in research as a sensitive measure of ageing upon RT and IIV and attentional shifting, yet not examined particularly in relation to subjective memory function and perceived test difficulty. Results indicated that information processing speed was significantly slowed in older compared to younger adults and attentional shifting was poorer in older adults. There was no relationship with subjective memory function whereas the influence of sex, education and perceived test difficulty were dependent on the condition and age.

In the second, larger study including the Trail Making Test (TMT), Simple reaction time (RT) test, Choice RT test and Multi-Item Localization test (MILO) [Chapters 35], the results indicated that in all tests older adults were significantly slower and more variable than young adults at group level. Person-related factors were influential depending on the test used. Subjective memory function and education were only influential within conditions of the MILO and perceived test difficulty influential in Trails B and Choice RT. Large effect sizes in visual search, MILO and the Choice RT suggested they were most sensitive to ageing effects.

In conclusion, we speculate which attentional tests may be more useful in research and than those already used in clinical settings i.e. TMT and highlight the need to take into consideration different factors depending on the attentional test used so as not to misinterpret normal levels of information processing speed in ostensibly healthy aging. 
This work has not previously been accepted in substance for any degree and is not being concurrently submitted in candidature for any degree.

Signed (candidate)

Date

\section{STATEMENT 1}

This thesis is the result of my own investigations, except where otherwise stated. Where correction services have been used, the extent and nature of the correction is clearly marked in a footnote(s).

Other sources are acknowledged by footnotes giving explicit references. A bibliography is appended.

Signed (candidate)

Date

\section{STATEMENT 2}

I hereby give consent for my thesis, if accepted, to be available for photocopying and for inter-library loan, and for the title and summary to be made available to outside organisations.

Signed (candidate)

Date

NB: Candidates on whose behalf a bar on access has been approved by the University (see Note 7), should use the following version of Statement 2:

I hereby give consent for my thesis, if accepted, to be available for photocopying and for inter-library loans after expiry of a bar on access approved by the Swansea University.

Signed (candidate)

Date 


\section{ACKNOWLEDGEMENTS}

I don't think completing my $\mathrm{PhD}$ would have been possible without the amazing support I have had along the way. To my wonderful parents for all their invaluable help and support, without them this $\mathrm{PhD}$ would not have been possible from the very beginning. I also want to thank my ever so patient partner who managed to keep me sane throughout the entire process and thank you to Frank and all my friends for having faith in me and keeping up words of encouragement.

Huge thanks to my supervisors Professor Andrea Tales, Dr Cristina Izura and Dr Megan Crawford for their valuable knowledge and advice throughout the project, in particular to Andrea for always being there to pick up the pieces and guide me in the right direction.

Many thanks to Neil Carter for all the IT support and to Dr Jade Norris for helping with participant recruitment. Finally, of course, thank you to all the young and older participants who were willing to take part in my study so that I was able to produce the rich amount of data I did. 


\section{TABLE OF CONTENTS}

Tables and Figures $\quad 8$

$\begin{array}{ll}\text { Abbreviations } & 12\end{array}$

$\begin{array}{ll}\text { Summary of Thesis } & 13\end{array}$

1.0. CHAPTER ONE: Introduction 31

1.1. Scientific background and context 32

1.1.1. Brain structure and neural changes and information processing speed in ageing

1.1.2. Brain structure and neural changes in ageing and Alzheimer's disease

1.1.3. Brain structure and neural changes related to information processing speed in Prodromal Stages of Alzheimer's disease: Mild Cognitive Impairment (MCI)

1.1.4. Brain structure and neural changes related to intraindividual variability (IIV) in ageing

1.1.5. Brain structure and neural changes related to intraindividual variability (IIV) in MCI and AD

1.1.6. Subjective Cognitive Impairment (SCI) and subjective memory function

1.1.7. DSM-5 Criteria

1.2. Selective Attention and information processing speed

1.2.1. Selective attention

1.3. Participant-related factors in information processing speed research

1.3.1. Sex and education

1.3.2. Anxiety and Depression

1.3.3. Perceived test difficulty

1.3.4. Number of trials

1.3.5. Errors

2.0. CHAPTER TWO: Visual Search (Study one)

2.1. Visual search: Introduction

2.2. Visual search: Methodology 66

2.3. Visual search: Results 69

2.4. Visual search: Discussion 84 
3.1. TMT: Introduction 106

3.2. TMT: Methodology 121

$\begin{array}{ll}\text { 3.3. TMT: Results } & 124\end{array}$

$\begin{array}{ll}\text { 3. 4. TMT: Discussion } & 131\end{array}$

4.0 CHAPTER FOUR: Simple \& Choice RT (Study two) 147

$\begin{array}{ll}\text { 4.1 Simple and Choice RT: Introduction } & 147\end{array}$

4.2 Simple RT (Experiment 1): Methodology 160

4.3 Simple RT: Results 162

$\begin{array}{ll}\text { 4.4 Choice RT (Experiment 2): Methodology } & 170\end{array}$

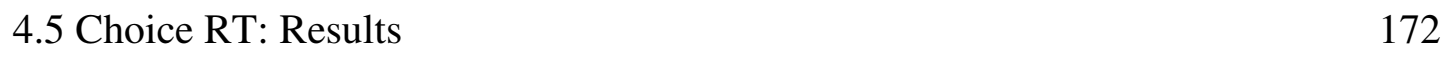

$\begin{array}{ll}\text { 4.6. Simple and Choice RT: Discussion } & 187\end{array}$

5.0. CHAPTER FIVE: IPad Testing [MILO] (Study two) 209

5.1. MILO: Introduction 209

5.2. MILO: Methodology 216

$\begin{array}{ll}\text { 5.3. MILO: Results } & 218\end{array}$

5.4. MILO: Discussion 232

6.0. CHAPTER SIX: Comparing tests - Effect sizes 251

6.1. Effect sizes and Information processing speed 257

6.2. Effect sizes and intraindividual variability 260

6.3. Effect sizes for correlations between information processing speed
and person-related factors

6.4. Effect sizes between intraindividual variability and person-related factors 266

$\begin{array}{ll}\text { 6.5. Comparing RT and IIV } & 269\end{array}$

6. 6. Choice RT: comparing blocks of trials 270

$\begin{array}{ll}\text { 6.7. Comparing errors between tests } & 272\end{array}$

$\begin{array}{ll}\text { 6.8. Limitations for comparing current effect sizes } & 275\end{array}$

6.9. Conclusion 275 
7.1. Findings in relation to the aims discussed and stated in the thesis introduction

$\begin{array}{ll}\text { 7.2. Outliers } & 278\end{array}$

$\begin{array}{ll}\text { 7.3. Effects of results on research and clinical settings } & 279\end{array}$

$\begin{array}{ll}\text { 7.4. Limitations across tests } & 280\end{array}$

$\begin{array}{ll}\text { 7.5. Future directions } & 282\end{array}$

$\begin{array}{lr}\text { 8.0. REFERENCES } & 285\end{array}$

$\begin{array}{lr}\text { 9.0 APPENDICES } & 330\end{array}$

$\begin{array}{ll}\text { Appendix A: Covering Letter } & 331\end{array}$

Appendix B: Confirmation email of ethics approval 332

Appendix C: Poster advertisement for older adults 333

Appendix D: Poster advertisement for young adults 334

Appendix E: Participant Information sheet 335

$\begin{array}{ll}\text { Appendix F: Participant Consent form } & 337\end{array}$

Appendix G: Participant Debrief form 338

Appendix H: Demographics questions 339

Appendix I: Copy of Montreal Cognitive Assessment (MoCA) 340

Appendix J: Copy of Memory Functioning Questionnaire (MFQ) 341

Appendix K: Copy of Patient Health Questionnaire (PHQ-9) 345

Appendix L: Copy of Anxiety Questionnaire (GAD-7) 346

Appendix M: Copy of Trails A \& B paper test 347

Appendix N: Likert scale of perceived test difficulty 351

Appendix O: Copy of paper -Haworth et al, 2016

Appendix P: Copy of paper - Torrens-Burton et al, 2017

$\begin{array}{ll}\text { Appendix Q: Parametric analysis } & 374\end{array}$

$\begin{array}{ll}\text { Appendix R: Correlations between tests } & 388\end{array}$ 


\section{TABLES AND FIGURES}

\section{Tables}

\section{Visual search}

Table 1: Normality of distribution for visual search data

Table 2: Mean demographic scores for older and young adult groups

Table 3: Information processing speed, intraindividual variability (IIV) and error rates- age comparison

Table 4: Mean number of errors for both conditions (target alone and target plus distractors) for young and older adults

Table 5: Mean number of errors for both conditions comparing age and sex (males and females)

\section{TMT}

Table 6: Normality of Distribution for TMT data

Table 7: Mean demographic scores for older and young adult groups

Table 8: Mean information processing speed and perceived test difficulty for both age groups

\section{Simple \& Choice RT}

Table 9: Normality of Distribution for Simple RT data

Table 10: Simple RT test Demographic scores for young and older adult groups

Table 11: Information processing speed, intraindividual variability, error rates and perceived test difficulty for both age groups in Simple RT test

Table 12: Overall mean number of errors in Simple RT for young and older adults.

Table 13: Overall mean number of errors in Simple RT for males and females in young and older adults.

Table 14: Normality of Distribution for Choice RT data

Table 15: Demographics of young and older adult groups for Choice RT test

Table 16: Information processing speed, intraindividual variability and perceived test difficulty for Choice RT 
Table 17: Information processing speed across Choice RT blocks in between age groups.

Table 18: Information processing speed across Choice RT blocks for age and sex

Table 19: Intraindividual variability across Choice RT blocks between age groups.

Table 20: Intraindividual variability across Choice RT blocks for age and sex

Table 21: Overall mean number of errors in Choice RT for young and older adults

Table 22: Overall mean number of errors comparing age and sex

Table 23: Mean number of errors across Choice RT blocks between age groups.

Table 24: Mean number of errors across Choice RT four blocks for age and sex

\section{MILO}

Table 25: Normality of distribution for MILO data

Table 26: Demographics for young and older adult groups.

Table 27: Information processing speed, intraindividual variability and perceived test difficulty between age groups and conditions

Table 28: Mean number of errors made in young and older adults

Table 29: Mean number of errors made - age and sex comparison

\section{Effect sizes}

Table 30: Effect sizes for the relationships found in each visual search condition

Table 31: Effect sizes comparing information processing speed between young and older adults in each second study tests and sub-tests

Table 32: Effect sizes of comparing intraindividual variability between young and older adults from each test or sub-test

Table 33: Effect sizes comparing information processing speed and metacognition between tests

Table 34: Effect sizes comparing information processing speed and educational level between tests

Table 35: Effect sizes comparing RT and IIV between young and older adults and between tests and subtests 
Table 36: Effect sizes comparing information processing speed of young and older adults between blocks of trials in Choice RT

Table 37: Effect sizes comparing IIV of young and older adults between blocks of trials in Choice RT

Table 38: Mean number of errors made by young and older adults between each test or sub-test

Table 39: Effect sizes comparing the mean number of errors between young and older adults in the Choice RT and MILO test

\section{Figures}

\section{Visual search}

Figure 1: Screen shot of visual search stimuli

Figure 2: Box plot of information processing speed comparing age and conditions

Figure 3: Box plot of mean difference in information processing speed $\left(\mathrm{RT}_{\text {Distractors }}-\mathrm{RTTarget}_{\text {alone }}\right.$ between sex and age

Figure 4: Box plot of mean information processing speed between age, sex and condition

Figure 5: Box plot of mean intraindividual variability (IIV) between age and conditions

Figure 6: Box plot of mean difference in intraindividual variability (IIV) (IIV $V_{\text {Distractors }}$ - IIVTarget ${ }_{\text {alone }}$ between sex and age

Figure 7: Box plot of mean intraindividual variability (IIV) between age, sex and condition

\section{TMT}

Figure 8: Screen shot of Trails A and Trails B stimuli

Figure 9: Box plot of information processing speed comparing age and condition

Figure 10: Box plot of information processing speed comparing age and sex

\section{Simple \& Choice RT}

Figure 11: Screenshot of Simple RT test stimuli

Figure 12: Screenshot of Choice RT stimuli 
Figure 13: Simple RT box plot of Information processing speed- age comparison

Figure 14: Simple RT box plot of Information processing speed- age and sex comparison

Figure 15: Simple RT box plot of intraindividual variability - age comparison

Figure 16: Simple RT box plot of Intraindividual variability - age and sex comparison

Figure 17: Choice RT box plot of Information processing speed- age comparison

Figure 18: Choice RT box plot of Information processing speed for Choice RT - age and sex comparison

Figure 19: Choice RT box plot of intraindividual variability - age comparison

Figure 20: Choice RT box plot of intraindividual variability - age and sex comparison.

Figure 21: Choice RT bar chart of mean number of errors across the four blocks age comparison

Figure 22: Choice RT bar chart Mean number of errors across the four blocks comparing age and sex

\section{MILO}

Figure 23: Screenshot of MILO stimuli

Figure 24: Box plot of Information processing speed for RT1- age comparison

Figure 25: Box plot of Information processing speed for RT1 - age and sex comparison

Figure 26: Box plot of Information processing speed for RT8 minus RT1- age comparison

Figure 27: Box plot of Information processing speed for RT8 minus RT1- age and sex comparison

Figure 28: Box plot of Intraindividual variability for IIV 1- age comparison

Figure 29: Box plot of Intraindividual variability for IIV 1 - age and sex comparison

Figure 30: Box plot of Intraindividual variability for IIV8 minus IIV1- age comparison

Figure 31: Box plot of Intraindividual variability for IIV8 minus IIV1- age and sex comparison 


\section{ABBREVIATIONS}

MCI: Mild cognitive impairment

AD: Alzheimer's disease

DSM-5: Diagnostic and Statistical Manual of Mental Disorders, 5th Edition

NCD: Neurocognitive disorders

TMT: Trail Making Test

SCI: Subjective Cognitive Impairment

RT: Reaction time

WMH: White matter hyperintensities

aMCI: Amnestic mild cognitive impairment

DTI: Diffusion tensor imaging

IIV: Intraindividual variability

CNS: Central nervous system

MMSE: Mini mental state exam

ERP: Event-related potential

IOR: Inhibition of return

APOE, e4: Apolipoprotein E, E4 allele

SMC: Subjective Memory Complaints

V1: primary visual cortex, striate cortex or area 17

V5/MT: middle temporal visual area

V4: fourth visual area

MoCA: Montreal cognitive assessment

MFQ: Memory Functioning Questionnaire

PHQ-9: Patient health questionnaire

GAD-7: Generalized Anxiety Disorder 7-item

CANTAB: Cambridge Neuropsychological Test Automated Battery 


\section{Thesis Background and Summary of Aims}

It has been widely reported by an abundance of research studies that information processing speed slows during ageing and disproportionately so in Alzheimer's disease compared to cognitively healthy ageing. Information processing speed (RT) is a factor traditionally measured in the clinical diagnosis of mild cognitive impairment (MCI), dementia (of all aetiologies but particularly Alzheimer's disease $[\mathrm{AD}])$ and as a marker of disease progression and response to intervention. Its importance is highlighted by the relatively new DSM-5 criteria for neurocognitive disorders which include a measure of information processing speed, and in particular a measure of such speed with respect to attentional function [American Psychiatric Association, 2013]. However, what is not addressed by the DSM-5 criteria is research evidence indicating that information processing speed is not always significantly slowed in AD or in MCI compared to cognitively healthy ageing [Landy et al, 2015; Tales et al, 2002; Grady et al, 1993] or that outcome variability can occur in such studies. This makes the relationship between dementia and information processing speed difficult to ascertain and interpret especially across different research studies and translation to clinical practice.

As described in detail in the following chapters, a significant body of evidence now indicates that the outcome of studies examining the functional integrity of information processing speed in ageing, cognitive impairment and dementia, can be influenced by a variety of factors in addition to the effects of ageing, dementia and cognitive impairment, which if not taken into account such factors could influence study outcome.

Different methodological factors, such as the type of test used to measure RT and its variability, can influence study outcome in the same group of individuals [Tales, Bayer, Haworth et al, 2010; Haworth, Philips, Newson et al, 2016]. Different tasks, ostensibly of the same function, may recruit different aspects of brain function; some of which may be either impaired or normal within the same individual depending upon the presence or absence of pathology or its distribution. It may also be the case that the integrity of information speed of certain brain functions (as recruited and measured by different tests) is more sensitive to ageing and/or disease than others. 
Such an effect can be observed in studies of visual attention where several different tasks ostensibly measure similar function i.e. executive and selection attention, but produce different outcome of results, namely the difference of RT and its variability between young and older adults. For example, the Trail Making test (TMT) [Reitan, 1971], a single trial pen and paper test, and the visual search test, [Tales et al, 2010] a multi-trial computer-based psychophysics test, both measure executive function and the ability to shift attention, yet the visual search test has been observed to better differentiate RT between aMCI (amnestic Mild cognitive impairment) and cognitively healthy ageing compared to the TMT. However both tests may present different results for the integrity of RT in terms of performance outcome depending on which test has been used [Haworth et al, 2016; see also Yung, Cardoso-Leite, P., Dale, et al, 2015]. Methodological factors also include factors such as whether the response is one of detection or discrimination and the processing load of the test [Tales et al, 2011).

In addition to these methodological factors, there are participant-related factors such as age, education, objectively measured cognitive function and sex (female/male). Despite there being some research into the potential impact of such factors i.e. sex and education upon the outcome of information processing speed, emerging evidence indicates that there is still outcome variability between previous research studies which have used smaller participant groups and different tests, thus there is a necessity to examine these factors in greater detail [Haworth et al, 2016; Phillips, Rogers, Haworth et al, 2013]. Moreover, other previously unacknowledged or unknown factors, namely subjective memory function and perceived test difficulty (both of which are discussed in the following sections) may also affect the results of such studies and thus need to be addressed.

In view of this increasingly emerging research evidence one should ask whether the DSM-5 statement needs to highlight such potential outcome variability and to state the type of test that should be used to clinically measure the integrity of information processing speed. It is important to investigate and account for the potential effects these different factors may have on information processing speed particularly in individuals used in control groups within dementia research. Subsequent results may not relate to the level of slowing expected from a healthy ageing sample thus not truly representing a typical control group used to compare against a dementia sample. 
As a result this may have an impact on interpreting results of ageing and dementia research as well as a clinical diagnosis of neurocognitive disorders (i.e. MCI or dementia). This thesis aims to address this issue with a novel approach of measuring information processing speed in relation to sex, education, subjective memory function and perceived test difficulty, using a variety of different visual attention tests, the number of trials within tests and error analysis.

\section{Subjective memory impairment (SCI)}

It is common in research measuring information processing speed in ageing to examine the potential influence of objectively ascertained cognitive function.

However, a potential confounding effect in such studies is that the potential influence of subjective memory impairment has not been addressed in great detail. Subjective memory impairment is important because although its aetiology is heterogeneous, it represents an increased risk factor for the development of MCI and AD in some people [Chen, Chen \& Chiu, 2017; Lehrner, Moser, Klug et al, 2014; Jessen, Wiese, Bachmann et al, 2010] and irrespective of aetiology, it may detrimentally effect information processing speed and its variability. Therefore SCI may be characterised by disproportionate slowing of information processing speed compared to levels expected in ostensibly healthy ageing. Using individuals with SCI, or variations of subjective feelings (i.e. subjective memory function) within control groups in dementia research would bias results as the true difference between control and dementia group results is obscured. In addition, characteristics of an individuals' RT performance during a clinical diagnosis such as for MCI or dementia may be misinterpreted thus lead to an incorrect or missed diagnosis. The level of slowing in these individuals may in fact overlap with the level of slowing which represents pathological ageing i.e. dementia. If information processing speed is indeed poorer in subjective memory function, the control group would not represent a cognitively healthy sample thus the results may be misrepresented. Therefore the current research allows observation whether SCI, in particular subjective memory function, may be associated with slower and more variable information processing speed. 


\section{Perceived test difficulty}

As briefly suggested above, the integrity of information processing speed in an individual is likely to be related, not simply to the presence of pathology and what aspects of brain function are specifically affected by such pathological processes, but may also be affected by other factors i.e. anxiety [Frick et al, 2014; Berggren \& Derakshan, 2013] and depression [Singh-Manoux et al, 2014; Rapp et al, 2006] or by psychological factors such as perceived test difficulty; a factor not examined in great detail in previous ageing and dementia research. Perceived test difficulty is an example of psychological self-assessment where individuals hold beliefs about themselves i.e. their own abilities or beliefs about the demands of a task which can, as a result, have an effect on the outcome of a cognitive activity [Flavell, 1979] i.e. slower information processing speed.

There are many examples of psychological self-assessment although in this thesis we focus on peoples' perception about how difficult a task is to perform. Believing a task is difficult to perform may be related to the propensity for individuals to think they performed badly despite their actual performance relating to what is expected in healthy ageing. Such beliefs may have a negative effect on information processing speed thus we ask whether the more difficult a task is perceived to be, the slower performance is. Some evidence demonstrates that aspects of self-perception of the integrity of cognition can negatively affect cognitive performance [Rounis, Maniscalco, Rothwell et al, 2010; Yokoyama, Miura, Watanabe, 2010; Bolmont, Thullier \& Abraini et al, 2000]. However this is still a novel factor to be examined in relation to information processing speed in visual attention, in particular the aspect of self-perceived difficulty of a test.

If perceived test difficulty influences information processing speed but is not taken into account, this may have significant implications on the integrity of the results i.e. of control groups and impact how past ageing and dementia studies have been interpreted. It also has clinical relevance as not taking perceived test difficulty into account may lead to clinicians misinterpreting the reason for slowed RT (such as underlying structural problems) thus misdiagnosing an individual. Different aspects of psychological self-assessment may also be responsive to intervention thus their effects, particularly in relation to RT may therefore be reversed. 
The influence of this novel factor of perceived test difficulty on information processing speed in relation to visual attention will be examined alongside other factors of subjective memory function sex and education. An initial study will examine the potential influence of perceived test difficulty on information processing speed and its variability (IIV) of a commonly used test (visual search, see Chapter Two). In a second larger study, it will be examined whether the influence of perceived test difficulty on information processing speed is similar across a number of visual attention tests in, where possible, a group of the same individuals (see Chapter Three to Five).

\section{Intraindividual variability of information processing speed}

Many previous research studies have examined RT in relation to ageing however fewer have examined both RT and its intraindividual variability (IIV) within the same study. Intraindividual variability of information processing speed reflects the variation of behavioural responses within a single persons' overall performance over the test period i.e. over a given number of trials. IIV is associated with the functional integrity of information processing speed and in particularly associated with fluctuations or deficits in attentional function and control [Ribeiro et al, 2016; Prado et al, 2011; MacDonald et al, 2006].

IIV can be sensitive to ageing as well as information processing speed, with older adults being more variable in their performance compared to young adults [MacDonald, Nyberg, \& Backman, 2006; Hultsch, MacDonald, \& Dixon, 2002] and increased IIV in MCI and AD compared to healthy ageing [Philips et al, 2013; Kälin, Pfluger, Gietl et al, 2014; Jackson et al, 2012] although this has not always been supported [Waugh, Fozard, Talland \& Erwin, 1973]. If information processing speed is potentially influenced by methodological factors i.e. type of test or person-related factors of sex, education and perceived test difficulty and subjective memory function, this may also be the case for IIV thus may have a similar impact on results of control groups in ageing and dementia research. 


\section{Number of Trials}

In RT research it is common to measure mean RT performance and IIV by using multiple trials (e.g. psychophysics techniques) during the test. However it is rare for RT studies to examine how the number of trials per se may have an effect on RT performance and IIV. More trials can invoke fatigue [Woods et al, 2015] or reduced sustained attention [Fernaeus et al 2013] and result in slower RT performance particularly by the end of the testing period. Controversially, in contrast high numbers of trials can have opposite effect and improve RT performance due to practice effects [Yotsumoto et al 2015; Siettos and Smyrnis 2017].

In the Choice RT test, information processing speed was examined across four blocks in order to observe whether participants slow or become more varied throughout the test (possibly due to fatigue) or speed up or become less varied (possibly due to practice effects). The effect of the number of trials is analysed and discussed in more detail in Choice test (see Chapter Four). The number of trials could have in measured in the other tests in this $\mathrm{PhD}$ using multiple trials [visual search, simple and MILO] however the way in which they were programmed made this difficult.

\section{Errors}

Errors during RT studies occur, for example, when the incorrect button for a response is pressed or the response is given too soon (i.e. prompting when to respond). Not all previous RT studies have included errors in their analysis however this measure may be important measure for a number of reasons. For example, making more errors may relate to a lack of concentration or fatigue while performing the test, problems with the test itself i.e. too difficult to complete [Cavaco et al, 2013; Seo et al, 2006], or commonly recognised issues with performing RT tests such as the speed accuracy trade-off [van Veen, Krug, \& Carter, 2008; Salthouse, 1979] where an individual places more emphasis on either speed (completing the test as quickly as possible) or accuracy (completing the test as accurately as possible).

For each test in this thesis the mean number of errors made was compared between young and old and between males and females to see if error rates associated with age or sex. The effect and implications of errors are discussed in more detail in relation to each attention test in the corresponding Chapters Two to Five. 
In addition, mean errors were compared between each test (see Chapter Six) to determine whether some tests of similar attention function are performed more accurately than others.

\section{Aims}

One of the aims of this thesis was to investigate the functional integrity of information processing speed and its intraindividual variability between younger and older adults in relation to a variety of different visual attention-related processing tests i.e. how the use of different tests in the same people may produce different results. From consideration of previous research, it was hypothesized that older adults would produce slower and more variable reaction time scores compared to young adults across each attention test. In addition the aim was to determine whether the number of trials may influence performance thus outcome measure (RT, IIV) and accuracy (number of errors). It is hypothesised that any influence of trial number on older adults would have a negative effect on information processing speed i.e. increase fatigue thus produce slower RT and in younger adults any potential trial influence will relate to practice effects thus produce faster RT.

A further aim was to investigate what influence sex and education may have on information processing speed and IIV (examined a little previously yet will be reexamined) and whether the result outcome is similar across different attentional tests. From previous research on sex and education, it is hypothesized that males would be faster and less variable compared to females in both young and older adults and higher levels of education would relate to faster and less variable RT.

A final aim was to examine, in a novel approach, the potential influence of subjective memory function and perceived test difficulty upon information processing speed and its intraindividual variability across different attentional tests. In addition, measuring subjective memory function allows us to observe whether subjective feelings are associated with slower and more variable information processing speed. Subjective memory function and perceived test difficulty, have not been subject to investigation in many previous RT and IIV and attention-related studies. It is hypothesized that older adults perceiving greater change to memory function would produce slower RT. As found in SCI research (and if indeed subjective memory function relates to SCI) these perceived changes may be highlighting underlying structural change to brain function which may relate to slower information processing speed in cognitive functions i.e. attention. 
Alternatively subjective memory function may be heterogeneous thus the influence on RT may also be psychological i.e. older adults notice changes to memory function thus assume their poorer memory results in them being poorer at performing the test (i.e. slow RT).

In addition, from previous findings, we hypothesise that perceiving the test to be more difficult may slow RT scores. This may relate to lower motivation or mood due to finding a test more difficult as suggested by past research (i.e. Bolmont et al, 2000) although it must be noted that we have not measured mood or motivation at this time. Any interactions between methodological and person-related factors were also examined as well as examining individuals within the young and older adults groups i.e. the importance of outliers and what they may tell us.

An initial study (Chapter 2) will examine information processing speed and its variability and attentional shifting efficiency in a group of young and a group of older adults using a common research attentional task, namely visual search [Tales, Bayer, Haworth et al, 2010]. This task has been used frequently in previous studies to measure differences in information processing speed between healthy ageing and MCI or AD. In addition we include an examination of the potential effect of sex, education, and unknown factors of subjective memory function and perceived test difficulty as previous research of visual search in relation to ageing and MCI or AD have not addressed how such factors may have affect study outcome. How these previously ignored factors may affect outcome may influence in future how we in fact interpret these results and especially in terms of what constitutes an older adults control group in studies of MCI and dementia.

A second, large study (see Chapters 3 to 5) will include a variety of different visual attention-related tests of information processing speed and individual variability. Using, where possible, the same large group of younger and older adults in each test, the aim is to examine how the use of a wide range of tests may influence study outcome i.e. does the outcome in similar groups of participants change depending on the type of attention test used?

In addition, the performance from each of these different tests will be examined with respect to education, sex, subjective memory function and perceived test difficulty and in the Choice reaction time test, and the number of trials. 
This is important to determine as the DSM-5 highlights the importance of measuring information processing speed for a diagnosis of MCI or AD yet does not provide further information as to the types of test to use, nor does the DSM address how factors such as education, sex, subjective memory or perceived test difficulty may affect information processing speed being measured per se or by a specific test.

\section{Thesis Limitations}

When reading the results of the two studies within this thesis it is important to take into account potential limitations which may have had an influence on how results were interpreted. When examining young and older adults, factors such as medication could not be controlled for, nor were other factors which may potentially have an effect on RT and IIV, taken into consideration i.e. sleep, occupation. We did not include a full medical analysis of general cognition or a detailed measure of overall individual memory function. In relation to subjective memory function in older adults, we were unable to include brain scans to observe whether subjective feelings indeed related to any underlying structural change.

In addition, the mean number of older adults fell at the younger end of the age range (65 years of a maximum of 80 years) thus this may be why there were a limited number of older adults with low MFQ scores i.e. not many adults who perceived many detrimental changes to memory function. The sample of young and older adults may have not represented the population since the young adult group consisted of mainly University students taking a Psychology degree. The older adult group included individuals who were willing to participate thus this may have limited the sample to a particular type of older adult participant and perhaps those of a particular level of education. A significant limitation in the second study was that not every young and older adult performed each of the tests because some were too tired to complete the battery or the test programme failed to work or broke, meaning the test could not be completed. This may have made it difficult to make direct comparisons between the results of each attention tests. 


\section{Summary of chapters}

What follows is a brief over view of the aims, research and outcome described in each chapter. Full details can be found in each chapter.

\section{Chapter One: Introduction}

This introduction and literature review introduces the concept of information processing speed (RT), intraindividual variability (IIV) and their importance with respect to the DSM-5. The introduction provides a background of the biological, clinical and research basis for the current research. Evidence of slowed and varied RT during healthy and abnormal ageing is discussed and related to cognitive impairment with the focus in this thesis being on visual attention related tests of RT and IIV. Subjective Cognitive Impairment (SCI) is explained in terms of its importance to characterise this stage for people whom it is the very early stage of further decline or just has a detrimental effect on everyday life. Non-clinical SCI, namely subjective memory function is also introduced and discussed in relation to its potential effect on RT and IIV studies.

This chapter introduces the concept that a variety of person related factors may influence information processing speed but they have been dismissed in previous studies of RT and visual attention. Details of the thesis aims are also explained.

Some of the information from this introduction was previously published in Haworth and colleagues [2016] (copy included in appendices)

\section{Chapter Two: Visual search (research test)}

A popular paradigm for measuring such visual attention-related RT processing and IIV is the visual search test [Chun \& Wolfe, 2001; Tales et al, 2010] which measures the time it takes to respond to a target with or without surrounding distractors. This visual search paradigm consists of multiple trials of two conditions. A target alone condition measures automatic attentional capture speed and processing and a target plus distractors condition measures how efficiently (i.e. quickly) attention can be shifted to find a particular target. Subtracting the target alone trials from the target with distractors trials provides a measure of how influential distracting information has been upon the search for a particular object [Tales et al 2010]. 
Visual search appears to display age effects with older adults performing slower compared to young adults due to taking longer to process information [Scialfa \& Joffe, 1997]. This is particularly true for visual search tasks which include distracting information or unexpected targets which can slow older adults' performance [Tales et al, 2010; Zeef et al, 1996; Nissen \& Corken, 1985; Cohn et al, 1984; Eriksen \& Eriksen, 1974].

The aim of this initial study is to investigate the functional integrity of information processing speed and its intraindividual variability (IIV) in younger and older adults using a visual search paradigm known to be sensitive to ageing effects [Tales et al, 2010]. In addition, a novel factor is to measure the influence of subjective memory function, perceived test difficulty, sex and education upon study outcome i.e. RT and IIV. This study finds slower information processing speed and greater IIV in older adults compared to young adults. Subjective memory function has no influence on information processing speed and its variability although significant outliers are observed in RT and IIV which may be of interest for further assessment and follow up.

Perceived test difficulty influences information processing speed only in older adults but does not influence intraindividual variability. Sex only influences information processing speed and its variability in young adults and education had an influence on older adults' information processing speed and its variability.

Finding a relationship between factors and information processing speed or IIV appears to be dependent on the condition within the visual search test possibly due to the differences in processing demands required [Phillips et al, 2013]. Therefore the results provide further evidence for the importance of the test on study outcome since results were observed to vary even within a given test. The findings in this study can only account for selective attention as measured by visual search therefore a second larger study is conducted with other tests of similar attentional function (selective attention) to examine whether the study outcomes on RT \& IIV i.e. the influence of sex, education, perceived test difficulty and subjective memory function are similar across different visual attention tests. This visual search already found factors (sex, education and perceived test difficulty) to affect RT and IIV which depended of the condition within the test thus emphasising the aim to determine whether similar findings are observed within other attention tests. 


\section{Chapter Three: Trail Making test (TMT) [typical clinical test]}

The Trail Making Test (TMT) is a typically used clinical task to measure information processing speed in relation to a variety of executive functions i.e. selective attention, switching attention and number sequencing. The task includes two conditions; Trails A and Trails B.

Trails A involves connecting numbers 1 to 25 in numerical order and Trails B involves connecting numbers 1 to 12 and letters A to L in order alternately i.e. 1A, $2 \mathrm{~B}, 3 \mathrm{C}$. The TMT is similar to the visual search task in terms of cognitive functions measured (switching and selecting attention on each target) but contains a single pen and paper trial for each condition.

There is evidence for slowing of information processing speed during the TMT, particularly in Trails B in older adults compared to young adults [Hashimoto, Meguro, Lee, et al., 2006; Zalonis, Kararizou et al., 2008; Periáñez, Rios-Lago, Rodriguez-Sanchez et al, 2007] and disproportionately slower information processing speed in MCI [Bezdicek, Motak, Axelrod et al, 2014; Silveri, Reali, Jenner, \& Puopolo, 2007] and AD [Johnstone, Hogg, Schopp, et al, 2002; Chen, Ratcliff, Phil et al, 2000] compared to healthy ageing. Yet further study is required to examine the influence of different factors on information processing speed in TMT. Sex and education have partially been addressed in previous TMT studies however perceived test difficulty and subjective memory function have been ignored.

In this chapter, the functional integrity of visual attention and information processing speed and IIV between young and older adults is measured using the clinical TMT. In addition the influence of other factors of sex, education, perceived test difficulty and subjective memory function are measured in relation to study outcome i.e. RT and IIV. This is the first test within a second larger study examining whether the integrity of information processing speed and the influence of different factors vary between different visual attention tests.

Results indicate that older adults are significantly slower compared to young adults for both TMT A and TMT B. There is no influence of subjective memory function on information processing speed for both Trails A and Trails B. In older adults, perceived test difficulty correlates with information processing speed (Trails B only) i.e. older adults who perceived Trails B to be difficult were slower. There is no effect of sex and education on information processing speed in both young and older adults. 
Subjective memory function correlates with education i.e. older adults who perceived greater changes to their memory had lower levels of education. Subjective memory function also correlates with perceived test difficulty in older adults; the easier both Trails A and B are perceived to be, the less change to memory function older adults perceive to have. Older adults with greater levels of education also report the test to be more difficult.

As found in the visual search (first study), finding a relationship between factors and information processing speed appears to be dependent on the condition within the TMT thus the results provide evidence for the importance of the test on the study outcome. Therefore further types of visual attention tests are examined in this second study to observe whether similar outcomes occur between tests and whether the same brain functions are affected by ageing and various person-related factors to the same or different degree.

Some of the data from Chapter 2 and 3 of this thesis has been published in TorrensBurton, A., Basoudan, M., Bayer, A., et al (2017) Perception and reality of cognitive Information processing speed, perceived memory function, and perceived task difficulty in older adults. Journal of Alzhiemer's disease, 60(4), 1601-1609.doi: 10.3233/JAD-170599 (copy included in appendices).

\section{Chapter Four: Simple and Choice RT (research test)}

Both the Simple and Choice RT tests measure slowing of information processing speed in relation to detrimental changes in encoding ability [Simon \& Pouraghabagher, 1978] in particular the ability to prepare, organise and execute a response [Vrtunski, Patterson et al, 1983]. The Choice RT test differs to that of the Simple RT test by the addition of a decision component i.e. a choice of what response to make depending on which of two targets are displayed. These tests have been used within ageing and MCI and AD research with significant slowing of information processing speed found in older adults compared to young adults [Bugg, Zook, Delosh et al, 2006; Krieg, Chrislip, Letz et al, 2001; Woods, Wyma, Yund et al, 2015] and disproportionately slower in MCI and AD compared to healthy ageing [Chen et al, 2017; Sano, Rosen, Stern et al, 2009; Tales \& Porter, 2008]. Comparing the two tests we observe outcome variability depending on which test is used which has been examined in ageing studies [Der \& Deary, 2006; Inui, 1997] and MCI and AD studies [Storandt \& Beaudreau, 2004; Nestor et al, 1991]. 
In this study the functional integrity of visual attention, information processing speed and IIV between young and older adults is examined in both Simple and Choice RT tests as well as measuring the influence of other factors of sex, education, perceived test difficulty and subjective memory function which have not been examined in detail previously in ageing studies using research tests of RT and IIV, namely Simple and Choice RT. In addition, a novel approach in the Choice RT test examines the potential effect of the number of trials on RT and IIV and error rate.

Overall, both the Simple and Choice RT tests reveal significant differences in information processing speed and IIV between young and older adults. Older adults are significantly slower and more variable compared to young adults. A greater difference in information processing speed between young and older adults (i.e. ageing) is observed in the Choice RT test due to larger effect sizes compared to the Simple RT test. When examining overall effects of different factors on information processing speed and IIV both tests find no significant effect of sex, education and subjective memory function in both young and older adults.

Finding no influence of these factors on information processing speed and IIV highlights the outcome variability can differ between visual attention tests since sex and education were found to be influential during the visual search test (Chapter 2). Perceived test difficulty reveals no influence on information processing speed in Simple RT test but significantly positively correlates with RT and IIV in young adults only in the Choice RT test. This also highlights outcome variability between simple and choice tests which may depend on the paradigm used and the level of complexity i.e. the added decision making function and processing multiple stimuli.

In the Choice RT test, information processing speed in young adults increases significantly i.e. got slower across trials speculated to reflects fatigue in repeating the same action multiple times in multiple trials rather than reflecting any cognitive impairment. In contrast, information processing speed in older adults decreases significantly across trials i.e. sped up speculated to relate to older adults benefitting from practice i.e. repeating the same action multiple times in multiple trials. IIV in young adults does not significantly differ across trials implying that their RT performance is consistent throughout the test. Older adults are more variable in their RT performance at the beginning of the test then remained consistently less varied until the end of the test again implying the benefit of practice. 
In relation to errors, in both young and older adults there are no significant change in accuracy throughout the test although overall older adults are more accurate (i.e. make significantly fewer errors) compared to young adults.

\section{Chapter 5: Multi-item localization (MILO) [research test]}

The MILO is an iPad based research test of eight billiard ball-type stimuli, each containing a number from 1 to 8 randomly distributed around the screen. The objective is to tap each ball in consecutive order (from number 1 to number 8). As each ball is tapped it disappears from the screen and once all 8 balls are tapped, they all appear again for the next trial in a different position around the screen.

MILO measures attentional shifting in a similar way to the visual search test but in addition it measures working memory for maintaining a target sequence and allows for the execution of direct responses (finger tap on an iPad). In addition, the MILO measures how quickly and accurately a sequence of stimuli can be processed [Horowitz \& Thornton, 2008]. The MILO is chosen for its similarity to attentional processing measured in Trails A yet in addition it contains multiple trials thus allowing for IIV to be measured which is a limitation of the TMT being a single trial. Furthermore multiple responses within a single trial allows different aspects of the test to be examined i.e. overall RT and IIV, hesitancy effects for beginning the test and the RT and IIV of a single response less affected by hesitancy effects. In depth explanations behind each aspect of the MILO measured is included in this chapter.

The MILO task has not been used extensively to measure information processing in ageing or to distinguish changes in $\mathrm{MCI}$ and $\mathrm{AD}$ compared to healthy ageing nor has this test been examined in detail in relation to the effects of subjective memory function as well as the inclusion of examining a variety of person-related factors i.e. sex, education and a psychological factor of perceived test difficulty.

In this study, information processing speed and IIV in relation to attentional function similar to the visual search and TMT is examined using the MILO to determine whether the use of a different medium (iPad compared to computer based visual search) and the use of multiple trials in comparison to TMT affects result outcome. In addition any influence of sex, education, perceived test difficulty and subjective memory function on RT and IIV is examined to determine whether the result outcome is similar to the other attention tests in the second study. 
Information processing speed is found to be significantly slower in older adults compared to young adults across conditions (RT1 and RT8- RT1) although older adults are only more varied when beginning the test (RT1). In older adults, subjective memory function (i.e. perceiving greater changes to memory function) is associated with slower information processing speed for the RT1 condition but does not have an effect on IIV. Education only influences overall RT performance (RT8 minus RT1) in older adults. Perceived test difficulty and sex has no influence on information processing speed and its variability in both young and older adults.

The results of the MILO, as found in the previous chapters, highlight the effects of person related factors may be dependent on the test or condition within the test, particularly subjective memory function which only has an effect on RT within the MILO and not in the visual search, Simple RT or Choice RT tests.

\section{Chapter 6: Comparing tests [effect sizes]}

This chapter highlights the differences in effect sizes from the relationships between RT, IIV and person-related factors between each test examined in both studies. This is to determine whether the effects of person related factors on RT or IIV were more robust (i.e. a large effect size) in some tests than others. In addition, the effect sizes of tests which produced significant differences of error rates between young and older adults are compared to determine which test may produce greater differences in accuracy in ageing.

Small effects sizes between young and older adult RT and IIV are observed in the Simple RT test and the TMT. In contrast, the tests with large effect sizes between young and older adult RT and IIV are the visual search test, the iPad Milo test and the Choice RT test implying the results are robust and differences in RT between young and older adults are more pronounced in these particular attention tests. This is an interesting finding particularly small effect sizes in the TMT as this test is used to measure information processing speed in memory clinics and diagnosis of MCI and dementia.

There is a dichotomy between the types of tests used within research studies and clinical settings [Haworth et al, 2016] thus finding larger effect sizes in the current research tests (i.e. visual search) may imply that research tests such as visual search should be used in clinical settings instead of the TMT. 
If tests such as the visual search find robust differences between young and older adult RT in research, if used in memory clinics they may also find robust differences of RT between healthy ageing and MCI or dementia.

Most effects of person-related factors are found to occur in the visual search with RT associated with sex, education and perceived test difficulty. Furthermore, a large effect size of the relationship between education and RT in the visual search implies the result was robust thus the effect of education should be considered when measuring RT or IIV using the visual search test. Only the Choice RT test (between trial blocks) and the MILO test reveal a significant difference in the mean number of errors made between young and older adults with effect sizes being larger in the MILO. This implies a large difference of accuracy between young and older possibly due to a speed/accuracy trade-off (see chapter for further discussion).

Noticing that even amongst the tests used in this thesis there are significant differences of information processing speed between young and older adults, could demonstrate that measuring information processing speed and the effects of different person-related factors during ageing is important but possibly test dependent thus not as simple as the DSM-5 suggests.

\section{Chapter 7: General discussion}

This chapter includes a very brief summary of results from the thesis in relation to the aims outlines in the thesis summary and introduction and what it might mean in relation to information processing speed and visual attention in ageing research and clinical practice. The significance of outliers is discussed as well as overall study limitations and future research. 


\section{Dissemination of results}

The following have been presented or published:

- Haworth, J., Phillips, M., Newson, M., Rogers, P. J., Torrens-Burton, A., \& Tales, A. (2016). Measuring information processing speed in mild cognitive impairment: Clinical versus research dichotomy. Journal of Alzheimer's Disease, 51(1), 263-275. doi: 10.3233/JAD-150791- examination of TMT and visual search

- $\quad$ Torrens-Burton, A., Basoudan, M., Bayer, A., et al (2017) Perception and reality of cognitive Information processing speed, perceived memory function, and perceived task difficulty in older adults. Journal of Alzhiemer's disease, 60(4), 1601-1609.doi: 10.3233/JAD-170599.- results from chapter two and three

- Findings of visual search chapter two presented as a poster in Vitae Poster competition, Swansea University

- Study aims and methodology of current research presented as oral presentation at 46th Annual Conference of the British Society of Gerontology, Swansea University

- Torrens-Burton, A., Bayer, A., \& Tales, A. Trial number in ageing studies of reaction time and intra-individual variability: Older, but not younger adults benefit from practice - in preparation 


\subsection{CHAPTER ONE: Introduction}

Information processing speed and the functional integrity of the brain (as measured through reaction time [RT]) has been applied to the study of ageing and the diagnosis and characterisation of dementias such as Alzheimer's disease (AD) and prodromal stages such as mild cognitive impairment (MCI), compared to cognitively healthy ageing [Bilello, Doshi, Nabvizadeh et al, 2015; Radanovic, Pereira, Stella et al, 2013; Verhaeghen \& Cerella, 2002; Foster, Behrmann \& Stuss, 1999]. During the diagnosis of MCI or $\mathrm{AD}$, the relatively new DSM-5 criteria for neurocognitive disorders (NCD) suggest the importance of measuring information processing speed in relation to attentional function as an indication of the integrity of brain structure and function. However as found by previous research, study outcome may vary, i.e. information processing speed may be influenced by a number of different factors including person-related factors such as sex and education and objectively measured cognitive function and methodological factors such as the type of test used [Tales, Bayer, Haworth et al, 2010; Haworth, Philips, Newson et al, 2016]. This makes measuring information processing speed less straightforward than anticipated as more factors may need to be taken into account than previously assumed.

Information processing speed and IIV can be compared between healthy ageing and conditions such as MCI and AD to observe whether levels are significantly different between healthy ageing and disease. This can help characterise MCI or dementia if information processing speed is disproportionately slower and more variable compared to what is expected during healthy ageing. However person-related factors i.e. sex and education may also be influential on information processing speed as well as psychological factors such as perceived test difficulty and also subjective memory function (subjective cognitive complaints in ostensibly healthy older adults). These factors have either been poorly addressed or not at all in previous ageing studies however is it important to observe how different factors may be affecting RT and IIV in healthy ageing (control groups) before comparing healthy ageing with pathological ageing i.e. MCI or dementia.

If these factors are influential on information processing speed this may make these older adults disproportionately slower than expected in healthy ageing. Failing to take this account may have a significant impact on how results are interpreted (i.e. possible incorrect interpretation of brain integrity in ageing). 
It is important that the data from older adult control groups are robust and related to the integrity of brain function rather than any extraneous variables.

The aim of the research presented in this thesis was to investigate how using different tests of attentional function in similar groups of young and older adults may affect the outcome measure of information processing speed and its variability (IIV). Within this aim is to determine whether the number of trials may influence performance thus outcome measure (RT, IIV) and accuracy (number of errors). Another aim is to determine whether result outcome in similar large groups is effected similarly across tests by a variety of person-related factors i.e. sex, education, objective cognition measure (MoCA), particularly previously un-tested factors of subjective memory function and perceived test difficulty which may help determine whether subjective feelings are associated with slower and more variable information processing speed.

A large study (Chapter 3 onwards) included a variety of different visual attention tests and using the same groups of young and older adults where possible to determine whether result outcome is dependent on the type of attention test used. However an initial study (Chapter 2) examined how factors of sex, education, subjective memory function and perceived test difficulty may influence RT and IIV using a typical research test of information processing speed and attention, namely visual search. All these factors are examined in more detail in this introduction and in the following chapters.

\subsection{Scientific background and context.}

\subsubsection{Brain structure and neural changes related to information processing speed in ageing}

As ageing advances, there is a general or 'natural' decrease in the speed (i.e. slowing) at which information can be processed [Birren, 1974; Birren, Riegel, \& Morrison, 1962; Salthouse, 1992]. It becomes more difficult for older adults to process information quickly which is observed by slower reaction times [RTs] (i.e., information processing speed) when performing behavioural tasks [Craik \& McDowd, 1987; Salthouse, 1992]. Furthermore, slowing may increase as the task increases in complexity. Tasks of greater complexity require larger amounts of information to be processed thus require a larger number of mental operations which produces a greater strain on mental resources such as attentional processing. 
Compared to young adults, older adults have less ability to deal with processing larger amounts of information from more complex tasks at one time so instead process smaller amounts of information consecutively [Kertchner et al, 2012; McDowd \& Craik 1988]. As a result, this produces a larger difference of processing speed between young and old adults i.e. older adults' information processing speed is slower [e.g. Papp et al, 2014; Kertchner et al, 2012; Deary et al, 2011; Bugg et al, 2006; Kirby \& Nettelbeck, 1991].

In cognitively normal ageing, death of neuronal cells results in brain volume decrease during the lifespan at a rate of $5 \%$ per decade after the age of 40 years [Svennerholm, Bostrom, \& Jungbjer, 1997], and this rate increasing over the age of 70 years [Scahill, Frost, Jenkins et al., 2003]. Grey matter volume also decreases [Raz, Gunning, Head et al., 1997] together with the density of synapses [Terry, 2000; Good, Johnsrude, Ashburner et al., 2001], although the most significant volume loss relates to white matter density [Chen, $\mathrm{Li}, \&$ Hindmarsh, 2001] which can reduce by up to $28 \%$ [Pakkenberg \& Gundersen, 1997, see also Madden , 2007].

An increasing body of research evidence indicates that ageing-related reduction of the integrity of white and gray matter, is significantly related not only to the decline in cognitive and other aspects of information processing (such as executive function), but also the slowing of such processing [Papp, Kaplan, Springate et al, 2014; Nilsson, Thomas, O'Brien, \& Gallagher, 2014; Kerchner, Racine, Hale et al, 2012; Yarkoni, Barch, Gray et al, 2009; Charlton, Barrick, McIntyre, et al., 2006; Sternberg, 1969; Rabbitt, 2015; de Groot, de Leeuw, Oudkerk et al., 2000; Guttmann, Jolesz, Kikinis et al., 1998; Mella, de Ribaupierre, Eagleson \& de Ribaupierre, 2013; Salami, Eriksson, Nilsson, \& Nyberg, 2012; Rabbitt, Scott, Lunn et al., 2007, Kubicki, Niznikiewicz, Connor et al, 2009; Charlton, Barrick, McIntyre et al, 2006; , Madden, Spaniol et al, 2008]. There is also substantial evidence to indicate that as white matter changes occur in areas of the brain which support different cognitive functions [Kubicki et al, 2009], disruption to, and slowing of, numerous and very specific aspects of information processing can be observed. For example, Tuch and colleagues [2005] found that poor integrity of visual tracts, such as the right posterior thalamus and the right optic radiation, was correlated with the slower processing speed of visuospatial functioning. It is important to know what happens to information processing speed primarily in healthy ageing as it included as an important measure in the DSM -5 for NCD i.e. comparing MCI or dementia to healthy controls. 


\subsubsection{Brain structure and neural changes related to information processing speed in Alzheimer's disease.}

The term dementia describes a variety of symptoms including, but not restricted to, loss of memory and difficulties with language, thinking and problem solving [Alzheimer's Society, 2017]. Dementia develops as a result of disease, the most common being Alzheimer's disease (AD) [Alzheimer's Association, 2017; Ferri, Pronce, Brayne et al, 2005]. AD is a neurodegenerative disease arising from, or associated with the presence of amyloid- $\beta$ plaques and neurofibrillary tangles in the brain [Hardy \& Selkoe, 2002; Delacourte, David, Sergeant et al, 1999]. These abnormal proteins result in the loss of connections in the brain and eventually the loss of brain tissue and the death of nerve cells and abnormal function.

The loss of connections in AD is eventually widespread, affecting several structural and functional regions such as the parietal, frontal and temporal lobes [Feller, 2016; Martin, 2015; Pritchard, 2011; Arnold, Hyman, Flory et al, 1991] resulting in abnormal function and slowing in areas such as cognition, attention, language, memory [Peters, 2006; Hyman, Van Hoesen, Kromer \& Damasio, 1986], perception [Rizzo \& Nawrot, 1998; Gilmore, Wenk, Naylor \& Koss, 1994] and problem solving [Willis, Allen-Burge, Dolan et al, 1998; Morris, 2005] thus resulting in detrimental effects upon the ability to carry out daily activities.

As $\mathrm{AD}$ is associated with an even greater degree of white matter deterioration than that related to cognitively normal ageing [Bartzokis, Cummings, Sultzer et al, 2003] i.e. white matter hyperintensities [Brickman, Zahodne, Guzman et al, 2015; Yoshita, Fletcher, Harvey et al, 2006; de Leeuw, Korf, Barkhof et al, 2006; Capizzano, Acion, Bekinschtein et al, 2004; Paus, Collins, Evans et al, 2001], one would therefore expect to observe a disproportionate slowing of information processing speed, in AD across a great many aspects of brain function. Indeed early research revealed that white matter lesions found in AD [Burns, Church, Johnson et al, 2005; Barber, Scheltens, Gholkar et al, 1999; O’Brien, Desmond, Ames et al, 1996] are associated with slower information processing speed [Burns et al, 2005; Amar, Bucks, Lewis et al, 1996; Skoog, Berg, Johansson et al, 1996]. In addition, information processing speed has been found to be significantly slower and more variable in relation to visual attention-related processing in AD compared to healthy ageing [Verhaeghen \& Cerella, 2002; Foster et al, 1999]. 
The substantial evidence for a relationship between the slowing of information processing speed particularly in relation to attention-related processing and $\mathrm{AD}$, has led to the inclusion of slowed information processing speed, specifically in relation to attention-related function, as a component in diagnostic criteria (particularly in the newer DSM-5 criteria).

As a result of the relationship between information processing speed and AD, information processing speed is increasingly examined in prodromal stages of dementia such as mild cognitive impairment (MCI) in order to determine whether it can predict the development of dementia thus the diagnostic aspect of information processing speed is clear. What is perhaps less obvious however, is how slowing of information processing speed in ageing and AD can also significantly negatively influence everyday activities, behaviour and well being. The importance of accurately assessing and interpreting information processing speed integrity is not just related therefore to a diagnostic context.

\subsubsection{Brain structure and neural changes related to information processing speed in Prodromal Stages of Alzheimer's disease: Mild Cognitive Impairment (MCI)}

MCI is a condition where an individual reports a complaint with their memory which, when measured objectively, is impaired compared to what is expected for someone of their age. General cognition is relatively preserved and there are only minor issues of performing daily activities. The final criterion is that individuals are not classed as demented when assessed from examining the previous four criteria [see Petersen, 2004; Petersen, Smith, Waring et al, 1999].

For some people, MCI represents a prodromal stage of Alzheimer's disease and indeed there is some evidence that at least in some people with MCI, white matter integrity and function is compromised [Zhang, Schuff, Camacho et al, 2013; Bilello et al, 2015; Radanovic et al, 2013; Olsson, Klasson, Berge et al, 2013, Bombois, Debette, Delbeuck et al, 2007; Yoshita et al, 2006], in the frontal, parietal, occipital, and temporal lobes [Defrancesco, Egger, Marksteiner et al, 2014; Huang \& Auchus, 2007; Babiloni, Frisoni, Steriade et al, 2006]. As in AD, one might therefore expect to find significant slowing in MCI (at least for some people) in various aspects of brain function especially those associated visual attention-related processing, compared to cognitively healthy ageing. 
Indeed it has been demonstrated that behavioural information processing speed can disproportionately slow in MCI [Ballesteros, Mayas \& Reales, 2013; Gorus, de Raedt, Lambert et al, 2008; Nestor, Parasuraman \& Haxby 1991] with some evidence of greater slowing in MCI cases which develop into dementia [Tales, Leonards, Bompas et al, 2012], although there is a relative lack of research in this area to allow comparison of results and the determination of any robust effects. Regardless of MCI heterogeneity it is important for research to observe how it differs to healthy ageing i.e. in relation to information processing speed to help better MCI and clinically, help distinguish between individuals ageing healthily and pathological ageing. Therefore in order to achieve this, RT needs to be examined firstly in healthy ageing to understand how information processing speed is affected thus understand when levels of slowing become abnormal.

In a series of studies by Tales and colleagues [Tales, Snowden, Phillips et al, 2011; Tales, Bayer, Haworth et al, 2010; Tales, Haworth, Nelson et al, 2005] and other research groups [Binetti, Cappa, Magni et al, 1998; 1996] either slowing of RT has been found in MCI or slowing of MCI has been found to be similar to levels expected in healthy ageing. This outcome variability may relate to differences in methodology i.e. sample sizes or differences in the type of test used. Few studies have shown significant group mean differences in information processing speed between cognitively healthy older adults and individuals with MCI [Petersen, 2004; Petersen et al, 2001]. Arguably, a reason for such variability of outcome is that MCI is aetiologically heterogeneous (i.e., having many other causes than neurodegenerative disease, such as lack of sleep, anxiety or depression) and it may only be those who have prodromal AD that exhibit slowed information processing speed and thus the proportion of such people in anyone research group would affect study outcome. What is also apparent from some other studies [e.g. Haworth et al, 2016; Phillips et al, 2013] that have published box plots of the results, is that any given MCI group can contain some people who have a slowing or greater variability of information processing speed far greater than other group members and which resembles more closely the performance of those people living with $\mathrm{AD}$.

Incidentally, this may also be found in ageing studies [e.g. Torrens-Burton et al. 2017; Haworth et al, 2016] that some older adults perform disproportionately slower than expected in ostensibly healthy ageing and may resemble more closely performance of individuals with MCI or MCI as a precursor to dementia. 
Arguably as slowed information processing is a characteristic of AD; it is possible that the individuals with MCI who show significant slowing may be at the greatest risk of developing $\mathrm{AD}$ and there is some evidence to indicate that this is the case [Tales et al, 2010]. As a result of such evidence, the measurement of information processing speed has been included in the clinical diagnosis and follow-up of MCI and AD and the relatively new DSM-5 document states that measuring processing speed is valuable for a dementia diagnosis, especially when measured in relation to attention.

What remains uncertain and not addressed by the DSM-5 is whether individual differences in factors such as sex, educational level, subjective memory complaints and perceived test difficulty, influence the results, and particularly can account for disproportionate slowing (i.e., outliers), i.e., are people who have outlying performance more likely to have early dementia or a neurodegenerative or disease basis for their slowing or is it related instead to these other factors? Evidence from previously published box plots also reveals that irrespective of cause, some people with MCI and some ostensibly cognitively healthy older adults show a greater than expected level of slowing (i.e. they are outliers) and thus potential for a poorer life quality.

\subsubsection{Brain structure and neural changes related to intraindividual variability (IIV) in ageing}

It is relatively rare for RT and ageing studies to routinely include a measure of intraindividual variability and it is relatively rare for IIV to be used clinically and it does not figure in the DSM-5 for neuro-cognitive disorders. Therefore a novel aspect of this PhD was to examine both RT and IIV in ageing.

Intraindividual variability (IIV) of information processing speed reflects the variation of behavioural responses within a single persons' overall performance thus the functional integrity the central nervous system (CNS). As is the case for information processing speed, IIV is associated with white matter integrity [Walhovd \& Fjell, 2007; Jackson, Balota, Duchek et al, 2012] more so than grey matter integrity [Moy, Millet, Haller et al, 2011; Walhovd \& Fjell, 2007] and associated with frontal lobe white matter hyperintensities in healthy ageing [Bunce, Anstey, Christensen et al, 2007]. 
As a result, an increase in IIV has been observed to be associated with impaired cognition [MacDonald et al, 2008; 2006] such switching attention and working memory [Head, Jackson, Balota et al, 2011] as well as the processing speed of such functions [Mazerolle, Wojtowicz, Omisade et al, 2013; Garrett, MacDonald \& Craik, 2012].

In ageing studies, older adults have been found to have more varied processing speed compared to young adults [Mella et al, 2013; Dykiert et al, 2012; Fozard et al, 1994; Inui, 1997] despite previous opposition [Waugh et al, 1973] thus implying IIV is effected by age [MacDonald, Nyberg, \& Backman, 2006; Hultsch, MacDonald, \& Dixon, 2002]. In addition some there is some evidence for IIV being more sensitive to ageing and dementia compared to the effects of information processing speed [Phillips et al, 2013; Jackson et al, 2012; Kelly et al, 2008].

As with information processing speed, IIV may be influenced by a variety of factors which has not been fully investigated previously. Thus alongside information processing speed, the functional integrity of intraindividual variability of processing speed will be investigated in this thesis, related to attention-related processing in both young and older adults and with respect to subjective memory function, education, sex and perceived test difficulty. In order to measure IIV, some attention tests (used in research or in clinical practice) using multiple trials (i.e. visual search and MILO) are included in order to efficiently highlight individual differences in information processing speed variability.

\subsubsection{Brain structure and neural changes related to intraindividual variability (IIV) in MCI and AD}

As mentioned for ageing studies it is relatively rare for RT and aMCI and AD studies to routinely include a measure of intraindividual variability despite some recognition that an increase in IIV is related to neurological disorders [e.g. Jackson et al, 2012; Anstey et al, 2007; MacDonald et al, 2006].

In healthy ageing IIV has been found to relate to white matter integrity [Walhovd \& Fjell, 2007; Jackson et al, 2012] and associated with frontal lobe white matter hyperintensities [Bunce, et al, 2007] which has also been found in MCI and AD along with white matter atrophy in temporal and parietal areas [Huang \& Achus, 2007]. 
The integrity of IIV has also been associated with atophy of the corpus callosum in MCI [Wang et al, 2005; Teipel et al, 2002; Hensel et al, 2002] and in AD [Gootjes et al, 2006; Thomann et al, 2006; Wang et al, 2005]. Therefore as with healthy ageing, there is some evidence that pro-dromal AD at the MCI stage is associated with increased IIV [Philips et al, 2013; Kälin, Pfluger, Gietl et al, 2014; Wang et al, 2005; Teipel et al, 2002; Hensel et al, 2002] as well as in early AD [Jackson et al, 2012. In addition, an increase in IIV may predict a decline from aMCI to AD within 2.5 years [Tales et al, 2012].

\subsubsection{Subjective Cognitive Impairment (SCI) and subjective memory function}

The potential influence of MCI upon information processing speed is acknowledged in both research and clinical arenas of ageing research. When information processing speed is measured in 'normal' ageing, individuals with MCI are excluded i.e. MCI is an exclusion criterion for older adult control groups when young and older adults are investigated. In addition, even in the absence of clinical diagnosis, older adults recruited for ageing and dementia-related studies are required to show objectively measured normality in general cognitive function (e.g. as measured by the MoCA [Nasreddine, Phillips, Bedirian et al, 2005]). However, it is now clear that there is another condition called Subjective Cognitive Impairment (SCI), which although aetiologically heterogeneous, can for some people represent an earlier prodromal stage of Alzheimer's disease than MCI. Therefore SCI may also be characterised (for some people) by disproportionately slower and more varied information processing speed compared to healthy ageing. Some older adults recruited for healthy control groups may exhibit SCI but has not been considered in previous RT studies examining MCI or dementia.

SCI is a condition where older adults report problems with their cognition yet when measured objectively i.e. in memory clinics, there is an absence of any verified cognitive decline [Garcia-Ptacek,Cavallin, Kareholt et al, 2014; Desai \& Schwartz, 2011; Reisberg, Shulman, Torossian et al, 2010; Reisberg, Prichep, Mosconi et al, 2008]. Despite an absence of objectively verified cognitive impairment, these subjective cognitive complaints have been associated with experiencing poorer verbal memory [Schaafsma, Homewood and Taylor, 2010], impaired executive function [Genziani, Stewart, Béjot et al, 2013] and a general slowing of information processing speed [Mol, van Boxtel, Willems et al, 2006]. 
SCI represents individuals who have been to their GP about their memory concerns i.e. it is a clinical diagnosis. However in many research studies, ostensibly older adults used in control groups may report subjective memory problems but have not visited their GP (namely subjective memory function) and these subjective problems may also act as confounds on task performance i.e. detrimentally affecting RT and IIV. Prevalence of individuals in the general population experiencing concern with their cognitive ability is high [Desai \& Scwartz, 2011; Cooper, Bebbington, Lindesay et al., 2011; Lautenschlager, Flicker, Vasikaran et al, 2005] with figures in the older adult population reaching as high as $88 \%$ [Smith, Peterson, Ivnik et al, 1996]. Despite this high prevalence, possible subjective memory impairment (clinically diagnosed or reported by people in the general population not visiting their GP) remains relatively unexplored and understood particularly the relationship between subjective feelings and a wide range of other brain functions i.e. attention and information processing speed [see Tales, Jessen, Butler et al, 2015] and the potential impact this may have upon daily life.

One issue with fully understanding SCI (thus subjective memory function) is that it is aetiologically heterogeneous [Cheng, Chen \& Chiu, 2017] and cause cannot be established in some cases [Gifford, Liu, Damon et al, 2015; Silva, Guerreiro, Faria et al, 2014; Reid \& Maclullich, 2006]. SCI and its influences on cognitive performance i.e. memory, may be associated with other conditions such as anxiety and depression [Caselli, Locke, Dueck et al, 2014; Montejo, Montenegro, Fernandez, \& Maestu, 2011] or with higher social class and worse physical health [Begum, Morgan, Chiu et al, 2012]. In these cases cognitive impairment may be reversed [Balash, Mordechovich, Shabtai et al, 2013; Wehling, Lundervold, Standnes et al, 2007] as these conditions can be easily treated [Tales et al, 2015].

Alternatively, SCI may relate to underlying pathological causes e.g. Alzheimer's disease or other forms of dementia and represent a greater risk of developing MCI and or AD [Cheng et al, 2017; Jacinto, Brucki, Porto et al, 2014; Jessen, Wolfsgruber, Wiese et al, 2014; Mitchell, Beaumont, Ferguson et al, 2014; Steinberg et al., 2013; Scheef et al., 2012; Jessen, Wisse et al, 2010; Reisberg et al., 2010; Glodzik-Sobanska, Reisberg, De Santi et al, 2007; Geerlings, Jonker, Bouter et al, 1999] and studies have found evidence that SCI can show similar patterns of impairment to brain function as found in AD [Caselli, Chen, Locke et al, 2014; Rodda, Dannhauser, Cutinha, et al, 2011; Johannsen, Jakobsen, Bruhn et al, 1999]. 
Such possible effects mean that if older adults in ostensibly healthy control groups have a variation of SCI, this may have an effect on cognitive function i.e. disproportionately slower information processing speed. However it is unclear whether this may also occur in individuals who report subjective issues with their memory but have not visited a GP, namely subjective memory function. Thus for this reason the studies in this thesis examined subjective memory function and its effects on information processing speed and IIV in older adults in the general population. It is clear that the majority, if not all, of previous ageing studies comparing young and older adults and also studies comparing healthy ageing to and MCI or AD have not tested their older adult control group for subjective feelings of memory (whether SCI or subjective memory function). If such either such subjective feelings in these 'control' participants are associated with slowed information processing speed but not taken into account, this could affect study outcome i.e. bias results and impact how ageing is characterised in research studies, and in clinical settings impact how the differences between healthy ageing and MCI or dementia are interpreted.

Currently for a SCI diagnosis, memory clinics conduct the same neuropsychological tests used to measure and diagnose MCI and AD. This includes the Mini mental state exam (MMSE) measuring for overall cognition and used for dementia diagnosis. However, there is a scarcity of research examining SCI and information processing speed, particularly in relation to visual attention which is stated to be important to measure in relation to information processing speed (DSM-5 criteria). Tests of attention are not measured in great detail during a diagnosis in a clinical setting and only information processing speed is measured (and not IIV) using the Trail Making Test if at all. This is definitely the case if individuals are not referred to a specialist memory clinic.

In addition it is also unclear whether similar effects on information processing speed are found for subjective memory function as with SCI. Understanding how information processing speed and IIV is associated with subjective memory function in different cognitive functions such as visual attention (and how it may or may not differ between tests) may incidentally help to further characterise clinically diagnosed SCI if indeed the two are related. Past research has found mixed findings for whether there is a relationship between subjective memory and objective cognitive performance [Reed, 2010]. 
Self rated measures have been associated with cognitive performance in healthy adults thus implying that subjective measures are valid tools for predicting cognitive decline [Reed, 2010; Cook \& Marsiske, 2005; Earles \& Salthouse, 1995]. A relationship has been found between subjective complaints and a diagnosis of baseline cognitive impairment [Schofield, Marder, Dooneief et al., 1997] or dementia [Grut, Jorm, Fratiglioni et al., 1993]. In contrast, other studies have found no relationship between subjective memory complaints and cognitive performance thus related any impairment down to other factors such as depression [Barker, Carter, \& Jones, 1994].

It is also argued that no relationship is found between dementia and subjective memory complaints due to anosognosia; the patient having the inability to judge their own cognition due to denial or unawareness that anything is wrong [Ansell \& Bucks, 2006; Kalbe, Salmon, Perani et al, 2005; Sevush \& Leve, 1993]. Therefore, of particular interest in this thesis was to examine information processing speed and IIV in relation to subjective memory function i.e. individuals reporting subjective changes to memory function who have not been to the GP thus do not have a formal/clinical SCI diagnosis (as measured by the Memory Functioning Questionnaire (MFQ) [Gilewski, Zelinski \& Schaie et al, 1990]). We aimed to determine whether RT and IIV performance is associated with perceiving changes to memory function and whether this is dependent on the type of attention test used.

\subsubsection{DSM-5 criteria}

The diagnostic criteria within the DSM-5 classes MCI and AD under the categories of mild to major Neurocognitive Disorder (NCD); a preferable, broader term to incorporate conditions which effect younger individuals i.e. brain injury or HIV and individuals with similar substantial decline in specific cognitive domains but may relate to a different condition i.e. amnestic disorder [American Psychiatric Association, 2013].Within these two categories of NCD, a number of symptoms need to be present for a diagnosis which incorporates different cognitive domains such as impairments with memory, language and social cognition. 
Specifically in relation to information processing speed and attention (our focus within this thesis) initial symptoms delirium are acknowledged which is categorized as having a disturbance in attention (such as shifting or sustaining attention) and awareness (i.e. orientation) which has developed over a short time period and alongside an additional cognitive disturbance (i.e. memory deficit). Individuals experiencing these symptoms of delirium are further diagnosed with symptoms within the domain of 'complex attention'. For mild NCD (MCI) these symptoms include finding errors in routine tasks, taking longer than usual to complete tasks and required double-checking of work more often. For major NCD (i.e.AD), attentional symptoms include being easily distracted by competing events, inability to attend unless input is restricted or simplified and having difficulty in retaining new information.

The older adult samples included within this thesis, are ostensibly healthy older adults who have not visited a GP with any cognitive complaints similar to the ones described above; not even delirium symptoms. Therefore they do not fit the criteria for mild or major NCD as routine performance is relatively ok i.e. no significant issues or errors made when performing routine tasks. These individuals would be potentially classed in clinical terms as cognitively healthy and used as control groups to compare healthy ageing with pathological ageing (i.e. MCI or AD). Although as stated through the thesis, there may be individuals who have not seen their GP but may be beginning to experience issues with their cognition (subjective memory function) which may already be having a negative influence on processing speed and daily functioning. These individuals may later be categorised as having SCI once visiting their GP. The DSM-5 does not state SCI directly but as mentioned above, individuals with SCI would begin to experience such as the types of problems described for mild NCD i.e. noticing more errors when performing tasks but not to the extent required for a diagnosis within the DSM-5 criteria (i.e. still performing normally during objective testing of cognitive domains). Individuals with subjective memory function or SCI within an ostensibly healthy older adults control group may subsequently become outliers thus skew the results leading to a misleading characterisation of what is considered to be healthy ageing. 


\subsection{Selective Attention and information processing speed}

There are an abundance of studies examining the functional integrity (i.e. in relation to information processing speed) of many different aspects of visual attention i.e. divided attention, inhibition of return, phasic alerting and associated executive function [Sventina, 2016; Fraser \& Bherer, 2013; Pratt \& Chasteen, 2007; McAuliffe et al, 2006; Langley et al, 2001; Finke et al, 2012; Tales et al, 2011; Festa-Martino et al, 2004; Tales et al, 2002; Plotek et al, 2014; Tales et al, 2010; Traykov et al, 2007; Yuspeh et al, 2002]. Therefore the importance of measuring information processing speed with respect to visual attention is increasingly emphasised [Gordon, Zacks, Blazey et al., 2015] i.e. as mentioned in the DSM-5 criteria [Sachs-Reicsson \& Blazer, 2015].

In addition, many studies indicate that attentional function and information processing speed in attention can be affected in ageing [Sofko, Boettcher, Hoadley, et al, 2014; Potter, Grealy, Elliott et al, 2012; Colcombe, Kramer, Erickson et al, 2005; Verhaeghen \& Cerella, 2002; Brink \& McDowd, 1999; Faust \& Balota, 1997] as well as in MCI and AD compared to cognitively healthy ageing [Gordon et al, 2015; Bayer, Philips, Porter et al, 2014; Sinai, Phillips, Chertkow \& Kabani, 2010; McGuinness, Barrett, Craig, et al, 2010; Tales et al, 2010; Tales et al, 2005; Tales Muir, Bayer \& Snowden, 2002; Binetti et al, 1998; Nebes \& Brady, 1993; Pate, Margolin, Friedrich, \& Bentley, 1994; Grady, Haxby, Horwitz et al, 1993; Nestor et al, 1991].

Previous research also indicates that irrespective of cognitive impairment, information processing speed can vary in the same group of people and even within a single attentional function depending on the methodology i.e. type of test used [Torrens-Burton, Basoudan, Bayer et al, 2017; Haworth et al, 2016; Tales et al, 2010; Fernandez-Duque \& Black 2006; Hartley, 1993] and in relation to the specific aspect(s) of brain function, i.e., attention, that is being measured. The DSM-5 criteria fail to take into account the potential outcome variability which would make it difficult to obtain a true understanding as to the level of slowing expected during ageing and thus recognise when levels are disproportionately slower implying pathological change related to dementia i.e. AD. 


\subsubsection{Selective attention}

Although there are, in research terms, many different aspects and definitions of visual attention (which can be affected in aging i.e. slower information processing speed), one of its most important functions is that of selective attention [Gordon et al, 2015]. Due to limited resources, the brain is unable to process all the information within the visual environment to a high, behaviour-influencing, level. Only information at the centre of our attentional field (attentional focus) can be processed to a high level while information outside of this focus cannot be processed to such a high level or at all. Information falling outside attentional focus within our visual environment can capture the focus of our attention or we can voluntarily shift the focus of attention to it.

The focus of our attention can be automatically captured by salient information (pop out) or controlled to different non-salient items i.e. attending to distractors in turn until the target is found. Aspects of selective attention and information processing speed, and its effects in ageing, MCI and dementia i.e. AD are discussed in more detail in the chapters (see particularly visual search in Chapter 2, pages 51-60). Evidence has found information processing speed relating particularly to selective attention to slow in ageing [Owsley, 2011; Madden, 2007; Zeef, Sonke, Kok et al, 1996; Zeef \& Kok, 1993; Nissen \& Corkin, 1985; Cohn, Dustman \& Bradford, 1984; Eriksen \& Eriksen, 1974]. Therefore in the following studies in this thesis information processing speed and IIV will be examined in relation to selective attention.

\subsection{Participant-related factors in information processing speed research}

The sections above highlighted various areas of attentional function which may become impaired during ageing, MCI or AD. However it has been highlighted that outcome variability exists and that this may be due to methodological factors such as the type of test used. Of course these factors may not act in isolation and a significant body of evidence, examined in the sections below, indicates that several, personrelated factors i.e. sex and education or unknown psychological factors i.e. perceived test difficulty may also influence whether information processing speed is slowed or not in healthy ageing either independently or in association with methodological factors. 
This $\mathrm{PhD}$ aimed to determine whether information processing speed and IIV are associated with these different person-related factors in ageing and whether performance outcome depends on the type of test used.

\subsubsection{Sex and education}

Information processing speed in visual attention can be influenced by sex [Dykiert, Der, Starr \& Deary, 2012; Karia, Ghuntla, Mehta, Gokhale, \& Shah, 2012; Fozard, Vercruyssen, Reynolds et al, 1994] and education [Hamdan \& Hamdan, 2009; Tombaugh, 2004; Deary, Der, \& Ford, 2001; Houx \& Jolles, 1993]. Males have been found to perform some tasks significantly faster compared to males as found in young adults [Karia et al, 2012; Blough and Slavin 1987] and throughout ageing [Murphy, DeCarli, McIntosh et al., 1996]. A notable sex difference has also been found in the intraindividual variability of information processing speed i.e. variation of behavioural responses within a single persons' overall performance.

Females have been found to be more varied in their responses compared to men, particularly at an older age [Reimers \& Maylor, 2006; Deary \& Der, 2005] although sex variability can depend on the length of the task i.e. women begin the task slowly then speed up [Reimers \& Maylor, 2006].

The level or years of education can have an effect on performance level on neuropsychological tests, with low levels of education recognized to have a negative association of cognition in general [Ardila, Ostrosky-Solis, Rosselli, \& Gómez, 2000], predict cognitive decline [Christensen, Korten, Jorm et al., 1997] or be an effective moderator of cognitive ability in older adulthood [Kramer, Bherer et al, 2004] although this has not always been supported for information processing speed [Christensen et al, 1997]. Bornstein \& Suga [1988] suggested that education itself relates to the onset of cognitive decline during ageing rather than the amount of decline. It also needs to be established whether education has an effect on cognitive processing alongside other factors for example occupation and socioeconomic status [Kramer et al, 2004].

It has been argued that both sex and education are not measured in enough detail during studies measuring information processing speed in visual attention during ageing [Haworth et al, 2016; Phillips, Rogers, Haworth et al, 2013; Cangoz, Karakoc \& Selekler, 2009]. 
Therefore the current research will measure the influence of sex and education on information processing speed in each visual attention-related information processing speed test to observe whether influences are equal (or indeed what the influences may be) in all visual attention tests. This helps to determine how older adults used as typical controls during AD and MCI studies (as well as the patients with MCI or AD) and in addition individuals with subjective memory complaints, are affected by changes to processing speed in visual attention or other factors such as education and sex. Within each chapter sex and education are examined in greater detail according to the attentional test being examined.

\subsubsection{Anxiety and Depression}

Cognitive impairment and information processing speed can be influenced by other psychological disorders for example anxiety [Frick, Engman, Alaie et al, 2014; Berggren \& Derakshan, 2013; Sadeh \& Bredemeier, 2011; Coombes, Higgins, Gamble et al, 2009; Hainaut \& Bolmont, 2006; Clarnette, Almeida, Forstl, et al, 2001] or depression [Singh-Manoux, Dugravot, et al., 2014; Steinberg et al, 2013; Mascherek, Zimprich, et al, 2011; Rapp, Schnaider-Beeri, Grossman et al, 2006].

In terms of SCI, noticing significant slowing of processing has been argued to be a risk factor of psychological distress occurring [Gale, Harris, \& Deary, 2016]. Some have argued therefore, that SCI or subjective memory complaints are more likely to be related to anxiety and depression rather than cognitive impairment [Yates, Clare, \& Woods, 2015; Silva, Silva, Falcao et al., 2014]. This may also be the case for subjective memory function as older adults in the general community perceiving negative changes to their memory function and despite not visiting their GP for different reasons, may experience significant distress as a result.

These factors may have in fact confounded previous studies measuring information processing speed during healthy or pathological ageing but have failed to be included [Tales \& Basoudan, 2016] particularly in association to subjective memory function Therefore it is important to note that current research measuring subjective memory function ensured that people tested were within the normal range thus reducing any effects of anxiety and depression to a minimum (discussed in more detail for each test in Chapters 2 to 5). 


\subsubsection{Perceived test difficulty}

Perceived test difficulty is an example of psychological self-assessment where individuals hold beliefs about themselves i.e. their own abilities or beliefs about the demands of a task which can, as a result, have an effect on the outcome of a cognitive activity i.e. slower information processing speed [Flavell, 1979]. There are many examples self-assessment and individual may hold however in this thesis it was examined whether perceived task difficulty is related to actual (objectively measured) speed of information processing. Believing a task to be difficult to perform may give a person the propensity to think they performed badly despite their actual performance being as expected in healthy ageing and similar to other people. This may have an effect on their information processing speed for example the more difficult the task is perceived to be, the slower it is performed. If information is found to be associated with this aspect of psychological self-assessment it may be more likely that effects (i.e. slowed RT) could be responsive to intervention and possibly reversed.

The level of difficulty perceived may depend on the test being performed and this may change depending on the individual (i.e. highlighting individual differences). Perception of difficulty may not reflect the designed level of difficulty of the task i.e. a test designed to be simple can be considered difficult or a complex task considered easy to complete. This may depend on other factors such as the level of education and individual has (i.e. level of skill). In addition self -perception of test difficulty may depend on perception of subjective memory function (i.e. if memory is perceived to be poor individuals may perceive the test to be more difficult as a result).Therefore the current thesis also aimed to examine whether there is any relationship between reported memory performance, education levels (in years) and the perception of task difficulty i.e., whether high levels of perceived memory dysfunction or lower levels of education are associated with greater perception of task difficulty [see Torrens-Burton et al, 2017 for similar analysis and discussion].

Some studies have found that psychological factors of self-assessment in general can negatively affect cognitive performance [Rounis, Maniscalco, Rothwell et al, 2010; Yokoyama, Miura, Watanabe, 2010; Bolmont, Thullier \& Abraini et al, 2000]. Perceiving a test to be difficult can negatively affect mood which in turn can have a negative impact on information processing speed [Bolmont et al, 2000]. 
Nonetheless, this factor of self-perception can differ between individuals and has not been taken into account during many previous studies of information processing speed in particular in relation to different visual attention tests in ageing and MCI or dementia studies.

If psychological factors have an influence on information processing speed but is not taken into account, this may have significant implications on the integrity of the results i.e. of control groups and impact how past ageing and dementia studies have been interpreted. The influence of this novel factor of self-assessment on information processing speed in relation to visual attention will be examined alongside other factors of subjective memory function sex and education. Firstly it will examined whether perceived test difficulty is related information processing speed of a commonly used test (visual search in an initial study, see Chapter Two) but also whether its influence on outcome is similar across a number of visual attention tests in a group of the same individuals (second larger study, see Chapter Three to Five).

\subsubsection{Number of trials}

In RT research it is common to measure mean RT performance and IIV by using multiple trials (e.g. psychophysics techniques) during the test. However it is rare for RT studies to examine how the number of trials per se may have an effect on RT performance and IIV as well as how the number of trials interacts with participantrelated factors such as sex (males/females). Multiple trials have been separated into blocks e.g. during choice a RT test [Ballesteros et al, 2013; Brown et al, 2005; Michaels, 1988] however this was part of the test design and not specifically for measuring RT and IIV over time thus we aimed to examine potential influence of trial number on RT and IIV.

A high number of trials in a single study can invoke fatigue [Woods et al 2015], reduce sustained attention particularly towards the end of the test period [Fernaeus et al 2013] or reduce the level of alerting required with sustained performance [Voelker, Rothbart and Posner 2016] and as a result RT performance can become slower throughout the test especially towards the end. In contrast a high number of trials may, to some degree, improve RT performance and reduce age-related decline in cognitive function due to repeated practice from performing multiple trials [Yotsumoto et al 2015; Siettos and Smyrnis 2017]. 
This may have an effect on how information processing speed is examined in research or in clinics i.e. how many trials are included in RT tests if performance or age related decline may be dependent on trial numbers.

In the Choice RT test (Chapter 4), information processing speed was examined across four blocks modelled on the test methodology of Ballesteros and colleagues [2013] in order to observe whether the number of trials related to participants slowing and/or becoming more varied throughout the test (possibly due to fatigue) or whether it related to participants speeding up and/or becoming less varied (possibly due to practice effects). The effect of the number of trials is analysed and discussed in more detail in Choice test (see Chapter 4). The effect of trial numbers could have been examined during the other attention tests in this $\mathrm{PhD}$ which used multiple trials (Visual search, Simple reaction time test and the Multi item localization test) however the way in which the tests were programmed made this examination difficult.

In addition, during the Simple RT test and the MILO trials which were performed incorrectly (i.e. pressing the button too soon or pressing the wrong ball in the sequence) were programmed to be repeated thus making it difficult to examine trial numbers and possible effects on RT and IIV. Future studies using the same tests would benefit from changing the configuration of the test design to make this analysis possible.

\subsubsection{Errors}

Errors can occur while measuring reaction time (information processing speed) and refer to making the incorrect response during the test, such as pressing the wrong button on a keyboard corresponding to the required response. Not all RT and ageing studies include error rates within their RT analysis which makes it difficult to make comparisons between studies using a similar or different test of a particular cognitive function or between studies measuring different cognitive functions. In addition, studies failing to include error rates may be missing important analysis i.e. what the number of errors represents. For example, some errors made during RT tests can be accidental due to a lack of concentration or fatigue which results in an individual not paying attention to which response they should be giving. 
Errors of this nature do not necessarily relate to significant cognitive impairment an individual may have but more likely can be rectified by changing aspects of the test or the environment i.e. time of day testing or breaks between blocks of trials (although this presents its own issues as discussed above).

Error rates may highlight that the test cannot be completed accurately enough, or even completed at all by some people accurately enough due to a fault in the test design which may suggest it is not a sufficient test of RT. Other factors may also be associated with error rates such as the level of education (higher educated individuals may have more skills thus make less errors) or, as examined in the current thesis, the effect of sex i.e. whether males or females make more mistakes. In contrast the number of errors made may be highlighting significant cognitive dysfunction relating to especially in older adults (it is more likely young adults' cognitive function is at 'normal' levels). If this is the case, it may be important to include error rates when examining RT performance as it helps identify which individuals whose performance may not be reflecting healthy ageing. The effect and implications of errors are discussed in more detail in relation to each attention test in the corresponding Chapters 2 to 5 .

Errors can also relate to a speed/accuracy trade off [van Veen, Krug, \& Carter, 2008; Salthouse, 1979] where during an RT test an individual places more emphasis on either speed (completing the test as quickly as possible) or accuracy (completing the test as accurately as possible). Individual differences can occur even between age groups with older adults placing more emphasis on accuracy rather than speed [Brébion, 2001; Salthouse, 1979]. Individual differences of the speed/accuracy tradeoff between participants are recognised and anticipated during RT studies [Brébion, 2001; Yellott, 1971; Pachella \& Pew, 1968]. As with the current research, studies try and control for this speed/accuracy trade off by instructing individuals to be as fast and as accurate as possible however it but may still occur regardless of instruction. Implications of speed/accuracy trade-off are discussed in more detail in relation to attention tests in this thesis (see Chapter 6, pages 272-273). In the current research, the numbers of errors were compared between young and older adults and between males and females as well as compared between the different attention tests to determine whether error rates differ with age and sex or the type of test used. 


\subsection{CHAPTER TWO: Visual Search}

\subsection{Introduction}

As mentioned in the introduction, the first aim of this $\mathrm{PhD}$ was to investigate the functional integrity of information processing speed and its intraindividual variability (IIV) in younger and older adults in relation to a variety of different visual attention-related selective attention tests i.e. how the use of different tests of information processing speed in the same people might produce different results. The second aim is to investigate in more detail what influence sex and education may have on information processing speed and IIV and in a novel approach, the potential influence of subjective memory function and perceived test difficulty; both factors of which have been subject to very little previous investigation.

In this chapter the initial study is described, which examines the potential effects of subjective memory function (SMF), perceived test difficulty, educational level and sex, in relation to attention-related information processing speed in younger and older adults, using the commonly used research task of visual search [Tales, Bayer, Haworth et al, 2010]. This task has been used frequently in previous studies to measure potential differences in attentional shifting and information processing speed associated with visual attention-related processing in healthy ageing, MCI and AD. Unlike the previous studies in this area [Landy et al, 2015; Kiss et al, 2012; Tales et al, 2010; Madden, Gottlob et al, 1999] the research forming part of this $\mathrm{PhD}$, measures these aspects of information processing in larger groups of both younger and older adults and examines in greater detail the potential influence upon such results of such factors either poorly addressed (sex and education) or not addressed in previous studies (perceived test difficulty and subjective memory function). How these previously ignored factors may affect information processing speed may influence in future how we in fact interpret the results of visual search tests and especially in terms of what constitutes an ostensibly cognitively health older adult control group in studies of visual attention-related function and information processing speed in MCI and dementia. 


\section{The visual search test}

The brain has a limited information processing capacity and is thus unable to processes all the information in our visual field in parallel. Information processing resources therefore need to be directed primarily to information in the environment which is potentially important and sequentially moved around if necessary.

Selective attention refers to the process of focussing attention on a goal-related target or potentially important information, whilst ignoring surrounding irrelevant information [Lavie, Hirst, De Fockert \& Viding, 2004] and shifting that attentional throughout the environment when necessary i.e. it is 'selectively' choosing or automatically directing attention to what is or might be the most important thing in the environment.

The focus of attention can be likened to the beam of a torch. Where this focus or beam falls is the area where the majority of the brain's processing capacity is focused and applied to the processing of the information at that location to a level which reaches consciousness and can affect behaviour. Processes include perception and cognition as opposed to minimal basic information processing which might tell the brain that something is there but not what it is. The focus of attention can be used to process information at will, i.e., what we choose to concentrate upon. However, to raise awareness of potential important changes within our environment that may be potentially important or relevant to current behaviour, our focus of attention can also be automatically captured by potentially important stimuli thus causing such stimuli to be processed.

As the focus of attention is unable to cover all the information within a scene, it has to be directed to regions or objects of greatest importance in some circumstances e.g. when searching for something. There are two main ways in which the focus of attention can be directed to information within the scene. Automatically (or preattentive, i.e. beyond our control) by virtue of the high saliency of an object compared to its surroundings via an automatic change detection system or via top down control (endogenous or volitional control/choice of processing) in which the focus of attention is, at will, moved or shifted throughout the environment and the information contained within it until a given pre-defined stimulus object or target is located and processed. Both of these processes per se and the speed of such processing are commonly investigated by the Visual Search paradigm which uses specific stimuli representing the environmental factors that elicit shifts of attention. 
The 'Feature Search' component of the paradigm represents the conditions under which the focus of attention can be automatically shifted to a given salient object within the environment. The 'Conjunction component' of the paradigm represents the conditions under which the focus of attention has to be shifted 'at will' to the location of given object within the environment when the object is not salient but instead is made up of conjoined features present in both the object and the environment.

In automatic, feature search [Treisman \& Gelade, 1980] the participant is asked to find a pre-defined target and make a response to it. This target is designed to be highly salient so that it captures attention automatically, by virtue of its unique feature i.e. colour or shape [Wang, Cavanagh, et al, 1994] compared to its surroundings; this distinctive feature creating a 'pop out effect' [Posner, Nissen, \& Ogden, 1978]. There is no need for the serial shifting of the focus of attention, so it is usually a very fast response [Huang \& Hsieh, 2013; Ruz \& Lupiáñez, 2002], i.e. the focus of attention is directed straight to the location of the information thus the time taken from the appearance of the target and the response to it is very rapid.

In Conjunction search [Treisman \& Sato, 1990; Posner \& Cohen, 1984] the participant is asked to find and to make a response to a target that is similar to its surroundings. In this condition, various features are joined together i.e. the target isn't defined by a simple salient feature but the conjoining of features from the target and the surroundings (e.g. a red horizontal bar target surrounded by non-target red vertical bars [Arguin, Joanette \& Cavanagh, 1993; Triesman \& Schmidt, 1982]. Because the target is not salient it cannot summon attentional focus automatically, i.e., directly to it's location. Instead, the focus of attention has to be serially shifted throughout the visual scene in order to locate and respond to the target [Kiss et al, 2012] i.e. attention shifted from object to object 'examining' each one until the predefined one is found and can be responded to. As a result, conjunction search takes significantly longer to perform compared to feature search.

Conjunction search provides an indication (in terms of information processing speed) of the detrimental effect of distracting but irrelevant information on the identification of specific objects within our environment i.e. generally speaking RT is slower when irrelevant distractors are present. The difference in information processing speed between conjunction search reaction time (RT) and the feature search RT is an indicator of how much distracting information slows down the processing of a given object i.e. the efficiency of attention shifting. 
In addition it relates to the efficiency by which attention is serially disengaged, shifted and re-engaged and how well, once the target is found, it is processed. This function is a highly important one for everyday environmental interaction and thus behaviour, and if it is significantly slowed it can have detrimental effects upon everyday life.

The visual search test paradigm [Tales et al, 2010] employed in this (and many previous studies) to measure attention and attention-related information processing speed is a computer-based, multi-trial psychophysics test used to measure both feature and conjunction search, i.e. measures of automatic attentional capture and attentional shifting respectively. The visual search test is also a measure of executive function i.e. attentional control, cognitive flexibility, planning and working memory and functions such as eye movements, shifting attention and decision making. In each trial a target symbol of either a left or right pointing arrow is presented for participants to respond to (see Figure 1). The target symbol appears either alone or with seven distractors of arrows pointing up or down. Each of the arrows is presented equally spaced within a clock-face configuration. Participants are asked to respond as quickly and as accurately as possible to the required targets (left or right arrows) by pressing the corresponding arrow on a keyboard.

Figure 1: Representation of the stimuli in which the target arrow ' $<$ ' is presented alone (automatic feature search) or surrounded by 7 distractors (conjunction search)
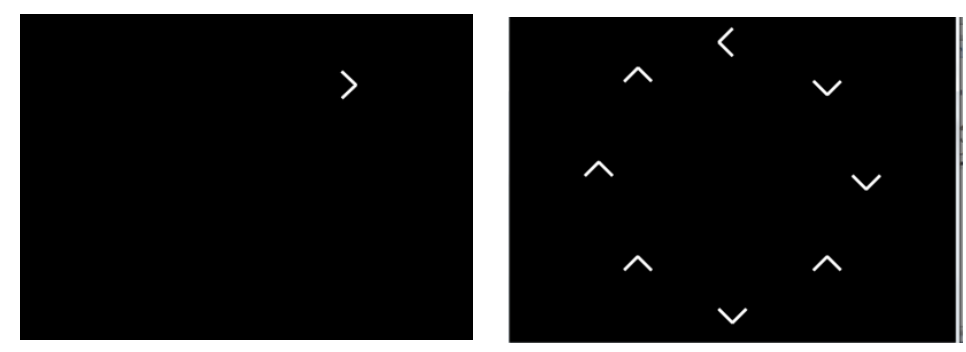

The target when surrounded by distracters constitutes a conjunction search condition, i.e., the conditions under which the target does not pop out; it does not automatically capture attention, but instead the distractors are similar to the target in colour (all white) but different in orientation (target arrow left or right surrounded by distractor arrows up and down). The focus of attention has to be sequentially shifted throughout the visual field (i.e., through each stimulus) before the target can be found and then processed (i.e., determine which way it is pointing). 
The task also contains a target alone condition that enables the efficiency (speed) of the automatic processing of the same target (feature search and 'pop out' effect). The target alone condition also allows us to compare baseline choice RT between and within groups [Tales et al, 2010], i.e., it is a simple choice reaction time measure). Subtracting the target alone condition from the target plus distractors condition (target plus distractors-target alone) allows for a measure of the overall efficiency of attention shifting i.e. how much of an effect distractors are on attention shifting. In addition the task contains multiple trials enabling the measurement of intraindividual variability (IIV) i.e. the variability of RT performance.

\section{Biological basis for feature and conjunction visual search}

Feature search, i.e., the automatic capture of attention by salient information, is associated with activity in posterior regions of the attentional network i.e. the superior parietal cortex and angular gyrus [Madden, Spaniol, Whiting et al, 2007] and in the frontal cortex [Lavie \& Fockert, 2006]. Pollmann, and colleagues [2006] argue that the anterior prefrontal components underpin the transient bottom up activation that are involved in feature detection, although they also found evidence to suggest that other areas are also involved in processing the features of a stimulus i.e. the V4 area processing colour and the V5/MT area processing motion. The importance of specific visual areas in this aspect of processing has also been supported by Zhaoping \& Snowden [2006] who proposed the V1 visual area is important for coding salient information. Therefore coding salient information does not just occur in areas of the extra striate cortex as previously assumed [Moran \& Desimone, 1985].

For conjunction search i.e. shifting attention throughout the visual scene, areas such as the parietal cortex, are activated. Damage to the left parietal lobe has been found to have a detrimental effect on visual orienting [Han, Jiang, Gu et al., 2004], therefore suggesting the importance of the parietal cortex for this type of attentional processing. Decreased activation has been found in the prefrontal cortex in older adults resulting in them displaying difficulty in ignoring non-salient information, i.e., difficulty in efficiently shifting attention throughout the scene [Harpur, Scialfa, \& Thomas, 1995; Connelly \& Hasher, 1993; Rabbitt, 1965;] particularly as the number of distractors increases [Guest, Howard, Brown, \& Gleeson, 2015; Scialfa, \& Joffe, 1997]. However, some evidence indicates that the mechanisms, anatomical area and networks underlying both feature and conjunction search may be very similar [Tales $\&$ Porter, 2008] as attention is composed of separate but inter-related functions. 
For example it has been observed that different areas of the parietal cortex can be involved in feature search as well as in conjunction search [Fan, McCandliss, Fossella, Flombaum, \& Posner, 2005; Posner \& Boies, 1971]. These attentional processes may become impaired and slowed as a result of detrimental changes in the integrity of white matter associated in areas of the brain involved in such processing [Bendlin, Fitzgerald, Ries et al, 2010; Michielse, Coupland, Camicioli et al, 2010; Kennedy \& Raz; Grieve, Williams, Paul et al, 2007]. Detrimental changes to white matter integrity may be an effect of ageing in which case it would be expected that these groups display disproportionate abnormality and behavioural slowing in tasks that recruit these functions (e.g. feature and conjunction search).

In addition, disproportionate slowing may be greater in $\mathrm{MCI}$ and $\mathrm{AD}$ compared to cognitively healthy ageing as underlying anatomical and functional areas are more impaired during MCI and AD than in cognitively normal ageing such as the parietal cortex. Originally the visual cortex was thought to be less affected in AD or MCI thus it was expected that in AD and MCI 'pop out' feature search would be 'normal' but abnormality found in conjunction search. The sections below describe evidence from previous research of impairment of selective attention (feature and conjunction search) and resulting slowing of information processing speed using visual search during ageing and $\mathrm{MCI}$ and $\mathrm{AD}$.

\section{Visual search performance in ageing}

Substantial research-based evidence indicates that ostensibly cognitively healthy older adults (as determined by objectively measured cognitive function) tend to display slower information processing speed (reaction time) during feature and conjunction search compared to young adults [e.g. Torrens-Burton et al, 2017; Self, Handelman, Le, \& Sigler, 2016; Muller-Oehring et al, 2013, Potter et al, 2012; Lorenzo-Lopez et al, 2008; Madden, 2007; Hommel et al, 2004] thus implying poorer efficiency of attentional shifting in ageing. Intraindividual variability (IIV) of information processing speed has also been found to increase with age [Mella et al, 2013; Dykier et al, 2012; Fozard et al, 1994; Inui, 1997] indicating poorer variability of information processing speed and poorer integrity of brain function i.e. CNS. However visual search was not used in these studies to compare young and older adults thus the inclusion of IIV as well as RT in the current visual search study as well the inclusion of young adults to compare with older adults i.e. effects of RT and IIV in ageing. 
Activation in the angular gyrus and superior parietal lobule was found to be greater in older adults compared to young adults during conjunction search [Madden, Spaniol et al, 2007]. This, according to the authors, [Madden, 2007] suggests that older adults require greater effort to perform conjunction search as effectively as young adults. Levels of acetylcholine play a vital role in orienting attention required during selective attention tasks i.e. conjunction search [Klinkenberg, Sambeth \& Blokland, 2011; Muir, Everitt, \& Robbins, 1994]. When levels decrease during aging [Gibson \& Peterson, 1981] due to damage to the cholinergic basal forebrain system [Muir, 1997], attention orienting (conjunction search) becomes impaired and behavioural slowing is observed [Klinkenberg et al, 2011].

Comparing both feature and conjunction search using a visual search task has also produced variation in study outcome, with some studies revealing a lack of agerelated slowing for feature based search compared to age related slowing in conjunction search [Muller-Oehring et al, 2013; Trick \& Enns, 1998; Scialfa \& Joffe, 1997]. This implies that the attentional mechanisms used for feature and conjunction search may change differently during ageing. Variation between feature and conjunction search suggests that older adults can profit from early parallel stages of visual information processing (feature search), but begin to fail with later serial processing (conjunction search) as some aspects of this type of processing become less efficient; for example the ability to, selectively and sequentially shift attention throughout the scene and to ignore distracting information [Greenwood, Parasuraman \& Alexander, 1997]. Indeed orienting attention overtly i.e. physical eye movement (which tend to represent and show the location or movement of the focus of attention) also shows age related differences [Veiel, Storandt, \& Abrams, $2006]$ as the number of eye saccades used increases slightly in older adults causing searching for a target to take longer [Port, Trimberger, Hitzeman et al, 2016]. More fixations are also needed on each target or location in conjunction search for older adults compared to young [Porter, Tales, et al., 2010].

\section{Visual search performance in mild cognitive impairment (MCI) and} Alzheimer's disease.

A substantial body of evidence using a visual search test also indicates that in MCI and $\mathrm{AD}$, information processing speed and attentional performance (e.g. shifting) is disproportionately poorer compared to cognitively health ageing [e.g. Haworth et al, 2016; Tales et al, 2010; McLaughlin, Borrie \& Murtha, 2010; Tales, Snowden, Haworth, \& Wilcock, 2005; Greenwood et al, 1997]. 
In $\mathrm{AD}$, pathology change is evident within parietal cortices [Arrington, Carr, Mayer, \& Rao, 2000; Tales \& Porter, 2008] and the primary visual cortex [Leuba \& Saini, 1995] as well as a significant decrease in acetylcholine levels [Herholz, Weisenbach, $\&$ Kalbe, 2008] and one would expect therefore that people living with AD would show slower information processing speed in both feature and conjunction search. In addition they would display poorer attentional shifting ability (i.e. target plus distracters - target alone ) performance and indeed individuals with AD have been found to perform slower i.e. higher reaction time speeds during feature search task compared to healthy controls [Foster et al, 1999; Meltzer, Zubieta, Brandt et al., 1996].

As mentioned above the visual cortex was originally thought to be less affected in $\mathrm{AD}$ or MCI thus why visual search studies first performed with the expectation of finding normal 'pop out' feature but abnormal conjunction search in AD and MCI. Finding the primary visual cortex to still be active in AD patients during visual search suggests feature search is relatively preserved in AD albeit slower compared to healthy ageing [Landy et al, 2015; Tales et al 2002; Grady et al., 1993]. This implies that in AD patients, the ability to automatically processes a stimulus (such as in feature search) is not compromised. It appears therefore that what is compromised in $\mathrm{AD}$ to a greater degree than in cognitively healthy ageing is the ability to shift attention efficiently through distracting information i.e. the ability to ignore distracting information and instead shift attention towards the intended target [Tales et al., 2002; Greenwood et al, 1993; Mendez, Cherrier, \& Cymerman, 1997; Greenwood et al, 1997; Parasuarman, Greenwood, Haxby et al, 1992]. These findings have been supported despite studies using different search paradigms [Tales et al, 2010; Greenwood et al, 1997; 1993; Mendez et al, 1997] thus results may not be directly comparable.

Different components of attention are required in different paradigms which involve searching through the environment such as shifting attention, disengagement, along with other markers of attention i.e. as well as search and shifting problems, people with $\mathrm{AD}$ may have associated problems e.g. eye movements, the integrity of which is also needed to be able to do search properly. These components can become abnormal during $\mathrm{AD}$. Indeed $\mathrm{AD}$ patients have demonstrated difficulties in visually exploring their environment, disengaging attention from one object in order to attend to the next object [Vallejo, Cazzoli, Rampa et al, 2016; Tales, Snowden, Brown, \& Wilcock, 2006; Perry \& Hodges, 1999; Mendez, Cherrier \& Perryman, 1997]. 
This is partly due to requiring a greater number of longer eye fixations while searching for a target [Chau, Herrmann, Eizenman et al, 2015; Rösler, Mapstone, Hays-Wicklund, et al, 2005] more so than healthy adults [Porter, Leonards, et al., 2010]. Although healthy older adults make more eye fixations and for a longer time period compared to young adults [Rösler et al, 2005], the effect is still greater in AD.

As in $\mathrm{AD}$, pathology in attention-related brain areas can detrimentally affect slowing of visual attention in MCI. It has been suggested that parietal lobe systems are responsible for the ability to disengage attention from one target and shift attention to another [Pardo et al, 1990; Posner and Driver, 1992]. Therefore if these attentional functions are impaired, it is speculated that the ability to disengage from and shift to stimuli will slow. Indeed dysfunction in the fronto-parietal networks is suggested by Redel and colleagues [2012] to impair reaction time performance in MCI and AD patients.

Individuals with aMCI, as well as those who decline further i.e. develop $\mathrm{AD}$, have been found to have problems with disengaging attention from an incorrectly cued location (invalid cue i.e. distractors) and engaging in valid cues. The inability to disengage attention and shift to the next location is reflected in the slower information processing speed between valid (target) and invalid (distractor) cues, known as the 'validity effect' [Tales et al, 2005]. This has been found in a variety of different tests which measure this aspect of attention i.e. Posner cueing paradigm [Tales et al, 2005], the Stroop paradigm [Stroop, 1935] as well as the visual search paradigm [Tales et al, 2010] therefore these different tests of attention validate each other i.e. they measure similar attention but in different ways.

Levinoff, Saumier, \& Chertkow [2005] also found signficant slowing in MCI providing evidence for impaired attentional processing and indeed they went so far as to suggest that differences in reaction time can be used as early diagnosis of cognitive impairments particularly in attention in the elderly. Although the magnitude of the difference in slowing and performance between AD and cognitively health ageing is generally large and robust, it is not so for MCI. If differences in MCI are found the effect sizes are often much smaller, and sometimes no difference in information processing speed is found between MCI and cognitively health older adults [Tales et al, 2002]. However there is much evidence that attention related $\mathrm{RT}$ is poor in $\mathrm{MCI}$ and $\mathrm{AD}$ although it needs to be taken into account outcome variability between studies may make it difficult to compared results. 
It is also important to note that not all individuals with MCI decline further to AD. Aetiology may differ between those with MCI who decline further and those with MCI who do not decline to a dementia diagnosis. Therefore these differences in aetiologies may also relate to differences in how information processing speed is effected i.e. level of slowing. This is supported by Tales and colleagues [2011] who found that in aMCI patients who developed within 2.5 years of their baseline measurement, their mean performance of conjunction and feature search was significantly poorer i.e. slower information processing speed, compared to those remaining stable at the MCI stage or who had returned to a normal level of functioning.

Even when differences in performance between older adult controls and patients with MCI are not significant it is usual to find performance outliers within the MCI group; i.e., various people with much slower than expected performance. Arguably, if performance at group level is normal, these individuals may have a greater risk of developing AD (as evidence suggests that slowing is a clinical characteristic of this disease) or at least have personally significant detrimental changes to their information processing speed that might detrimentally affect their quality of life. Such outliers if not acknowledged or taken into account can influence results or our understanding of the disease and its effects. Mean group differences in MCI may not show very much yet there may be some people with the disease whose RT, IIV and attention is very poor and the consequence of this are such outliers.

The same may occur in healthy ageing groups as there may be some individuals with unknown impairment i.e. subjective memory function or SCI and these people have disproportionately slower and varied IIV presented as outliers within group mean RT. Therefore in the current study of RT and ageing we took any outliers into account and discussed what they might mean.

\section{Intraindividual variability}

Intraindividual variability reflects the variation of behavioural responses within a single persons' overall performance and if increased reflects poorer integrity of the central nervous system (CNS) and greater variability of RT performance. IIV is also of interest when measuring information processing speed in ageing, MCI and AD. In relation to visual search, IIV can measure within-person variability of processing speed in behavioural performance. 
Variability has found to be greater in older adults compared to young adults [Mella et al, 2013; Dykier et al, 2012; Fozard et al, 1994; Inui, 1997] although not in relation to visual search whereas IIV has been found to be significantly greater in MCI and AD compared to healthy controls [Phillips et al, 2013; Tractenberg \& Pietrzak, 2011]. Gorus and colleagues [2008] examined variability of information processing speed in both motor and cognitive components with aMCI and AD patients. Both aMCI and AD patients demonstrated greater variability in their processing speed compared to healthy controls [see also Philips et al, 2013 and McLaughlin et al 2010].

However within visual search research, to our knowledge IIV has not been examined in as much detail in healthy aging (i.e. between young and older adults) and in relation to different factors (particularly subjective memory function and perceived test difficulty). Previous studies may be confounded by not taking the influence of different factors i.e. sex and education and particularly subjective memory function and perceived test difficulty into account which may have had an impact of how the results of assumed healthy older adult control groups were interpreted in MCI and AD studies. Therefore the relationship between different factors and IIV will be examined in detail in the current study (and throughout the thesis).

\section{Visual search performance in Subjective Cognitive Impairment (SCI) and subjective memory function.}

Information processing speed has been demonstrated to slow during in MCI and AD compared to healthy ageing in the visual search test [Haworth et al, 2016; Tales et al, 2010; McLaughlin et al, 2010; Tales et al, 2005; Greenwood et al, 1997].

Within these studies MCI or AD are compared to ostensibly healthy older adults taken from the local community and considered to display 'normal' levels of information processing speed. Typically in most studies objective cognitive function of the control group is tested and taken into account i.e. if an individual score is too low (considered to be impaired cognition) these individuals are excluded from the control group. However some individuals within the control group may be reporting problems with tier cognition which may relate to disproportionate slowing which reflects the type of slowing found in abnormal ageing i.e. MCI or AD, namely Subjective Cognitive Impairment (SCI). 
In the current thesis we focused on subjective memory function i.e. older adults perceiving change to memory function but have not visited their GP (thus not diagnosed with SCI). In research control older adults taken from general public may have subjective memory function which has not been detected but again this may be reflecting the type of slowing attributed to abnormal ageing thus these individuals should not be included in healthy adult groups.

A relationship has previously been found between subjective complaints and a diagnosis of baseline cognitive impairment [ Reed, 2010; Cook \& Marsiske, 2005; Earles \& Salthouse, 1995; Schofield, Marder, Dooneief et al., 1997] however this relates to SCI and as far as we are aware, no ageing studies using visual search have examined subjective memory function in relation to information processing speed and IIV in community dwelling older adults. Therefore this initial study examined whether subjective memory function can be characterised by slow RT and increased IIV thus contribute towards the characterisation of SCI.

\section{Summary}

Information processing speed and attention has been demonstrated to slow in the visual search paradigm during ageing, MCI and AD [Self et al, 2016; Haworth et al, 2016; Tales et al, 2010; McLaughlin et al, 2010; Tales et al, 2005]. However age effects have not always been found [Muller-Oehring et al, 2013] due to the variability between visual search studies i.e. the type of paradigm used, small participant sizes or failing to include young adults to measure ageing. Outcome variability may also relate to the influence of other person- related factors on information processing speed i.e. sex, education or subjective memory function and perceived test difficulty (as described in further detail in the sections below).

Previous visual search studies have examined sex and education on information processing speed [Fozard et al, 1994; Dykiert et al, 2012] although not all previous studies have considered these factors. Therefore sex and education should probably be examined in more detail during a visual search paradigm [as discussed in Haworth et al, 2016]. Therefore one of the aims of the current study of visual search was to examine sex and education in relation to RT and IIV in more detail with increased subject numbers. In addition, visual search studies have not addressed subjective memory function and perceived test difficulty as a potential influence on information processing speed. 
These factors have either been dismissed previously as not deemed to be potentially important or had not been considered (particularly dismissed subjective memory function and perceived test difficulty) thus examining these factors in relation to RT and IIV in the current visual search study. Failing to take these factors into consideration may result in biased study outcome thus misinterpret what constitutes ostensibly 'normal' information processing speed in cognitively healthy older adult control groups during visual search studies comparing ageing, MCI and AD thus may be masking a greater difference between cognitively normal functions and AD or MCI.

\section{Person-related factors}

\section{Sex}

Previous visual search studies found information processing speed to be faster in males compared to females, not just in younger adults [Karia et al, 2012] but across age groups [Fozard et al, 1994; Dykiert et al , 2012] as well as IIV significantly increasing in females compared to males [Philips et al, 2013]. Finding sex effects imply that if slowing of information processing speed is dependent on sex and not considered as a factor, results may vary and have an influence on how information processing speed between men and women is characterised. What constitutes 'normal' levels of information processing speed in healthy control groups, may have been misinterpreted in previous MCI and dementia studies. In the current visual search paradigm in an initial study using more participants than previously used, sex will be examined as a potential factor influencing information processing speed, IIV and attention.

\section{Education}

Lower education has been associated with slower information processing speed during visual search [Tales et al, 2010; Tun \& Lachman, 2008]. It has been argued that more future studies would benefit taking education into consideration [Haworth et al, 2016; Tales et al, 2010] when measuring information processing speed in selective attention using visual search in order to produce robust and replicable results. Finding education effects imply that if slowing of information processing speed is dependent on education and not considered as a factor, results may vary and have an influence on how information processing speed during ageing is characterised. 
In older adults, finding an association between education and information processing speed may relate their levels of cognitive reserve i.e. the brains barrier to cognitive impairment. It has been found that higher levels of education can help with cognitive reserve [Sattler, Toro, Schonknecht, \& Schroder, 2012; Meng \& D'Arcy, 2012] thus in relation to the current study, higher education in older adults may relate to their cognition function (i.e. those required in visual search) being more resilient to the effects of disease or even age. Therefore RT performance may be faster and less varied as a result.

In the current visual search paradigm in an initial study, education will be examined as a potential factor influencing information processing speed and IIV alongside sex, subjective memory function and perceived test difficulty.

\section{Perceived test difficulty}

As mentioned in the in introduction to this thesis perceived test difficulty relates to psychological factors i.e. self -beliefs an individual may have about the demands of the task which may have an effect on cognitive performance i.e. slower information processing speed [Flavell, 1979]. The research in this thesis focused on the perceived difficulty of different attention tests with the assumption that if an individual perceives a test to be more difficult, this may have a negative effect on information processing speed i.e. slower RT.

Some studies argue that perceiving a test to be difficult can negatively affect mood which in turn can have a negative impact on information processing speed [Setti, Loughman, Savva \& Kenny, 2015; Bolmont, et al, 2000]. However, perceived test difficulty not examined in much detail in relation to RT and IIV and using the visual search in both young and older adults. Failing to take this factor (into account may impact how levels of slowing are interpreted in healthy ageing in visual search and possibly highlight how the integrity of information processing speed of control groups in past visual search studies may have been misinterpreted.

In the current study perceived test difficulty (measured after test completion i.e. retrospectively) was examined in relation to RT performance and IIV in young and older adults as well as any interactions with other factors (education and subjective memory function) to determine whether perceived test difficulty may be associated with additional factors other than age which may consequently effect information processing speed. The results may help determine whether this factor should be taken into account in research studies using the visual search. 


\subsection{METHODOLOGY}

\section{$\underline{\text { Participants }}$}

Swansea university students ( $\mathrm{n}=48$; age 18-26 years; 19 males: 29 females) were recruited through the Psychology department credit system and through advertising around the university and social network. Community-dwelling older adults ( $n=54$; 50-80 years; 24 males: 30 females) were recruited through advertisements given out to older adult social clubs and local papers in Swansea, through email, word of mouth and via a volunteer database of older adults set up by the Swansea Psychology Department. Ethical approval was provided by the Swansea University Psychology departmental ethics committee and the study conducted to the principles in the Declaration of Helsinki. Formal written consent was obtained from each participant.

\section{$\underline{\text { Inclusion criteria }}$}

Participants (both young and older adults) self-reported to be in good general health, with no history of serious head injury, cognitive, visual or neurological impairments, colour blindness or have any condition which might be affected by flashing images on a screen. Although medication could not be controlled for, it was attempted to exclude individuals whose medication was likely to affect attention and RT. The vision of all participants was normal or 'corrected-to-normal'. All participants were requested to bring any corrective lenses they require for reading and computer work.

All participants ( young and older adults) general cognition was measured by the Montreal Cognitive Assessment (MoCA) [Tiffin-Richards, Costa, Holschbach et al, 2014; Nasreddine, Phillips, Bedirian et al, 2005] (a 'normal' score being 26 or above $^{1}$ ). They had no significant levels of depression or anxiety as measured by the Patient Health Questionnaire (PHQ-9) [Kroenke, Spitzer \& Williams, 2001] (score of 9 and below from a maximum of 27) and the Generalized Anxiety Disorder 7-item (GAD-7) [Löwe et al, 2008] (score of 5 and below from a maximum of 21). For older adults, subjective memory function was measured using the Memory Functioning Questionnaire (MFQ) [Gilewski et al, 1990] (higher scores, equal to lower concern). Age (16 to 25 years for young adults; 50 to 80 years for older adults), sex and years of full time education were also recorded.

\footnotetext{
${ }^{1}$ Note that the scores of the majority of participants were within the normal value of 26 or above. However for 13 young and older adult participants the score was lower than 26 . These were included because we were interested in a range of MoCa scores and whether the score has in influence on RT or IIV.
} 
Education was matched across both groups and for males and females as closely as possible making the results very similar between both age groups. However a slight range in years of education was included which produced slight variability in results which is why the effects of education are examined.

\section{$\underline{\text { Stimuli }}$}

The stimuli were presented on a Dell Precision PC running on Windows XP X86 CPU, viewed at a distance of $57 \mathrm{~cm}$. In each trial a target symbol of either a left pointing arrow ' $<$ ' or a right pointing arrow ' $>$ ' was presented for participants to respond to. The target symbol appeared either alone or with seven distractors which were the arrows pointing up ' $\wedge$ ' or down ' $v$ ' (see Figure 1). Each of the arrows was presented equally spaced within a clock-face configuration. All targets and distractors were white against a black background with each line measuring $1 \mathrm{~mm}$ in width and $5 \mathrm{~mm}$ in length.

A fixation cross appeared in the centre of the screen for $1000 \mathrm{~ms}$ then removed until the next trial. The target followed either alone or with distractors and the participant pressed the left arrow key on the keyboard if the target arrow was facing left and the right arrow key for a right facing target Once the target was responded to, the next trial began. The trials were randomized between trials with targets presented on their own and trials with targets presented along with distractor arrows. Both target arrows were presented 8 times in each of the clock locations in order to eliminate any processing differences between upper or lower and left and right visual fields. 4 of the 8 times the target was presented alone and the other 4 with distractors. This gave 64 trials in total.

\section{Procedure}

Participants were instructed to focus their attention on the centre of the cross between trials. They were to respond as quickly and as accurately as possible to the required targets (left or right arrows) by pressing the corresponding arrow on a keyboard. Participants were given five or six trials as a practice before the programme was restarted for testing. Additional practice was provided to any participant who required it by giving them another 5 trails. The testing phase was then conducted with a total of 64 trials. Researcher supervision of participants continued throughout the task to check the correct buttons were being pressed. 
Once testing had ended, participants were given a Likert scale from 1 to 7 to rate how difficult they found the test with 1 very easy to complete and 7 very difficult. Debriefing followed with the opportunity to ask any questions.

\section{Data Processing}

In alignment with typical practice and previous research, any responses were eliminated if they were incorrect or outliers i.e. below 150ms (faster than 'natural' reaction time therefore pre-empting the stimulus) or above 10,000ms (associated with lapses of attention, see Tales and colleagues, 2010). No participants failed to respond to a trial. Trials (for both old and young adults) were split into two conditions: 'target alone' and 'target plus distractors' trials.

The median RT and inter-quartile range (for IIV) was determined for each individual for each condition together with the group mean attained. The error rate for each condition was also determined i.e. number of incorrect responses. For each individual the 'distractor effect' was calculated $\left[\mathrm{RT}_{\text {Distractors }}-\mathrm{RT}_{\text {Target alone }}\right]$ (a measure of the attention shifting efficiency) and overall group means obtained. In response to the non-normal distribution of the data (see Table 1) SPSS non parametric analysis was conducted (as in several previous studies by Tales and colleagues). Factors i.e. sex and education, subjective memory fucntion and perceived test difficulty were grouped into separate families for analysis. The RT and IIV and attention effects were different data sets and thus did not require Bonferroni correction for multiple analyses. In addition, previous studies using a visual search paradigm also excluded correlations for multiple comparisons were not made.

Table 1. Normality of Distribution (Shapiro Wilkes test) ${ }^{2}$

\begin{tabular}{|c|c|c|c|c|c|c|c|}
\hline & \multicolumn{3}{|c|}{ Older } & \multicolumn{3}{|c|}{ Young } \\
\hline & & Statistic & $d f$ & Sig. & statistic & $d f$ & Sig. \\
\hline \multirow[t]{2}{*}{ Target Alone } & $\begin{array}{c}\text { Information } \\
\text { processing speed }\end{array}$ & .887 & 54 & .000 & 620 & 48 & .000 \\
\hline & $\begin{array}{c}\text { Intraindividual } \\
\text { Variability }\end{array}$ & .785 & 54 & .000 & .602 & 48 & .000 \\
\hline \multirow{2}{*}{$\begin{array}{l}\text { Target plus } \\
\text { distractors }\end{array}$} & $\begin{array}{c}\text { Information } \\
\text { processing speed }\end{array}$ & .939 & 54 & .009 & .913 & 48 & .002 \\
\hline & $\begin{array}{l}\text { Intraindividual } \\
\text { Variability }\end{array}$ & .984 & 54 & .736 & .922 & 48 & .004 \\
\hline
\end{tabular}

\footnotetext{
${ }^{2}$ Note: data classified as a non-normal due to the positively skewed distribution of the data and in some cases bimodal distributions. There is a natural limit to the data i.e. how fast RT can be.
} 


\subsection{RESULTS}

\section{$\underline{\text { Demographics }}$}

Mann-Whitney analysis revealed a significant difference in mean depression (PHQ-

9) score and mean anxiety (GAD-7) score. Depression level was significantly greater for young adults compared to older adults $[\mathrm{U}=878.5, p=.005$, effect size $(\mathrm{r})=.28]$ and anxiety level was significantly greater for young adults compared to older adults $[\mathrm{U}=943.5, p=.017$, effect size $(\mathrm{r})=.24]$. There was no significant difference in mean MoCA score or mean years of education $[p>.05]$.

Table 2: Mean demographic scores for the older adult and younger adult groups. Standard deviation in parenthesis.

\begin{tabular}{|c|c|c|c|c|c|c|}
\hline & $\begin{array}{c}\text { Age } \\
\text { (years) }\end{array}$ & $\begin{array}{c}\text { Education } \\
\text { (years) }\end{array}$ & $\begin{array}{c}\text { MoCA } \\
\text { (score) }\end{array}$ & $\begin{array}{c}\text { MFQ } \\
\text { (score) }\end{array}$ & $\begin{array}{c}\text { PHQ-9 } \\
\text { (score) }\end{array}$ & $\begin{array}{c}\text { GAD-7 } \\
\text { (score) }\end{array}$ \\
\hline $\begin{array}{c}\text { All } \\
\text { young } \\
\text { Adults } \\
\text { (n= 48) }\end{array}$ & $20(2.0)$ & $15(1.9)$ & $27(2.4)$ & - & $5(3.3)$ & $4(3.5)$ \\
\hline $\begin{array}{c}\text { Young } \\
\text { Male } \\
\text { (n=19 }\end{array}$ & $20(2.1)$ & $15(1.9)$ & $26(2.1)$ & - & $2(2.8)$ & $1(1.2)$ \\
\hline $\begin{array}{c}\text { Young } \\
\text { Female } \\
\text { (n= 29) }\end{array}$ & $20(2.0)$ & $15(1.9)$ & $27(1.9)$ & - & $5(3.2)$ & $5(3.4)$ \\
\hline $\begin{array}{c}\text { All } \\
\text { Older } \\
\text { Adults } \\
(\mathbf{n = 5 4 )}\end{array}$ & $66(5.2)$ & $15(3.7)$ & $27(2.4)$ & $\begin{array}{c}290 \\
(46.5)\end{array}$ & $4(3.0)$ & $3(3.0)$ \\
\hline $\begin{array}{c}\text { Older } \\
\text { Male } \\
\text { (n=23) }\end{array}$ & $66(5.0)$ & $15(4.6)$ & $26(2.6)$ & $\begin{array}{c}282 \\
(42.0)\end{array}$ & $4(3.2)$ & $3(3.1)$ \\
\hline $\begin{array}{c}\text { Older } \\
\text { Female } \\
\text { (n=31) }\end{array}$ & $55(5.4)$ & $15(2.9)$ & $28(2.1)$ & $\begin{array}{c}295 \\
(49.4)\end{array}$ & $3(2.8)$ & $3(3.0)$ \\
\hline
\end{tabular}

MoCA, Montreal Cognitive Assessment; MFQ, Memory Functioning Questionnaire; PHQ-

9, Patient Health Questionnaire; GAD-7, Generalized Anxiety Disorder 7-item scale. 
Table 3: Group mean information processing speed and Intraindividual Variability (IIV) (from individual participant median RT scores) and mean number of errors (incorrect key presses) for the young and older adult groups for the target alone and target plus distractors conditions in visual search. Standard deviation in parenthesis.

\begin{tabular}{|c|c|c|c|c|c|c|c|}
\hline & \multicolumn{2}{|c|}{$\begin{array}{l}\text { Mean information } \\
\text { processing speed in } \\
\text { ms (SD) }\end{array}$} & \multicolumn{2}{|c|}{$\begin{array}{l}\text { Intraindividual } \\
\text { Variability (ms) }\end{array}$} & \multicolumn{2}{|c|}{$\begin{array}{c}\text { Mean Errors } \\
\text { (incorrect key presses) }\end{array}$} & \multirow[b]{2}{*}{$\begin{array}{c}\text { Perceived } \\
\text { performance } \\
\text { Likert Scale }\end{array}$} \\
\hline & $\begin{array}{l}\text { Target } \\
\text { alone }\end{array}$ & $\begin{array}{c}\text { Target }+ \\
\text { Distractors }\end{array}$ & $\begin{array}{l}\text { Target } \\
\text { alone }\end{array}$ & $\begin{array}{c}\text { Target }+ \\
\text { Distractors }\end{array}$ & $\begin{array}{c}\text { Target } \\
\text { alone }\end{array}$ & $\begin{array}{c}\text { Target }+ \\
\text { Distractors }\end{array}$ & \\
\hline $\begin{array}{c}\text { All } \\
\text { Young } \\
\text { adults } \\
(\mathrm{n}=48)\end{array}$ & $\begin{array}{l}526.85 \\
(116.89)\end{array}$ & $\begin{array}{l}1038.33 \\
(270.85)\end{array}$ & 101.89 & 495.27 & $\begin{array}{c}0.60 \\
(1.01)\end{array}$ & $\begin{array}{c}0.63 \\
(1.14)\end{array}$ & $\begin{array}{l}3(1.4) \\
\text { (range 1-5) }\end{array}$ \\
\hline $\begin{array}{c}\text { Male } \\
(\mathrm{n}=19)\end{array}$ & $\begin{array}{l}502.68 \\
(45.27) \\
\end{array}$ & $\begin{array}{c}926.76 \\
(217.65)\end{array}$ & 79.14 & 435.58 & $\begin{array}{c}0.29 \\
(0.47) \\
\end{array}$ & $\begin{array}{c}0.12 \\
(0.33) \\
\end{array}$ & $\begin{array}{c}3(1.4) \\
\text { (range 1-5) }\end{array}$ \\
\hline $\begin{array}{l}\text { Female } \\
(n=29)\end{array}$ & $\begin{array}{c}542.69 \\
(144.78)\end{array}$ & $\begin{array}{l}1111.43 \\
(280.54)\end{array}$ & 116.79 & 534.38 & $\begin{array}{c}0.89 \\
(1.28) \\
\end{array}$ & $\begin{array}{c}1.11 \\
(1.41) \\
\end{array}$ & $\begin{array}{c}2(1.0) \\
\text { (range 1-4) }\end{array}$ \\
\hline $\begin{array}{c}\text { All } \\
\text { Older } \\
\text { Adults } \\
(\mathrm{n}=54)\end{array}$ & $\begin{array}{c}743.02 \\
(164.91)\end{array}$ & $\begin{array}{l}1658.55 \\
(314.23)\end{array}$ & 170.96 & 822.63 & $\begin{array}{c}0.50 \\
(0.75)\end{array}$ & $\begin{array}{c}0.38 \\
(0.58)\end{array}$ & $\begin{array}{c}3(1.4) \\
\text { (range 1-6) }\end{array}$ \\
\hline $\begin{array}{c}\text { Male } \\
(n=23)\end{array}$ & $\begin{array}{c}747.17 \\
(175.33)\end{array}$ & $\begin{array}{c}1631.98 \\
(352)\end{array}$ & 179.91 & 794.93 & $\begin{array}{c}0.63 \\
(0.90)\end{array}$ & $\begin{array}{c}0.64 \\
(0.67)\end{array}$ & $\begin{array}{c}3(1.4) \\
\text { (range 1-5) }\end{array}$ \\
\hline $\begin{array}{l}\text { Female } \\
(\mathrm{n}=31)\end{array}$ & $\begin{array}{c}739.94 \\
(159.62)\end{array}$ & $\begin{array}{l}1678.26 \\
(287.46)\end{array}$ & 164.32 & 843.18 & $\begin{array}{c}0.38 \\
(0.59)\end{array}$ & $\begin{array}{c}0.15 \\
(0.38)\end{array}$ & $\begin{array}{c}3(1.4) \\
\text { (range 1-6) }\end{array}$ \\
\hline
\end{tabular}




\section{Information processing speed}

\section{Age Comparison: Information Processing Speed}

Figure. 2: Box plot of mean information processing speed (ms) comparing Target plus distractors and Target alone trials in older and young adults.

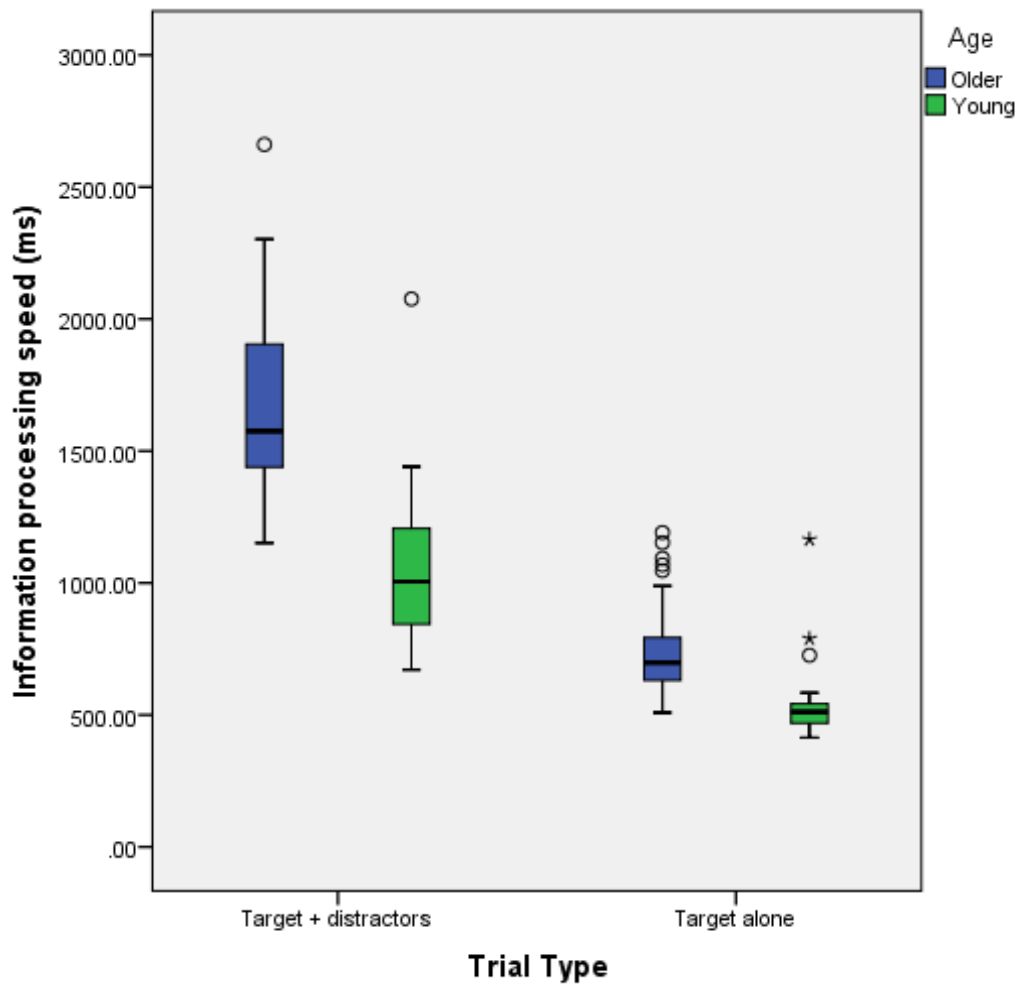

Figure 3. Box plot of mean difference in information processing speed $\mathrm{RT}_{\text {Distractors }}-$ $\mathrm{RT}_{\text {Target alone }}$ (distractor effect i.e. attentional efficiency) between sex (male vs. female) and age (young vs. older adults).

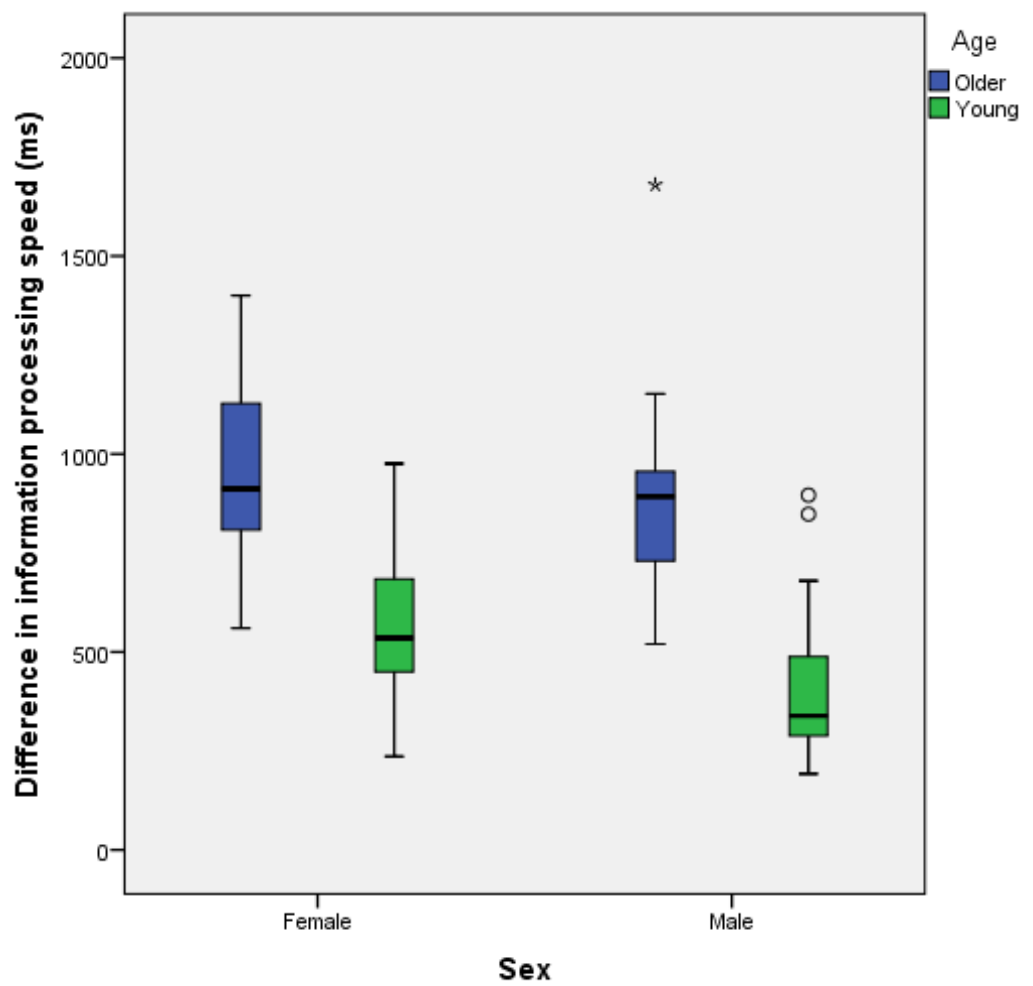


As displayed in Figure 2, at group level the older adults were slower over both target alone and target plus distracter conditions, and as expected, information processing speed was slower for both groups in the target plus distracter compared to the target alone conditions. The effect of distracting information upon information processing speed $\left[\mathrm{RT}_{\text {Distractors }}-\mathrm{RT}_{\text {Target alone }}\right.$ (a measure of the attention shifting efficiency, see Figure 3) was also greater for the older compared to the younger adults. Note however, that there are outliers in this data in both target conditions but particularly in the Target Alone condition.

\section{Information processing speed between conditions}

There was a significant difference in information processing speed between the two visual search conditions; RT performance was significantly slower in the target plus distractors condition compared the target alone condition in young adults $[\mathrm{U}=49, p$ $<.001$, effect size $\mathrm{r}=.82]$ and older adults $[\mathrm{U}=3, p<.001$, effect size $\mathrm{r}=.86]$.

\section{Target Alone information processing speed.}

Mann-Whitney statistical analysis revealed a significant difference in information processing speed between young and older adults; with young faster than older adults $[\mathrm{U}=194, p<.001$, effect size $\mathrm{r}=.73]$.

\section{Target plus distractors information processing speed.}

Mann-Whitney analysis revealed a significant difference of information processing speed between young and older adults (see Figure 2); young adults were significantly faster than older adults $[\mathrm{U}=129, p<.001$, effect size $\mathrm{r}=.77]$.

\section{Distractor effect (attentional efficiency) $\left[R \mathrm{~T}_{\text {Distractors }}-\mathrm{RT}_{\text {Target alone }}\right]$ : Information processing speed}

Mann-Whitney analysis revealed a significantly greater difference of RT between the two conditions in older adults; older adults were more affected by distractors (i.e. have poorer attentional shifting efficiency) compared to young adults $[\mathrm{U}=222, p<$ .001 , effect size $r=.71]$. 


\section{Information processing speed and subjective memory function}

In a novel approach, the information processing speed for each target condition and the distracter effect (attentional integrity) was examined with respect to subjective memory function in the older adult group.

Correlational analysis using Spearman's rho revealed no significant correlation between information processing speed and total MFQ score (subjective memory function) for target alone or the target plus distracters conditions [ $p$ values $>.05]$ nor with the distractor effect (attentional integrity) $\left[\mathrm{RT}_{\text {Distractors }}-\mathrm{RT}_{\text {Target alone }}\right][p>.05]$.

\section{Information processing speed and post- hoc measured perceived test difficulty}

In another novel approach the information processing speed for each target condition and the distracter effect was examined with respect to how difficult the participant reported the task to be (when asked after the test was completed). Correlational analysis using Spearman's rho revealed that for young adults, information processing speed did not significantly correlate with perceived test difficulty for target alone or the target plus distracters conditions [ $p$ values $>.05]$ or for the distractor effect (attentional integrity [all $p$ values $>0.05$ ]. For the young adults therefore, how difficult they perceived the task to be was not related to their actual performance, i.e., perceived task difficulty was not related to actual information processing speed.

In older adults, perceived test difficulty was significantly negatively correlated with information processing speed for the target alone condition $[\mathrm{r}=-.294, p=.031]$. Perceived test difficulty was significantly related to their actual performance i.e. the faster they performed the harder they perceived the test to be afterward. However there was no significant correlation between perceived task difficulty and information processing speed for the target plus distracters condition $[p>.05]$ or for the distractor effect (attentional integrity $[p>0.05]$.

RT for the two conditions (target alone and target plus distractors) was analysed in relation to perceived test difficulty being judged on the test as a whole. There was no significant difference in mean perceived test difficulty scores between young and older adults $[\mathrm{p}>.05]$. 


\section{Information processing speed and educational level}

Mean educational level was matched across both groups and for males and females as closely as possible making the results very similar between both age groups.

However a slight range in years of education was in evidence and thus we examined the results with respect to this narrow range of educational level.

In young adults, information processing speed did not significantly correlate with education for target alone or the target plus distractors condition or the distractor effect (attentional integrity) [ $\mathrm{RT}_{\text {Distractors }}-\mathrm{RT}_{\text {Target alone }}$ condition [all $p$-values $\left.>.05\right]$.

In older adults, there was no significant correlation in the target alone condition $[p>$ $.05]$, but information processing speed was significantly negatively correlated with education for the target plus distractors condition with faster information processing speed related to a higher level of education $[\mathrm{r}=-.398, \mathrm{p}=.003]$ and the mean distractor effect (attentional efficiency) was significantly negatively correlated with education, with greater education related with less distraction, i.e., less detrimental effect of distracting information $[\mathrm{r}=-.337, p=.013]$.

\section{Sex Comparison: Information processing Speed in males and females}

Figure. 4. Box plot of mean information processing speed between males and females for both young and older adults in target alone and target plus distractors trials.

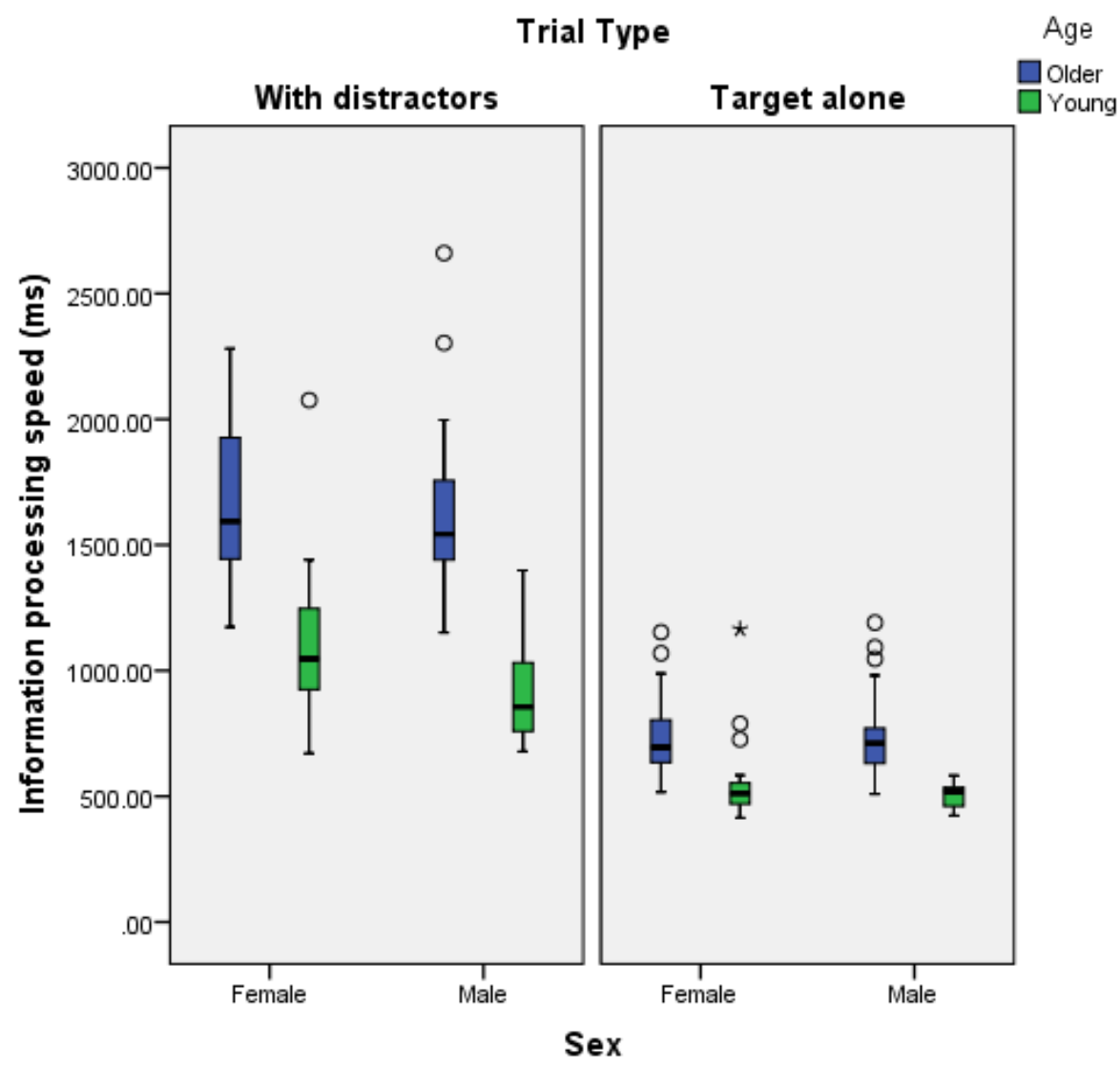


$\underline{\text { Target Alone }}$

Mann-Whitney analysis revealed no significant difference in reaction time between males and females for young adults [ $p>.05]$ or older adults $[p>.05]$.

\section{$\underline{\text { Target plus distractors }}$}

In young adults, Mann-Whitney analysis revealed a significant sex-related difference of information processing speed (see Figure 4); males were significantly faster in their reaction time performance compared to females $[\mathrm{U}=162, p=.021$, effect size $\mathrm{r}$ $=.33$ ]. In older adults, Mann-Whitney analysis revealed no significant difference in reaction time between males and females $[p>.05]$.

\section{Distractor effect (attentional integrity) $\left[\mathrm{RT}_{\text {Distractors }}-\mathrm{RT}_{\text {Target alone }}\right]$}

In young adults, Mann-Whitney analysis revealed that the effect of distracters was greater (i.e. attentional integrity poorer) for females compared to males $[\mathrm{U}=145, p=$ .008 , effect size $r=.38$ ]; females' attentional shifting was less efficient when distracters are present than males.

In older adults Mann-Whitney analysis revealed no significant difference of the distractor effect (attentional integrity) between males and females [ $p>.05]$.

\section{Information processing speed, anxiety and depression levels}

In young adults information processing speed significantly negatively correlated with depression levels; higher levels of depression related to faster information processing speed for the target alone condition $[\mathrm{r}=-.290, p=.045]$ but not for the target plus distractors condition $[p>.05]$. Anxiety levels did not significantly correlate with information processing speed for the target alone condition $[p>.05]$ or the target plus distractors condition $[p>.05]$.

In older adults, information processing speed did not significantly correlate with anxiety levels or depression levels for both conditions [all $p$ values $>.05$ ] 


\section{Information processing speed and objective cognitive performance (MoCA)}

In both young and older adults, there was no significant correlation between information processing speed and MoCA score (objective measure of general cognition) for the target alone, target plus distractors conditions, or the $\left[\mathrm{RT}_{\text {Distractors }}-\right.$

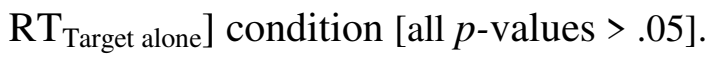

\section{Subjective memory function and perceived task difficulty in older adults.}

For the older adults, subjective memory function (total MFQ score) was not significantly correlated with perceived test difficulty for the target alone, target plus distractors conditions, or the $\left[\mathrm{RT}_{\text {Distractors }}-\mathrm{RT}_{\text {Target alone }}\right.$ condition [all $p$-values $\left.>.05\right]$.

\section{Educational level and perceived task difficulty.}

For the young adults educational level was not significantly correlated with perceived test difficulty $[p>.05]$. Difficulty was judged on the test as a whole thus the two conditions (target alone and target plus distractors) cannot be analysed separately.

For the older adults, educational level was significantly positively correlated with perceived test difficulty; the higher the level of education (in years), the more difficult the test was perceived to be $[\mathrm{r}=.440, p=.01$ effect size $=.44]$. The two conditions (target alone and target plus distractors) cannot be analysed separately as difficulty was judged on the test as a whole.

\section{Educational Level and subjective memory function in older adults.}

For the older adults educational level was not significantly correlated with subjective memory function $[p>.05]$.

\section{Anxiety and depression levels}

Anxiety levels significantly positively correlated with depression levels in young adults $[\mathrm{r}=.623, p<.001]$ and older adults $[\mathrm{r}=.534, p<.001]$. As anxiety levels increased so did depression levels. 
In older adults depression levels significantly positively correlated with education i.e. higher education related to higher levels of depression $[\mathrm{r}=.348, p=.010]$ and significantly positively correlated with perceived test difficulty; perceiving the test to be more difficult related to high levels of depression $[\mathrm{r}=.273, p=.046]$.

\section{Intraindividual variability (IIV)}

\section{Age Comparison: Intraindividual variability (IIV)}

Figure 5: Box plot of mean intraindividual variability (IIV) between target plus distractors and target alone in older and young adults.

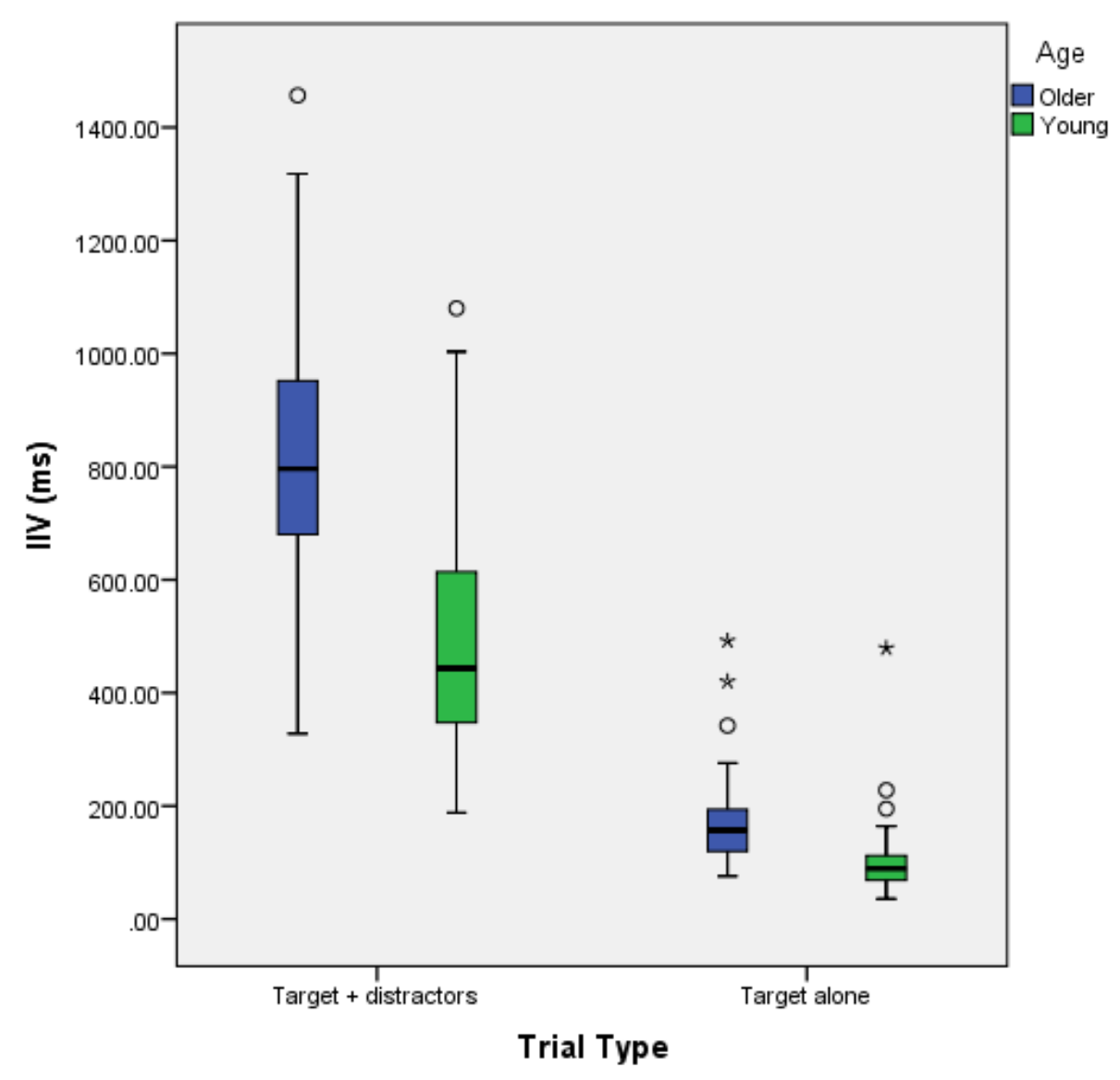


Figure 6. Box plot of mean difference in intraindividual variability (IIV) IIVDistractors - IIV $\mathrm{V}_{\text {Target alone }}$ (distractor effect i.e. attentional efficiency) between sex (male vs. female) and age (young vs. older adults).

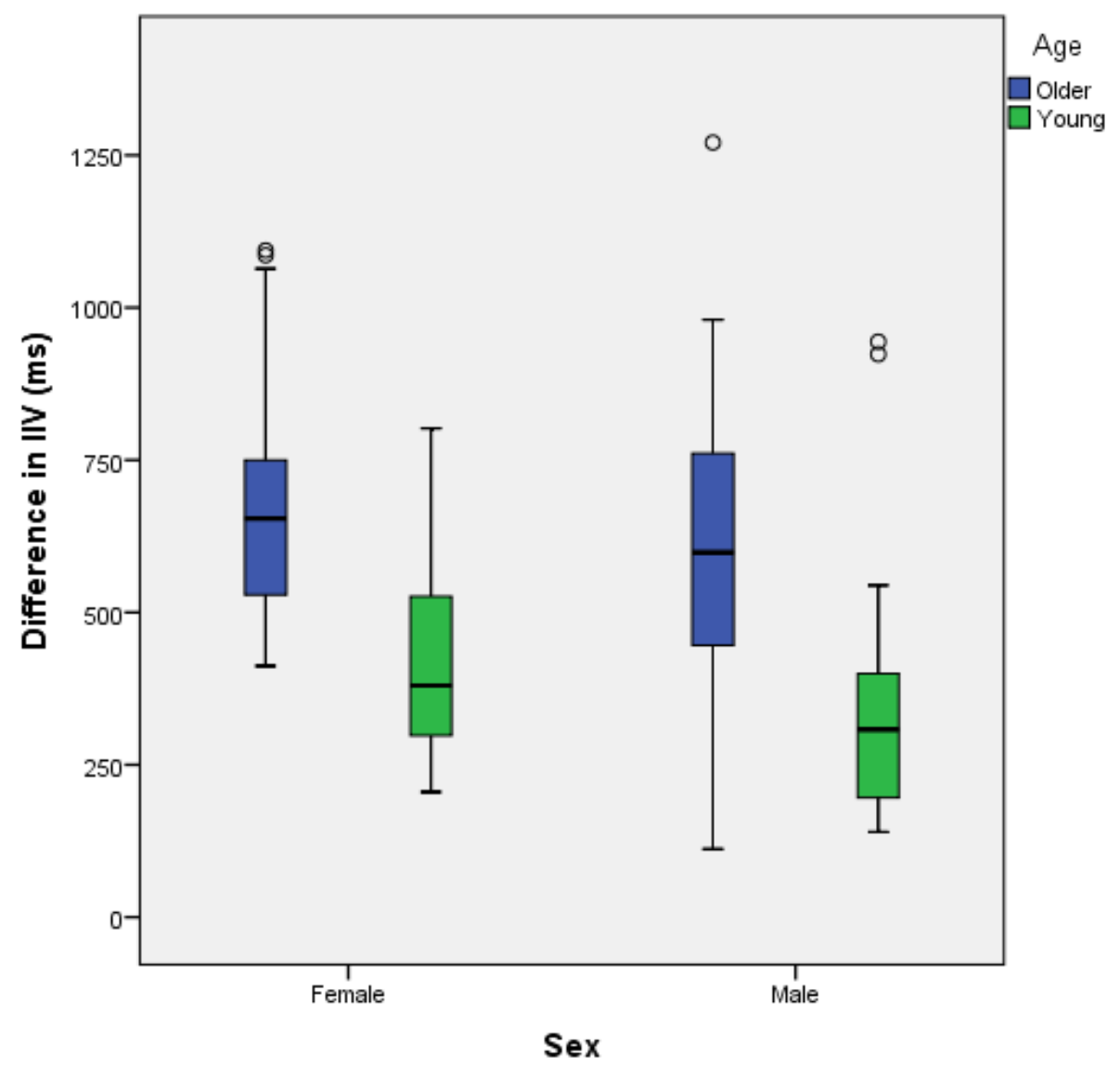

As displayed in Figure 5, at group level the older adults were more variable over both target alone and target plus distracter conditions, and intraindividual variability was greater for both groups in the target plus distracter compared to the target alone conditions. The effect of distracting information upon intraindividual variability $\left[\mathrm{IIV}_{\text {Distractors }}-\mathrm{IIV}_{\text {Target alone }}\right]$ (see Figure 6) was also greater (i.e. poorer attentional integrity) for the older compared to the younger adults. Note that there are outliers in this data in both target conditions but particularly in the Target Alone condition.

\section{Intraindividual variability between conditions}

There was a significant difference in IIV between the two visual search conditions; RT performance was significantly more variable in the target plus distractors condition compared the target alone condition in young adults $[\mathrm{U}=49, p<.001$, effect size $\mathrm{r}=.84]$ and older adults $[\mathrm{U}=3, p<.001$, effect size $\mathrm{r}=.86]$. 


\section{Target alone intraindividual variability}

Mann-Whitney analysis revealed a significant difference of IIV between young and older adults; young adults were less variable in their reaction time performance compared to older adults $[\mathrm{U}=341, p<.001$, effect size $\mathrm{r}=.63]$.

\section{Target plus distractors intraindividual variability}

Mann-Whitney analysis revealed a significant difference of IIV between young and older adults (see figure 3); young adults were less variable in their reaction time performance compared to older adults [ $\mathrm{U}=357.5, p<.001$, effect size $\mathrm{r}=.62]$.

\section{Distraction effect (attentional integrity) [IIV $\left.\mathrm{Distractors}_{-}-\mathrm{IIV}_{\text {Target alone }}\right]$ :} Intraindividual variability.

Mann-Whitney analysis revealed a significantly greater difference of RT variability between the two conditions in older adults; older adults were effected more by distractors (i.e. have poorer attentional integrity) compared to young adults [ $\mathrm{U}=472$, $p<.001$, effect size $\mathrm{r}=.55]$.

\section{Intraindividual Variability and subjective memory function in older adults}

Correlational analysis using Spearman's rho revealed that for older adults, the total MFQ score (subjective memory function) did not significantly correlate with intraindividual variability for target alone and target plus distractors conditions [all $p$ values $>.05]$ nor did the distractor effect (attentional integrity) [IIV $\mathrm{Vistractors}-\mathrm{IIV}_{\text {Target }}$ alone] [all $p$ values $>0.05$ ].

\section{Intraindividual Variability and perceived test difficulty}

Correlational analysis using Spearman's rho revealed that for young adults, intraindividual variability did not significantly correlate with perceived test difficulty for target alone or the target plus distracters conditions [ $p$ values $>.05]$ or for the distractor effect (attentional integrity) [all $p$ values $>0.05$ ]. For the young adults therefore, how difficult they perceived the task to be was not related to their actual performance, i.e., perceived task difficulty was not related to actual intraindividual variability. 
In older adults, intraindividual variability did not significantly correlate with perceived test difficulty for target alone or the target plus distracters conditions $[p$ values $>.05$ ] or for the distracter effect [all $p$ values $>0.05$ ]. In older adults, how difficult they perceived the task to be was not related to their actual performance, i.e., perceived task difficulty was not related to actual intraindividual variability.

The two conditions (target alone and target plus distractors) cannot be analysed separately as difficulty was judged on the test as a whole.

\section{Intraindividual Variability and educational level}

In young adults, intraindividual variability did not significantly correlate with education for target alone and target plus distractors conditions [ $p$ values $>.05]$ or the $\left[\mathrm{IIV}_{\text {Distractors }}-\mathrm{IIV}_{\text {Target alone }}\right.$ attentional function condition [all $p$-values $\left.>.05\right]$.

In older adults, intraindividual variability significantly negatively correlated with education for target plus distractors $[\mathrm{r}=-.354, p=.009]$ but not for the target alone condition $[p>.05]$ or the $\left[\mathrm{RT}_{\text {Distractors }}-\mathrm{RT}_{\text {Target alone }}\right]$ condition [all $p$-values $\left.>.05\right]$.

\section{Sex Comparison: Intraindividual variability}

Figure. 7. Box plot of mean intraindividual variability (IIV) between males and females for both young and older adults in target alone and target plus distractors trials.

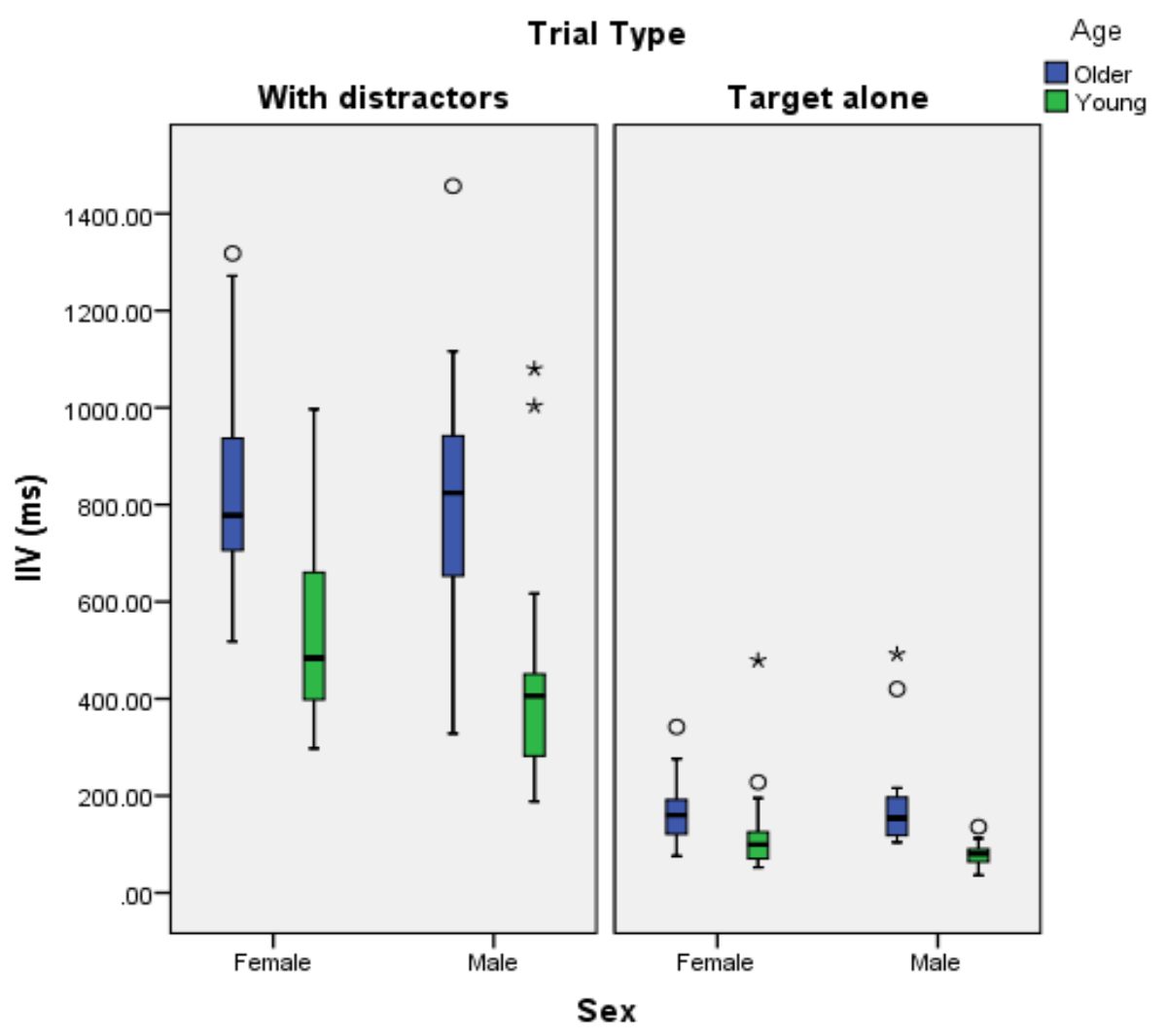


$\underline{\text { Target Alone }}$

In young adults, Mann-Whitney analysis revealed no significant difference in IIV between males and females [ $p>.05]$. In older adults, Mann-Whitney analysis revealed no significant difference in reaction time variability between males and females $[p>.05]$.

\section{$\underline{\text { Target plus distractors }}$}

In young adults, Mann-Whitney analysis revealed a significant difference of IIV between sex (see Figure 5); males were significantly less variable in their reaction time performance compared to females [ $\mathrm{U}=151, p=.011$, effect size $\mathrm{r}=.37$ ]. In older adults, Mann-Whitney analysis revealed no significant difference in reaction time variability between males and females $[p>.05]$.

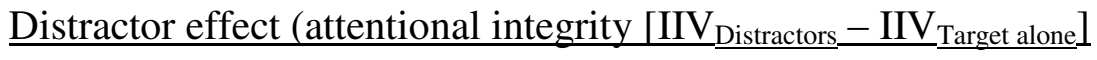

In young adults Mann-Whitney analysis revealed a significant difference of the mean effect of distractors on IIV between sex; the effect of distractors were greater (i.e. poorer attentional integrity) for females compared to males $[\mathrm{U}=173.5, p=.040$, effect size $r=.30]$.

In older adults, Mann-Whitney analysis revealed no significant difference of the distractor effect (attentional integrity) between males and females [ $p>.05]$.

\section{Intraindividual variability, anxiety and depression levels}

In young adults IIV significantly negatively correlated with depression levels; for the target plus distractors condition i.e. higher levels of depression related to less varied information processing speed $[\mathrm{r}=-.330, p=.008]$ and the effect of distractors (attentional integrity) on IIV i.e. better attentional integrity related to higher levels of depression [ $\mathrm{r}=-.304, p=.036]$. There was no significant correlation between IIV and depression for the target alone condition [ $p>.05]$. Anxiety levels did not significantly correlate with IIV for the target alone condition $[p>.05]$ or the target plus distractors condition $[p>.05]$.

In older adults, IIV did not significantly correlate with anxiety levels or depression levels for both conditions [all $p$ values $>.05$ ]. 


\section{Intraindividual variability and objective cognitive performance (MoCA)}

In young adults there was a significant positive correlation between IIV and MoCA score i.e. having better general cognition related to being less variable in RT performance for the target alone condition $[\mathrm{r}=.308, p=.033]$ but not for the target plus distractors condition, or the $\left[\mathrm{RT}_{\text {Distractors }}-\mathrm{RT}_{\text {Target alone }}\right]$ condition [all $p$-values $>$ $.05]$.

In older adults, there was no significant correlation between information processing speed and MoCA score (objective measure of general cognition) for the target alone condition, the target plus distractors condition, or the $\left[\mathrm{RT}_{\text {Distractors }}-\mathrm{RT}_{\text {Target alone }}\right]$ condition [all $p$-values $>.05]$.

\section{RT and IIV}

In young adults, RT significantly positively correlated with IIV; as RT increased (i.e. slowed), IIV also increased (i.e. RT performance was more variable) for the target alone condition $[\mathrm{r}=.517, p<.001]$ and the target plus distractors condition $[\mathrm{r}=.805$, $p<.001]$.

In older adults, RT significantly positively correlated with IIV; as RT increased (i.e. slowed), IIV also increased (i.e. RT performance was more variable) for the target alone condition $[\mathrm{r}=.638, p<.001]$ and the target plus distractors condition $[\mathrm{r}=.528$, $p<.001]$.

\section{Errors in visual search}

Table 4. Mean number of errors (incorrect button presses) for both conditions of visual search (target alone and target plus distractors) for young and older adults

\begin{tabular}{|l|c|c|}
\hline & $\begin{array}{c}\text { Target } \\
\text { alone }\end{array}$ & $\begin{array}{c}\text { Target }+ \\
\text { Distractors }\end{array}$ \\
\hline $\begin{array}{l}\text { Young } \\
\text { adults }\end{array}$ & $0.60(1.01)$ & $0.63(1.14)$ \\
\hline $\begin{array}{l}\text { Older } \\
\text { Adults }\end{array}$ & $0.50(0.75)$ & $0.38(0.58)$ \\
\hline
\end{tabular}




\section{Target alone}

Mann Whitney analysis revealed no significant difference in the mean number of errors made between young and older adults [p $>.05]$.

$\underline{\text { Target plus distractors }}$

Mann Whitney analysis revealed no significant difference in the mean number of errors made by young and older adults [p > .05].

Table 5. Mean number of errors (incorrect button presses) for both conditions of visual search (target alone and target plus distractors) for males and females in young and older adults

\begin{tabular}{|l|c|c|}
\hline & $\begin{array}{c}\text { Target } \\
\text { alone }\end{array}$ & $\begin{array}{c}\text { Target }+ \\
\text { Distractors }\end{array}$ \\
\hline Young males & $0.29(0.47)$ & $0.12(0.33)$ \\
\hline $\begin{array}{l}\text { Young } \\
\text { females }\end{array}$ & $0.89(1.28)$ & $1.11(1.41)$ \\
\hline Older males & $0.63(0.90)$ & $0.64(0.64)$ \\
\hline $\begin{array}{l}\text { Older } \\
\text { females }\end{array}$ & $0.38(0.59)$ & $0.15(0.38)$ \\
\hline
\end{tabular}

\section{$\underline{\text { Target alone }}$}

In young adults, Mann Whitney analysis revealed no significant difference in the number of errors made between males and females [ $p>05]$. In older adults there was no significant difference in the number of errors made between males and females $[p>.05]$.

\section{$\underline{\text { Target plus distractors }}$}

In young adults, Mann Whitney analysis revealed a significant difference in the number of errors made between males and females; females made significantly more errors compared to males [ $\mathrm{U}=186.5, p=.019$, effect size $\mathrm{r}=.32]$. In older adults there was no significant difference in the number of errors made between males and females $[\mathrm{p}>.05]$. 


\subsection{DISCUSSION}

In this chapter a visual search test was used to examine the integrity of attentional function i.e. ability to shift around focus of attention, information processing speed and its variability in young and old adults as well as the potential effects of subjective memory function, perceived test difficulty, educational level and sex. This task has been used frequently in previous studies to measure potential differences in information processing speed associated with visual attention-related processing in healthy ageing, MCI and AD. However unlike previous studies, this current study measures information processing in larger groups of both younger and older adults and examines in greater detail the potential influence upon such results of such factors either poorly addressed (sex and education) nor not addressed in previous studies (perceived test difficulty and subjective memory function). How these previously ignored factors may affect may influence in future how we in fact interpret the results of visual search test and especially in terms of what constitutes an ostensibly cognitively health older adult control group in studies of MCI and dementia.

\section{Information Processing Speed}

\section{Age comparison in information processing speed}

The target alone condition represented feature search i.e. automatic processing of the same target with no distracting information (Choice RT). Older adults were significantly slower in their reaction time (RT) performance (i.e. slower information processing speed) compared to young adults as reflected by a large effect size. The target alone condition allows for a measure of basic reaction time between groups [Tales et al, 2010] thus what the results imply is that older adults' information processing speed is significantly slower compared to young adults. This result supports previous evidence that information processing speed slows with age [Mella et a, 2013; Dykiert et al, 2012; Fozard et al, 1994] and that older adults perform feature search slower in a visual search test compared to younger adults [Self et al, 2016] which has not always been found in feature search [Muller-Oehring et al, 2013]. This result implies that older adults are slower at processing a single target compared to young adults despite attention towards the target being more automatic i.e. 'pop out' effect. 
The target plus distractors condition represented conjunction search i.e. shifting the focus of attention throughout the visual field (i.e., through each stimulus) before finding and processing the target. Young adults were significantly faster at conjunction search compared to older adults (as demonstrated by a large effect size) which suggests that older adults take longer to shift their attention through the same number of distractors compared to young adults and take longer to process the information at each object stopped at. As a result, searching for a target takes longer thus performance (information processing speed). This supports the findings and conclusions drawn from previous visual search studies [Port et al; 2016; MullerOehring et al., 2013; Porter et al, 2010; Madden et al, 2007; Greenwood et al, 1997; Scialfa \& Joffe, 1997].

Anxiety did not associate with RT in young and older adults implying that ageing effects were more influential on information processing speed than levels of anxiety although levels were reported to be low. In contrast, higher levels of depression in young adults was associated with faster RT which may have influenced mean RT in young adults being faster compared to older adults although depression levels were within 'normal' levels. We are uncertain why higher depression would improve information processing speed thus requires further investigation.

When comparing feature and conjunction feature search within both young and older adults, target alone trials (feature search) produced faster reaction times compared to when distractors were present (conjunction search). This supports the notion that salient information is processed quicker than non-salient information [Tales et al, 2004]. Salient information can quickly and automatically guide our attention towards it, whereas when distractors are present, this requires shifting through the irrelevant information which takes more time on a limited attentional processing system, therefore produces greater reaction times (i.e. slower information processing speed) [Plude \& Doussard-Roosevely; Treisman \& Gelade. 1980]. Differences between feature and conjunction search may also be due to the differences in processing demands of the two tasks [Phillips et al, 2013]. Including distractors increases the complexity of the task which requires more information processing resources i.e. greater processing load. An increase of processing load results in greater effects of different factors i.e. age on information processing speed. This was observed in this study with older adults being affected greatly by distractors thus their information speed was significantly slower compared to young adults. 
One reason for using the visual search test was to examine a larger number of participants compared to some previous studies which included smaller samples and recognised as a limitation. However, the results of the current study supported the results of these smaller studies. This implies that the sample size may not be as important and the visual search test itself i.e. visual search is a sensitive test to distinguish differences of RT between young and older adults.

\section{Distractor effect (attentional efficiency)}

In older adults, there was a greater difference of RT between the target plus distractor condition and the target alone condition compared to young adults, as reflected by the large effect size. This implies that older adults are affected more by distractors i.e. for the same amount of distracters it takes a lot longer for older adults to shift their attention through them and orient attention towards the intended target [Langley, 2011; Trick et al 1998]. Therefore attentional function (i.e. shifting and disengagement) is less efficient with age as reflected by slower information processing speed in older adults compared to young adults [as supported by Greenwood et al, 1997].

Overall, the results suggest that information processing speed in relation to visual search (i.e. components of selective attention) is negatively affected by ageing which supports previous findings of information processing speed increasing with age during visual search [Self et al., 2016] and general findings that information processing slows with age [Nilsson et al, 2014; Papp et al, 2014; Kerchner et al, 2012; Ylikoski, Ylikoski, Erkinjuntti et al, 1993].

\section{Subjective memory function in older adults}

In older adults, information processing speed performance had no significant relationship to subjective memory function in either condition (target alone or target plus distractors). Since there is a close relationship between information processing speed and structural change i.e. reduced white matter, finding no relationship between information processing speed and subjective memory function in these community dwelling adults, may suggest that any slowing of information processing speed is unlikely to relate to detrimental structural change particularly since the majority participants in this study performed to 'normal' levels in objective testing (MoCA score)[see Torrens-Burton et al, 2017 for similar discussion]. 
Any slowing of information processing speed is more likely to relate to healthy ageing and thus at group level, these older adults accurately represent a healthy control group. However there were a minority of older adults with lower MoCA scores which may imply abnormality with general cognition although we only used one test of general cognition thus further assessment is required.

These results support previous findings that there is no direct relationship between subjective memory complaints and cognitive performance i.e. information processing speed [Minett et al, 2007; ] however the previous studies examined SCI and not subjective memory function which was a novel approach in the current study. Since the current study did not find subjective memory function to relate to information processing speed, it may be the case that subjective complaints in these ostensibly healthy older adults are not having significant effect on RT thus information processing speed remains at 'normal levels' expected in ageing. In an attempt to help with the characterisation of SCI, the results may suggest that SCI may not disproportionately slow information processing speed in the older adults taken from the general population. This can only be speculated at present as the older adults who perceived changes to their memory function could not be followed up to observe whether they were later diagnosed with SCI.

Past studies suggest that slowing of information processing speed may be influenced by anxiety and depression as a result of subjective cognitive complaints [ Gale et al, 2016; Yates, et al 2015; Ansell \& Bucks, 2006; Barker et al,1994; Sevush \& Leve, 1993 ] although this has not been examined in relation to subjective memory fucntion. Anxiety and depression are treatable conditions thus once levels are low, 'normal' levels of slowing expected in healthy adults may be observed thus older adults no longer perceive as many memory changes. However this study recognised that depression and anxiety can confound RT studies [as discussed in Tales \& Basoudan, 2016] thus levels were controlled for and were low and no relationship was found between neither anxiety nor depression and information processing speed nor was a relationship found between depression levels and subjective memory function.

Alternatively, finding a lack of a relationship of subjective memory function in visual search test may suggest that visual search test is not sensitive enough to detect any changes of information processing speed in this population of older adults with perceived changes to their memory function. 
However, it must be noted that the ratio of subjective memory function scores was uneven with less people perceiving changes to their memory compared to those who did not perceive memory changes. Therefore there may have been too few older adults with perceived memory changes in order to find a relationship with information processing speed.

This uneven ratio may have occurred either because these older adults have no memory changes to notice or there is the possibility that any changes are not being recognised for what they are, especially if changes are to other aspects of cognition other than memory. It may have been of use to measure memory in more detail to determine whether subjective perceptions about memory function were legitimate or any memory changes were missed. In addition it may have been of use to measure older adults' perception of attentional function since RT in relation to attention was being measured.

Despite finding no relationship there were still some extreme RT values (i.e. outliers) i.e. some individuals with perceived changes to memory (low MFQ score) were performing significantly slower. These individuals may be of clinical significance and highlight valid subjective concerns which may represent undiagnosed SCI or subjective memory function. These individuals may have affected the results and misrepresenting a healthy older adult control group. Therefore further assessment and follow up in these particular individuals will be of interest to observe whether they should be diagnosed with SCI and information processing speed is significantly slowed as a result.

\section{Perceived test difficulty and information processing speed}

In young adults, there was no relationship between information processing speed and perceived test difficulty. How difficult the test (as a whole) was perceived to be was not related to their information processing speed. Therefore we speculate that this particular psychological factor/self-assessment does not influence information processing speed in young adults during visual search. However, in this study only one example of a psychological factor was examined against information processing speed (perceived test difficulty). It may be possible that if young adults were examined against other factors of self-assessment, the relationship with information processing speed may differ. 
In older adults, perception of test difficulty significantly negatively correlated with information processing speed in the target alone condition. The more difficult the test was perceived to be, the faster their performance was.

This result implies that older adults' self assessment or judgement of the demands of the task (during performing the test but asked about it afterwards) may be related to information processing speed. This supports Flavell [1979] that the demands of the task can affect the outcome of a task (in this case the speed of performance). People can have the propensity to believe a test to be difficult despite performing well (as discussed in the introduction to this thesis). Therefore peoples' perception influences information processing speed but not in a way that reflects actual performance.

On group level, the average level of difficulty perceived by older adults was not significantly greater to young adults i.e. both groups perceived visual search to be of a similar level of difficulty. Despite this, perceived test difficulty only influenced information processing speed in older adults possibly as older adults have greater expectations than young adults of how difficult the test may be and how they are likely to perform i.e. more critical or alternatively more unfamiliar with the test. However, it must be noted that the effect size was small and it is difficult to judge the relationship completely since the participants were scoring the test as a whole and not judging the two conditions separately. Older adults finding the visual search be more difficult also reported higher levels of depression which may relate to lower mood influencing perception of the test thus having an effect on information processing speed [as discussed in Setti et al, 2015; Bolmont, et al, 2000]. However perceived test difficulty was found to have a positive effect on information processing speed i.e. faster RT and there was no significant relationship between depression levels and information processing speed in older adults. In addition the relationship between perceived difficulty and depression levels was only just within significant level.

For older adults in the local community, psychological factors such as perceived test difficulty, specifically perceived test difficulty, may be influencing information processing speed during attentional processing in visual search rather than physical changes to brain function (albeit a small effect). This suggests that past research measuring selective attention and information processing speed using visual search in control groups may be flawed by neglecting to include perceived test difficulty as an influential factor i.e. healthy older adult control group are inaccurately represented. 
However at present we could only speculate that no physical changes were present in older adults since having no access to brain scans and follow up assessment.

\section{Information processing speed and Educational level}

In young adults, education did not relate to information processing speed suggesting that education does not influence young adults during a visual search test. In older adults, education (even within the narrow ranges used) significantly negatively correlated with information processing speed during the target plus distractors condition (medium level effect sizes) and with the distractor effect but not during the target alone condition.

A greater level of formal education (in years) was related to faster reaction times and being less affected by the presence of distractors, i.e., more efficient attentional shifting and disengagement. The older adult result supports Tun and colleagues [2008] who found lower education to be associated with slower responses although in this current study, education was not split between low and high levels in comparison as the range of education (in years) was narrow. Higher education in older adults related to high levels of depression although demographics displayed depression to be at normal levels and depression was not associated with RT (or IIV).

The influence of education with older adults' reaction time implies that education can be a 'barrier' towards cognitive decline i.e. increases cognitive reserve. Cognitive reserve is the brains' protective barrier against disease which can explain how an individual with a high level of cognitive reserve does not manifest symptoms at the same time as an individual with low levels of cognitive reserve. People naturally have different levels of cognitive reserve with higher amounts allowing for a more effective use of cerebral networks [Sole-Padulles, Bartres-Faz, Junque et al., 2009] and different factors are said to increase cognitive reserve including but not restricted to education [Sattler, Toro, Schonknecht, \& Schroder, 2012; Meng \& D'Arcy, 2012].

Finding an effect of education was an important finding as although education was matched between both groups (young and older adults) as closely as possible, there was slight variation between both age groups. Even within the narrow range, educational level had an effect on information processing speed in older adults although the range was not varied enough to find an education effect in young adults. 
The results highlight that education is a factor to take into account when measuring information processing speed in older adults (i.e. control groups) during visual search [as suggested by Haworth et al, 2016].

Despite the influence of education, it must be noted that older adults were still slower with poorer attentional integrity compared to young adults suggesting that the factor of age has a stronger effect on information processing speed. This is reflected in greater effect sizes for age effects on information processing speed compared to education effects.

\section{Sex and information processing speed}

In the target alone condition (feature search), there was no difference of information processing speed between males and females which was found with both young and older adults. In the target plus distractors condition (conjunction search) there was no difference between males and females in older adults but in young adults, information processing speed was significantly faster in males compared to females (medium level effect size). In addition, young adult females were affected more by distractors i.e. poorer attentional integrity compared to males but again no effect was found in older adults.

Sex differences found in young adults supports past studies of selective attention finding that males are faster at disengaging their attention an invalidly cued location and redirecting attention towards the target location [Merritt et al, 2005; Frederikse, Lu, Aylward et al, 1999; Collins \& Kimura, 1997]. Males are more efficient at attentional shifting which is required during visual search particularly during conjunction search which requires shifting attention from distractor to distractor until the target is found. It is argued that the difference between male and female performance is due to hormone levels. It has been found that testosterone is associated with better cognitive performance [Muller et al, 2005] including RT performance [Fontani et al, 2004; Muller, 1994] which supports why the current results found young males to be faster than females. In addition, hormone levels in males level out in older adults which may have less influence on cognitive performance as a result. Therefore older male RT performance may not be as significantly different to females (as found in the current study). However this is simply speculation thus further investigation is required. 
Finding no sex difference in older adults supports some previous evidence [Solanik, Brazatis \& Skurvydas, 2016; Libzda, Ebner, Haiener et al, 2013] but contradicts some other previous findings that older adult males are faster than (older) females [Dykiert et al, 2012; Fozard et al, 1994].

Geary [1998] previously attempted to explain why sex differences may occur in young adults but not in older adults. From an evolutionary point of view, young adult males require better attentional processing and faster reactions compared to females i.e. for hunting, which may potentially explain why young males are faster than females and faster than older adults (particularly males). Alternatively, finding few sex effects may imply that the influence of sex on information processing speed is test specific thus the importance of examining a variety of different visual attention tests. This will be addressed in this thesis by examining a variety of different visual attentional tests in a second study to observe whether study outcome in relation to sex effects are similar across tests.

Sex effects were only discovered in young adults for the target plus distractors condition and not the target alone condition. This implies that sex may be dependent on conditions within an attention test as well as between different tests themselves. Sex differences of information processing speed may have reached significant levels in older adults if more males were included the sample as the majority of older adults were females. Having more males would have made for a better comparison with females thus possible finding a significant difference of RT between the two. However it may be the case that individuals' age is a more influential factor on the integrity of information processing speed and sex is not an important factor. This may imply that in research studies using visual search, sex should be taken into account depending on whether young adults are included as a comparison to older adults. Previous studies of attention-related information processing speed and ageing using visual search have either poorly addressed sex as a factor or failed to compare the effects of sex on information processing speed. In addition, the DSM 5 which has highlighted the importance of measuring information processing speed does not discuss sex effects in detail and its potential influence on the results of RT studies.

\section{Subjective memory function and Education}

The level of education (in years) older adults had did not relate to whether or not they perceived changes to their memory function. 
The fact that education had an influence on processing speed and not subjective memory function is an important finding as it suggests that it may not matter what people think about their cognition. Instead information processing speed is influenced by other factors i.e. education.

The positive influence of education on information processing speed supports that this factor can be beneficial for producing a barrier against cognitive deterioration i.e. cognitive reserve [Sattler et al, 2012; Meng \& D'Arcy, 2012]. Having higher levels of cognitive reserve results in the brain being more protected against disease and any symptoms of cognitive impairment are delayed. A lack of symptoms (such as impaired memory function) would reflect in older adult's perception of their memory i.e. they do not perceive anything is wrong thus report very few memory changes. Further examination using other attention tests are included in the current study to determine whether the level of education in older adults is similarly more influential on information processing speed and IIV and not subjective memory function.

\section{Subjective memory function and Perceived test difficulty}

In older adults there was no significant relationship between subjective memory function and perceived test difficulty. How difficult the visual search test was perceived to be did not relate to having perceived changes of memory function. This is an interesting finding as we would presume that if a person believes their cognition is poor, they would assume that tests will be more difficult to perform as a result. This may be because they believe that poor cognition will impair the ability to perform a cognitive task well enough thus making the task more difficult to complete. Since this direction was not found it may highlight further that subjective memory function is only a person's perception of their own ability with no practical influence (as discussed earlier with information processing speed).

\section{Education and perceived test difficulty}

In young adults there was no relationship between education and perceived test difficulty. The number of years in education had no influence on how difficult young adults perceived the visual search test to be. In older adults however, education positively correlated with perceived test difficulty; the higher the level of education (in years), the more difficult the test was perceived to be (medium level effects size). 
As discussed in the introduction to this thesis, the level of education an individual has may influence how difficult the test is perceived to be. If this is the case, it would be expected that higher levels of education to be beneficial i.e. higher levels of motor skill or concentration thus help tests appear less difficult. However, older adults found the test to be more difficult despite having higher education.

In addition better general cognition (MoCA score) in older adults also related to finding the test more difficult. This may be due to older adults being unfamiliar with the test thus expecting it to be more difficult than it was actually designed to be. Alternatively older adults may have different expectations i.e. be more critical of the test and thus their performance.

\section{Intraindividual variability (IIV)}

\section{Age comparison of IIV}

In the target alone condition, older adults were significantly more variable in their reaction time performance compared to young adults (reflected by a large effect size) implying that the integrity of information processing speed in older adults is poorer during feature search (finding a salient target) thus poorer integrity of CNS. This supports previous evidence that IIV increases with age [Mella et al, 2013; Dykier et al, 2012; Fozard et al, 1994; Inui, 1997] although these previous study findings were in relation to visual search. Lower IIV i.e. less varied RT performance in young adults may have related to finding an association between lower RT and better general cognition (high MoCA score) in the target alone condition i.e. having good general cognition may be reflecting good integrity of CNS thus less variability of RT performance.

In the target plus distractors condition, older adults were also significantly more variable (reflected by a large effect size) in their reaction time performance compared to young adults implying that the integrity of information processing speed (and brain function) in older adults is poorer in conjunction search. Older adults find it more difficult to focus attention away from distracting information thus are slower at shifting attention from one location to another until the target is found. 
Less variable RT performance and better attention integrity found in young compared to older adults may have been influenced by depression levels as higher levels of depression were related to lower IIV i.e. less varied RT performance although why higher depression would improve IIV requires further investigation.

When comparing the two conditions within both young and older adults, target alone trials (feature search) produced less varied reaction times compared to when distractors were present (conjunction search). This result implies that both young and older adults are less variable when shifting attention towards salient information than disengaging attention from non-salient information (distractors) and shifting attention towards a target [Plude \& Doussard-Roosevely; Treisman \& Gelade. 1980].

\section{Integrity of Attention (distractor effect)}

In older adults, there was a greater difference of IIV between the distractor condition and the target alone condition compared to young adults. This is a strong effect as the effect size was large. This implies that older adults had a poorer ability to shift attention away from distracting information and focus attention on the target thus increasing variability of RT performance.

Again this finding is similar to the findings with information processing speed suggesting that the integrity of attentional shifting and disengagement is poorer as age increases. As mentioned above, the inclusion of distractors increases the complexity of the task which in turn requires more information processing resources i.e. greater processing load. An increase of processing load results in greater effects of different factors i.e. age on information processing speed and IIV, in this case older adults being affected greatly by distractors thus their information speed was significantly slower and more varied compared to young adults.

In both young and older adults as RT increased (i.e. slowed), IIV also increased (i.e. RT performance was more variable) in both the target alone and target plus distractors condition. Despite this significant correlation between RT and IIV, both measures were not influenced in the same way by person-related factors i.e. perceived test difficulty significantly correlated with RT but not with IIV. This implies that information processing speed may be more effected by perceived test difficulty compared to IIV thus this relationship should be focused on in future visual search studies. 
In addition, effect sizes were greater when comparing RT between young and older adults suggesting that information processing speed better distinguishes differences between young and older adults.

\section{Subjective memory function and intraindividual variability}

In older adults, IIV performance had no significant relationship to subjective memory function. Any changes in IIV were not reflected in their subjective perception about their memory (SCI measure).

The same finding was found for information processing speed thus suggesting that any variation of information processing speed was unlikely to be the result of an influence of peoples' perception of their memory function. Arguably therefore, it would not have mattered at mean group level if older adults reported perceived changes to their memory before visiting their GP (no formal SCI diagnosis). Variation of information processing speed is more likely to relate to healthy ageing and thus at group level, these older adults accurately represent a healthy control group. However a limitation in the current study was failing to include specific memory tests and only a score of overall general cognition (MoCA). More specific memory tests may have provided more detail of memory function thus we may have found more relationships with subjective memory function.

There were still some extreme IIV values (i.e. outliers) i.e. some individuals with perceived changes to memory were performing significantly more variably lower. These individuals may be of clinical significance and highlight valid subjective concerns which may represent undiagnosed SCI. These individuals may have affected the results and misrepresenting a healthy older adult control group. Therefore further assessment and follow up in these particular individuals will be of interest to observe whether further examination leads to a SCI diagnosis of which information processing speed is significantly more varied as a result (implying poorer integrity of information processing speed and related brain function).

\section{Perceived test difficulty and intraindividual variability}

There was no significant relationship between IIV and the perceived difficulty of both conditions in visual search for both young and older adults. This is interesting as older adult information processing speed, did relate to perceived test difficulty albeit a small relationship (i.e. small effect size thus possibly not very robust). 
Since IIV has not been investigated as thoroughly in relation to visual search as information processing speed, understanding why only information processing speed was found to be influenced by perceived test difficulty requires further examination although one explanation may be that information processing speed is greatly more effected by perceived test difficulty compared to IIV.

\section{Education and intraindividual variability}

In young adults, there was no association between educational level and IIV which was also found between education and information processing speed. In older adults, education significantly negatively correlated with intraindividual variability for the target plus distractors condition. A greater level of formal education (in years) was related to less variation of reaction times (medium level effect size). This implies that a greater level of education helps the integrity of the CNS thus variability is low. It has been argued that higher levels of education relates to greater cognitive reserve i.e. brain's resilience to disease [Sattler et al, 2012; Meng \& D'Arcy, 2012].

The findings are similar to those for information processing speed although in information processing speed a relationship was also found between education and attentional integrity (distractor effect). This is also an important finding as although education was matched between both groups (young and older adults) as closely as possible, there was some variation and even in this narrow range, educational level had an effect on IIV in older adults.

\section{Sex and intraindividual variability}

In the target alone condition (feature search), there was no difference of IIV between males and females which was found with both young and older adults. In the target plus distractors condition (conjunction search) there was no difference of IIV between males and females in older adults. In contrast, information processing speed in young adults was significantly less varied in males compared to females. In addition, young adult females were affected more by distractors i.e. poorer attentional integrity compared to males but again no effect was found in older adults (medium level effect sizes). Similar results were found for information processing speed therefore sex appears to be only influential on young performance and young females during visual search as well as being dependent on components within the visual search. 
One suggestion is that sex differences in RT variability may be due to the effect of sex hormones on the brain [Dykiert et al, 2012; McEwan, 2001; Hampson, 1990]. For example oestrogen levels may affect attention systems [McEwen, 2001] and as oestrogen levels are high, cognitive performance in tasks which males tend to be better at performing i.e. spatial ability, is poorer in females [Hampson, 1990].

Therefore we speculate that this supports finding a sex difference in young adults and not in older adults as in older adulthood, hormone levels have levelled out. However, oestrogen has not always been found to influence RT variability [Low, Anstey, Jorm et al, 2006; Wegesin \& Stern, 2004). This outcome variability is argued to reflect study limitations i.e. small sample sizes and further replication required [Dykiert et al, 2012].

\section{Outliers}

As with information processing speed, outliers were more prominent in the target alone condition and the distractor effect (attentional efficiency) in older adults but with extreme values (see Figure 2) in young adults. When examining sex and information processing speed outliers were also predominantly in older adults (male) target alone condition but more extreme in young (female).

With intraindividual variability, outliers were more prominent in the target alone condition and distractor effect in older adults (and with one extreme value) but also with extreme values in young adults (see Figure 5). There was no single individual highlighted as a single outlier in each condition but a number of different participants were highlighted as outliers with significantly slower and more varied information processing speed. These outliers may have influenced the level of difference between young female and male information processing speed thus finding young males to be significantly faster compared to young females. Therefore, it will be of interest to examine these individual young females in greater detail to determine why their information processing speed is significantly slower compared to the mean result.

Outliers i.e. disproportionately slower RT in young adults are speculated to be the result of a lack of concentration or settling into the task (if the outlier occurred at the very beginning of the task) rather than any significant deterioration of cognitive processing [discussed in Torrens-Burton et al, 2017, Haworth et al, 2016. 
However it cannot be ignored that these adults may be poor at the task or despite being young, may be displaying early signs of further cognitive decline.

Understanding which explanation is more likely requires further examination and follow up. Large outliers, particularly the extreme values, in older adult information processing speed and IIV may be highlighting discrete issues in cognitive functioning which require further assessment and follow up.

\section{Errors}

Errors were related to pressing the wrong button for the designated response i.e. responding incorrectly to the direction of the arrow stimulus. In both the target alone condition and the target plus distractors conditions there was no significant difference in the number of errors made between young and older adults. This does not support past research finding more errors made by older adults in visual search [Madden et al, 1999; Madden, Turkington, Provenzale et al, 2002]. When comparing sex, in the target alone condition there was no significant difference in the number of errors made between females and males in both young and older adults. In the target plus distractors condition, there was no significant difference in the number of errors made between older adult males and females but in young adult females made significantly more errors compared to males.

Errors which were made by young adults (in particular you females) may have been attributed to a brief slip of concentration or making a simple mistake due to performance anxiety although there was so significant correlation between anxiety levels and the number of errors in each condition. In contrast, errors made by older adults may have reflected impairment of cognitive processing, in this case selective attention resulting in the inability to respond correctly. If cognitive impairment is present in a particular individual this may raise the mean number of errors although there were no one individual older adult singled out for making a significant amount of errors compared to others. In addition, for both young and older adults the individuals presenting outlying RT or IIV did not relate to the individuals who made the greatest number of errors. This implies that outliers made by individuals related to responses which were correct but were disproportionately slower or more varied.

Alternatively the errors made by both young and older adults may have related to a speed/accuracy trade-off which is found to occur in RT studies [van Veen, Krug \& Carter, 2008; Brebien, 2001]. 
This trade-off refers to individual differences in whether a person places more emphasis on speed or accuracy when performing RT tests. Young adults were significantly faster compared to young adults thus may have placed more emphasis on speed resulting in errors being made.

In contrast older adults were significantly slower and made fewer errors compared to young adults (although on group level this difference was not significant). Older adults may have placed more emphasis on performing the test more accurately than performing quickly. Indeed it has been observed previously that older adults place more emphasis on accuracy rather than speed during RT tests [Brébion, 2001;

Salthouse, 1979]. We tried to control for this speed/accuracy trade off by instructing all participants to be as fast and as accurate as possible. However the results highlight that there still individual differences in which aspect of the RT test a person holds more value to.

A greater number of errors made (particularly in young adult females) appeared to depend on the condition in the visual search test which may relate to the differences in processing demands of the two tasks [Phillips et al, 2013]. The more complex the task is (i.e. target plus distractors condition compared to target alone condition), the more information processing resources are required as more brain areas have to be recruited. The result of an increase of processing load may increase strain on mental resources which may provide greater capacity for making an error.

It could have been useful to look at practice and fatigue effects i.e. the number of trials having effect on RT or IIV. This analysis was not possible due to the way the visual search paradigm was programmed. Therefore examining the number of trials was included in a different test with multiple trials within the current research (Choice reaction time test).

\section{Study Limitations}

Despite this study trying to address the limitations from other methodologies, it still has its potential limitations. There was a gender bias towards females who were willing to participate in psychological research as well as variability between the participants themselves, for example the level of education although this was matched as far as possible. 
When beginning this study, the inclusion criteria for older adults included a maximum age cut off of 70 years however this was limiting the number of participants available to achieve larger sample sizes compared to some previous visual search studies. Therefore increasing the age limit improved the number of participants however it did not change the ratio of females to males. Similar issues occurred within recruiting both sexes for the young and older adult populations. Attaining the required number of males was difficult due to more females of both age groups being willing to participate which may have made the results not as generalizeable to both the young and older adult populations. Therefore, this should be taken into account when comparing sex in both older and young adults thus the requirement to recruit a greater number of young and older adult males.

In addition there were not enough older adults with or without subjective memory complaints in order compare RT and IIV between those with subjective memory complaints and those without subjective memory complaints. This may also have explained why no relationship was not found between subjective memory function and information processing speed or IIV i.e. a higher number of older adults perceived no changes to their memory function compared to those perceiving no memory changes.

A longitudinal study design would be beneficial to observe whether the older adults used in the study went on to develop SCI, especially those individuals who perceived changes to their memory function or those who produced disproportionately slower and more varied RT i.e. outliers. This may help to further characterise how RT and IIV of attention is affected in SCI if a relationship is found between subjective memory function and the development of SCI. A longitudinal study could not be achieved since only having a limited time to conduct the current research studies.

Other factors may have had an effect on reaction time performance but were difficult to control for. For example, older adults were likely to be on different medications or have other conditions which may have an effect on reaction time performance (e.g. diabetes) or which can cause difficulties in using the equipment available i.e. trying to press the spacebar for a long period of time with arthritic hands. This may have had an effect on the generalizability of results i.e. not reflecting true RT performance for cognitively healthy older adults. Medication could not be controlled for but those most likely to affect attention were excluded from the study. 
Acquiring an accurate and consistent reaction time from responding to the trial relied on the participant keeping their eyes focused on the cross at the beginning of each trial before searching for the target. This required the researcher to keep a close watch during the test which may have caused small levels of performance anxiety for the participant thus being unable to perform to their optimum level. The effects of distraction could have been transferred onto having a researcher in the room [Tales et al, 2011] therefore compromising the performance of the participant. It would have been beneficial to measure eye movement with the use of an eye tracker to check they were on track which is a potential measure for future testing.

With computers there is always a minute delay between a persons' natural reaction time to a stimulus and the time it takes for the software to record the RT when a keyboard button is pressed. This is a latency effect although it is usually not significant enough to make a significant effect on results as it is corrected for by computer programmes [Woods et al, 2015] although it may have an effect on 'true' RT performance of an individual. The programme used for this test tried to minimise this effect and the same computer was used for all participants thus any latency effect which was present would have effected all participants similarly. Nevertheless it is important to keep this latency effect in mind when measuring and comparing computer based reaction time tasks. There is also a question of the reliability of subjective score raising queries as to the honesty of the participants' responses about their cognitive concerns.

It would be of interest to ask participants about how they feel about their attentional processing and compare this with their actual attentional performance during the visual search. Although direct questions about memory can usually provide more accurate reflection of cognition as people do not often recognise other issues i.e. attentional problems for what they are.

\section{Future directions}

Having a greater number of older adults with subjective memory complaints (low MFQ score) would make it easier to compare their RT and IIV with individuals with no subjective memory complaints (high MFQ score). This may help to determine how RT and IIV in selective attention are affected in subjective memory function and possibly help to characterise how RT and IIV in selective attention are affected in SCI although this would also require further assessment and follow up to determine whether the older adults in the general population with subjective memory complaints go on to develop SCI after visiting their GP. 
Detailed measures of objective memory would be of use alongside subjective measures of memory function as subjective questionnaires rely on individuals accurately reporting changes. Objective measures of memory may help to determine whether individuals were correctly interpreting the types of changes they perceived to be having with their memory i.e. forgetfulness.

The subjective measure of memory function used in the current study (Memory Functioning Questionnaire, [MFQ]) has been validated as a reliable measure of memory self-appraisal although it is recognised that it is not a substitute for objective memory tests [Gilewski et al, 1990]. It may have been of use to include questionnaires measuring subjective perceptions of everyday attention to examine how individuals perceive their attentional function by have an effect on their RT and IIV of attention. However questions on poor attentional function need to be worded carefully as individuals in the general public may not recognise changes to attention for what they are. Conducting focus groups would be helpful in this case to determine whether individuals perceive attention in similar ways.

Other factors not taken into account in the current study should also be compared with information processing speed and IIV such as sleep, occupation and personality, to observe whether they are influential on information processing speed and variability or whether ageing effects are more influential.

It would be an interesting direction to compare saccades (eye movements) between older and young adults. This would provide more of a neurological comparison of information processing speed attentional processing during ageing; increased saccades have already been found in AD [Rösler et al., 2005]. In addition, examining brain images of the same older participants i.e. physical changes to brain structure such as reduced white matter integrity, may provide a clearer explanation as to why older adults were producing significantly slower RT compared to younger adults. In addition bran scan would determine whether older adults who perceived changes to their memory function indeed had physical impairment to memory function.

\section{Conclusion}

This study found slower information processing speed and greater IIV in older adults compared to young adults. Subjective memory function had no influence on information processing speed and its variability suggesting that in community dwelling older adults without a formal SCI diagnosis, perception of memory function has no impact on the interpretation of control group results in a visual 
search selective attention test. Despite this there were some individuals highlighted as outliers (significantly slower information processing speed), which may have an influence on the results. Therefore subjective memory function should be taken into account in older adult control groups as well as all outlying results followed up with further assessment. Subjective memory function in these individuals may be due to significant cognitive impairment as related to SCI which is being reflected in significantly slower information processing speed although this is only one explanation for slower information processing speed in older adults.

Perceived test difficulty influenced information processing speed in older adults but did not influence intraindividual variability. Despite a small effect size, psychological factors may need to be considered if they have an influence on information processing speed and thus included when investigating control groups in visual search studies. This measure needs to be repeated as the current result may not have been robust enough.

Sex only influenced information processing speed and its variability in young adults and education only had an influence on older adults' information processing speed and its variability i.e. faster and less variable RT. In addition, education was influential on information processing speed regardless of perceived changes implying that it does not appear to matter how people perceive their memory function to be. Therefore, education is important to take into consideration when measuring information processing speed and its variability of control groups in visual search studies.

What this study did observe is that finding a relationship between information processing speed or IIV and sex, education and perceived test difficulty did appear to depend on the condition within the visual search test. The majority of correlations were found in the target plus distractors condition possibly due to the differences in processing demands of the two tasks [Phillips et al, 2013]. The more complex the task is (i.e. target plus distractors condition compared to target alone condition), the more information processing resources are required as more brain areas have to be recruited. The result of an increase of processing load may be a greater effect of different factors i.e. age on information processing speed and IIV. The variation of task processing demands needs to be considered therefore as a factor influencing study outcome [as discussed in Phillips et al, 2013]. 
The findings in this study can only account for selective attention in visual search therefore different tests of similar attentional function need to be examined. It important to observe whether effects of different factors are task dependent as although there was no effect of subjective memory function on information processing speed and its variability (IIV) during visual search, it may be the case that effect of these factor is sensitive to other tests of visual attention. Therefore a second larger study in this thesis was conducted with other tests of similar attentional function i.e. selective attention to examine whether study outcomes i.e. the influence of sex, education, perceived test difficulty and subjective memory function are similar across different visual attention tests. In addition the effect of the number of trials on RT and IIV was examined in the Choice RT test. 


\subsection{CHAPTER THREE: The Trail Making Test}

\subsection{INTRODUCTION}

In the first study (see Chapter 2) factors of sex, education, subjective memory function and perceived test difficulty) were examined with respect to a single visual search test of attentional related information processing speed and attentional function, commonly applied for research in this area but for which such factors had not been fully investigated. The results of this visual search study found that information processing speed in older adults was slower and more variable compared to young adults. Information processing speed was influenced by sex in young adults (males performing faster compared to females) but not in older adults. Sex influenced IIV in young adults but only in the Target plus distractors condition. In older adults, subjective memory function did not significantly influence either information processing speed or intraindividual variability, i.e., whether or not older adults perceived changes to their memory, did not have an influence on their actual performance speed or variability.

Perceived test difficulty had a significant influence on information processing speed in older adults but not in young adults. Older adults who perceived the test to be difficult were in fact quicker at performing the test (faster information processing speed) thus their judgement of how difficult the task was did not reflect in their actual performance. In older adults, how difficult they perceived the test to be was significantly related to their level of education with those older adults with higher education (in years) judging the test to be more difficult compared to those with lower levels of education. Perceived test difficulty had no influence on IIV in both young and older adults thus how variable young and older adults' performance was did not relate to how difficult or easy they perceived the test to be. Visual searchrelated information processing speed and IIV was influenced by education in older adults with those with higher levels of education performing faster and less variably than those with lower levels of education, but not in young adults.

Finding an influence of sex, education and perceived test difficulty on information processing speed and its variability during visual search were dependent on the condition i.e. finding a correlation in only target alone condition or only in target plus distractors condition indicating that even sub-tests of paradigms can influence study outcome. 
Despite outcome variability within sub-tests, the visual search test in this current research suggests that older adult RT and IIV (in particular RT with larger effects sizes) may be influenced by different factors such as sex, education and perceived test difficulty other than the effects of age. Therefore, this implies that previous studies using a similar visual search paradigm but not including sex, education and perceived test difficulty may have misinterpreted their results of how information processing speed is affect in ageing.

Finding no relationship of subjective memory function in the visual search may indicate that information processing speed and its variability as measured by the visual search test remains at 'normal' levels in a healthy older adult group despite those who perceive changes to their memory (and since performed to 'normal' levels in objective testing (MoCA score), see Torrens-Burton et al, 2017 for similar discussion]. Alternatively, the visual search test measuring information processing speed may not be sensitive enough to detect any cognitive changes in older adults experiencing subjective memory changes. However, a lack of an effect of subjective memory function was found at group level yet the presence of outliers of information processing speed and IIV (separate from those outliers removed before analysis) may indicate that visual search is sensitive enough for some individuals who perceive memory changes. These people may be displaying poor function thus not a good representation of healthy older adults so cannot be used in healthy control groups. Further assessment and follow up would be useful in these individuals to determine whether they have significantly poorer function than expected during healthy ageing.

The results (i.e. influences on information processing speed) found in this visual search study can only account for how information processing speed is effected in young and older adults in relation to this particular research attention test. It may be the case that some tests are more sensitive to the influence of these different factors than others in the same group of people. Therefore, result outcome may vary depending on the type of test used. This makes it difficult to characterise what is happening to information processing speed (i.e. what factors influence it) during ageing. The importance of information processing speed is highlighted in the DSM-5 yet if it is not specified clearly in clinics what research tells us about as to how information processing speed is associated by which factors and by which test, or type of test. This makes it difficult for clinics or research to replicate studies and may hinder understanding of RT and IIV in ageing. 
Therefore, a second study was designed to include a larger battery of attention tests (research and clinical) to examine how the use of different tests of information processing speed in the same people (where possible) might produce different results. The type of test used by influence Whether RT and IIV is significantly reduced or not in ageing or whether factors such as sex and education, subjective memory function and perceived test difficulty have an effect on RT.

Testing the same young and older adults in each test helps to determine whether information processing speed is influenced in the same way (i.e. by the same factors) between tests or whether the outcome varies depending on the type of test used. Using the same people where possible helps to control any confounding variables which may occur so that they are the same for each test otherwise it is unclear whether the results are due to the test or to the people themselves if different groups are used. There are a number of different factors which can influence results i.e. medication, mood, thus using the same people attempts to minimise these biases.

In this second study information processing speed and intraindividual variability and the influence of different factors (sex, education, subjective memory function and perceived test difficulty) will be measured in the same groups of young and older adults (where possible) using the Trail Making test (TMT) [clinical], Simple reaction time test, (research) Choice RT research test (in which the effect of trial numbers can be examined which was not possible in visual search) and the Multi-item localization (MILO) test (research). In the current Chapter, the results will be described from the Trail Making Test; a test typically used to measure information processing speed in clinical settings.

\section{The Trail Making Test}

The TMT has been used in research to compare information processing speed and executive function between healthy ageing, MCI and AD and is typically used in memory clinics in the diagnosis of AD, other aetiologies of dementia and MCI. However, study outcome may vary between TMT studies due to methodological differences. For example, previous studies have used a wide age range (age continuum) rather than comparing young adults with older adults [Bezdicek et al. 2012; Rasmusson et al, 1998; Cangoz et al, 2009] and within those participant groups different demographics have been measured [e.g. Wright et al, 2016] along with differences in inclusion and exclusion criteria. 


\section{Problems with previous studies addressed in this study}

In some TMT studies a young control group has not been included to compare to older adult groups. Failing to include a young adult group to compare with older adults (i.e. healthy ageing control groups in MCI and AD studies) may result in an inaccurate representation of how information processing speed slows during healthy ageing.

Different factors may differentially influence young and older adults and also clinical populations. If not taken into account, these factors may lead to a misinterpretation of RT results thus affecting the clinical interpretation of cognitive function in individuals. In addition some previous studies have used small or unequal numbers of participants when comparing healthy older adults with MCI or AD on the TMT [Hagen, Ehlis, Haeussinger et al, 2014; Sanchez-Cubillo, Perianez, Adrover-Roig et al., 2009; Ashendorf et al, 2008] or when comparing young and older adults [Hagen et al, 2014]. Not having a sufficient enough sample size may result in a poor representation of the population and thus affect the validly of the results. This may impact how information processing is characterized within healthy ageing (the difference of processing speed between young and older adults) or characterised between healthy ageing and MCI or AD. To address these two issues, the current study included large samples of both young and older adults which were compared to each other with similar numbers in each group.

Sex and education have been examined previously using TMT with some studies finding slower information processing speed in females compared to males in both young adults [Karia et al, 2012] and older adults [Dykiert et al, 2012; Fozard et al, 1994] and slower information speed associated with lower education [Plotek et al, 2014; Cangoz et al, 2009] found in both young and older adults [Hamdan et al, 2009]. However variability of outcome in TMT studies have also found no association between sex and RT [Wright et al, 2016; Stuss, Stethem \& Poirier, 1987 (older research)] or only a sex effect in Trails A [e.g. Giovagnoli et al, 1996, although results of low significance] and no association of education on RT [Hashimoto et al, 2006] or only and education effect in Trails B [Hashimoto et al., 2006; Tombaugh, 2004]. Variability of methodology occurs between these studies which may explain differences between results i.e. scoring methods, type of analysis and the interpretation of errors [Haworth et al, 2016], small number of participants [Wright et al, 2016; Bezdicek et al, 2012]. 
Not many studies, if any, have examined subjective memory function and psychological factors i.e. perceived test difficulty. How these previously ignored factors may affect information processing speed may influence results and in future how researchers interpret the results of TMT studies and what clinicians take into account when measuring RT using the TMT especially in terms of what constitutes an ostensibly cognitively health older adults. In addition, the DSM-5 highlights the importance of measuring information processing speed [Sachs-Reicsson \& Blazer, 2015] yet does not provide details particularly in relation to the potential influence of sex, education and especially novel factors of subjective memory function and psychological factors i.e. perceived test difficulty.

\section{One trial versus multiple trials}

A significant aspect of the clinical TMT is that this pen and paper test typically uses a single trial as its measure of information processing speed. In contrast, research studies typically use tests including multiple trials for a mean score of RT performance. After an initial investigation of TMT and visual search, a paper [Haworth et al, 2016] was produced (using a different study of Dr Tales) examining the dichotomy between tests used in research i.e. the visual search and tests used in clinics i.e. the TMT. This study compared RT between aMCI patients and healthy controls for both the visual search and the TMT. It was found that the TMT could only distinguish differences between aMCI and healthy ageing in the Trails B condition but not the Trails A condition. In contract both conditions of the visual search (target alone and target plus distractors) found aMCI patients to be significantly slower compared to healthy ageing.

The conclusion drawn was that despite the clinical TMT being a simple test to administer, the use of one trial may not be sufficient enough to make comparisons of RT between healthy and pathological ageing. Arguably therefore, the TMT may not be sufficient enough to make comparisons of RT in healthy ageing i.e. between young and older adults. Haworth and colleagues also argue that a greater number of trials (used in research tests) may provide more sensitive results which is an argument that has already been made before [i.e. Salthouse \& Fristoe, 1995].

Research tests using multiple trials can also measure intraindividual variability (IIV) i.e. measuring the functional integrity of information processing speed which a single trial test such as the TMT cannot achieve. 
Another issue with the TMT is that in research versions, the test could not be finished by healthy ageing individuals for different reasons i.e. giving up, [Kim et al, 2014] language barriers [Seo et al, 2006] or low levels of education [Cavaco et al, 2013; Seo et al, 2006]. This highlights that if the TMT cannot be finished in research settings by healthy individuals this may be a problem for clinics using the test as failed attempts may be incorrectly associated with cognitive dysfunction and not with any other factor.

Finding limitations with the TMT begs the question whether clinically we should be using more research tests such as the visual search rather than the TMT if the visual search appears to be a more sensitive test. In addition it questions whether clinical practice (i.e. using the TMT) is in accord with what is known about the test from research studies such as failed attempts of the TMT in healthy individuals or the association of RT with other factors i.e. sex and particularly education. It may be necessary to raise awareness to clinicians as to what is being found in research studies as to which RT tests may be more sensitive to the effects of ageing or best distinguish between healthy and pathological ageing.

Despite all these issues above, the second study in this thesis included an examination of the TMT (and compare results with visual search) for two reasons. Firstly, information processing speed has not been compared in much detail between research visual search test and clinical TMT for healthy ageing i.e. comparing young and older adults. Secondly, factors person-related factors of subjective memory function and perceived test difficulty, along with sex and education, have not been examined in relation to RT in relation to the visual search and the TMT (and IIV in the visual search). Therefore we examined whether factors that influence RT using a multi-trail research test (visual search) also have a similar effect on a one trial clinical test (TMT).

\section{Details of the Trail Making Test (TMT)}

The TMT measures information processing speed in relation to executive function; a compilation of cognitive processes required for the cognitive control of behaviour including attentional control, cognitive flexibility, planning and working memory and functions such as eye movements, shifting attention and decision making. Some functions overlap in the visual search task making the TMT similar in many ways to visual search. 
During the TMT the focus of attention has to be serially shifted throughout the visual scene in order to locate and respond to the target (number or letter) while

disengaging attention from the distracting information (unwanted numbers or letters). The TMT differs from executive function measured by the visual search task as it measures number sequencing, switching attention from numbers to letters and motor movement of drawing a line to connect both numbers and letters. Another difference in the TMT is that it only includes one trial (thus IIV cannot be measured) whereas the visual search is a multiple trial test therefore, due discrepancies between clinical tests (single trials) and research tests (multiple trials) mentioned above, we were interested in examining TMT (a clinical test) and comparing with visual search (a research test).

The TMT is a pen and paper task consisting of two parts (see Figure 8). Part A (Trails A) involves drawing a continuous line connecting numbered circles in numerical order which are distributed around the paper. Part B (Trails B) involves drawing a continuous line connecting numbers and letters in alternating order i.e.1A, 2-B etc.

Figure 8: Representation of the stimuli for Trails A and Trails B
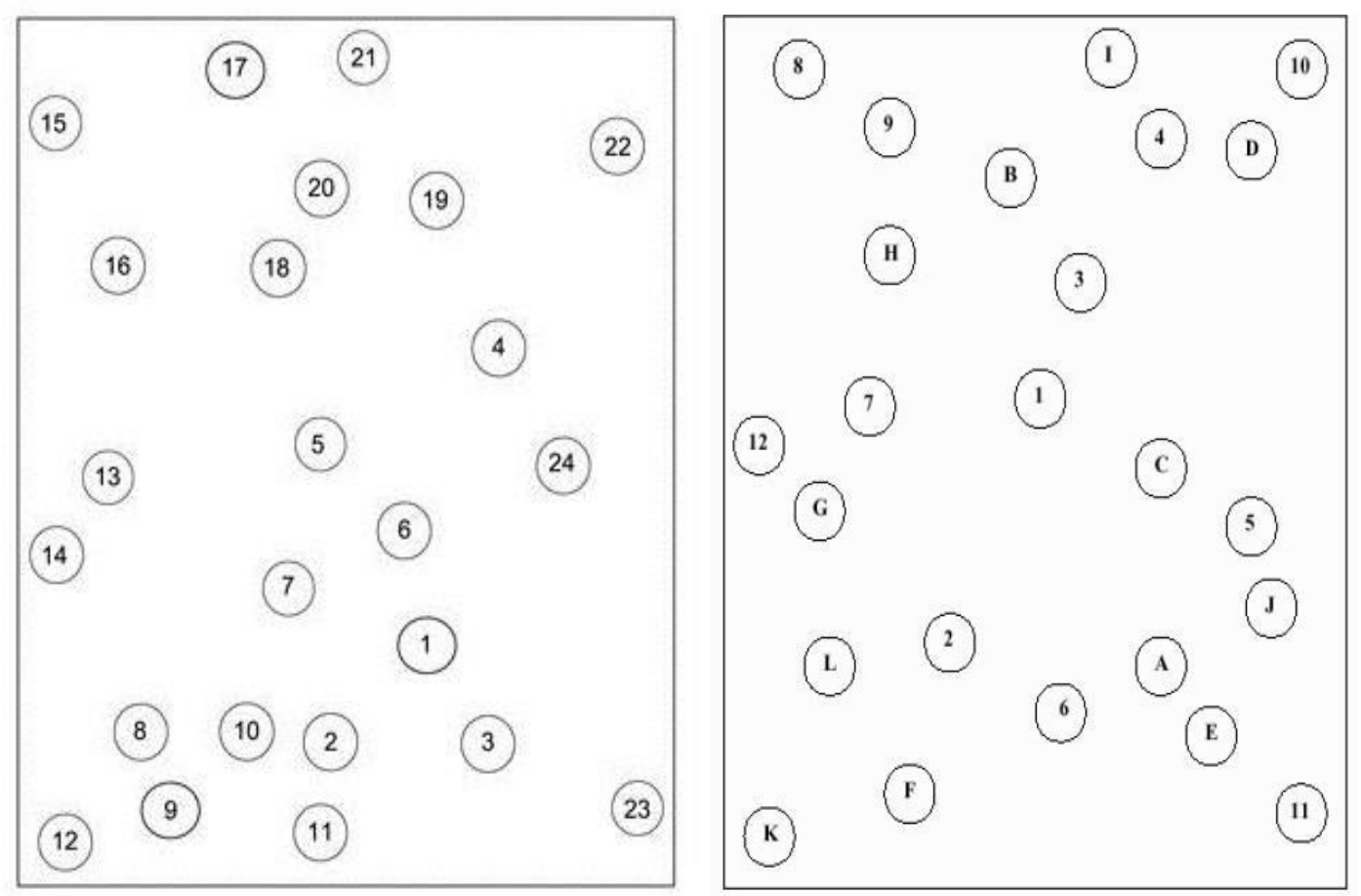
Trails A is typically described as measuring information processing speed in relation to attentional processing as well as number recognition, numeric sequencing and motor speed whereas the Trails B task is typically described as measuring the efficiency of attention switching, simultaneous maintenance of two sequences, working memory and cognitive flexibility (which make up executive function) though measuring information processing speed [Salthouse, 2011; Sanchez-Cubillo et al., 2009].

During Trails B participants are required to mentally maintain two separate sequences (consecutive numbers and consecutive letters) and constantly switch between the two. This requires greater cognitive flexibility and the processing of larger amounts of information (two separate sequences compared to one). Processing greater levels of information in Trails B requires greater processing load compared to Trails A [Haworth et al, 2016] which puts greater strain on cognitive resources thus it would be expected that Trails B would produce slower reaction times compared to Trails A and indeed this is the case [Haworth et al; 2016; Sofko et al, 2014; Rasmussen et al, 1998].

\section{Brain activity occurring during the TMT}

TMT is associated with activation of the pre-frontal cortex (PFC) [Shibuya-Tayoshi, Sumitani, Kikuchi et al., 2007; Zakzanis, Mraz, \& Graham, 2005; Moll, de OliveiraSouza, Moll, Bramati, \& Andreiuolo, 2002] as found to be activated when performing the Trails A [Hagen et al, 2014] and Trails B conditions [Arbuthnott \& Frank, 2000] in both young adults [Crowe, 1998] and older adults [Hagen et al., 2014].

However, it has been argued that executive functioning is not restricted to the prefrontal area of the brain. This is because some individuals with damage to the PFC have displayed a lack of executive deficits and individuals without damage to the PFC still displaying deficits in executive functioning [Andres, 2003]. Supporting this assumption, Zakzanis and colleagues [2005] discovered activation of the left middle and superior temporal gyrus during TMT performance therefore suggesting that the PFC is not the sole area activated during the executive functioning during the TMT. It is useful to understand which areas of the brain are activated during the TMT. If brain areas associated with the TMT are damaged during ageing it can be observed whether TMT performance in healthy ageing is poor (i.e. slower information processing speed). 
In addition if similar brain areas are impaired in MCI or AD, TMT performance in MCI or AD patients compared to healthy ageing may provide a better characterisation of how information processing speed is affected between healthy and abnormal ageing thus useful for clinical settings i.e. diagnosis purposes.

\section{Past research measuring information processing speed and IIV using the TMT in ageing}

During ageing, white matter in the brain begins to shrink due to the loss of neurons and as a result, scars i.e. white matter lesions develop indicating areas of damage [Rabbitt, 2015]. Detrimental change to white matter have been observed in frontal areas of the brain [Damoiseaux, Smith, Witter et al., 2009; Perry, McDonald, Hagler et al., 2009; Bucur et al, 2008] with lesions found in the PFC in older adults which, as mentioned above, is an area found to be activated during the TMT [Demakis, 2004; Stuss, Bisschop, Alexander et al., 2001] therefore it would be expected that TMT performance would be poorer. Indeed, poorer white matter integrity during ageing has been demonstrated to result in impaired executive functioning thus negatively affect overall performance of the TMT (i.e. slower information processing speed) [Cook, Leuchter, Morgan et al, 2004; Keys \& White, 2000].

Many research studies have found information processing speed to slow in older adults during the TMT due to the effects of ageing [Hagen, 2014; Plotek et al, 2014; Sofko et al., 2014; Pálsson, 2013; Cangoz et al, 2009; Zalonis et al., 2008; Periáñez, Rios-Lago et al, 2007; Hashimoto et al, 2006; Demakis, 2004; Stuss et al., 2001; Keys \& White, 2000; Rasmusson et al, 1998; Ylikoski et al, 1993]. In addition, within older adults Trails B found to be performed slower compared to Trails A [Sofko et al, 2014; Mrazik, Millis \& Drane, 2010; Rasmusson et al, 1998]. This may be because Trails B is more complex (switching between numbers and letters), which requires greater cognitive flexibility and processing resources than that required during Trails A (connecting numbers only), as well as a greater range and depth of operations [Haworth et al, 2016].

In contrast, not all findings supported slowing in increased age [Boll \& Reitan, 1973] or significant slowing found for Trails A as well as Trails B in ageing [Rasmusson et al, 1998]. Therefore outcome variability occurs between studies (even supporting studies) which may reflect differences between methodologies. For example some studies excluded a young adult group to compare with their older adult control group [Cangoz et al; 2009; Rasmusson et al, 1998]. 
This may result in an inaccurate representation of healthy ageing i.e. how information processing speed in ageing is characterised. Previous TMT studies include differences in sample sizes which may impact on how representative the results may be i.e. smaller sample sizes may have meant that previous studies were underpowered. Other differences include the use of different paradigms i.e. oral versions [Mrazik et al, 2010] and differences between demographics i.e. education level, IQ and nationality as well as differences in the inclusion and exclusion criteria. Outcome variability may have an impact on how different study results are interpreted and may impact which tests are useful in a clinical setting for an accurate diagnosis i.e. comparing healthy ageing to $\mathrm{MCI}$ or dementia.

\section{Past research measuring information processing speed using the TMT in MCI and AD}

The TMT is commonly used within a battery of neuropsychological tests in memory clinics to measure attention-related information processing speed and executive function as part of the diagnosis of MCI or AD. White matter integrity decreases in ageing but is also found to disproportionally decrease in AD [Head et al, 2011; Johnson, Barrow, Anderson et al, 2010; Damoiseaux et al, 2009; Huang \& Auchus, 2007; Capizzano et al, 2004] in areas such as the left anterior temporal lobe [Damoiseaux et al, 2009; Borroni, Brambati, Agosti, \& et al., 2007] and the thalamus [Terada et al., 2013] and white matter lesions are also apparent in AD [Burns, Church, Johnson et al, 2005; Barber, Scheltens, Gholkar et al, 1999; O’Brien, Desmond, Ames et al, 1996] in brain areas such as in the bilateral superior parietal lobules [Shindo, Terada, Sato et al., 2013].

The areas of the brain described above are associated with the TMT fucntion therefore therefore is would be assumed that information processing speed would be disproportionately slow in AD compared to healthy ageing and indeed this has been observed [Bezdicek et al, 2014; Terada et al., 2013; Shindo et al., 2013; Silveri et al, 2007; Johnstone et al, 2002; Kortte et al, 2002; Reitan, 1971]. Therefore, it is implied that a good method for discriminating between AD and healthy ageing is measuring levels of slowing of information processing speed during executive function i.e. the TMT [Ashendorf, Jefferson, O'Connor et al, 2008; Chen et al, 2000]. However, finding a significant difference of information processing speed between $\mathrm{AD}$ and healthy ageing has been recognised to depend on which sub-test of the TMT was being performed (Trails A or Trails B). 
Significant differences between $\mathrm{AD}$ and healthy ageing were found more frequently during Trails B compared to Trails A [Bezdicek et al, 2014; Terada et al, 2013; Silveri et al, 2007; Johnstone et al, 2002]. Therefore outcome variability can occur between conditions within the TMT (although majority of these previous tests only tested Trails B) as well as between studies which may relate to differences in test protocol (how the test was conducted), whether other factors were considered (i.e. sex and education), small and unequal sample sizes (not generalizable), or the interpretation of results.

MCI has been documented for some people as a stage before the development dementia such as AD and within MCI pathology a decrease of white matter is also prominent [Liu, Wang, Shu et al, 2016; Zhuang, Wen, Zhu et al, 2010; Rogalski et al, 2009; Huang \& Auchus, 2007]. In addition, areas associated with the TMT i.e. prefrontal cortex have found to have a significantly lower level of white matter during MCI [Pa, Possin \& Wilson, 2010; Chao, Pa, Duarte et al, 2009; Whitwell, Petersen, Negash et al, 2007]. Therefore studies have found disproportionately slower information processing speed in MCI patients compared to healthy ageing during the TMT [Pa et al, 2010; Chao et al, 2009; Whitwell et al, 2007; Salthouse \& Fristoe, 1995; Breteler, van Amerongen et al., 1994]. However this has not always been supported [Baudic, Dalla Barba et al., 2006] thus highlighting the variability of results when measuring information processing speed in MCI. Again, contradictory results between TMT studies of MCI may relate to methodological differences i.e. interpretation of results, sample sizes and test procedure.

Slowing of TMT performance (information processing speed) has also been found in amnesic mild cognitive impairment (aMCI) [Bezdicek et al, 2014; Silveri et al, 2007] although Haworth and colleagues [2016] found that Trails A failed to significantly differentiate between aMCI and healthy ageing unlike Trails B, suggesting that the cognitive processes required during Trails A remain intact if healthy ageing declines to a diagnosis of aMCI. This implies that even between sub tests results may vary and both Trails A and B are not necessarily required for a diagnosis of aMCI. In clinics it is typically standard procedure to use both Trails A and B although this may not be required to find differences in ageing and between healthy ageing and MCI or AD (particularly the Trails A). 
The conclusion drawn by Haworth and colleagues (as discussed above) was that as the clinical TMT uses only one trial, it may not be sufficient enough to make comparisons of RT between healthy and pathological ageing. In the same study they examined a multiple trial research test of visual search and both conditions found significant difference of RT between aMCI patients and healthy controls thus argued to have produced more sensitive results. This questions whether clinically we should be using research tests rather than the TMT if the visual search appears to be a better test. It may be necessary to raise awareness to clinicians as to what is being found in research studies as to which RT tests may be more sensitive to the effects of ageing or best distinguish between healthy and pathological ageing. However, possible discrepancies between research and clinical tests have not been examined RT in relation to healthy ageing i.e. comparing RT between young adults and older adults as well as potential effects of different person-related factors on information processing speed. Therefore the current study examined RT between young and older adults and the potential effects of different factors (sex and education subjective memory function and perceived test difficulty) using the TMT, and compared findings with the results of a visual search test to determine whether effects of RT in ageing are similar across research and clinical tests.

\section{Subjective cognitive impairment, subjective memory function and TMT}

Information processing speed during TMT has been demonstrated to slow in MCI and AD compared to healthy ageing [Shindo et al, 2013; Ashendorf et al, 2008; Salthouse \& Fristoe, 1995; Breteler et al, 1994]. Therefore we speculate that similar effects occur on information processing speed during earlier stages before an MCI diagnosis i.e. in Subjective Cognitive Impairment (SCI) or in subjective memory function, referring to older adults perceiving change to memory function but have not visited their GP (as focused on in this thesis).

Older adults used in research versions of the TMT (i.e. control groups in MCI and AD studies) are recruited from the local community and are considered to display 'normal' levels of information processing speed. However some individuals may be exhibiting SCI or (as discussed in introduction to this thesis) some, if not all, of older adults in a control group may be perceiving changes to their memory function yet have not visited a GP about them (subjective memory function). 
Irrespective of aetiology, SCI or subjective memory function may influence information processing and its speed i.e. disproportionate slowing thus affect RT results of ostensibly healthy ageing thus these individuals should not be used within healthy older adult control groups.

Clinical SCI has failed to have an influence on TMT performance (information processing speed) of an elderly sample [Minett, da Silva, Ortiz et al, 2008] although as far as we are aware, there are no other TMT studies which have examined SCI in relation to TMT performance. In addition previous studies have not examined RT performance during the TMT in relation to subjective memory function in the general population. We previously found in a visual search test (chapter 2 of this thesis) that subjective memory function did not appear to influence information processing speed in older adults. However these results may differ depending on the type of attention test used. Therefore the aim of the current study was to determine whether subjective memory function is associated with RT in Trails A and Trails B. In addition results between attention tests may differ depending on whether test is clinical or research based (i.e. one trial or multiple trial issue) thus the results of this clinical TMT study were compared to the results of the research visual search (Chapter 2) to determine whether the effects of subjective memory function on RT were similar for a one trial clinical test compared to a multi trial research test. A further aim was to examine how subjective memory function is characterised by slowing in TMT in an attempt to better characterise SCI.

\section{Summary}

Information processing speed has been demonstrated to slow during the TMT during ageing, MCI and AD in earlier stages such as SCI. However age effects have not always been found [Haworth et al, 2016; Minett et al, 2008; Boll \& Reitan, 1973] due to the variability between TMT studies i.e. the type of test (use of TMT), small participant sizes or failing to include young adults to measure ageing. However, outcome variability may also relate to the influence of other person- related factors on information processing speed i.e. sex, education or subjective memory function and perceived test difficulty (as described in further detail in the sections below) therefore in this study the aim is to address these issues. 


\section{Person related factors in the TMT}

Sex

TMT studies have not always included sex (male/female) as a potential influence on information processing speed either because it has not been considered or has been dismissed as a contributing factor. Some TMT studies which have included looking at the potential effect of sex found faster information processing speed in males compared to females during the TMT [Foroozandeh 2014; Cangoz et al, 2009] although sometimes only in Trails A [Giovagnoli et al, 1996] or only in Trails B [Foroozandeh 2014]. The difference between males and females (particularly for Trails B) is implied to relate to males being more efficient at attentional shifting, flexible mental processing and motor speed (particularly required during Trail B) due to hormone levels i.e. testosterone associated with better cognitive performance [Muller et al, 2005; Cherrier et al, 2001].

In contrast, PŁotek and colleagues [2014] found females to be faster than males during the TMT implied to be as a result of females being better at sustaining their attention during the test. In addition some studies have not found sex to be influential on information processing speed in both Trails A and B [Haworth et al, 2016; Bezdicek et al, 2012; Ashendorf et al, 2008; Tombaugh, 2004; Waldmann, Dickson, Monahan et al, 1992] which has also been argued to be test dependent [Haworth et al, 2016]. Outcome variability between TMT tests may highlight that the influence of sex on study outcome (i.e. RT) may be test dependent. Studies finding sex effects imply that if slowing of information processing speed is dependent on sex and not considered as a factor, research results may be misinterpreted thus may impact how the test should be used in clinical settings. Therefore, in this research version of the TMT study, sex is examined as a potential factor influencing information processing speed to determine whether which may also be examined during clinical use of the TMT.

\section{Education}

There is evidence that TMT performance (information processing speed) can be influenced by the level of education [Ptotek et al, 2014; Cangoz, et al, 2009] in both young and older adults [Hamdan et al 2009; Tombaugh, 2004; Amodio, Wenin, Del Piccolo et al., 2002] with higher levels of education associated with faster information processing speed [Palsson, 2013; Bezdicek et al, 2012; Hamdan et al 2009; Tombaugh, 2004]. 
As with sex, if education has an influence on information processing speed and not considered as a factor, this may lead to misinterpreting differences between healthy control groups and MCI and dementia i.e. not properly characterizing TMT in relation to ageing. However, some TMT studies found no effect of education on information processing speed [Hashimoto et al, 2006] or the effect of education only influenced information processing speed in Trails B [Hashimoto et al., 2006; Tombaugh, 2004]. This highlights outcome variability between studies and even in sub-tests which has been argued to dependent on the study i.e. scoring methods, type of analysis and the interpretation of errors [as argued by Haworth et al, 2016] or number of participants [Bezdicek et al, 2012]. Therefore, this TMT study also examines education as a potential factor influencing information processing speed.

\section{Perceived Test Difficulty}

While performing an RT task, individuals may hold different perceptions about their own abilities or demands of the test itself which, as a result, may have an effect on how quickly the test is completed (discussed in more detail in the introduction to this thesis). In TMT studies it has been found that perception of visual acuity is associated with slowed information processing speed [Setti, Loughman, Savva \& Kenny, 2015; Bolmont et al, 2000] however this factor has not been examined in great detail in RT and ageing studies.

In the current study the psychological factor measured was the perceived difficulty of the TMT test with the assumption that if an individual perceives a test to be more difficult, this may have a negative effect on information processing speed i.e. slower RT. Any affects on RT were examined in young and older adults as well as any interactions with other factors (education and subjective memory function) to determine whether perceived test difficulty may be associated with additional factors other than age which may consequently effect information processing speed.

The results may help determine whether this factor should be taken into account in research studies using the TMT thus examined in clinical uses of the TMT. In addition, the results were compared the of visual search test from the previous chapter (Chapter 2) to determine whether research tests may be better to use in clinical setting. 


\subsection{METHODOLOGY}

\section{$\underline{\text { Participants }}$}

Swansea university students ( $\mathrm{n}=81$; age 18-26 years; 30 males: 51 females) were recruited through the Psychology department credit system and through advertising around the university and social network. Community-dwelling older adults $(n=87$; age 50-80 years; 33 males: 54 females) were recruited through advertisements given out to older adult social clubs and local papers in Swansea, through email, word of mouth and via a volunteer database of older adults set up by the Swansea Psychology Department. Ethical approval was provided by the Swansea University Psychology departmental ethics committee and the study conducted to the principles in the Declaration of Helsinki. Formal written consent was obtained from each participant.

\section{$\underline{\text { Inclusion \& exclusion criteria }}$}

Participants reported to be in good general health, had no any history of serious head injury, serious cognitive, visual or neurological impairments, colour blindness or have any condition which might be affected by flashing images on a screen, although medication could not be controlled for. All participants were requested to bring any corrective lenses they require for reading and computer work. Participants' overall cognition score ('normal' score being 26 or above) was measured using the Montreal Cognitive Assessment (MoCA) [Nasreddine et al, 2005] $]^{1}$. Both young and older adults and had no significant levels of depression or anxiety as measured by the Patient Health Questionnaire (PHQ-9) [Kroenke et al, 2001] (score of 9 and below) and the Generalized Anxiety Disorder 7-item (GAD-7) [Löwe et al, 2008] (score of 5 and below). For older adults, subjective memory function was measured using the Memory Functioning Questionnaire (MFQ) [Gilewski et al, 1990] (higher scores equal to perceiving less change to memory function). Age, sex and years of full time education were also recorded.

\footnotetext{
${ }^{1}$ Note that the scores of the majority of participants were within the normal value of 26 or above. However for 15 young and 16 older adult participants the score was lower than 26 . These were included because we were interested in a range of MoCa scores and whether the score has in influence on RT or IIV.
} 


\section{$\underline{\text { Stimuli }}$}

The first practice (Trails A) contained numbered circles from one to eight in a random order across the paper. The testing sheet contained 25 numbered circles distributed around the page (see Figure 8). In Trails B, the practice sheet contained 8 circles, four with numbers 1-4 and four with letters A-D.

They were positioned across the page alternating between numbers and letter in order i.e. 1-A, 2-B. For the testing the same format on the paper but with the number 1-12 and letters A-L distributed across the sheet (see Figure 8). A stopwatch was used to time each participant.

\section{Procedure}

Participants were firstly given the practice Trails A by the researcher where they were asked to draw a continuous line connecting the numbered circles in numerical order. Once this was completed they were given the testing sheet with the instruction that they were to do the same task but this time as quickly and as accurately as possible as it would be timed. A timer was started by the researcher as soon as they began drawing and stopped as soon and the pen was taken off the last number to be connected. Participants were then given the Trails B practice sheet with the instruction to daw one continuous line but to alternate between numbers then letters. Once participants stated verbally that they fully understood the test, the longer test version was given and participants went through the same timed procedure as previously carried out with Trails A.

Any errors made during testing could be corrected at the time by back tracking and re-drawing the line through the numbers or letters. Participants were given small prompts if they became stuck (i.e. repeating the numbers and letters they had already connected in order to prompt the next number or letter in the sequence) but it was essentially up to the participant to complete the test and not to be completed for them. Few participants required more than one prompt and prompt was usually near the end of the test. 


\section{Data cleaning}

For any trial from Trails A or B which was drawn incorrectly and not corrected by the participant by the end of the testing i.e. could not be completed correctly, the pair was initially eliminated from analysis. 10 young adults were eliminated leaving 71 participants and 6 older adults were eliminated leaving 81 overall.

Information processing speed (RT) was provided in seconds and for each age group (older and young adults) the mean, standard deviation and inter-quartile range (IIV) was calculated. In response to the non-normal distribution of the data (see Table 6) SPSS non parametric analysis was conducted in accordance with common practice. Factors i.e. sex and education, subjective memory function and perceived test difficulty were grouped into separate families for analysis. The RT and IIV and attention effects were different data sets and thus did not require Bonferroni correction for multiple analyses.

Table 6. Normality of Distribution (Shapiro Wilkes test) ${ }^{2}$

\begin{tabular}{|l|lll|lll|}
\cline { 2 - 8 } \multicolumn{1}{c|}{} & \multicolumn{3}{c|}{ Older adults } & \multicolumn{3}{c|}{ Young adults } \\
\cline { 2 - 8 } \multicolumn{1}{c|}{} & statistic & $d f$ & Sig. & statistic & $d f$ & Sig. \\
\hline Trails A & .927 & 81 & .000 & .933 & 71 & .001 \\
\hline Trails B & .894 & 81 & .000 & .932 & 71 & .001 \\
\hline
\end{tabular}

\footnotetext{
${ }^{2}$ Note: data classified as a non-normal due to the positively skewed distribution of the data and in some cases bimodal distributions. There is a natural limit to the data i.e. how fast RT can be.
} 


\subsection{RESULTS}

\section{$\underline{\text { Demographics }}$}

Mann-Whitney analysis revealed depression level was significantly greater for young adults compared to older adults $[\mathrm{U}=1763.5, p<.001$, effect size $(\mathrm{r})=.33]$ and anxiety level was significantly greater for young adults compared to older adults [U $=1809.5, p<.001$, effect size $(r)=.32]$. There was no significant difference between young and older adults in mean MoCA score or mean years of education [ $p>.05]$.

Table 7: Mean demographic scores for the older adult and younger adult groups. Standard deviation in parenthesis.

\begin{tabular}{|c|c|c|c|c|c|c|}
\hline & $\begin{array}{c}\text { Age } \\
\text { (years) }\end{array}$ & $\begin{array}{c}\text { Education } \\
\text { (years) }\end{array}$ & $\begin{array}{l}\text { MoCA } \\
\text { (score) }\end{array}$ & $\begin{array}{c}\text { MFQ } \\
\text { (score) }\end{array}$ & $\begin{array}{c}\text { PHQ-9 } \\
\text { (score) }\end{array}$ & $\begin{array}{l}\text { GAD-7 } \\
\text { (score) }\end{array}$ \\
\hline $\begin{array}{c}\text { All } \\
\text { Young } \\
\text { Adults }\end{array}$ & $\begin{array}{c}20 \\
(1.7)\end{array}$ & $15(3.2)$ & $27(2.0)$ & - & $6(4.5)$ & $4(3.8)$ \\
\hline $\begin{array}{c}\text { Young } \\
\text { Male } \\
(n=25)\end{array}$ & $\begin{array}{c}21 \\
(2.0)\end{array}$ & $15(2.9)$ & $27(2.2)$ & - & $7(5.8)$ & $4(4.4)$ \\
\hline $\begin{array}{l}\text { Young } \\
\text { Female } \\
(n=46)\end{array}$ & $\begin{array}{c}20 \\
(1.3)\end{array}$ & $14(3.3)$ & 27 (1.8) & - & $6(3.8)$ & $4(3.2)$ \\
\hline $\begin{array}{c}\text { All } \\
\text { Older } \\
\text { Adults } \\
\end{array}$ & $\begin{array}{c}65 \\
(5.5)\end{array}$ & $16(4.8)$ & $27(2.2)$ & $\begin{array}{c}295 \\
(49.1)\end{array}$ & $3(3.1)$ & $2(2.8)$ \\
\hline $\begin{array}{c}\text { Older } \\
\text { Male } \\
(n=31)\end{array}$ & $\begin{array}{c}65 \\
(5.9)\end{array}$ & $17(5.8)$ & $26(2.3)$ & $\begin{array}{c}292 \\
(44.0)\end{array}$ & $3(3.1)$ & $2(3.2)$ \\
\hline $\begin{array}{c}\text { Older } \\
\text { Female } \\
(n=50)\end{array}$ & $\begin{array}{c}64 \\
(5.3)\end{array}$ & $15(3.7)$ & $28(2.0)$ & $\begin{array}{c}297 \\
(52.4)\end{array}$ & $3(3.2)$ & $2(2.5)$ \\
\hline
\end{tabular}

MoCA, Montreal Cognitive Assessment; MFQ, Memory Functioning Questionnaire; PHQ-9, Patient Health Questionnaire; GAD-7, Generalized Anxiety Disorder 7-item scale. 
Table 8: Mean information processing speed and mean perceived test difficulty of Trails A and Trails B for both young and older adults who completed the TMT task. Standard deviation in parenthesis.

\begin{tabular}{|c|c|c|c|c|}
\hline & \multicolumn{2}{|c|}{$\begin{array}{l}\text { Information processing } \\
\text { Speed (Seconds) }\end{array}$} & \multicolumn{2}{|c|}{$\begin{array}{l}\text { Perceived Performance } \\
\text { (Likert Scale) }\end{array}$} \\
\hline & Trails A & Trails B & Trails A & Trails B \\
\hline $\begin{array}{c}\text { All } \\
\text { Young } \\
\text { Adults }\end{array}$ & $\begin{array}{c}24.49 \\
(8.2)\end{array}$ & $40.92(8.5)$ & $\begin{array}{c}2(1.1) \\
\text { (Range 1-6) }\end{array}$ & $\begin{array}{c}3(1.8) \\
\text { (Range 1-7) }\end{array}$ \\
\hline $\begin{array}{l}\text { Young } \\
\text { Males }\end{array}$ & $\begin{array}{l}22.94 \\
(7.6)\end{array}$ & $42.27(10.7)$ & $\begin{array}{c}2(0.7) \\
\text { (Range 1-3) }\end{array}$ & $\begin{array}{c}3(1.9) \\
\text { (Range 1-7) }\end{array}$ \\
\hline $\begin{array}{c}\text { Young } \\
\text { Females }\end{array}$ & $\begin{array}{l}25.19 \\
(8.5)\end{array}$ & $40.35(7.5)$ & $\begin{array}{c}2(1.3) \\
\text { (Range 1-6) }\end{array}$ & $\begin{array}{c}3(1.7) \\
\text { (Range 1-6) }\end{array}$ \\
\hline $\begin{array}{l}\text { All Older } \\
\text { Adults }\end{array}$ & $\begin{array}{l}29.05 \\
(9.3)\end{array}$ & $43.43(9.4)$ & $\begin{array}{c}2(1.2) \\
\text { (Range 1-6) } \\
\end{array}$ & $\begin{array}{c}3(1.6) \\
\text { (Range 1-6) }\end{array}$ \\
\hline $\begin{array}{l}\text { Older } \\
\text { Males }\end{array}$ & $\begin{array}{l}29.93 \\
(9.6)\end{array}$ & $44.73(9.8)$ & $\begin{array}{c}2(1.3) \\
\text { (Range 1-5) }\end{array}$ & $\begin{array}{c}3(1.7) \\
\text { (Range 1-6) }\end{array}$ \\
\hline $\begin{array}{c}\text { Older } \\
\text { Females }\end{array}$ & $\begin{array}{c}28.57 \\
(9.2)\end{array}$ & $42.72(9.3)$ & $\begin{array}{c}2(1.1) \\
\text { (Range 1-6) }\end{array}$ & $\begin{array}{c}3(1.7) \\
\text { (Range 1-6) }\end{array}$ \\
\hline
\end{tabular}




\section{Information Processing Speed}

\section{Age comparison: Information processing speed}

Figure. 9: Box plot of mean information processing speed (secs) between Trails A and $\mathrm{B}$ in young adults and older adults. Note the presence of outliers in the performance of this task.

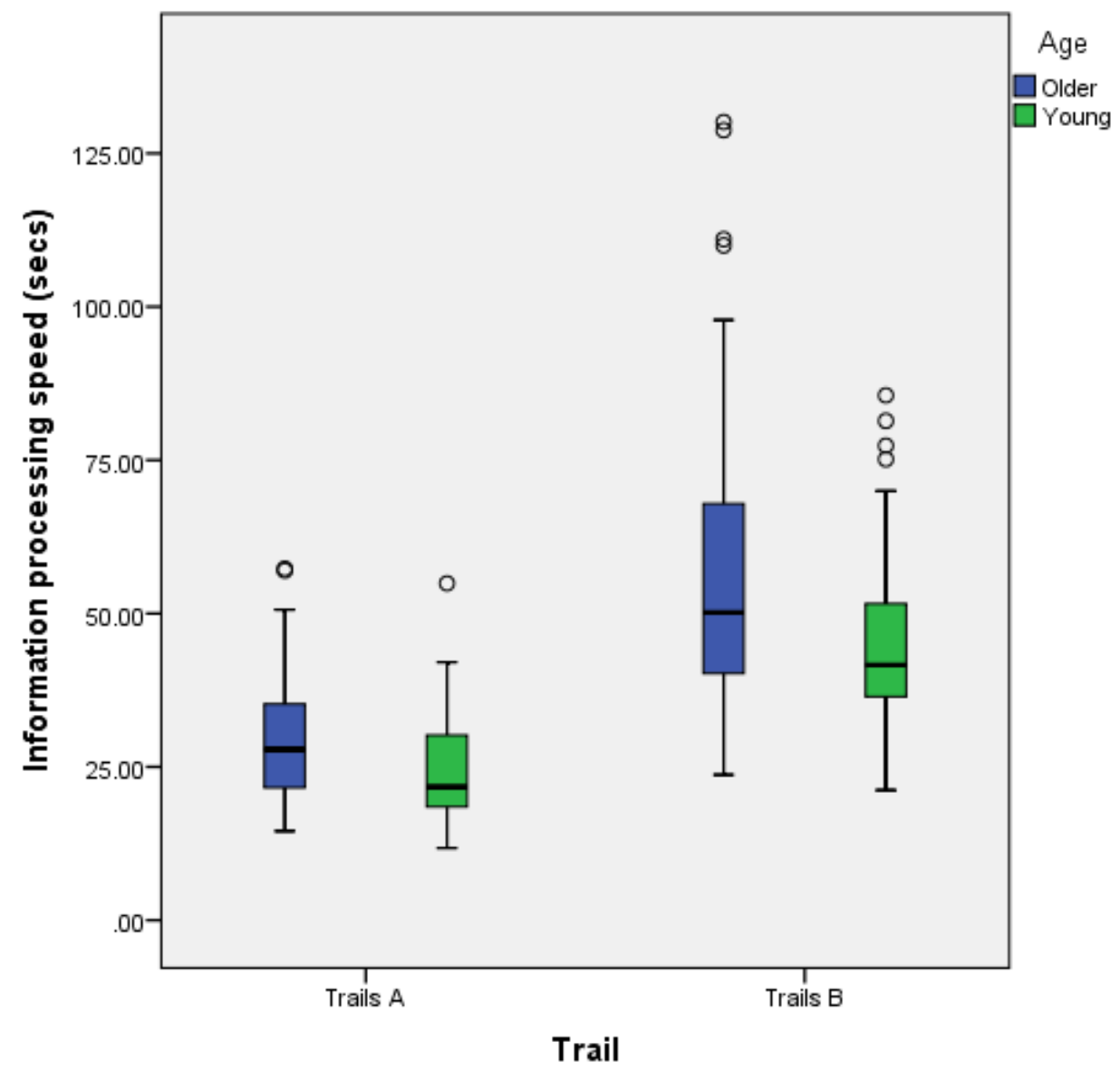

As displayed in Figure 9, at group level, older adults were slower compared to young adults over both Trails A and Trails B and information processing speed was slower for both groups in Trails B compared to Trails A.

Mann Whitney analysis revealed a statistically significant difference of reaction time between Trails A and Trails B; Trails B was performed significantly slower compared to Trails $\mathrm{A}$ in young adults $[\mathrm{U}=379.5, p<.001$, effect size $\mathrm{r}=.73$ ] and older adults [U $=570, p<.001$, effect size $\mathrm{r}=.69]$. 


\section{Trails A information processing speed}

For Trails A, Mann-Whitney analysis revealed a statistically significant difference of reaction time between young and older adults; young adults were faster in their reaction time performance compared to older adults [ $\mathrm{U}=1991.5, p=.001$, effect size $r=.26]$.

\section{Trails B information processing speed}

For Trails B, Mann-Whitney analysis revealed a statistically significant difference of reaction time between young and older adults; young adults were faster in their reaction time performance compared to older adults [ $\mathrm{U}=1962.5, p=.001$, effect size $r=.27]$.

\section{Information processing speed and subjective memory function}

In a novel approach, the information processing speed for each target condition and the distracter effect was examined with respect to subjective memory function in the older adult group. Correlational analysis using Spearman's rho revealed no significant correlation between information processing speed and total MFQ score (subjective memory function) for either Trails A or Trails B [both $p$ values > .05].

\section{Information processing speed and perceived test difficulty}

In another novel approach the information processing speed for both Trails A and Trails B was examined with respect to how difficult the participant reported the task to be. Correlational analysis using Spearman's rho revealed that for young adults, information processing speed did not significantly correlate with perceived test difficulty for Trails A and Trails B [both $p$ values > .05].

For older adults, information processing speed was significantly positively correlated with perceived test difficulty for Trails B; as perceived difficulty increased reaction time also increased (i.e. slower performance) $[\mathrm{r}=.293, p=.008]$. Information processing speed did not significantly correlate with perceived test difficulty for Trails A $[p>.05]$. 


\section{Information processing speed and education level}

Education was matched across both groups and for males and females as closely as possible making the educational level similar for both age groups. However a slight range in years of education was included which produced slight variability in results which is why the effects of education are examined. In both young and older adults, information processing speed did not significantly correlate with educational level for both Trails A and Trails B [ $p$ values > .05].

\section{Sex comparison: Information Processing Speed in males and females}

Figure. 10: Box plot of mean information processing speed (seconds) between males and females for both young and older adults in Trails A \& B.

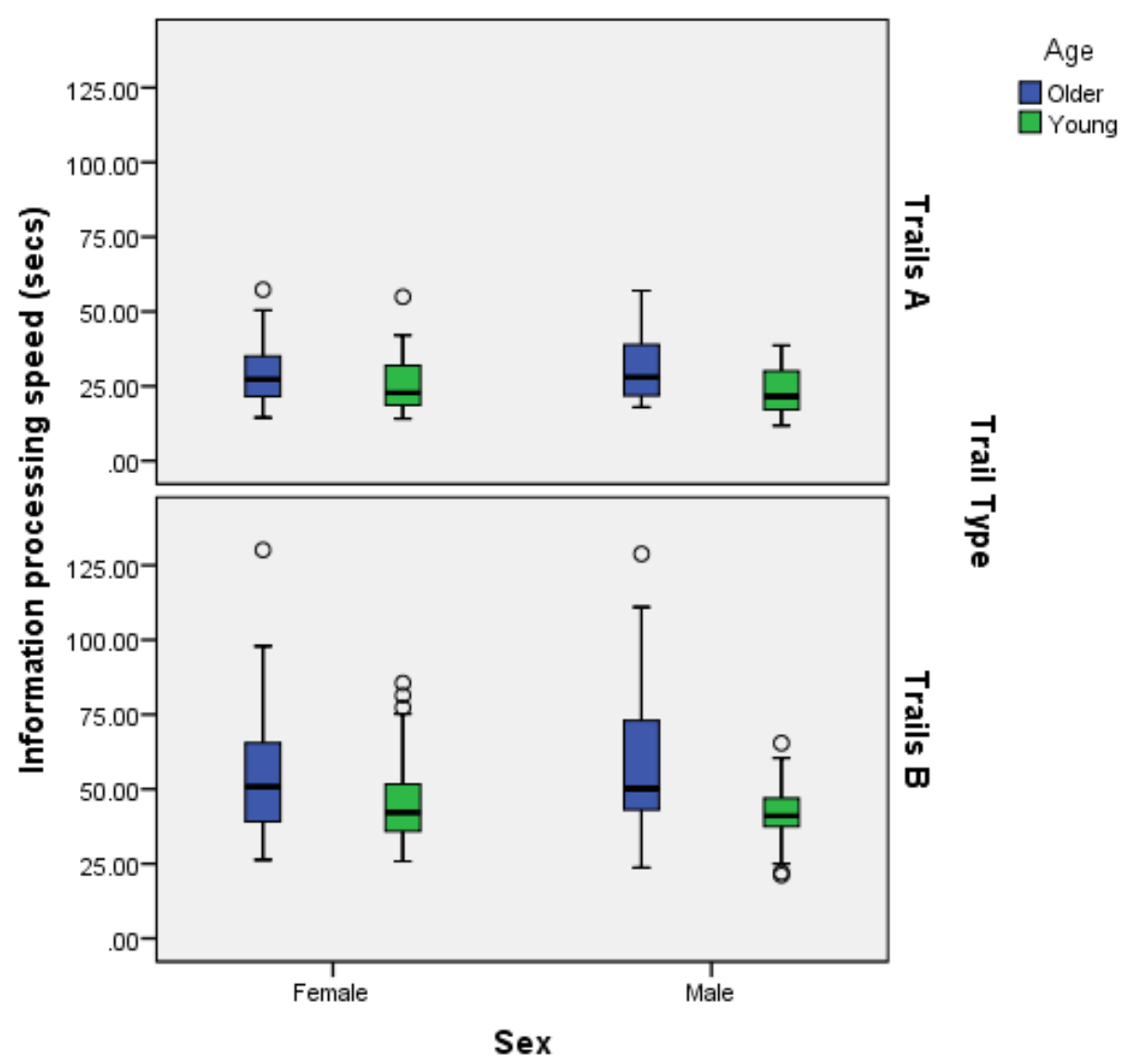

\section{Trails A}

Mann-Whitney analysis revealed no significant difference of information processing speed between males and females in young adults $[p>.05]$ and in older adults $[p>.05]$ (see Figure 10). 


\section{$\underline{\text { Trails B }}$}

There was no significant difference of information processing speed between males and females in young adults $[p>.05]$ and in older adults $[p>.05]$.

\section{Information processing speed, anxiety and depression levels}

In young adults information processing speed did not significantly correlate with anxiety or depression levels for both Trails A and B [all $p$ values $>.05$ ]. In older adults information processing speed did not significantly correlate with anxiety or depression levels for both Trails A and B [all $p$ values > .05].

\section{Information processing speed and objective cognitive performance (MoCA)}

In both young and older adults, there was no significant correlation between information processing speed and MoCA score (objective measure of general cognition) for Trails A [p > .05] or Trails B [p > .05]

\section{Subjective memory function and perceived test difficulty}

In older adults, subjective memory function (total MFQ score) was significantly negatively correlated with perceived task difficulty for Trails $\mathrm{A}[\mathrm{r}=-.275, \mathrm{p}=.013]$ and for Trails B [r = -.334, $\mathrm{p}=.002]$ : the higher the total MFQ score (i.e. less perceived change of memory function) the easier Trails A or Trails B was perceived to have been.

\section{Education and perceived test difficulty}

For young adults, educational level did not significantly correlate with perceived test difficulty of both Trails A and Trails B [both $p$ values > .05]. In older adults, education level was significantly positively correlated with perceived test difficulty of Trails A; the greater the level of education (years), the harder Tails A was perceived to be $[r=.238, p=.033]$ despite information processing speed not correlating with perceived test difficulty. However there was no significant correlation between education and the perceived difficulty of Trails $\mathrm{B}[p>.05]$. 


\section{Perceived test difficulty and objective cognitive performance (MoCA)}

In young adults there was no significant correlation between perceived test difficulty and MoCA score; how difficult the test was perceived to be did not relate to the overall quality of general cognition for Trails A $[p>.05]$ or Trails B $[p>.05]$.

In older adults perceived test difficulty significantly negatively correlated with MoCA for Trails A; perceiving Trails A to be more difficult related to having poorer general cognition $[\mathrm{r}=-.245, p=.027]$ but not for Trails $\mathrm{B}[p>.05]$.

\section{Subjective memory function and education level}

For older adults, educational level was significantly negatively correlated with subjective memory function (total MFQ score), i.e., lower levels of education were related to increase in MFQ score i.e. less perceived change of memory function $[r=-$ $.238, p=.032]$.

\section{Anxiety and depression levels}

Anxiety levels significantly positively correlated with depression levels in young adults $[\mathrm{r}=.778, p<.001]$ and older adults $[\mathrm{r}=.601, p<.001]$. As anxiety levels increased so did depression levels. In young adults, depression levels significantly negatively correlated with MoCA score; higher levels of depression related to having poorer general cognition $[\mathrm{r}=-.227, p=.042]$. In older adults depression levels significantly negatively correlated with subjective memory function (total MFQ score); in older adults perceiving fewer changes to memory function, depression levels were lower $[\mathrm{r}=-.253, p=.047]$. 


\section{4. DISCUSSION}

This chapter examined the integrity of information processing speed and IIV between young and older adults and the potential effects of educational level and sex, subjective memory function and perceived test difficulty using a typical clinical test; the Trail Making Test (TMT). The TMT has also been used in previous research to compare attention-related information processing speed between healthy ageing, $\mathrm{MCI}$ and $\mathrm{AD}$.

\section{Information processing speed}

\section{Age comparison in information processing speed}

Trails B was performed more slowly compared to Trails A in both young and older adults. This supports past evidence that Trails B produces slower information processing speed compared to Trails A [Sofko et al, 2014; Rasmusson et al, 1998]. Trails B is a more complex task compared to Trails A; as well as visual search, motor speed and numeric sequencing required during Trails A, Trails B requires switching between two separate sequences (consecutive numbers and consecutive letters). This required greater cognitive flexibility and producing larger amount of information. As a result greater processing load is required which outs greater strain on cognitive resources thus information processing speed significantly slows [Haworth et al, 2016].

When comparing age groups, older adults were significantly slower compared to young adults in both Trails A and Trails B (similar effect sizes) thus supports some previous research that ageing effects occur within both conditions of the TMT [Ashendorf et al, 2008; Chen et al, 2000]. This suggests older adults have poorer executive function compared to young adults i.e. number recognition, number sequencing visual search, switching attention, and motor speed. This result supports previous TMT studies comparing age groups that information processing speed slows during ageing [Ferreira, Molina, Machado et al 2014; Hagen, 2014; Plotek et al, 2014; Hashimoto et al., 2006; Demakis, 2004; Drane, Yuspeh, Huthwaite et al, 2002; Stuss et al, 2001; Keys \& White, 2000] Slowing of information processing speed in older adults further supports previous findings of impaired performance of executive function required during TMT [Cook et al, 2004; Keys \& White, 2000]. 
This slowing has been argued to relate to a decrease in white matter occurring during ageing and in areas of the brain associated with the TMT [Perry et al, 2009; Damoiseaux et al, 2009; Bucur et al, 2008; Demakis, 2004; Stuss et al, 2001] although brain scans were not available in the current study to support this.

The current results contradict past TMT studies which found no effect of age on information processing speed [Rasmusson et al, 1998; Boll \& Reitan, 1973] or finding younger adults displaying slower reaction times compared to older adults [Hamdan \& Hamdan, 2009]. This may highlight variability of outcome between studies due to, for example, different proportions of young and older adults, and smaller sample sizes which may result in the sample being underpowered.

It has been found in some TMT studies that slowing of information processing speed and executive function can be influenced by anxiety and depression [Emerson et al, 2005; Naismith et al, 2003; Barker et al, 1994] thus may explain differences in RT between young and older adults if one age group is more anxious or depressed than the other. In addition, in young adults higher levels of depression were associated with poorer general cognition (objective measure) which may relate to slower information processing speed. However, RT performance for both young and older adults did not relate to levels of anxiety and depression (levels were low) and young adults general cognition (MoCA score) was within normal healthy levels (the relationship was only just significant). Therefore this implies that slowing of RT is associated with increased age.

It must be noted that when comparing RT between young and older adults, effect sizes were small thus the result may not be very robust. In comparison, the effect sizes of the visual search were large which may suggest that the visual research produces more significant results for the difference of RT in ageing. Therefore in relation to clinical uses of RT tests, it may be more advantageous use multi trial research tests (i.e. visual search) compared to single trial clinical tests (i.e. TMT).

\section{Subjective memory function and information processing speed in older adults}

There was no correlation between information processing speed and subjective memory function for both Trails A and Trails B. Whether or not older adults perceived changes to their memory, this did not appear to influence information processing speed for either Trails A or B. 
These results support Minett and colleagues [2008] who found no association between subjective feelings and TMT performance although this was in clinical SCI and not subjective memory function as measured in this study. In addition the work of Minett et al is only one study to compare the current results with.

A lack of an association between subjective memory function and RT in the current research TMT study may help understand which factors should also be considered when using TMT as a measure of RT in clinical settings. Subjective memory function i.e. perceived changes of memory function in the general population may not be an important factor to consider when using the TMT in clinics to measure information processing speed in healthy ageing to compare with pathological ageing i.e. MCI or dementia. The research test of visual search also found no association between RT and subjective memory function. This implies that in relation to the effects of subjective memory function there appears to be no difference between a single trial clinical test (TMT) or a multiple trial research test (visual search).

As a result of finding no relationship between subjective memory function and information processing speed, we speculate that in individuals taken from the general population, information processing speed in during the TMT remains at 'normal' levels despite some of these individuals perceiving problems with their cognition. Since there is a close relationship between information processing speed and structural change i.e. reduced white matter, we speculate that any slowing of information processing speed or any perceived changes to memory function is unlikely to relate to detrimental structural change. In addition since there is an association between MCI or AD and slowed information processing speed, a lack of a relationship between perceived memory function and information processing speed is unlikely to indicate a neuro-degenerative basis for these complaints especially since the majority of participants in this study reported subjective memory changes performed to 'normal' levels in objective testing (MoCA score) [as discussed in Torrens-Burton et al, 2017]. However there were a minority of older adults with lower MoCA scores which may imply abnormality with general cognition although we only used one test of general cognition thus further assessment is required.

Finding no relationship between subjective memory function and may not be helpful for the further characterization of SCI if indeed the effects on RT are similar between the two (see introduction to this thesis for further discussion). 
However, we did not have access to brain scans thus cannot determine any structural change in these older adults.

In addition, we were unable to follow up these older adults for further assessment thus it cannot be said whether those with perceived changes to memory function indeed decline further. We also did not include detailed measures of memory which may have validated any perceived changes to memory function older adults may have had.

Some previous research associated subjective memory complaints (albeit clinical SCI and not subjective memory function) with high levels of anxiety and depression rather than structural change [Caselli, et al, 2014; Montejo et al, 2011]. However, in both young and older adults there was no relationship between RT and depression or anxiety levels. Therefore, anxiety and depression levels did not appear to relate to how poor some older adults perceived their memory function to be. This may relate to the levels of anxiety and depression levels being low, possibly because those older adults perceiving poor memory function were not too anxious or depressed as a result. For some older adults this may have been the first time they had contemplated any changes to their memory function thus not displaying any underlying anxiety to affect RT. In addition, since subjective memory function was measured after the TMT was completed, any possible concern with memory would not have had an effect on RT performance.

There were some older adults producing RT values which were significantly slower than the mean score. These outliers differed from the extreme scores removed before analysis as they did not relate to any extraneous noise. Two older adults produced significantly slow RT scores and a low MFQ score (greater perceived changes to memory function). This may suggest that their perception of memory function reflects their actual RT performance i.e. poorer memory function thus poorer performance (slower information processing speed). In contrast the other significantly slow RT scores were in older adults who perceived little changes to memory function thus highlighting individual differences between participants. This may imply disproportionate slowing regardless of subjective memory function measure which may be reflecting underlying structural change. If this is the case, these individuals cannot be used within healthy control groups as they may be skewing the mean result of what is considered to be a 'normal' level of slowing in healthy older adults during the TMT. 
Consequently, results of healthy control groups in TMT may be misinterpreted. Further assessment and follow up in these particular individuals will be of interest to observe whether these individuals are of clinical significance.

The current results imply that subjective memory function does effect underlying cognitive function in the TMT although there is the possibility that finding no relationship between information processing speed and subjective memory function is due to the TMT task itself not being sensitive enough to detect any functional changes between older adults form the general population despite them perceiving changes to their memory.

\section{Perceived test difficulty and information processing speed}

In young adults information processing speed did not significantly correlate with perceived test difficulty for Trails A and Trails B. How difficult young adults perceived either Trails A or Trails B to be did not reflect how quickly they performed each task. Therefore we speculate that this particular example of self-assessment does not influence information processing speed in young adults during TMT thus it may be beneficial to measure other psychological factors i.e. self perception of abilities to observe any potential effects they may have on RT.

In contrast, in older adults, perceived test difficulty significantly correlated with information processing speed for Trails B. Older adults perceiving Trails B to be more difficult performed more slowly compared to those perceiving the test to be easy. This result implies that, the judgement of test difficulty is related to the nature of the test (i.e. the complexity of conditions) and related to actual performance. In addition since there was no relationship found between subjective memory function and RT, older adults' self assessment of test demands may be having an effect on slowing information processing speed and not the result of underlying cognitive dysfunction. This supports a previous finding that previously that self-reporting is associated with poor TMT performance [Setti et al, 2015] (although the selfreporting measure related to visual acuity and not perceived test difficulty and this is only one study to compare the current results with). 
A study by Torrens-Burton and colleagues [2017] also examined perceived test difficulty and RT in TMT and found no correlation between perceived test difficulty and RT in Trails A but a significant positive correlation in Trails B i.e. perceiving the test to be more difficult related to slower information processing speed. This study also concluded that judgement of test difficulty is related to the nature of the test.

Perceived test difficulty had an influence on older adult information processing speed thus we can speculate that other psychological factors may also influence information processing speed. This factor appeared to effect young and older adults differently which may affect how differences in information processing speed during the TMT are interpreted throughout ageing. Reasons for why perceived test difficulty differed between young and old may relate to factors such as personality differences, occupation, hand dexterity or mood however these factors were not measured in the current study thus need to be examined in greater detail in future studies. Judgement of test difficulty may also depend on individual differences between older adults such as the level of education i.e. levels of education may influence how demanding the test is perceived to be. This relationship between perceived test difficulty and education is examined in a later section. An association between perceived test difficulty and RT in the current research TMT study may further help understand how RT should be measured in clinical settings i.e. perceived test difficulty should be taken into account if measuring RT in healthy older adults.

The research test of visual search also found an association between RT and perceived test difficulty which was also dependent of the condition within the test (see Chapter 2). This implies that in relation to the effects of perceived test difficulty there appears to be no difference between a single trial clinical test (TMT) or a multiple trial research test (visual search).

\section{Education and information processing speed}

In both older and young adults, there was no significant effect of education on information processing speed performance in Trails A and Trails B. This supports previous TMT studies finding no influence of education on information processing speed in older adults and young adults [Hashimoto et al, 2006; Seo, Lee, Kim et al, 2006; Tombaugh, 2004]. 
The current results are however in contrast to previous TMT studies finding an influence of education with higher levels of education associated with faster information processing speed [Ptotek et al, 2014; Palsson, 2013; Bezdicek et al, 2012; Cangoz, et al, 2009; Hamdan et al 2009; Tombaugh, 2004] in both young and older adults [Hamdan et al 2009; Tombaugh, 2004; Amodio, Wenin, Del Piccolo et al., 2002].

This outcome variability may reflect differences in the way previous TMT studies were conducted compared to the current study. Not all TMT studies have included young adults to compare with older adult information processing speed [Bezdicek et al, 2014; Palsson, 2013; Tombaugh, 2004; Waldermann et al, 1992] or age ranges have differed or not been separated [Plotek et al, 2014; Palsson et al, 2013]. In addition the range of education was relatively narrow in the current study compared to ranges used in previous studies [Bezicek et al, 2014; Plotek et al, 2014; Hamdan et al, 2009' Tombough, 2004]. These differences make it difficult to compare education effects on information processing speed in ageing between studies.

Not finding a relationship of education with RT in the current TMT study may relate to the range of education (in years) in the current study being relatively too narrow to find a correlation of education level on information processing speed in young and older adults. In young adults this narrow range may relate to all young adults being university students (considered as a previous limitation [Tombaugh, 2004]) and in older adults, this may relate to the older adults participating in the study being of a particular level of education. This may imply that the TMT is not sensitive enough to find an effect of education within this educational range in comparison to other attentional tests as interestingly, there was an influence of education on information processing speed for older adults found in visual search despite this narrow educational range (see previous chapter). This is interesting as although participants groups in this test were different than the visual search in previous chapter the demographics were similar i.e. similar education range.

\section{Sex and information processing speed}

There was no significant effect of sex on information processing speed in both Trails A and Trails B and for both young adults and older adults. This supports previous TMT studies who found no influence of sex on information processing speed in older adults [Haworth et al, 2016; Bezdicek et al, 2012; Ashendorf et al, 2008] and young adults [Palsson, 2013; Tombaugh, 2004; Waldmann et al, 1992]. 
In contrast other TMT studies found a sex effect in older adults during Trails A [Seo et al, 2006] or in both A \& B with males to produce faster reaction times [ Cangoz et al, 2009; Dodrill, 1979].

Mixed results of sex effects between TMT studies including the results of the current study (and between Trails A and B) may have related to methodological differences between studies i.e. no inclusion of young adults [Palsson, 2013; Tombaugh, 2004; Waldermann et al, 1992] and smaller sample sizes in previous studies [Tombaugh, 2004; Ashendorf et al, 2008]. It may also related to including different sample characteristics i.e. IQ, socioeconomic status, sleeping patterns [Wright et al, 2016] or greater range of education [Ashendorf et al, 2008] which were not included in the current TMT thus a limitation of the current study.

The current results also differ to the visual search in the previous chapter. A sex effect was found in young adults during visual search with males being faster than females however the same was not found in the young adults during the TMT study. It must be highlighted that both tests contained different participants despite their demographics being similar which may relate to why outcome variability of sex effects between visual search and TMT. A relationship between sex and RT may also depend on the type of test used hence why we aim to examine the influence of sex between different attention tests to see if effects differ between tests. These sex effects may have varied between the visual search and the TMT due to the way both tests were conducted. The visual search was a research test containing multiple trials whereas the TMT was a simple pen and paper test of a single trial. Multi trial tests such as the visual search may relate to an increase of complexity when performing the test thus may explain why differences where found between males and females although this was only observed in young adults. Therefore when measuring RT it may be better to use research tests such as the visual search rather than clinical tests such as the TMT.

\section{Subjective memory function and perceived test difficulty}

In older adults, subjective memory function correlated with perceived test difficulty for both Trails A and Trails B. As older adults perceived greater changes to their memory (low MFQ score) the more difficult both Trails A and Trails B were perceived to be. 
This supports our speculation that if older adults believe their cognition to be poor, they would assume that tests will be more difficult to perform as a result as poor cognition will impair the ability to perform a cognitive task well enough. It must be noted that the relationship between subjective memory function and perceived test difficulty produced a small effect size in trails A but a medium effect size in Trails B which implies that the results of Trails B result are more robust thus more reliable.

As far as we are aware, subjective memory function has not been correlated or examined with perceived test difficulty in previous TMT studies. Therefore further examination is required to compare subjective memory function with perceived test difficulty as well as other psychological factors, in order to compare results with the current study. Further examination is required why psychological factors i.e. perceived test difficulty (test demands) influences information processing speed in older adults during the TMT but not subjective memory function. In addition further examination is required to determine subjective judgement is more influential on information processing speed in other aspects of attention thus in the current study interactions between subjective memory function and perceived test difficulty are examined in different attention tests.

\section{Education and perceived test difficulty}

In young adults there was no relationship between education and perceived test difficulty. The number of years in education had no influence on how difficult young adults perceived the TMT to be. This finding was also found in the visual search test (see previous chapter) and may relate to the education range being too narrow to find a relationship (since all young adults were university students). In addition, finding no relationship of education in young adults may relate to finding no influence of perceived test difficulty on information processing speed i.e. perceived test difficulty is not an influential factor in young adults since we speculated perceived test difficulty relates to education level.

In older adults, education significantly correlated with perceived test difficulty although only during Trails A. Higher levels of education (in years) related to perceiving Trails A to be more difficult. This result was also found in the visual search test (see previous chapter) and supports our assumption that judgement of TMT difficulty relates to the level of education although not in the direction we anticipated. 
We assumed high levels of education may help to mentally switch between numbers and letters during the TMT (i.e. greater practice of cognitive flexibility) thus the TMT would be considered easy to perform.

In addition we assumed Trails B would have been considered more difficult due to having to switch between two sequences and since only in Trails B was there was a negative relationship between perceived test difficulty and RT. Instead, perceived test difficulty was high in older adult with higher levels of education and this was only observed in Trails A. Therefore, we speculated that older adult were unfamiliar with the task thus were judgemental about the TMT (particularly Trails A) thus assuming the test to be more difficult that it was. Alternatively older adults perceiving Trails A to be difficult may have been justified as these individuals were related to having poorer general cognition (i.e. having a lower MoCA score). However it must be noted that overall cognition was still within the 'normal' range for healthy ageing and effect sizes was low implying the relationship was not so robust or meaningful.

It must be noted that the effect size of the significant result of Trails A was small therefore the findings may not be robust enough to be reliable. As far as we are aware there are few, if any, TMT studies which have examined education against perceived test difficulty. Therefore further research is required to examine whether other psychological factors other than perceived test difficulty relate to the level of education in older adults and whether similar findings occur across different visual attention tests.

\section{Education level and subjective memory function}

In older adults, educational level significantly correlated with subjective memory function. Lower levels of education related to fewer perceptions of change to memory function. Therefore, older adults with higher levels of education are likely to perceive their memory is getting worse. As far as we are aware, education and subjective memory function have not been compared previous during the TMT thus the result in the current study cannot be compared to previous TMT studies. The result may suggest that older adults with higher education are detrimentally judgemental about their memory function. 
In contrast, it may suggest that older adults with higher education are possibly more aware or have greater understanding of the quality of their memory function.

However, a limitation of this study is that did not include specific memory tests but just a general score of overall cognition (MoCA score). If specific memory tests were included this may have provided more information on their actual memory function thus we may have found different relationships with subjective memory function.

A similar relationship had also been found between education and perceived test difficulty. It was speculated that subjective memory function is also a psychological factor i.e. type of self assessment (of memory function) [as discussed above]. This may provide an explanation as to why a relationship was also found between educational level and subjective memory function i.e. psychological self-assessment relates to educational level. It is interesting that education had an effect on subjective memory function but not on information processing speed. This suggests that in the TMT, education has an influence on what older adults think about their memory function (and in addition, how demanding they perceive the TMT to be) but does not influence actual performance (slowing of information processing speed).

This result differs to what was found in the visual search where education did not correlate with perceived changes to memory function. Despite similar demographics of older adults in the TMT and visual search, the use of different participants between tests may relate to the variability of result outcome. In addition outcome variability may depend of the type of test examined although again the sample size was small thus the result may not be very robust and reliable. Therefore further examination of different visual attention tests is required to determine whether a relationship between education and subjective memory function is similar across tests.

\section{Errors}

Previously it has been assumed that older adults make significantly more errors during the task compared to young adults especially in Trails B as the strain on executive processing increased [Rasmussen et al, 1998]. Seo and colleagues [2006] suggested that the fact that a large number of elderly participants in their study were unable to complete the test was an indication of the limited applicability of Trails B in particular for elderly participants especially if they had a lower educational background. 
In the current study the number of errors per se were not measured between Trails A and B or between young and older adults because they were considered at the time of testing difficult to quantify although we did observe after the test was completed some papers showed lines going to the incorrect number or letter in the sequence then corrected. It was found that 10 young adults and 6 older adults could not complete Trails B (i.e. gave up) thus were eliminated from analysis. In previous research studies of TMT, older adults failing to complete the test related to low levels of education (i.e. 6 years) [Cavaco et al, 2013; Seo et al, 2006] however in the current study older adults had higher levels of education in comparison to these previous studies. Test incompletion may instead indicate impaired cognitive ability in older adults i.e. switching attention reflecting an inability to move from number to letter alternatively in the allotted time for performance to be considered 'normal'.

Alternatively, we proposed task incompletion may have related to methodological problems. For example, as a pen and paper task, the TMT relies on fully functioning motor control as well as sufficient vision in order to be able to complete it successfully. We speculated that any issues with motor movement i.e. hand dexterity or visual impairment, however minor, may make the test difficult to complete. Indeed the older adults did find the format of the test a challenge to physically move their hand around the page while having to search for the next number or letter. If motor performance during a particular test (such as the TMT in the current study) is significantly more influential on RT performance other than the attended measure of attentional function, the test in question may not be the best one to measure RT in ageing. This may depend on the type of motor movement. For example motor movement was also required during the visual search (pressing two buttons) however it is possibly easier to press buttons compared to drawing lines across a page in the TMT, particularly if hand dexterity is an issue such as having arthritis, although we did not measure hand dexterity thus a possible limitation of the current study.

Interestingly, the young group contained more individuals who could not complete the test. Previous study found participants (older adults) failing to complete the TMT due to language barriers [Seo et al, 2006]. Indeed within the young group in the current study there were young adults whose first language was not English which may have contributed to test incompletion i.e. not understanding the instructions. 
Other reasons for not completing the test may be associated with not concentrating properly on the test thus giving up. However we could only speculate the reasons as a limitation of the current study was not to ask directly for details as to why young adults did not complete the test.

Failure to complete the test highlights the issue that even in research settings, the TMT cannot be completed by both young and older adults i.e. individuals considered cognitively healthy from the objective measure of general cognition (high MoCA score) thus this questions whether the TMT should therefore be used in clinics, test incompletion may be incorrectly associated with cognitive dysfunction. Alternatively it may be better to use a research test in clinical settings such as the visual search examined in the previous chapter (Chapter 2) since no participants failed to complete the test.

\section{Outliers}

Outliers other than those removed before analysis (because of extraneous noise) occurred in young and older adult data for both Trails A and Trails B. Although more outliers occurred in so in Trails B possibly due to greater processing load (switching between numbers and letters). In older adults, outliers may depict disproportionately slower information processing speed compared to mean level in healthy ageing as a result of underlying structural change i.e. a diagnosis of SCI. These individual scores may be skewing the mean result of older adults and lead to a misinterpretation of what constitutes 'normal' levels of information processing speed in healthy ageing thus are of interest for further examination and follow up.

Outliers in young adults may also imply these individual young adults are significantly worse at performing the TMT due to early signs of underlying cognitive dysfunction. However we had no way of testing this (i.e. no brain scans or longitudinal studies) thus we speculate these slow RT scores are more likely due to extraneous noise i.e. lack of concentration but were below our cut off scores thus not removed before analysis.

There were a greater number of outliers in females compared to males in Trails A and B for both young and older adults. We would speculate that these higher RT values in females may increase the mean score compared to males. However since no sex affect was found on information processing speed in both young and older adults, we therefore speculate that these outliers did not significantly affect the mean difference between female and male RT scores thus influence the overall result. 


\section{Study Limitations}

Individual differences occurred during test performance despite the same instructions given. For example some participants did not make a continuous trail but instead stopped after each letter or number taking the pen off the paper (despite being given explicit instructions not to do this) while others did what was instructed. In addition, regardless of clear instruction, some participants were unable to complete the test but instead stated they could not finish thus testing stopped. All these participants were excluded from analysis since the test wasn't performed correctly or fully which did mean that the number of people who could be used in analysis reduced slightly. This is important issue as without appropriate and strict instructions, people will use different strategies that may affect outcome. This may also be observed in clinics with individuals, particularly those with any cognitive dysfunction forgetting instructions. If the TMT is causing problems due to the way in which it must be performed it may not be the best measure of cognition to use on older adult participants and even on a younger cohort.

Another significant limitation of the trail making test is that it only requires one trial per participant and when used in memory clinics, that becomes their cognitive measure towards a diagnosis. Despite the ease of administration, it has been argued that one trial is not sufficient enough to make comparisons between healthy and pathological ageing and a task with a greater number of trials will provide more sensitive results [Haworth et al, 2016] and also be able to measure intra-individual variability (IIV) in order to measure the functional integrity of information processing speed. It had already been recommended from a previous TMT study that multi-trial type tasks should be administered rather than a single trial test [Salthouse \& Fristoe, 1995]. Haworth et al [2016] performed the TMT on MCI patients and healthy controls as well as a computerized visual search paradigm which contained significantly more trials.

The visual search task used within research settings, not only showed to be more sensitive compared to the TMT when finding a difference between the two groups, but demonstrated that tasks used during a research setting may prove to be superior to those used in a clinical setting therefore the gap between methods used in clinical settings and research laboratories requires closing. 
This difference between visual search and TMT was also found in the current study as highlighted by small effect sizes in the TMT compared to larger effect sizes in the visual search test (see Chapter 2). This implies that the TMT results may not be as robust as the visual search thus supporting the argument that tasks used during a research setting (visual search) may prove to be superior to those used in a clinical setting (TMT). Therefore, the challenge is to develop tests which are sensitive to detect change to information processing speed and IIV but which can be effectively administered in a clinical environment.

\section{Conclusion}

The current study examined the functional integrity visual attention and information processing speed and IIV between young and older adults using the clinical TMT as well as measuring the influence of other factors of sex, education perceived test difficulty and subjective memory function which have not been examined in detail previously. The results indicated that older adults were significantly slower compared to young adults on both TMT A and TMT B. There was no influence of subjective memory function on information processing speed for both Trails and Trails B, although subjective memory function did correlate with education as older adults who perceived greater changes to their memory had lower levels of education.

Subjective memory function also correlated with perceived test difficulty in older adults; the easier both Trails A and B were the perceived to be, the less change to memory function older adults perceived to have. Perceived test difficulty correlated with information processing speed (Trails B only) in older adults i.e. older adults were slower when finding Trails B to be more difficult. Older adults with greater levels of education also found the test to be more difficult. There was no effect of sex and education to information processing speed in both young and older adults.

Similarities (effects of perceived test difficulty but not subjective memory function) and differences (effects of sex and education) between the current TMT study and the previous visual search test (visual search examined in Chapter 2), research may help further understanding as to how RT should be measured in healthy ageing i.e. what are the effects of ageing and person related factors on information processing speed. 
To speculate, (as in Torrens-Burton et al, 2017) significant, disproportionate slowing of information processing speed in some older adults is likely to be related to possible neurodegenerative change or structural abnormality [see Torrens-Burton et al, 2017 for similar discussion] (bearing in mind the relationship between white matter changes, and slowed RT in AD). However it must be noted that there was no access to participant brain scans thus we cannot be sure of the structural and functional integrity of the brain. What must also be highlighted is that the majority of effect sizes were small suggesting any relationships between information processing speed and education or between information processing speed and perceived test difficulty are not very robust thus potentially unreliable.

As speculated above, test outcome i.e. influence of different factors on information processing speed, may be test specific (and specific to conditions within tasks) since finding correlations between single conditions only (either Trails A or Trails B). Therefore further types of visual attention tests need to be examined in this second study to observe whether similar outcome occurs between tests. With this in mind, we will use a different test (Multi-item Localization test) in a later chapter similar to the TMT in its measure of information processing speed in relation to executive function but containing multiple trials thus determine whether multiple trials increase test sensitivity.

Some of the older adult data from Chapters 2 and 3 of this thesis was published in a study by Torrens-Burton and colleagues [2017]. This study examined relationships between a typical clinical test (TMT) and commonly used research test (visual search) and educational level, subjective memory function and perceived difficulty for each test. Information processing speed did not significantly correlate with subjective memory function for both the TMT and the visual search although some individuals displayed significantly slower information processing which may be of clinical significance and require further assessment and follow up. Greater perception of task difficulty was associated with higher perception of subjective memory dysfunction in the TMT only and both tests found a relationship between actual information processing speed and perceived task difficulty although the direction of this relationship varied between tests. The results from this study highlight the importance of taking into account perceived test difficulty factors and the type of task when measuring integrity of information processing speed in older adults. The researchers argued that this is important since information processing speed is now specifically cited as a significant measure i.e. in the DSM-5 for NCD. 


\subsection{CHAPTER FOUR: Simple \& Choice Reaction Time}

\subsection{INTRODUCTION}

In brief, results from the visual search (Chapter 2) and the TMT (Chapter 3) found information processing speed was significantly slower in older adults compared to young adults in both tests and more variable in the visual search. For both tests subjective memory function did not significantly influence information processing speed (or IIV in visual search), i.e., whether or not older adults perceived changes to their memory, did not have an influence on their actual performance. In older adults, perceived test difficulty correlated with information speed adults in target alone condition of the visual search and Trails B of the TMT however the direction of the correlation varied between tests. In visual search perceiving the test to be difficult was associated with faster information processing speed however in the TMT perceiving the test to be difficult was associated with slower information processing speed.

In visual search information processing speed and IIV was influenced by education in older adults with higher levels of education associated with faster and less varied information processing speed. Sex was associated with information processing speed (males performing faster and less variably compared to females) only in young adults and only in the target plus distractors condition. In contrast, the TMT revealed no relationship between information processing speed and sex or education in young or older adults in the TMT.

So far from the TMT and visual search results highlight the potential importance of considering different participant-related factors when measuring information processing speed in ageing studies. In addition, result outcome with such factors can be dependent on a specific condition of the test or the type of test itself i.e. a research test with multiple trials (visual search) compared to a single trial clinical test (TMT). In addition effect sizes of RT between young and older adults were larger in the visual search compared to the TMT suggesting visual search better distinguished differences of information processing speed in ageing. This implies that when measuring information processing speed in ageing, tests used in clinical settings such as the single trial TMT should be replaced with research tests of multiple trials i.e. visual search. 
Therefore, in the current chapter we continued examining information processing speed using other research attention tests with multiple trials namely Simple reaction time and Choice RT. to determine whether these research tests also produce greater difference of RT in ageing as well as the opportunity to measure IIV from using multiple trials.

\section{Simple and Choice RT}

In this chapter information processing speed and its variability (IIV) was examined using a Simple reaction time and a Choice RT test; both commonly used in research but not in clinical practice. The Simple RT test designed for the current study involved making a response as quickly as possible to a single stimulus whereas the Choice RT designed for the current study required making an appropriate response to one of a number of possible stimuli [Deary et al, 2011] which requires additional cognitive functions i.e. executive decision making. In the current study both tests included measuring of processing which occurs when being pre warned of a stimulus appearing thus allowing a response to be prepared for.

The Simple and Choice RT tests differ (in relation to attention function) in comparison to the other research test used in the current research so far (visual search) since in this research the aim was to compare RT and IIV in different attention tests. The target alone condition of the visual search test (feature search) involved automatically shifting the focus of attention to a given salient object on the screen i.e. finding a pre-defined stimulus because its salient feature captures attention. In contrast, in Simple RT the target stimulus is not specifically designed to be salient as it automatically captures attention being the only stimulus on the screen. The Target alone condition does allow for a measure of comparing baseline choice RT between and within age groups however in comparison to the Choice RT test in the current study, the stimuli differ (symbols or letters) which may activate different areas of cognitive function. The 'Conjunction component' of the visual search paradigm (Target plus distractors condition) involves shifting attention 'at will' to the location of given object within the environment and ignoring distracting information. In comparison in the Simple and Choice RT tests, this aspect of selective attention is not required to the same extent as only one stimulus is presented at one given time. 
As part of the second larger study, the same young and older adult participants from the TMT were used (where possible), we could examine whether information processing speed and IIV differs in ageing depending on the test used. The Simple and Choice RT results were examined in relation to the influence, if any, of different factors (sex, education, subjective memory function, perceived test difficulty) and whether outcome varies to results of the TMT and visual search. In addition the Choice RT examined how the number of trials may be associated with information processing speed and IIV by splitting the test into four separate blocks of trials.

An issue with the TMT was that the integrity of IIV could not be measured due to the test involving only a single trial. Due to multiple trials the Simple and Choice RT tests are able to measure the variability of RT performance in young and older adults. Unlike many research tests of information processing speed and its variability, these tasks are very simple and easy to perform. Both tests (measuring different aspects of attention) were examined to determine whether the integrity of information processing speed and IIV between older and young adults is similar between tests and whether there are similar influences of sex, education, perceived test difficulty and subjective memory function.

\section{The Simple RT test}

The Simple RT test designed in the current study included three coloured circles (red, orange, green) with instructions of 'Ready', 'Steady' and 'Go!' appearing consecutively on a screen (see Figure 11). The aim was to respond to the target green 'GO!' as quickly as possible after the first two cues by pressing a single button. Including visual cues before presenting the target was designed for an increase in alertness i.e. preparing the brain for the arrival of information that needs a response. Therefore the test measures an aspect of processing which occurs when an individual is pre-warned of a target thus allowing them to prepare their response. This aspect of processing was examined by measuring reaction time (RT) from the target appearing to the response being given (button press).

In addition, the length of time between the two cues and the target stimulus differed during each trial. The use of these visual cues as well as varying the time they were presented was designed to reduce the number of anticipatory responses i.e. pressing the button too soon [as described in Dykiert, Der, Starr \& Deary, 2012]. This may occur as participants learn the order of each cued and target stimuli thus try to predict when the target will appear. 
Figure 11: Representation of the Simple RT stimuli in which 'Ready', 'Steady' and 'Go!' are displayed consecutively.

\section{1.}

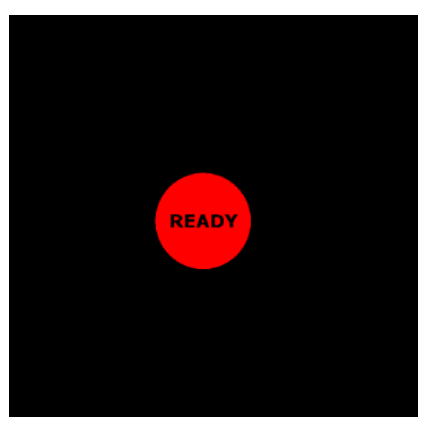

\section{2.}

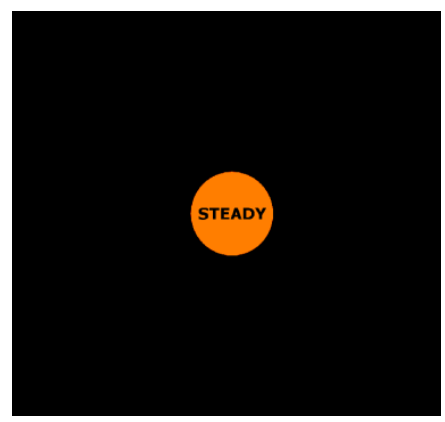

3.

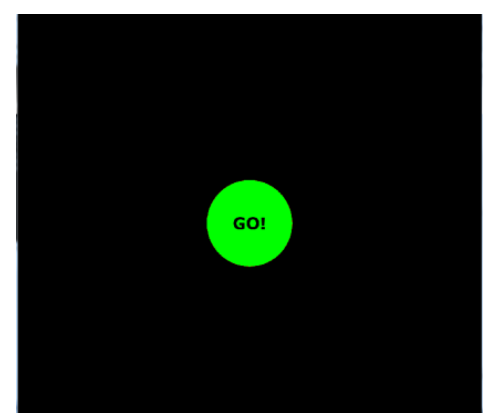

The Simple RT test measures the integrity of information processing speed of detecting/finding and attending to a designated target i.e. the ability to prepare, organise and execute a response [Vrtunski et al, 1983]. In this Simple RT test the preparation relates to the visual cues presented before the target stimulus warning the target arrival and that a response is required thus allowing the individual to prepare for said response. Following the cue, the target appears on the screen which is processed and understood that this is the target to respond to thus such response is executed i.e. a button press. Reaction time is measured from the time it takes for the target to appear on the screen (i.e. recognise the target) and to respond (press a button).

It is common for Simple RT studies to include a visual cue before the target is presented i.e. prompting an individual to prepare for the target to arrive [Dykiert et al, 2012]. The brain has limited processing resources thus in order to draw a persons' attention towards a particular location or object, a warning is given in advance in order to prepare the individual for the upcoming stimulus and required response. This helps the individual to be more efficient at processing the stimulus and respond to the target i.e. faster RT. This visual cue allows a person to react quickly when a target stimulus appears shortly afterwards due to a phasic increase in alertness [Posner, 1986]. This operation can enhance the processing of the target stimulus thus producing a faster response [Fernandez-Duque \& Posner, 1997]. This alerting effect is mediated by the noradrenergic system [Tales et al, 2002] which plays an important role in sustaining a persons' sensory readiness for external stimuli [Tales et al, 2002; Robbins, 1997; Posner, 1993]. 
The Simple RT test in this study does not measure the alerting effect per se since trials did not differ between cued and non-cued responses i.e. we did not measure differences in RT between warned (cued) or non-cued target performance. However, the test uses visual cues ('Ready' and 'Steady' circles) in order to increase some alertness thus prompt individuals to prepare for a response. Two visual cues of different colours (red and orange) were used as they simulated a familiar traffic light sequence thus participants would be prepared/alert for a green Target stimulus thus able to respond quickly once it appeared on the screen. Therefore this represented normal everyday aspect of attention-related info processing speed i.e. we learn that every day events follow each other and know when to prepare for a specific response.

\section{The Choice RT test}

The current Choice RT test used stimuli and methodology modelled on the study by Ballesteros and colleagues [2013]. In each trial, a visual cue of an asterisk was presented on a computer screen followed by one of two target stimuli, ' $\mathrm{X}$ ' or ' $\mathrm{O}$ ' (see Figure 12). Participants respond to each target stimulus with a designated response key: pressing ' $Z$ ' on a keyboard with the appearance of the ' $\mathrm{X}$ ' target and the ' $\mathrm{M}$ ' key with the appearance of the ' $\mathrm{O}$ ' target.

Figure 12: Representation of the choice RT stimuli by responding with either the $\mathrm{z}$ or m keys.

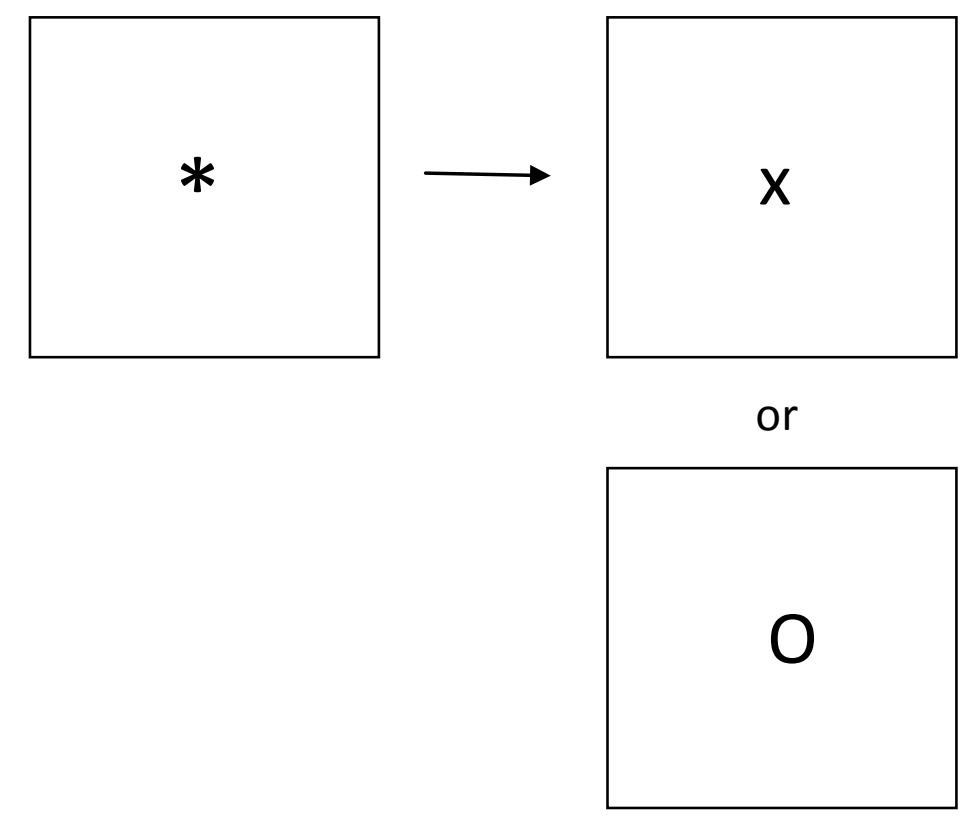


A visual cue of an asterisk was designed to increase alertness i.e. prepare the brain to detect the arrival of a target stimulus which requires a response. Reaction time was then measured for the time it takes to process the target stimulus once it appears on the screen and then respond.

How the Choice RT differs from the Simple RT to prepare for and execute a response is that it includes multiple target stimuli, each with a designated response. This requires further processing as once a target appears on the screen an executive decision needs to be made before responding as to which response relates to which target stimulus. This needs to be done very quickly as although the individual is pre warned of the stimulus appeared thus are prepared for the response, they do not know which of the two responses will appear. Therefore the Choice RT test measures the time taken to process the response once it appears, quickly make a decision then respond correctly.

An increase of mental operations (i.e. executive decision making) may put greater strain on mental resources thus affecting how quickly the stimulus can be responded to i.e. slower RT. Therefore, Choice RT tests may produce slower reaction time performance compared to Simple RT tests which only requires responding to a single stimulus. Indeed this has been observed in previous choice RT studies when comparing information processing speed with Simple RT tests [i.e. Woods et al., 2015; Bugg et al, 2006; Der \& Deary, 2006; Krieg et al, 2001]. Note however that in the current Simple and Choice RT tests are not directly comparable since different stimuli were used.

\section{Additional measures in the Choice RT test: Number of Trials}

It is common for RT studies to use multiple trials to measure RT and IIV but rare to examine how the number of trials per se influence these measures and whether influence, if any, is affected by other factors i.e. differences between males and females. Any influence of trial number is generally reported in terms of fatigue [Woods et al, 2015], practice [Edwards, 2017; Yotsumoto et al, 2015; Kuang et al, 2016; Siettos \& Smyrnis, 2016] or related to reduced sustained attention resulting slower RT by the end of the test [Fernaeus et al, 2013]. Previous Choice RT tests (although aged) have split trials into blocks [Ballesteros et al, 2013; Brown et al, 2005; Michaels, 1988; Craik \& McDowd, 1987; Logan et al, 1984; Posner et al, 1980] however this was only part of their design and not used for deliberately measuring the potential affect of the number of trials on RT or IIV. 
Therefore in the current Choice RT test, as well as measuring overall RT and IIV and any effects of factors in ageing trials were also separated into separate blocks in a novel approach to examine how the number of trials may have an effect on RT performance and IIV between young and older adults whether any such variation differs between sexes of a particular age group (young or older males and females).

This measure may provide greater detail of how information processing speed and IIV during Choice RT (and the attentional functions it measures) is affected in ageing. For example Edwards [2017] found that more trials related to prolonged practice in RT tests and over repeated sessions this can improve RT performance However in the current study, time was limited thus any influence of practice on RT and IIV could only be examined over the course of the test.

\section{Information processing speed and intraindividual variability using Simple RT and Choice RT tests during ageing: Previous studies}

\section{Simple RT}

Information processing speed has been observed to increase (i.e., slow) with age during a Simple RT test [Bugg et al, 2006; Der \& Deary, 2006; Krieg al, 2001; Wilkinson \& Allison, 1989]. This has been found in different paradigms i.e. the Deary-Liewald reaction time task [Deary, Liewald, \& Nissan, 2011] and also in auditory versions of the test [Fozard et al, 1994].

In addition, the inclusion of visual cues has revealed differences between young and older adults. When the target stimulus was presented shortly after a cue, older adults were significantly slower compared to young adults [Lahtela, Niemi \& Kuusela, 1985]. This may relate to older adults finding it more difficult to perform behavioural tasks such as the Simple RT quickly thus perform slower compared to young adults [Kerchner et al, 2012; Craik \& McDowd, 1987] In contrast, visual cues (of 200ms) have been observed to improve RT performance in both young and older adults [Tales, et al, 2002] which may highlight an increase in alertness [Posner, 1986] by which processing of the target stimulus can be enhanced thus produce a faster response [Fernandez-Duque \& Posner, 1997]. 


\section{Choice RT}

In the Choice RT task, older adults have too found to show significant slowing of information processing speed [Woods et al., 2015; Bugg et al, 2006; Der \& Deary, 2006; Deary \& Der, 2005] as well as a decline in accuracy between young and older adults [Vaportzis, Georgiou-Karistianis, \& Stout, 2013]. Slowing during ageing in the Choice RT has been suggested [i.e. in Simon \& Pouraghabagher, 1978] to highlight detrimental changes to encoding ability i.e. the ability to process each target and quickly execute a response.

Although we could not find examples to contradict these results, previous study results may have varied slightly compared to each other due to differences in sample sizes or participant demographics or differences in test methodology i.e. stimuli. For example, four stimuli (thus four different responses)[i.e. in Deary et al, 2011; Der \& Deary, 2006] require greater processing load i.e. greater executive decision making compared to when only two stimuli are present [i.e. Bugg et al, 2006]. As a result, the greater number of response choices may increase the time it takes to make a response compared to when fewer choices are required.

\section{Comparing Simple and Choice RT}

Information processing speed has been examined using both Choice RT and Simple RT tests within a single ageing study. Age effects on information processing speed has been found to vary between Simple and Choice RT [Woods et al., 2015; Bugg et al, 2006; Der \& Deary, 2006; Krieg, Chrislip, Letz et al, 2001] with the difference in slowing between young and older adults found to be greater in Choice RT compared to the Simple RT test [Woods et al., 2015; Bugg et al, 2006; Der \& Deary, 2006; Krieg et al, 2001].

Previous studies [i.e. Yordanova, Kolev, Hahnsbein et al, 2004; Inui, 1997; Benton, 1986] argue that the Choice RT is more sensitive to differences between young and older adults. This may be because older adults find the decision component of a Choice RT more challenging compared to young adults [Bugg, et al, 2006] and require processing multiple stimuli separately and consecutively [Kertchner et al, 2012; McDowd \& Craik 1988]. As a result, responses take longer to perform thus larger differences of information processing speed are observed between young and old adults i.e. older adults are slower [Woods et al., 2015; Bugg et al, 2006; Der \& Deary, 2006; Krieg et al, 2001]. 
In the current study the Choice RT test was compared to the Simple RT to determine whether Choice RT associated with greater differences between RT and IIV between young and older adults. In addition in a new approach both tests were compared in relation to which produced greater relationships between RT, IIV and different person-related factors i.e. sex, education, subjective memory function and perceived test difficulty.

\section{Intraindividual variability}

Intraindividual variability was measured to examine the functional integrity of information processing speed in Simple RT and Choice RT. This could not be achieved in the TMT (Chapter 3 ) since the pen and paper test only consistent of a single trial.

For Simple RT tasks, variability of information processing speed (IIV) has been found to be stable until around aged 50 years [Der \& Deary, 2006] then increase in older adulthood [Bielak, Cherbuin, Bunce, \& Anstey, 2014]. In contrast IIV measured using choice RT has been found to increase throughout adulthood [Dykiert et al, 2012] and even slow significantly in middle age [Bielak et al, 2014].

Additionally, age effects on IIV have been found to be larger in a Choice RT test compared to a Simple RT test [Dykiert et al., 2012; Dixon, Garrett, Lentz et al, 2007; West et al, 2002] which Dykiert and colleagues suggest relates to the level of complexity required for task completion. Choice RT requires further processing once a target appears as an executive decision needs to be made very quickly before responding i.e. which response corresponds to the stimulus. An increase of mental operations (i.e. executive decision making) may put greater strain on mental resources thus affecting how quickly the stimulus is responded to and vary RT performance i.e. increased IIV.

Therefore the current study aimed to determine whether young and older adults are more variable in their RT performance in the Choice RT compared to the Simple RT test as well as whether IIV is affected by different person related factors (sex, education, subjective memory function and perceived test difficulty). It is important to measure RT and IIV in healthy ageing to understand how they are affected in ostensibly healthy control groups, before comparing healthy ageing with pathological ageing i.e. MCI or dementia. 


\section{Information processing speed and intraindividual variability using Simple RT and Choice RT tests in MCI and AD: Previous studies}

\section{Simple RT}

Past Simple RT studies have found information processing speed to be significantly slower and more variable in MCI [Cheng, et al, 2017; Gorus et al, 2008; Levinoff, Saumier, \& Chertkow, 2005] and in AD [Cheng et al, 2017; Sano et al, 2009; Gorus et al, 2008; Tales et al, 2008] compared to healthy ageing. In addition, past studies have found responses after a visual cue to be significantly slower in MCI and AD compared to healthy controls [Pate et al, 1994; Sano et al, 1995] due to an inability to benefit from a given cue and focus attention on a subsequent target [Pate et al, 1994]. However an effect of visual cues on information processing speed in AD patients has not always been observed [Tales et al, 2002].

AD patients are insufficient at processing and responding to rapid changes in the environment [Tales et al, 2002] even when they are warned that something may happen shortly, thus this may significantly negative impact everyday tasks [Perry \& Hodges, 1999] particularly those tasks which require a rapid response. The stimuli in the current Simple RT test mirror these types of real world situations i.e. changing traffic lights although we only measured how they may be affected in healthy ageing i.e. between young and older adults.

In contrast, not all research has found processing speed to be a significant dementia related impairment during Simple RT [Nettleback et al, 2014; Dodonova \& Dodonov, 2013; Tales et al, 2011]. Storandt \& Beaudreau [2004] used a simple reaction time task on individuals with very mild dementia and mild dementia. They found that not all patients showed slowing therefore reaching the assumption is that slowing is not always universal. This was also found by Nestor and colleagues [1991] who found reaction time in a simple RT test to be normal in AD patients.

In addition IIV has not always been observed to differentiate abnormal ageing i.e. aMCI to healthy ageing using Simple RT [Tales et al, 2011] which may relate to the use of different simple RT tests used i.e. different stimuli. This highlights the fact that information processing speed and IIV may be affected differently depending on the type of Simple RT test paradigm used. 
Outcome variability may also relate to differences in sample size and the inclusion of other factors i.e. lower IQ associated with slower information processing speed [as discussed in Nettleback et al, 2014] or sex and education [as found by Dykiert et al., 2012; Reimers \& Maylor, 2006].

The current study aimed to take into account sex and education alongside factors not considered previously in Simple RT studies (namely subjective memory function and perceived test difficulty).

\section{Choice RT}

Information processing speed measured using the Choice RT test has been observed to be significantly slower and more variable in MCI [Cheng, et al, 2017; Levinoff, Saumier, \& Chertkow, 2005] and in AD [Cheng et al, 2017; Sano et al, 2009; Tales et al, 2008; Hultsch et al, 2000; Christensen, Ogle et al, et al, 1981] compared to healthy ageing. In addition, the difference of information processing speed between normal ageing and Alzheimer's disease has been argued to be more significant when tasks include making a choice [Pirozzolo et al, 1981; Nestor et al, 1991].

Therefore, past studies argue that Choice RT tests can be more sensitive to the severity of dementia [Storandt \& Beaudreau, 2004; Flicker, Bartus, Crook et al, 1984] and to a pre dementia diagnosis i.e. MCI [Levinoff et al, 2005]. A more complex task such as the Choice RT recruits more areas of the brain i.e. executive control and requires greater processing load which produces a great strain on these mental resources. As more mental processes are required, this increases the chance for some, if not all cognitive functions to be affected by disease as well as ageing.

\section{Person-related factors}

\section{Sex and education}

In both Simple and Choice RT, faster information processing speed in males compared to females has been found not just in younger adults [Karia et al, 2012; Krieg Jr et al., 2001; Bleecker, Bolla-Wilson, Agnew, \& Meyers, 1987; ] but in older adult groups [Dykiert, et al, 2012; Lahtela, Niemi, \& Kuusela,1985]. However in Choice RT, it has also been found that in healthy adults, females started slower than males at the beginning of the choice RT task but then quicken throughout the trials until they were in fact faster than males [Reimers \& Maylor, 2006; Landauer, Armstrong \& Digwood, 1980]. 
This may highlight sex differences due to task complexity (i.e. the Choice RT required greater processing load) or differences in processing with respect to the number of trials and thus why trial numbers are examined in this study (alongside comparing single trials and multiple trials). In addition, sex differences may reflect differences in processing strategies with females choosing serial processing and males choosing parallel processing [Adam, Paas, Buekers et al., 1999].

Sex differences have been found in intraindividual variability with females being more variable in their RT performance compared to males in Simple RT tests [Dykiert et al., 2012] and in Choice RT tests [Dykiert et al., 2012; Reimers \& Maylor, 2006]. Again these sex differences may reflect differences in processing strategies between males and females particularly during the Choice RT test using multiple stimuli.

Education has also been examined previously during Simple and Choice RT tests finding both young and older adults with higher education performing faster compared to medium and low levels of education [Deary et al, 2001; Houx \& Jolles, 1993]. However, this is not supported in all research findings [Woods, et al, 2015; Hooyman, 2005] therefore it has been suggested that further research is required with a focus on the influence of education on information processing speed during these Simple and Choice RT tests [de Jager, 2003, Houx \& Jolles, 1993].

\section{Subjective memory function}

When measuring RT and IIV in ageing and MCI or AD studies typically, objective cognitive function of healthy older adult control groups is tested and taken into account. However there may be some individuals with complaints to their memory function (SCI) and this may have an effect on their RT performance thus have an effect on group mean RT. Previous Simple and Choice RT tests have not taken into account these subjective memory complaints but also, of particular interest to the current research, have not taken into consideration subjective memory function i.e. memory complaints in older adults in general public who have not visited their GP. Therefore the results of ostensibly healthy older adults' information processing speed in Simple and Choice RT tests may have been misinterpreted.

So far in the current research both the visual search (research) and the TMT (clinical) did not find an association between subjective memory function and information processing speed (or IIV in the visual search) suggesting no effect of perceived changes to memory function on RT and IIV in ostensibly healthy older adults. 
However this outcome may still differ depending on the type of test i.e. the type of research test or research tests with multiple trials compared to clinical tests a single trial. Therefore the current study aimed to determine whether subjective memory function is associated with information processing speed or IIV in Simple and Choice RT tests in a similar way (i.e. to other research and clinical tests).

Any affect of subjective memory function may suggest the need to take this factor into account in future ageing studies (and in control groups in MCI and AD studies) and highlight the differences between different aspects of attention (as well as between research and clinical tests). In addition any affects of subjective memory function on RT and IIV may help the characterisation of how RT and IIV are affected in SCI.

\section{Perceived test difficulty}

Perceived test difficulty will be examined in the current study to determine whether any possible effect on study outcome is similar between both the Simple and Choice RT tests. If perceived test difficulty has an influence on information processing speed in Simple and Choice RT, this may suggest that other psychological factors i.e. judgement of ability of performance or mood influence information processing speed. Failing to take this factor (or factors) into account may impact how we interpret levels of slowing in healthy ageing and possibly highlight how the integrity of information processing speed of control groups in past Simple and Choice RT studies may have been misinterpreted. 


\subsection{SIMPLE RT: Experiment 1}

\section{METHODOLOGY}

\section{$\underline{\text { Participants }}$}

Swansea university students ( $\mathrm{n}=82,18-25$ years; 32 males: 50 females) were recruited through psychology department credit system and through advertising around the university and social network. Community dwelling older adults $(\mathrm{n}=90$; 50-80 years; 38 males: 52 females) were recruited through advertisements given out to older adult social clubs and local papers in Swansea, through email, word of mouth and via a volunteer database of older adults set up by the Swansea Psychology Department. Ethical approval was provided by the departmental ethics committee and formal written consent was obtained from each participant. The inclusion and exclusion criteria were the same as previous chapters.

\section{$\underline{\text { Simple RT Materials }}$}

The stimuli were presented on a Dell Precision PC running on Windows XP X86 CPU, viewed at a distance of $57 \mathrm{~cm}$. Two visual cues were presented consecutively in the middle of a black computer screen (1920 x 1080 pixels). These two cues consisted of a red circle with 'Ready' written in the centre and an orange circle with 'Steady' written in the centre. Following the visual cues was a target cue of a green circle with 'Go!' presented in the middle [see Figure 11]. The delay in-between each circle appearing varied between each trial set (between 1 to 4 seconds). This was designed so that participants would not anticipate when each circle would appear thus respond before the target circle appeared on the screen.

Participants were asked to press the spacebar on the keyboard with their index finger of their dominant hand as quickly as possible when the target stimulus of Green 'Go!' appeared on the screen. Participants were given 5-6 trials as a practice before the programme was restarted for the main testing. There were 35 trials in total. If an error was made i.e. the spacebar pressed too early, the trial would repeat until the response was made correctly but no feedback was given to the participant at the end of each trial regardless if the button was pressed correctly or incorrectly. 


\section{Data Cleaning}

For both young adults and older adults, any reaction times which were under 150ms were removed as this is faster than 'natural' RT (i.e. pre-empting the stimulus) and over 2000ms observed as a lack of concentration. The programme recorded the number of 'errors' i.e. the participants pressing too early resulting in the trial repeating although the number of errors did not affect elimination procedures.

The median was calculated for each individual and the group mean RT and Interquartile range (for IIV) was calculated for both age groups. In response to the non-normal distribution of the data (see Table 9) SPSS non parametric analysis was conducted. Factors i.e. sex and education, perceived test difficulty and subjective memory function were grouped into separate families for analysis. The RT and IIV and attention effects were different data sets and thus did not require Bonferroni correction for multiple analyses.

Table 9. Normality of Distribution (Shapiro Wilkes test) ${ }^{1}$

\begin{tabular}{|l|ccc|crc|}
\cline { 2 - 8 } \multicolumn{1}{c|}{} & \multicolumn{3}{c}{ Older } & \multicolumn{3}{c|}{ Young } \\
\cline { 2 - 8 } \multicolumn{1}{c|}{} & Statistic & $d f$ & Sig. & statistic & $d f$ & Sig. \\
\hline $\begin{array}{l}\text { Information } \\
\text { processing speed }\end{array}$ & .953 & 90 & .003 & .775 & 82 & .000 \\
\hline $\begin{array}{l}\text { Intraindividual } \\
\text { variability }\end{array}$ & .937 & 90 & .000 & .884 & 82 & .000 \\
\hline
\end{tabular}

\footnotetext{
${ }^{1}$ Note: data classified as a non-normal due to the positively skewed distribution of the data and in some cases bimodal distributions. There is a natural limit to the data i.e. how fast RT can be.
} 


\subsection{SIMPLE RT RESULTS}

\section{$\underline{\text { Demographics }}$}

Mann-Whitney analysis revealed no significant difference in mean education or MoCA score between young and old adult groups [ $p>.05]$. Depression level was significantly greater for young adults compared to older adults $[\mathrm{U}=1887, p<.001$, effect size $(r)=.38$ ] and anxiety level was significantly greater for young adults compared to older adults $[\mathrm{U}=2204.5, p<.001$, effect size $(\mathrm{r})=.30]$.

Table 10: Mean demographic scores for the older adult and younger adult groups. Standard deviation in parenthesis.

\begin{tabular}{|c|c|c|c|c|c|c|}
\hline & $\begin{array}{c}\text { Age } \\
\text { (Years) }\end{array}$ & $\begin{array}{c}\text { Education } \\
\text { (Years) }\end{array}$ & $\begin{array}{c}\text { MoCA } \\
\text { score } \\
\end{array}$ & $\begin{array}{l}\text { MFQ } \\
\text { Score }\end{array}$ & $\begin{array}{c}\text { PHQ-9 } \\
\text { score }\end{array}$ & $\begin{array}{c}\text { GAD-7 } \\
\text { score }\end{array}$ \\
\hline $\begin{array}{c}\text { All } \\
\text { Young }\end{array}$ & $\begin{array}{c}20 \\
(2.1)\end{array}$ & $\begin{array}{c}15 \\
(3.5)\end{array}$ & $\begin{array}{c}27 \\
(2.1)\end{array}$ & - & $\begin{array}{c}6 \\
(4.0)\end{array}$ & $\begin{array}{c}5 \\
(4.0)\end{array}$ \\
\hline $\begin{array}{l}\text { Young } \\
\text { males } \\
(\mathrm{n}=32)\end{array}$ & $\begin{array}{c}20 \\
(2.0)\end{array}$ & $\begin{array}{c}15 \\
(3.0)\end{array}$ & $\begin{array}{c}27 \\
(2.3)\end{array}$ & - & $\begin{array}{c}5 \\
(5.1)\end{array}$ & $\begin{array}{c}4 \\
(4.6)\end{array}$ \\
\hline $\begin{array}{l}\text { Young } \\
\text { female } \\
(n=50)\end{array}$ & $\begin{array}{c}20 \\
(2.2)\end{array}$ & $\begin{array}{c}14 \\
(3.9)\end{array}$ & $\begin{array}{c}27 \\
(2.0)\end{array}$ & - & $\begin{array}{c}6 \\
(3.7)\end{array}$ & $\begin{array}{c}5 \\
(4.0)\end{array}$ \\
\hline $\begin{array}{c}\text { All } \\
\text { Older }\end{array}$ & $\begin{array}{c}65 \\
(5.8) \\
\end{array}$ & $\begin{array}{c}15 \\
(4.9) \\
\end{array}$ & $\begin{array}{c}27 \\
(2.3) \\
\end{array}$ & $\begin{array}{c}291 \\
(48.3) \\
\end{array}$ & $\begin{array}{c}3 \\
(3.1) \\
\end{array}$ & $\begin{array}{c}3 \\
(2.8) \\
\end{array}$ \\
\hline $\begin{array}{c}\text { Older } \\
\text { males } \\
(n=38)\end{array}$ & $\begin{array}{c}65 \\
(5.9)\end{array}$ & $\begin{array}{c}17 \\
(6.0)\end{array}$ & $\begin{array}{c}27 \\
(2.5)\end{array}$ & $\begin{array}{c}286 \\
(42.3)\end{array}$ & $\begin{array}{c}3 \\
(3.1)\end{array}$ & $\begin{array}{c}2 \\
(2.4)\end{array}$ \\
\hline $\begin{array}{l}\text { Older } \\
\text { female } \\
(\mathrm{n}=52)\end{array}$ & $\begin{array}{c}66 \\
(6.3)\end{array}$ & $\begin{array}{c}14 \\
(3.5)\end{array}$ & $\begin{array}{c}28 \\
(1.8)\end{array}$ & $\begin{array}{c}300 \\
(52.3)\end{array}$ & $\begin{array}{c}3 \\
(3.1)\end{array}$ & $\begin{array}{c}3 \\
(3.0)\end{array}$ \\
\hline
\end{tabular}

MoCA, Montreal Cognitive Assessment; MFQ, Memory Functioning; PHQ, Patient Health questionnaire; GAD, Generalized Anxiety Disorder 7-item (GAD-7) scale. 
Table. 11. Mean information processing speed, intraindividual variability, mean number of errors (pressing too early) and mean perceived test difficulty for both older and young adults. Standard deviation in parenthesis.

\begin{tabular}{|c|c|c|c|c|}
\hline & $\begin{array}{c}\text { Information } \\
\text { processing } \\
\text { Speed (ms) }\end{array}$ & $\begin{array}{c}\text { Intra- } \\
\text { Individual } \\
\text { Variability } \\
(\text { ms) }\end{array}$ & $\begin{array}{c}\text { Errors } \\
\text { (early } \\
\text { key } \\
\text { presses) }\end{array}$ & $\begin{array}{c}\text { Perceived } \\
\text { Difficulty } \\
\text { (Likert } \\
\text { Scale) }\end{array}$ \\
\hline $\begin{array}{c}\text { All } \\
\text { Young } \\
\text { adults }\end{array}$ & $342.73(47.2)$ & 56.82 & $\begin{array}{c}0.63 \\
(1.24)\end{array}$ & $\begin{array}{c}2(0.9) \\
\text { (Range 1-5) }\end{array}$ \\
\hline $\begin{array}{c}\text { Young } \\
\text { Male }\end{array}$ & $336.96(42.4)$ & 54.19 & $\begin{array}{c}0.93 \\
(1.70)\end{array}$ & $\begin{array}{c}2(0.8) \\
(\text { Range 1-5) }\end{array}$ \\
\hline $\begin{array}{c}\text { Young } \\
\text { Female }\end{array}$ & $346.42(50.0)$ & 58.5 & $\begin{array}{c}0.48 \\
(0.81)\end{array}$ & $\begin{array}{c}2(1.0) \\
(\text { Range 1-5) }\end{array}$ \\
\hline $\begin{array}{c}\text { All } \\
\text { Older } \\
\text { Adults }\end{array}$ & $370.77(64.0)$ & 74.99 & $\begin{array}{c}0.42 \\
(0.75)\end{array}$ & $\begin{array}{c}1(0.8) \\
(\text { Range 1-5) }\end{array}$ \\
\hline $\begin{array}{c}\text { Older } \\
\text { Male }\end{array}$ & $354.43(53.4)$ & 73.12 & $\begin{array}{c}0.45 \\
(0.60)\end{array}$ & $\begin{array}{c}1(0.0) \\
(\text { Range 1-5) }\end{array}$ \\
\hline $\begin{array}{c}\text { Older } \\
\text { Female }\end{array}$ & $382.72(68.8)$ & 76.31 & $\begin{array}{c}0.40 \\
(0.85)\end{array}$ & $\begin{array}{c}2(0.9) \\
(\text { Range 1-5) }\end{array}$ \\
\hline
\end{tabular}




\section{Information processing speed: Simple RT}

\section{Age comparison: Information processing speed}

Figure 13: Box plot of mean information processing speed between older adults and younger adults during Simple RT test. Note the presence of outliers in the performance of this task.

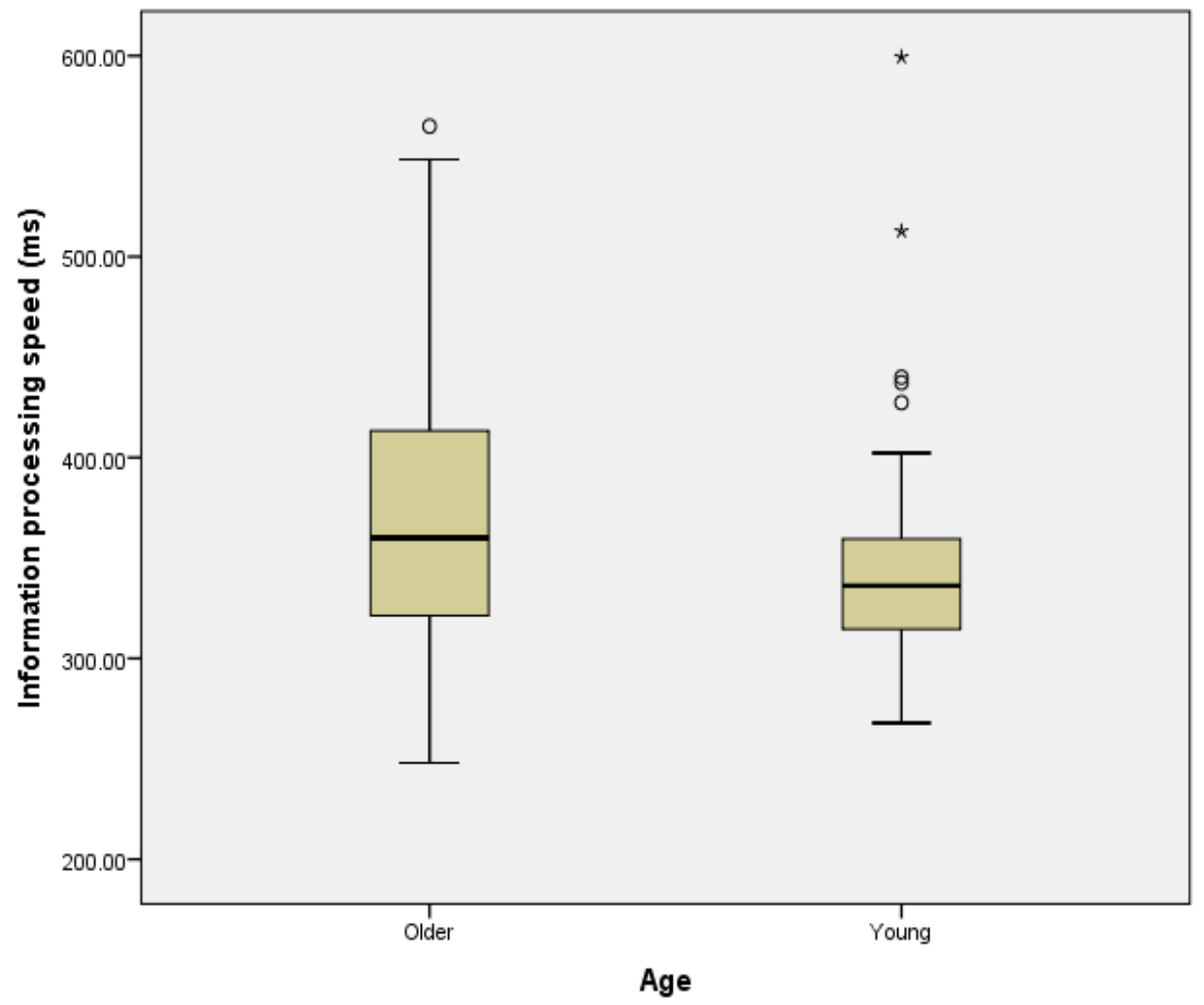

Mann-Whitney analysis comparing information processing speed for age group (older adults and young adults) revealed a significant difference between age groups; older adults produced slower reaction times compared to young adults [ $\mathrm{U}=2655, p$ $=.002$, effect size $(r)=.24]$.

\section{Information processing speed and subjective memory function}

Correlational analysis using Spearman's rho revealed no significant correlation between information processing speed and total MFQ score (subjective memory function) $[p>.05]$. 


\section{Information processing speed and perceived test difficulty}

Spearman's rho revealed no significant correlation between information processing speed and perceived test difficulty in both young and older adults [all $p$ values $>.05$ ].

\section{Information processing speed and education level}

In both young and older adults, Spearman's rho revealed no significant correlation between information processing speed and educational level [all $p$ values $>.05$ ].

\section{Sex Comparison: Information processing speed}

Figure 14: Box plot of mean information processing speed between sex (male and female) in young and older adults.

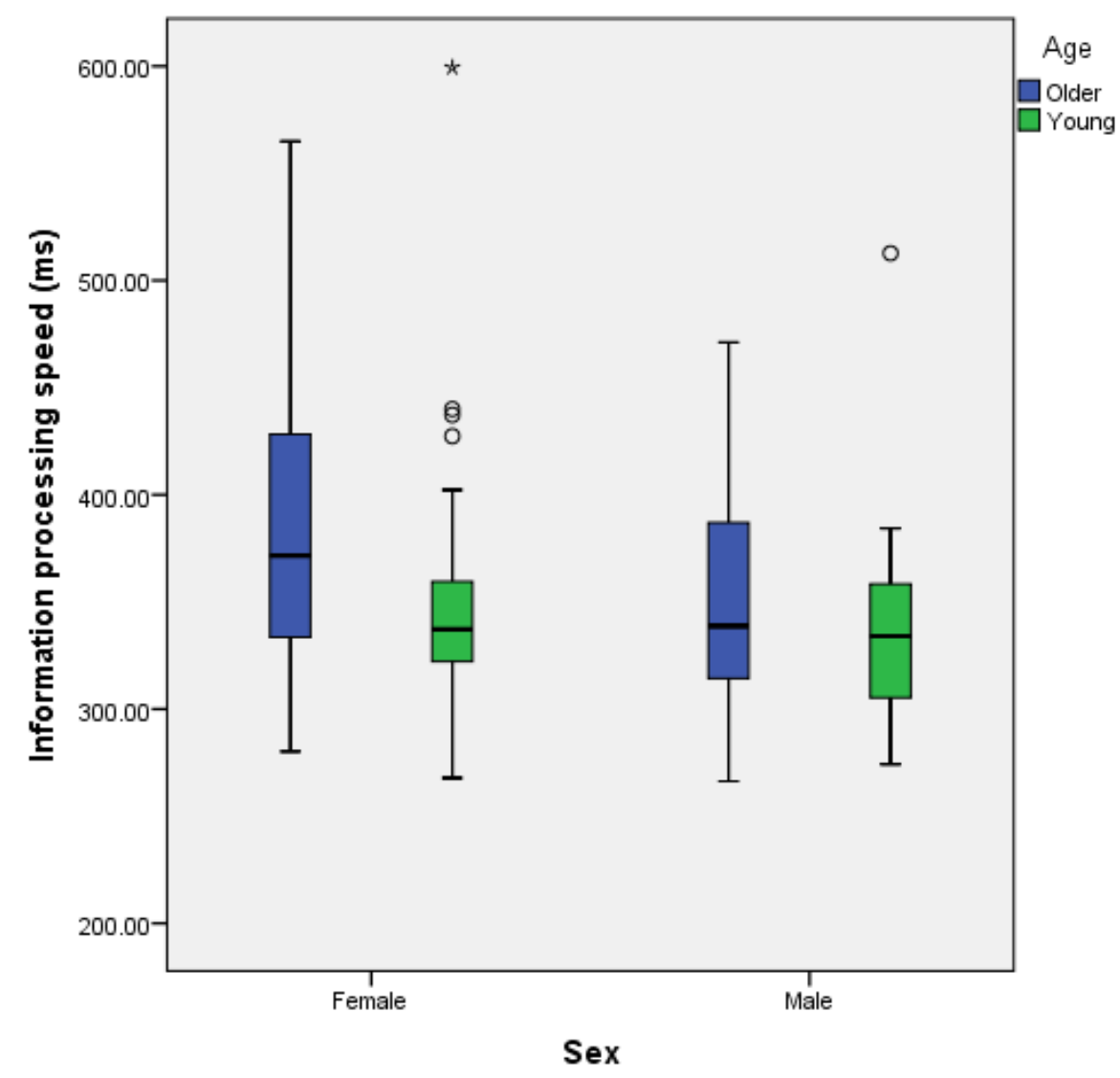

Mann-Whitney analysis revealed no significant difference of reaction time between males and females in young adults $[\mathrm{U}=693.5, p=.311]$ or in older adults $[\mathrm{U}=760$, $p=.063]$. 
Information processing speed, anxiety and depression levels

For both young and older adults, information processing speed did not significantly correlate with anxiety levels $[p>.05]$ or depression levels $[p>.05]$.

\section{Information processing speed and objective cognitive performance (MoCA)}

In both young and older adults, there was no significant correlation between information processing speed and MoCA score (objective measure of general cognition) [all $p$-values > .05]. In older adults MoCA score significantly negatively correlated with perceived test difficulty, finding the simple RT test more difficult related to poorer general cognition $[\mathrm{r}=-.208, p=.049]$.

\section{Subjective memory function and perceived test difficulty}

In older adults, subjective memory function (Total MFQ score) was significantly negatively correlated with perceived test difficulty $[\mathrm{r}=-.241, p=.022]$. As older adults perceived greater changes to their memory (low MFQ score) the more difficult the Simple RT was perceived to be.

\section{Education level and subjective memory function}

In older adults, Spearman's rho revealed no significant correlation between educational level and subjective memory function (total MFQ score) [ $p>.05]$.

\section{Education level and perceived test difficulty}

Spearman's rho revealed no significant correlation between educational level and perceived test difficulty for both young and older adults [all $p$ values $>.05$ ]

\section{Anxiety and depression levels}

Anxiety levels significantly positively correlated with depression levels in young adults $[\mathrm{r}=.743, p<.001]$ and older adults $[\mathrm{r}=.618, p<.001]$. As anxiety levels increased so did depression levels. 


\section{Intraindividual variability: Simple RT}

\section{Age comparison: Intraindividual variability}

Figure 15: Box plot of mean intraindividual variability (IIV) between older adults and younger adults

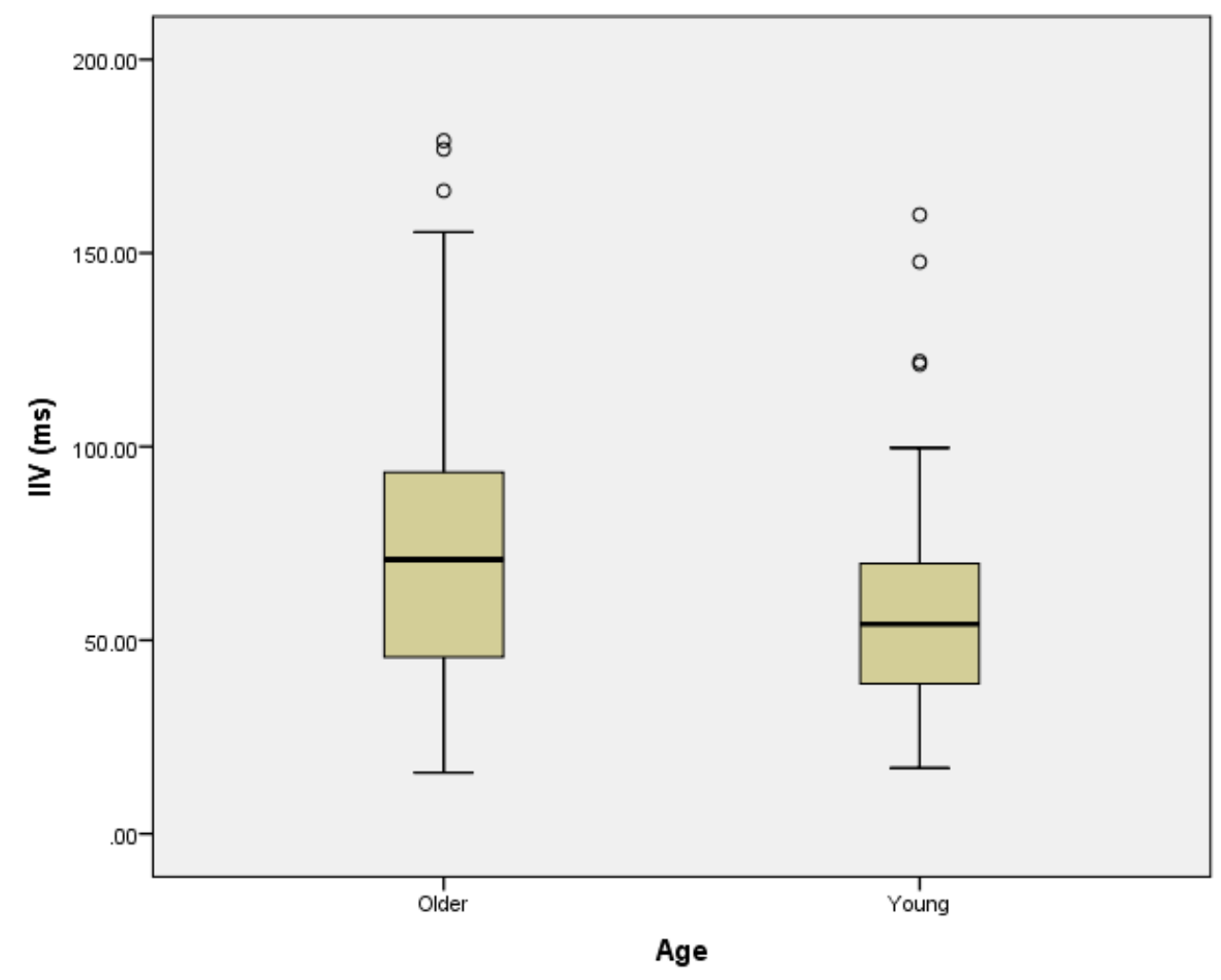

Mann-Whitney analysis comparing mean reaction time variability revealed a significant difference between age groups; older adults were more variable in their reaction time performance compared to young adults $[\mathrm{U}=2593, p=.001$, effect size $(\mathrm{r})=.26]$.

\section{Intraindividual variability and Subjective memory function}

In older adults, Spearman's rho revealed no significant correlation between subjective memory function (Total MFQ score) and intraindividual variability $[p>$ $.05]$.

\section{Intraindividual variability and Perceived test difficulty}

For both young and older adults, intraindividual variability did not significantly correlate with perceived test difficulty [all $p$ values $>.05$ ]. 


\section{Intraindividual variability and Education level}

For both young and older adults, intraindividual variability did not significantly correlate with education [all $p$ values $>.05$ ].

\section{Sex Comparison: Intraindividual Variability}

Figure 16: Box plot of mean intraindividual variability between sex (male and female) in young and older adults.

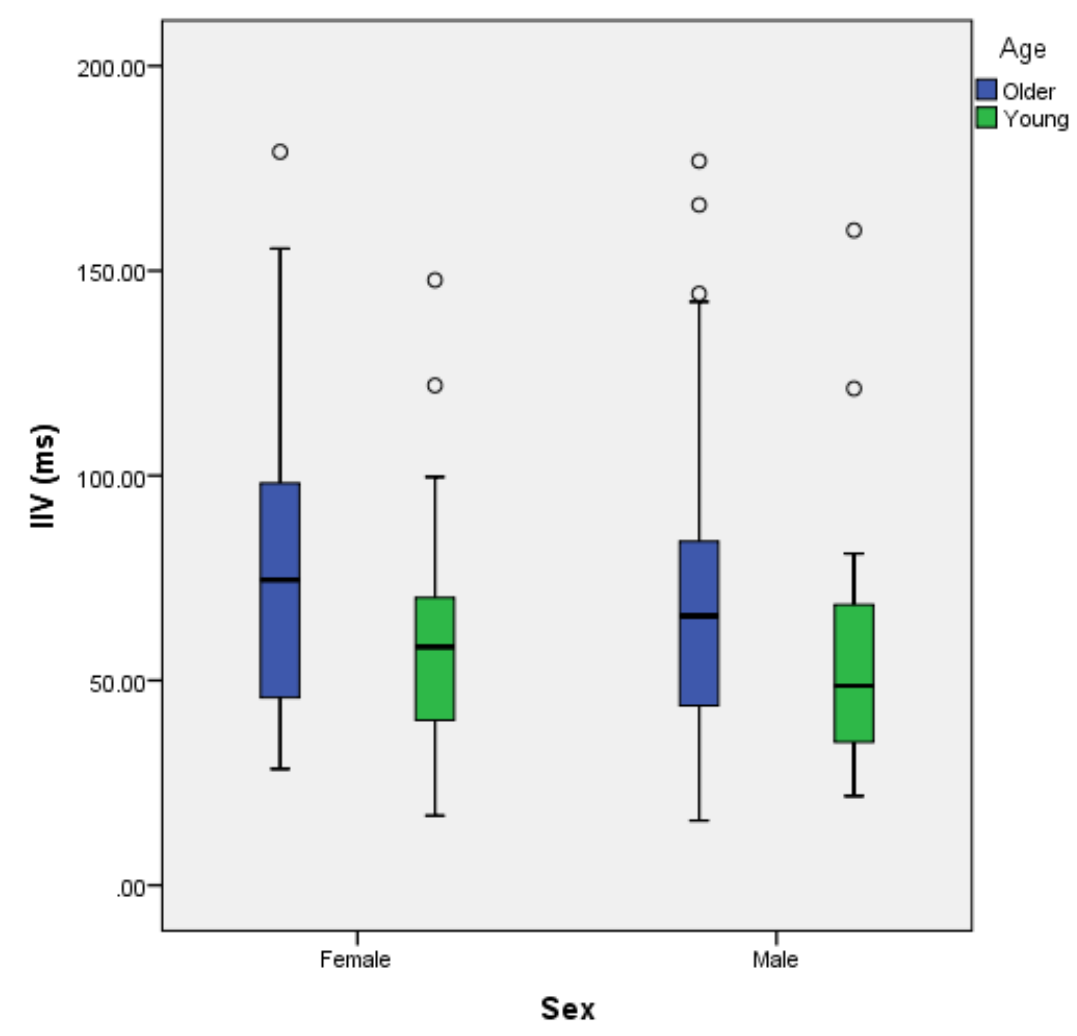

Mann-Whitney analysis revealed no significant difference of reaction time variability between males and females in young adults $[\mathrm{U}=659.5, p=.182]$ or in older adults $[\mathrm{U}=904, p=.493]$.

\section{Intraindividual variability, anxiety and depression levels}

For both young and older adults, IIV did not significantly correlate with anxiety levels $[p>.05]$ or depression levels $[p>.05]$. 
Intraindividual variability and objective cognitive performance (MoCA)

In both young and older adults, there was no significant correlation between IIV and MoCA score (objective measure of general cognition) [all $p$-values $>.05$ ].

\section{Simple RT: Errors}

\section{Overall mean number of errors: Age comparison}

Table 12: Overall mean number of errors in Simple RT for young and older adults. Standard deviation in parenthesis.

\begin{tabular}{|c|c|}
\hline & $\begin{array}{l}\text { Total } \\
\text { Mean }\end{array}$ \\
\hline & 0.42 \\
Older & $(0.75)$ \\
\hline & 0.63 \\
Young & $(1.24)$ \\
\hline
\end{tabular}

Mann Whitney analysis revealed no significant difference in overall mean errors between young and older adults $[\mathrm{U}=3473.5, p=.424]$

\section{Overall mean number of errors: Age comparison}

Table 13: Overall mean number of errors in Simple RT for males and females in young and older adults. Standard deviation in parenthesis.

\begin{tabular}{|r|c|}
\hline & Overall Mean \\
\hline & 0.88 \\
Young Males & $(0.9)$ \\
\hline & 0.48 \\
Young Females & $(0.81)$ \\
\hline & 0.45 \\
Older Males & $(0.6)$ \\
\hline & 0.4 \\
Older Females & $(0.85)$ \\
\hline
\end{tabular}

Mann Whitney analysis revealed no significant difference in overall mean errors between males and females in both young adults $[\mathrm{U}=724, p=.394]$ and older adults $[\mathrm{U}=871.5, p=.242]$. 


\subsection{CHOICE RT: Experiment 2}

\section{METHODOLOGY}

\section{$\underline{\text { Participants }}$}

79 young adults (30 males: 49 females) and 82 older adults (34 males: 48 females) were recruited. The recruitment protocol was the same as in Experiment 1; there were some participants who performed the choice task without performing the simple RT task which is why participant and sex ratios differ to experiment 1 and why the demographic details for the younger and older adults for experiment 2 are shown in Table 13.

\section{$\underline{\text { Stimuli }}$}

The task was created using Superlab Pro software on a Dell Precision PC running on Windows XP, X86 CPU, viewed at a distance of $57 \mathrm{~cm}$. A black asterisk flashed in the centre of a white screen (dimensions) for $1000 \mathrm{~ms}$ followed by either an ' $\mathrm{X}$ ' or ' $O$ ' in black, font Times New Roman, size 20 (see Figure 12). There was an instruction screen explaining the task in which could be proceeded by pressing ' $S$ ' on the computer keyboard. There were four experimental blocks of 30 trials each, giving a total of 120 trials. In each block 15 trials were the ' $X$ ' target and 15 trials were the ' $\mathrm{O}$ ' target presented in a randomized order. Each block was separated with a screen indicting the end of the block which could be preceded by pressing ' $S$ ' on the keyboard. A final instruction screen appeared at the end of the task to indicate it had ended.

\section{Procedure}

The participant was asked to respond as quickly and as accurately as possible with pressing ' $Z$ ' on the computer keyboard with their left index finger when the ' $X$ ' target appeared on the screen and the ' $M$ ' key with their right index finger when the 'O' target appeared. Participants were given a practice block of 20 trails followed by a screen which repeated the instructions. In the testing phase there were 4 blocks of 30 trials with a break screen in-between. 


\section{Cleaning data}

For both age groups trials under 150ms (which are faster than natural RT) were eliminated. Individuals who produced more than $20 \%$ of incorrect trials across the four blocks were also omitted from analysis as they were considered not to have performed the test correctly. Individual median RT and IQR across all trials were calculated as well as the median RT and IQR for each of the four blocks. Overall group and block medians were calculated for both young and older adults. In response to the non-normal distribution of the data (see Table 12) SPSS non parametric analysis was conducted. Factors i.e. sex and education, perceived test difficulty and subjective memory function were grouped into separate families for analysis. The RT and IIV and attention effects were different data sets and thus did not require Bonferroni correction for multiple analyses.

Table 14. Normality of Distribution (Shapiro Wilkes test) ${ }^{2}$

\begin{tabular}{|l|lcc|lccc|}
\cline { 2 - 8 } \multicolumn{1}{c|}{} & \multicolumn{3}{c|}{ Older } & \multicolumn{3}{c|}{ Young } \\
\cline { 2 - 8 } \multicolumn{1}{c|}{} & statistic & $d f$ & Sig. & statistic & $d f$ & Sig. \\
\hline $\begin{array}{l}\text { Information } \\
\text { processing speed }\end{array}$ & .962 & 82 & .015 & .974 & 79 & .111 \\
\hline $\begin{array}{l}\text { Intraindividual } \\
\text { variability }\end{array}$ & .898 & 82 & .000 & .913 & 79 & .000 \\
\hline
\end{tabular}

\footnotetext{
${ }^{2}$ Note: data classified as a non-normal due to the positively skewed distribution of the data and in some cases bimodal distributions.
} 


\subsection{CHOICE RT RESULTS}

\section{Demographics}

Mann-Whitney analysis revealed no significant difference in mean education or MoCA score between young and old adult groups $[p>.05]$. Depression level was significantly greater for young adults compared to older adults $[\mathrm{U}=1646, p<.001$, effect size $(r)=.38$ ] and anxiety level was significantly greater for young adults compared to older adults $[\mathrm{U}=1696.5, p<.001$, effect size $(\mathrm{r})=.37]$.

Table 15: Baseline demographics for the older adult and younger adult groups.

\begin{tabular}{|c|c|c|c|c|c|c|}
\hline & $\begin{array}{c}\text { Age } \\
\text { (Years) }\end{array}$ & $\begin{array}{c}\begin{array}{c}\text { Education } \\
\text { (Years) }\end{array} \\
\end{array}$ & MoCA & MFQ & PHQ-9 & GAD-7 \\
\hline $\begin{array}{c}\text { All } \\
\text { Young }\end{array}$ & $\begin{array}{c}20 \\
(2.1) \\
\end{array}$ & $\begin{array}{c}15 \\
(3.6)\end{array}$ & $\begin{array}{c}27 \\
(2.1) \\
\end{array}$ & - & $\begin{array}{c}6 \\
(4.0) \\
\end{array}$ & $\begin{array}{c}5 \\
(4.0) \\
\end{array}$ \\
\hline $\begin{array}{l}\text { Young } \\
\text { males } \\
(\mathrm{n}=30)\end{array}$ & $\begin{array}{c}20 \\
(1.7)\end{array}$ & $\begin{array}{c}15 \\
(2.9)\end{array}$ & $\begin{array}{c}27 \\
(2.3)\end{array}$ & - & $\begin{array}{c}5 \\
(5.5)\end{array}$ & $\begin{array}{c}5 \\
(4.5)\end{array}$ \\
\hline $\begin{array}{l}\text { Young } \\
\text { female } \\
(n=49)\end{array}$ & $\begin{array}{c}20 \\
(2.3)\end{array}$ & $\begin{array}{c}14 \\
(3.9)\end{array}$ & $\begin{array}{c}27 \\
(2.0)\end{array}$ & - & $\begin{array}{c}6 \\
(3.7)\end{array}$ & $\begin{array}{c}5 \\
(4.0)\end{array}$ \\
\hline $\begin{array}{c}\text { All } \\
\text { Older }\end{array}$ & $\begin{array}{c}65 \\
(6.2) \\
\end{array}$ & $\begin{array}{c}15 \\
(4.9) \\
\end{array}$ & $\begin{array}{c}27 \\
(2.2) \\
\end{array}$ & $\begin{array}{c}291 \\
(49.4) \\
\end{array}$ & $\begin{array}{c}3 \\
(3.1) \\
\end{array}$ & $\begin{array}{c}2 \\
(2.7) \\
\end{array}$ \\
\hline $\begin{array}{c}\text { Older } \\
\text { males } \\
(\mathrm{n}=34)\end{array}$ & $\begin{array}{c}65 \\
(6.2)\end{array}$ & $\begin{array}{c}17 \\
(6.3)\end{array}$ & $\begin{array}{c}26 \\
(2.4)\end{array}$ & $\begin{array}{c}287 \\
(44.1)\end{array}$ & $\begin{array}{c}4 \\
(3.5)\end{array}$ & $\begin{array}{c}2 \\
(3.3)\end{array}$ \\
\hline $\begin{array}{l}\text { Older } \\
\text { female } \\
(n=48)\end{array}$ & $\begin{array}{c}66 \\
(6.2)\end{array}$ & $\begin{array}{c}14 \\
(3.5)\end{array}$ & $\begin{array}{c}28 \\
(1.8)\end{array}$ & $\begin{array}{c}294 \\
(52.9)\end{array}$ & $\begin{array}{c}3 \\
(2.8)\end{array}$ & $\begin{array}{c}2 \\
(2.2)\end{array}$ \\
\hline
\end{tabular}

MoCA, Montreal Cognitive Assessment; MFQ, Memory Functioning Questionnaire; PHQ-9, Patient Health Questionnaire; GAD-7, Generalized Anxiety Disorder 7-item scale. 
Table 16: Group mean information processing speed and Intraindividual Variability (from individual participant median RT scores) and mean perceived test difficulty for the young and older adult groups for the choice RT test. Standard deviation in parenthesis.

\begin{tabular}{|c|c|c|c|}
\hline & $\begin{array}{c}\text { Information } \\
\text { Processing } \\
\text { Speed (ms) }\end{array}$ & $\begin{array}{c}\text { Intra- } \\
\text { Individual } \\
\text { Variability } \\
\text { (ms) }\end{array}$ & $\begin{array}{c}\text { Perceived } \\
\text { Performance } \\
\text { (Likert Scale) }\end{array}$ \\
\hline $\begin{array}{c}\text { All } \\
\text { Young } \\
\text { adults }\end{array}$ & $\begin{array}{c}456.37 \\
(55.9)\end{array}$ & 108.81 & $\begin{array}{c}3(1.4) \\
\text { (Range 1-6) }\end{array}$ \\
\hline $\begin{array}{c}\text { Young } \\
\text { Male }\end{array}$ & $\begin{array}{c}463.62 \\
(57.0)\end{array}$ & 104.1 & $\begin{array}{c}3(1.1) \\
\text { (Range 1-5) }\end{array}$ \\
\hline $\begin{array}{c}\text { Young } \\
\text { Female }\end{array}$ & $\begin{array}{c}451.93 \\
(55.4)\end{array}$ & 111.7 & $\begin{array}{c}3(1.5) \\
\text { (Range 1-6) }\end{array}$ \\
\hline \multicolumn{1}{|c|}{$\begin{array}{c}\text { All } \\
\text { Older } \\
\text { Adults }\end{array}$} & $\begin{array}{c}542.98 \\
(83.5)\end{array}$ & 146.11 & $\begin{array}{c}3(1.4) \\
\text { (Range 1-6) }\end{array}$ \\
\hline $\begin{array}{c}\text { Older } \\
\text { Male }\end{array}$ & $\begin{array}{c}548.82 \\
(86.9)\end{array}$ & 150.23 & $\begin{array}{c}3(1.5) \\
\text { (Range 1-6) }\end{array}$ \\
\hline $\begin{array}{c}\text { Older } \\
\text { Female }\end{array}$ & $\begin{array}{c}538.83 \\
(81.6)\end{array}$ & 143.19 & $\begin{array}{c}3(1.4) \\
\text { (Range 1-6) }\end{array}$ \\
\hline
\end{tabular}




\section{Choice RT: Information Processing Speed}

\section{Age Comparison: Information Processing Speed}

Figure 17: Mean information processing speed (ms) between young adults and older adults.

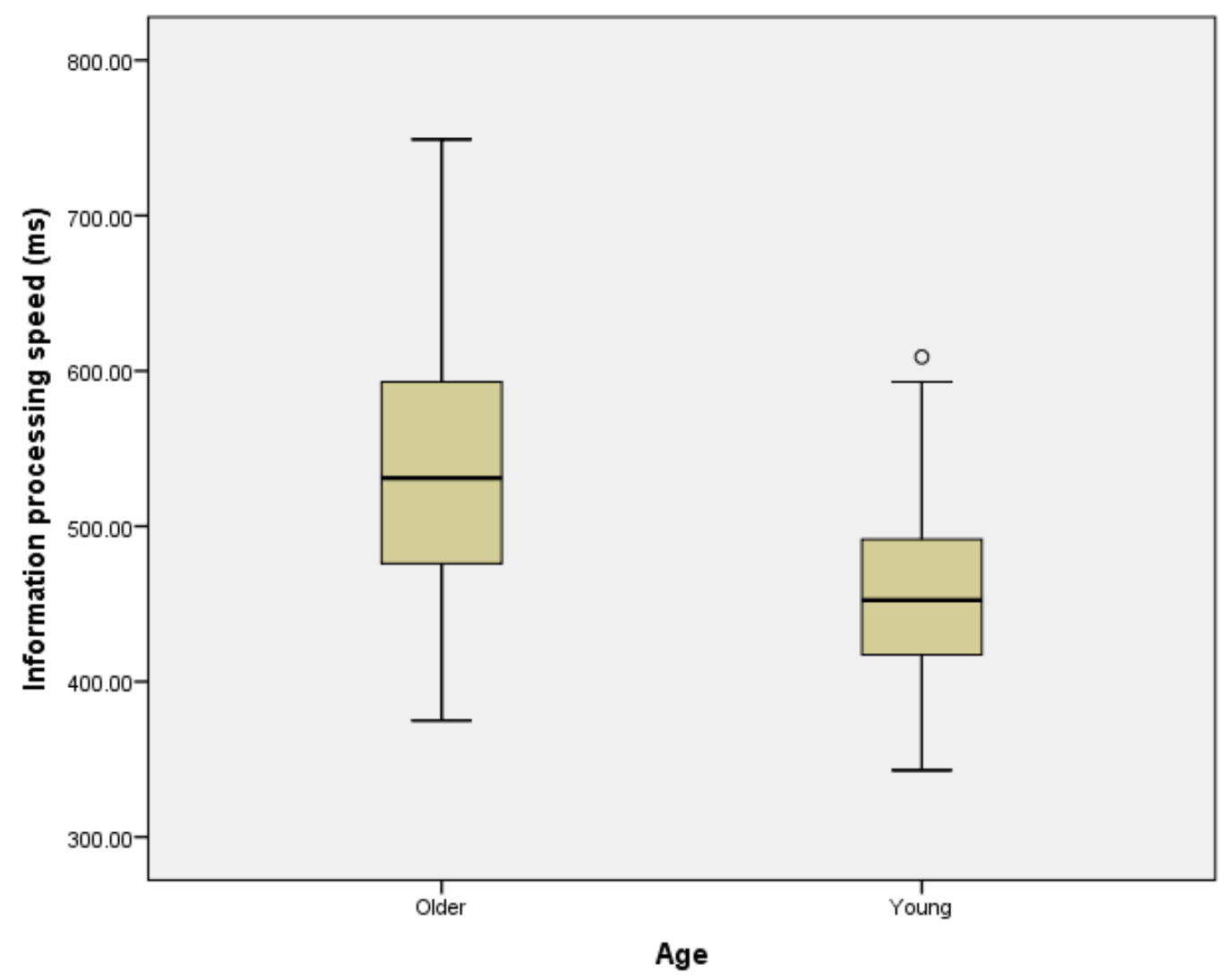

Mann-Whitney analysis revealed a significant difference of information processing speed between age groups; older adults information processing speed was significantly slower compared to young adults $[\mathrm{U}=1228, p<.001$, effect size $(\mathrm{r})=$ $.54]$.

\section{Information processing speed and Subjective memory function}

Correlational analysis using Spearman's rho revealed no significant correlation between information processing speed and total MFQ score (subjective memory function) $[p>.05]$. 


\section{Information processing speed and Perceived test difficulty}

In young adults, information processing speed significantly positively correlated with perceived test difficulty $[\mathrm{r}=.273, p=.015]$. As young adults found the test to be more difficult, their information processing speed slowed. In older adults, there was no significant correlation between information processing speed and perceived test difficulty for older adults $[p$ value $>.05]$.

\section{Information processing speed and education level}

Spearman's rho revealed no significant correlation between information processing speed and educational level in both young and older adults [ $p$ values $>.05]$.

\section{Sex comparison: Information Processing Speed}

Figure 18: Box plot of mean information processing speed (ms) between age (young and older adults) and sex (male and female).

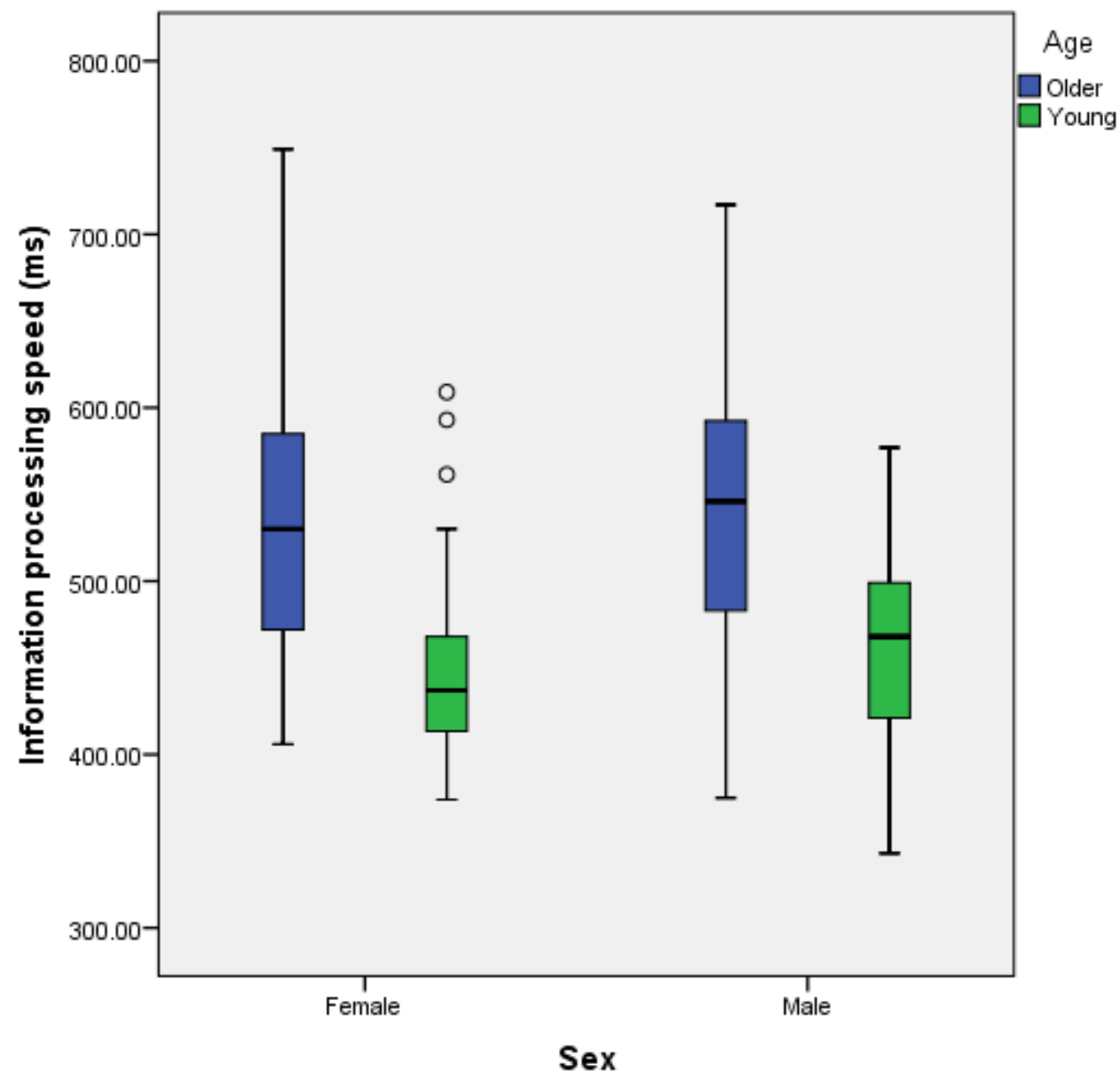


Mann-Whitney analysis revealed no significant difference in reaction time between males and females both in young adults $[\mathrm{U}=604.5, p=.187]$ and in older adults [U $=766.5, p=.599]$.

\section{Information processing speed, anxiety and depression levels}

For both young and older adults, information processing speed did not significantly correlate with anxiety levels $[p>.05]$ or depression levels $[p>.05]$.

\section{Information processing speed and objective cognitive performance (MoCA)}

In young adults, there was no significant correlation between information processing speed and MoCA score (objective measure of general cognition) [ $p>.05]$. In older adults MoCA score significantly negatively correlated with information processing speed; faster RT related to better general cognition [ $\mathrm{r}=-.221, p=.047]$.

\section{Subjective memory function and perceived test difficulty}

Despite aiming to examine the same participants in the Choice RT test to compare with the Simple RT test, there were a few differences in individuals who performed both tests thus this analysis (and the following two sections) is repeated.

In older adults, there was a significant negative correlation between subjective memory function and perceived test difficulty $[\mathrm{r}=-.389, p<.001]$. As older adults perceived greater changes to their memory (low MFQ score) the more difficult the Choice RT was perceived to be.

\section{Education level and subjective memory function}

In older adults, Spearman's rho revealed a significant negative correlation between subjective memory function (total MFQ score) and educational level $[\mathrm{r}=-.266, p=$ .018]. Lower levels of education were related to increase in MFQ score i.e. less perceived change of memory function 


\section{Education level and perceived test difficulty}

Spearman's rho revealed no significant correlation between educational level and perceived test difficulty for both young and older adults [all $p$ values $>.05$ ].

\section{Anxiety and depression levels}

Anxiety levels significantly positively correlated with depression levels in young adults $[\mathrm{r}=.713, p<.001]$ and older adults $[\mathrm{r}=.626, p<.001]$. As anxiety levels increased so did depression levels. In older adults depression levels significantly negatively correlated with subjective memory function (total MFQ score); in older adults perceiving fewer changes to memory function (high MFQ score), depression levels were lower $[\mathrm{r}=-.311, p=.005]$.

\section{Choice RT Comparison of age across blocks: Information Processing Speed}

Table 17. Mean information processing speed (ms) across the four blocks for older and young adults. Standard deviation in parenthesis.

\begin{tabular}{|c|c|c|c|c|}
\hline & Block 1 & Block 2 & Block 3 & Block 4 \\
\hline & 558.43 & 540.26 & 544.85 & 538.28 \\
Older & $(89.29)$ & $(87.17)$ & $(85.35)$ & $(93.43)$ \\
\hline & 448.22 & 455.15 & 461.54 & 462.34 \\
Young & $(61.06)$ & $(59.08)$ & $(64.64)$ & $(62.90)$ \\
\hline
\end{tabular}

In young adults, the Friedman test revealed a significant difference in reaction time across the four blocks $\left[\mathrm{X}^{2}(3, \mathrm{n}=79)=8.27, p=.041\right.$, effect size $($ Kendall's W $)=$ .035]. Post hoc analysis revealed reaction time to significantly increase between block 1 and block $3[\mathrm{Z}=-2.41, p=.016$, effect size $(\mathrm{r})=.27]$ and between block 1 and block $4[\mathrm{Z}=-2.318, p=.020$, effect size $(\mathrm{r})=.26]$ i.e. young adults become slower throughout the test.

In older adults, the Friedman test revealed a significant difference in reaction time across the four blocks $\left[\mathrm{X}^{2}(3, \mathrm{n}=82)=23.60, p<.001\right.$, (Kendall's $\left.\left.\mathrm{W}\right)=.096\right]$. Post hoc analysis revealed reaction time to differ significantly decrease between block 1 and block $2[\mathrm{Z}=-3.473, p=.001$, effect size $(\mathrm{r})=.38]$ between block 1 and block 3 $[\mathrm{Z}=-3.127, p=.002$, effect size $(\mathrm{r})=.35]$ and between block 1 and block $4[\mathrm{Z}=-$ $3.622, p<.001$, effect size $(r)=.40]$ i.e. older adults became faster throughout the test. 


\section{Comparison of sex across blocks: Information processing speed}

Table 18. Mean information processing speed (ms) across the four blocks in males and females for older and young adults. Standard Deviation in parenthesis.

\begin{tabular}{|c|c|c|c|c|}
\hline & Block 1 & Block 2 & Block 3 & Block 4 \\
\hline $\begin{array}{c}\text { Older } \\
\text { male }\end{array}$ & 566.71 & 543.01 & 546.65 & 545.54 \\
$(97.80)$ & $(82.35)$ & $(86.33)$ & $(94.66)$ \\
\hline Older & 553.56 & 538.31 & 543.57 & 533.14 \\
female & $(83.30)$ & $(91.24)$ & $(85.54)$ & $(93.21)$ \\
\hline Young & 462.87 & 465.93 & 465.08 & 464.07 \\
male & $(60.48)$ & $(60.37)$ & $(68.32)$ & $(63.68)$ \\
\hline Young & 439.24 & 448.55 & 459.37 & 461.28 \\
female & $(60.26)$ & $(57.90)$ & $(62.90)$ & $(63.06)$ \\
\hline
\end{tabular}

\section{$\underline{\text { Young adults }}$}

In males, the Friedman test revealed no significant difference in RT across the four blocks $[p>.05]$. In females, the Friedman test revealed a significant difference in RT across the four blocks $\left[\mathrm{X}^{2}(3, \mathrm{n}=49)=14.96, p=.002\right.$, $($ Kendall's W) $=.102]$. Post hoc analysis revealed reaction time to increase significantly between block 1 and block $3[\mathrm{Z}=-3.006, p=.003$, effect size $(\mathrm{r})=.43]$, between block 1 and block $4[\mathrm{Z}=$ $-2.715, p=.007$, effect size $(r)=.39]$ and between block 2 and block $4[\mathrm{Z}=-2.755, p$ $=.006$, effect size $(\mathrm{r})=.39 \mathrm{]}$ thus implies that young females are significantly slower (and less consistent) throughout the test compared to young males.

\section{$\underline{\text { Older adults }}$}

In older males, the Friedman test revealed a significant difference in RT across the four blocks $\left[\mathrm{X}^{2}(3, \mathrm{n}=34)=16.43, p=.001,(\right.$ Kendall's $\left.\mathrm{W})=.161\right]$. Post hoc analysis revealed reaction time to get faster significantly between block 1 and block $2[\mathrm{Z}=-3.333, p=.001$, effect size $(\mathrm{r})=.57]$, between block 1 and block $3[\mathrm{Z}=-$ $2.506, p=.012$, effect size $(r)=.43]$ and between block 1 and block $4[\mathrm{Z}=-2.261, \mathrm{p}$ $=.024$, effect size $(r)=.39]$.

In females, the Friedman test revealed a significant difference in RT across the four blocks $\left[\mathrm{X}^{2}(3, \mathrm{n}=48)=12.29, p=.006\right.$, $($ Kendall's $\left.\mathrm{W})=.085\right]$. Post hoc analysis revealed reaction time to get faster significantly between block 1 and block $2[\mathrm{Z}=-$ $2.191, p=.028$, effect size $(r)=.32]$ and between block 1 and block $4[\mathrm{Z}=-2.647, p$ $=.008$, effect size $(\mathrm{r})=.38$ ] thus both older adult males and females (unlike young adults) become faster as the number of trials increase. 


\section{Intraindividual Variability: Choice RT}

\section{Age Comparison: Intraindividual Variability}

Figure 19: Mean intraindividual variability (IIV) (ms) between young adults and older adults.

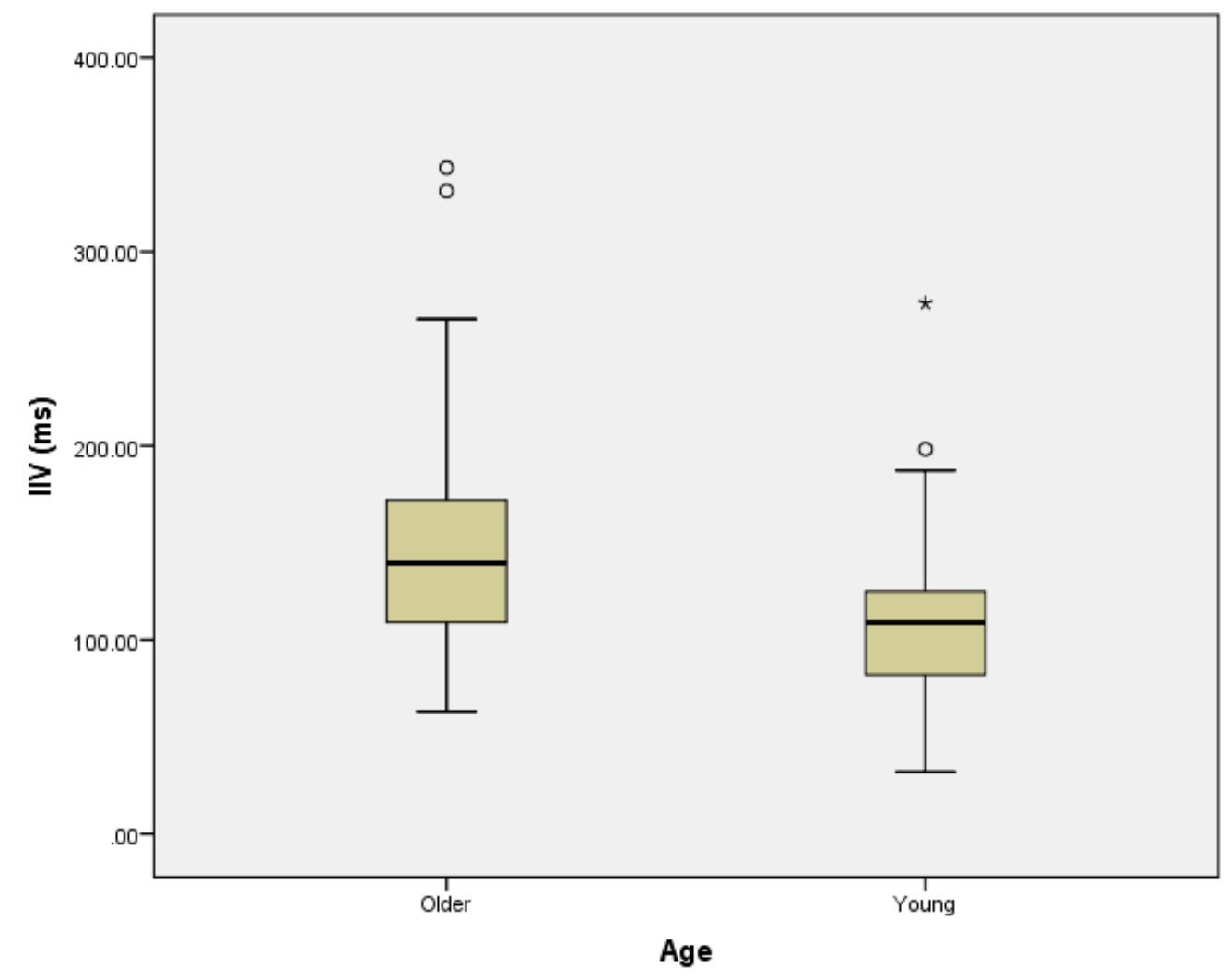

Mann-Whitney analysis revealed a significant difference between age groups; young adults were significantly less variable compared to older adults $[\mathrm{U}=1716, p<.001$, effect size $(r)=.41]$.

\section{Intraindividual variability and Subjective memory function}

In older adults, Spearman's rho revealed no significant correlation between intraindividual variability and subjective memory function (Total MFQ score) $[p>$ $.05]$.

\section{Intraindividual variability and education level}

For both young and older adults, intraindividual variability did not significantly correlate with education [ $p$ values $>.05]$. 


\section{Intraindividual variability and Perceived test difficulty}

In young adults, intraindividual variability significantly positively correlated with perceived test difficulty $[\mathrm{r}=.344, p=.002]$. As young adults found the test to be more difficult, their information processing speed was more varied.

In older adults, there was no significant correlation between intraindividual variability and perceived test difficulty $[p>.05]$.

\section{Sex comparison: Choice RT Intra-individual Variability}

Figure 20: Box plot of mean intraindividual variability (IIV) between age (young and older adults) and sex (male and female).

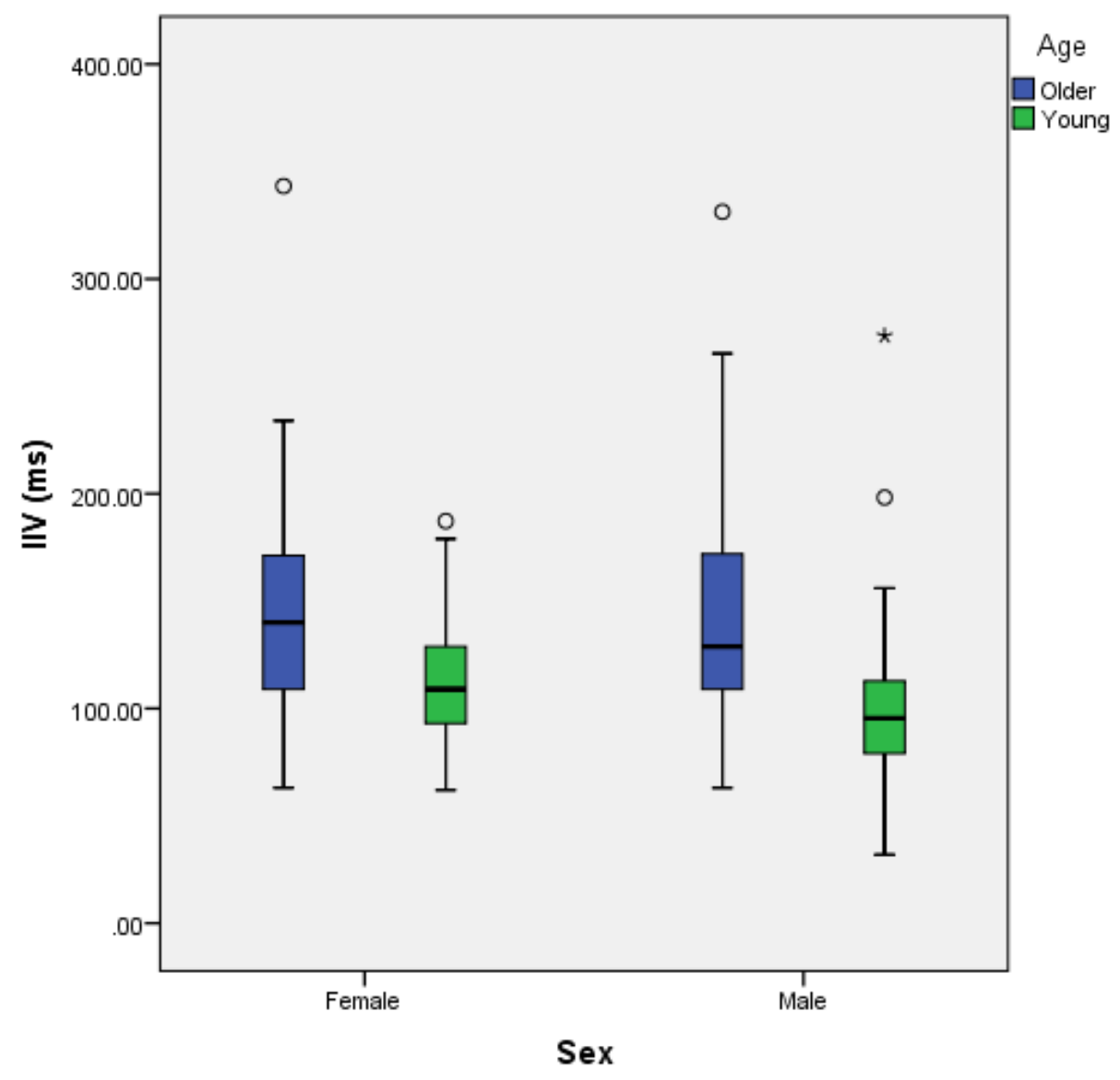

Mann-Whitney analysis revealed no significant difference in reaction time variability between males and females both in young adults $[\mathrm{U}=585, p=.129]$ or in older adults [U $=800, p=.833]$. 


\section{Intraindividual variability, anxiety and depression levels}

For both young and older adults, intraindividual variability did not significantly correlate with anxiety levels $[p>.05]$ or depression levels $[p>.05]$.

\section{Intraindividual variability and objective cognitive performance (MoCA)}

In both young and older adults, there was no significant correlation between IIV and MoCA score (objective measure of general cognition) [all $p$-values $>.05$ ].

\section{Comparison of age across blocks: Intraindividual Variability}

Table 19. Mean intraindividual variability (ms) across the four blocks for older and young adults.

\begin{tabular}{|c|c|c|c|c|}
\hline & Block 1 & Block 2 & Block 3 & Block 4 \\
\hline Older & 153.41 & 138.14 & 132.37 & 131.08 \\
\hline Young & 97.34 & 107.44 & 101.06 & 104.58 \\
\hline
\end{tabular}

In young adults, the table indicates some evidence of increasing variability across the whole test. However the Friedman test revealed no significant difference in IIV across the four blocks $\left[\mathrm{X}^{2}(3, \mathrm{n}=79)=7.42, p=.060\right]$.

In older adults, the table indicates some evidence of reduced variability across the four blocks. The Friedman test revealed a significant difference in IIV across the four blocks $\left[\mathrm{X}^{2}(3, \mathrm{n}=82)=16.55, p=.001\right.$, $($ Kendall's $\left.\mathrm{W})=.067\right]$. Post hoc analysis revealed reaction time variability to significantly decrease between block 1 and block $2[\mathrm{Z}=-2.429, p=.015$, effect size $(\mathrm{r})=.27]$, between block 1 and block $3[\mathrm{Z}=-$ $3.367, p=.001$, effect size $(\mathrm{r})=.37]$ and between block 1 and block $4[\mathrm{Z}=-3.682, p$ $<.001$, effect size $(r)=.41]$ thus older adults become less varied by the end of the test. 


\section{Comparison of sex across blocks: Intraindividual Variability}

Table 20. Mean intra individual variability $(\mathrm{ms})$ across the four blocks for males and females in older and young adults.

\begin{tabular}{|c|c|c|c|c|}
\hline & Block 1 & Block 2 & Block 3 & Block 4 \\
\hline $\begin{array}{c}\text { Older } \\
\text { male }\end{array}$ & 162.38 & 139.43 & 137.31 & 131.76 \\
\hline $\begin{array}{c}\text { Older } \\
\text { female }\end{array}$ & 147.06 & 137.22 & 128.88 & 130.59 \\
\hline $\begin{array}{c}\text { Young } \\
\text { male }\end{array}$ & 100.93 & 108.96 & 102.93 & 97.26 \\
\hline $\begin{array}{c}\text { Young } \\
\text { female }\end{array}$ & 95.14 & 106.52 & 99.91 & 109.07 \\
\hline
\end{tabular}

\section{$\underline{\text { Young adults }}$}

In males, the Friedman test revealed no significant difference in IIV across the four blocks $[p>.05]$. In females, the Friedman test revealed a significant difference in RT variability across the four blocks $\left[\mathrm{X}^{2}(3, \mathrm{n}=49)=10.28, p=.016\right.$, (Kendall's W) $=$ .07]. Post hoc analysis revealed reaction time variability to increase significantly between block 1 and block $2[\mathrm{Z}=-2.462, p=.014$, effect size $(\mathrm{r})=.35]$ and between block 1 and block $4[Z=-2.180, p=.029$, effect size $(r)=.31]$ i.e. females becoming more variable throughout the test.

\section{$\underline{\text { Older adults }}$}

In males, the Friedman test revealed a significant difference in IIV across the four blocks $\left[\mathrm{X}^{2}(3, \mathrm{n}=34)=10.52, p=.015\right.$, (Kendall's W $\left.)=.103\right]$. Post hoc analysis revealed IIV to decrease significantly between block 1 and block $2[\mathrm{Z}=-2.325, p=$ .020 , effect size $(r)=.40]$, between block 1 and block $3[\mathrm{Z}=-2.082, p=.037$, effect size $(r)=.36]$ and between block 1 and block $4[\mathrm{Z}=-2.855, p=.004$, effect size $(\mathrm{r})=$ .49] i.e. males becoming less variable throughout the test. In females, the Friedman test revealed no significant difference in IIV across the four blocks $[p>.05]$. 


\section{Choice RT: Errors}

\section{Overall mean number of errors: Age comparison}

Table 21: Overall mean number of errors in Choice RT for young and older adults. Standard deviation in parenthesis.

\begin{tabular}{|c|c|}
\hline & $\begin{array}{l}\text { Total } \\
\text { Mean }\end{array}$ \\
\hline Older & $\begin{array}{c}2.55 \\
(3.22)\end{array}$ \\
\hline & 4.10 \\
Young & $(3.30)$ \\
\hline
\end{tabular}

Mann Whitney analysis revealed a significant difference in overall mean errors between young and older adults $[\mathrm{U}=2107, p<.001$, effect size $\mathrm{r}=.30]$. Young adults made significantly more errors overall compared to older adults despite being faster.

Spearman's rho analysis revealed no significant correlations between overall mean number of errors and mean information processing speed, IIV, educational level (in years), subjective memory function or perceived test difficulty [all $p$ values $>.05$ ].

\section{Overall mean number of errors: Age and sex comparison}

Table 22. Overall mean number of errors for young males and females and older adult males and females. Standard deviation in parenthesis.

\begin{tabular}{|r|c|}
\hline & Overall Mean \\
\hline & 3.67 \\
Young Males & $(3.40)$ \\
\hline & 4.37 \\
Young Females & $(3.24)$ \\
\hline & 4.37 \\
Older Males & $(3.24)$ \\
\hline & 2.75 \\
Older Females & $(3.56)$ \\
\hline
\end{tabular}

Mann Whitney analysis revealed no significant difference in overall mean errors between males and females in both young adults $[\mathrm{U}=608, p=.195]$ and older adults $[\mathrm{U}=795.5, p=.844]$. 
Mean number of errors across blocks: age comparison

Table 23: Mean number of errors (incorrect responses) across the four blocks in young and older adults. Standard deviation in parenthesis.

\begin{tabular}{|c|c|c|c|c|}
\hline & Block 1 & Block 2 & Block 3 & Block 4 \\
\hline & 0.55 & 0.70 & 0.71 & 0.60 \\
Older & $(0.80)$ & $(1.84)$ & $(1.76)$ & $(1.73)$ \\
\hline & 0.95 & 1.08 & 0.90 & 1.18 \\
Young & $(1.00)$ & $(1.11)$ & $(0.94)$ & $(1.84)$ \\
\hline
\end{tabular}

Figure 21. Bar chart of the mean number of errors across the four blocks for older and young adults

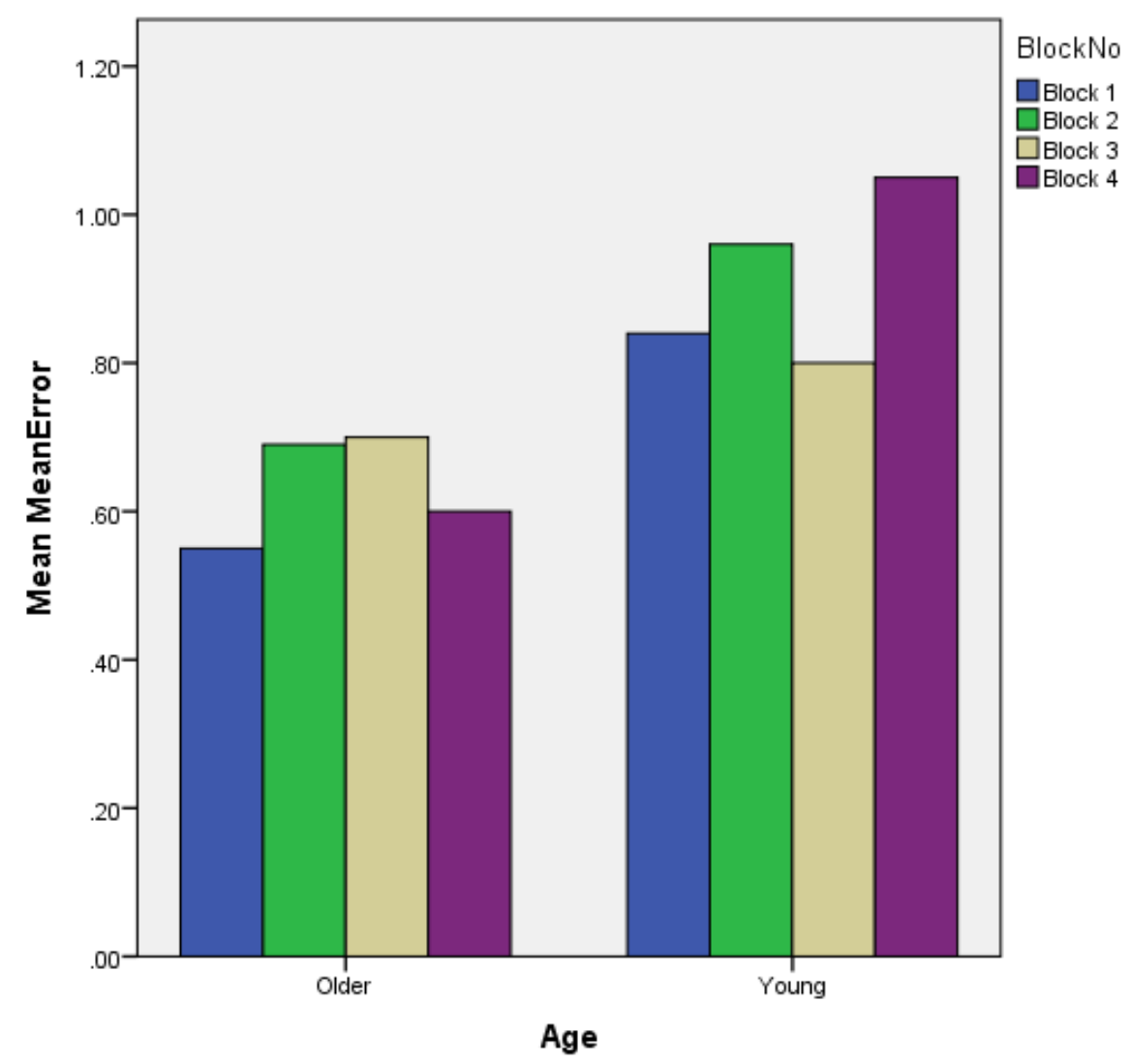

In young adults Table 21 and Figure 21 indicate a greater number of errors were made in Block 4 implying young adults was less accurate in their performance by the end of the test. However the Friedman test revealed no significant difference in the mean number of errors across the four blocks $\left[\mathrm{X}^{2}(3, \mathrm{n}=79)=1.44, p=.967\right]$ implying no significant difference in accuracy throughout the test. 
In older adults it was indicated that a greater number of errors were made in Block 2 and Block 3 (see Table 21 and Figure 21). However the Friedman test revealed no significant difference in the mean number of errors across the four blocks $\left[X^{2}(3, n=\right.$ $82)=1.13, p=.770]$ implying no significant difference in accuracy throughout the test. .

\section{Mean number of errors across blocks: age and sex comparison}

Table 24: Mean number of errors (incorrect responses) across the four blocks for males and females in young and older adults

\begin{tabular}{|c|c|c|c|c|}
\hline & Block 1 & Block 2 & Block 3 & Block 4 \\
\hline $\begin{array}{c}\text { Older } \\
\text { male }\end{array}$ & 0.38 & 0.41 & 1.03 & 0.44 \\
$(0.65)$ & $(0.50)$ & $(2.61)$ & $(0.56)$ \\
\hline Older & 0.66 & 0.9 & 0.5 & 0.71 \\
female & $(0.88)$ & $(2.35)$ & $(0.65)$ & $(2.22)$ \\
\hline Young & 0.7 & 1.13 & 1.0 & 0.9 \\
male & $(0.88)$ & $(1.01)$ & $(0.96)$ & $(1.28)$ \\
\hline Young & 1.1 & 1.04 & 0.9 & 1.4 \\
female & $(1.05)$ & $(1.17)$ & $(0.94)$ & $(2.10)$ \\
\hline
\end{tabular}

Figure 22. Bar chart of the mean number of errors across the four blocks for males and females in young and older adults

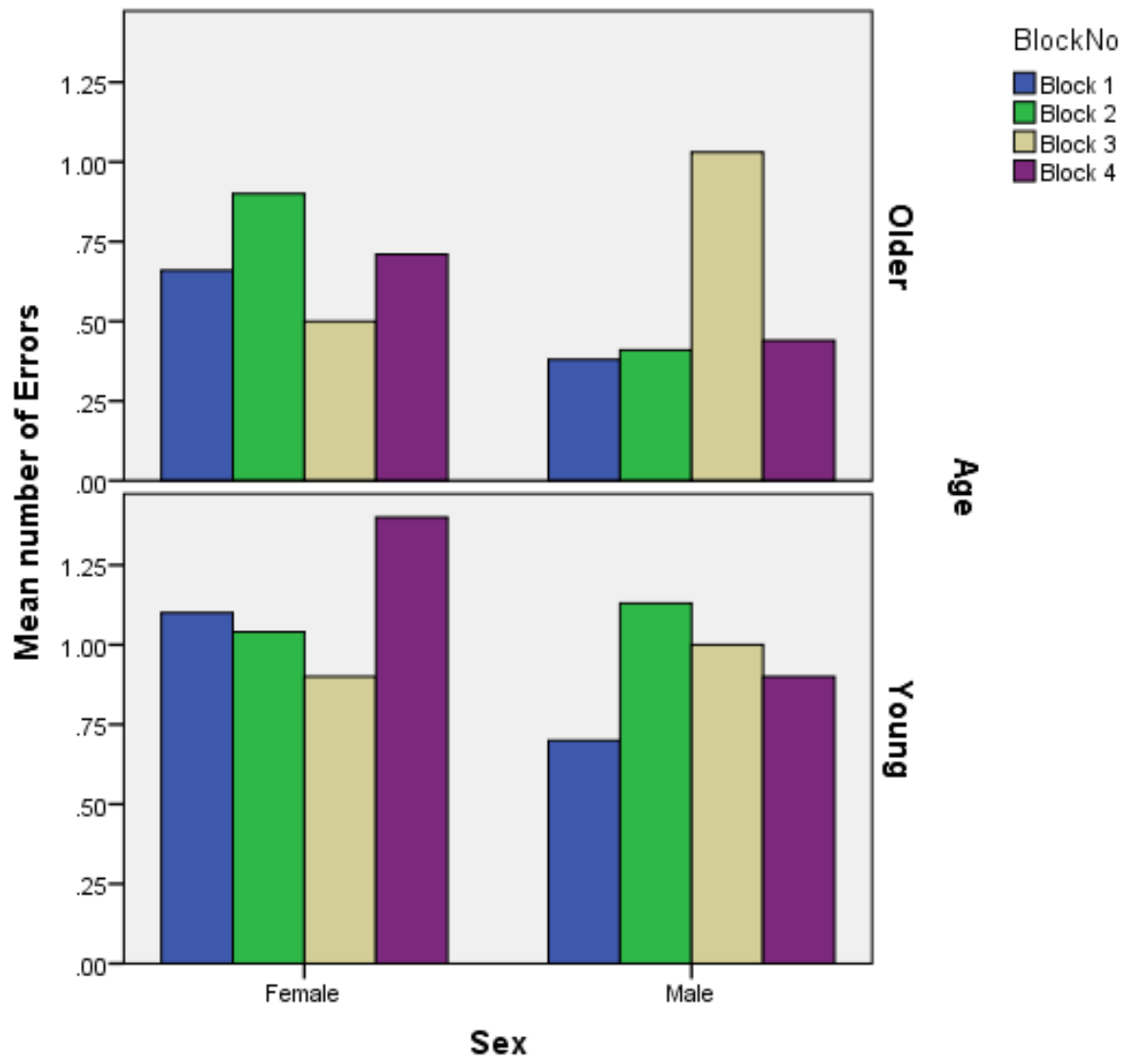




\section{$\underline{\text { Young adults }}$}

In males, the Friedman test revealed no significant difference in the mean number of errors across the four blocks $\left[\mathrm{X}^{2}(3, \mathrm{n}=30)=5.82, p=.121\right]$ implying no significant difference in accuracy throughout the test. In females, the Friedman test revealed no significant difference in the mean number of errors across the four blocks $\left[X^{2}(3, n=\right.$ $49)=2.47, p=.480]$ implying no significant difference in accuracy throughout the test.

\section{$\underline{\text { Older adults }}$}

In males, the Friedman test revealed no significant difference in the mean number of errors across the four blocks $\left[\mathrm{X}^{2}(3, \mathrm{n}=34)=2.14, p=.544\right]$ implying no significant difference in accuracy throughout the test. In females, the Friedman test revealed no significant difference in the mean number of errors across the four blocks $\left[X^{2}(3, n=\right.$ $48)=1.82, p=.611]$ implying no significant difference in accuracy throughout the test. 


\subsection{DISCUSSION}

In the current study Simple RT and Choice RT tests were used to examine the integrity of information processing speed and IIV between young and older adults and the potential effects of subjective memory function, perceived test difficulty, educational level, sex and trial number (Choice RT only).

\section{Information processing speed}

\section{Age comparison: information processing speed}

\section{Simple RT}

In the Simple RT test, information processing speed was significantly slower in older adults compared to young adults in response to a target which was pre-warned with a visual cue of coloured circles and written instruction. These age effects support past research finding information processing speed associated with pre-warned arrival of objects to slow with age during a Simple RT test [Ferreira et al, 2014; Deary et al, 2011; Bugg et al, 2006; Krieg et al, 2001; Wilkinson \& Allison, 1989]. The slowing of information processing speed implies the slowing of encoding ability in older adults [Simon \& Pouraghabagher, 1978] i.e. the ability to prepare, organise and execute a response [Vrtunski et al, 1983]. In addition, the result indicates that this type of processing significantly slows in older compared to younger adults even when the target arrival has been pre-warned.

In contrast, the results contradict past studies which found no significant differences between young and older adults [Nettleback et al, 2014; Dodonova \& Dodonov, 2013]. This may highlight variability of outcome between studies i.e. participant demographics and largely due to the use of different Simple RT paradigms in previous studies compared to the current Simple RT study i.e. the inclusion of visual cues. Visual cues may have enhanced the processing of the target stimulus [Fernandez-Duque \& Posner, 1997] thus producing faster responses compared to studies which did not use cues before a target response thus reducing ageing effects (contrary to ageing effects found in the current study). 


\section{Choice RT}

In the Choice RT test, older adults were also found to be significantly slower in their RT performance compared to young adults. This result supports previous studies finding information processing speed slowing with age during a Choice RT test [Woods et al, 2015; Bugg et al, 2006; Der \& Deary, 2006]. Again the slowing in older adults implies slowing in the ability to prepare, organise and execute a response i.e. encoding ability [Vrtunski et al, 1983; Simon \& Pouraghabagher, 1978] even when the target arrival has been pre-warned with a visual cue. In addition since the current choice RT test included an added decision component, slowed information processing speed in older adults implies that older adults find it more challenging to make quick decisions between stimuli and responses [as discussed in Bugg et al, 2006] i.e. deciding which keyboard response corresponded to each stimulus.

Although we could not find any contradicting studies i.e. finding no age effect during ageing, previous supporting studies (and in comparison to the current study) contained variations to methodology. For example, the current paradigm was designed to replicate the method used by Ballesteros and colleagues [2013] however differences in methodology included smaller sample sizes and the inclusion of other tests and participant groups (i.e. Mild cognitive impairment). Other studies used narrow or age ranges (age groups not separated) or included larger participant groups compared to the current study [Woods et al, 2015; Der \& Deary, 2006] despite our intention to include large groups of both young and older adults. This may have resulted in the current study being less representative of the population. In contrast, not all past Choice RT studies examined the potential influence of other factors on information processing speed i.e. sex, education and particularly did not address the potential influence of subjective memory function and perceived test difficulty.

\section{Choice RT Information processing speed across blocks: age and sex}

In the Choice RT test, information processing speed was examined across each of the four blocks in order to examine how the number of trials per se influences information processing speed and whether influence, if any, is affected by sex i.e. differences between males and females.

Information processing speed in young adults increased significantly from the first block to the last block i.e. young adults slowed down throughout the test. In contrast, information processing speed in older adults decreased significantly from the first block to the last block i.e. sped up. 
We speculated that in the first block, older adults were not familiar with the task thus using different strategies to make sense of it. However by the second block, they had settled into the task and benefitted from practice. Arguably therefore, adults are not necessarily faster per se but are influenced more so than young adults by different factors contributing to task performance i.e. practice. The number of trials for older adults may have helped improve performance or learning, enough to improve information processing speed. This may have implications on the number of trials which should be used in RT tests or in clinics using RT as a measure (single trials or multiple trials) if older adults require greater exposure i.e. more trials to reach maximum efficiency.

Despite a decrease in processing speed, older adults started off with slower RT and were significantly slower in their overall RT performance compared to young adults. This may suggest the factor of the number of trials may have an effect (and should be taken into account) within young or older adult performance but does not have a greater effect on overall RT performance between young and older adults. Indeed effect sizes for the differences in RT between young and older adults were similarly large between trial blocks and for overall mean RT performance in Choice RT.

When comparing sex across the blocks, information processing speed in young males did not differ significantly across the four blocks of trials. However, information processing speed in young females significantly increased across the four blocks of trials i.e. started faster than slowed throughout the trials. This may imply that young males are faster than females during a Choice RT test however when comparing sex independently of age, there was no significant sex effect on RT found between young males and females.

In older adults, information processing speed significantly decreased across the four blocks of trials i.e. started slower then became faster throughout the trials in both males and females although in females decrease was not as gradual. This implies that older adult male and female information processing speed is similar to one another. This was supported when comparing sex independently of age as there was no significant sex effect found between older adult males and females. This implies that neither sex benefit from the number of trials but rather older adults in general may benefit i.e. improve their RT across trials. 
To our knowledge, information processing speed and has not be examined across blocks in relation to differences between young and older adults and between sexes thus the results in this study cannot be compared with previous Choice RT studies. Further investigation is required to determine whether the results in this study are replicable.

\section{Subjective memory function and information processing speed}

There was no significant relationship between information processing speed and subjective memory function in both the Simple RT test and the Choice RT test. Whether or not older adults perceived changes to their memory, their judgement did not appear to influence actual performance i.e. information processing speed for either the Simple RT or Choice RT test.

Therefore, we speculate that since there is a close relationship between information processing speed and structural change i.e. reduced white matter, any slowing of information processing speed is unlikely to relate to detrimental structural change. This is supported by the fact that the majority participants in this study reporting subjective memory changes performed to 'normal' levels in objective testing (MoCA score) [see similar discussion in Chapter $2 \& 3$ and in Torrens-Burton et al, 2017]. Consequently, we speculate that information processing speed in ostensibly healthy older adults perceiving memory changes, remains at 'normal' levels during the both the Simple and Choice RT tests. However there were a minority of older adults with lower MoCA scores which may imply abnormality with general cognition although we only used one test of general cognition thus further assessment is required.

In addition we speculate that subjective memory function did not relate to greater levels of anxiety as levels in older adults were within a normal range and did not correlate with RT and IIV scores. In contrast subjective memory function did correlate with depression levels with older adults who perceived greater changes to memory function had higher levels of depression. This is plausible as individuals noticing changes to memory function, particularly negative changes, would have an effect on mood. However the levels of depression in these older adults were still within a normal range and neither anxiety or depression levels had a significantly effect on information processing speed or IIV in older adults. The results contradict previous studies finding a relationship between subjective complaints and cognitive impairment [Reed, 2010; Cook \& Marsiske, 2005; Earles \& Salthouse, 1995;

Schofield et al., 1997; Grut et al., 1993]. 
It is however, difficult to make direct comparisons between these studies which compared different cognitive functions to the current study i.e. information processing speed as well as examined SCI and not subjective memory function. In contrast, the current results support the findings from the visual search test (see Chapter 2) and the TMT (see Chapter 3) by not finding an association between subjective memory function and information processing speed. Therefore, this supports the speculation that perceived memory changes in ostensibly healthy older adults are not influential on information processing speed in visual attention tests due to any underlying detrimental structural change. However, we did not have access to brain scans and we were unable to perform any follow up assessment therefore it cannot be said whether those with perceived changes to memory function indeed decline further. In addition different participants were used in the visual search test thus it is difficult to make direct comparisons with Simple and Choice RT.

There is the possibility that finding no relationship between information processing speed and subjective memory function relates to the Simple and Choice RT tests not being sensitive enough to detect any functional changes between older adults despite them perceiving changes to their memory. In addition finding an effect of subjective memory function may still depend on the type of visual attention test being measured since the current results can only account for the Simple RT test and the Choice RT test. Therefore, other visual attention tests will be examined to determine whether similar findings between information processing speed and subjective memory function are found across tests.

However, in the Simple RT test (in contrast to the Choice RT test), some older adults produced RT values which were significantly slower than the mean score. These outliers differed from the extreme scores removed before analysis as they did not relate to any extraneous noise. This may imply disproportionate slowing regardless of perceiving changes to memory function and may be reflecting underlying structural change. If this is the case, these individuals cannot be used within healthy control groups as they may be skewing the mean result of what is considered to be a 'normal' level of slowing in healthy older adults during the Simple RT test. Consequently, results of healthy control groups in the Simple RT test may be misinterpreted. Further assessment and follow up in these particular individuals will be of interest to observe whether these individuals are of clinical significance. 


\section{Information processing speed and perceived test difficulty}

\section{Simple RT}

Information processing speed did not significantly correlate with perceived test difficulty for both young and older adults. However difficult young and older adults perceived the Simple RT test to be, this did not reflect in how quickly they performed the test.

As far as we are aware, perceived test difficulty has not been examined with information processing speed of young and older adults in previous Simple RT studies thus we cannot make any comparisons with the current study. However, finding no influence of perceived test difficulty in young adults supports the findings from the visual attention tests examined in previous chapters (see Chapter 2 and 3) which also did not find perceived test difficulty to be associated with RT in young adults. In contrast, finding no relationship in older adult results contradicts the finding from the previous chapters i.e. visual search (Chapter 2) and the TMT (Chapter 3) which found an association between information processing speed and perceived test difficulty. This highlights outcome variability between different visual attention tests i.e. results depend on the type of test used which we aimed to examine by comparing different visual attention tests to each other.

We speculate that self-assessment or judgement i.e. how people perceive the demands of the test to be, may not always reflect actual performance thus other factors influence slowed information processing speed during Simple RT i.e. ageing effects. In addition, the Simple RT was considered easy to perform by both young and older adults thus the range of test difficulty scores was narrow which may explain why no relationship was found. However, in this Simple RT study only one example of psychological factors was examined against information processing speed (perceived test difficulty). It may be possible that if young and older adults were examined against other factors of self-assessment, the relationship with information processing speed may differ.

\section{Choice RT}

In young adults, there was a relationship between perceived test difficulty and information processing speed. As young adults found the test to be more difficult, their information processing speed slowed. This result implies that young adults' self assessment or judgement of the demands of the task (during test performance) is related to information processing speed. 
This supports the concept that demands of the task (as well as judgement of own abilities) can affect the outcome of a task (in this case the speed of performance) [Flavell, 1979]. Perceiving a test to be difficult can negatively affect mood which in turn can have a negative impact on information processing speed [Bolmont, et al, 2000].

In contrast, this result contradicts finding no relationship between perceived test difficulty and information processing speed in Simple RT test (as described above) and in the TMT (Chapter 3) and the visual search test (Chapter 2). As with the Simple RT test, this highlights outcome variability between different visual attention tests i.e. results depend on the type of test used which we aimed to examine by comparing different visual attention tests to each other. As far as we are aware, perceived test difficulty has not examined with information processing speed in previous Choice RT studies thus we cannot make any comparisons of older adults with the current study.

We speculated that judgement of test difficulty reflected actual performance in young during Choice RT (and not Simple RT) due to the Choice RT test being more complex compared to the Simple RT test i.e. including additional executive decision making function. In addition, the Choice RT test produced a greater range of test difficulty scores thus may provide an explanation as to why a relationship was found between perceived test difficulty and information processing speed. Past Choice RT studies examining information processing speed in young adults may have been misinterpreted if young adults' judgement about test difficulty appears to influence how quickly they perform the test. However, it must be noted that the effect size of this result was small therefore the result may not be very robust thus may not be very reliable.

In older adults, information processing speed did not significantly correlate with perceived test difficulty. However difficult older adults perceived the Choice RT test to be, this did not reflect in how quickly they performed the test. We speculate that no relationship was found between perceived test difficulty and information processing speed in older adults because other factors are more influential on slowed information processing speed during Simple RT i.e. ageing effects. Although in the current Choice RT study only one example of a psychological factor was examined against information processing speed (perceived test difficulty). It may be possible that if older adults were examined against other factors of self-assessment, the relationship with information processing speed may differ. 


\section{Education and information processing speed}

In both the Simple RT and Choice RT tests, there was no significant effect of education on information processing speed in both young and older adults. This result supports some studies who found no education effect in both the Simple RT test [Deary et al, 2001] and in the Choice RT test [Woods et al, 2015; Deary et al, 2001] and contradicts other past evidence that higher education is associated with faster information processing speed during both the Simple RT test and the Choice RT test in young and older adults [Houx \& Jolles, 1993]. This outcome variability may reflect different methodology used between studies. Different paradigms have been used for both the Simple RT and Choice RT tests which may have varied in complexity i.e. type and speed of stimuli. Past studies [Woods et al, 2015; Der \& Deary, 2006] have also included larger sample sizes compared to the current study (despite our intention to include large groups) which may provide a greater representation of the population.

Some studies [Deary et al, 2001; Lahtela et al, 1985] only examined information processing speed in older adults with no comparison to young adults i.e. a measure of ageing. Therefore it may be difficult to make conclusions about the effects of education on information processing speed during ageing.

A great difference when examining the effect of education on information processing is that past studies grouped education between low levels (0- 11 years) and high levels (11 years +) [following Verhage, 1965]; a method which the current study did not replicate. There is an issue with splitting education in this way as in young adults there would be a narrower range of education levels compared to older adults [as suggested by Tun \& Lachman, 2008] due to the few years of further education young adults have reached compared to older adults. This is especially true for the current study as the young adult population were not as advanced in their years of further education compared to the older adult population.

We attempted to match education as far as possible across both groups thus the range was narrow although still varied enough to be investigated. Interestingly there was in influence of education on information processing speed for older adults found in visual search despite this narrow educational range (see Chapter 2). This may imply that the Simple and Choice RT tests are not sensitive enough to find an effect of education within this narrow educational range as found with the TMT test (see Chapter 3). 
Alternatively, different tests have components which are differentially affected by education. Therefore, we may speculate that finding no effect of education in the Simple and Choice RT tests may be due to other factors such as age having a greater effect on information processing speed.

\section{Sex and information processing speed}

In both the Simple RT and Choice RT tests, there was no significant effect of sex on information processing speed for young and older adults. The results do not support previous findings that males are faster than females during the Simple RT [Karia et al; 2012; Roivainen, 2011; Krieg et al, 2001; Fozard et al, 1994; Bleecker et al, 1987; Lahtela et al, 1985] and Choice RT [Dykiert et al, 2012; Karia et al, 2012] and as found in young adults [Karia et al, 2012] and older adults [Dykiert et al, 2012; Fozard et al, 1994].

Differences in methodology between the previous studies and the current study may account for the variability in outcome. For example in the current Simple RT test, the paradigm contained different stimuli compared to previous simple RT studies i.e. stimuli mirroring real world situations which require a rapid response. Using real life stimuli may be more relatable thus responded to more efficiently. Sample sizes in some previous studies were smaller thus previous results to compare with the current study may have not been generalizable. In addition, finding no sex effect on information processing speed may have been due to the ratio of males and females available for comparison. For both young and older adults, there was a female bias in the participant sample thus it would be useful to repeat the test with a greater number of males to observe whether the same findings are still true.

The results in the current Simple and Choice RT tests support each other and the TMT in the previous chapter (Chapter 3). Similar influences of sex related to information processing speed between both tests and the TMT may relate to the fact that the majority of the same young and older participants completed both tests. Despite there being a small number of individuals (both older and young adults) who differed between the TMT, Simple and Choice RT, this did not appear to result in significant differences in results when comparing each test with one another. This indicates that any differences, if any, are related to the teak and not the participants. In contrast finding contradicting results of sex in the Simple RT, Choice RT tests and the TMT compared to the visual search test (Chapter 2) may relate to completely different participant samples used during the visual search test. 


\section{Subjective memory function and Perceived test difficulty}

In both the Simple RT test and the Choice RT test, a relationship was found between subjective memory function and perceived test difficulty. As older adults perceived greater changes to their memory (low MFQ score) the more difficult the Simple RT test and the Choice RT test was perceived to be. This result supports our speculation that if older adults believe their cognition to be poor, they would assume that tests will be more difficult to perform as a result as poor cognition will impair the ability to perform a cognitive task well enough.

This relationship was examined separately between both tests as there were a few participants who differed between them despite trying to use same people in order to determine whether results are similar between tests. Since both tests found a relationship between subjective memory function and perceived test difficulty, the slight differences in participants may not have been influential on the overall result. It must be noted that the effect size of the relationship in Simple RT was small therefore the result may not be very robust thus the outcome being very reliable. In comparison, the effect size of the relationship in Choice RT was greater which may imply that the Choice RT test is more robust thus more reliable than the Simple RT.

\section{Educational level and perceived test difficulty}

In both the Simple RT test and the Choice RT test, there was no relationship found between educational level and perceived test difficulty in both young and older adults. How difficult the Simple or Choice RT test was perceived to be, this did not relate to the level of education (in years) either young or older adults had.

We speculated that those with greater years of education would judge the demands of the test to be low due to higher levels of education being beneficial for test performance i.e. higher levels of motor skill or concentration help tests appear less difficult. However, finding no relationship between educational level and perceived test difficulty may be due to the Simple and Choice tests being designed to be relatively simple to complete. As a result, education level may not account for how difficult the both tests were perceived to be by both young and older adults. Indeed, young and older adults rated the Simple and Choice RT test to be easier to complete compared to other tests i.e. the TMT and visual search. 
As far as we are aware there are few, if any, Simple and Choice RT studies which have examined education against perceived test difficulty or other psychological factors. Therefore, further research is required to examine whether other psychological factors other than perceived test difficulty may relate to the level of education in young and older adults and whether similar findings occur across other visual attention tests.

\section{Educational level and subjective memory function}

\section{Simple RT}

In the Simple RT test, there was no relationship between subjective memory function and educational level in older adults. This result supports the findings of the visual search test (see Chapter 2) where education did not correlate with perceived changes to memory function although the participant sample differ between the two tests. In contrast, the result contradicts those found in the TMT (see Chapter 3) i.e. lower levels of education related to less perceptions of change to memory function.

This variability of outcome between tests may highlight that finding a relationship between subjective memory function and educational level in older adults may depend on the type of test used. A similar relationship had also been found between education and perceived test difficulty. It was speculated that subjective memory function is also a psychological factor i.e. self-assessment of memory function [as discussed above]. This may provide an explanation as to why no relationship was also found between educational level and subjective memory function i.e. psychological factors do not relate to educational level.

\section{Choice RT}

For the Choice RT test, educational level significantly correlated with subjective memory function in older adults. Lower levels of education related to less perceptions of change to memory function. Therefore, older adults with higher levels of education are likely to perceive their memory is getting worse. This result differs to the Simple RT test (above) and the visual search (Chapter 2) finding no relationship but supports the TMT (Chapter 3 ) finding a negative correlation between educational level and subjective memory function. 
As with the TMT, the result may suggest that older adults with higher education are more judgemental about their memory function. In contrast, it may suggest that older adults with higher education are possibly more aware or have greater understanding of the quality of their memory function.

Finding a relationship in the Choice RT (and not in the Simple RT) again highlights outcome variability between tests and implies differences due to test complexity (the Choice RT being more complex compared to the Simple RT). It must be noted that the sample size was small thus the result may not be very robust and reliable.

\section{Intraindividual variability}

\section{Age comparison: intraindividual variability}

In the Simple RT test, older adults produced greater IIV i.e. more variable information processing speed compared to young adults. This supports previous studies finding older adults to be more variable in simple RT tasks [Bielak et al, 2014; Inui et al, 1997] compared to young adults [Fozard et al, 1994].

In the Choice RT test older adults also produced greater IIV compared to young adults. This supports previous Choice RT studies finding an increase in IIV as age increased [Bielak et al, 2014; Dykiert et al, 2012; Der \& Deary, 2006] thus implying poorer variability of information processing speed during ageing thus poorer integrity of underlying central nervous system (CNS). Greater variation of information processing speed during the Choice RT has been argued to be a reflection of the task being more complex thus requiring greater processing load [Der \& Deary, 2006].

\section{Intraindividual variability across blocks: age and sex}

IIV was examined across each of the four blocks in order to examine how the number of trials per se influences IIV and whether influence, if any, is affected by sex i.e. differences between males and females.

In young adults, IIV did not significantly differ between the four blocks implying that their RT performance was consistent throughout the test thus were not affected by any practice effects or fatigue which may influence variation of information processing speed. 
In contrast, a significant difference of IIV was found between blocks in older adults. Older adults were more variable in their RT performance during the first block but became less varied by the second block and remained consistently less varied until the end of the test thus improved information processing speed. This suggests, as with information processing speed, that in the first block older adults were not used to the task thus having different strategies to make sense of the task. However by the second block, they had settled into the task and benefitted from practice which again may suggest that the number of trials for older adults may have helped improve performance or learning, enough to improve IIV. Arguably therefore, older adults are not necessarily less variable per se but are influenced more so than young adults by different factors contributing to task performance i.e. practice.

In contrast when examining the test overall, older adults were significantly more varied in their overall RT performance compared to young adults. We speculate that greater IIV in older adults highlights poorer variability of information processing speed thus poorer integrity of underlying CNS. Finding effect of IIV in ageing may depend on whether measuring overall mean IIV or examining the number of trials. Therefore this may have to be taken into account in future Choice RT research studies and possibly clinical studies.

When comparing sex across blocks, IIV in young males did not differ significantly across the four blocks of trials implying that their RT performance remained consistent. However, IIV in young females significantly increased i.e. RT performance became more varied across the four blocks of trials. This may imply that young males are more consistent during the Choice RT test compared to females. However, when comparing sex with overall IIV independently of age, there was no significant sex effect found between young males and females which may imply that the numbers of trials need to be examined specifically to find a sex effect. In older adults, IIV significantly decreased i.e. RT performance was less varied across the four blocks of trials in males implying their performance improved throughout the test. In contrast there was no significant difference in IIV across blocks in older adult females implying their RT performance remained consistent throughout the Choice RT test. The number of trials does not appear to assist performance or learning and thus have an influence on IIV. 
As with young adults, when comparing sex with IIV independently of age, there was no significant sex effect found in overall test IIV between older adult males and females, again implying that examining the number of trials has an effect on finding differences of IIV between males and females.

\section{Subjective memory function and intraindividual variability}

In older adults, there was no significant relationship between IIV and subjective memory function in both the Simple RT test and the Choice RT test. Whether or not older adults perceived changes to their memory, this did not appear to influence the variation of participants' information processing speed for either the Simple RT test or the Choice RT test.

We would speculate that if the integrity of information processing speed is poor (i.e. increased IIV) this may reflect impairment to cognitive function thus older adults would perceive their memory function to be worse. However perceived changes to memory did not influence IIV. Therefore, IIV remains at 'normal' levels expected in ostensibly healthy older adults. However as mentioned above, we did not have access to brain scans and we were unable to perform any follow up assessment therefore it cannot be said whether those with perceived changes to memory function indeed decline further.

As far as we are aware, past Simple and Choice RT studies have not included a measure of subjective memory function and IIV therefore we cannot make comparisons compare the current results. The current IIV result supports finding no relationship between subjective memory function and IIV during the visual search test (see Chapter 2) although it must be highlighted that visual search used a different sample of participants to the Simple and Choice RT tests.

However as similar results were found (i.e. a lack of relationship between IIV and subjective memory function), this supports the speculation that perceived memory changes in ostensibly healthy older adults are not influential on the variation of information processing speed (IIV) in visual attention tests. In contrast, finding an effect of subjective memory function on IIV may still depend on the type of visual attention test being measured. We cannot make comparisons with the TMT since the test contained a single trial thus IIV could not be measured. Therefore, other visual attention tests using multiple trials will be examined to determine whether similar findings between IIV and subjective memory function are found. 
It must also be noted that as with the RT scores, there were some older adults producing significantly greater IIV during the Simple and Choice RT tests i.e. these individuals were significantly more varied than the mean score. Again these outliers differed from the extreme scores removed before analysis as they did not relate to any extraneous noise.

This may imply significantly poorer integrity of information processing speed regardless of perceiving changes to memory function and may be reflecting underlying structural change. If this is the case, these individuals cannot be used within healthy control groups as they may be skewing the mean result of what is considered to be a 'normal' level of variation in healthy older adults during the Simple and Choice RT tests. Consequently, results of healthy control groups in the Simple and Choice RT test may be misinterpreted. Further assessment and follow up in these particular individuals will be of interest to observe whether these individuals are of clinical significance.

\section{Perceived test difficulty and intraindividual variability}

\section{Simple RT}

In the Simple RT test, there was no significant relationship found between perceived test difficulty and intraindividual variability for both young and older adults. How difficult the Simple RT test was perceived to be did not relate to how varied RT performance was in young and older adults. This result was similar to the relationship found between in information processing speed thus as speculated above, self-assessment or judgement i.e. how people perceive the demands of the test to be, does not reflect actual performance. Also mentioned above, Simple RT was considered easy to perform by both young and older adults thus the range of test difficulty scores was narrow which may also explain why no relationship was found with IIV.

As far as we are aware, perceived test difficulty has not been examined with IIV of young and older adults in previous Simple RT studies thus we cannot make any comparisons with the current study. The current result supports the visual search test which also found no relationship between IIV and perceived test difficulty in young and older adults (see Chapter 2) although it must be noted that participant samples differed between the two tests. 
Finding an effect of perceived test difficulty on IIV may still depend on the type of visual attention test and the type of psychological factor being measured. Therefore, other visual attention tests using multiple trials will be examined to determine whether similar findings between IIV and perceived test difficulty are found.

\section{Choice RT}

In the Choice RT test, perceived test difficulty positively correlated with IIV in young adults. As young adults perceived the Choice RT test to be more difficult, IIV (variation of RT performance) increased. This result relates to the concept that the demands of the task can affect the outcome of a task (in this case the variation of performance). As with information processing speed, finding a relationship during the Choice RT and not the Simple RT test may relate to the Choice RT being more complex in comparison.

The Choice RT test produced a slightly greater range of test difficulty scores thus may provide an explanation as to why a relationship was found between perceived test difficulty and information processing speed in the Choice RT test. This result does not support the visual search test (see Chapter 2) which found no relationship between IIV and perceived in young adults (although participant samples differed between tests). The Choice RT test cannot support or contradict the TMT as IIV could not be measured since it contained only one trial. In addition, to our knowledge perceived test difficulty has not been examined with IIV of young adults in previous Choice RT studies thus we cannot make any comparisons with the current study. In older adults, IIV did not significantly correlate with perceived test difficulty. How difficult older adults perceived the Choice RT test to be, did not reflect in how varied their information processing speed was throughout the test. This result supports the visual search test (Chapter 2) finding no relationship between IIV and perceived test

difficulty in older adults thus highlighting that finding a relationship between IIV and perceived test difficulty in older adults may depend on the type of visual attention test used.

As speculated with information processing speed, no relationship between perceived test difficulty and IIV may be a result of other factors being more influential on the variation of information processing speed during the Choice RT i.e. ageing effects. 
However, in the current Choice RT study only one example of a psychological factor was examined against information processing speed (perceived test difficulty). It may be possible that if older adults were examined against other factors of selfassessment, the relationship with information processing speed may differ.

\section{Education and intraindividual variability}

In both the Simple RT and Choice RT tests, there was no significant relationship between educational level (in years) and IIV for young and older adults. A lack of relationship was also found between educational level and information processing speed.

The current result supports the TMT (Chapter 3) finding no influence of education on IIV in young adults. In contrast, the results contradict the findings of older adults during visual search which found a greater level of formal education (in years) was related to less variation of reaction time. Outcome variability in the older adult population may relate to the sample size being larger in the Simple and Choice RT tests and a different population of older adults included in the visual search test.

We could only make speculations about the current results at present as, few, if any, previous Simple and Choice RT studies to our knowledge have examined education level and IIV thus comparisons cannot be made with the current results.

\section{Sex and intraindividual variability}

In both the Simple RT and Choice RT tests, there was no effect of sex on IIV for young and older adults. This result was also found in information processing speed for both tests and age groups.

The current result suggests that in young and older adults, males are as consistent as females in their RT performance during Simple and Choice RT. This contradicts past Simple and Choice RT studies finding IIV to be greater in females [Bielak et al, 2014; Dykiert et al., 2012; Der \& Deary, 2006; Silverman, 2006; Reimers \& Maylor, 2006]. The current result also contradicts the visual search test in this thesis (Chapter 2) again highlighting the variability of study outcome possibly due to uneven ratio of males and females in the current study, differences in sample sizes and participant groups during the visual search. 


\section{Number of Errors}

\section{Simple RT}

During the Simple RT test, errors were associated with responding before the stimulus appeared on the screen once both visual cues were presented. These anticipatory presses were greater in young adults compared to older adults thus may be highlighting greater alerting affects in young adults although the difference did not reach significant level not did the difference in error numbers between males and females in both young and older adults. However information processing speed of impulsive responses does not necessarily represent the speed of processing a target if the target has not yet been presented on the screen after the visual cues.

The Simple RT test was designed for participants to be prepared for the target (i.e. being alert after visual cues) but not to respond until the target was presented. The style of the task itself provided greater opportunity for impulsive responses i.e. use of the traffic lights in visual cues and target stimulus. As the order was very recognisable for participants this made it easy to anticipate when the next target would appear and therefore press before the target actually appeared. Future versions of the current Simple RT paradigm may benefit from a warning message to participants if they responded too early.

It must be noted that with computers there is a minute delay between a persons' natural reaction time to a stimulus and the time it takes for the software to record the RT when keyboard button is pressed. This is not a significant amount and usually corrected for during programme development however it is important to keep in mind when measuring and comparing computer based reaction time tasks.

\section{Choice RT}

During the Choice RT test, there were six older adults and two young adults who had to be eliminated from analysis due to their error rate being too high (over 20\%). In older adults, this may be a sign of significant negative changes occurring in their cognitive processing although in young adults error rates may reflect impulsivity to complete the task quickly despite the need for accuracy or a lack of concentration to which response was required for each stimulus. Only one of the eliminated older adult participants had a low MFQ score i.e. expressed memory concerns. Therefore it may be of interest to further assess this participant to observe whether any further cognitive decline is occurring. 
In error trials, when they saw either the ' $\mathrm{X}$ ' or ' $\mathrm{O}$ ' stimulus, the participant pressed the key designated to the opposite stimulus instead of the one designated to the target at that point on the screen. Errors were likely due to forgetting which key was designated to which stimulus thus we speculated that later blocks would have had fewer errors as participants were reminded which keys to use in their response. Accuracy of the test may have been improved by having response buttons that match the letters of the stimuli. Indeed it was mentioned by older adult participants especially that the incongruence between the stimuli and the response key were confusing. This confusion could highlight older adults demonstrating detrimental change in their ability to process multiple pieces of information.

The mean number of errors was not significantly different across blocks in older adults despite it being indicated that a greater number of errors in Block 2 and Block 3 (see Table 21 and Figure 21) nor was there a significant difference in males and females. In addition mean number of errors were not significantly different across blocks in young adults although it was indicated that a greater number of errors were made in Block 4 (see Table 21 and Figure 21) nor was there a significant difference in males and females (see Table 22 and Figure 22)

In contrast, when comparing young and older adults with overall mean error, young adults made significantly more errors compared to older adults. This implies that error rate in young and older adults may depend on how the numbers of trials are examined (whether examined overall or across blocks of trials). Therefore, it may be important for future Choice RT studies to include the effects of trial numbers on error rates and on RT as how the numbers of trials are analysed may produce different effects on information processing speed.

When comparing mean error to RT, in young adults females were slower throughout the test which implies a speed/accuracy trade-off with females focusing on accuracy rather than speed. In older adults, males and females were faster throughout the test this implying a focus on both accuracy and speed. Overall, young adults were significantly faster compared to older adults but made significantly more errors. This implies that in relation to the speed/accuracy trade-off, young adults may have a tendency to focus on speed rather than accuracy whereas older adults focus on performing the test correctly rather than quickly. 


\section{Outliers}

In the Simple RT test, outliers were observed in information processing speed and IIV for both older and young adults, with more extreme scores found in young adults. In the Choice RT tests, outliers were observed in RT in young adults only and in both age groups outliers were observed in RT variability. These outliers differed from the extreme scores removed before analysis as they did not relate to any extraneous noise. The outlier values in the young adults during both tests may be less likely to reflect significant cognitive impairment but possibly due to fatigue or a lack of concentration. Although it must also be considered that these young adults producing significantly slower information processing speed were poor at performing the test and possibly experiencing further cognitive impairment.

In older adults, these outlier values may imply disproportionate slowing regardless of perceiving changes to memory function and may be reflecting underlying structural change. If this is the case, these individuals cannot be used within healthy control groups as they may be skewing the mean result of what is considered to be a 'normal' level of slowing in healthy older adults during the Choice RT test. Consequently, results of healthy control groups in the Choice RT test may be misinterpreted. Further assessment and follow up in these particular individuals will be of interest to observe whether these individuals are of clinical significance thus it may be important to highlight outliers in RT research.

\section{Study Limitations}

In the Simple RT test, the paradigm was programmed to repeat the trial if the participants responded before the target stimulus appeared on the screen i.e. errors. This is a potential limitation as it may have has an impact on the group mean of information processing speed thus how integrity of alerting effect is interpreted. Some individuals (particularly young adults) made a greater number of errors thus ended up performing more trials compared to others. This may either lead to greater practice thus faster RT performance in following trials and faster overall mean RT. In contrast, more trials may lead to feelings of fatigue thus slower RT performance in following trials and slower overall mean RT. As a result the difference between young and older adults may have been misinterpreted. 
This change of RT performance across trials was particularly observed in the Choice RT when examining trials in blocks as finding differences in RT between young and old appeared to depend on whether RT was measured overall or across the number of trials (i.e. separate blocks). Previous ageing research using Choice RT have not included a measure of the number of trials particularly in relation to RT and IIV thus this may be a useful measure in future Choice RT studies and even other RT tests using multiple trials.

Different motor movement was required to respond to the target stimuli during both Simple and Choice RT. This required different practice periods in order to reach optimum speed particularly the Choice RT, since it the response procedure was more complex i.e. matching the response button to the corresponding stimulus on the screen. The Simple RT involved one finger pressing one button whilst the Choice RT to use two fingers, one for each response although it has been observed that multiple fingers can produce faster responses compared to using the same hand [Annett \& Annett, 1979]. The Choice RT test therefore may require a longer practive period in order for participants ot become efficient at the task. In addition, motor movement in both simple and choice RT may also be impaired in older adults whether due to possible stiffness in hand movement or arthritic pain. As a result this may reflect slower reaction time in older adults compared to young adults.

An issue with comparing results of the Simple RT with the Choice RT is that the visual cues and target stimuli were different between both tests. Different stimuli i.e. shapes or words may activate different components of attentional function thus it may not be possible to directly compare information processing speed between the two tests. It may be useful to redesign the current Simple and Choice RT tests using similar stimuli thus directly comparing the same attentional function (alerting effect) and what influence an added decision component (multiple trials in Choice RT) has on the altering effect and information processing speed.

\section{Conclusion}

The current study examined the functional integrity visual attention and information processing speed and IIV between young and older adults using both Simple and Choice RT tests as well as measuring the influence of other factors of sex, education perceived test difficulty and subjective memory function which have not been examined in detail previously. Both the Simple and Choice RT tests revealed significant differences in information processing speed and IIV between young and older adults. 
Older adults were significantly slower and more variable compared to young adults. A greater difference in information processing speed between young and older adults (i.e. ageing) was observed in the Choice RT test due to larger effect sizes compared to the Simple RT test.

When examining different factors on information processing speed and IIV both tests found no significant effect of sex, education and subjective memory function in both young and older adults. Finding no influence of these factors on information processing speed and IIV highlights the outcome variability can differ between visual attention tests since sex and education were found to be influential during the visual search test (Chapter 2). Perceived test difficulty revealed no influence on information processing speed in Simple RT test but significantly positively correlated with RT and IIV in young adults only in the Choice RT test. This also highlights outcome variability between simple and choice tests which may depend on the paradigm used and the level of complexity i.e. the use of a 'choice' entity. Variability is less likely to relate to sample size and sample characteristics as majority of the same people used in both tests.

In the Choice RT, past studies may have been misguided in dismissing the inclusion of perceived test difficulty as the results of information processing speed and IIV in older adult control groups may have been misinterpreted.

Reduced ability in older adults to process and respond to stimuli may imply that older adults may have trouble responding to sudden changes in the environment. This may prove troublesome when performing particular daily tasks thus have a negative impact on their quality of life i.e. giving up driving due to an inability to respond efficiently to sudden obstacles. 


\subsection{CHAPTER FIVE. iPad -based visual search testing: The Multi-item Localization (MILO) test.}

\subsection{INTRODUCTION}

In summary, the results so far indicate that information processing speed is significantly slower and more variable in older adults compared to young adults across all the tests employed. The effects of different person-related factors on RT or IIV however differed between tests. In the visual search test (Chapter 2) higher educational level and perceiving the test to be more difficult related to faster information processing speed in older adults, and sex was influential on young adult information processing speed with males being faster and less variable compared to females. In contrast, the TMT (Chapter 3) and the Simple RT and Choice RT tests (Chapter 4) revealed no significant relationship between information processing speed and sex or education for both young and older adults. In the TMT, perceiving the test to be more difficult related to slower information processing speed in older adults whereas in the Choice RT, the same relationship was found in young adults and not older adults. In the Simple RT there was no relationship between perceived test difficulty and information processing speed. All these tests did not find a significant relationship between information processing speed and subjective memory function.

So far in this series of studies, the visual attention-related information processing speed has been investigated using the common administration platforms, namely a desk-top computer (Visual search, Simple and Choice RT) or pen and paper (Trails A and B) typically used in clinical diagnosis and/ or research. It can be argued that both presentation modes may bias against older adults because of difficulties holding or using a pen and /or pressing specific keys on a computer keyboard. Thus if similar tests were used by using a different response mechanism, and one which may be more representative of real life performance, older adults' RT performance may actually provide a more representative measure of the functional integrity of information processing speed in ageing. Therefore, the aim of the present study was to determine whether differences in RT and IIV between young and older adults could also be found on an iPad based test namely the Multi-item localization (MILO) test [Thornton \& Horowitz, 2004; Horowitz \& Thornton, 2008]; a test we had redesigned for the purpose of resembling a visual search task and the TMT in order measure information processing speed, attentional function (shifting attention), feature search and executive function. 
In addition the aim was to determine if the difference in RT and IIV and errors during a different type of visual search task and using a different response mechanism, resembled those in the original search task [Tales et al, 2010] and the TMT [Reitan, 1971].

A further aim was to determine whether the outcome using this MILO-type test would be affected by sex, education, perceived test difficulty and subjective memory function in a similar way to the other tests examined in previous chapters. Any effects of person-related factors may indicate the requirement to take them into account in further studies using the MILO test.

\section{IPad testing}

The use of tablet technology such as iPads [Rentz, Dekhtyar, Sherman et al., 2016; Dalmaijer, Van der Stigchel, Nijboer et al, 2015; Georgsson \& Staggers, 2016; Zapata, Fernández-Alemán, Idri, \& Toval, 2015] is gaining popularity in both clinical and research arenas to measure cognitive function (e.g. the Cambridge Neuropsychological Test Automated Battery (CANTAB) similar to neuropsychological tests used in dementia screening batteries i.e. the Mini Mental State Exam (MMSE) [Barnett, Blackwell, Sahakian, \& Robbins, 2016; JuncosRabadan, Pereiro, Faucal et al, 2014; Junkkila, Oja, Laine, \& Karrasch, 2012] and a series of studies have indicated that both younger and older adults are comfortable in using this mode of technology i.e. the use of touch screens [Jenkins, Lindsay, Eslambolchilar et al, 2016; Collerton et al, 2007] and are motivated to perform well when using an iPad [Cox, Cains, Shah \& Carroll, 2012; Green \& Bavelier, 2006; Yee, 2006].

The use of iPads or other tablet based devices are being developed as a cheap and portable method of cognitive testing (as mentioned in the introduction) and are widely available thus can be used easily in both research and clinical settings. Test programming can be tailored to the specific areas of cognition and processing speed being measured. IPads can be used at home or in clinics without taking up as much space as computers. In addition, test performance can be immediately uploaded to peoples' clinical notes thus making a diagnosis more available to patients/participants and tests can be easily repeated if any further assessment is required. 
Using advanced technology can therefore be a useful tool for screening cognitive health of an older adult population in a more flexible setting [Onoda, Hamano, Nabika et al., 2013; Rentz et al., 2016; Cole, Mennemeier, Bost et al, 2011]. Arguably therefore, iPad tests such as the MILO may be useful for clinical use however it first needs to be determined whether the test is as effective as other similar clinical (or research) tests in relation to ageing and what factors the test may be influenced by.

IPads have been demonstrated to be useful devices to measure the integrity of measuring cognitive function but have not been used extensively to measure information processing speed and its intraindividual variability between young and older adults in relation to visual attention and examining other factors (i.e. sex, education, and particularly perceived test difficulty and subjective memory function). Therefore the present chapter examined the integrity of information processing speed between young and older adults and potential influence of sex, education, perceived test difficulty and subjective memory function using the MILO test.

The MILO is a test developed by Horowitz \& Thornton [ Horowitz \& Thornton, 2008; Thornton \& Horowitz, 2004] for research into similar aspects of brain function as measured by the visual search test, and Trails A and B i.e. information processing speed, attentional function (shifting attention), feature search and executive function. However the MILO is also more sophisticated to both visual search tests and TMT as it includes more direct responses (finger tap on the iPad) designed to closely reflect everyday interactions with objects around us [Thornton \& Horowitz, 2004] with each response producing a separate RT which cannot be achieved in the visual search and TMT. The MILO test in the current was modified to be a simpler test and allow us to examine RT and IIV in relation to visual search thus permitting comparisons to be made between the MILO and both the visual search and TMT tests from the current research.

In this current MILO test, a representation of eight billiard ball-type stimuli were presented on an iPad screen, each containing a number from 1 to 8 randomly distributed around the screen. As each ball was tapped it disappeared from the screen. Once all 8 balls were tapped, they appeared again for the next trial in a different position around the screen. Participants were instructed to tap each ball in consecutive order (from number 1 to number 8 ) as shown in Figure 23. 
Figure 23. Representation of MILO stimuli of eight numbered billiard balls.

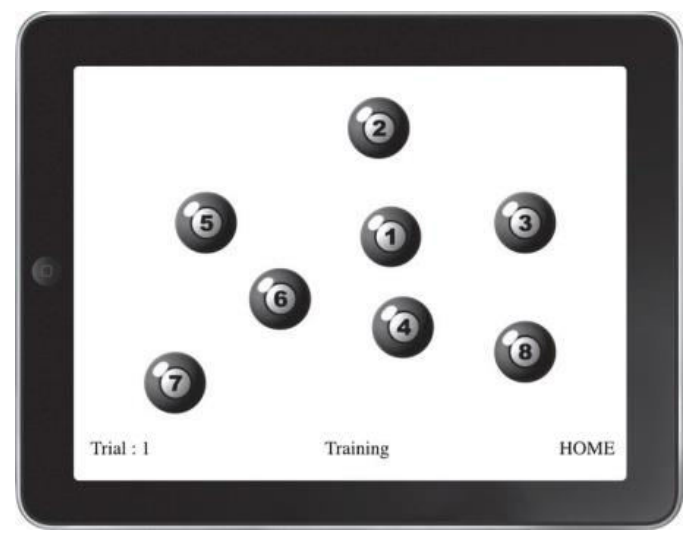

Each ball tap response produced a separate RT score and within these separate responses, the MILO measures the reaction time from the stimulus appearing and tapping the first response [RT1]. The first test trial began once the participant pressed a 'start' button on the screen thus RT1 score related to the time from all 8 balls appeared to the participant tapping the first ball. For the following 30 test trials, the RT score for RT1 related to the time taken to tap the $1^{\text {st }}$ ball from the ball sequence appearing again after finishing the previous trial.

This response is similar to a Simple RT test as reaction time is measured from the time the stimuli appear to the tapping of the first response. In addition is the need for visual search i.e. searching through all the balls on the screen repeatedly for the next number in the sequence, in effect searching continuously through distracting balls for the correct one in the sequence. Potential effects of the first response (RT1) were examined separately by eliminating RT1 from overall performance (i.e.RT8-RT1). This has been singled out by other RT studies (across a long time span) [e.g. Jentzsch, Leuthold \& Ulrich, 2007; Ratcliff, 1979], as the overall reaction time score may possibly be slower due to participants hesitating before responding even when this first response is located (reasons for this hesitancy differ between individuals).

This is another factor which may have an influence on performance outcome and may also contribute to outcome heterogeneity. Typically, practice trials are provided to participants before testing, in an attempt to reduce this hesitancy to a certain extent i.e. the participant verbally telling the researcher that they understood how to perform the test. 
However, hesitancy effects may still be apparent per se (which may still differ between individuals) despite the participants understanding the test procedure. Including this potentially slower RT score may have a significant effect on the overall RT performance i.e. the time taken to tap from the first ball to the last ball in the sequence. Therefore we also measured overall RT performance i.e. shifts of attention from item to item from beginning to end whilst excluding the first response in an attempt to eliminate (or at least in some part) some of these hesitancy effects (i.e. RT8 - RT1) assuming that tapping ball 1 to ball 2 and onwards until finishing with the $8^{\text {th }}$ ball tap is less affected by hesitancy effects). This overall measure is similar to the score produced in Trails A or Trails B of the TMT. Measuring possible hesitancy effects from the first response cannot be achieved by the TMT as only one RT score is produced from connecting the first number to the last number (Trails A) or letter (Trails B) in the sequence. Individuals performing the TMT may also hesitate before beginning the test thus their single trial performance may not be representative of their true performance.

Eliminating the first response in the MILO endeavours to determine whether hesitations may affect information processing speed thus have an impact on interpreting result outcome of the whole test i.e. results being much slower than they should be. This may also impact how the results from other similar reaction time tests have been interpreted (i.e. visual search or TMT). It may be the case that incorporating a measure of first response hesitancy as included the current MILO are useful when measuring information processing speed and IIV in ageing research or in clinics comparing healthy ageing controls to pathological gearing such as MCI or dementia.

As for the search test, the MILO contains multiple trials to measure the variability of information processing speed which may (as discussed in Chapter 3) increase the sensitivity of the test finding age related change and pathology compared to measuring a single trial of RT (as in the TMT) [Haworth et al, 2016; Mella et al, 2013; Dykier et al, 2012; Moy et al, 2011; Walhovd et al, 2007; Salthouse \& Fristoe, 1995]. An IIV score can be produced for each stimulus response firstly to measure the variability of beginning the test to determine whether the response to the first ball is more variable compared to responses of the following balls i.e. whether IIV improves by the time the $8^{\text {th }}$ ball is responded to. 
Participants during the MILO test are required to tap exactly on the stimulus for reaction time to register which may make the RT of each ball tapped more accurate compared to the TMT where participants may skim past or only settle on the individual stimulus briefly.

Drawing lines between numbers and letters in the TMT by hand can be a problem if hand control is not sufficient i.e. arthritic hands or poor hand- eye coordination (although tapping can become a problem if for example nails are too long). The inability to complete the pen and paper test has been observed by Schmid and colleagues [2013] and in the TMT chapter (see Chapter 3) where both older and young adults had to be removed from analysis due to being unable to complete the entire test.

In addition, recording test completion time for the TMT relies on a crude measure of reaction time through the use of a timer controlled by the clinician or researcher. Deciding when to press the timer to start it and when to stop the timer depends on the person administering the TMT, thus the overall RT may vary each time the test is administered even with the same participant. This is open to human error whereas using an iPad eliminates this by being more precise. Computer based devices are of course not without error; there may be a slight delay between human RT and the device recording the response. However, this difference is usually very small and can be controlled for during programming.

Arguably the MILO may be excellent for clinical use compared to typically used tests i.e. TMT (for reasons such as the use of multiple trials) however it is important to examine first what occurs during healthy ageing and what effects, if any, different factors have on performance outcome which may need to be considered. The current MILO test will compare any potential influence of such factors to the results of the same participants in previous tests (particularly the TMT which the MILO test is similar to) so as to better understand how information processing speed during ageing vary depending on different factors or the type of test used.

\section{Person-related factors}

As with previous tests in this thesis, the MILO test will also be examined with respect to the influence of various person-related factors on information processing speed and IIV, i.e. sex, education, subjective memory function and perceived test difficulty. 
Sex has been examined alongside age using an IPad as a presentation mode [Barnett et al, 2016; Rentz et al, 2016; Blackwell et al, 2010] and education has been examined in relation to errors with higher education related to making fewer errors Blackwell et al, 2010]. These two factors as well as subjective memory function and perceived test difficulty has not been examined in relation to information processing speed and its variability using the MILO test thus a novel aspect in the current study.

So far the results in previous test chapters (Chapters 2 to 4) have been mixed in relation to finding effects of person-related factors on RT or IIV (as summarized above) highlighting that study outcome may depend on age and the visual attention test (or sub-test) used. Measuring different person-related factors in the MILO, and compared to a variety of different attentional tests may help to further understand whether these factors influence information processing speed across attention function. In addition this may help characterise how information processing speed may be affected in ageing. Failing to take into account any influence of these personrelated factors may negatively impact how information processing speed results of MILO and ageing studies are interpreted. 


\subsection{METHODOLOGY}

\section{$\underline{\text { Participants }}$}

Swansea university students $\left(n=58,18-25\right.$ years, 13 males: 45 females ${ }^{1}$ ) were recruited through the Psychology department credit system and through advertising around the university and social network. Community dwelling older adults ( $n=80$, 50-80 years; 32 males: 48 females) were recruited through advertisements given out to older adult social clubs and local papers in Swansea, through email, word of mouth and via a volunteer database of older adults set up by the Swansea Psychology Department. The inclusion \& exclusion criteria were the same as previous chapters and the same participants tested in the TMT, Simple and Choice were tested.

\section{$\underline{\text { Stimuli \& procedure }}$}

The modified MILO test is composed of a representation of eight billiard ball-type stimuli each containing a number from 1 to 8 randomly distributed around the screen (see Figure 27). As each ball was tapped by the participant with the index finger of their dominant hand, it disappeared from the screen. Once all 8 balls were tapped, they appeared again on screen after a two second interval in a different distribution around the screen, ready for the next trail, producing 30 trails in total. Participants were shown the stimuli in full in a practice phase and instructed that the aim of the test was to tap each ball in consecutive order (from number 1 to number 8 ) using their dominant index finger as quickly but as accurately as possible. This involved searching around the screen for each number. The researcher completed one trial as a demonstration then the participant was given an opportunity to practice for 2-3 trials or until they fully understood the test. There were no young or older adult participants who required more than 3 practice trials to understand the test. When the practice phase was complete, the programme was set to testing and the first test trial began once the participant pressed a 'start' button on the screen. The following 29 trials began once the previous trial ended.

\footnotetext{
${ }^{1} 55$ of the young adults who completed the MILO also completed the TMT, Simple RT and Choice RT. 54 older adults completed all four tests.
} 


\section{Data cleaning}

For both age groups, any extra trials in which the balls were tapped in the wrong order and had to be repeated i.e. errors were eliminated. Each trial produced a reaction time for each ball tapped which allowed for a variety of measures to be analyzed.

Two RT measures (conditions) were included in analysis. 'RT1' referred to the response (RT) from the start of the trial notice to pressing the first ball tap i.e. the time taken from the stimulus appearing and tapping the first response. Overall RT performance was measured i.e. the time taken from tapping the first ball to the $8^{\text {th }}$ ball sequentially referred to as RT8 - RT1 because in an attempt to eliminate (or at least in some part) some hesitancy effects from the first response (RT1). Due to multiple trials, the inter-quartile range was also calculated for both RT conditions to measure intraindividual variability (IIV). IIV for RT1 measured the variability of beginning the test (tapping the first response) to determine whether the first response is also more varied as well as slower due to hesitancy effects. This measure was labelled as 'IIV1'. The variability of overall RT performance measured and excluding the potential effects from the first response was labelled as 'IIV8 - IIV1'.

In response to the non-normal distribution of the data in some conditions (see Table 23) SPSS non parametric analysis was conducted. Not all conditions were nonnormally distributed however we wanted consistency in our analysis. Factors i.e. sex and education, perceived test difficulty and subjective memory function were given a-priori hypotheses and were grouped into separate families for analysis. The RT and IIV effects were different data sets and thus did not require Bonferroni correction for multiple analyses.

Table 25. Normality of Distribution (Shapiro Wilkes test) ${ }^{2}$

\begin{tabular}{|l|lll|lll|}
\cline { 2 - 7 } \multicolumn{1}{c|}{} & \multicolumn{3}{c}{ Older } & \multicolumn{3}{c|}{ Young } \\
\cline { 2 - 8 } \multicolumn{1}{c|}{} & statistic & $d f$ & Sig. & statistic & $d f$ & Sig. \\
\hline RT1 & .719 & 80 & .000 & .743 & 58 & .000 \\
\hline RT8 - RT1 & .932 & 80 & .000 & .951 & 58 & .021 \\
\hline IIV1 & .922 & 80 & .000 & .766 & 58 & .000 \\
\hline IIV8 - IIV1 & .968 & 80 & .044 & .973 & 58 & .232 \\
\hline
\end{tabular}

\footnotetext{
${ }^{2}$ Note: data classified as a non-normal due to the positively skewed distribution of the data and in some cases bimodal distributions.
} 


\subsection{RESULTS}

\section{Demographics}

Mann-Whitney analysis revealed depression level was significantly greater for young adults compared to older adults $[\mathrm{U}=1468, p<.001$, effect size $(\mathrm{r})=.38]$ and anxiety level was significantly greater for young adults compared to older adults $[\mathrm{U}=1278.5, p<.001$, effect size $(\mathrm{r})=.32]$. There was no significant difference in mean MoCA score or mean years of education $[p>.05]$.

Table 26. Mean baseline demographics for the older adult and younger adult groups. Standard deviation in parenthesis.

\begin{tabular}{|c|c|c|c|c|c|c|}
\hline & $\begin{array}{c}\text { Age } \\
\text { (Years) }\end{array}$ & $\begin{array}{c}\text { Education } \\
\text { (Years) }\end{array}$ & MoCA & MFQ & PHQ-9 & GAD-7 \\
\hline All Young & 20 & 14 & 27 & - & 6 & 5 \\
Young & 20 & 15 & 27 & & 6 & $(4.0)$ \\
males (n=13) & $(2.0)$ & $(2.2)$ & $(2.8)$ & - & $(5.7)$ & $(4.7)$ \\
\hline Young & 20 & 14 & 27 & & 6 & 5 \\
female (n=45) & $(1.6)$ & $(3.4)$ & $(1.9)$ & - & $(3.7)$ & $(4.0)$ \\
\hline All Older & 66 & 15 & 27 & 293 & 3 & 2 \\
\hline Older & $(5.6)$ & $(4.8)$ & $(2.3)$ & $(50.6)$ & $(3.2)$ & $(2.5)$ \\
\hline males (n=32) & $(5.6)$ & $(5.6)$ & $(2.6)$ & $(43.8)$ & $(2.9)$ & $(2.8)$ \\
\hline Older & 66 & 14 & 28 & 300 & 3 & 2 \\
female (n=48) & $(5.5)$ & $(4.0)$ & $1.8)$ & $53.7)$ & $(3.3)$ & $(2.3)$ \\
\hline
\end{tabular}

MoCA, Montreal Cognitive Assessment; MFQ, Memory Functioning Questionnaire; PHQ-

9, Patient Health Questionnaire; GAD-7, Generalized Anxiety Disorder 7-item scale. 
Table 27. Group mean information processing speed, intraindividual variability (from individual participant median RT scores) and mean perceived test difficulty for the young and older adult groups for both conditions in the MILO (RT1 and RT8-RT1). Standard deviation in parenthesis.

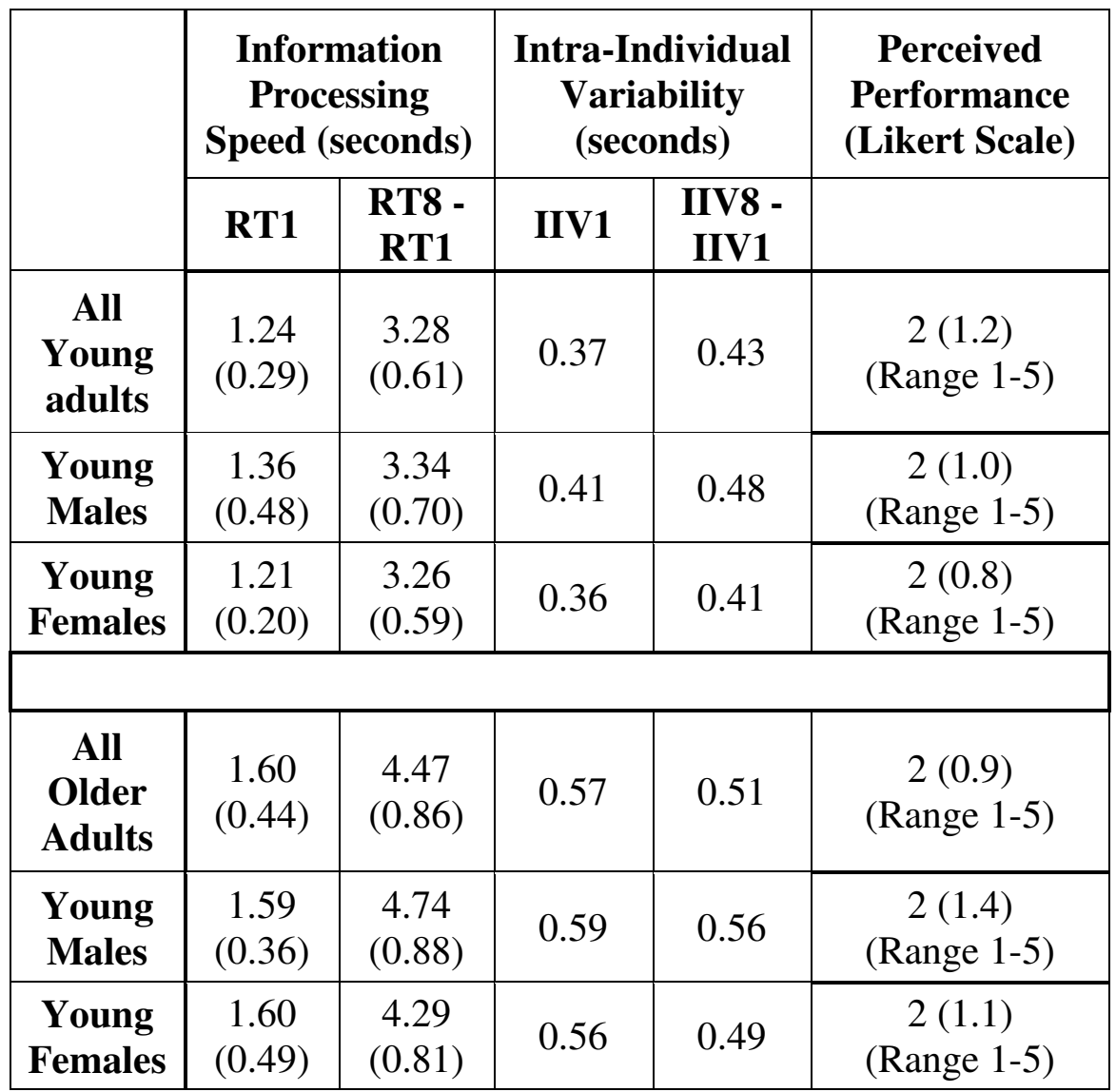




\section{Information Processing Speed}

\section{Comparison of age and sex: Information Processing Speed}

\section{RT 1}

The time taken to tap the 1st ball was measured to determine the RT of starting the test i.e. from when the stimuli appeared to tapping the first ball.

Figure. 24. Box plot of mean information processing speed of tapping the first ball (RT1) (secs) for young and older adults. Note the presence of outliers in the performance of this task.

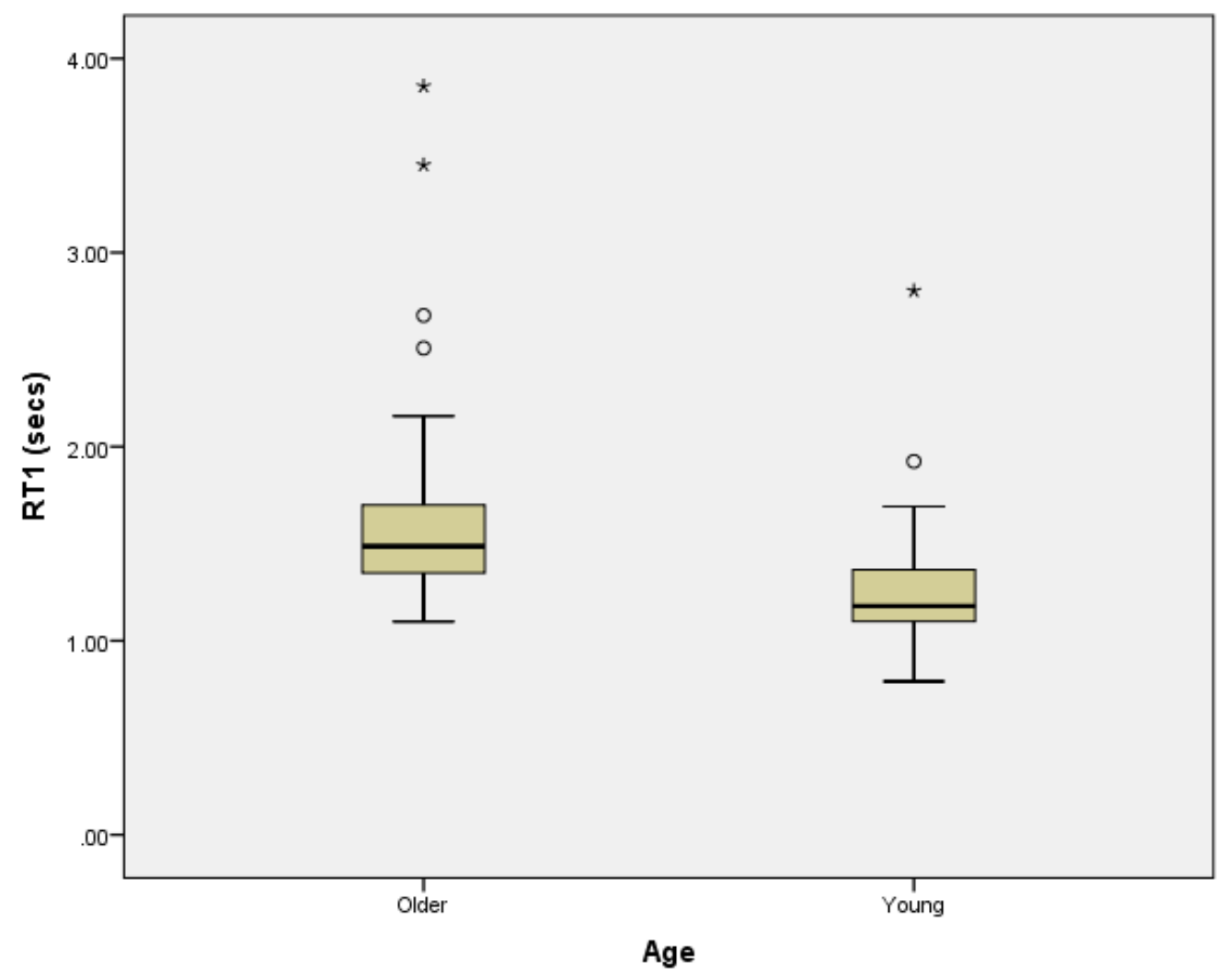

Mann-Whitney analysis revealed a significant age-related difference in information processing speed; older adults were significantly slower compared to young adults at starting the test i.e. from when the stimuli appeared to tapping the first ball $[\mathrm{U}=737$, $p<.001$, effect size $r=.58$ ] implying that older adults were slower at beginning the test compared to young adults, possibly due there being a hesitancy component. 
Figure. 25. Box plot of mean information processing speed of tapping the first ball (RT1) (secs) for males and females in young and older adults. Note the presence of outliers in the performance of this task.

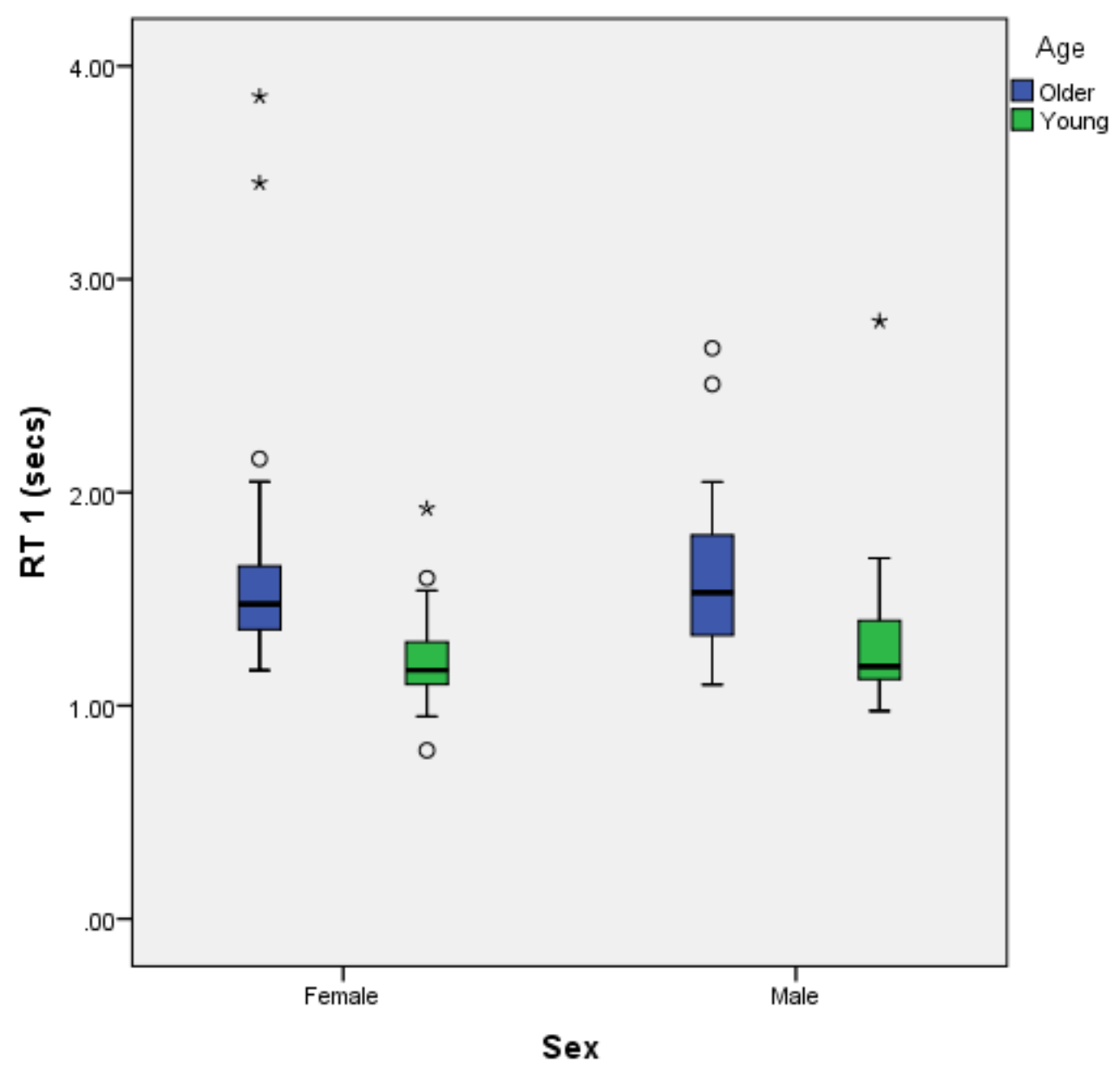

Mann-Whitney analysis revealed no significant difference of information processing speed between males and females in young adults $[p>.05]$ or in older adults $[p>$ $.05]$ at beginning the test. 


\section{RT 8 - RT1}

Overall RT performance was calculated i.e. the time taken from tapping the first ball to the $8^{\text {th }}$ ball sequentially and excluding the first response (RT1) to remove the possible hesitancy effects.

Figure 26. Box plot of mean information processing speed of overall RT performance (RT8 - RT1) (secs) for young and older adults

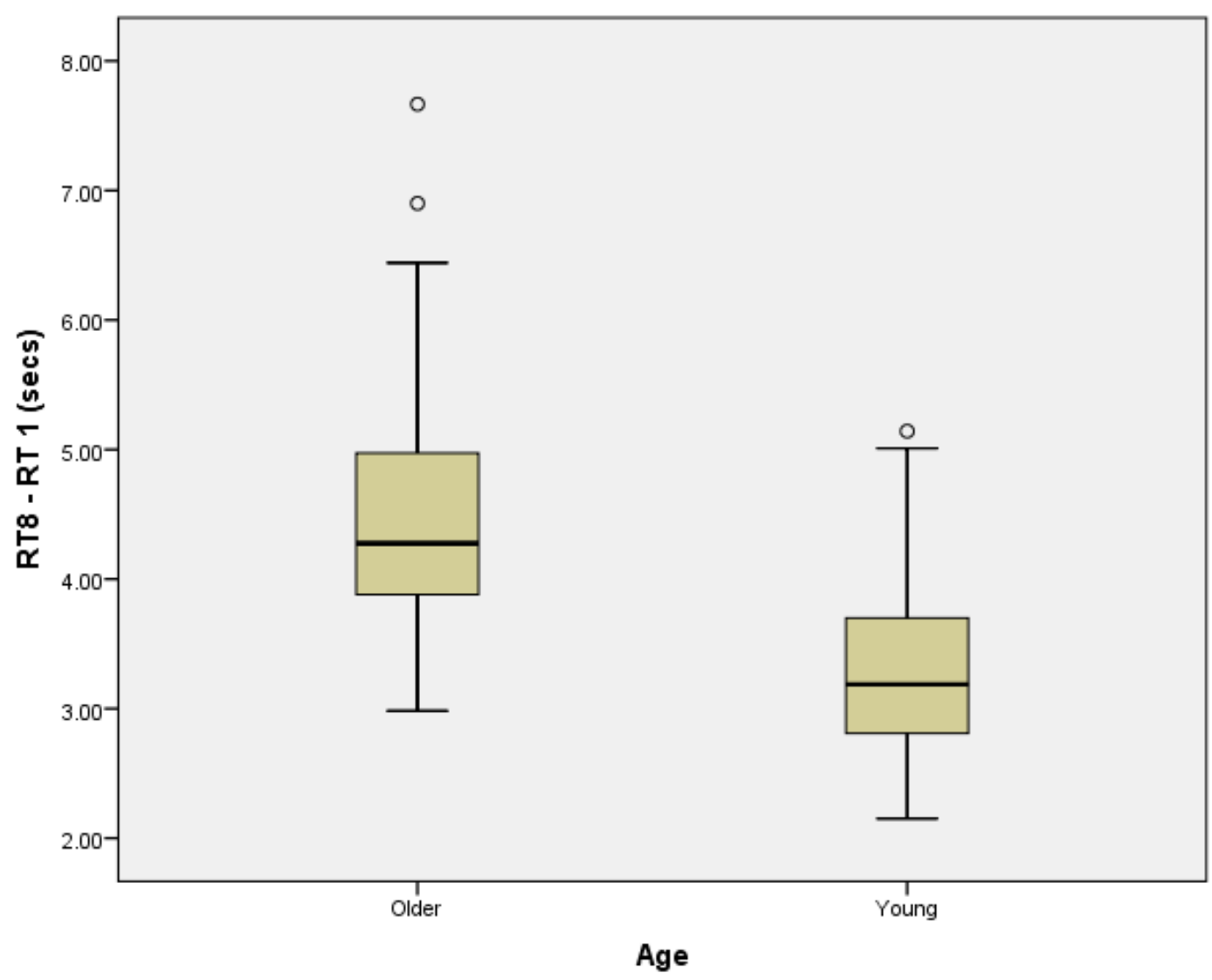

Mann-Whitney analysis revealed a significant difference of information processing speed between young and older adults; older adults were significantly slower compared to young adults in their overall $\mathrm{RT}$ performance $[\mathrm{U}=512.5, p<.001$, effect size $r=.66]$. 
Figure 27. Box plot of mean information processing speed of overall RT performance (RT8 - RT1) (secs) for males and females in young and older adults

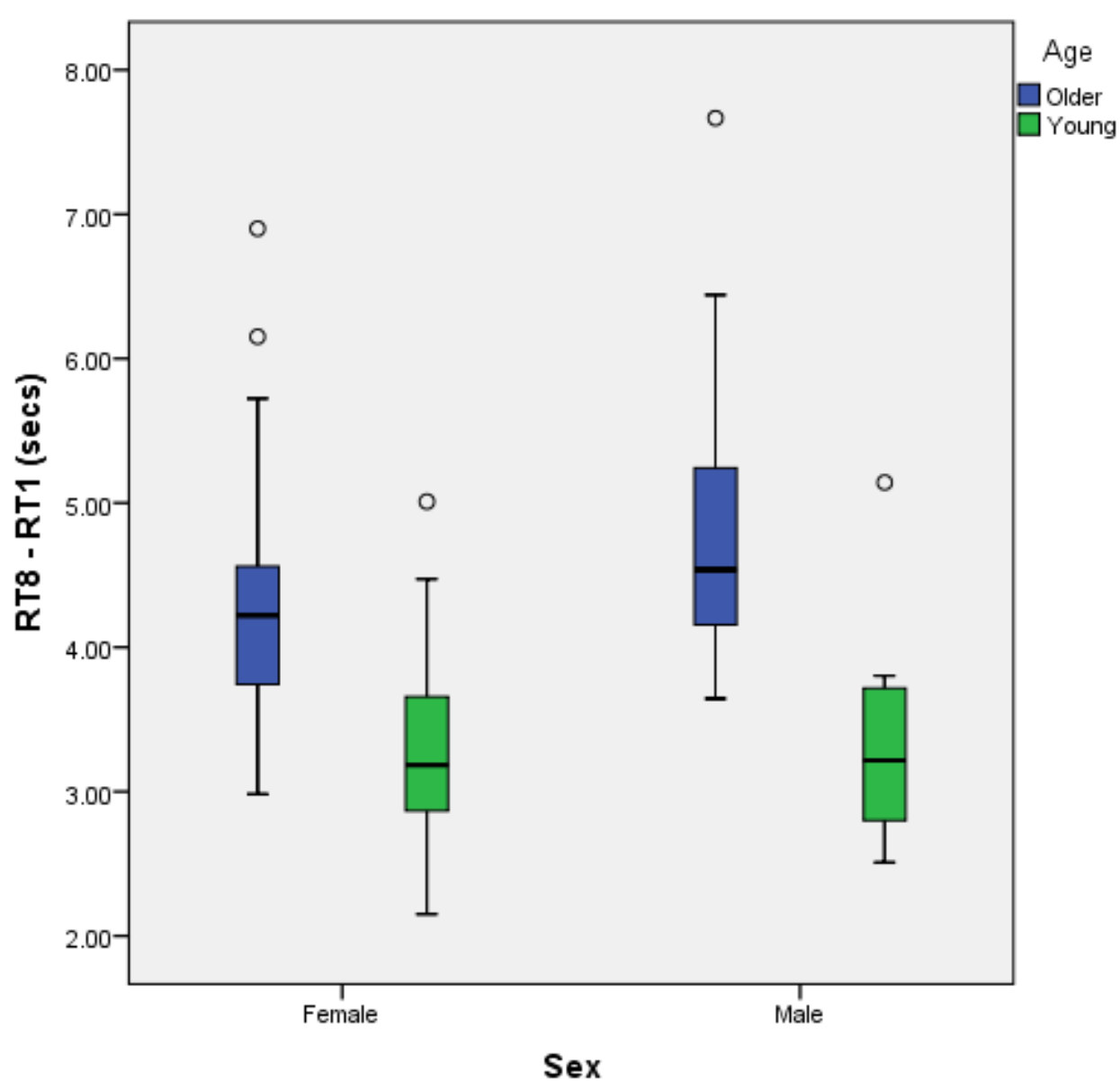

Mann-Whitney analysis revealed no significant difference of information processing speed between males and females in young adults $[p>.05]$ and in older adults $[p>$ $.05]$ in overall RT performance (excluding RT1).

\section{Information processing speed and Subjective memory function}

Subjective memory function (Total MFQ score) significantly negatively correlated with information processing speed for RT $1[\mathrm{r}=-.263, p=.019]$; less perceived changes to memory function (higher MFQ score) related to faster information processing speed. There was no significant correlation between subjective memory function and information processing speed for RT8 - RT1 $[p>.05]$ i.e. overall information processing speed performance excluding RT1. 


\section{Information processing speed and Perceived test difficulty}

For both young and older adults information processing speed did not significantly correlate with perceived test difficulty for RT1 [ $p>.05]$ or RT8 - RT1 [ $p>.05]$.

\section{Information processing speed and education level}

In young adults, there was no significant correlation between education and information processing speed for RT1 [ $p>.05]$ or RT8 - RT1 [ $p>.05]$.

In older adults, there was a significant negative correlation between education information processing speed for RT8 - RT1 i.e. time taken to complete the test; higher education related to faster information processing speed [ $\mathrm{r}=-.225, p=.044]$. There was no significant correlation between educational level and information processing speed for beginning the test (RT1) $[p>.05]$.

\section{Information processing speed, anxiety and depression levels}

In young adults, information processing speed did not significantly correlate with anxiety levels or depression levels for RT1 [all $p$ values > .05] or RT8 - RT1 [all $p$ values $>.05]$.

In older adults, information processing speed did not significantly correlate with anxiety levels or depression levels for RT1 [all $p$ values > .05] or RT8 - RT1 [all $p$ values > .05].

\section{Information processing speed and objective cognitive performance (MoCA score)}

In young and adults, information processing speed did not significantly correlate with MoCA score for RT1 [ $p>.05]$ or RT8 - RT1 $[p>.05]$.

In older adults information processing speed was significantly negatively correlated with MoCA score; faster RT performance related to better general cognition for overall MILO performance RT8 - RT1 [r = -.309, $p=.005]$ but not for beginning the test (RT1) [ $p>.05]$. 


\section{Subjective memory function and perceived test difficulty}

In older adults there was a significant negative correlation between subjective memory function and perceived test difficulty; perceiving greater changes in memory function (lower MFQ score) related to perceiving the test to be more difficult $[\mathrm{r}=-.260, p=.02]$.

\section{Education level and subjective memory function}

In older adults education significantly negatively correlated with subjective memory function (total MFQ score) with i.e., lower levels of education related to increase in MFQ score i.e. less perceived change of memory function [ $\mathrm{r}=-.243, p=.03]$.

\section{Education level and perceived test difficulty}

There was no significant correlation between education and perceived test difficulty for both young and older adults [p values $>.05]$.

\section{Anxiety and depression levels}

Anxiety levels significantly positively correlated with depression levels in young adults $[\mathrm{r}=.763, p<.001]$ and older adults $[\mathrm{r}=.584, p<.001]$. As anxiety levels increased so did depression levels. 


\section{Intra-individual Variability}

\section{Comparison of age and sex: Intra-individual Variability}

\section{IIV 1}

Figure. 28. Box plot of mean intraindividual variability of tapping the first ball (IIV 1) (secs) for young and older adults.

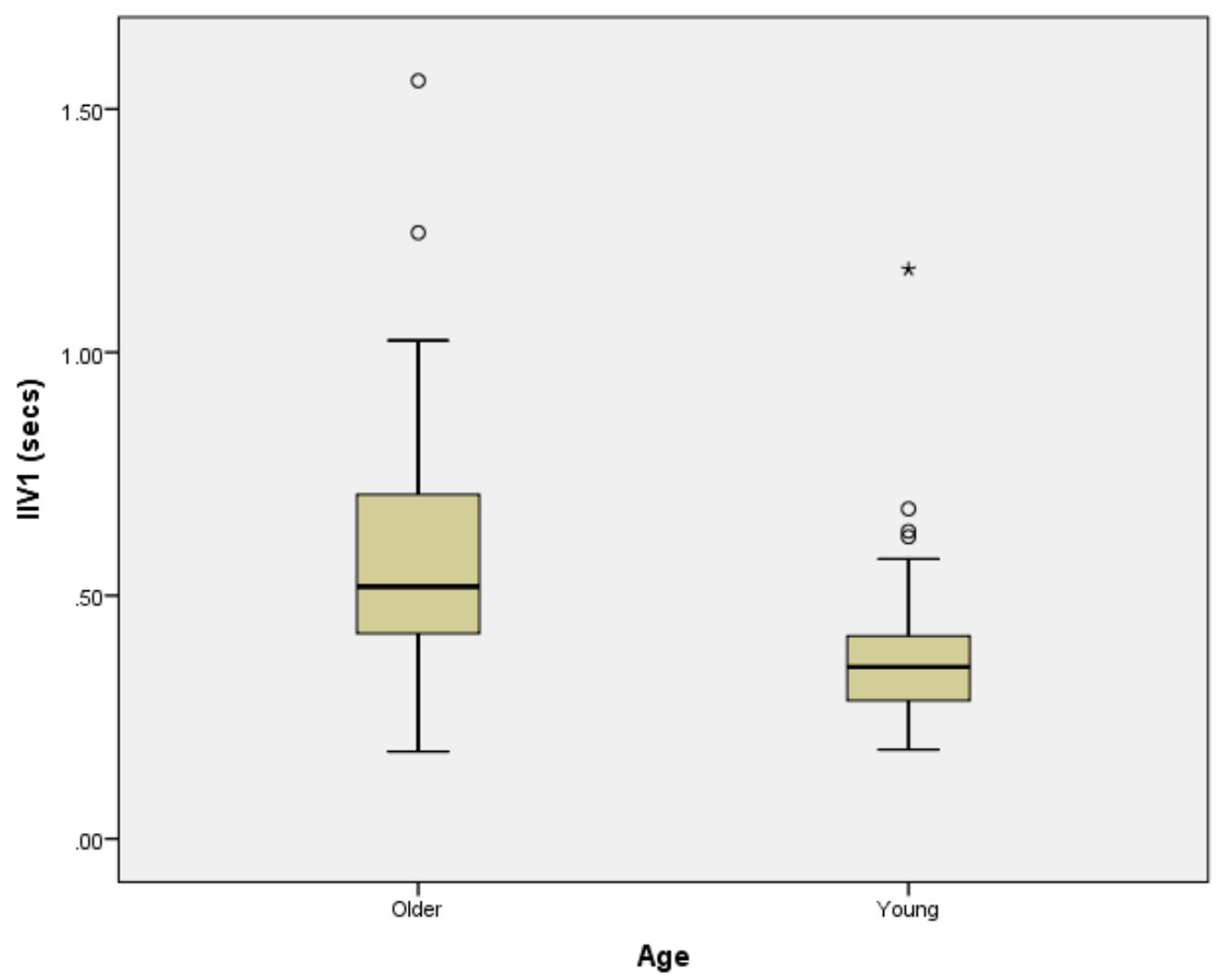

Mann-Whitney analysis revealed a significant difference of IIV between young and older adults; older adults were significantly more variable when beginning the test compared to young adults $[\mathrm{U}=958, p<.001$, effect size $\mathrm{r}=.50]$ possibly due to some contribution of hesitancy effects. 
Figure. 29. Box plot of mean intraindividual variability of the first ball press (IIV 1) (secs) for males and females in young and older adults.

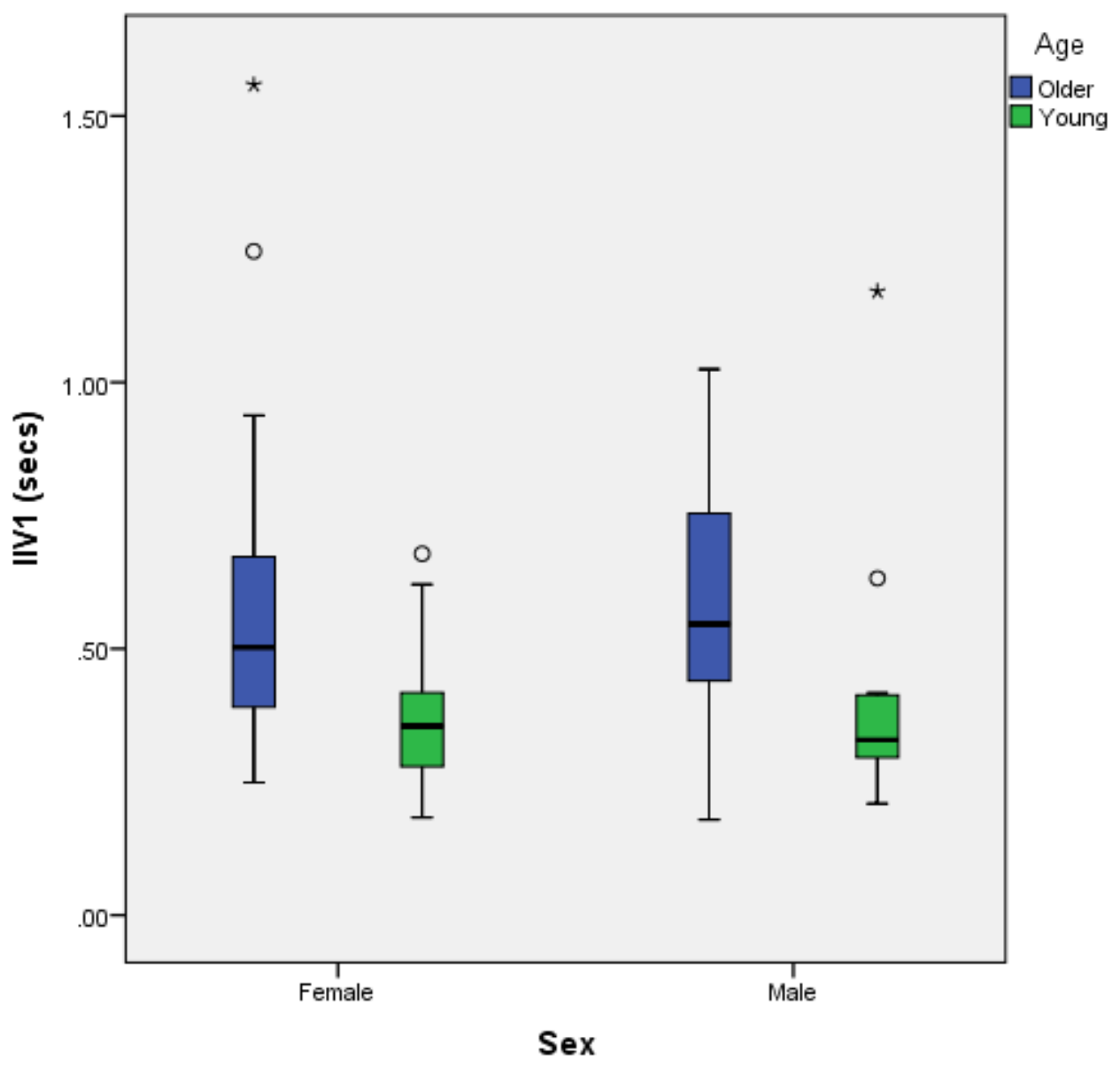

Mann-Whitney analysis revealed no significant difference of IIV between males and females in young adults $[p>.05]$ and in older adults $[p>.05]$. 


\section{IIV8 - IIV1}

Figure 30. Box plot of mean intraindividual variability of overall RT performance (IIV8 - IIV1) (secs) for young and older adults.

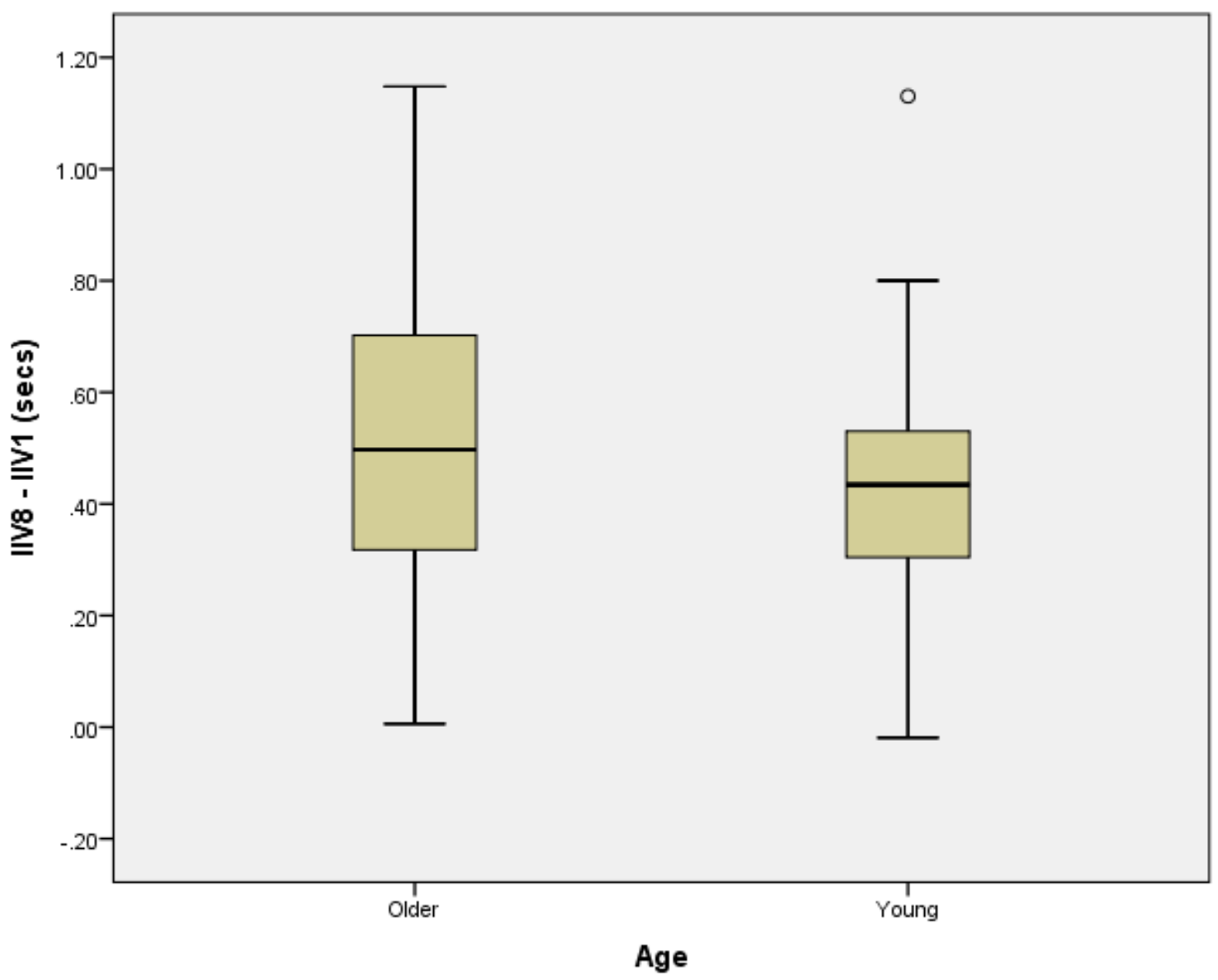

Mann-Whitney analysis revealed no significant difference of IIV between young and older adults $[p>.05]$. 
Figure 31. Box plot of mean intraindividual variability of overall RT performance (IIV8 - IIV1) (secs) for males and females in young and older adults.

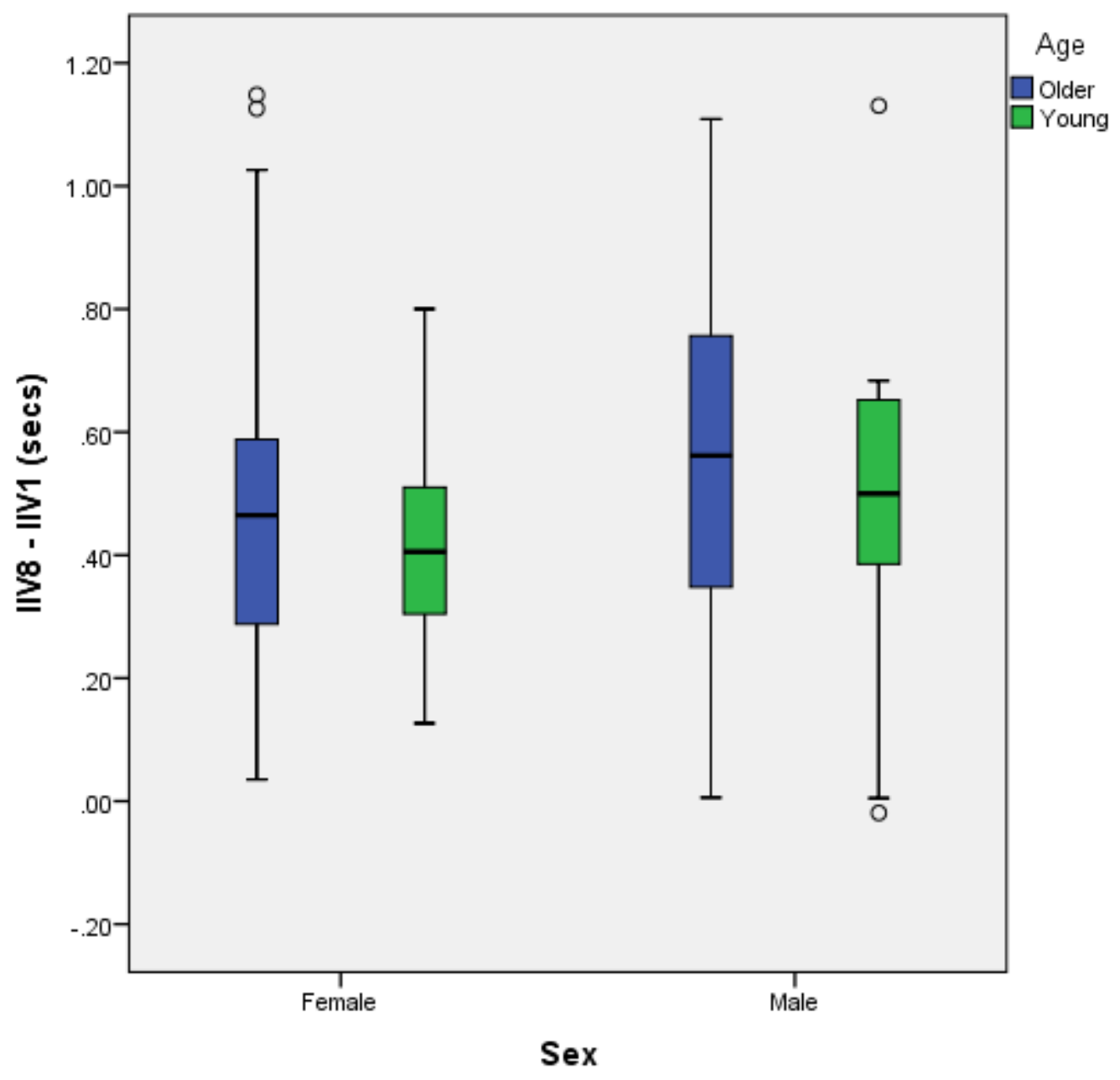

Mann-Whitney analysis revealed no significant difference of IIV between males and females in young adults $[p>.05]$ or in older adults $[p>.05]$.

\section{Intraindividual variability and Subjective memory function}

In older adults, subjective memory function (Total MFQ score) did not significantly correlate with intraindividual variability for IIV1 [ $p>.05]$ or IIV8 - IIV1 [ $p>.05]$.

\section{Intraindividual variability and Perceived test difficulty}

For young and older adults, intraindividual variability did not significantly correlate with perceived test difficulty for IIV1 [all $p$ values > .05] or IIV8 - IIV1 [all $p$ values $>.05]$. 


\section{Intraindividual variability and Education level}

For young and older adults, intraindividual variability did not significantly correlate with education for IIV1 [all $p$ values $>.05$ ] or IIV8 - IIV1 [all $p$ values > .05].

\section{Intraindividual variability, anxiety and depression levels}

In young adults, intraindividual variability did not significantly correlate with anxiety or depression levels for IIV1 [all $p$ values > .05] or IIV8 - IIV1 [all $p$ values $>.05]$.

In older adults, intraindividual variability significantly positively correlated with anxiety levels; higher levels of anxiety related to more variable performance for IIV1 $[\mathrm{r}=.258, p=.021]$ but not for IIV8 -IIV1 [ $p>.05]$. Intraindividual variability did not significantly correlate with depression levels for IIV1 $[p>.05]$ or IIV8 - IIV1 [ $p>$ $.05]$.

\section{Intraindividual variability and objective cognitive performance (MoCA)}

In young and adults, intraindividual variability did not significantly correlate with MoCA score for IIV1 $[p>.05]$ or IIV8 - IIV1 $[p>.05]$.

In older adults IIV significantly negatively correlated with MoCA score; less variable performance related to better general cognition for overall MILO performance IIV8 - IIV1 [ $\mathrm{r}=-.280, p=.012]$ but not for beginning the test i.e. IIV1 $[p>.05]$.

\section{Errors in the MILO}

\section{Mean number of errors throughout the test: Age comparison}

Table 28. Mean number of errors (tapping incorrect number sequence) in young and older adults. Standard deviation in parenthesis.

\begin{tabular}{|l|c|}
\hline & Mean no. of errors \\
\hline & 2.57 \\
Young & $(1.74)$ \\
\hline & 1.25 \\
Older & $(1.59)$ \\
\hline
\end{tabular}


Mann Whitney analysis revealed that young adults' mean number of errors was significantly greater compared to older adults $[\mathrm{U}=1150, p<.001$, effect size $(\mathrm{r})=$ .44] implying that young adults made significantly more errors compared to older adults. Young adults were also significantly faster compared to older adults implying a speed/accuracy trade off i.e. young adults appeared to trade accuracy for speed.

\section{Mean number of errors throughout the test: Age and sex comparison}

Table 29. Mean number of errors (tapping incorrect number sequence) between males and females in young and older adults. Standard deviation in parenthesis.

\begin{tabular}{|l|c|}
\hline & Mean no. of errors \\
\hline Young Males & $2.46(1.85)$ \\
\hline Young Females & $2.60(1.72)$ \\
\hline Older Males & $1.22(1.45)$ \\
\hline Older Females & $1.27(1.69)$ \\
\hline
\end{tabular}

\section{$\underline{\text { Young adults }}$}

In young adults, there was no significant difference in the mean number of errors between males and females implying no significant difference between sexes in how many errors (i.e. tapping incorrect number order ) were made $[\mathrm{U}=273, p=.709]$

\section{$\underline{\text { Older adults }}$}

In older adults, there was no significant difference in the mean number of errors between males and females implying no significant difference between sexes in how many errors (i.e. tapping incorrect number order ) were made [U $=745.5, p=.816]$. 


\subsection{DISCUSSION}

The current study aimed to determine the functional integrity of information processing speed and IIV with respect to visual attention-related function between young and older adults using the iPad-based modified MILO test; a different medium to the previous computer based and pen and paper tests examined so far in this thesis. The study also aimed to examine whether factors such as subjective memory function, perceived test difficulty (meta-cognition), sex and education have an influence on information processing speed and IIV in both young and older adults as well as the possible effect of hesitancy on the first trial.

\section{Information processing speed}

\section{Age comparison: information processing speed}

The time taken for the stimuli to appear and the first tap on the screen (RT1) was significantly slower in older adults compared to young adults implying that older adults were slower at the beginning the test. Both young and older adult participants were allowed to practice the test before-hand which should have reduced any hesitancy of starting each trial yet despite this, older adults were still slower per se.

This result is in accordance with those from all our other studies but what is not clear is whether ageing-related RT and IIV effects are exacerbated by a greater influence of hesitancy to the first trial in older than younger adults and whether this might influence the overall difference in RT between young and older adults. It is unclear whether these possible hesitancy effects are the result of general hesitancy which may occur for RT type tests or whether it related to the method of testing i.e. using an iPad. For example, it may be the case that older adults require more time to process all the information on the screen before starting thus resulting in a slower first response. In contrast, slower RT for the first response may have reflected a hesitation in tapping the screen despite given practice. In addition older adults may have different strategies when performing the test i.e. wanting to respond accurately thus examining the screen first to get an advantage. Indeed older adults made significantly less errors than young adults (as discussed in a later section). It would have been useful to obtain feedback from participants on using the iPad in an attempt to understand the reasons for any hesitancy thus a limitation of the current study. 
Slower RT1 in older adults is a significant finding as if there are such hesitations in older adults compared to young adults this may highlight the importance of taking hesitancy effects into account when measuring information processing speed using the MILO. This may have an impact on interpreting result outcome of the whole test. The RT from the stimulus appearing to tapping the first ball may not be representative of older adults' overall performance yet may be skewing the results to being much slower than they should be. Therefore it may be useful to measure hesitancy effects in the other RT tests within the current research to determine whether ageing-related RT and IIV effects are exacerbated by slower first response.

Overall RT performance (RT8 - RT1) i.e. time to complete the whole test was measured after eliminating the first response which may have introduced a longer RT thus not representing 'true' performance. Older adults were significantly slower in their overall performance compared to young adults implying ageing has a detrimental effect on information processing speed and feature search, shifting attention and executive function. In addition the result suggests that older adults are still slower compared to young adults despite removing any hesitancy effects from the first response. Therefore any possible hesitancy of tapping the first ball does not appear to reduce ageing effects on information processing speed thus does not explain overall slowing of test performance between young and older adults. The effect size for overall test performance (0.66) was not much different to the effect size for RT1 (0.58) although suggests that removing the first response may improve information processing speed slightly.

Slower RT in older adults' overall performance supports the findings of the previous tests examined i.e. visual search, TMT, Simple RT and Choice RT, (see Chapters 2 to 4 ) in that older adults are significantly slower (mean RT performance) compared to young adults. Therefore, as concluded in previous tests, the modified MILO test also appears to be sensitive differences of RT in to ageing although the other attentional tests did not include eliminating the first response from overall performance due to possible hesitancy effects. To our knowledge the MILO test has not been examined in relation to information processing speed and executive function thus the current study cannot, at present, be compared to previous ageing studies. 


\section{Information processing speed and Subjective memory function}

In older adults, there was only a significant negative correlation between subjective memory function and information processing speed for the first stimulus tap (RT1) Greater perceived changes to memory function related to slower information processing speed when beginning the test. Older adults who perceived their memory to be poorer may have been less confident in beginning the test compared to older adults perceiving no changes to their memory function i.e. being more hesitant.

In contrast, there was no significant relationship between information processing speed and subjective memory function for the overall RT performance (RT8 -RT1). How intact older adults perceived their memory function to be did not appear to relate to overall information processing speed i.e. from tapping the $2^{\text {nd }}$ ball to the $8^{\text {th }}$ ball. This may suggest that older adults who display concerns with memory function are only less confident in beginning the test enough to slow the RT of first response. We speculated that once the test began, older adults settled into the task thus any concerns with memory function did not affect slowing of overall performance but instead overall RT between young and older adults differed for other reasons i.e. ageing effects.

Finding no relationship between subjective memory function and overall RT performance is in accordance with the results from the visual search, TMT, Simple RT and Choice RT tests discovery no relationship between information processing speed and subjective memory function (see Chapters 2 to 4 ). As discussed previously this suggests that slowing of information processing speed is unlikely to relate to detrimental structural change but remains at levels expected in healthy older adults. This is speculated since there is a close relationship between information processing speed and structural change i.e. reduced white matter associated with slower information processing speed. In addition, the majority of participants performed to 'normal' levels in objective testing (MoCA score) although a few participants' MoCA scores were lower $(<26)$ which may imply cognitive impairment thus require further assessment.

Interestingly we found that RT1 is the only condition in which there was a significant relationship between information processing speed and subjective memory function across all the visual attention tests examined in the current research. 
This may relate to fact that the MILO test differed to other tests of similar attentional function i.e. TMT and visual search by including an RT measure for each stimulus response. Alternatively this MILO test results in people feeling less confident in beginning the test, possibly due to the method of testing i.e. using an $\mathrm{iPad})$.

An effect of subjective memory function on information processing speed may only be present in certain aspects of an RT/attentional test rather than in overall test performance. This may have a significant impact on how information processing speed should be measured in attentional tests i.e. which aspect of the test should be measured although it is possible that this result could have occurred by chance.

There were some extreme RT values in older adults for RT1 and in overall RT performance, different to the outliers eliminated before analysis due to extraneous variables. These disproportionally slower RT scores from the RT1 measure related to subjective memory in two older adults function i.e. they perceived greater changes to memory function. In contrast, there were two older adults who perceived little to no change to their memory function thus do not explain why a significant relationship was found between subjective memory function and RT1.

Disproportionally slower RT scores in overall RT performance may reflect underling significant impairment to cognition regardless of subjective feelings since not all disproportionate slowing related similarly to how older adults perceived their memory function to be. All these significantly slower scores may be of clinical significance thus it will be of interest to examine further.

\section{Information processing speed and Perceived test difficulty}

In both young and older adults, there was no significant correlation between information processing speed and perceived test difficulty. How difficult participants perceived the test did not relate to their actual RT performance for beginning the test or overall RT performance. This result supports the finding of overall RT performance in Simple RT, Trails A of the TMT and the Target plus distractor condition in the visual search test (Chapters 2 to 4 ). In contrast, the results contradict the Target alone condition in the visual search test and the Choice RT in older adults and Trails B in young adults. In addition the results contradict previous studies finding perceived test difficulty to have a negative influence on information processing speed [Setti et al, 2015; Bolmont, et al, 2000; Flavell, 1979]. 
A lack of a relationship between perceived test difficulty and information processing speed implies that actual RT performance is not relative to individuals' judgement of the MILO test. Differences in RT between young and older adults when beginning the test (RT1) may have occurred due to factors other than perceptions of test demands i.e. as discussed above having less confidence in beginning the test (hesitancy effects) or perceiving changes to memory function (for some older adults). Differences in overall RT (excluding RT1) may not relate to how judgement of the test is associated with actual performance but related to the effect age has on information processing speed.

Outcome variability between attention test results highlights that the influence of perceived test difficulty on information processing speed may depend on the test and the aspect of self-assessment being conducted. It may be possible that if young and older adults were examined against other factors of self-assessment, the relationship with information processing speed may differ.

\section{Information processing speed and Educational level}

In young adults, there was no significant relationship between educational level (in years) and information processing speed for RT1 (beginning the test) and for overall RT performance (RT8 - RT1). This supports the lack of influence of education in young adults in TMT, Simple and Choice RT and the Visual search test. As discussed in previous chapters, it is possible that a relationship was not discovered in young adults due to the narrow range of education years.

In older adults, there was no significant relationship between educational level and information processing speed of the first response implying that education did not influence how beginning of test was performed i.e. the level of slowing. In addition the implication is that education did not influence how concerned individuals may have been to begin the test (thus hesitancy effects). Less confidence in starting the test may have related to perceiving changes to memory function since subjective memory function correlated with RT for the first response i.e. perceiving greater changes to memory function related to slower RT of the first response.

There was a significant relationship between overall test performance (RT8 - RT1) and educational level with higher education related to faster information processing speed. 
This finding supports the Target plus distractors condition in the visual search test (see Chapter 2) which may imply that older adults with higher education are better at feature search and executive function as well as number sequencing and attentional switching. However, we did not ascertain similar findings between education level and information processing speed in the TMT despite the test measuring similar attentional function. This implying education effects on overall RT performance may be dependent on the type of attentional test used.

As discussed in the visual search (Chapter 2) we can highlight this as is an important finding as although education was matched between both groups (young and older adults) as closely as possible, there was slight variation between both age groups. Even within the narrow range, educational level had an effect on information processing speed in older adults although the range was not varied enough to find an education effect in young adults. Therefore, this further supports the speculation that the influence of education on information processing speed is dependent on age as well as the type of attentional test.

\section{Information processing speed and Sex}

There was no significant relationship between sex and information processing speed for both young and older adults. This implies that sex is not influential on information processing speed when beginning the MILO or performing the test overall. Therefore males and females of both age groups began and performed the test similarly to each other.

This result supports the findings from across the larger second study (Chapters 3 and 4). Only in the visual search test (Target plus distractors condition) were males significantly faster compared to females in young adults although the participant sample differed between the initial study (visual search) and the second study. The influence this factor has on how information processing speed is characterised in ageing may depend on the type of visual attention test and the age of the participant (i.e. young adults) although sample sizes between tests make results not completely comparable.

As far as we are aware, sex has not been examined in the MILO test in relation to information processing speed between young and older adults. Therefore, further research is required in order to determine whether this result can be replicated. 
The young adult sample in the current MILO test was small in comparison to the sample of young adults used in the other tests in this research (i.e. TMT) thus examining a similar participant sample in the MILO may be more useful to make comparisons of the influence of sex on information processing speed.

\section{Information processing speed, anxiety and depression levels}

In young adults, anxiety or depression levels did not relate to RT performance for beginning the test or overall RT performance. This implies that levels were not significant enough to have a negative effect on performance which, indeed anxiety and depression in young adult demographics were low to normal levels.

In older adults, neither anxiety nor depression levels related to overall RT performance or for beginning the test (RT1). For RT1 this suggests that hesitancy effects were not associated with slower RT in the first response due to, in particularly how anxious older adults may have been. Alternatively, hesitancy may be associated with slow RT due to a lack confidence to begin the test as a result of perceiving memory function to be poor.

No correlation between anxiety and depression on older adults' overall performance suggests that significant slowing between young and older adults was not associated to older adults feeling anxious or depressed when performing the test. As with young adults anxiety and depression were low to normal levels thus may explain why RT performance was not affected. Differences of overall RT between young and older adults may instead be associated with the effects of age on information processing speed.

\section{Information processing speed and objective cognitive performance (MoCA)}

In young adults, there was no significant correlation between objective cognitive performance and information processing speed. The integrity of overall cognition did not appear to relate to the integrity of information processing speed in the MILO test i.e. how quickly young adults began the test (RT1) or performed the whole test (RT8 $-\mathrm{RT} 1)$.

In older adults, better performance in the MoCA test i.e. better general cognition related to faster information processing speed for overall RT performance (RT8 RT1). 
This implies that the outcome of older adults performing the whole test being slower compared to young adults may not be associated with any cognitive impairment (whether due to ageing or pathology). Instead slower RT may be associated with other factors such as ageing effects or lower levels of education.

However, the MoCA was only one test of general cognition measured in the current study and there was no inclusion of more detailed measures of cognitive function i.e. memory. In addition the current study did not have access to older adult brain scans to determine the integrity of physical brain structure to determine whether there was indeed no underlying cognitive impairment in the older adults sample as suggested by 'normal' MoCA scores or whether there was any underlying impairment in individuals with low MoCA scores (score of 25 and below).

Finding no relationship between information processing speed and MoCA score for RT1 implies that the integrity of general cognition did not have an effect on how quickly the older adults began the test i.e. whether older adults were hesitant at tapping the first response. As mentioned above, slow RT for the first response and possible hesitancy may relate to other factors i.e. subjective memory function.

\section{Subjective memory function and Perceived test difficulty}

In older adults, the result revealed a significant negative correlation between subjective memory function and perceived test difficulty; greater perceived changes to memory function related to judging the test to be more difficult to perform. This finding supports the relationship revealed in the TMT, Simple and Choice RT chapters (Chapters 3 and 4) although the effect size in the MILO was small compared to Trails B of the TMT and Choice RT. As discussed in previous chapters this negative correlation supports our speculation that if older adults believe their cognition to be poor, they would assume that tests will be more difficult to perform by assuming poor cognition impairs the ability to perform a cognitive task adequately.

In contrast, the result contradicts the visual search test (Chapter 2) finding no relationship between subjective memory function and perceived test difficulty thus this outcome variability may depend on the type of attentional test. Although it must be highlighted that the participant sample of the visual search differed to the other tests as well as the MILO. 
It must also be noted that the relationship between subjective memory function and perceived test difficulty produced a small effect size thus the results may not be particularly reliable and thus require replication.

\section{Educational level and perceived test difficulty}

In both young and older adults there was no relationship between educational level and perceived test difficulty. The number of years of education young and older adults had did not have a significant influence on how difficult they perceived the MILO test was to perform.

For young adults, the current results support the findings from each of the previous tests examined in this thesis. For older adults, Trails B of the TMT and the Simple RT test also found no relationship between these factors as with the MILO (see Chapters 3 and 4). However, in the Visual search test and Trails A, older adults with higher levels of education perceived the tests to be more difficult. It must be noted that the sample sizes differed between the MILO and the other tests particularly in the number of young adults thus these results are not completely comparable. The difference in results implies that a relationship between educational level and perceived test difficulty is dependent upon age. In addition, the type of test thus the areas of attention function examined also relates to the variability of outcome between the visual attention tests examined in this research.

As mentioned in previous chapters, we speculate that those with greater years of education would judge the demands of the test to be lower because of higher levels of education being beneficial for test performance i.e. higher levels of motor skill or concentration help tests appear less difficult. However since a relationship was not found, the perceived difficulty of the MILO test was too low to have a significant influence (as observed with the relationship between perceived test difficulty and information processing speed). Indeed, young and older adults rated the MILO test to be easier to complete compared to other tests i.e. the TMT and visual search. Therefore the levels of education young or older adults have is not an influential factor in the MILO test. 


\section{Subjective memory function and educational level}

In older adults, there was a negative correlation between subjective memory function and educational level. Lower levels of education related to less perceptions of detrimental change in memory function. Therefore, older adults with higher levels of education are likely to perceive their memory is getting worse.

This result supports the Choice RT test and the TMT, but however contradicts the Simple RT and the Visual search test findings. This implies that interactions between different factors are dependent on the visual attention test thus dependent on the area of visual attention being measured. However, it must be also be noted here that the sample sizes differed between the MILO and the other tests particularly in the number of young adults thus these results are not completely comparable.

As discussed with the TMT (Chapter 3) and Choice RT (Chapter 4), the result may suggest that older adults with higher education are more judgemental about their memory function. In contrast, older adults with higher education are possibly more aware or have greater understanding of the quality of their memory function although this can only be speculated as memory function was not examined in great detail during the study.

\section{Intraindividual variability}

\section{Age comparison: intraindividual variability}

Older adults were significantly more variable at beginning the test compared to young adults i.e. the time taken for the stimuli to appear and the first tap on the screen (IIV1). This implies that older adults spend more time compared to young adults looking around the screen before beginning the test. This may be a sign of poorer integrity of processing the stimuli in older adults or, as discussed with information processing speed, it is a possible relation to hesitancy effects due to being less confident with beginning the test and using the iPad. The results of IIV1 support finding more variable IIV between young and older adults in the Simple RT, Choice RT and the visual search test (Chapters 2 to 4) although IIV1 in the MILO measured beginning the test whereas result for the other attention tests were for overall RT performance. 
In comparison to the result for information processing speed, there was no significant difference of IIV between young and older adults for overall RT performance (excluding RT1) i.e. tapping balls 2 to 8 in sequence. Overall, older adults were no more varied in their RT performance completing the test compared to young adults.

This is an interesting finding as it suggests that it is the response to the first ball and not overall RT performance that explains any variability between young and older adults in the MILO. Therefore in the MILO, the speed of information processing rather than its variability is more sensitive to the effects of ageing thus a more importance measure to distinguish between young and older adults overall performance. This result does not support the results for overall RT performance from the other tests examined (visual search, Simple RT and Choice RT) nor could this pattern be observed in the TMT since the TMT contained a single trial thus IIV could not be measured. Outcome variability for differences in IIV between young and older adults implies that finding age effects of IIV is dependent on the attention test used. In addition any age effects of IIV may have been reduced by making errors during the test. Trials were repeated if any were performed incorrectly which may have helped improve performance or learning i.e. practice affects thus less variability in overall RT performance. However this does not explain why information processing speed was not influenced by potential practice i.e. older adults being significantly slower compared to young adults.

\section{Intraindividual variability and Subjective memory function}

In older adults, there was no significant relationship between subjective memory function and IIV for both conditions. How older adults perceived their memory function was not reflected in the actual variability of their overall RT performance (excluding RT1) or beginning the test.

As far as we are aware, subjective memory function has not been examined with IIV of young and older adults using the MILO test thus we cannot make any comparisons with the current study. However in relation to the visual attention tests from previous chapters (Chapters 2 to 4 ), the MILO supports findings that there is no significant relationship between subjective memory function and IIV. 
As with information processing speed, a lack of relationship proposes that during ageing associated with perceived changes, there is no underlying structural change and RT function in relation to visual attention does not differ from what is expected during healthy ageing during the MILO task. However as in RT scores, there were extreme IIV scores i.e. outliers depicting significantly poorer integrity of information processing speed which may be highlighting underlying structural change and significant cognitive impairment thus require further assessment.

\section{Intraindividual variability and Perceived test difficulty}

There was no significant relationship between perceived test difficulty and IIV in both young and older adults. How young and older adults may judge the difficulty of the MILO does not appear to reflect the actual integrity of information processing speed. This supports the findings from the visual search (Chapter 2) and the Simple RT test (Chapter 4) but contradicts the Choice RT test (Chapter 4) which found young adults to be more varied when perceiving the test to be more difficult.

This variability between the MILO and the Choice RT test may highlight outcome depends on the test (and attentional function) since the same participants were used within both tests. This is particularly the case for older adults whereas the young adult sample between tests (in size) thus may be more difficult to compare directly. As far as we are aware, perceived test difficulty has not been examined previously with IIV in young and older adults using the MILO test thus we cannot make any comparisons with the current study at present.

\section{Intraindividual variability and Educational level}

There was no significant relationship between educational level and IIV in both young and older adults. This result supports the TMT (Chapter 3) and Simple and Choice RT tests (Chapter 4) finding no influence of education on IIV in young adults (despite different sample populations).

In contrast, the results contradict the findings of older adults during visual search which found a greater level of formal education (in years) was related to less variation of reaction time. Outcome variability in the older adult population suggests that the effect of education depends on the type of test used (i.e. visual search and not TMT, Simple RT or choice RT) and the age of the participants (i.e. older adults and not young adults). 
We can only make speculations about the current results at present as, to our knowledge, the MILO test has not been used in ageing studies to examine intraindividual variability between young and older adults.

\section{Intraindividual variability and Sex}

There was no significant relationship between sex and IIV in both young and older adults. The current result suggests that in young and older adults, males are as consistent as females in their RT performance during the MILO test.

As with information processing speed, IIV has not been examined between young and older adults using the MILO test thus the current results cannot be compared to previous research. However the result supports the findings of previous chapters (see Chapter 3 and 4) males are as consistent as females in their RT performance for both males and females. In addition, the MILO also contradicts the visual search test (as did the other attention tests) which observed young males to be less varried in their RT performance compared to females. This further supports our assumption that the influence of sex on IIV depends on a particular visual attention test (i.e. visual search).

\section{Intraindividual variability, anxiety and depression levels}

In young adults, anxiety or depression levels did not relate to how varied the participants were at beginning the test (IIV1) or the variability for overall test performance. As mentioned above, in young adult demographics, anxiety levels were between low and normal levels thus may explain why no relationship was found.

In older adults, anxiety levels related to RT variability of the first ball tap (despite overall levels being low) with higher levels of anxiety relating to greater variability of RT performance when beginning the test. Levels of anxiety may therefore be associated with hesitancy effects i.e. being anxious about beginning the test thus exacerbating the speed of information processing. Anxiety did not correlate with RT implying that anxiety has more of an influence on how variable older adults are at beginning the test compared to how fast they are.

Finding no effect of anxiety or depression on IIV for completing the test (IIV8 IIV1) is in addition to finding no relationship between IIV and age, subjective memory function, education or perceived test difficulty. 
Therefore this may indicate that for overall performance, information processing speed is more sensitive to effects of different person related factors i.e. educational level, as well as being more sensitive to the effects of ageing.

\section{Intraindividual variability and objective cognitive performance (MoCA)}

In young adults, there was no significant correlation between objective cognitive performance and IIV. The integrity of overall cognition did not appear to relate to the variability of information processing speed in the MILO test.

In older adults, better performance in the MoCA test i.e. better general cognition related to less varied information processing speed in completing the whole test. (IIV8 - IIV1). This implies that the integrity of the CNS (related to IIV) remains intact which is also highlighted by finding no significance of IIV between young and older adults. However as mentioned above, we could not include brain scans to confirm the integrity of brain structure and function.

Finding no relationship between IIV and MoCA score for RT1 implies that the integrity of general cognition did not have an effect on how variable older adults were at beginning the test i.e. whether older adults were hesitant at tapping the first response. As mentioned above, factors such as subjective memory function may be associated with hesitancy effects and slow information processing speed and not its variability.

\section{Errors}

Errors related to tapping the incorrect numbered ball during the sequence and resulted in the trial being repeated. Young adults made significantly more errors on group level compared to older adults possibly due to a lack of concentration other than being unable to complete the correct number sequence. Making errors resulted in the trial being repeated thus young adults performed more trials thus providing more practice. Practice effects may have improved performance or learning per se which may have reduced age effects of IIV since IIV was not significantly different between young and older adults for overall performance. However this does not explain why age effects were not reduced for information processing speed since a significant difference in RT was observed between young and older adults. 
Since young adults were faster at performing the test compared to older adults this implies that there was a speed/accuracy trade-off with young adults focusing on speed rather than accuracy i.e. to complete these extra trials and finish the test.

In relation to participant gender, in both young and older adults, there was no significant difference between males and females in the mean number of errors made thus implying no effect of sex on the level of accuracy of performance in both young and older adults.

\section{Outliers}

The results of information processing speed and IIV produced some significantly greater (i.e. slower and more variable) scores in both young and older adults. These outliers differed from those eliminated from analysis due to extraneous variables. In addition, outliers occurred across each condition measured in the MILO test, namely the time to begin the test (RT1) and overall test performance excluding the possible hesitancy effects of the first response (RT8 - RT1).

In older adults, these outliers may be depicting disproportionate slowing and variability due to underlying structural change in attention function the MILO test measures (i.e. executive function and information processing speed). If this is the case, these individuals cannot be used within healthy control groups as they may be skewing the mean result of what is considered to be a 'normal' level of slowing in healthy older adults during the MILO test.

Some outliers from the first response (RT1) and (RT8 - RT1) were produced by the same older adults across the MILO test conditions. This highlights their potential clinical significance i.e. underlying cognitive dysfunction thus further examination and follow up will be of interest. Alternately, significantly slower and more variable RT scores may depict poor performance due to the method of testing i.e. the use of an iPad. The older adults in the current study may have been unfamiliar with using tablet technology which may have exacerbated their performance and increased hesitancy effects i.e. being uncertain about beginning the test. A limitation of the current study was not to ask older adult participants whether iPads were unfamiliar as well as general feedback about completing the MILO test to determine whether this had an effect on RT performance. 
We speculate that outliers in young adults may be associated with young adults being distractible or due to methodological issues i.e. finger tap not recorded quickly enough associated with outliers; these outliers being too low to be eliminated automatically. However it cannot be ignored that these adults may be poor at the task or despite being young, may be displaying early signs of further cognitive decline i.e. in the attentional test measured (executive function).

\section{MILO Study Limitations}

The main limitation was the unequal sample sizes between young and older adults with the sample of younger adults being significantly smaller compared to older adults. However despite the smaller young adult group size, there was a significant difference of information processing speed between young and older adults implying the sample size was robust enough within the test and information processing speed is sensitive to aging in the MILO test although what the results did observe was that some aspects of the MILO may have been more sensitive than others particularly the to the effects of IIV.

The aim during the MILO was to use the same young and older adults from the previous tests (TMT, Simple RT and Choice RT) to compare outcome variability of information processing speed and the effect of person-related factors between different attentional tests. However, not all participants completed each test particularly the young adults as only 58 young adults from the previous sample of 80 were measured during the MILO test due to running out of time to test participants. Therefore it may be difficult to make direct comparisons between the MILO and the other attentional tests particularly in young adults although arguably, there was a large overlap in the people who completed each test. In addition young adults had performed more trials due to making more errors since incorrect trials resulted in the trial being repeated. This may have improved performance or learning i.e. practice, enough to improve RT performance which may have influenced the resulting comparison between young and older adults.

It is important that people can keep up with new technology and an issue to highlight would have been older adult participants not performing as well as young adults due to them not being as familiar with using an iPad. Indeed we speculated that being unfamiliar with the iPad may have related to the potential hesitancy effects when beginning the test. 
However all participants responded well to the technology as observed before by Collerton and colleagues [2007] who observed computerized tests to be more acceptable than pen and paper tests and by Jenkins et al [2016] who explored young and old attitudes to iPad technology and received a positive response.

Many of the older adult participants were familiar with using iPads, having one themselves which highlights that an increasing amounts of older adults are utilizing electronic devices due to technology is moving forward at a fast pace [Rentz et al, 2016]. However a limitation of the current study was not to ask both young and older adults what they thought about using the iPad.

Finding no significant relationship between reaction time and subjective feelings for overall performance may have related to not including enough older adults perceiving changes to their memory function to compare RT and IIV with individuals perceiving no changes to their memory function. Splitting older adults (those with memory complaints compared to those with no memory complaints) and with larger samples may have found a different relationship. However a relationship was found in the first two conditions suggesting the effects of subjective memory function are dependent on the test or condition used.

Alternatively, finding no relationship of subjective memory function in each condition i.e. overall RT performance may have related to the age of the older adult sample. The older adults on average in this MILO study are quite young with the average age of 66 years with respect to the maximum age of older adults which could have been included ( 80 years). It may be expected that older adults at the younger end of the age range would not report as many changes to their memory function compared to the older individuals thus information processing speed being to 'normal' levels expected in healthy ageing. It may have been of use to separate the older adult age range into groups (i.e. 50s, 60s, 70s and 80s) and include a larger sample in each groups to determine whether the older range (i.e. 70s and 80s) perceive more changes to memory function and whether this has an effect on information processing speed and IIV. 


\section{Conclusion}

The iPad test (MILO) used in this study contributed to a battery of attention tests designed to examine the integrity of information processing speed and IIV in young and older adults and examine the influence, if any, person-related (RT) factors (sex, education, subjective memory function and the psychological factor of perceived test difficulty) had on RT and IIV . This was in the attempt to determine whether the characterisation of information processing speed in ageing is dependent on the type of visual attention test used.

Information processing speed was significantly slower in older adults compared to young adults across conditions although older adults were only more varied when beginning the test (RT1). Subjective memory function did not influence IIV however, greater perceived changes to memory function related to slower information processing speed when beginning the test (RT1). Finding this result in the MILO test may imply that these aspects the test are sensitive to subjective memory function thus need to be taken into account when measuring information processing speed in MILO studies.

Perceived test difficulty had no influence on information processing speed and its variability thus individual judgement of the MILO in young and older adults bore no relationship to the actual test performance. Contradictory findings to that of the Choice RT, Trails B and the Target alone condition of the visual search test highlights that the influence of perceived test difficulty may be dependent upon the attentional test i.e. attentional function measured. Education only influenced overall RT performance (RT8 - RT1) and not IIV in the MILO, whereas there was no relationship of sex and information processing speed or IIV.

The MILO was examined particularly to compare with the TMT (as mentioned in Chapter 3) in its measure of information processing speed in relation to executive function but containing multiple trials thus determine whether multiple trials increase test sensitivity. Multiple trials appear to be more sensitive to the difference on information processing speed as observed by a greater effect size of age effects in the MILO compared to that of the TMT. This has already been argued by Haworth and colleagues [2016] that using a research test of multiple trials (i.e. visual search) may be more significant for finding ageing effects of information processing speed compared to the clinically used TMT. 
In addition, we argue that tapping the iPad screen may be more accurate compared to drawing a line through the numbers and letters in the TMT and can be completed successfully. This relates to finding both young and older adult participants failing to complete the TMT [see Chapter 3] but not failing to complete the current MILO test.

Outcome variability of results within the MILO and between each of the tests examined in this thesis appears to be dependent on the type of test (and attentional function) and also dependent on age as person-related factors influenced information processing speed differently in young adults (sex) and in older adults (education and perceived test difficulty). This may impact which test to use in research for the characterisation of information processing speed and IIV in ageing. Therefore in the following chapter, effect sizes will be examined between tests to determine which test(s) may best discriminate differences of RT and IIV between young and older adults as well as which tests show the biggest effects of personrelated factors on RT and IIV. 


\subsection{CHAPTER SIX: Comparing Tests - Effect sizes}

One of the main aims of this thesis was to investigate whether differences in RT and IIV between young and older adults (ageing effects) were similar across a variety of different attention-related tests. Another aim was to determine how person-related factors, namely sex, education, subjective memory function and perceived test difficulty may differentially affect, or not, the outcome of RT and IIV and error performance. Comparing the potential effects of a variety of factors as well as any interaction between them, was examined in much greater detail than in previous studies of ageing. Especially novel was the inclusion of subjective memory function and perceived test difficulty as factors.

Another novel aspect of this research overall was the inclusion of a study designed to measure the influence of trial number upon RT, IIV and errors (a block design choice RT test, i.e. the Choice RT was split into four separate blocks to measure how RT performance in both young and older adults may change throughout the test whether due to fatigue (slower RT), practice effects (faster RT) or any other factors or their combinations.

An additional new initiative was an attempt using a modified MILO task to examine the influence of 'first response hesitancy and whether this might significantly influence any differences between young and older adults in overall test performance (comprised of numerous trials) i.e. results being much slower than they should be. It may be the case that taking first response hesitancy into account is useful when measuring information processing speed in RT studies in order to obtain a more accurate difference between young and older adults. The MILO was modified to measured similar function to that of TMT and visual search and used as another attention-related test to compare RT and IIV but using different stimuli and administered via an iPad platform to determine whether a different response mechanism provides a better representation of information processing speed integrity.

In order to compare test outcome in the second larger study (TMT, Simple RT, Choice and MILO), ideally the same participants were to complete each test so direct comparisons could be made between tests as to which were more sensitive to ageing effects and the influence of person-related factors. 
However, not all the same participants (both young and older adults) completed each test for a variety of reasons. Some older adults were too tired to complete the test battery or were only available to be tested for some tests and not others.

Occasionally equipment malfunctions occurred (for both young and older adults) thus the test could not be completed and the participant was unable to return to repeat the test. This is a potential limitation of the second study since the aim was to directly compare each test. However there was a large enough overlap of young and older adults to provide large enough samples in order to compare RT, IIV and errors between tests. In addition, in an attempt to reduce the effect of this potential limitation, the demographics of both young and older adults (education level, anxiety and depression levels and objective cognition score [MoCA]) were very similar for all tests and with a narrow range of scores.

In this chapter we compared effect sizes of information processing speed and IIV between young and old in each of the attentional tests examined in the first study (visual search) and the second study (TMT, Simple RT, Choice RT and MILO) to statistically observe which test(s) appear most sensitive to ageing effects. In addition we compared effect sizes of the significant relationships between information processing speed, IIV and different person related factors (sex, education, perceived test difficulty and subjective memory function) to observe which results are more robust and what may affect RT and IIV results in each test thus what might need to be taken account of when undertaking studies and clinical testing in this area. Information processing speed is regarded as an important measure of the integrity of brain structure and function (as stated in the relatively new DSM-5) but there is a lack of detail as to which RT test may best measure this integrity and not considering the possible influence of different person related factors. Effect sizes between information processing speed and IIV were also compared to determine whether RT or IIV is a more sensitive measure of the differences between young and older adults as well as any effects of person-related factors.

It is difficult to compare the outcome many previous studies of RT and IIV because tests and participant groups are different but we used the same participant where possible to directly compare tests. In addition previous studies have not always provided effect sizes within their results thus making it difficult to compare them to new studies and to each other. 
Therefore in the current chapter we examined the tests with respect to their effect size i.e. the effect size of the difference between old and young information processing speed, IIV and errors which can then be compared to each other to observe which test may be more robust thus more useful in ageing studies.

\section{Visual search findings and effect sizes}

In chapter two, a single study examining ageing with respect to a typically used visual search test of attention-related information processing speed and its variability (IIV) was reported. Information processing speed was found to be significantly slower and significantly more variable in older adults compared to young adults. Information processing speed and IIV were found to be associated with education, sex, and perceived test difficulty although these effects were associated with the task conditions (e.g. whether distracting information was present or not) and the age of the participant (young or older adults). Greater perceived difficulty of the test related to faster information processing speed only in the target alone condition and in older adults. Higher levels of education in older adults were associated with faster and less varied information processing speed but only in the target plus distractors condition.

In contrast, only in young adults (and target plus distractors condition) was there an effect of sex on information processing speed and its variability with males performing significantly faster and less variably compared to females.

In relation to the factor of subjective memory function in older adults, there was no relationship with information processing speed or IIV in either condition. Finding older adults to be significantly slower compared to young adults suggests that the visual search test is sensitive to the effects of ageing per se i.e. the test components affected by ageing. This is supported by finding very large effect sizes $(>0.49)$ for the difference of information processing speed and IIV between young and older adults (see Table 30). 
Table 30. Effect sizes for the relationships found for visual search in each condition (Target alone and Target plus distractors)

\begin{tabular}{|c|c|c|}
\hline $\begin{array}{c}\text { RT difference of old vs } \\
\text { young }\end{array}$ & 0.73 & Target alone \\
\hline $\begin{array}{c}\text { Perceived test } \\
\text { difficulty + RT } \\
\text { correlation }\end{array}$ & 0.29 (Older adults) & No relationship \\
\hline $\begin{array}{c}\text { Education + RT } \\
\text { correlation }\end{array}$ & No relationship & 0.40 (Older adults) \\
\hline Sex + RT Correlation & No relationship & 0.33 (Young adults) \\
\hline \multicolumn{2}{|c|}{0.63} & 0.35 (Older adults) \\
\hline $\begin{array}{c}\text { IIV difference of old } \\
\text { vs young }\end{array}$ & 0.63 \\
\hline $\begin{array}{c}\text { Education + IIV } \\
\text { correlation }\end{array}$ & No relationship & 0.37 (Young adults) \\
\hline Sex + IIV correlation & No relationship \\
\hline \multicolumn{2}{|c}{0.44 (Older adults) } \\
\hline $\begin{array}{c}\text { Perceived test } \\
\text { difficulty + education } \\
\text { correlation }\end{array}$ & \multicolumn{2}{|c}{} \\
\hline
\end{tabular}

Visual search has been examined in multiple studies and effect sizes have been found to be large (0.63 to 1.1) [e.g. Tales et al, 2010; Tales et al, 2005; Phillips et al, 2013 ] as found in the current study (0.73 and 0.77). When comparing with the current study, some of these previous studies used smaller sample sizes [e.g. Tales et al, 2010] and arguably this may have affected results and how robust they are.

However, even though the current study used larger age groups the effect sizes were also large. This may imply that smaller numbers of participants is not important and the visual search test is very sensitive to the ageing process.

These previous visual search studies did not take into consideration factors such as perceived test difficulty, or subjective memory function alongside sex and education as well any correlations between them. We examined these factors in the current visual search study to determine whether these previously ignored factors (particularly perceived test difficulty and subjective memory function) may affect information processing speed and IIV. In addition, observing how subjective memory function may affect information processing speed may help to characterise SCI per se. 
If effects of different factors are found, future visual search tasks should take the effects of different factors into account so as not to misinterpret changes (i.e. slowing) to information processing speed in healthy ageing thus inaccurately characterising healthy ageing.

An effect of perceived test difficulty may imply other aspects of self-assessment i.e. perceived ability may also have an influence on information processing speed. Finding an effect of perceived test difficulty on RT in visual search implies that previous visual search studies may have exaggerated the size of the differences of RT between young and older adults by not taking this factor into consideration. However the effect size of the relationship between perceived test difficulty and information processing speed was small $(<0.29)$ [see Table 30]. This suggests the result is not very robust thus finding an effect may not be as significant as the $p$ value suggests.

Moderate effect sizes (>0.29 and < 0.49) were found in correlations between information processing speed or IIV and sex and education and from correlations between subjective memory function, perceived test difficulty and education. These moderate effect sizes imply the resulting relationships are fairly robust thus the significant effect of sex and education should be considered when measuring information processing speed and IIV during ageing studies using visual search. In addition an effect of education was found for RT in older adults despite a narrow range of education scores within the visual search test. RT between young and older adults gave a larger effect size compared to IIV (see Table 30) which implies RT in visual search is more affected by ageing than IIV.

The DSM-5 does not specify which tests to use in relation to information processing speed nor the effects different factors may have on information processing speed and IIV within these tests. This makes it unclear as to which RT tests are best to use i.e. more sensitive to the effects of ageing or whether influenced by different factors. The effects of factors found in the current results may impact how information processing in ageing is characterised in visual search thus impact how visual search is measured in research studies i.e. consider sex and education depending on the condition or the age of the individual. In addition, finding visual search to be sensitive to the effects of ageing (large effect size) may impact which tests are used in clinical settings. Typically clinics include the TMT in their neuropsychological battery as the measure of information processing speed. 
However the current visual search test was observed to be more sensitive i.e. more affected to differences in RT between young and older adult information processing speed (i.e. larger effect sizes) compared to the current TMT which produced very small effect sizes (See Table 31) and compared to other TMT studies with small effect sizes [e.g. 0.21 for Trails B in Haworth et al, 2016]. This suggests that visual search may be a better test to use in clinical settings to compare disproportionately slower information processing speed (possible cognitive impairment i.e. MCI or dementia) with healthy controls.

A second study was designed to examine a variety of different attentional tests and whether they would produce similar relationships and similar effect sizes to the visual search (Chapter 2). The tests used in this second study are similar to the visual search in relation to the aspects of attentional function they measure i.e. executive function, selective attention, attentional shifting and decision making. Therefore comparing the outcome of each test with each other and with the visual search we may find a test more sensitive to ageing other than the visual search and test(s) more or less affected by factors such as sex and education. Tests less affected by other factors suggest that any changes of information processing speed (i.e. slowing) are more likely to relate to the effects of age.

\section{Second study findings and effect sizes: comparing to visual search}

In chapter 3, 4 and 5 different visual attention tests were used in a larger multi-test type study; the TMT, the Simple RT and the Choice RT tests and the modifiedMILO test. As in the visual search test outcome was related to several factors and their combinations. Information processing speed was found to be slower in each test (and more variable in Simple and Choice RT and RT1 of the MILO) in older adults compared to young adults. In the TMT, RT was associated with the task conditions (e.g. RT effected in Trails B and not Trails A) and perceived test difficulty (greater perceived test difficulty relating to slower RT) but not with sex, education or subjective memory function. In the Simple and Choice RT test, older adults were significantly slower and more variable compared to young adults. RT and IIV were associated with perceived test difficulty (greater perceived test difficulty relating to slower RT) but not with sex, education or subjective memory function. 
In the MILO test RT was associated with the task conditions (e.g. RT effected in RT8 - RT1 and not RT1) subjective memory function (perceiving changes to memory function related to slower RT) and education (higher education related to faster RT) but not with sex or perceived test difficulty. It is clear therefore that the results of the second, larger study highlight outcome variability in relation to the effect of different factors appear dependent on the type of test or sub-test used particularly in relation to subjective memory function as an effect was only found in MILO and not any of the other tests (including visual search).

In addition, outcome variability appeared dependent on the age of the participant as found in visual search i.e. person- related factors effecting RT in older adults and not young adults thus should be taken into account when comparing information processing speed in young and older adults. The variability of results between tests also suggests the design of the paradigm may be influential i.e. using different stimuli or having multiple trials compared to a single trial. The attention-related tests used in the current study ostensibly measured similar aspects of attention. However, the use of different stimuli or trial numbers may have recruited slightly dissimilar aspects of brain function which may be differentially sensitive to ageing thus result outcome varied between tests. Different samples especially between initial study (visual search) and second larger study (TMT, Simple RT, Choice RT and MILO), may have accounted for outcome variability between tests for whether person-related factors were associated with differences in RT or IIV. However it must be noted that demographics were similar across young and older adult groups.

\subsection{Effect sizes and Information processing speed}

Table 31. Comparing the Effect sizes (Cohen's d) in information processing speed between young and older adults in each test or sub-test

\begin{tabular}{|l|c|c|c|c|c|c|c|c|}
\hline & \multicolumn{2}{|c|}{ Visual Search } & \multicolumn{2}{c|}{ TMT } & & & \multicolumn{2}{c|}{ MILO } \\
\cline { 2 - 9 } & $\begin{array}{c}\text { Target } \\
\text { alone }\end{array}$ & $\begin{array}{c}\text { Target + } \\
\text { distractors }\end{array}$ & Trails A & Trails B & $\begin{array}{c}\text { Simple } \\
\text { RT }\end{array}$ & $\begin{array}{c}\text { Choice } \\
\text { RT }\end{array}$ & $\begin{array}{c}\text { RT1 } \\
\text { RT1 }\end{array}$ \\
\hline $\begin{array}{l}\text { Effect } \\
\text { size }\end{array}$ & 0.73 & 0.77 & 0.26 & 0.27 & 0.24 & 0.54 & 0.58 & 0.66 \\
\hline
\end{tabular}


The MILO (overall RT and the first response) and Choice RT produced large effect sizes although they were not as large as the visual search (target alone and target plus distractors). Large effect sizes imply that these tests may be considered as highly sensitive to the effects of ageing. In contrast, the TMT and Simple RT produced small effect sizes implying these tests were less affected by ageing thus a lack of sensitivity. It must be noted that not all participants completed each of the tests however there was enough overlap and similar demographics to make comparisons between tests. In addition the participant sample in the visual search differed to the participant sample in the other tests thus effect sizes may not be directly comparable although demographics were similar.

\section{$\underline{\text { MILO }}$}

In the MILO test the effect size of overall RT (RT8-RT1) was larger than effect size of first response (RT1). As mentioned in the MILO chapter (Chapter 5), excluding the first response may increase the difference between young and older adults. Older adults were still slower in their overall RT response compared to young adults despite removing any hesitancy effects from the first response. Therefore any initial hesitancy of tapping the first ball does not appear to reduce ageing effects on information processing speed thus does not explain overall slowing of test performance between young and older adults.

\section{Comparing TMT and Visual search}

The dichotomy between tests has been examined between TMT and visual search and it has been argued that multiple trial computer based RT tests other than the TMT (i.e. the visual search) may be better to distinguish differences of information processing speed in ageing as observed by larger effect sizes [e.g. Haworth et al, 2016]. This was supported in the current study as the effect sizes for the visual search were also larger compared to TMT suggesting visual search is more sensitive to the effects of ageing.

In clinical settings the TMT is commonly used as an RT measure yet in the current study effect sizes were small compared to other research tests such as the visual search, MILO and Choice RT. Therefore, research tests may be a better measure of the effect of age on RT and IIV. 
In view of increasingly emerging evidence of outcome variability one should ask whether the DSM-5 statement needs to highlight such potential outcome variability and to state the type of test(s) that should be used to clinically measure the integrity of information processing speed. This emphasises the need to raise awareness that different types of test provide different age-related results and therefore the different brain functions they recruit may be differentially affected by the ageing process which may impact which tests should be used in research and clinical settings.

\section{Comparing MILO and TMT}

Finding differences in sensitivity between multiple and single trial tests provided a rationale for why the MILO is compared with the TMT (as discussed in Chapter 3) since both tests measure similar attentional functioning but differ in terms of methodology i.e. number of trials.

The MILO (overall performance) was found to better distinguish differences in RT between young and older adults compared to the TMT (i.e. greater effect sizes were observed in the MILO). The TMT contained a single trial which may not be a true representation of attentional function required since the test can be greatly affected by an individual making a one-off mistake. Therefore, the TMT may not be as sensitive in distinguishing differences of RT between young and older adults (as established by the smaller effect sizes [see Chapter 3 ).

\section{Simple RT compared to Choice RT}

The current research found the Choice RT test produced larger effect size thus a stronger association between the reaction time of young and older adults compared to the Simple RT test which was considered to be poor (small effect size). This supports the conclusion drawn by Der \& Deary [2006] that the cognitive functions from a choice RT test display greater differences i.e. slowing during ageing compared to a simple RT test. The Choice RT in current study recruited additional function i.e. decision making and these additional functions may be more sensitive to ageing effects. In addition older adults older adults have less ability to deal with processing larger amounts of information from more complex tasks at one time so instead process smaller amounts of information consecutively thus produce slower information processing speed compared to young adults. 


\section{$\underline{\text { Differences between tests }}$}

Differences of effect sizes between the tests in the current research supports previous arguments that irrespective of any cognitive impairment or lack of, information processing speed can vary in the same group of people depending on the type of task or methodology used [e.g. Torrens-Burton et al, 2017; Haworth et al, 2016; Tales et al, 2010; Der \& Deary, 2006].

For example the use of different stimuli or using multiple or single trials may activate slightly different brain functions which may be differently affected by ageing. Effect size may also have depended on the specific aspect(s) of brain function, i.e., attention, that is being measured thus differences of effect sizes between tests suggests that not all cognitive functions are effected by ageing in the same way as suggested by Robbins and colleagues [1997]. Different visual attention tests require a greater number of brain functions during test performance thus there is greater chance that a greater number of components will be more affected by ageing although between tests some results were very similar i.e. RT was always found to be slower in older adults compared to young adults.

It must be highlighted that within the differences of information processing speed and IIV between young and older adults, there were outliers in the data. These outliers were different to those removed before analysis due to extraneous variables. It is possible that these outliers represent disproportionately slower and more varied information processing speed in certain individuals which may account for the significant differences between young and older adults.

\subsection{Effect sizes and intraindividual variability}

Table 32. Effect sizes of comparing intraindividual variability between young and older adults from each test or sub-test

\begin{tabular}{|l|c|c|c|c|c|}
\hline & $\begin{array}{l}\text { Visual search } \\
\text { Target alone }\end{array}$ & $\begin{array}{c}\text { Visual Search } \\
\text { Target }+ \\
\text { distractors }\end{array}$ & $\begin{array}{c}\text { Simple } \\
\text { RT }\end{array}$ & $\begin{array}{c}\text { Choice } \\
\text { RT }\end{array}$ & $\begin{array}{c}\text { MILO } \\
\text { RT1 }\end{array}$ \\
\hline $\begin{array}{l}\text { Effect } \\
\text { size }\end{array}$ & 0.63 & 0.62 & 0.26 & 0.41 & 0.5 \\
\hline
\end{tabular}


The large effect sizes were observed in the visual search conditions (target alone and target plus distractors) and the RT1 condition of the MILO implying robust results of information processing speed between young and older adults. Moderate effect sizes were observed in the Choice RT implying the results were fairly robust. A small effect size was found in the Simple RT test implying less robust results.

Visual search may be more sensitive to differences in RT variability between young and older adults compared to the MILO and especially the Simple and Choice RT tests. Previous studies using visual search have not always included IIV alongside RT thus the current results may suggest that IIV is a useful measure to distinguish between young and older adults' performance in RT tests. However, the effect sizes were larger compared to the effect sizes for IIV implying that information processing speed is more sensitive to the effects of ageing.

Multiple trials in the MILO allow for a measure of variability of RT performance which the TMT could not achieve thus one reason for using the MILO test. Older adults were more variable at beginning the test compared to young adults demonstrated by a moderate effect size thus examining the first response in the MILO may be useful for distinguishing differences of IIV in ageing thus may be a useful measure in other RT tests using multiple trials i.e. visual search.

In contrast, overall MILO performance did not find a significant difference of IIV between young and older adults. This result differs to the results from the each of the other tests in the current research which found a difference of IIV between young and older adults. Finding an ageing effect on IIV only when beginning the test (RT1) may imply that excluding the first response from the overall RT eliminated the majority of the variance between young and older adults.

Therefore we speculate that finding an ageing effect on IIV in the other attention tests in the current studies may have related to including the first response in each of these tests. It is possible that greater variability occurs when beginning the test (possibly due to hesitancy) thus if not excluded this first response influences the overall test performance i.e. the test being more variable than it should be. 


\subsection{Effect sizes for correlations between information processing speed and person-related factors}

\section{Information processing speed and subjective memory function}

For most of the tests perceived memory function did not correlate with information processing speed. Only the RT1 condition of the MILO (time taken to begin the test) showed a relationship with subjective memory function with less perceived changes to memory function (higher MFQ score) associated with faster information processing speed. This may suggest that perceived memory change relates to possible hesitancy effects when beginning the test i.e. perceiving memory function to be worse thus feeling less confident in beginning the trial. The results also indicate that SCI per se (at group level) is characterized by an association with hesitancy effects for the first response in MILO. Therefore, we speculate that subjective memory function and SCI may also be related to the first response in the other attention tests in the current studies. Excluding the first response from RT tests may indicate that any perceived changes to memory function no longer have an effect on information processing speed thus do not misrepresent the level of slowing which occurs in ostensibly healthy adult control groups.

It must be noted however, that the effect size in the MILO test was small (0.26) implying a less robust result despite a significant result i.e. a weak association between subjective memory function and information processing speed in the RT1 condition in the MILO. Therefore subjective memory function may need to be taken into account for this condition but other factors may be more sensitive to the differences in RT between young and older adults i.e. the effects of age.

\section{Information processing speed and perceived test difficulty}

Not all tests found a relationship between perceived test difficulty and information processing speed. The effect sizes of those tests and sub-tests which found a relationship are displayed in the table below.

Table 33. Effect sizes comparing correlations of information processing speed and perceived test difficulty between Target alone condition (visual search), Trails B condition (TMT) and Choice RT

\begin{tabular}{|l|c|c|c|}
\hline & $\begin{array}{c}\text { Visual search } \\
\text { Target alone }\end{array}$ & $\begin{array}{c}\text { TMT } \\
\text { Trails B }\end{array}$ & Choice RT \\
\hline $\begin{array}{l}\text { Effect } \\
\text { size }\end{array}$ & $\begin{array}{c}0.29 \text { (older } \\
\text { adults) }\end{array}$ & $\begin{array}{c}0.29 \text { (older } \\
\text { adults) }\end{array}$ & $\begin{array}{c}0.27 \text { (young } \\
\text { adults) }\end{array}$ \\
\hline
\end{tabular}


Effect sizes for the Target alone condition (visual search) and Trails B (TMT) were marginally larger compared to the Choice RT however all three effect sizes were small implying a weak association between information processing speed and perceived test difficulty.

It must be noted that the Target alone and Trails B results related to older adults whereas the Choice RT result related to young adults thus effect sizes may not be directly comparable to each other. In addition, in the visual search perceiving the test to be more difficult related to faster RT compared to slower RT in the TMT and Choice RT. Taking perceived test difficulty into account may be dependent on which attentional test is used when measuring information processing speed in research and clinical studies as well as the age of the participant i.e. whether young and older adults find the test more difficult.

Despite a small effect size there may be an aspect of judgement influencing how quickly the tests are performed thus any slowing of RT may not necessary relate to any cognitive impairment. However any affects of perceived test difficulty on RT may be depended on the participant sample used in future studies using the same attention tests from the current studies (e.g. visual search, TMT, Choice RT). For example how difficult individuals perceive the test to be may depend on factors such as their level of education (having additional skill) or dexterity of motor movement (poor movement results in test completion being challenging). Alternately as effect sizes between perceived test difficulty and RT were small, there may be other explanations for information processing speed differences between young and older adults i.e. age effects or the type of attention test or aspect of attentional function measured.

We can only make speculations as to why outcome variability for perceived test difficulty and RT occurs between tests. Different psychological factors i.e. selfassessment such as perceived test difficulty have not been examined in great detail in the attention tests used in the current studies thus replication is needed.

\section{Information processing speed and educational level}

Not all tests found a relationship between educational level and information processing speed. The effect sizes of those sub-tests which found a relationship are displayed in the table below. 
Table 34. Effect sizes comparing information processing speed and educational level between Target plus distractors condition (visual search) and RT8-RT1 (MILO)

\begin{tabular}{|l|c|c|}
\hline & $\begin{array}{c}\text { Visual search } \\
\text { Target + } \\
\text { distractors }\end{array}$ & $\begin{array}{c}\text { MILO } \\
\text { RT8-RT1 }\end{array}$ \\
\hline $\begin{array}{l}\text { Effect } \\
\text { size }\end{array}$ & 0.40 & 0.23 \\
\hline
\end{tabular}

The effect size for the Target plus distractors condition was of medium strength whereas the effect for the RT8-RT1 condition was small. A larger effect size for the Target plus distractors condition implies a more robust association between information processing speed and educational level in the visual search test compared to that of the MILO test.

In the visual search, faster information processing speed related to higher education in older adults which may indicate that education can improve cognitive reserve i.e. the brain's resilience to any pathology which may occur due to disease, such as dementia. Higher education has been demonstrated to produce a significant reduction in the prevalence of dementia [Sattler et al, 2012; Meng \& D'Arcy, 2012] thus implying that higher education is associated with higher cognitive reserve [Mourany \& Pillai, 2014]. This may provide an explanation for why information processing speed was faster in older adults with higher education. High levels of education are potentially protecting against any possible cognitive impairment thus information processing speed appears to remain at normal levels for ostensibly healthy ageing. However, this does not explain why a correlation was not found between RT and education in the other conditions within visual search and MILO or in the other attention tests in the current studies. Levels of education in older adults were similar across the attention tests thus we would speculate that similar effects of education would thus occur in each test. In addition, the majority of the same participants completed the TMT, Simple RT, Choice RT and MILO therefore we would speculate that the same levels of cognitive reserve would have been present when performing each test.

Therefore, outcome variability between tests suggests education should be taken into account when measuring information processing speed depending on the attention test or brain function measured i.e. visual search. A possible limitation of the current studies was not including a measure of cognitive reserve but only educational level. 
In addition, education was not split into high and low levels as used previously [Verhage, 1965] which may provide a clearer measure of whether there is a relationship between high education and faster information processing speed in other aspects of attention i.e. the other attention tests in the current research.

\section{Information processing speed: Sex comparison}

An influence of sex on information processing speed was only found in young adults and in the target plus distractors condition in the visual search test. The effect size was of moderate strength (0.33) implying a moderately robust association between sex and information processing speed i.e. young males being faster compared to females.

This result suggests that in visual search, young males and females perform the test at difference speeds, one speculation being due to hormone levels. For example oestrogen levels may affect attention systems [McEwen, 2001] and as oestrogen levels are high, cognitive performance in tasks which males tend to be better at performing i.e. spatial ability, is poorer in females [Hampson, 1990]. This may explain differences in cognitive performance i.e. information processing speed in visual search between young and as hormone levels balance out in older age, this may explain why no difference in RT was found between males and females in older adults. However, we are only speculating at present and further investigation is required to explain differences between young and older adult males and females i.e. measuring hormone levels. In addition further investigation is required as to why sex effects were only found in the visual search test and not the other attention tests in the current studies (TMT, Simple RT, Choice RT and MILO). At present we speculate that this may have related to the aspect of attention being measured i.e. the type of attention test used. Alternatively differences between tests may have related to having a different sample of participants and numbers of males and females in the visual search compared to the other attention tests. 


\subsection{Effect sizes between intraindividual variability and person-related factors}

\section{Intraindividual variability and subjective memory function}

There was no significant correlation between subjective memory function and IIV in any test or condition within each test. This implies that perceived changes to memory function is not characterized by a greater variability of information processing speed in visual search, TMT, Simple RT, Choice RT or MILO in ostensibly healthy older adults perceiving changes to their memory function.

The results also indicate that SCI per se (at group level) is not characterized by a greater variability of information processing speed in these attention tests the same ostensibly healthy older adults perceiving changes to their memory function. However finding no effects of subjective memory function may have related to the inclusion of fewer older adults in the sample perceiving changes to memory function (low MFQ score) compared to perceiving changes to memory function. Therefore using the attention tests from the current study, further investigation is required including a better comparison between older adults who perceive changes to memory function and those who do not for a clearer measure of whether there is a relationship between subjective memory function and IIV.

\section{Intraindividual variability and perceived test difficulty}

Only Choice RT found a relationship between perceived test difficulty and IIV of moderate strength (effect size $=0.34$ ). This implies that the result is fairly robust i.e. in young adults, greater perceived difficulty related to more variable RT performance in the Choice RT. Taking perceived test difficulty into account may be dependent on which attentional test is used when measuring IIV in research studies and should also be considered in clinical studies.

For the Choice RT, the effects size was smaller when measuring RT compared to measuring IIV thus implying that IIV may be more sensitive to the effects of perceived test difficulty in this test. To reiterate, perceived test difficulty has not been examined in great detail in previous attention tests such as the tests examined in the current studies as well as including a measure of IIV alongside information processing speed. Therefore, further examination is required as to why relationships are found between IIV and perceived test difficulty in the Choice RT and not in other attention tests i.e. visual search, Simple RT and the MILO. 


\section{Intraindividual variability and educational level}

Only the target plus distractors condition in the visual search test found a relationship between educational level and IIV of moderate strength (effect size = 0.35 ) implying a fairly robust result that higher education relates to less variable RT performance (in this condition of the visual search). Lower IIV may relate to higher education being associated with better cognitive reserve i.e. preserving cognitive function from any impairment. However as argued above, we would expect similar effects of education on IIV in the TMT, Simple R, Choice RT and MILO since the majority of the same participants completed each test.

In the visual search test, the effect size for correlating RT with education was larger compared to the effect size comparing education with IIV. In addition, IIV did not significantly correlate with education level in overall performance in the MILO whereas there was a significant correlation with RT. This suggests that information processing speed is more sensitive to the effects of education than IIV.

Finding an effect of education on IIV may be dependent on the attention test used and aspect of attention being measured thus should be taken account in research studies including IIV as a measure. Including IIV and using visual search may also be useful in clinical studies alongside measuring information processing speed thus in these clinical studies the effect of education should also be taken into account.

\section{Intraindividual variability: sex comparison}

Only the target plus distractors condition in the visual search test found a significant difference in IIV between males and females and this sex effect was only in young adults. The effect size was of moderate strength (0.37) and only slightly larger compared to the effect size for RT (0.33) thus implying a fairly robust result that young males are less varied (as well as faster) compared to females possibly due to hormone differences (as suggested in the RT section above). Therefore, in the visual search test the variability of performance between young males and females should be taken into account to better characterise differences of IIV in young adults compared to older adults. Variability between young males and females may relate to differences in brain functions i.e. males better at spatial ability. 
Only finding sex effects on IIV in the visual search and not the other tests in the current studies (as also found with RT) may suggest that differences of IIV between young males and females may be dependent on the test thus may indicate why IIV in young males and females was similar in the TMT, Simple RT, Choice RT and the MILO.

Overall, the visual search test was affected by the most number of factors (i.e. sex, education and perceived test difficulty) despite being the most sensitive test to ageing effects although this did appear to be dependent on age (education effects in older adults but sex effects in young adults). This implies that the visual search (and the aspects of attention measured) may be the most sensitive test to the effects of age but is also sensitive to variation caused by these factors. This finding may be useful in future use of the visual search test in research studies or even in clinical studies so that these factors can be taken into account as possible influences on information processing speed and IIV.

Arguably, when comparing young and older adults, it may be best to use the types of attentional tests in which information processing speed and IIV are least effected by different factors (i.e. Choice RT not effected by sex nor education). This suggests we can be more certain that any changes to information processing speed is indeed due to healthy ageing effects and when RT is disproportionately slower it may relate to pathological ageing i.e. MCI or dementia. Therefore if it is important not to have many effects of person-related factors, we may be able to use tasks such as Simple RT which found fewer effects of factors despite a small effect size (i.e. less sensitive) to age effects on information processing speed and IIV. Additionally, the Choice RT found large effects of age similar to the visual search but in comparison information processing speed and IIV were not affected by sex and education. Consequently, the Choice RT may be a better test to use in ageing and RT studies if differences in RT and IIV appear to relate to age effects and not person-related factors. However we understand that the effects of person-related factors cannot always be controlled for or excluded completely thus must simply be taken into account when comparing RT and IIV between young and older adults. Alternatively finding effects for a greater number of factors in visual search compared to the other attention tests may reflect individual differences in the participants used. 
If this is the case, how information processing speed and IIV is measured should be based on the individual person. This has implications especially in clinical studies as it implies that we should use person centred diagnosis and treatment to acknowledge individual differences.

\subsection{Comparing RT and IIV}

Effect sizes for the difference in RT and IIV between young and older adults were compared within each test as well as between tests to see which may be more sensitive to ageing. The TMT could not measure IIV thus was not included in this comparison.

Table 35. Effect sizes comparing information processing speed (RT) and intraindividual variability (IIV) between young and older adults between tests and subtests

\begin{tabular}{|c|c|c|c|c|c|}
\hline \multirow{2}{*}{} & \multicolumn{2}{|c|}{ Visual Search } & & & MILO \\
\cline { 2 - 6 } & Target alone & $\begin{array}{c}\text { Target + } \\
\text { distractors }\end{array}$ & $\begin{array}{c}\text { Simple } \\
\text { RT }\end{array}$ & $\begin{array}{c}\text { Choice } \\
\text { RT }\end{array}$ & RT1 \\
\hline $\begin{array}{c}\text { Effect size } \\
\text { RT }\end{array}$ & 0.73 & 0.77 & 0.24 & 0.54 & 0.58 \\
\hline $\begin{array}{c}\text { Effect size } \\
\text { IIV }\end{array}$ & 0.63 & 0.62 & 0.26 & 0.41 & 0.50 \\
\hline
\end{tabular}

Effect sizes were larger when measuring the difference of RT between young and older adults suggesting that information processing speed is more sensitive to the effects of ageing compared to IIV. This appears to support the statement by the DSM-5 that information processing speed is a significant measure in ageing and particularly when comparing healthy ageing control groups and abnormal ageing i.e. MCI and dementia. However in the Simple RT test, the effect size for measuring IIV was larger compared to the effect size for RT, although only slightly. IIV has not been considered by the DSM-5 as a measure alongside information processing speed in different attention tests thus it may be the case that how young and older adults are compared (whether using RT or IIV) should be evaluated based on which attention test is being used.

In addition person related factors correlated with RT more so than correlating with IIV implying information processing speed is more sensitive to the effects of person related factors (also not considered in the DSM-5). 
Therefore different factors should be taken into account in research and clinical studies depending on whether they are measuring information processing speed or IIV. In addition, whether person-related factors should be considered should be based on the attention test used as in the current studies, effects of factors on RT or IIV appeared to be test dependent.

It has not been common practice in previous research or in clinical studies to include both RT and IIV. We argue that since both measures were found to be affected by age in the current studies, they are both useful for characterising differences between young and older adults. Therefore RT and IIV may also be useful for characterising differences between ostensibly healthy ageing and pathological ageing i.e. MCI or dementia.

\section{6. Choice RT: comparing blocks of trials}

\section{Information processing speed}

It is common to use multiple trials to measure RT and IIV but rare to examine how the number of trials per se may influence RT performance. We included novel design of the Choice RT test paradigm by splitting the trials into blocks to examine how the number of trials per se may influence RT performance and whether these changes, if any, are due to fatigue (slowed) or practice effects (sped up) any other factors or their combinations.

Table 36. Effect sizes comparing information processing speed of young and older adults between blocks of trials in Choice RT

\begin{tabular}{|l|c|c|c|c|}
\hline & Block 1 & Block 2 & Block 3 & Block 4 \\
\hline Effect size & 0.59 & 0.49 & 0.51 & 0.45 \\
\hline
\end{tabular}

Block 1 and Block 3 produced large effect sizes implying that these blocks best differentiated differences in RT between young and older adults. This result from Block 1 in particular supports previously finding RT to be significantly affected by age in earlier trials compared to later trials [Fernaeus et al, 2013]. The smallest effect size occurred in Block 4 although this was still a moderate effect size thus implying a moderately robust result. 
The effect size in Block 1 suggests there was a large difference in RT between young and older adults i.e. older adults significantly slower compared to young adults. We speculate that this may relate to the influences of the first response as discussed in the MILO test (Chapter 5). Older adults are possibly slower at the beginning of the Choice RT test due to hesitancy effects thus having effect on the overall information processing speed in the first block i.e. a greater difference in RT between young and older adults than there should be. It would be useful to examine the first trial in future Choice RT tests to determine whether it indeed has an impact on the first block when separating trials or for overall RT performance during Choice RT.

In Block 3 a large difference was found between young and older adult information processing speed. From post hoc analysis it was suggested that young adults were slowing throughout the test and older adults were getting faster. Young adults were possibly slowing due to fatigue although we would have expected greater fatigue in older adults due to their poorer ability to process multiple stimuli (as required in the current Choice RT) thus putting a greater strain on mental resources.

Information processing speed in older adults decreased i.e. sped up throughout the test. This implies that older adults are not faster per se but are influenced more so than young adults by different factors contributing to task performance i.e. practice. Practice may improve performance or learning thus decreasing information processing speed. Therefore older adults' RT performance may be dependent on the number of trials included which may need to be taken into account in research and clinical studies when measuring information processing speed in older adults.

The large effect sizes when comparing separate blocks of trials (i.e. Block 1 and Block 3) were similarly as large as the effect size examining overall RT performance between young and older adults (i.e. a single RT score). Therefore, we speculate that the number of trials is as sensitive to the effects of age thus may be a useful measure to consider in RT research or in clinical studies by providing more detail of how RT is influenced in young and older adults i.e. fatigue or practice effects. 


\section{Intraindividual variability}

Table 37. Effect sizes comparing IIV of young and older adults between blocks of trials in Choice RT

\begin{tabular}{|l|c|c|c|c|}
\hline & Block 1 & Block 2 & Block 3 & Block 4 \\
\hline Effect size & 0.52 & 0.26 & 0.31 & 0.29 \\
\hline
\end{tabular}

Block 1 produced large effect sizes implying a robust result thus this block best differentiates differences of IIV between young and older adults. This may relate to the first trial being more variable due to hesitancy effects (as discussed for information processing speed) thus producing a greater difference in IIV between young and older adults than there should be.

Small effect sizes from the other blocks imply less significant differences of IIV between young and older adults. This was supported by post hoc analysis which revealed that throughout the test, both young and older adults produced little variability in their RT performance.

The effect sizes from comparing separate blocks of trials were greater when measuring information processing speed compared to measuring IIV. This may indicate that information processing speed is more sensitive to the effects of the number of trials and should be included in RT studies to better differentiate between young and older adults.

\subsection{Comparing errors between tests}

The mean number of errors was calculated for young and older adults for each test and each sub test where possible. The number of errors for TMT could not be quantified so this test is not included.

Table 38. Mean number of errors made by young and older adults between each test or sub-test

\begin{tabular}{|c|c|c|c|c|c|c|c|c|c|}
\hline & & \multicolumn{2}{|c|}{ Visual Search } & \multirow[b]{2}{*}{$\begin{array}{c}\text { Simple } \\
\text { RT }\end{array}$} & \multicolumn{4}{|c|}{ Choice RT } & \multirow[b]{2}{*}{ Milo } \\
\hline & & $\begin{array}{c}\text { Target } \\
\text { alone }\end{array}$ & $\begin{array}{c}\text { Target + } \\
\text { distractors }\end{array}$ & & $\begin{array}{c}\text { Block } \\
1\end{array}$ & $\begin{array}{c}\text { Block } \\
2 \\
\end{array}$ & $\begin{array}{c}\text { Block } \\
3 \\
\end{array}$ & $\begin{array}{c}\text { Block } \\
4\end{array}$ & \\
\hline \multirow{2}{*}{$\begin{array}{l}\text { Mean } \\
\text { error }\end{array}$} & Young & 0.44 & 0.46 & 0.63 & 0.95 & 1.08 & 0.90 & 1.18 & 2.57 \\
\hline & Old & 0.37 & 0.33 & 0.42 & 0.55 & 0.70 & 0.71 & 0.60 & 1.25 \\
\hline
\end{tabular}


The mean number of errors was compared between young and older adults. Again the TMT could not be measured as number of errors could not be quantified. Only in the Choice RT test (between trial blocks) and the MILO did Mann Whitney analysis reveal a significant difference in the mean number of errors made between young and older adults. The effect sizes for these two tests were calculated and are presented in the table below.

Table 39. Effect sizes comparing the mean number of errors between young and older adults in the Choice RT across blocks and the MILO test

\begin{tabular}{|l|c|c|c|c|c|}
\hline & $\begin{array}{c}\text { Choice Block } \\
\mathbf{1}\end{array}$ & $\begin{array}{c}\text { Choice Block } \\
\mathbf{2}\end{array}$ & $\begin{array}{c}\text { Choice Block } \\
\mathbf{3}\end{array}$ & $\begin{array}{c}\text { Choice Block } \\
\mathbf{4}\end{array}$ & MILO \\
\hline $\begin{array}{l}\text { Effect } \\
\text { size }\end{array}$ & 0.22 & 0.28 & 0.19 & 0.29 & 0.44 \\
\hline
\end{tabular}

The effect size for the Choice RT was small across the four blocks implying the differences of errors between age groups are significant but the result is not very robust. In contrast the effect size for the MILO test was of moderate size implying a more robust result with younger adults making a greater number of errors compared to older adults.

In these two tests, young adults made significantly more errors compared to older adults. We speculate this is unlikely to reflect impairment to their attentional function resulting in making errors during the test. Young adults are possibly trading accuracy for speed thus making more errors (speed/accuracy trade off [van Veen, et al, 2008; Salthouse, 1979]). This may be supported by also finding large effect sizes in the difference of RT between young older adults i.e. young adults may perform the test quickly but respond randomly (press any key or button) thus they are not necessarily activating the attentional areas required to processes the stimuli correctly and make the required response.

Older adults may not necessarily be slower due to age effects but instead they aim to perform the test correctly i.e. tap the stimuli in the correct order) thus slow their performance in order to achieve this. Indeed it has been observed previously that older adults place more emphasis on accuracy rather than speed [Brébion, 2001; Salthouse, 1979]. 
Individual differences of the speed/accuracy trade-off between participants is recognised and anticipated during RT studies [Brébion, 2001; Yellott, 1971; Pachella $\&$ Pew, 1968]. We tried to control for this speed/accuracy trade off by instructed everyone to be as fast and as accurate as possible. However our results highlight that there are individual differences in which aspect of the RT test a person holds more value to.

Older adults appeared to make a great number of errors in the MILO compared to the Choice RT (although this is only speculated at present) which may be reflecting impairment in attentional function required to perform the test. However we speculate that there are greater chances to make more errors (by both young and older adults) during the MILO compared to the Choice RT as there are more actions to carry out in each trial i.e. moving around the screen and tapping multiple stimuli which gives more opportunity to make mistakes. If errors relate to the test design, the attention test itself may not be an accurate measure of information processing speed and the aspect of attention the test measures. We speculate this may have been the case for the current MILO test as young adults made significantly more errors compared to older adults.

For the Choice RT test and the MILO test effect sizes for RT and IIV were larger compared to the effect sizes for the number of errors. This implies that information processing speed and IIV are more sensitive to the effects of ageing. This implication may be supported by not finding a significant difference in error rates between young adults in the other attention tests (visual search, TMT and Simple RT) but finding a significant difference in RT and IIV.

Therefore it may be more useful to measure RT and IIV rather than the number of errors in attention tests to determine what occurs in ageing. Tests such as visual search produced fewer errors by both young and older adults (difference was not significantly different) but was still sensitive to ageing effects (large effect size). Although ceiling effects need to be avoided, perhaps tests such as the visual search should be used in future ageing studies which produce fewer errors in older adults and particularly young adults but are still sensitive to ageing effects. 


\subsection{Limitations for comparing current effect sizes}

A potentially significant limitation, particularly in relation to the second study, was that not all young and older adults completed each test. This was due to a number of reasons i.e. some participants were too tired to finish the battery, equipment malfunctions, time constraints or loss of data. Different sample sizes and different participants between tests may have made it difficult to directly compare results although demographics were very similar in each age group. However there was enough overlap young and older adults completing each test to provide large enough samples to compare RT, IIV and errors. It must also be noted that the sample in the visual search differed to the second larger study of attention tests thus any differences in results from the visual search may have been a result of this.

Analysis was conducted at group level however, it would be interesting to examine at individual level. Having a rich amount of data for one person for a number of different areas of visual attention can provide a more detailed picture of what changes are occurring to that individual as each individual is different, their baseline performance would be different so their decline would be different (i.e. different levels of cognitive reserve). This requires conducting a longitudinal study to examine how change in RT performance, if any, may occur over time.

\subsection{Conclusion}

Outcome variability of the effect of person-related factors (sex, education, perceived test difficulty, subjective memory function) was found to be dependent on the attention test or condition within a single test. In addition, differences in effect sizes for the difference of information processing speed between young and older adults imply some tests are more sensitive to the effects of ageing than others.

The most sensitive tests to age effects appear to be the visual search test, the iPad modified-MILO test and the Choice RT test whereas the poorer tests being the TMT and the Simple RT test. This is interesting as the tests considered to be poor, particularly the TMT, are already used during neurological assessment thus there is the possibility that clinical studies should reconsider which tests are used when measuring information processing speed. 
Noticing that even amongst the tests used in this thesis there are significant differences of information processing speed between young and older adults, could demonstrate that measuring information processing speed during ageing is important but possibly test dependent thus not as simple as the DSM-5 suggests. It also highlights the importance of using particular tests if they are to be used to discriminate either processing speed changes in healthy aging, or potentially processing speed changes between healthy and pathological ageing.

Note there was some correlation between tests of RT although thus may depend on what aspect of brain function information processing speed is related to. The main aim was not to correlate between tests although in a brief examination of RT and IIV between tests from the second study (i.e. those with similar participant sample), in both young and older adults the tests producing smaller effect sizes correlated with each other (Simple RT and TMT) and the tests producing larger effect sizes correlated with each other (MILO and Choice RT). These results are summarized in the appendices. 


\subsection{CHAPTER SEVEN: General summary and discussion of the thesis}

\subsection{Findings in relation to the aims discussed and stated in the thesis introduction}

One main aim was to measure the integrity of information processing speed and its variability between young and older adults along with the aim to determine whether effects of RT and IIV were similar across difference selective attention tests. The results of information processing speed were similar in all attention tests in the current research as older adults were significantly slower in their RT performance compared to young adults. What varied between tests were the effect sizes for how robust the differences between Young and older adults' information processing speed may be. The results for IIV were similar between the attention tests in the current research apart from one (modified- iPad based MILO test). As with the RT results, effect sizes for IIV varied between those tests which did find a significant difference of IIV.

Another main aim was to determine any potential effects of sex education subjective memory function and perceived test difficulty on information processing speed and its variability. Within this aim was to determine whether the number of trials has an effect on RT and IIV which was examined in the Choice RT test. Whether these factors correlated with RT or IIV appeared to be dependent on the attention test used or the condition within a single test, as well the age i.e. factors effecting older adults more so than young adults. Effect sizes between RT or IIV and factors also differed between tests suggesting differences in how robust the relationships were i.e. how much of an effect factors had on RT or IIV in different tests. In the Choice RT test, the number of trials appeared to influence RT and IIV in young and older adults suggesting that Choice RT (and maybe the other RT tests) should include an examination of the number of trials when used in research and the method of testing information processing speed (and IIV) should be re-evaluated in clinical studies.

Test dependency and differences in effect sizes highlight that information processing speed is not as simple a measure as suggested (i.e. in the DSM-5). Both factors have not been considered in previous RT studies despite previously finding effects on information processing speed to vary within ageing and RT research and even in AD and MCI research. 
It must be noted that participants differed between the initial visual search study and the second larger study (TMT, Simple RT, Choice RT and MILO) which may explain outcome variability between tests although there were a number of reasons why results may have differed (discussed in more detail in each study chapter) i.e. individual differences in cognitive reserve, uneven sample of males and females, the type of test used, multiple trials compared to single trials.

There has been much debate as to whether to use parametric or non parametric testing with RT data. In this thesis we used non parametric testing as normality testing stated that data not normally distributed requires non parametric testing. However, it has been argued [Lumley, Diehr, Emerson \& Chen, 2002] that a large enough data set like this suitable for parametric testing, therefore we include parametric analysis in the thesis (see appendices). The results were similar when conducting parametric testing as well as the non parametric testing used. If non parametric and parametric are in agreement, it can be argued that both statistical analyses can be used and any significant comparisons made to information processing speed in this research are indeed significant.

\subsection{Outliers}

When comparing outliers across the attention tests used in this thesis, in older adults, participant 28 displayed significantly greater and more variable information processing speed in the visual search test and the MILO test. Otherwise all older adults' participants presenting as outliers adults as well as outliers within young adults differed between tests. Participant 28 may be of interest to examine further as they may be reflecting greater abnormal function and possible neurodegenerative change if information processing speed is significantly slower in more than one test. Interestingly this participant perceived few changes to their memory function which may suggest there is no underlying impairment although alternatively, any impairment was not recognised for what it was.

Access to brain scans are required to determine whether the participant in question has physical structural change thus possible underlying cognitive impairment to warrant such disproportionately slower and more variable RT scores. 
The number of errors made by Participant 28 may be an additional reason for suspected cognitive dysfunction. However when comparing the number of errors made between tests, Participant 28 did not make the greatest number of errors compared to other participants in the visual search, Simple RT, Choice RT and the MILO test although errors in the Choice RT test were still greater than the mean error rate. It is a possibility that this individual put more emphasis on accuracy rather than speed to the extent of producing significantly slower RT in order to complete the test correctly.

\subsection{Effects of results on research and clinical settings}

The results of the current study may impact which tests should be used to best examine differences in information processing speed and IIV between young and older adults in ageing studies. In addition results highlight which person- related factors should be taken into account when measuring RT and IIV in ageing so as not to misinterpret any slowing or variability. The findings from the current research may impact upon how information processing speed should be measured in clinical settings between healthy controls and disease associated with cognitive dysfunction such as MCI or dementia.

Effect sizes were greatest in the visual search test and the Choice RT; research based tests containing multiple trials. In contrast the TMT, a clinical single trial test produced small effect sizes thus less robust results. This implies that research tests using multiple trials may best distinguish RT differences in ageing (young and older adults) thus also should be used in clinics to compare MCI or AD and healthy controls. It has been argued by other research that a greater number of trials (used in RT research tests) may provide more sensitive results [Haworth et al, 2016; Salthouse \& Fristoe, 1995]. Finding an effect of sex, education and perceived test difficulty in visual search suggests these factors should be taken into account during $\mathrm{RT}$ research thus also should be included measuring information processing speed in clinics.

An additional benefit of using multiple trials is the ability to measure intraindividual variability. The current studies found older adults' RT performance to be significantly more variable compared to young adults thus highlighting that IIV is also a useful measure in distinguishing between young and older adults. 
Not all RT studies have also included IIV when comparing young and older adults or MCI and dementia to healthy ageing.

\subsection{Limitations across tests}

In MILO and Choice RT (across blocks) young adults made significantly more errors compared to older adults. We speculate this is unlikely to reflect impairment to their attentional function resulting in making errors during the test. Young adults are possibly trading accuracy for speed thus making more errors (speed/accuracy trade off [van Veen, et al, 2008; Salthouse, 1979]). This may be supported by also finding large effect sizes in the difference of RT between young older adults i.e. young adults are significantly faster and making significantly more errors. It has also been observed previously that older adults place more emphasis on accuracy rather than speed [Brébion, 2001; Salthouse, 1979] which is supported by the current results from the MILO and Choice RT as older adults made fewer errors compared to young adults thus were more accurate but were slower.

Individual differences of the speed/accuracy trade-off between participants is recognised and anticipated during RT studies [Brébion, 2001; Yellott, 1971; Pachella $\&$ Pew, 1968]. We tried to control for this speed/accuracy trade off by instructed everyone to be as fast and as accurate as possible. However our results highlight that there are individual differences in which aspect of the RT test a person holds more value to.

Some tests may not have been a good measure of information processing speed for example, the TMT could not be completed by a few young and older adults and the Simple RT version contained distracting stimuli before the target which should have been removed in order to better reflect previous Simple RT tests. The tasks themselves attempted to replicate validated methods where possible instead of using already tested versions (due to access) although this was limited to the programmes and resources available.

All young and older participants were analysed as one group sample. For the young adults the age range spanned seven years and all were psychology university students so similar in terms of education and mainly students of psychology discipline.

Therefore, significant individual differences were not expected. In contrast, the older adults age range spanned three decades and the average age of the older participants was 65 years therefore a young sample in comparison to the maximum age range. 
It would be expected that the younger end of the spectrum (50 years) would perform better during RT tests thus having faster information processing speed compared to the older end of the spectrum (80 years). The samples of both older adults and young are limited to psychology students and older adults in the local community who are interested in participating thus reducing the generalizability of the participant sample. This has been commented on before [Lachman, 2004] yet could not be avoided due to study restrictions i.e. limited funding and participant availability.

It would be expected that older adults in their 50s would have better perception of their memory and less concerns compared to those in their 80s which may explain the current MFQ score ratio with more individuals declaring no memory concerns compared to having memory concerns. There were not enough individuals with memory concerns within the older adult sample to correlate with information processing speed.

Participants were only tested on one occasion and could not take into account the possibility that perceived memory may fluctuate, due to temporary conditions such as fatigue, or everyday stressful life events. In addition, older adults from the community may not have had ongoing concerns or notice significant change prior to being tested until asked to think about their cognition during the study. There was no use of brain scans therefore it is unclear as to the extent of physiological changes to link with subjective complaints which is still a factor which needs be included (as mentioned in Torrens-Burton et al, 2017).

It would have been preferable to test exactly the same participants performing the entire battery as having all the same young and old adults would provide a better characterisation of which test best differentiates between young and older adults to strengthen the comparison of each test. Differences in participant numbers between tests occurred due to some participants growing tired or unwilling to complete every task, impaired equipment (i.e. older computer software) or due to time constraints. The visual search test measured a different sample of young and older adults thus it would be useful to observe whether including the visual search in the larger test battery with the same participants, would result in a similar outcome.

Factors not measured in this research were sleep complaints which can influence reaction time [Gagnon, Baril, Gagnon et al., 2014; Altena, Ramautar, Van Der Werf \& Van Someren, 2010], nor occupation the level of which has also been related to an individuals' susceptibility to age related cognitive decline [Schooler, Malatu \& 
Oates, 1999] or pathology such as Alzheimer's disease [Stern, Albert et al, 1999]. Occupations which are lower in complexity are argued to be a higher risk for cognitive decline compared to more cognitively challenging professions [Capurso, Panza, Solfrizzi et al, 2000]. In relation to sex, males have been found to be faster compared to females suggested to relate to higher levels of testosterone related to improved RT performance [Fontani et al, 2004; Muller, 1994]. Furthermore, medication was not considered. Larson and colleagues [1987] identified having an adverse reaction to drugs i.e. benzodiazepines is associated with cognitive impairment in an elderly population. The cognitive impairment caused by drug intake is more sensitive in older adults [Molchan, Martinez et al, 1992]. Drugs can also cause changes to i.e. metabolic structure which mimics what also occurs in Alzheimer's disease [Tonn, Bartenstein, \& Dahmen, 2005; Riepe, Walther, Vonend $\&$ Beer, 2015].

Asking participants to rate perceived test difficulty may not have been the correct question despite correlating with information processing speed although it is a novel factor so has room for refining. For a better relationship between self reporting and true information processing speed, it may be more pertinent to ask participants to rate their perceived performance of the task instead of test difficulty and correlate with true information processing speed. Using self-rated measures have been found to relate to cognitive performance in healthy adults implying that subjective measures can predictive of cognitive decline [Jessen et al, 2014; Reed, 2010; Earles \& Salthouse, 1995].

Furthermore, it may have been more appropriate to ask about perceived visual attention function instead of memory function, after all it was visual attention being examined. This could observe whether peoples' perceived changes in visual attention reflects actual slowing of visual attention. People do not tend to understand slowing of visual attention function for what it is but if given scenarios, they would be able to report any difficulty, thus understand the types of daily struggles which constitute the slowing of visual attention.

\subsection{Future directions}

This research has the potential for expansion and further analysis firstly to measure other areas of visual attention i.e. perception, divided attention using the same novel methodology to observe whether similar differences are observed in information processing speed during ageing and whether RT in these areas are influenced by 
subjective measures or other factors such as perceived test difficulty, sex and education. Furthermore, further analysis may include additional factors such as sleep and occupation, which have been discovered to influence information processing speed as mentioned earlier. Measuring impulsitivity would also be beneficial, particularly for the young adults to observe whether this was a significant influence on their faster information processing.

As discussed throughout the current research, the young adults tend to make more errors and it was suggested that this may be due to them being more impulsive with their responses. It would be interesting to measure eye saccades through eye tracking particularly during the visual search to examine exactly where participants are orienting their attention and observe whether distractors are indeed inhibiting attention to a target directly. Furthermore, including brain scans would help provide a better idea of any structural and functional impairment to the brain and how this reflects in information processing speed. Further research would benefit from separating the older adults into decades in order to compare them to each other. Tombaugh [2004] measured information processing speed during the TMT in older adults by decade and RT increased as they got older in both Trails A and Trails B which may be true for other visual attention tests. Additionally, improving the generalizability of the sample would involve expanding the young adult population to include non university students of the same age and include a greater number of males in both young and older adult groups due to the current sample being female biased which may explain sex effects.

In order to measure education effects more thoroughly, years of education may benefit from being split between high and low levels of education i.e. up to 11 years vs. 11 years onwards reflecting compulsory and further education [Verhage, 1965]. However, there is an issue with splitting education in this way as in young adults there would be a poor ratio of low to high education levels in order to make a comparison due to the few years of further education young adults have reached compared to older adults [Tun et al, 2008]. This was especially true for the current study as the young adult population were not as advanced in years of further education compared to the older adult population. Furthermore, the ration of low and high education was also poor in older adults due to there being significantly more highly educated therefore making it difficult to make a comparison with lower levels of education. Perhaps it may be better to compare education levels between age groups rather than within age groups. 
It may also be useful to split MFQ scores (no concerns with memory vs. high concern) and correlate with information processing speed, seeing as correlating total MFQ found no relationship across tests. This would require recruiting additional older adults to achieve a better ratio of individuals with no concerns and individuals with high concerns.

A longitudinal study of same people tested in this research would observe any further decline on attentional function and what effect would that have on information processing speed and IIV. Currently, visual attention appears to decline naturally in community dwelling adults therefore it would be useful to explore the relationship between information processing speed and IIV, subjective memory function, perceived test difficulty, sex and education [i.e. using the same parameters] in individuals clinically diagnosed with SCI, to observe whether differences in slowing at this stage can characterize between slowing in healthy ageing and slowing in potential neurodegenerative change. 


\subsection{REFERENCES}

Adam, J. J., Paas, F. G., Buekers, M. J., Wuyts, I. J., Spijkers, W. A., \& Wallmeyer, P. (1999). Gender differences in choice reaction time: evidence for differential strategies. Ergonomics, 42(2), 327-335. doi:10.1080/001401399185685

Altena, E., Ramautar, J. R., Van Der Werf, Y. D., \& Van Someren, E. J. W. (2010). Do sleep complaints contribute to age-related cognitive decline? Progress in brain research, 185, 181-205. doi:10.1016/b978-0-444-53702-7.00011-7

Alzhiemer's Society. (2017). What is dementia? Retrieved on $6^{\text {th }}$ November 2017 at: https://www.alzheimers.org.uk/info/20007/types_of_dementia/1/what_is_dementia

Alzheimer's Association. (2017). Alzheimer's Disease Facts and Figures. Alzheimers Dement, 13, 325-373.

Amar, K., Bucks, R. S., Lewis, T., Scott, M., \& Wilcock, G. K. (1996). The effect of white matter low attenuation on cognitive performance in dementia of the Alzheimer type. Age and ageing, 25(6), 443-448.doi: https://doi.org/10.1093/ageing/25.6.443

American Psychiatric Association. (2013). Diagnostic and statistical manual of mental disorders (DSM-5®). American Psychiatric Pub.

Amodio, P., Wenin, H., Del Piccolo, F., Mapelli, D., Montagnese, S., Pellegrini, A., . . Umiltà, C. (2002). Variability of Trail Making Test, Symbol Digit Test and Line Trait Test in normal people. A normative study taking into account age-dependent decline and sociobiological variables. Aging Clinical and Experimental Research, 14(2), 117-131. doi:10.1007/BF03324425

Andres, P. (2003). Frontal cortex as the central executive of working memory: time to revise our view. Cortex, 39(4-5), 871-895. doi: https://doi.org/10.1016/S00109452(08)70868-2

Annett, M., \& Annett, J. (1979). Individual differences in right and left reaction time. British Journal of Psychology, 70(3), 393-404. doi: 10.1111/j.20448295.1979.tb01709.x

Ansell, E. L., \& Bucks, R. S. (2006). Mnemonic anosognosia in Alzheimer's disease: a test of Agnew and Morris (1998). Neuropsychologia, 44(7), 1095-1102.

doi:10.1016/j.neuropsychologia.2005.10.019

Anstey, K. J., Mack, H. A., Christensen, H., Li, S. C., Reglade-Meslin, C., Maller, J., ... \& Sachdev, P. (2007). Corpus callosum size, reaction time speed and variability in mild cognitive disorders and in a normative sample. Neuropsychologia, 45(8), 19111920.doi: 10.1016/j.neuropsychologia.2006.11.020 
Arbuthnott, K., \& Frank, J. (2000). Trail Making Test, Part B as a Measure of Executive Control: Validation Using a Set-Switching Paradigm. Journal of Clinical and Experimental Neuropsychology, 22(4), 518-528. doi:10.1076/13803395(200008)22:4;1-0;FT518

Ardila, A. (1998). A Note of Caution: Normative Neuropsychological Test Performance: Effects of Age, Education, Gender and Ethnicity: A Comment on Saykin et al. (1995). Applied Neuropsychology, 5(1), 51-53. doi:10.1207/s15324826an0501_8

Arguin, M., Joanette, Y., \& Cavanagh, P. (1993). Visual search for feature and conjunction targets with an attention deficit. Journal of Cognitive Neuroscience 5(4): 436-452.

Arnold, S.E., Hyman, B. T., Flory, T., Damasio, A.R., \& Van Hoesen, G.W. (1991). The Topographical and Neuroanatomical Distribution of Neurofibrillary Tangles and Neuritic Plaques in the Cerebral Cortex of Patients with Alzheimer's Disease, Cerebral Cortex, 1(1), 103-116, https://doi.org/10.1093/cercor/1.1.103

Arrington, C. M., Carr, T. H., Mayer, A. R., \& Rao, S. M. (2000). Neural mechanisms of visual attention: object-based selection of a region in space. Journal of cognitive neuroscience, 12(Supplement 2), 106-117.doi:

https://doi.org/10.1162/089892900563975

Ashendorf, L., Jefferson, A. L., O'Connor, M. K., Chaisson, C., Green, R. C., \& Stern, R. A. (2008). Trail Making Test errors in normal aging, mild cognitive impairment, and dementia. Archives of Clinical Neuropsychology, 23(2), 129-137. doi:10.1016/j.acn.2007.11.005

Babiloni, C., Frisoni, G., Steriade, M., Bresciani, L., Binetti, G., Del Percio, C., ... \& Zappasodi, F. (2006). Frontal white matter volume and delta EEG sources negatively correlate in awake subjects with mild cognitive impairment and Alzheimer's disease. Clinical Neurophysiology, 117(5), 1113-1129. doi: https://doi.org/10.1016/j.clinph.2006.01.020

Balash, Y., Mordechovich, M., Shabtai, H., Giladi, N., Gurevich, T., \& Korczyn, A. D. (2013). Subjective memory complaints in elders: depression, anxiety, or cognitive decline? Acta Neurologica Scandinavica, 127(5), 344-350. doi:10.1111/ane.12038

Ballesteros, S., Mayas, J., \& Reales, J. M. (2013). Cognitive function in normal aging and in older adults with mild cognitive impairment. Psicothema, 25(1), 1824.doi: $10.7334 /$ psicothema2012.181 
Barber, R., Scheltens, P., Gholkar, A., Ballard, C., McKeith, I., Ince, P., ... \& O'brien, J. (1999). White matter lesions on magnetic resonance imaging in dementia with Lewy bodies, Alzheimer's disease, vascular dementia, and normal aging. Journal of Neurology, Neurosurgery \& Psychiatry, 67(1), 66-72. doi: http://hdl.handle.net/1871/23028

Barker, A., Carter, C., \& Jones, R. (1994). Memory performance, self-reported memory loss and depressive symptoms in attenders at a GP-referral and a selfreferral memory clinic. International Journal of Geriatric Psychiatry, 9(4), 305-311. doi:10.1002/gps.930090407

Barnett, J. H., Blackwell, A. D., Sahakian, B. J., \& Robbins, T. W. (2016). The Paired Associates Learning (PAL) Test: 30 Years of CANTAB Translational Neuroscience from Laboratory to Bedside in Dementia Research. In T. W. Robbins \& B. J. Sahakian (Eds.), Translational Neuropsychopharmacology (pp. 449-474). Cham: Springer International Publishing.

Bartzokis, G., Cummings, J. L., Sultzer, D., Henderson, V. W., Nuechterlein, K. H., \& Mintz, J. (2003). White matter structural integrity in healthy aging adults and patients with Alzheimer disease - A magnetic resonance imaging study. Archives of Neurology, 60(3), 393-398. doi:10.1001/archneur.60.3.393

Baudic, S., Dalla Barba, G., Thibaudet, M. C., Smagghe, A., Remy, P., \& Traykov, L. (2006). Executive function deficits in early Alzheimer's disease and their relations with episodic memory. Archives of Clinical Neuropsychology, 21(1), 15-21. doi: https://doi.org/10.1016/j.acn.2005.07.002

Bayer, A., Phillips, M., Porter, G., Leonards, U., Bompas, A., \& Tales, A. (2014). Abnormal Journal of Alzheimers Disease, 40(1), 177-189. doi:10.3233/jad-131934

Begum, A., Morgan, C., Chiu, C.-C., Tylee, A., \& Stewart, R. (2012). Subjective memory impairment in older adults: aetiology, salience and help seeking. International Journal of Geriatric Psychiatry, 27(6), 612-620. doi:10.1002/gps.2760

Bendlin, B. B., Fitzgerald, M. E., Ries, M. L., Xu, G., Kastman, E. K., Thiel, B. W., ... \& Johnson, S. C. (2010). White matter in aging and cognition: a cross-sectional study of microstructure in adults aged eighteen to eighty-three. Developmental neuropsychology, 35(3), 257-277.doi: 10.1080/87565641003696775

Benton, A. (1986). Reaction time in brain disease: some reflections. Cortex, 22(1), 129-140.doi: https://doi.org/10.1016/S0010-9452(86)80037-5

Berggren, N., \& Derakshan, N. (2013). Attentional control deficits in trait anxiety: why you see them and why you don't. Biological Psychology, 92(3), 440-446. doi: https://doi.org/10.1016/j.biopsycho.2012.03.007 
Bezdicek, O., Motak, L., Axelrod, B. N., Preiss, M., Nikolai, T., Vyhnalek, M., .. . Ruzicka, E. (2012). Czech Version of the Trail Making Test: Normative Data and Clinical Utility. Archives of Clinical Neuropsychology, 27(8), 906-914. doi:10.1093/arclin/acs084

Bielak, A. A., Cherbuin, N., Bunce, D., \& Anstey, K. J. (2014). Intraindividual variability is a fundamental phenomenon of aging: evidence from an 8-year longitudinal study across young, middle, and older adulthood. Dev Psychol, 50(1), 143-151. doi:10.1037/a0032650

Bilello, M., Doshi, J., Nabavizadeh, S. A., Toledo, J. B., Erus, G., Xie, S. X., ... Davatzikos, C. (2015). Correlating Cognitive Decline with White Matter Lesion and Brain Atrophy MRI Measurements in Alzheimer's Disease. Journal of Alzheimer's Disease : JAD, 48(4), 987-994. http://doi.org/10.3233/JAD-150400

Binetti, G., Cappa, S. F., Magni, E., Padovani, A., Bianchetti, A., \& Trabucchi, M. (1998). Visual and spatial perception in the early phase of Alzheimer's disease. Neuropsychology, 12(1), 29-33. doi:10.1037//0894-4105.12.1.29

Binetti, G., Cappa, S. F., Magni, E., Padovani, A., Bianchetti, A., \& Trabucchi, M. (1996). Disorders of visual and spatial perception in the early stage of Alzheimer's disease. Neurobiology of Alzheimer's Disease, 777, 221-225. doi:10.1111/j.17496632.1996.tb34422.x

Birren, J. E. (1974). Translations in gerontology: From lab to life: Psychophysiology and speed of response. American Psychologist, 29(11), 808.doi http://dx.doi.org/10.1037/h0037433

Birren, J. E., Riegel, K. F., \& Morrison, D. F. (1962). Age differences in response speed as a function of controlled variations of stimulus conditions: evidence of a general speed factor. Gerontologia, 6, 1-18.doi: 10.1159/000211102

Blackwell, A. D., Barnett, J. H., Hayat, S., Luben, R., Moore, S., Dalzell, N., ... \& Brayne, C. (2010). The effect of age, sex and education on visuospatial paired associates learning ability: Preliminary data from a British population study. Alzheimer's \& Dementia: The Journal of the Alzheimer's Association, 6(4), S485.doi: https://doi.org/10.1016/j.jalz.2010.05.1617

Bleecker, M. L., Bolla-Wilson, K., Agnew, J., \& Meyers, D. A. (1987). Simple visual reaction time: Sex and age differences. Developmental neuropsychology, 3(2), 165-172. doi:10.1080/87565648709540372

Blough, P. M., \& Slavin, L. K. (1987). Reaction time assessments of gender differences in visual-spatial performance. Perception \& Psychophysics, 41(3), 276281.doi: http://dx.doi.org/10.3758/BF03208225 
Boll, T. J., \& Reitan, R. M. (1973). Effect of age on performance of the Trail Making Test. Percept Mot Skills, 36(3), 691-694.doi:

http://dx.doi.org/10.2466/pms.1973.36.3.691

Bolmont, B., Thullier, F., \& Abraini, J. H. (2000). Relationships between mood states and performances in reaction time, psychomotor ability, and mental efficiency during a 31-day gradual decompression in a hypobaric chamber from sea level to 8848 m equivalent altitude. Physiology \& behavior, 71(5), 469-476.doi: https://doi.org/10.1016/S0031-9384(00)00362-0

Bombois, S., Debette, S., Delbeuck, X., Bruandet, A., Lepoittevin, S., Delmaire, C., ... \& Pasquier, F. (2007). Prevalence of subcortical vascular lesions and association with executive function in mild cognitive impairment subtypes. Stroke, 38(9), 25952597.doi https://doi.org/10.1161/STROKEAHA.107.486407

Bornstein, R. A., \& Suga, L. J. (1988). Educational level and neuropsychological performance in healthy elderly subjects. Developmental neuropsychology, 4(1), 1722. doi: http://dx.doi.org/10.1080/87565648809540386

Borroni, B., Brambati, S., Agosti, C., \& et al. (2007). Evidence of white matter changes on diffusion tensor imaging in frontotemporal dementia. Archives of Neurology, 64(2), 246-251. doi:10.1001/archneur.64.2.246

Brébion, G. (2001). Language processing, slowing, and speed/accuracy trade-off in the elderly. Experimental Aging Research, 27(2), 137-150.doi:

https://doi.org/10.1080/036107301750073999

Breteler, M. M., van Amerongen, N. M., van Swieten, J. C., Claus, J. J., Grobbee, D. E., van Gijn, J., . . van Harskamp, F. (1994). Cognitive correlates of ventricular enlargement and cerebral white matter lesions on magnetic resonance imaging. The Rotterdam Study. Stroke, 25(6), 1109. doi: https://doi.org/10.1161/01.STR.25.6.1109

Brickman, A. M., Zahodne, L. B., Guzman, V. A., Narkhede, A., Meier, I. B., Griffith, E. Y., ... \& Luchsinger, J. A. (2015). Reconsidering harbingers of dementia: progression of parietal lobe white matter hyperintensities predicts Alzheimer's disease incidence. Neurobiology of aging, 36(1), 27-32.

https://doi.org/10.1016/j.neurobiolaging.2014.07.019

Brink, J. M., \& McDowd, J. M. (1999). Aging and selective attention: an issue of complexity or multiple mechanisms? The Journals of Gerontology Series B:

Psychological Sciences and Social Sciences, 54(1), 30-33. doi:

https://doi.org/10.1093/geronb/54B.1.P30

Brown, S., \& Heathcote, A. (2005). A ballistic model of choice response time. Psychological review, 112(1), 117.doi: 10.1037/0033-295X.112.1.117 
Bucur, B., Madden, D. J., Spaniol, J., Provenzale, J. M., Cabeza, R., White, L. E., \& Huettel, S. A. (2008). Age-related slowing of memory retrieval: contributions of perceptual speed and cerebral white matter integrity. Neurobiology of aging, 29(7), 1070-1079. doi: 10.1016/j.neurobiolaging.2007.02.008

Bugg, J. M., Zook, N. A., DeLosh, E. L., Davalos, D. B., \& Davis, H. P. (2006). Age differences in fluid intelligence: contributions of general slowing and frontal decline. Brain and Cognition, 62(1), 9-16. doi: https://doi.org/10.1016/j.bandc.2006.02.006

Bunce, D., Anstey, K. J., Christensen, H., Dear, K., Wen, W., \& Sachdev, P. (2007). White matter hyperintensities and within-person variability in community-dwelling adults aged 60-64 years. Neuropsychologia, 45(9), 2009-2015. https://doi.org/10.1016/j.neuropsychologia.2007.02.006

Burns, J. M., Church, J. A., Johnson, D. K., Xiong, C., Marcus, D., Fotenos, A. F., ... \& Buckner, R. L. (2005). White matter lesions are prevalent but differentially related with cognition in aging and early Alzheimer disease. Archives of Neurology, 62(12), 1870-1876. doi:10.1001/archneur.62.12.1870

Cangoz, B., Karakoc, E., \& Selekler, K. (2009). Trail Making Test: normative data for Turkish elderly population by age, sex and education. J Neurol Sci, 283(1-2), 7378. doi:10.1016/j.jns.2009.02.313

Capizzano, A. A., Acion, L., Bekinschtein, T., Furman, M., Gomila, H., Martinez, A., ... \& Starkstein, S. E. (2004). White matter hyperintensities are significantly associated with cortical atrophy in Alzheimer's disease. Journal of Neurology, Neurosurgery \& Psychiatry, 75(6), 822-827. http://dx.doi.org/10.1136/jnnp.2003.019273

Capurso, A., Panza, F., Solfrizzi, V., Torres, F., Capurso, C., Mastroianni, F., \& Del Parigi, A. (2000). Age-related cognitive decline: evaluation and prevention strategy. Recenti Progressi in Medicina, 91(3),127-34.

Caselli, R. J., Chen, K. W., Locke, D. E. C., Lee, W., Roontiva, A., Bandy, D., . . Reiman, E. M. (2014). Subjective cognitive decline: Self and informant comparisons. Alzheimers \& Dementia, 10(1), 93-98. doi:10.1016/j.jalz.2013.01.003

Caselli, R. J., Locke, D. E. C., Dueck, A. C., Knopman, D. S., Woodruff, B. K., Hoffman-Snyder, C., . . Reiman, E. M. (2014). The neuropsychology of normal aging and preclinical Alzheimer's disease. Alzheimers \& Dementia, 10(1), 84-92. doi:10.1016/j.jalz.2013.01.004

Cavaco, S., Gonçalves, A., Pinto, C., Almeida, E., Gomes, F., Moreira, I., ... \& Teixeira-Pinto, A. (2013). Trail Making Test: Regression-based norms for the Portuguese population. Archives of Clinical Neuropsychology, 28(2), 189-198.doi: https://doi.org/10.1093/arclin/acs115 
Cerella, J., Poon, L. W., \& Williams, D. M. (1980). Age and the complexity hypothesis. In L. W. Poon (Ed.), Aging in the 1980s: Psychological issues (pp. 332340).http://dx.doi.org/10.1037/10050-024

Chao, L. L., Pa, J., Duarte, A., Schuff, N., Weiner, M. W., Kramer, J. H., ... \& Johnson, J. K. (2009). Patterns of cerebral hypoperfusion in amnestic and dysexecutive MCI. Alzheimer disease and associated disorders, 23(3), 245.doi: 10.1097/WAD.0b013e318199ff46

Chau, S. A., Herrmann, N., Eizenman, M., Chung, J., \& Lanctôt, K. L. (2015). Exploring Visual Selective Attention towards Novel Stimuli in Alzheimer's Disease Patients. Dementia and geriatric cognitive disorders extra, 5(3), 492-502. doi: https://doi.org/10.1159/000442383

Charlton, R. A., Barrick, T. R., McIntyre, D. J., Shen, Y., O'Sullivan, M., Howe, F. A., . . Markus, H. S. (2006). White matter damage on diffusion tensor imaging correlates with age-related cognitive decline. Neurology, 66(2), 217-222. doi:10.1212/01.wnl.0000194256.15247.83

Chen, Z. G., Li, T. Q., \& Hindmarsh, T. (2001). Diffusion tensor trace mapping in normal adult brain using single-shot epi technique - A methodological study of the aging brain. Acta Radiologica, 42(5), 447-458. doi:10.1034/j.1600-

0455.2001.420504.x

Chen, P., Ratcliff, G., Belle, S. H., Cauley, J. A., DeKosky, S. T., \& Ganguli, M. (2000). Cognitive tests that best discriminate between presymptomatic AD and those who remain nondemented. Neurology, 55(12), 1847-1853. doi: http://dx.doi.org/10. 1212/WNL.55.12.1847

Cheng, Y. W., Chen, T. F., \& Chiu, M. J. (2017). From mild cognitive impairment to subjective cognitive decline: conceptual and methodological evolution.

Neuropsychiatr Dis Treat, 13, 491-498. doi:10.2147/ndt.s123428

Cherrier, M. M., Asthana, S., Plymate, S., Baker, L., Matsumoto, A. M., Peskind, E., ... \& LaTendresse, S. (2001). Testosterone supplementation improves spatial and verbal memory in healthy older men. Neurology, 57(1), 80-88.doi:

https://doi.org/10.1212/WNL.57.1.80

Christensen, H., Korten, A. E., Jorm, A. F., Henderson, A. S., Jacomb, P. A., Rodgers, B., \& Mackinnon, A. J. (1997). Education and decline in cognitive performance: compensatory but not protective. International Journal of Geriatric Psychiatry, 12(3), 323-330. doi: 10.1002/(SICI)1099-1166(199703)12:3<323::AIDGPS492>3.0.CO;2-N 
Chun, M.M., \& Wolfe, J.M. (2001). Visual Attention. In Goldstein B, editor. Handbook of perception. Oxford, UK: Blackwell Publishers Ltd, 272-310.

Clarnette, R. M., Almeida, O. P., Forstl, H., Paton, A., \& Martins, R. N. (2001). Clinical characteristics of individuals with subjective memory loss in Western Australia: Results from a cross-sectional survey. International Journal of Geriatric Psychiatry, 16(2), 168-174. doi:10.1002/1099-1166(200102)16:2<168::aidgps291>3.0.co;2-d

Cohn, N. B., Dustman, R. E., \& Bradford, D. C. (1984). Age-related decrements in stroop color test performance. Journal of clinical psychology, 40(5), 1244-1250. doi: 10.1002/1097-4679(198409)40:5<1244::AID-JCLP2270400521>3.0.CO;2-D

Colcombe, S. J., Kramer, A. F., Erickson, K. I., \& Scalf, P. (2005). The implications of cortical recruitment and brain morphology for individual differences in inhibitory function in aging humans. Psychology and Aging, 20(3), 363. doi: http://dx.doi.org/10.1037/0882-7974.20.3.363

Cole, C. S., Mennemeier, M., Bost, J. E., Smith-Olinde, L., \& Howieson, D. (2011). Measurement of Reaction Time in the Home for People With Dementia. Biological Research For Nursing, 15(2), 179-184. doi:10.1177/1099800411420862

Collerton, J., Collerton, D., Arai, Y., Barrass, K., Eccles, M., Jagger, C., . . . the Newcastle 85+ Study Core, T. (2007). A Comparison of Computerized and Penciland-Paper Tasks in Assessing Cognitive Function in Community-Dwelling Older People in the Newcastle 85+ Pilot Study. Journal of the American Geriatrics Society, 55(10), 1630-1635. doi:10.1111/j.1532-5415.2007.01379.x

Collins, D. W., \& Kimura, D. (1997). A large sex difference on a two-dimensional mental rotation task. Behav Neurosci, 111(4), 845-849. doi: 10.1037/07357044.111.4.845

Connelly, S. L., \& Hasher, L. (1993). Aging and the inhibition of spatial location. $J$ Exp Psychol Hum Percept Perform, 19(6), 1238-1250. doi: http://dx.doi.org/10.1037/0096-1523.19.6.1238

Cook, I. A., Leuchter, A. F., Morgan, M. L., Dunkin, J. J., Witte, E., David, S., ... \& Abrams, M. (2004). Longitudinal progression of subclinical structural brain disease in normal aging. The American journal of geriatric psychiatry, 12(2), 190-200. doi: https://doi.org/10.1097/00019442-200403000-00010

Cook, S., \& Mariske, M. (2006). Subjective memory beliefs and cognitive performance in normal and midly impaired older adults. Aging and Mental Health, 10(4), 413-423. doi: http://dx.doi.org/10.1080/13607860600638487 
Coombes, S. A., Higgins, T., Gamble, K. M., Cauraugh, J. H., \& Janelle, C. M. (2009). Attentional control theory: Anxiety, emotion, and motor planning. Journal of anxiety disorders, 23(8), 1072-1079.doi:

https://doi.org/10.1016/j.janxdis.2009.07.009

Cooper, C., Bebbington, P., Lindesay, J., Meltzer, H., McManus, S., Jenkins, R., \& Livingston, G. (2011). The meaning of reporting forgetfulness: a cross-sectional study of adults in the English 2007 Adult Psychiatric Morbidity Survey. Age and Ageing, 40(6), 711-717. doi:10.1093/ageing/afr121

Cox, A., Cairns, P., Shah, P., \& Carroll, M. (2012, May). Not doing but thinking: the role of challenge in the gaming experience. In Proceedings of the SIGCHI Conference on Human Factors in Computing Systems (pp. 79-88). ACM.doi: $10.1145 / 2207676.2207689$

Craik, F. I. M., Bialystok, E., \& Freedman, M. (2010). Delaying the onset of Alzheimer disease Bilingualism as a form of cognitive reserve. Neurology, 75(19), 1726-1729. doi:10.1212/WNL.0b013e3181fc2a1c

Craik, F. I. M., \& McDowd, J. M. (1987). Age-differences in recall and recognition. Journal of Experimental Psychology-Learning Memory and Cognition, 13(3), 474479. doi:10.1037//0278-7393.13.3.474

Crowe, S. F. (1998). The differential contribution of mental tracking, cognitive flexibility, visual search, and motor speed to performance on parts A and B of the Trail Making Test. J Clin Psychol, 54(5), 585-591. doi: 10.1002/(SICI)10974679(199808)54:5<585::AID-JCLP4>3.0.CO;2-K

Dalmaijer, E. S., Van der Stigchel, S., Nijboer, T. C. W., Cornelissen, T. H. W., \& Husain, M. (2015). CancellationTools: All-in-one software for administration and analysis of cancellation tasks. Behavior Research Methods, 47(4), 1065-1075. doi: 10.3758/s13428-014-0522-7.

Damoiseaux, J. S., Smith, S. M., Witter, M. R., Sanz-Arigita, E. J., Barkhof, F., Scheltens, P., . . Rombouts, S. (2009). White Matter Tract Integrity in Aging and Alzheimer's Disease. Human Brain Mapping, 30(4), 1051-1059. doi:10.1002/hbm.20563

Deary, I. J., \& Der, G. (2005). Reaction Time, Age, and Cognitive Ability: Longitudinal Findings from Age 16 to 63 Years in Representative Population Samples. Aging, Neuropsychology, and Cognition, 12(2), 187-215. doi:10.1080/13825580590969235

Deary, I. J., Der, G., \& Ford, G. (2001). Reaction times and intelligence differences: A population-based cohort study. Intelligence, 29(5), 389-399.

doi:https://doi.org/10.1016/S0160-2896(01)00062-9 
Deary, I. J., Liewald, D., \& Nissan, J. (2011). A free, easy-to-use, computer-based simple and four-choice reaction time programme: the Deary-Liewald reaction time task. Behavior Research Methods, 43(1), 258-268. doi:10.3758/s13428-010-0024-1

Defrancesco M, Egger K, Marksteiner J, Esterhammer R, Hinterhuber H, Deisenhammer EA, et al. (2014) Changes in White Matter Integrity before Conversion from Mild Cognitive Impairment to Alzheimer's Disease. PLoS ONE, 9(8): e106062. doi: https://doi.org/10.1371/journal.pone.0106062

Delacourte, A., David, J. P., Sergeant, N., Buee, L., Wattez, A., Vermersch, P., ... \& Petit, H. (1999). The biochemical pathway of neurofibrillary degeneration in aging and Alzheimer's disease. Neurology, 52(6), 1158-1158. doi: http://dx.doi.org/10. 1212/WNL.52.6.1158

Demakis, G. J. (2004). Frontal Lobe Damage and Tests of Executive Processing: A Meta-Analysis of the Category Test, Stroop Test, and Trail-Making Test. Journal of Clinical and Experimental Neuropsychology, 26(3), 441-450.

doi:10.1080/13803390490510149

Der, G., \& Deary, I. J. (2006). Age and sex differences in reaction time in adulthood: Results from the United Kingdom Health and Lifestyle Survey. Psychology and Aging, 21(1), 62-73. doi:10.1037/0882-7974.21.1.62

Desai, A. K., \& Schwartz, L. (2011). Subjective cognitive impairment: When to be concerned about 'senior moments'. Current Psychiatry, 10(4).

Dixon, R. A., Garrett, D. D., Lentz, T. L., MacDonald, S. W., Strauss, E., \& Hultsch, D. F. (2007). Neurocognitive markers of cognitive impairment: exploring the roles of speed and inconsistency. Neuropsychology, 21(3), 381.doi: 10.1037/08944105.21.3.381

Dodonova Y. A., Dodonov Y. S. (2013). Is there any evidence of historical slowing of reaction time? No, unless we compare apples and oranges. Intelligence $41674-$ 687.doi: https://doi.org/10.1016/j.intell.2013.09.001

Dodrill, C. B. (1979). Sex differences on the Halstead-Reitan Neuropsychological Battery and on other neuropsychological measures. Journal of clinical psychology, 35(2), 236-241. doi:10.1002/1097-4679(197904)35:2<236::AIDJCLP2270350202>3.0.CO;2-9

Drane, D. L., Yuspeh, R. L., Huthwaite, J. S., \& Klingler, L. K. (2002). Demographic characteristics and normative observations for derived-trail making test indices. Cognitive and Behavioral Neurology, 15(1), 39-43. 
Dykiert, D., Der, G., Starr, J. M., \& Deary, I. J. (2012). Age Differences in IntraIndividual Variability in Simple and Choice Reaction Time: Systematic Review and Meta-Analysis. Plos One, 7(10). doi:10.1371/journal.pone.0045759

Dykiert, D., Der, G., Starr, J. M., \& Deary, I. J. (2012). Sex differences in reaction time mean and intraindividual variability across the life span. Dev Psychol, 48(5), 1262-1276. doi:10.1037/a0027550

Earles, J. L., \& Salthouse, T. A. (1995). Interrelations of age, health, and speed. Journals of Gerontology Series B-Psychological Sciences and Social Sciences, 50(1), 33-41.doi: https://doi.org/10.1093/geronb/50B.1.P33

Edwards, J. D., Xu, H., Clark, D. O., Guey, L. T., Ross, L. A., \& Unverzagt, F. W. (2017). Speed of processing training results in lower risk of dementia. Alzheimer's \& Dementia: Translational Research \& Clinical Interventions, 3(4), 603-611.doi: https://doi.org/10.1016/j.trci.2017.09.002

Emerson, C. S., Mollet, G. A., \& Harrison, D. W. (2005). Anxious-depression in boys: an evaluation of executive functioning. Archives of Clinical Neuropsychology, 20(4), 539-546. doi:http://dx.doi.org/10.1016/j.acn.2004.10.003

Eriksen, B. A., \& Eriksen, C. W. (1974). Effects of noise letters upon the identification of a target letter in a nonsearch task. Perception \& psychophysics, 16(1), 143-149.doi: http://dx.doi.org/10.3758/BF03203267

Fan, J., McCandliss, B. D., Fossella, J., Flombaum, J. I., \& Posner, M. I. (2005). The activation of attentional networks. Neuroimage, 26(2), 471-479.

doi:https://doi.org/10.1016/j.neuroimage.2005.02.004

Faust, M. E., \& Balota, D. A. (1997). Inhibition of return and visuospatial attention in healthy older adults and individuals with dementia of the Alzheimer type.

Neuropsychology, 11(1), 13-29. doi:10.1037/0894-4105.11.1.13

Feller, S. (2016). First brain region affected by Alzheimer's disease identified, retrieved on $28^{\text {th }}$ March 2017 at

https://www.upi.com/Health_News/2016/02/17/First-brain-region-affected-byAlzheimers-disease-identified/4191455730408/

Fernaeus, S. E., Östberg, P., \& Wahlund, L. O. (2013). Late reaction times identify MCI. Scandinavian journal of psychology, 54(4), 283-285.doi: 10.1111/sjop.12053

Fernandez-Duque, D., \& Black, S. E. (2006). Attentional networks in normal aging and Alzheimer's disease. Neuropsychology, 20(2), 133. doi:

http://dx.doi.org/10.1037/0894-4105.20.2.133 
Fernandez-Duque, D., \& Posner, M. I. (1997). Relating the mechanisms of orienting and alerting. Neuropsychologia, 35(4), 477-486.doi: https://doi.org/10.1016/S00283932(96)00103-0

Ferreira, D., Molina, Y., Machado, A., Westman, E., Wahlund, L. O., Nieto, A., . . . Barroso, J. (2014). Cognitive decline is mediated by gray matter changes during middle age. Neurobiol Aging, 35(5), 1086-1094.

doi:10.1016/j.neurobiolaging.2013.10.095

Ferri, C.P., Pronce, M., Brayne, C., Brodaty, H.,Fratiglioni, L., Ganguli, M., Hall, K., Hasegawa, K., Hendrie, H., Huang, Y., Jorm, A., Mathers, C., Menedez, P.R., Rimmer, E., \& Scazufca, M. (2006). Global prevalence of dementia: a Delphi consensus study. The Lancet, 366 (9503), 2112-2117. doi: https://doi.org/10.1016/S0140-6736(05)67889-0

Festa-Martino, E., Ott, B. R., \& Heindel, W. C. (2004). Interactions between phasic alerting and spatial orienting: effects of normal aging and Alzheimer's disease. Neuropsychology, 18(2), 258. doi: http://dx.doi.org/10.1037/0894-4105.18.2.258

Finke, K., Matthias, E., Keller, I., Müller, H. J., Schneider, W. X., \& Bublak, P. (2012). How does phasic alerting improve performance in patients with unilateral neglect? A systematic analysis of attentional processing capacity and spatial weighting mechanisms. Neuropsychologia, 50(6), 1178-1189.

doi: $10.1098 /$ rstb.2013.0054

Flavell, J. H. (1979). Metacognition and cognitive monitoring: A new area of cognitive-developmental inquiry. American psychologist, 34(10), 906.doi@ http://dx.doi.org/10.1037/0003-066X.34.10.906

Flicker, C., Bartus, R. T., Crook, T. H., \& Ferris, S. H. (1984). Effects of aging and dementia upon recent visuospatial memory. Neurobiology of Aging, 5(4), 275-283. doi:10.1016/0197-4580(84)90003-4

Fontani, G., Lodi, L., Felici, A., Corradeschi, F., \& Lupo, C. (2004). Attentional, emotional and hormonal data in subjects of different ages. European Journal of Applied Physiology, 92, 452- 461. doi:10.1007/ s00421-004-1108-3

Foroozandeh, E. (2014). Gender Differences in Trail Making Test Performance in a Nonclinical Sample of Adults. International Journal of Clinical and Experimental Neurology, 2(1), 1-3. DOI: 10.12691/ijcen-2-1-1

Foster, J. K., Behrmann, M., \& Stuss, D. T. (1999). Visual attention deficits in Alzheimer's disease: Simple versus conjoined feature search. Neuropsychology, 13(2), 223-245. doi: http://dx.doi.org/10.1037/0894-4105.13.2.223 
Fozard, J. L., Vercruyssen, M., Reynolds, S. L., Hancock, P. A., \& Quilter, R. E. (1994). Age Differences and Changes in Reaction Time: The Baltimore Longitudinal Study of Aging. Journal of gerontology, 49(4), P179-P189.

doi:10.1093/geronj/49.4.P179

Fraser, S., \& Bherer, L. (2013). Age-related decline in divided-attention: from theoretical lab research to practical real-life situations. Wiley Interdisciplinary Reviews: Cognitive Science, 4(6), 623-640. doi:10.1002/wcs.1252

Frederikse, M. E., Lu, A., Aylward, E., Barta, P., \& Pearlson, G. (1999). Sex Differences in the Inferior Parietal Lobule. Cerebral Cortex, 9(8), 896-901.doi: https://doi.org/10.1093/cercor/9.8.896

Frick, A., Engman, J., Alaie, I., Björkstrand, J., Faria, V., Gingnell, M., ... \& Morell, A. (2014). Enlargement of visual processing regions in social anxiety disorder is related to symptom severity. Neuroscience letters, 583, 114-119.doi: https://doi.org/10.1016/j.neulet.2014.09.033

Gagnon, K., Baril, A. A., Gagnon, J. F., Fortin, M., Decary, A., Lafond, C., . . . Gosselin, N. (2014). Cognitive impairment in obstructive sleep apnea. Pathologie Biologie, 62(5), 233-240. doi:10.1016/j.patbio.2014.05.015

Gale, C. R., Harris, A., \& Deary, I. J. (2016). Reaction time and onset of psychological distress: the UK Health and Lifestyle Survey. Journal of Epidemiology and Community Health, 70(8), 813-817. doi:10.1136/jech-2015-206479

Garcia-Ptacek, S., Cavallin, L., Kåreholt, I., Kramberger, M. G., Winblad, B., Jelic, V., \& Eriksdotter, M. (2014). Subjective Cognitive Impairment Subjects in Our Clinical Practice. Dementia and Geriatric Cognitive Disorders EXTRA, 4(3), 419430. http://doi.org/10.1159/000366270

Garrett, D. D., MacDonald, S. W., \& Craik, F. I. (2012). Intraindividual reaction time variability is malleable: feedback-and education-related reductions in variability with age. Frontiers in human neuroscience, 6. Doi:

https://doi.org/10.3389/fnhum.2012.00101

Geary, D. C. (1998). Male, female: The evolution of human sex differences. American Psychological Association.doi: http://dx.doi.org/10.1037/10370-000

Geerlings, M. I., Jonker, C., Bouter, L. M., Ader, H. J., \& Schmand, B. (1999). Association between memory complaints and incident Alzheimer's disease in elderly people with normal baseline cognition. American Journal of Psychiatry, 156(4), 531537. doi: http://hdl.handle.net/1871/22432 
Genziani, M., Stewart, R., Bejot, Y., Amieva, H., Artero, S., \& Ritchie, K. (2013).

Subjective memory impairment, objective cognitive functioning and social activity in French older people: Findings from the Three Cities study. Geriatrics \& Gerontology International, 13(1), 139-145. doi:10.1111/j.1447-0594.2012.00873.x

Georgsson, M., \& Staggers, N. (2016). Quantifying usability: an evaluation of a diabetes mHealth system on effectiveness, efficiency, and satisfaction metrics with associated user characteristics. J Am Med Inform Assoc, 23(1), 5-11.

doi:10.1093/jamia/ocv099

Gibson, G. E., \& Peterson, C. (1981). Aging decreases oxidative metabolism and the release and synthesis of acetylcholine. Journal of neurochemistry, 37(4), 978984.doi: 10.1111/j.1471-4159.1981.tb04484.x

Giebel, C. M., Challis, D. J., \& Montaldi, D. (2016). A revised interview for deterioration in daily living activities in dementia reveals the relationship between social activities and well-being. Dementia (London), 15(5), 1068-1081.

doi:10.1177/1471301214553614

Gilewski, M. J., Zelinski, E. M., \& Schaie, K. W. (1990). The Memory Functioning Questionnaire for assessment of memory complaints in adulthood and old age. Psychology and aging, 5(4), 482.doi: http://dx.doi.org/10.1037/08827974.5.4.482

Gilmore, G. C., Wenk, H. E., Naylor, L. A., \& Koss, E. (1994). Motion perception and Alzheimer's disease. Journal of Gerontology, 49(2), 52-57.doi: https://doi.org/10.1093/geronj/49.2.P52

Gifford, K. A., Liu, D. D., Damon, S. M., Chapman, W. G., Romano, R. R., Samuels, L. R., . . Alzheimer's Dis Neuroimaging, I. (2015). Subjective Memory Complaint Only Relates to Verbal Episodic Memory Performance in Mild Cognitive Impairment. Journal of Alzheimers Disease, 44(1), 309-318. doi:10.3233/jad-140636

Giovagnoli, A. R., Del Pesce, M., Mascheroni, S., Simoncelli, M., Laiacona, M., \& Capitani, E. (1996). Trail making test: normative values from 287 normal adult controls. The Italian journal of neurological sciences, 17(4), 305-309.doi: 10.1007/BF01997792

Glodzik-Sobanska, L., Reisberg, B., De Santi, S., Babb, J. S., Pirraglia, E., Rich, K. E., . . de Leon, M. J. (2007). Subjective Memory Complaints: Presence, Severity and Future Outcome in Normal Older Subjects. Dementia and Geriatric Cognitive Disorders, 24(3), 177-184.doi: http://dx.doi.org/10.1159/000105604 
Good, C. D., Johnsrude, I. S., Ashburner, J., Henson, R. N. A., Friston, K. J., \& Frackowiak, R. S. J. (2001). A voxel-based morphometric study of ageing in 465 normal adult human brains. Neuroimage, 14(1), 21-36. doi:10.1006/nimg.2001.0786

Gootjes, L., Bouma, A., Van Strien, J. W., Van Schijndel, R., Barkhof, F., \& Scheltens, P. (2006). Corpus callosum size correlates with asymmetric performance on a dichotic listening task in healthy aging but not in Alzheimer's disease. Neuropsychologia, 44(2), 208-217.doi: 10.1016/j.neuropsychologia.2005.05.002

Gordon, B. A., Zacks, J. M., Blazey, T., Benzinger, T. L. S., Morris, J. C., Fagan, A. M., . . Balota, D. A. (2015). Task-evoked fMRI changes in attention networks are associated with preclinical Alzheimer's disease biomarkers. Neurobiology of Aging, 36(5), 1771-1779. doi:http://dx.doi.org/10.1016/j.neurobiolaging.2015.01.019

Gorus, E., De Raedt, R., Lambert, M., Lemper, J.-C., \& Mets, T. (2008). Reaction Times and Performance Variability in Normal Aging, Mild Cognitive Impairment, and Alzheimer's Disease. Journal of Geriatric Psychiatry and Neurology, 21(3), 204218. doi:10.1177/0891988708320973

Grady, C. L., Haxby, J. V., Horwitz, B., Gillette, J., Salerno, J. A., Gonzalez-Aviles, A., . . Rapoport, S. I. (1993). Activation of cerebral blood flow during a visuoperceptual task in patients with Alzheimer-type dementia. Neurobiology of aging, 14(1), 35-44. doi: http://dx.doi.org/10.1016/0197-4580(93)90018-7

Green, C. S., \& Bavelier, D. (2006). Effect of action video games on the spatial distribution of visuospatial attention. Journal of experimental psychology: Human perception and performance, 32(6), 1465.doi: 10.1037/0096-1523.32.6.1465

Greenwood, P. M., Parasuraman, R., \& Alexander, G. E. (1997). Controlling the focus of spatial attention during visual search: effects of advanced aging and Alzheimer disease. Neuropsychology, 11(1), 3. doi: http://dx.doi.org/10.1037/08944105.11.1.3

Greenwood, P. M., Parasuraman, R., \& Haxby, J. V. (1993). Changes in visuospatial attention over the adult lifespan. Neuropsychologia, 31(5), 471-485.doi: https://doi.org/10.1016/0028-3932(93)90061-4

Grieve, S. M., Williams, L. M., Paul, R. H., Clark, C. R., \& Gordon, E. (2007). Cognitive aging, executive function, and fractional anisotropy: a diffusion tensor MR imaging study. American Journal of Neuroradiology, 28(2), 226-235.doi:

de Groot, J. C., de Leeuw, F.-E., Oudkerk, M., van Gijn, J., Hofman, A., Jolles, J., \& Breteler, M. M. B. (2000). Cerebral white matter lesions and cognitive function: The Rotterdam scan study. Annals of Neurology, 47(2), 145-151. doi: 10.1002/15318249(200002)47:2<145::AID-ANA3>3.0.CO;2-P 
Grut, M., Jorm, A. F., Fratiglioni, L., Forsell, Y., Viitanen, M., \& Winblad, B. (1993). Memory complaints of elderly people in a population survey: variation according to dementia stage and depression. J Am Geriatr Soc, 41(12), 12951300.doi: 10.1111/j.1532-5415.1993.tb06478.x

Guest, D., Howard, C. J., Brown, L. A., \& Gleeson, H. (2015). Aging and the rate of visual information processing. Journal of Vision, 15(14), 10-10.

doi:10.1167/15.14.10

Guttmann, C. R. G., Jolesz, F. A., Kikinis, R., Killiany, R. J., Moss, M. B., Sandor, T., \& Albert, M. S. (1998). White matter changes with normal aging. Neurology, 50(4), 972-978.doi: http://dx.doi.org/10.1212/WNL.50.4.972

Hagen, K., Ehlis, A. C., Haeussinger, F. B., Heinzel, S., Dresler, T., Mueller, L. D., . . Metzger, F. G. (2014). Activation during the Trail Making Test measured with functional near-infrared spectroscopy in healthy elderly subjects. Neuroimage, $85 \mathrm{Pt}$ 1, 583-591. doi:10.1016/j.neuroimage.2013.09.014

Hainaut, J. P., \& Bolmont, B. (2006). Moderate state-anxiety differently modulates visual and auditory response times in normal-and very low trait-anxiety subjects. Neuroscience letters, 395(2), 129-132.doi: https://doi.org/10.1016/j.neulet.2005.10.071

Hamdan, A. C., \& Hamdan, E. M. L. R. (2009). Effects of age and education level on the Trail Making Test in A healthy Brazilian sample. Psychology \& Neuroscience, 2, 199-203. doi: http://dx.doi.org/10.3922/j.psns.2009.2.012

Hampson, E. (1990). Variations in sex-related cognitive abilities across the menstrual cycle. Brain and Cognition, 14, 26 - 43. doi:10.1016/0278- 2626(90)90058-V

Han, S., Jiang, Y., Gu, H., Rao, H., Mao, L., Cui, Y., \& Zhai, R. (2004). The role of human parietal cortex in attention networks. Brain, 127(Pt 3), 650-659.

doi:10.1093/brain/awh071

Hardy, J., \& Selkoe, D. J. (2002). The amyloid hypothesis of Alzheimer's disease: progress and problems on the road to therapeutics. Science, 297(5580), 353-356. doi: 10.1126/science. 1072994

Harpur, L. L., Scialfa, C. T., \& Thomas, D. M. (1995). Age Differences in Feature Search as a Function of Exposure Duration. Experimental Aging Research, 21(1), 115. doi:10.1080/03610739508254264

Hartley, A. A. (1993). Evidence for the selective preservation of spatial selective attention in old age. Psychology and Aging, 8(3), 371. doi:

http://dx.doi.org/10.1037/0882-7974.8.3.371 
Hashimoto, R., Meguro, K., Lee, E., Kasai, M., Ishii, H., \& Yamaguchi, S. (2006). Effect of age and education on the Trail Making Test and determination of normative data for Japanese elderly people: the Tajiri Project. Psychiatry Clin Neurosci, 60(4), 422-428. doi:10.1111/j.1440-1819.2006.01526.x

Haworth, J., Phillips, M., Newson, M., Rogers, P. J., Torrens-Burton, A., \& Tales, A. (2016). Measuring information processing speed in mild cognitive impairment:

Clinical versus research dichotomy. Journal of Alzheimer's Disease, 51(1), 263-275. doi: 10.3233/JAD-150791

Head, D., Jackson, J., Balota, D., \& Duchek, J. (2011). White matter integrity and intraindividual variability in aging and early-stage Alzheimer disease. Alzheimer's \& Dementia: The Journal of the Alzheimer's Association, 7(4), S736.

doi: http://dx.doi.org/10.1016/j.jalz.2011.05.2119

Hensel, A., Wolf, H., Kruggel, F., Riedel-Heller, S. G., Nikolaus, C., Arendt, T., \& Gertz, H. J. (2002). Morphometry of the corpus callosum in patients with questionable and mild dementia. Journal of Neurology, Neurosurgery \& Psychiatry, 73(1), 59-61.doi: 10.1136/jnnp.73.1.59

Herholz, K., Weisenbach, S., \& Kalbe, E. (2008). Deficits of the cholinergic system in early AD. Neuropsychologia, 46(6), 1642-1647.

doi:http://dx.doi.org/10.1016/j.neuropsychologia.2007.11.024

Hill, N., Mogle, J., Kitko, L., Gilmore-Bykovskyi, A., Wion, R., Kitt-Lewis, E., \& Kolanowski, A. (2017). Incongruence of Subjective Memory Impairment Ratings and the Experience of Memory Problems in Older Adults Without Dementia: A Mixed Methods Study. Aging \& Mental Health, 1-8, doi:

10.1080/13607863.2017.1337715

Hommel, B., Li, K. Z., \& Li, S. C. (2004). Visual search across the life span. Developmental Psychology, 40(4), 545.doi: 10.1037/0012-1649.40.4.545

Hoogendijk, W. J., Feenstra, M. G., Botterblom, M. H., Gilhuis, J., Sommer, I. E., Kamphorst, W., ... \& Swaab, D. F. (1999). Increased activity of surviving locus ceruleus neurons in Alzheimer's disease. Annals of neurology, 45(1), 82-91.doi: 10.1002/1531-8249(199901)45:1<82::AID-ART14>3.0.CO;2-T

Horowitz, T. S., \& Thornton, I. M. (2008). Objects or locations in vision for action? Evidence from the MILO task. Visual cognition, 16(4), 486-513. doi: $10.1080 / 13506280601087356$

Houx, P. J., \& Jolles, J. (1993). Age-Related Decline of Psychomotor Speed: Effects of Age, Brain Health, Sex, and Education. Percept Mot Skills, 76(1), 195-211. doi:10.2466/pms.1993.76.1.195 
Huang, J., \& Auchus, A. P. (2007). Diffusion Tensor Imaging of Normal Appearing White Matter and Its Correlation with Cognitive Functioning in Mild Cognitive Impairment and Alzheimer's Disease. Annals of the New York Academy of Sciences, 1097(1), 259-264. doi:10.1196/annals.1379.021

Huang, Y. F., \& Hsieh, P. J. (2013). The mere exposure effect is modulated by selective attention but not visual awareness. Vision research, 91, 56-61.doi: https://doi.org/10.1016/j.visres.2013.07.017

Hultsch, D. F., MacDonald, S. W. S., \& Dixon, R. A. (2002). Variability in reaction time performance of younger and older adults. Journals of Gerontology Series BPsychological Sciences and Social Sciences, 57(2), P101P115.doi:https://doi.org/10.1093/geronb/57.2.P101

Hultsch, D. F., MacDonald, S. W. S., Hunter, M. A., Levy-Bencheton, J., \& Strauss, E. (2000). Intraindividual variability in cognitive performance in older adults: Comparison of adults with mild dementia, adults with arthritis, and healthy adults. Neuropsychology, 14(4), 588-598.http://dx.doi.org/10.1037/08944105.14.4.588

Hyman, B. T., Van Hoesen, G. W., Kromer, L. J., \& Damasio, A. R. (1986). Perforant pathway changes and the memory impairment of Alzheimer's disease. Annals of neurology, 20(4), 472-481.doi: 10.1002/ana.410200406

Inui, N. (1997). Simple reaction times and timing of serial reactions of middle-aged and old men. Percept Mot Skills, 84(1), 219-225. doi:10.2466/pms.1997.84.1.219

Jacinto, A. F., Brucki, S. M., Porto, C. S., de Arruda Martins, M., \& Nitrini, R. (2014). Subjective memory complaints in the elderly: a sign of cognitive impairment? Clinics (Sao Paulo), 69(3), 194-197. doi:10.6061/clinics/2014(03)09

Jackson, J. D., Balota, D. A., Duchek, J. M., \& Head, D. (2012). White matter integrity and reaction time intraindividual variability in healthy aging and early-stage Alzheimer disease. Neuropsychologia, 50(3), 357-366. doi: https://doi.org/10.1016/j.neuropsychologia.2011.11.024

Jenkins, A., Lindsay, S., Eslambolchilar, P., Thornton, I. M., \& Tales, A. (2016). Administering Cognitive Tests Through Touch Screen Tablet Devices: Potential Issues. Journal of Alzheimer's Disease(Preprint), 1-14.

Jentzsch, I., Leuthold, H., \& Ulrich, R. (2007). Decomposing sources of response slowing in the PRP paradigm. Journal of Experimental Psychology: Human Perception and Performance, 33(3), 610-626.doi: http://dx.doi.org/10.1037/00961523.33.3.610 
Jessen, F., Amariglio, R. E., van Boxtel, M., Breteler, M., Ceccaldi, M., Chetelat, G., ... Grp, S. I. W. (2014). A conceptual framework for research on subjective cognitive decline in preclinical Alzheimer's disease. Alzheimers \& Dementia, 10(6), 844-852. doi:10.1016/j.jalz.2014.01.001

Jessen, F., Wiese, B., Bachmann, C., Eifflaender-Gorfer, S., Haller, F., Koelsch, H., . .. German Study Aging, C. (2010). Prediction of Dementia by Subjective Memory Impairment Effects of Severity and Temporal Association With Cognitive Impairment. Archives of General Psychiatry, 67(4), 414-422.

Jessen, F., Wolfsgruber, S., Wiese, B., Bickel, H., Moesch, E., Kaduszkiewicz, H., . . . Dem. (2014). AD dementia risk in late MCI, in early MCI, and in subjective memory impairment. Alzheimers \& Dementia, 10(1), 76-83. doi:10.1016/j.jalz.2012.09.017

Johannsen, P., Jakobsen, J., Bruhn, P., \& Gjedde, A. (1999). Cortical responses to sustained and divided attention in Alzheimer's disease. Neuroimage, 10(3), 269281.doi: https://doi.org/10.1006/nimg.1999.0475

Johnson, D. K., Barrow, W., Anderson, R., Harsha, A., Honea, R., Brooks, W. M., \& Burns,J. M. (2010). Diagnostic utility of cerebral white matter integrity in early Alzheimer's disease. International Journal of Neuroscience, 120(8), 544-550.doi: https://doi.org/10.3109/00207454.2010.494788

Johnstone, B., Hogg, J. R., Schopp, L. H., Kapila, C., \& Edwards, S. (2002). Neuropsychological deficit profiles in senile dementia of the Alzheimer's type. Archives of Clinical Neuropsychology, 17(3), 273-281.doi: https://doi.org/10.1016/S0887-6177(01)00112-3

Juncos-Rabadan, O., Pereiro, A. X., Facal, D., Reboredo, A., \& Lojo-Seoane, C. (2014). Do the Cambridge Neuropsychological Test Automated Battery episodic memory measures discriminate amnestic mild cognitive impairment? Int J Geriatr Psychiatry, 29(6), 602-609. doi:10.1002/gps.4042

Junkkila, J., Oja, S., Laine, M., \& Karrasch, M. (2012). Applicability of the CANTAB-PAL Computerized Memory Test in Identifying Amnestic Mild Cognitive Impairment and Alzheimer's Disease. Dementia and Geriatric Cognitive Disorders, 34(2), 83-89. doi: 10.1159/000342116

Kalbe, E., Salmon, E., Perani, D., Holthoff, V., Sorbi, S., Elsner, A., . . Herholz, K. (2005). Anosognosia in Very Mild Alzheimer's Disease but Not in Mild Cognitive Impairment. Dementia and Geriatric Cognitive Disorders, 19(5-6), 349-356. 
Kälin, A. M., Pflüger, M., Gietl, A. F., Riese, F., Jäncke, L., Nitsch, R. M., \& Hock, C. (2014). Intraindividual variability across cognitive tasks as a potential marker for prodromal Alzheimer's disease. Frontiers in aging neuroscience, 6.

doi: 10.3389/fnagi.2014.00147

Kang, J. I., Huppé-Gourgues, F., \& Vaucher, E. (2014). Boosting visual cortex function and plasticity with acetylcholine to enhance visual perception. Frontiers in systems neuroscience, 8. doi: 10.3389/fnsys.2014.00172

Karia, R. M., Ghuntla, T. P., Mehta, H. B., Gokhale, P. A., \& Shah, C. J. (2012). Effect of gender difference on visual reaction time: A study on medical students of Bhavnagar region. IOSR Journal of Pharmacy, 2(3), 452-454.

Kelly, A. C., Uddin, L. Q., Biswal, B. B., Castellanos, F. X., \& Milham, M. P. (2008). Competition between functional brain networks mediates behavioral variability. Neuroimage, 39(1), 527-537.doi:

https://doi.org/10.1016/j.neuroimage.2007.08.008

Kennedy, K. M., \& Raz, N. (2009). Aging white matter and cognition: differential effects of regional variations in diffusion properties on memory, executive functions, and speed. Neuropsychologia, 47(3), 916-927.doi:

https://doi.org/10.1016/j.neuropsychologia.2009.01.001

Kerchner, G. A., Racine, C. A., Hale, S., Wilheim, R., Laluz, V., Miller, B. L., \& Kramer, J. H. (2012). Cognitive Processing Speed in Older Adults: Relationship with White Matter Integrity. Plos One, 7(11). doi:10.1371/journal.pone.0050425

Keys, B. A., \& White, D. A. (2000). Exploring the relationship between age, executive abilities, and psychomotor speed. Journal of International Neuropsychological Society, 6(1), 76-82.doi: http://dx.doi.org/10.1017/S1355617700611098

Kirby, N. H., \& Nettelbeck, T. (1991). Speed of information-processing and age. Personality and Individual Differences, 12(2), 183-188. doi:10.1016/01918869(91)90101-g

Kiss, M., Grubert, A., Petersen, A., \& Eimer, M. (2012). Attentional capture by salient distractors during visual search is determined by temporal task demands. Journal of cognitive neuroscience, 24(3), 749-759.

doi: 10.1162/jocn_a_00127

Klinkenberg, I., Sambeth, A., \& Blokland, A. (2011). Acetylcholine and attention. Behav Brain Res, 221(2), 430-442. doi:10.1016/j.bbr.2010.11.033 
Kortte, K. B., Horner, M. D., \& Windham, W. K. (2002). The trail making test, part B: cognitive flexibility or ability to maintain set? Applied Neuropsychology, 9(2), 106-109.doi: 10.1207/S15324826AN0902_5

Kramer, A. F., Bherer, L., Colcombe, S. J., Dong, W., \& Greenough, W. T. (2004). Environmental influences on cognitive and brain plasticity during aging. The Journals of Gerontology Series A: Biological Sciences and Medical Sciences, 59(9), M940-M957.doi: https://doi.org/10.1093/gerona/59.9.M940

Krieg Jr, E. F., Chrislip, D. W., Letz, R. E., Otto, D. A., Crespo, C. J., Stephen Brightwell, W., \& Ehrenberg, R. L. (2001). Neurobehavioral test performance in the third National Health and Nutrition Examination Survey. Neurotoxicology and Teratology, 23(6), 569-589. doi:http://dx.doi.org/10.1016/S0892-0362(01)00177-5

Kroenke, K., Spitzer, R. L., \& Williams, J. B. (2001). The phq-9. Journal of general internal medicine, 16(9), 606-613. doi: 10.1046/j.1525-1497.2001.016009606.x

Kubicki, M., Niznikiewicz, M., Connor, E., Ungar, L., Nestor, P. G., Bouix, S., ... \& Shenton, M. E. (2009). Relationship between white matter integrity, attention, and memory in schizophrenia: a diffusion tensor imaging study. Brain imaging and behavior, 3(2), 191-201.

doi: $10.1007 / \mathrm{s} 11682-009-9061-8$

Lachman, M. E. (2004). Development in midlife. Annu Rev Psychol, 55, 305-331. doi:10.1146/annurev.psych.55.090902.141521

Lahtela, K., Niemi, P., \& Kuusela, V. (1985). Adult visual choice reaction time, age, sex and preparedness: A test of Welford's problem in a large population sample. Scandinavian journal of psychology, 26(1), 357-362. doi: 10.1111/j.14679450.1985.tb01175.x

Landauer, A. A., Armstrong, S., \& Digwood, J. (1980). Sex difference in choice reaction time. British Journal of Psychology, 71(4), 551-555. doi:10.1111/j.20448295.1980.tb01766.x

Landy, K. M., Salmon, D. P., Filoteo, J. V., Heindel, W. C., Galasko, D., \& Hamilton, J. M. (2015). Visual search in Dementia with Lewy Bodies and Alzheimer's disease. Cortex, 73, 228-239.doi: 10.1016/j.cortex.2015.08.020.

Langley, L. K., Fuentes, L. J., Hochhalter, A. K., Brandt, J., \& Overmier, J. B. (2001). Inhibition of return in aging and Alzheimer's disease: Performance as a function of task demands and stimulus timing. Journal of Clinical and Experimental Neuropsychology, 23(4), 431-446. doi:10.1076/jcen.23.4.431.1235 
Langley, L. K., Friesen, C. K., Saville, A. L., \& Ciernia, A. T. (2011). Timing of reflexive visuospatial orienting in young, young-old, and old-old adults. Attention, Perception, \& Psychophysics, 73(5), 1546-1561.

Larson, E. B., Kukull, W. A., Buchner, D., \& Reifler, B. V. (1987). Adverse drug reactions associated with global cognitive impairment in elderly persons. Annals of Internal Medicine, 107(2), 169-173.doi: 10.1059/0003-4819-107-2-169

Lautenschlager, N. T., Flicker, L., Vasikaran, S., Leedman, P., \& Almeida, O. P. (2005). Subjective memory complaints with and without objective memory impairment - Relationship with risk factors for dementia. American Journal of Geriatric Psychiatry, 13(8), 731-734. doi:10.1176/appi.ajgp.13.8.731

Lavie, N., \& Fockert, J. d. (2006). Frontal control of attentional capture in visual search. Visual Cognition, 14(4-8), 863-876.doi:

http://dx.doi.org/10.1080/13506280500195953

Lavie, N., Hirst, A., De Fockert, J. W., \& Viding, E. (2004). Load theory of selective attention and cognitive control. Journal of Experimental Psychology:

General, 133(3), 339.doi: http://dx.doi.org/10.1037/0096-3445.133.3.339

de Leeuw, F. E., Korf, E., Barkhof, F., \& Scheltens, P. (2006). White matter lesions are associated with progression of medial temporal lobe atrophy in Alzheimer disease. Stroke, 37(9), 2248-2252.

https://doi.org/10.1161/01.STR.0000236555.87674.e1

Lehrner, J., Moser, D., Klug, S., Gleiss, A., Auff, E., Dal-Bianco, P., \& Pusswald, G. (2014). Subjective memory complaints, depressive symptoms and cognition in patients attending a memory outpatient clinic. International Psychogeriatrics, 26(3), 463-473. doi:10.1017/s1041610213002263

Leuba, G., \& Saini, K. (1995). Pathology of subcortical visual centres in relation to cortical degeneration in Alzheimer's disease. Neuropathology and applied neurobiology, 21(5), 410-422. doi: 10.1111/j.1365-2990.1995.tb01078.x

Levinoff, E. J., Saumier, D., \& Chertkow, H. (2005). Focused attention deficits in patients with Alzheimer's disease and mild cognitive impairment. Brain and Cognition, 57(2), 127-130. doi:http://dx.doi.org/10.1016/j.bandc.2004.08.058

Lidzba K, Ebner K, Hauser T-K, Wilke M (2013) Complex Visual Search in Children and Adolescents: Effects of Age and Performance on fMRI Activation. PLoS ONE, 8(12): e85168. https://doi.org/10.1371/journal.pone.0085168

Liu, D., Wang, Z., Shu, H., \& Zhang, Z. (2016). Disrupted white matter integrity is associated with cognitive deficits in patients with amnestic mild cognitive impairment: An atlas-based study. SAGE open medicine, 4, 2050312116648812.doi: $10.1177 / 2050312116648812$ 
Logan, G. D., Cowan, W. B., \& Davis, K. A. (1984). On the ability to inhibit simple and choice reaction time responses: a model and a method. Journal of Experimental Psychology: Human Perception and Performance, 10(2), 276.doi:

http://dx.doi.org/10.1037/0096-1523.10.2.276

Lorenzo-López, L., Amenedo, E., \& Cadaveira, F. (2008). Feature processing during visual search in normal aging: electrophysiological evidence. Neurobiology of Aging, 29(7), 1101-1110.doi: https://doi.org/10.1016/j.neurobiolaging.2007.02.007

Low, L.F., Anstey, K. J., Jorm, A. F., Christensen, H., \& Rodgers, B. (2006). Hormone replacement therapy and cognition in an Australian representative sample aged 60 - 64 years. Maturitas, 54, 86 -94. doi: 10.1016/j.maturitas.2005.09.001

Löwe, B., Decker, O., Müller, S., Brähler, E., Schellberg, D., Herzog, W., \& Herzberg, P. Y. (2008). Validation and standardization of the Generalized Anxiety Disorder Screener (GAD-7) in the general population. Medical care, 46(3), 266-274. doi: 10.1097/MLR.0b013e318160d093

Lumley, T., Diehr, P., Emerson, S., \& Chen, L. (2002). The importance of the normality assumption in large public health data sets. Annual review of public health, 23(1), 151-169.

doi: https://doi.org/10.1146/annurev.publhealth.23.100901.140546

MacDonald, S. W. S., Nyberg, L., \& Backman, L. (2006). Intra-individual variability in behavior: links to brain structure, neurotransmission and neuronal activity. Trends in Neurosciences, 29(8), 474-480. doi:10.1016/j.tins.2006.06.011

MacDonald, S. W., Nyberg, L., Sandblom, J., Fischer, H., \& Bäckman, L. (2008). Increased response-time variability is associated with reduced inferior parietal activation during episodic recognition in aging. Journal of Cognitive Neuroscience, 20(5), 779-786. doi: 10.1162/jocn.2008.20502

Madden, D. J. (2007). Aging and Visual Attention. Current directions in psychological science, 16(2), 70-74. doi:10.1111/j.1467-8721.2007.00478.x

Madden, D. J., Gottlob, L. R., \& Allen, P. A. (1999). Adult age differences in visual search accuracy: Attentional guidance and target detectability. Psychology and Aging, 14(4), 683-694. doi:10.1037/0882-7974.14.4.683

Madden, D. J., Spaniol, J., Whiting, W. L., Bucur, B., Provenzale, J. M., Cabeza, R., ... Huettel, S. A. (2007). Adult age differences in the functional neuroanatomy of visual attention: a combined fMRI and DTI study. Neurobiol Aging, 28(3), 459-476. doi:10.1016/j.neurobiolaging.2006.01.005 
Madden, D. J., Turkington, T. G., Provenzale, J. M., Denny, L. L., Langley, L. K., Hawk, T. C., \& Coleman, R. E. (2002). Aging and attentional guidance during visual search: functional neuroanatomy by positron emission tomography. Psychol Aging, 17(1), 24-43.doi: http://dx.doi.org/10.1037/0882-7974.17.1.24

Martin, D. (2015). 9 Brain Regions Affected by Alzheimer's. retrieved 5th Aug 2017 at http://www.healthcentral.com/slideshow/9-areas-brain-affectedalzheimers\#slide $=8$

Mascherek, A., Zimprich, D., Rupprecht, R., \& Lang, F. R. (2011). What do cognitive complaints in a sample of memory clinic outpatients reflect? GeroPsych: The Journal of Gerontopsychology and Geriatric Psychiatry, 24(4), 187.doi: https://doi.org/10.1024/1662-9647/a000046.

Mazerolle, E. L., Wojtowicz, M. A., Omisade, A., \& Fisk, J. D. (2013). Intraindividual variability in information processing speed reflects white matter microstructure in multiple sclerosis. NeuroImage: Clinical, 2, 894-902. doi: https://doi.org/10.1016/j.nicl.2013.06.012

McAuliffe, J., Chasteen, A. L., \& Pratt, J. (2006). Object- and location-based inhibition of return in younger and older adults. Psychology and Aging, 21(2), 406410.http://dx.doi.org/10.1037/0882-7974.21.2.406

McDowd, J. M., \& Craik, F. I. (1988). Effects of aging and task difficulty on divided attention performance. Journal of Experimental Psychology: Human Perception and Performance, 14(2), 267.

McEwen, B. S. (2001). Genome and hormones: Gender differences in physiology: Invited review: Estrogens effects on the brain: Multiple sites and molecular mechanisms. Journal of Applied Physiology, 91, 2785- 2801. Retrieved from http://jap.physiology.org/content/91/6/2785.full .pdf

McGuinness, B., Barrett, S. L., Craig, D., Lawson, J., \& Passmore, A. P. (2010). Attention deficits in Alzheimer's disease and vascular dementia. Journal of Neurology Neurosurgery and Psychiatry, 81(2), 157-159. doi:10.1136/jnnp.2008.164483

McLaughlin, P. M., Borrie, M. J., \& Murtha, S. J. (2010). Shifting efficacy, distribution of attention and controlled processing in two subtypes of mild cognitive impairment: Response time performance and intraindividual variability on a visual search task. Neurocase, 16(5), 408-417. doi: 10.1080/13554791003620306.

Medina, D., Urresta, F., Gabrieli, J. D., Moseley, M., Fleischman, D., Bennett, D. A., ... \& Stebbins, G. T. (2006). White matter changes in mild cognitive impairment and AD: A diffusion tensor imaging study. Neurobiology of aging, 27(5), 663-672. https://doi.org/10.1016/j.neurobiolaging.2005.03.026 
Mella, N., de Ribaupierre, S., Eagleson, R., \& de Ribaupierre, A. (2013). Cognitive Intraindividual Variability and White Matter Integrity in Aging. Scientific World Journal, 16. doi:10.1155/2013/350623

Meltzer, C. C., Zubieta, J. K., Brandt, J., Tune, L. E., Mayberg, H. S., \& Frost, J. J. (1996). Regional hypometabolism in Alzheimer's disease as measured by positron emission tomography after correction for effects of partial volume averaging. Neurology, 47(2), 454-461.

Mendez, M. F., Cherrier, M. M., \& Cymerman, J. S. (1997). Hemispatial neglect on visual search tasks in Alzheimer's disease. Neuropsychiatry Neuropsychol Behav Neurol, 10(3), 203-208.

Mendez, M. F., Cherrier, M. M., \& Perryman, K. M. (1997). Differences between Alzheimer's disease and vascular dementia on information processing measures. Brain and Cognition, 34(2), 301-310.doi: http://dx.doi.org/10.1006/brcg.1997.0923

Meng, X., \& D'Arcy, C. (2012). Education and Dementia in the Context of the Cognitive Reserve Hypothesis: A Systematic Review with Meta-Analyses and Qualitative Analyses. Plos One, 7(6). doi:10.1371/journal.pone.0038268

Merritt, P., Hirshman, E., Wharton, W., Devlin, J., Stangl, B., Bennett, S., \& Hawkins, L. (2005). Gender differences in selective attention: Evidence from a spatial orienting task. Journal of Vision, 5(8), 1000-1000. doi:10.1167/5.8.1000

Michaels, C. F. (1988). SR compatibility between response position and destination of apparent motion: Evidence of the detection of affordances. Journal of Experimental Psychology: Human Perception and Performance, 14(2), 231.doi: 10.1037//0096-1523.14.2.231

Michielse, S., Coupland, N., Camicioli, R., Carter, R., Seres, P., Sabino, J., \& Malykhin, N. (2010). Selective effects of aging on brain white matter microstructure: a diffusion tensor imaging tractography study. Neuroimage, 52(4), 1190-1201.doi: https://doi.org/10.1016/j.neuroimage.2010.05.019

Minett, T. S. C., Da Silva, R. V., Ortiz, K. Z., \& Bertolucci, P. H. F. (2008). Subjective memory complaints in an elderly sample: a cross-sectional study. International Journal of Geriatric Psychiatry, 23(1), 49-54. doi:10.1002/gps.1836

Mitchell, A. J., Beaumont, H., Ferguson, D., Yadegarfar, M., \& Stubbs, B. (2014). Risk of dementia and mild cognitive impairment in older people with subjective memory complaints: meta-analysis. Acta Psychiatrica Scandinavica, 130(6), 439451. doi:10.1111/acps. 12336 
Moll, J., de Oliveira-Souza, R., Moll, F. T., Bramati, I. E., \& Andreiuolo, P. A. (2002). The cerebral correlates of set-shifting: an fMRI study of the trail making test. Arq Neuropsiquiatr, 60(4), 900-905.doi: http://dx.doi.org/10.1590/S0004282X2002000600002

Mol, M. E. M., van Boxtel, M. P. J., Willems, D., \& Jolles, J. (2006). Do subjective memory complaints predict cognitive dysfunction over time? A six-year follow-up of the Maastricht Aging Study. International Journal of Geriatric Psychiatry, 21(5), 432-441. doi:10.1002/gps.1487

Molchan, S. E., Martinez, R. A., Hill, J. L., Weingartner, H. J., Thompson, K., Vitiello, B., \& Sunderland, T. (1992). Increased cognitive sensitivity to scopolamine with age and a perspective on the scopolamine model. Brain Research Reviews, 17(3), 215-226.doi: https://doi.org/10.1016/0165-0173(92)90017-G

Montejo, P., Montenegro, M., Fernández, M. A., \& Maestú, F. (2012). Memory complaints in the elderly: Quality of life and daily living activities. A population based study. Archives of Gerontology and Geriatrics, 54(2), 298-304. doi:http://dx.doi.org/10.1016/j.archger.2011.05.021

Montejo, P., Montenegro, M., Fernandez, M. A., \& Maestu, F. (2011). Subjective memory complaints in the elderly: Prevalence and influence of temporal orientation, depression and quality of life in a population-based study in the city of Madrid. Aging Ment Health, 15(1), 85-96. doi:10.1080/13607863.2010.501062

Moran, J., \& Desimone, R. (1985). Selective attention gates visual processing in the extrastriate cortex. Frontiers in cognitive neuroscience, 229, 342-345. doi: http://dx.doi.org/10.1126/science.4023713

Morris, J.C. (2005). Clinical Dementia Rating: A Reliable and Valid Diagnostic and Staging Measure for Dementia of the Alzheimer Type. International Psychogeriatrics, 9(1), 173-176. doi: https://doi.org/10.1017/S1041610297004870

Moy, G., Millet, P., Haller, S., Baudois, S., De Bilbao, F., Weber, K., ... \& Delaloye, C. (2011). Magnetic resonance imaging determinants of intraindividual variability in the elderly: combined analysis of grey and white matter. Neuroscience, 186, 88-93. https://doi.org/10.1016/j.neuroscience.2011.04.028

Mourany, L., \& Pillai, J. (2014). Education Effects On Rate Of Cognitive Decline Compared In Autopsy Confirmed Alzheimer's, Lewy Body And Vascular Dementia (P5. 239). Neurology, 82(10 Supplement), P5-239.

Mrazik, M., Millis, S., \& Drane, D. L. (2010). The oral trail making test: effects of age and concurrent validity. Archives of Clinical Neuropsychology, 25(3), 236243.doi: 10.1093/arclin/acq006 
Muller, M. J. (1994). Salivary testosterone and simple reaction time parameters. Neuropsychobiology, 30, 173-177. doi:10.1159/000119157

Muller, M., Aleman, A., Grobbee, D. E., De Haan, E. H. F., \& van der Schouw, Y. T. (2005). Endogenous sex hormone levels and cognitive function in aging men. Is there an optimal level?. Neurology, 64(5), 866-871. doi:

https://doi.org/10.1212/01.WNL.0000153072.54068.E3

Muller-Oehring, E. M., Schulte, T., Rohlfing, T., Pfefferbaum, A., \& Sullivan, E. V. (2013). Visual search and the aging brain: discerning the effects of age-related brain volume shrinkage on alertness, feature binding, and attentional control.

Neuropsychology, 27(1), 48-59. doi:10.1037/a0030921

Muir, J. L. (1997). Acetylcholine, aging, and Alzheimer's disease. Pharmacology Biochemistry and Behavior, 56(4), 687-696.doi: https://doi.org/10.1016/S00913057(96)00431-5

Muir, J. L., Everitt, B. J., \& Robbins, T. W. (1994). AMPA-induced excitotoxic lesions of the basal forebrain: a significant role for the cortical cholinergic system in attentional function. Journal of Neuroscience, 14(4), 2313-2326.doi:

Murphy, D. G., DeCarli, C., Mclntosh, A. R., Daly, E., Mentis, M. J., Pietrini, P., ... \& Rapoport, S. I. (1996). Sex differences in human brain morphometry and metabolism: an in vivo quantitative magnetic resonance imaging and positron emission tomography study on the effect of aging. Archives of general psychiatry, 53(7), 585-594. doi:10.1001/archpsyc.1996.01830070031007

Myerson, J., Hale, S., Poon, L. W., Wagstaff, D., \& Smith, G. A. (1990). The information-loss model - a mathematical-theory of age-related cognitive slowing. Psychological Review, 97(4), 475-487. doi:10.1037/0033-295x.97.4.475

Naismith, S. L., Hickie, I. B., Turner, K., Little, C. L., Winter, V., Ward, P. B., . . . Parker, G. (2003). Neuropsychological performance in patients with depression is associated with clinical, etiological and genetic risk factors. J Clin Exp Neuropsychol, 25(6), 866-877. doi:10.1076/jcen.25.6.866.16472

Nasreddine, Z. S., Phillips, N. A., Bedirian, V., Charbonneau, S., Whitehead, V., Collin, I., . . . Chertkow, H. (2005). The Montreal Cognitive Assessment, MoCA: a brief screening tool for mild cognitive impairment. J Am Geriatr Soc, 53(4), 695699. doi:10.1111/j.1532-5415.2005.53221.x

Nebes, R. D., \& Brady, C. B. (1993). Phasic and Tonic Alertness in Alzheimer's Disease. Cortex, 29(1), 77-90. doi:https://doi.org/10.1016/S0010-9452(13)80213-4

Nebes, R. D., \& Brady, C. B. (1989). Focused and divided attention in Alzheimer's disease. Cortex, 25(2), 305-315.doi: http://dx.doi.org/10.1016/S00109452(89)80045-0 
Nestor, P. G., Parasuraman, R., \& Haxby, J. V. (1991). Speed of information processing and attention in early Alzheimer's dementia. Developmental

Neuropsychology, 7(2), 243-256.doi: http://dx.doi.org/10.1080/87565649109540491

Nettelbeck T. J. (2014). Smarter but slower? A comment on Woodley, te Nijenhuis and Murphy (2013). Intelligence, 42 1-4.doi:

https://doi.org/10.1016/j.intell.2013.09.006

Nilsson, J., Thomas, A. J., O'Brien, J. T., \& Gallagher, P. (2014). White Matter and Cognitive Decline in Aging: A Focus on Processing Speed and Variability. Journal of the International Neuropsychological Society, 20(3), 262-267.

doi:10.1017/s1355617713001458

Nissen, M. J., \& Corkin, S. (1985). Effectiveness of attentional cueing in older and younger adults. Journal of gerontology, 40(2), 185-191.doi:

http://dx.doi.org/10.1093/geronj/40.2.185

O'Brien, J. O. H. N., Desmond, P., Ames, D., Schweitzer, I., Harrigan, S., \& Tress, B. R. I. A. N. (1996). A magnetic resonance imaging study of white matter lesions in depression and Alzheimer's disease. The British Journal of Psychiatry, 168(4), 477485.doi: 10.1192/bjp.168.4.477

Olsson, E., Klasson, N., Berge, J. et al., (2013). "White Matter Lesion Assessment in Patients with Cognitive Impairment and Healthy Controls: Reliability Comparisons between Visual Rating, a Manual, and an Automatic Volumetrical MRI MethodThe Gothenburg MCI Study," Journal of Aging Research, vol. 2013, Article ID 198471, 10 pages, 2013. doi:10.1155/2013/198471

Onoda, K., Hamano, T., Nabika, Y., Aoyama, A., Takayoshi, H., Nakagawa, T., . . . Yamaguchi, S. (2013). Validation of a new mass screening tool for cognitive impairment: Cognitive Assessment for Dementia, iPad version. Clinical Interventions in Aging, 8, 353-360. doi:10.2147/CIA.S42342

Onoda, K., \& Yamaguchi, S. (2014). Revision of the Cognitive Assessment for Dementia, iPad Version (CADi2). Plos One, 9(10), e109931.

doi:10.1371/journal.pone.0109931

Owsley, C. (2011). Aging and vision. Vision Research, 51(13), 1610-1622. doi:http://dx.doi.org/10.1016/j.visres.2010.10.020

Pa, J., Possin, K. L., Wilson, S. M., Quitania, L. C., Kramer, J. H., Boxer, A. L., ... \& Johnson, J. K. (2010). Gray matter correlates of set-shifting among neurodegenerative disease, mild cognitive impairment, and healthy older adults. Journal of the International Neuropsychological Society, 16(4), 640-650. doi: $10.1017 / \mathrm{S} 1355617710000408$ 
Pachella, R. G., \& Pew, R. W. (1968). Speed-Accuracy Tradeoff in Reaction Time: Effect of Discrete Criterion Times. Journal of Experimental Psychology, 76(1, Pt.1), 19-24.doi: http://dx.doi.org/10.1037/h0021275

Pakkenberg, B., \& Gundersen, H. J. G. (1997). Neocortical neuron number in humans: Effect of sex and age. Journal of Comparative Neurology, 384(2), 312-320. doi:10.1002/(sici)1096-9861(19970728)384:2<312::aid-cne10>3.0.co;2-k

Pálsson, Á. (2013). Normative Scores on the Trail Making Test for the Icelandic Population.doi: http://hdl.handle.net/1946/16643

Papp, K. V., Kaplan, R. F., Springate, B., Moscufo, N., Wakefield, D. B., Guttmann, C. R. G., \& Wolfson, L. (2014). Processing speed in normal aging: Effects of white matter hyperintensities and hippocampal volume loss. Aging Neuropsychology and Cognition, 21(2), 197-213. doi:10.1080/13825585.2013.795513

Parasuraman, R., Greenwood, P. M., Haxby, J. V., \& Grady, C. L. (1992). Visuospatial attention in dementia of the alzheimer type. Brain, 115, 711-733. doi:10.1093/brain/115.3.711

Pardo, J. V., Pardo, P. J., Janer, K. W., \& Raichle, M. E. (1990). The anterior cingulate cortex mediates processing selection in the Stroop attentional conflict paradigm. Proceedings of the National Academy of Sciences, 87(1), 256-259.doi: 1990PNAS...87..256P

Pate, D. S., Margolin, D. I., Friedrich, F. J., \& Bentley, E. E. (1994). Decisionmaking and attentional processes in ageing and in dementia of the Alzheimer's type. Cognitive Neuropsychology, 11(3), 321-339. doi:10.1080/02643299408251978

Paus, T., Collins, D.L., Evans, A.C., Leonard, G., Pike, B., \& Zijdenbos, A. (2001. Maturation of white matter in the human brain: a review of magnetic resonance studies. Brain Research Bulletin, 54 (3), 255-266. doi https://doi.org/10.1016/S03619230(00)00434-2

Periáñez, J. A., Ríos-Lago, M., Rodríguez-Sánchez, J. M., Adrover-Roig, D., Sánchez-Cubillo, I., Crespo-Facorro, B., . . B Barceló, F. (2007). Trail Making Test in traumatic brain injury, schizophrenia, and normal ageing: Sample comparisons and normative data. Archives of Clinical Neuropsychology, 22(4), 433-447. doi:http://dx.doi.org/10.1016/j.acn.2007.01.022

Perry, E. K., Tomlinson, B. E., Blessed, G., Perry, R. H., Cross, A. J., \& Crow, T. J. (1981). Neuropathological and biochemical observations on the noradrenergic system in Alzheimer's disease. Journal of the neurological sciences, 51(2), 279287.doi: https://doi.org/10.1016/0022-510X(81)90106-4 
Perry, R. J., \& Hodges, J. R. (1999). Attention and executive deficits in Alzheimer's disease. Brain, 122(3), 383-404.doi: https://doi.org/10.1093/brain/122.3.383

Perry, M. E., McDonald, C. R., Hagler, D. J., Gharapetian, L., Kuperman, J. M., Koyama, A. K., . . McEvoy, L. K. (2009). White Matter Tracts Associated with SetShifting in Healthy Aging. Neuropsychologia, 47(13), 2835-2842. doi:10.1016/j.neuropsychologia.2009.06.008

Peters, R. (2006). Ageing and the brain. Postgraduate Medical Journal, 82(964), 8488. doi:10.1136/pgmj.2005.036665

Petersen, R. C. (2004). Mild cognitive impairment as a diagnostic entity. Journal of internal medicine, 256(3), 183-194.doi: 10.1111/j.1365-2796.2004.01388.x

Petersen, R. C., Smith, G. E., Waring, S. C., Ivnik, R. J., Tangalos, E. G., \& Kokmen, E. (1999). Mild cognitive impairment: Clinical characterization and outcome. Archives of Neurology, 56(3), 303-308.doi: doi:10.1001/archneur.56.3.303

Petersen, R. C., Stevens, J. C., Ganguli, M., Tangalos, E. G., Cummings, J. L., \& DeKosky, S. T. (2001). Practice parameter: Early detection of dementia: Mild cognitive impairment (an evidence-based review) Report of the Quality Standards Subcommittee of the American Academy of Neurology. Neurology, 56(9), 11331142.doi: http://dx.doi.org/10.1212/WNL.56.9.1133

Phillips, M., Rogers, P., Haworth, J., Bayer, A., \& Tales, A. (2013). Intra-individual reaction time variability in mild cognitive impairment and Alzheimer's disease: gender, processing load and speed factors. Plos One, 8(6), e65712.

Pirozzolo, F. J., Christensen, K. J., Ogle, K. M., Hansch, E. C., \& Thompson, W. G. (1981). Simple and choice reaction time in dementia: Clinical implications. Neurobiology of Aging, 2(2), 113-117. doi:http://dx.doi.org/10.1016/01974580(81)90008-7

Plotek, W., Lyskawa, W., Kluzik, A., Grzeskowiak, M., Podlewski, R., Zaba, Z., \& Drobnik, L. (2014). Evaluation of the Trail Making Test and interval timing as measures of cognition in healthy adults: comparisons by age, education, and gender. Med Sci Monit, 20, 173-181. doi:10.12659/msm.889776

Plude, D. J., \& Doussard-Roosevelt, J. A. (1989). Aging, selective attention, and feature integration. Psychol Aging, 4(1), 98-105.doi: http://dx.doi.org/10.1037/08827974.4.1.98 
Pollmann, S., Weidner, R., Müller, H. J., Maertens, M., \& von Cramon, D. Y. (2006). Selective and interactive neural correlates of visual dimension changes and response changes. NeuroImage, 30(1), 254-265.doi:

https://doi.org/10.1016/j.neuroimage.2005.09.013

Port, N. L., Trimberger, J., Hitzeman, S., Redick, B., \& Beckerman, S. (2016). Micro and regular saccades across the lifespan during a visual search of "Where's Waldo" puzzles. Vision research, 118, 144-157.doi:

https://doi.org/10.1016/j.visres.2015.05.013

Porter, G., Leonards, U., Wilcock, G., Haworth, J., Troscianko, T., \& Tales, A. (2010). New insights into feature and conjunction search: II. Evidence from Alzheimer's disease. Cortex, 46(5), 637-649.doi: https://doi.org/10.1016/j.cortex.2009.04.014

Porter, G., Tales, A., Troscianko, T., Wilcock, G., Haworth, J., \& Leonards, U. (2010). New insights into feature and conjunction search: I. Evidence from pupil size, eye movements and ageing. Cortex, 46(5), 621-636.doi:

10.1016/j.cortex.2009.04.013

Posner, M. I. (1993). Interaction of arousal and selection in the posterior attention network. In A. D. Baddeley \& L. Weiskrantz (Eds.), Attention: Selection, awareness, and control: A tribute to Donald Broadbent (pp. 390-405). New York: Clarendon Press/Oxford University Press.

Posner, M. I. (1980). Orienting of attention. Quarterly journal of experimental psychology, 32(1), 3-25.

Posner, M. I. (1978). Chronometric explorations of mind. Lawrence Erlbaum.

Posner, M. I., \& Boies, S. J. (1971). Components of attention. Psychological Review, 78(5), 391. doi: http://dx.doi.org/10.1037/h0031333

Posner, M. I., \& Cohen, Y. (1984). Components of visual orienting. Attention and performance X, Control of language processes, 32, 531-556.

Posner, M. I., \& Driver, J. (1992). The neurobiology of selective attention. Current opinion in neurobiology, 2(2), 165-169.

Posner, M. I., Nissen, M. J., \& Ogden, W. C. (1978). Attended and unattended processing modes: The role of set for spatial location. Modes of perceiving and processing information, 137, 158.

Posner, M. I., Snyder, C. R., \& Davidson, B. J. (1980). Attention and the detection of signals. Journal of experimental psychology: General, 109(2), 160.doi:

http://dx.doi.org/10.1037/0096-3445.109.2.160 
Potter, L. M., Grealy, M. A., Elliott, M. A., \& Andres, P. (2012). Aging and performance on an everyday-based visual search task. Acta Psychologica, 140(3), 208-217. doi:10.1016/j.actpsy.2012.05.001

Prado, J., Carp, J., \& Weissman, D. H. (2011). Variations of response time in a selective attention task are linked to variations of functional connectivity in the attentional network. Neuroimage, 54(1), 541-549.doi:

https://doi.org/10.1016/j.neuroimage.2010.08.022

Pratt, J., \& Chasteen, A. L. (2007). Examining inhibition of return with multiple sequential cues in younger and older adults. Psychology and Aging, 22(2), 404409.http://dx.doi.org/10.1037/0882-7974.22.2.404

Pritchard, J. (2011). Parts of the Brain Affected by Alzheimer's.retrieved 5th Aug 2017 at http://www.livestrong.com/article/122035-parts-brain-affected-alzheimers/

Rabbitt, P. (2015). The Aging Mind: An Owner's Manual: Routledge.

Rabbitt, P. (1965). An age-decrement in the ability to ignore irrelevant information. $J$ Gerontol, 20, 233-238.doi: https://doi.org/10.1093/geronj/20.2.233

Rabbitt, P., Scott, M., Lunn, M., Thacker, N., Lowe, C., Pendleton, N., . . Jackson, A. (2007). White matter lesions account for all age-related declines in speed but not in intelligence. Neuropsychology, 21(3), 363-370. doi:10.1037/0894-4105.21.3.363

Radanovic, M., Pereira, F. R. S., Stella, F., Aprahamian, I., Ferreira, L. K., Forlenza, O. V., \& Busatto, G. F. (2013). White matter abnormalities associated with Alzheimer's disease and mild cognitive impairment: a critical review of MRI studies. Expert review of neurotherapeutics, 13(5), 483-493. doi:10.1586/ern.13.45

Rapp, M. A., Schnaider-Beeri, M., Grossman, H. T., Sano, M., Perl, D. P., Purohit, D. P., . . Haroutunian, V. (2006). Increased hippocampal plaques and tangles in patients with Alzheimer disease with a lifetime history of major depression. Archives of General Psychiatry, 63(2), 161-167. doi:10.1001/archpsyc.63.2.161

Rasmusson, X. D., Zonderman, A. B., Kawas, C., \& Resnick, S. M. (1998). Effects of age and dementia on the Making Test. The Clinical Neuropsychologist, 12(2), 169-178.doi: http://dx.doi.org/10.1076/clin.12.2.169.2005

Ratcliff, R. (1979). Group reaction time distributions and an analysis of distribution statistics. Psychological Bulletin, 86(3), 446-461.doi:http://dx.doi.org/10.1037/00332909.86.3.446 
Raz, N., Gunning, F. M., Head, D., Dupuis, J. H., McQuain, J., Briggs, S. D., . . . Acker, J. D. (1997). Selective aging of the human cerebral cortex observed in vivo: Differential vulnerability of the prefrontal gray matter. Cerebral Cortex, 7(3), 268282. doi:10.1093/cercor/7.3.268

Redel, P., Bublak, P., Sorg, C., Kurz, A., Forstl, H., Muller, H. J., . . Finke, K. (2012). Deficits of spatial and task-related attentional selection in mild cognitive impairment and Alzheimer's disease. Neurobiology of Aging, 33(1), 16. doi:10.1016/j.neurobiolaging.2010.05.014

Reed, N. M. (2010). The Relationship between Subjective Memory and Objective Cognition, Depression, and Anxiety by Dementia Status.

Reid, L. M., \& Maclullich, A. M. (2006). Subjective memory complaints and cognitive impairment in older people. Dementia and Geriatric Cognitive Disorders, 22. doi:10.1159/000096295

Reimers, S., \& Maylor, E. A. (2006). Gender Effects on Reaction Time Variability and Trial-to-Trial Performance: Reply to Deary and Der (2005). Aging, Neuropsychology, and Cognition, 13(3-4), 479-489. doi:10.1080/138255890969375

Reisberg, B., Prichep, L., Mosconi, L., John, E. R., Glodzik-Sobanska, L., Boksay, I., ... de Leon, M. J. (2008). The pre-mild cognitive impairment, subjective cognitive impairment stage of Alzheimer's disease. Alzheimers \& Dementia, 4(1), S98-S108. doi:10.1016/j.jalz.2007.11.017

Reisberg, B., Shulman, M. B., Torossian, C., Leng, L., \& Zhu, W. (2010). Outcome over seven years of healthy adults with and without subjective cognitive impairment. Alzheimers \& Dementia, 6. doi:10.1016/j.jalz.2009.10.002

Reitan, R. M. (1971). Trail making test results for normal and brain-damaged children. Percept Mot Skills, 33(2), 575-581. doi:10.2466/pms.1971.33.2.575

Rentz, D. M., Dekhtyar, M., Sherman, J., Burnham, S., Blacker, D., Aghjayan, S. L., ... Sperling, R. A. (2016). The Feasibility of At-Home iPad Cognitive Testing For Use in Clinical Trials. J Prev Alzheimers Dis, 3(1), 8-12. doi:10.14283/jpad.2015.78

Ribeiro, M. J., Paiva, J. S., \& Castelo-Branco, M. (2016). Spontaneous fluctuations in sensory processing predict within-subject reaction time variability. Frontiers in Human Neuroscience, 10, 200.doi: 10.3389/fnhum.2016.00200.

Richardson, M. (2009). Principal component analysis. URL: http://people. maths. ox. ac. uk/richardsonm/SignalProcPCA. pdf (last access: 3.5. 2013). Aleš Hladnik Dr., Ass. Prof., Chair of Information and Graphic Arts Technology, Faculty of Natural Sciences and Engineering, University of Ljubljana, Slovenia ales. hladnik@ ntf. unilj. si. 
Riepe, M. W., Walther, B., Vonend, C., \& Beer, A. J. (2015). Drug-induced cerebral glucose metabolism resembling Alzheimer's Disease: a case study. BMC psychiatry, 15(1), 157.doi: 10.1186/s12888-015-0531-9.

Rizzo, M., \& Nawrot, M. (1998). Perception of movement and shape in Alzheimer's disease. Brain: a journal of neurology, 121(12), 2259-2270.doi:

https://doi.org/10.1093/brain/121.12.2259

Robbins, T. W. (1997). Arousal systems and attentional processes. Biological psychology, 45(1-3), 57-71.doi: https://doi.org/10.1016/S0301-0511(96)05222-2

Rodda, J., Dannhauser, T., Cutinha, D. J., Shergill, S. S., \& Walker, Z. (2011). Subjective cognitive impairment: Functional MRI during a divided attention task. European Psychiatry, 26(7), 457-462. doi:10.1016/j.eurpsy.2010.07.003

Roivainen, E. (2011). Gender differences in processing speed: A review of recent research. Learning and Individual differences, 21(2), 145-149.doi: https://doi.org/10.1016/j.lindif.2010.11.021

Rösler, A., Mapstone, M., Hays-Wicklund, A., Gitelman, D. R., \& Weintraub, S. (2005). The "zoom lens" of focal attention in visual search: Changes in aging and Alzheimer's disease. Cortex, 41(4), 512-519.doi: https://doi.org/10.1016/S00109452(08)70191-6

Rounis, E., Maniscalco, B., Rothwell, J. C., Passingham, R. E., \& Lau, H. (2010). Theta-burst transcranial magnetic stimulation to the prefrontal cortex impairs metacognitive visual awareness. Cognitive Neuroscience, 1(3), 165-175.doi: $10.1080 / 17588921003632529$.

Ruz, M., \& Lupiáñez, J. (2002). A review of attentional capture: On its automaticity and sensitivity to endogenous control. Psicológica), 23, 283-309.

Sachs-Ericsson, N. \& Blazer, D. G. (2015).The new DSM-5 diagnosis of mild neurocognitive disorder and its relation to research in mild cognitive impairment. Aging Ment Health, 19, 2-12.

Sadeh, N., \& Bredemeier, K. (2011). Individual differences at high perceptual load: The relation between trait anxiety and selective attention. Cognition and Emotion, 25(4), 747-755. doi: http://dx.doi.org/10.1080/02699931.2010.500566

Salami, A., Eriksson, J., Nilsson, L.-G., \& Nyberg, L. (2012). Age-related white matter microstructural differences partly mediate age-related decline in processing speed but not cognition. Biochimica Et Biophysica Acta-Molecular Basis of Disease, 1822(3), 408-415. doi:10.1016/j.bbadis.2011.09.001 
Salthouse, T. A. (2011). What cognitive abilities are involved in trail-making performance? Intelligence, 39(4), 222-232.

doi:http://dx.doi.org/10.1016/j.intell.2011.03.001

Salthouse, T. (2000). A theory of cognitive aging (Vol. 28): Elsevier.

Salthouse, T. A. (1979). Adult age and the speed-accuracy tradeoff. Ergonomics, 22(7),811-

821.doi: http://dx.doi.org/10.1080/00140137908924659

Salthouse, T. A. (1992). What do adult age-differences in the digit symbol substitution test reflect. Journals of Gerontology, 47(3), P121-P128.

Salthouse, T. A., \& Fristoe, N. M. (1995). Process analysis of adult age effects on a computer-administered Trail Making Test. Neuropsychology, 9(4), 518.doi: http://dx.doi.org/10.1037/0894-4105.9.4.518

Salthouse, T. A., Rogan, J. D., \& Prill, K. (1984). Division of attention: Age differences on a visually presented memory task. Memory \& Cognition. 12, 613-620

Sanchez-Cubillo, I., Perianez, J. A., Adrover-Roig, D., Rodriguez-Sanchez, J. M., Rios-Lago, M., Tirapu, J., \& Barcelo, F. (2009). Construct validity of the Trail Making Test: role of task-switching, working memory, inhibition/interference control, and visuomotor abilities. J Int Neuropsychol Soc, 15(3), 438-450. doi:10.1017/s1355617709090626

Sano, M., Rosen, W., Stern, Y., Rosen, J., \& Mayeux, R. (2009). Simple reaction time as a measure of global attention in Alzheimer's disease. Journal of the International Neuropsychological Society, 1(1), 56-61.

doi:10.1017/S1355617700000102

Sarter, M., Hasselmo, M. E., Bruno, J. P., \& Givens, B. (2005). Unraveling the attentional functions of cortical cholinergic inputs: interactions between signaldriven and cognitive modulation of signal detection. Brain Res Brain Res Rev, 48(1), 98-111. doi:10.1016/j.brainresrev.2004.08.006

Sattler, C., Toro, P., Schonknecht, P., \& Schroder, J. (2012). Cognitive activity, education and socioeconomic status as preventive factors for mild cognitive impairment and Alzheimer's disease. Psychiatry Res, 196(1), 90-95.

doi:10.1016/j.psychres.2011.11.012

Scahill, R. I., Frost, C., Jenkins, R., Whitwell, J. L., Rossor, M. N., \& Fox, N. C. (2003). A longitudinal study of brain volume changes in normal aging using serial registered magnetic resonance imaging. Archives of Neurology, 60(7), 989-994. doi:10.1001/archneur.60.7.989 
Schaafsma, M., Homewood, J., \& Taylor, A. (2010). Subjective cognitive complaints at menopause associated with declines in performance of verbal memory and attentional processes. Climacteric, 13(1), 84-98. doi:10.3109/13697130903009187

Scheef, L., Spottke, A., Daerr, M., Joe, A., Striepens, N., Koelsch, H., . . Jessen, F. (2012). Glucose metabolism, gray matter structure, and memory decline in subjective memory impairment. Neurology, 79(13), 1332-1339.

doi:10.1212/WNL.0b013e31826c1a8d

Schmid, N. S., Taylor, K. I., Foldi, N. S., Berres, M., \& Monsch, A. U. (2013). Neuropsychological signs of Alzheimer's disease 8 years prior to diagnosis. $J$ Alzheimers Dis, 34(2), 537-546. doi:10.3233/jad-121234

Schofield, P. W., Marder, M., Dooneief, G., Jacobs, D. M., Sano, M., \& Stern, Y. (1997). Association of subjective memory complaints with subsequent cognitive decline in community-dwelling elderly individuals with baseline cognitive impairment. American Journal of Psychiatry, 154(5), 609-615.doi:

10.1176/ajp.154.5.609

Schooler C, Mulatu, M.S., \& Oates, G. (1999). The continuing effects of substantively complex work on the intellectual functioning of older workers. Psychology of Aging, 14, 483-506.doi: http://dx.doi.org/10.1037/08827974.14.3.483

Scialfa, C. T., \& Joffe, K. M. (1997). Age differences in feature and conjunction search: Implications for theories of visual search and generalized slowing. Aging, Neuropsychology, and Cognition, 4(3), 227-246. doi:10.1080/13825589708256649

Self, E., Handelman, S., Le, A., \& Sigler, M. (2016). Changes in Visual Attention with Normal Aging. Journal of Vision, 16(12), 787-787. doi:10.1167/16.12.787

Seo, E. H., Lee, D. Y., Kim, K. W., Lee, J. H., Jhoo, J. H., Youn, J. C., . . Woo, J. I. (2006). A normative study of the Trail Making Test in Korean elders. International Journal of Geriatric Psychiatry, 21(9), 844-852.doi: 10.1002/gps.1570

Setti, A., Loughman, J., Savva, G. M., \& Kenny, R. (2015). Trail Making Test performance contributes to subjective judgment of visual efficiency in older adults. PeerJ, 3, e1407.

Sevush, S., \& Leve, N. (1993). Denial of memory deficit in Alzheimer's disease. Am J Psychiatry, 150(5), 748-751. doi:10.1176/ajp.150.5.748

Shankar, A., Hamer, M., McMunn, A., \& Steptoe, A. (2013). Social isolation and loneliness: relationships with cognitive function during 4 years of follow-up in the English Longitudinal Study of Ageing. Psychosom Med, 75(2), 161-170.

doi:10.1097/PSY.0b013e31827f09cd 
Shibuya-Tayoshi, S., Sumitani, S., Kikuchi, K., Tanaka, T., Tayoshi, S. Y., Ueno, S.I., \& Ohmori, T. (2007). Activation of the prefrontal cortex during the Trail-Making Test detected with multichannel near-infrared spectroscopy. Psychiatry and Clinical Neurosciences, 61(6), 616-621. doi:10.1111/j.1440-1819.2007.01727.x

Shindo, A., Terada, S., Sato, S., Ikeda, C., Nagao, S., Oshima, E., . . Uchitomi, Y. (2013). Trail Making Test Part A and Brain Perfusion Imaging in Mild Alzheimer's Disease. Dementia and geriatric cognitive disorders extra, 3(1), 202-211. doi:10.1159/000350806

Siettos, C. I., \& Smyrnis, N. (2017). Reaction time as a stochastic process implemented by functional brain networks. Cognitive neuroscience, 8(2), 133135.doi: 10.1080/17588928.2016.1206519

Silva, D., Guerreiro, M., Faria, C., Maroco, J., Schmand, B. A., \& de Mendonca, A. (2014). Significance of Subjective Memory Complaints in the Clinical Setting. Journal of Geriatric Psychiatry and Neurology, 27(4), 259-265. doi:10.1177/0891988714532018

Silva, L. D. S. V. e., Silva, T. B. L. d., Falcão, D. V. d. S., Batistoni, S. S. T., Lopes, A., Cachioni, M., .. . Yassuda, M. S. (2014). Relations between memory complaints, depressive symptoms and cognitive performance among community dwelling elderly. Archives of Clinical Psychiatry (São Paulo), 41, 67-71.doi: http://dx.doi.org/10.1590/0101-60830000000013

Silveri, M. C., Reali, G., Jenner, C., \& Puopolo, M. (2007). Attention and memory in the preclinical stage of dementia. Journal of Geriatric Psychiatry and Neurology, 20(2), 67-75. doi:10.1177/0891988706297469

Silverman, I. W. (2006). Sex differences in simple visual reaction time: A historical meta-analysis. Sex roles, 54(1-2), 57-68.

Simon, J. R., \& Pouraghabagher, A. R. (1978). The effect of aging on the stages of processing in a choice reaction time task. Journal of Gerontology, 33(4), 553-561. doi:https://doi.org/10.1093/geronj/33.4.553

Sinai, M., Phillips, N. A., Chertkow, H., \& Kabani, N. J. (2010). Task Switching Performance Reveals Heterogeneity Amongst Patients With Mild Cognitive Impairment. Neuropsychology, 24(6), 757-774. doi:10.1037/a0020314

Singh-Manoux, A., Dugravot, A., Ankri, J., Nabi, H., Berr, C., Goldberg, M., . . . Elbaz, A. (2014). Subjective cognitive complaints and mortality: Does the type of complaint matter? Journal of Psychiatric Research, 48(1), 73-78.

doi:10.1016/j.jpsychires.2013.10.005 
Skoog, I., Berg, S., Johansson, B., Palmertz, B. and Andreasson, L.-A. (1996), The influence of white matter lesions on neuropsychological functioning in demented and non-demented 85-year-olds. Acta Neurologica Scandinavica, 93: 142-148. doi:10.1111/j.1600-0404.1996.tb00190.x

Smith, G. E., Petersen, R. C., Ivnik, R. J., Malec, J. F., \& Tangalos, E. G. (1996). Subjective memory complaints, psychological distress and longitudinal change in objective memory performance. Psychology and Aging, 11(2), 272-279. doi:10.1037/0882-7974.11.2.272

Sofko, C., Boettcher, A., Hoadley, R., \& Hill, B. (2014). A-21Differential Rates of Age-Related Decline on Trail Making Tests. Archives of Clinical Neuropsychology, 29(6), 511-511.doi: https://doi.org/10.1093/arclin/acu038.21

Solianik, R., Brazaitis, M., \& Skurvydas, A. (2016). Sex-related differences in attention and memory. Medicina, 52(6), 372-377.doi:

https://doi.org/10.1016/j.medici.2016.11.007

Sole-Padulles, C., Bartres-Faz, D., Junque, C., Vendrell, P., Rami, L., Clemente, I. C., .. . Luis Molinuevo, J. (2009). Brain structure and function related to cognitive reserve variables in normal aging, mild cognitive impairment and Alzheimer's disease. Neurobiology of Aging, 30(7), 1114-1124. doi:10.1016/j.neurobiolaging.2007.10.008

Steinberg, S. I., Negash, S., Sammel, M. D., Bogner, H., Harel, B. T., Livney, M. G., ... Arnold, S. E. (2013). Subjective Memory Complaints, Cognitive Performance, and Psychological Factors in Healthy Older Adults. American Journal of Alzheimers Disease and Other Dementias, 28(8), 776-783. doi:10.1177/1533317513504817

Stern, Y., Albert, S., Tang, M. X., \& Tsai, W. Y. (1999). Rate of memory decline in $\mathrm{AD}$ is related to education and occupation Cognitive reserve?. Neurology, 53(9), 1942-1942.doi: http://dx.doi.org/10.1212/WNL.53.9.1942

Sternberg, S. (1969). Memory scanning mental processes revealed by reaction time experiments. American Scientist, 57(4), 421-457.doi:

http://www.jstor.org/stable/27828738

Storandt, M., \& Beaudreau, S. (2004). Do reaction time measures enhance diagnosis of early-stage dementia of the Alzheimer type. Arch Clin Neuropsychol, 19(1), 119124.doi: https://doi.org/10.1016/S0887-6177(02)00220-2

Stroop, J. R. (1935). Studies of interference in serial verbal reactions. Journal of experimental psychology, 18(6), 643.doi: http://dx.doi.org/10.1037/h0054651 
Stuss, D. T., Bisschop, S. M., Alexander, M. P., Levine, B., Katz, D., \& Izukawa, D. (2001). The Trail Making Test: a study in focal lesion patients. Psychol Assess, 13(2), 230-239.doi: http://dx.doi.org/10.1037/1040-3590.13.2.230

Stuss, D. T., L. L. Stethem, and C. A. Poirier. "Comparison of three tests of attention and rapid information processing across six age groups." The Clinical

Neuropsychologist 1, no. 2 (1987): 139-

152.doi:https://doi.org/10.1080/13854048708520046

Svennerholm, L., Bostrom, K., \& Jungbjer, B. (1997). Changes in weight and compositions of major membrane components of human brain during the span of adult human life of Swedes. Acta Neuropathologica, 94(4), 345-352.doi: $10.1007 / \mathrm{s} 004010050717$

Svetina, M. (2016). The reaction times of drivers aged 20 to 80 during a divided attention driving. Traffic Injury Prevention, 17(8), 810-814.

doi:10.1080/15389588.2016.1157590

Tales, A., \& Basoudan, N. (2016). Anxiety in old age and dementia-implications for clinical and research practice. Neuropsychiatry, 6(4).

Tales, A., Bayer, A. J., Haworth, J., Snowden, R. J., Philips, M., \& Wilcock, G. (2010). Visual Search in Mild Cognitive Impairment: A Longitudinal Study. Journal of Alzheimers Disease, 24(1), 151-160. doi:10.3233/jad-2010-101818

Tales, A., Haworth, J., Nelson, S., Snowden, R. J., \& Wilcock, G. (2005). Abnormal visual search in mild cognitive impairment and Alzheimer's disease. Neurocase, 11(1), 80-84. doi:10.1080/13554790490896974

Tales, A., Jessen, F., Butler, C., Wilcock, G., Phillips, J., \& Bayer, T. (2015). Subjective Cognitive Decline. Journal of Alzheimer's Disease, 48(Suppl 1), S1-S3. http://doi.org/10.3233/JAD-150719

Tales, A., Leonards, U., Bompas, A., Snowden, R. J., Philips, M., Porter, G., . . . Bayer, A. (2012). Intra-Individual Reaction Time Variability in Amnestic Mild Cognitive Impairment: A Precursor to Dementia? Journal of Alzheimers Disease, 32(2), 457-466. doi:10.3233/jad-2012-120505

Tales, A., Muir, J. L., Bayer, A., Jones, R., \& Snowden, R. J. (2002). Phasic visual alertness in Alzheimer's disease and ageing. Neuroreport, 13(18), 2557-2560. doi:10.1097/01.wnr.0000047985.70540.9d

Tales, A., Muir, J. L., Bayer, A., \& Snowden, R. J. (2002). Spatial shifts in visual attention in normal ageing and dementia of the Alzheimer type. Neuropsychologia, 40(12), 2000-2012. doi:10.1016/s0028-3932(02)00057-x 
Tales, A., Muir, J., Jones, R., Bayer, A., \& Snowden, R. J. (2004). The effects of saliency and task difficulty on visual search performance in ageing and Alzheimer's disease. Neuropsychologia, 42(3), 335-345.

doi:10.1016/j.neuropsychologia.2003.08.002

Tales, A., \& Porter, G. (2008). Visual attention-related processing in Alzheimer's disease. Reviews in Clinical Gerontology, 18(03), 229-243.

doi: https://doi.org/10.1017/S0959259809002792

Tales, A., Snowden, R. J., Brown, M., \& Wilcock, G. (2006). Alerting and orienting in Alzheimer's disease. Neuropsychology, 20(6), 752-756. doi:10.1037/08944105.20.6.752

Tales, A., Snowden, R. J., Haworth, J., \& Wilcock, G. (2005). Abnormal spatial and non-spatial cueing effects in mild cognitive impairment and Alzheimer's disease. Neurocase, 11(1), 85-92. doi:10.1080/13554790490896983

Tales, A., Snowden, R. J., Phillips, M., Haworth, J., Porter, G., Wilcock, G., \& Bayer, A. (2011). Exogenous phasic alerting and spatial orienting in mild cognitive impairment compared to healthy ageing: Study outcome is related to target response. Cortex, 47(2), 180-190. doi:https://doi.org/10.1016/j.cortex.2009.09.007

Teipel, S. J., Bayer, W., Alexander, G. E., Teichberg, D., Kulic, L., Schapiro, M. B., ... \& Hampel, H. (2002). Progression of corpus callosum atrophy in Alzheimer disease. Archives of Neurology, 59(2), 243-248.doi: 10.1001/archneur.59.2.243

Terada, S., Sato, S., Nagao, S., Ikeda, C., Shindo, A., Hayashi, S., . . Uchitomi, Y. (2013). Trail making test B and brain perfusion imaging in mild cognitive impairment and mild Alzheimer's disease. Psychiatry Res, 213(3), 249-255. doi:10.1016/j.pscychresns.2013.03.006

Terry, R. D. (2000). Cell death or synaptic loss in Alzheimer disease. Journal of Neuropathology and Experimental Neurology, 59(12), 1118-1119.doi: https://doi.org/10.1093/jnen/59.12.1118

Thomann, P. A., Wüstenberg, T., Pantel, J., Essig, M., \& Schröder, J. (2006). Structural changes of the corpus callosum in mild cognitive impairment and Alzheimer's disease. Dementia and geriatric cognitive disorders, 21(4), 215-220.doi: https://doi.org/10.1159/000090971

Thornton, I. M., \& Horowitz, T. S. (2004). The multi-item localization (MILO) task: measuring the spatiotemporal context of vision for action. Percept Psychophys, 66(1), 38-50. doi:10.3758/BF03194859. 
Tiffin-Richards FE, Costa AS, Holschbach B, Frank RD, Vassiliadou A, Krüger T, et al. (2014) The Montreal Cognitive Assessment (MoCA) - A Sensitive Screening Instrument for Detecting Cognitive Impairment in Chronic Hemodialysis Patients. PLoS ONE, 9(10): e106700. https://doi.org/10.1371/journal.pone.0106700

Tombaugh, T. N. (2004). Trail Making Test A and B: normative data stratified by age and education. Archives of Clinical Neuropsychology, 19(2), 203-214.doi: https://doi.org/10.1016/S0887-6177(03)00039-8

Tonn, P., Bartenstein, P., \& Dahmen, N. (2005). Lithium inoxication imitates Alzheimer's dementia in PET and clinical findings. The nerve physician , 76(5), 613 616.doi: 10.1007 / s00115-004-1758-8

Torrens-Burton, A., Basoudan, M., Bayer, A., et al (2017) Perception and reality of cognitive Information processing speed, perceived memory function, and perceived task difficulty in older adults. Journal of Alzhiemer's disease, 60(4), 1601-1609.doi: 10.3233/JAD-170599.

Tractenberg, R. E., \& Pietrzak, R. H. (2011). Intra-individual variability in Alzheimer's disease and cognitive aging: definitions, context, and effect sizes. PLoS One, 6(4), e16973.doi: https://doi.org/10.1371/journal.pone.0016973

Traykov, L., Raoux, N., Latour, F., Gallo, L., Hanon, O., Baudic, S., ... \& Rigaud, A. S. (2007). Executive functions deficit in mild cognitive impairment. Cognitive and Behavioral Neurology, 20(4), 219-224. doi: 10.1097/WNN.0b013e31815e6254

Treisman, A. M., \& Gelade, G. (1980). A feature-integration theory of attention. Cognitive psychology, 12(1), 97-136.doi: https://doi.org/10.1016/00100285(80)90005-5

Treisman, A., \& Sato, S. (1990). Conjunction search revisited. Journal of experimental psychology: human perception and performance, 16(3), 459.doi: 10.1037//0096-1523.16.3.459

Treisman, A., \& Schmidt, H. (1982). Illusory conjunctions in the perception of objects. Cognitive Psychology, 14(1), 107-141. doi:10.1016/0010-0285(82)90006-8

Trick, L. M., \& Enns, J. T. (1998). Lifespan changes in attention: The visual search task. Cognitive Development, 13(3), 369-386.doi: https://doi.org/10.1016/S08852014(98)90016-8

Tuch, D. S., Salat, D. H., Wisco, J. J., Zaleta, A. K., Hevelone, N. D., \& Rosas, H. D. (2005). Choice reaction time performance correlates with diffusion anisotropy in white matter pathways supporting visuospatial attention. Proceedings of the National Academy of Sciences of the United States of America, 102(34), 12212-12217. doi: 10.1073/pnas.0407259102 
Tun, P. A., \& Lachman, M. E. (2008). Age Differences in Reaction time and Attention in a National Telephone Sample of Adults: Education, Sex, and Task Complexity Matter. Dev Psychol, 44(5), 1421-1429. doi:10.1037/a0012845

Vallejo, V., Cazzoli, D., Rampa, L., Zito, G. A., Feuerstein, F., Gruber, N., . . Nef, T. (2016). Effects of Alzheimer's Disease on Visual Target Detection: A "Peripheral Bias". Frontiers in Aging Neuroscience, 8.doi:10.3389/fnagi.2016.00200.

van Veen, V., Krug, M. K., \& Carter, C. S. (2008). The neural and computational basis of controlled speed-accuracy tradeoff during task performance. Journal of Cognitive Neuroscience, 20(11), 1952-1965.doi:

https://doi.org/10.1162/jocn.2008.20146

Vaportzis, E., Georgiou-Karistianis, N., \& Stout, J. C. (2013). Dual task performance in normal aging: a comparison of choice reaction time tasks. Plos One, 8(3), e60265.

Verhage, F. (1965). Intelligence and age in a Dutch sample. Human Development, 8(4), 238-245.doi: https://doi.org/10.1159/000270308

Verhaeghen, P., \& Cerella, J. (2002). Aging, executive control, and attention: a review of meta-analyses. Neuroscience and Biobehavioral Reviews, 26(7), 849-857. doi:10.1016/s0149-7634(02)00071-4

Veiel, L. L., Storandt, M., \& Abrams, R. A. (2006). Visual search for change in older adults. Psychology and aging, 21(4), 754.doi: http://dx.doi.org/10.1037/08827974.21.4.754

Voelker, P., Rothbart, M. K., \& Posner, M. I. (2016). A polymorphism related to methylation influences attention during performance of speeded skills. AIMS Neuroscience, 3(1), 40-55.doi: https://doi.org/10.3934/Neuroscience.2016.1.40

Vrtunski, P. B., Patterson, M. B., Mack, J. L., \& Hill, G. O. (1983).

Microbehavioural analysis of the choice reaction time response in senile dementia1. Brain, 106(4), 929-947. doi:10.1093/brain/106.4.929

Waldmann, B. W., Dickson, A. L., Monahan, M. C., \& Kazelskis, R. (1992). The relationship between intellectual ability and adult performance on the Trail Making Test and the Symbol Digit Modalities Test. Journal of Clinical Psychology, 48(3), 360-363. DOI: 10.1002/1097-4679(199205)48:3<360::AIDJCLP2270480314>3.0.CO;2-P

Walhovd, K. B., \& Fjell, A. M. (2007). White matter volume predicts reaction time instability. Neuropsychologia, 45(10), 2277-2284.doi:

https://doi.org/10.1016/j.neuropsychologia.2007.02.022

Wang, P. J., Saykin, A. J., Flashman, L. A., Wishart, H. A., Rabin, L. A., Santulli, R. B., ... \& Mamourian, A. C. (2006). Regionally specific atrophy of the corpus callosum in AD, MCI and cognitive complaints. Neurobiology of aging, 27(11), 1613-1617.doi: 10.1016/j.neurobiolaging.2005.09.035 
Wang, Q., Cavanagh, P., \& Green, M. (1994). Familiarity and pop-out in visual search. Attention, Perception, \& Psychophysics, 56(5), 495-500.doi:

http://dx.doi.org/10.3758/BF03206946

Waugh, N. C., Fozard, J. L., Talland, G. A., \& Erwin, D. E. (1973). Effects of age and stimulus repetition on two-choice reaction time. Journal of Gerontology, 28(4), 466-470.doi: https://doi.org/10.1093/geronj/28.4.466

Wegesin, D. J., \& Stern, Y. (2004). Inter- and intraindividual variability in recognition memory: Effects of age and estrogen use. Neuropsychology, 18, $646-$ 657. doi:10.1037/0894-4105.18.4.646

Wehling, E., Lundervold, A. J., Standnes, B., Gjerstad, L., \& Reinvang, I. (2007). APOE status and its association to learning and memory performance in middle aged and older Norwegians seeking assessment for memory deficits. Behavioral and Brain Functions, 3(1), 1-10. doi:10.1186/1744-9081-3-57

West, R., Murphy, K. J., Armilio, M. L., Craik, F. I. M., \& Stuss, D. T. (2002). Lapses of Intention and Performance Variability Reveal Age-Related Increases in Fluctuations of Executive Control. Brain and Cognition, 49(3), 402-419. doi:https://doi.org/10.1006/brcg.2001.1507

Whitwell, J. L., Petersen, R. C., Negash, S., Weigand, S. D., Kantarci, K., Ivnik, R. J., ... \& Jack, C. R. (2007). Patterns of atrophy differ among specific subtypes of mild cognitive impairment. Archives of Neurology, 64(8), 1130-1138.doi: 10.1001/archneur.64.8.1130

Willis, S. L., Allen-Burge, R., Dolan, M. M., Bertrand, R. M., Yesavage, J., \& Taylor, J. L. (1998). Everyday problem solving among individuals with Alzheimer's disease. The Gerontologist, 38(5), 569-577.

Wilkinson, R. T., \& Allison, S. (1989). Age and simple reaction time: Decade differences for 5,325 subjects. Journal of gerontology, 44(2), 29-35. doi:https://doi.org/10.1093/geronj/44.2.P29

Woods, D. L., Wyma, J. M., Yund, E. W., Herron, T. J., \& Reed, B. (2015). Agerelated slowing of response selection and production in a visual choice reaction time task. Front Hum Neurosci, 9, 193. doi:10.3389/fnhum.2015.00193

Wright, B. A., Peters, E. R., Ettinger, U., Kuipers, E., \& Kumari, V. (2016). Moderators of noise-induced cognitive change in healthy adults. Noise \& health, 18(82), 117.doi: 10.4103/1463-1741.181995

Wright, R. E. (1981). Aging, divided attention, and processing capacity. Journal of Gerontology,36, 605-614 
Yarkoni, T., Barch, D. M., Gray, J. R., Conturo, T. E., \& Braver, T. S. (2009). BOLD Correlates of Trial-by-Trial Reaction Time Variability in Gray and White Matter: A Multi-Study fMRI Analysis. Plos One, 4(1). doi:10.1371/journal.pone.0004257

Yates, J. A., Clare, L., \& Woods, R. T. (2015). Subjective memory complaints, mood and MCI: a follow-up study. Aging Ment Health, 1-9.

doi:10.1080/13607863.2015.1081150

Yee, N. (2006). Motivations for play in online games. CyberPsychology \& behavior, 9(6), 772-775.doi: https://doi.org/10.1089/cpb.2006.9.772

Yellott Jr, J. I. (1971). Correction for fast guessing and the speed-accuracy tradeoff in choice reaction time. Journal of Mathematical Psychology, 8(2), 159-199.doi: https://doi.org/10.1016/0022-2496(71)90011-3

Ylikoski, R., Ylikoski, A., Erkinjuntti, T., Sulkava, R., Raininko, R., \& Tilvis, R. (1993). White matter changes in healthy elderly persons correlate with attention and speed of mental processing. Archives of Neurology, 50(8), 818-824.doi: 10.1001/archneur.1993.00540080029009

Yokoyama, O., Miura, N., Watanabe, J., Takemoto, A., Uchida, S., Sugiura, M., ... \& Nakamura, K. (2010). Right frontopolar cortex activity correlates with reliability of retrospective rating of confidence in short-term recognition memory performance. Neuroscience research, 68(3), 199-206.doi: https://doi.org/10.1016/j.neures.2010.07.2041

Yordanova, J., Kolev, V., Hohnsbein, J., \& Falkenstein, M. (2004). Sensorimotor slowing with ageing is mediated by a functional dysregulation of motor-generation processes: evidence from high-resolution event-related potentials. Brain, 127( $\mathrm{Pt} 2)$, 351-362. doi:10.1093/brain/awh042

Yoshita, M., Fletcher, E., Harvey, D., Ortega, M., Martinez, O., Mungas, D. M., ... \& DeCarli, C. S. (2006). Extent and distribution of white matter hyperintensities in normal aging, MCI, and AD. Neurology, 67(12), 2192-2198. doi: http://dx.doi.org/ 10.1212/01.wnl.0000249119.95747.1f

Yotsumoto, Y., Chang, L. H., Ni, R., Pierce, R., Andersen, G. J., Watanabe, T., \& Sasaki, Y. (2014). White matter in the older brain is more plastic than in the younger brain. Nature communications, 5, 5504.doi: 10.1038/ncomms6504.

Yung, A., Cardoso-Leite, P., Dale, G., Bavelier, D., \& Green, C. S. (2015). Methods to Test Visual Attention Online. Journal of Visualized Experiments : JoVE, (96), 10.3791/52470. Advance online publication. http://doi.org/10.3791/52470 
Yuspeh, R. L., Vanderploeg, R. D., Crowell, T. A., \& Mullan, M. (2002).

Differences in executive functioning between Alzheimer's disease and subcortical ischemic vascular dementia. Journal of Clinical and Experimental Neuropsychology, 24(6), 745-754.doi: https://doi.org/10.1076/jcen.24.6.745.8399

Zakzanis, K. K., Mraz, R., \& Graham, S. J. (2005). An fMRI study of the Trail Making Test. Neuropsychologia, 43(13), 1878-1886.

doi:10.1016/j.neuropsychologia.2005.03.013

Zalonis, I., Kararizou, E., Triantafyllou, N. I., Kapaki, E., Papageorgiou, S., Sgouropoulos, P., \& Vassilopoulos, D. (2008). A Normative Study of the Trail Making Test A and B in Greek Adults. The Clinical Neuropsychologist, 22(5), 842850. doi:10.1080/13854040701629301

Zapata, B. C., Fernández-Alemán, J. L., Idri, A., \& Toval, A. (2015). Empirical studies on usability of mHealth apps: a systematic literature review. Journal of medical systems, 39(2), 1.doi: 10.1007/s10916-014-0182-2

Zeef, E. J., \& Kok, A. (1993). Age-related differences in the timing of stimulus and response processes during visual selective attention: Performance and psychophysiological analyses. Psychophysiology, 30(2), 138-151.doi: 10.1111/j.1469-8986.1993.tb01727.x

Zeef, E. J., Sonke, C. J., Kok, A., Buiten, M. M., \& Kenemans, J. (1996). Perceptual factors affecting age-related differences in focused attention: performance and psychophysiological analyses. Psychophysiology, 33(5), 555-565.doi:

10.1111/j.1469-8986.1996.tb02432.

Zhang Y, Schuff N, Camacho M, Chao LL, Fletcher TP, Yaffe K, et al. (2013) MRI Markers or Mild Cognitive Impairment: Comparisons between White Matter Integrity and Gray Matter Volume Measurements. PLoS ONE, 8(6): e66367. https://doi.org/10.1371/journal.pone.0066367

Zhuang, L., Wen, W., Zhu, W., Trollor, J., Kochan, N., Crawford, J., ... \& Sachdev, P.(2010).White matter integrity in mild cognitive impairment: a tract-based spatial statistics study. Neuroimage, 53(1), 16-25.doi: https://doi.org/10.1016/j.neuroimage.2010.05.068

Zhaoping, L., \& Snowden, R. J. (2006). A theory of a saliency map inprimaryvisualcortex(V1) tested by psychophysics of colour-orientation interference in texture segmentation. Visual cognition, 14(4-8), 911-933.doi: http://dx.doi.org/10.1080/1350628050019603 
9.0. APPENDICES 
Dear Members of the Ethics Committee,

Please see attached an emended copy of my ethics application form for the project titled 'Investigating the potential influence of perceived changes in memory and thinking skills upon reaction time and attention in ageing.'

The main change which has been made is in the Information sheet to include information about the memory testing component during the study which is included within the MoCa test (also attached). There is also a second poster to advertise for young people as well as older adults.

Many thanks and kind regards,

Anna Torrens-Burton

\& Professor Andrea Tales 


\section{Volunteers needed for psychology research on the potential influence of perceived changes in memory and thinking skills upon reaction time and attention in ageing}

You will be presented with a number of short computerized and pen and paper tasks.

Eligibility: Males and females between the ages of 50-80yrs in general good health.

Cannot have any history of serious head injury, serious cognitive, visual, neurological impairments, depression and no condition which might be affected by flashing images on a screen and not have seen your GP about your memory or thinking skills.

Time required: 2 hours (includes breaks)

$$
\begin{gathered}
\text { Venue: Department of Psychology } \\
\text { 8th }^{\text {th }} \text { Floor, Vivian Tower, } \\
\text { Swansea University, } \\
\text { Singleton Park, } \\
\text { Swansea, } \\
\text { SA2 8PP }
\end{gathered}
$$

\section{Local travel expenses reimbursed}

If you would like to take part or want further information, please contact 


\section{Volunteers needed for psychology research} on the potential influence of perceived changes in memory and thinking skills upon reaction time and attention in ageing

You will be presented with a number of short computerized and pen and paper tasks.

Eligibility: Males and females between the ages of 18-25 in general good health.

Cannot have any history of serious head injury, serious cognitive, visual, neurological impairments, depression and no condition which might be affected by flashing images on a screen and not have seen your GP about your memory or thinking skills.

Time required: 2 hours (includes breaks)

$$
\begin{gathered}
\text { Venue: Department of Psychology } \\
\text { 8 }^{\text {th }} \text { Floor, Vivian Tower, } \\
\text { Swansea University, } \\
\text { Singleton Park, } \\
\text { Swansea, } \\
\text { SA2 8PP }
\end{gathered}
$$

If you would like to take part or want further information, please 


\section{PARTICIPANT INFORMATION SHEET}

\section{Investigating the potential influence of perceived changes in memory and thinking skills upon reaction time and attention in ageing.}

You are being invited to take part in some research. Before you decide whether or not to participate, it is important for you to understand why the research is being conducted and what it will involve. Please read the following information carefully.

\section{What is the purpose of the research?}

We are conducting research on the relationship between reaction time, vision and visual attention and perceived changes in thinking and memory skills in ageing. The session should take no longer than 2 hours including breaks between tests.

\section{Who is carrying out the research?}

The data is being collected by Anna Torrens-Burton, PhD student at Department of Psychology, Swansea University, under the supervision of Prof. Andrea Tales, Department of Psychology, Swansea University. The research has been approved by the Department of Psychology's Research Ethics Committee.

\section{What happens if I agree to take part?}

You will be given a number of short tasks and questionnaires which measure your reaction time, vision and attention and some aspects of your memory. There will be a mixture of computer based and pen and paper tasks and questionnaires to complete. You will be given time to ask questions about each of the tasks and to practice them. In between each different computer-based task you will be offered a short break to rest your eyes and move about. You will also be asked to complete a range of questions designed to measure some aspects of your memory and you will also be will also be asked to complete a questionnaire about yourself, which includes questions about your age, gender, level of education, how you feel about your memory and thinking skills, occupation, general health (including levels of anxiety and or depression) and questions about the vision and attention tests you performed. The outcome of these tasks will be used for research purposes only; they will not be used for diagnostic purposes and we are unable to provide feedback upon the outcome of the tests or any interpretation of performance.

\section{Is participation voluntary and what if I wish to later withdraw?}

Your participation is entirely voluntary. You have no obligation to take part if you do not wish to do so. If you decide to participate, but later wish to withdraw from the study, then you are free to do so at any time. You do not have to give a reason and you will not be penalized. Just let the researcher know and you will be free to leave. 
What will happen to the information I provide?

All the data obtained will be confidential to the study and will be used only for research purposes. You will be identified via a participation number, not by name so that all personal details are held strictly confidential. Please note that because the data will be made anonymous, it will not be possible to identify and remove your data at a later date should you decide to withdraw from the study. Therefore, if at the end of this research you decide to have your data withdrawn, please let us know before you leave.

An analysis of the information will form part of our report at the end of the study and may be presented to interested parties and published in scientific journals and related media. Note that information presented in any reports or publications will be anonymous

\section{What if I have other questions?}

If you have further questions about this research please do not hesitate to contact us:

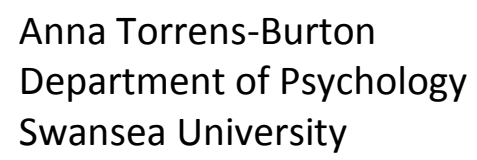

Prof. Andrea Tales

Department of Psychology

Swansea University

SA2 8PP 


\section{CONSENT FORM}

\section{Investigating the potential influence of perceived changes in memory and thinking skills upon reaction time and attention in ageing.}

I agree to take part in the above research. I have read the Participant Information Sheet, which is attached to this form. I understand what my role will be in this research, and all my questions have been answered to my satisfaction.

I understand that participation is voluntary and also that I am free to withdraw from the research at any time, for any reason and without prejudice.

I have been informed that the confidentiality of the information I provide will be safeguarded.

I am free to ask any questions at any time before and during the study.

I have been provided with a copy of the Participant Information Sheet.

I understand that it will not be possible to identify my data at a later date, and therefore if I wish to withdraw my data from the study, I will need to do so before leaving the room.

I am aged 18 years or above.

Data Protection: I agree to the University processing personal data that I have supplied. I agree to the processing of such data for any purposes connected with the Research Project as outlined to me.

Name of participant

(Print)

Office Use Only

Signed

Date

Participant No.

Study group/condition 


\section{APPENDIX G}

\section{DEBRIEF FORM}

\section{Investigating the potential influence of perceived changes in memory and thinking skills upon reaction time and attention in ageing.}

Thank you very much for taking part in our research! Now that we've finished, let us explain the rationale behind this work.

There is evidence from previous research of a general slowing of cognitive processing during the process of aging as a result of age-related changes to the structure and function of the brain. However, how ageing and any changes in our memory and thinking skills may affect how we see and attend to things and the speed with which this is performed, is unclear. We are interested therefore in whether there is any change in response times, vision and attention function during ageing and whether any such change is related to any changes in our memory and thinking skills we may experience.

In this research you completed different vision, attention and reaction time tasks. This data will be compared between individuals of different stages of ageing; in this case young adults aged 18-24 years and older adults aged 50-70 years. Although we expect young adults to be faster at responding (i.e. lower response times) compared to older adults, the results of these novel tests from each of our groups will be related to what individuals think about their memory and thinking skills, thus improving our knowledge about the potential links between memory and attention.

If you feel affected by issues raised by this research and would like to discuss any concerns, then please contact the study Supervisor: email: Phone:

You can also contact Swansea University's Wellbeing services, for advice: Wellbeing Services, Horton Building, Swansea University, Singleton Park, Swansea, SA2 8PP, Tel: 01792 295592, www.swansea.ac.uk/wellbeing/. Further information about healthy lifestyles can also be found at www.bbc.co.uk/health.

If you have any other questions about the research, please do not hesitate to contact us at:

Anna Torrens-Burton

Department of Psychology

Swansea University

SA2 8PP
Prof. Andrea Tales

Department of Psychology

Swansea University

SA2 8PP 
Participant ID:

Investigating the potential influence of perceived changes in memory and thinking skills upon reaction time and attention in ageing.

1. Age:

2. Gender: Male

Female

3. Left handed

Right handed

4. Current Occupation/Past occupation (if you have had a number of different occupations please state the one which you have been in the longest):

\section{Highest level of education:}

\begin{tabular}{ll}
$\square$ & GCSEs/O levels \\
\hline$\square$ & A levels \\
\hline$\square$ & Higher education certificates \\
\hline$\square$ & Bachelors Degree \\
$\square$ & Masters degree \\
\hline & $\mathrm{PhD}$ \\
\hline$\square$ & Doctoral certificates \\
\hline & Other (please specify)
\end{tabular}

6. Number of years in education: 


\section{Memory Functioning Questionnaire (MFQ)}

This is a questionnaire about how you remember information. There are no right or wrong answers. Circle a number between 1 and 7 that best reflects your judgment about your memory. Think carefully about your responses, and try to be as realistic as possible when you make them. Please answer all questions.

\section{GENERAL RATING SCALE}

1. How would you rate your memory in terms of the kinds of problems that you have? major problems some minor problems

$\begin{array}{lllll}1 & 2 & 3 & 4 & 5\end{array}$

no problems

\section{RETROSPECTIVE FUNCTIONING SCALE}

2. How is your memory compared to the way it was...

\begin{tabular}{|c|c|c|c|c|c|c|c|}
\hline \multirow[b]{2}{*}{ a. one year ago? } & \multicolumn{2}{|c|}{ much worse } & \multicolumn{3}{|c|}{ same } & \multicolumn{2}{|c|}{ much bette } \\
\hline & 1 & 2 & 3 & 4 & 5 & 6 & 7 \\
\hline b. five years ago? & 1 & 2 & 3 & 4 & 5 & 6 & 7 \\
\hline c. ten years ago? & 1 & 2 & 3 & 4 & 5 & 6 & 7 \\
\hline d. twenty years ago? & 1 & 2 & 3 & 4 & 5 & 6 & 7 \\
\hline e. when you were eighteen? & 1 & 2 & 3 & 4 & 5 & 6 & 7 \\
\hline
\end{tabular}

\section{FREQUENCY OF FORGETTING SCALE}

3. How often do these present a problem for you?

\begin{tabular}{|c|c|c|c|c|c|c|c|c|}
\hline \multirow[b]{2}{*}{ a. } & \multirow[b]{2}{*}{ names } & \multicolumn{2}{|l|}{ always } & \multicolumn{3}{|c|}{ sometimes } & \multirow[b]{2}{*}{6} & \multirow{2}{*}{$\begin{array}{c}\text { never } \\
7\end{array}$} \\
\hline & & 1 & 2 & 3 & 4 & 5 & & \\
\hline b. & faces & 1 & 2 & 3 & 4 & 5 & 6 & 7 \\
\hline c. & appointments $\ldots \ldots \ldots \ldots \ldots$ & 1 & 2 & 3 & 4 & 5 & 6 & 7 \\
\hline d. & where you put things (e.g., keys) & 1 & 2 & 3 & 4 & 5 & 6 & 7 \\
\hline e. & performing household chores & 1 & 2 & 3 & 4 & 5 & 6 & 7 \\
\hline f. & directions to places $\ldots \ldots \ldots \ldots \ldots \ldots \ldots$ & 1 & 2 & 3 & 4 & 5 & 6 & 7 \\
\hline g. & phone numbers you've just checked ........ & 1 & 2 & 3 & 4 & 5 & 6 & 7 \\
\hline h. & phone numbers you use frequently $\ldots \ldots \ldots$ & 1 & 2 & 3 & 4 & 5 & 6 & 7 \\
\hline i. & things people tell you & 1 & 2 & 3 & 4 & 5 & 6 & 7 \\
\hline j. & keeping up correspondence & 1 & 2 & 3 & 4 & 5 & 6 & 7 \\
\hline k. & personal dates (e.g., birthdays) & 1 & 2 & 3 & 4 & 5 & 6 & 7 \\
\hline I. & words $\ldots \ldots \ldots \ldots$ & 1 & 2 & 3 & 4 & 5 & 6 & 7 \\
\hline
\end{tabular}




\section{MFQ (continued)}

m. going to the store and forgetting what you wanted to buy $\ldots \ldots \ldots \ldots \ldots \ldots, 1$

n. taking a test $\ldots \ldots \ldots \ldots \ldots \ldots \ldots \ldots \ldots, 1$

o. beginning to do something and forgetting what you were doing

p. losing the thread of thought in conversation

q. losing the thread of thought in public speaking

23

3

4

$$
5
$$

$6 \quad 7$

23

4

5

6

$\begin{array}{lllllll}1 & 2 & 3 & 4 & 5 & 6 & 7\end{array}$

r. knowing whether you've already told someone something

2

2

3

4

$6 \quad 7$

REQUENCY OF FORGETTING DURING READING SCALE

4. As you are reading a novel, how often do you have trouble remembering what you have read...

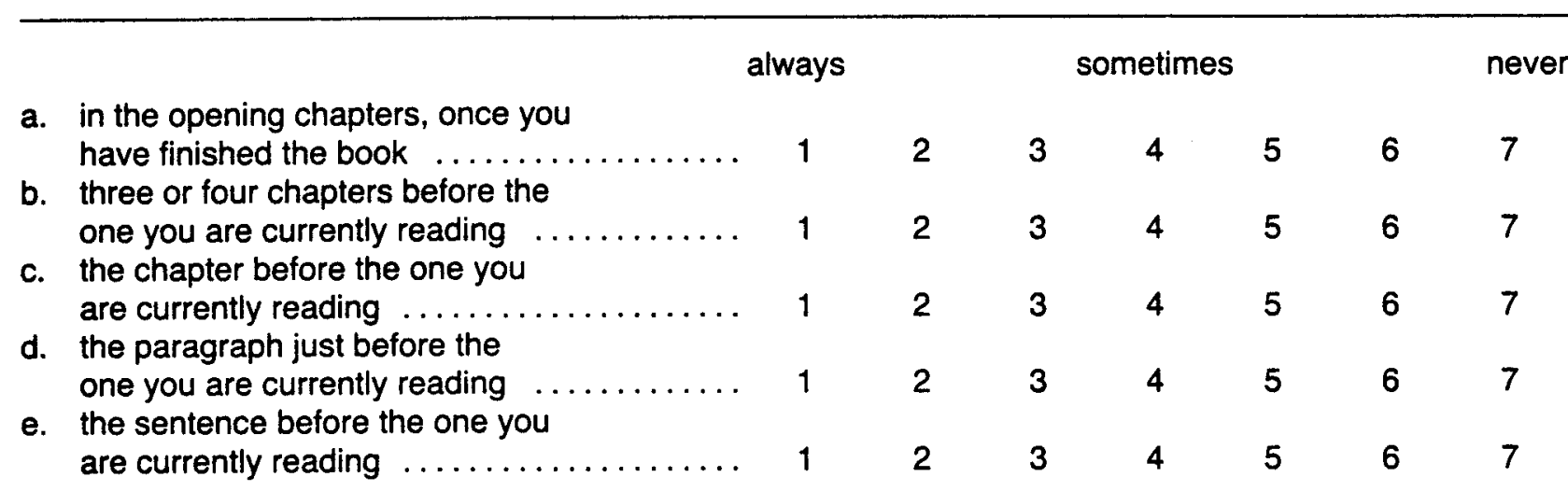

5. When you are reading a newspaper or magazine article, how often do you have trouble remembering what you have read...

always

sometimes

never

a. in the opening paragraphs, once you have finished the article...

b. three or four paragraphs before the one you are currently reading

c. the paragraph before the one you are currently reading ......

d. three or four sentences before the one you are currently reading

e. the sentence before the one you are currently reading

$\begin{array}{llllll}2 & 3 & 4 & 5 & 6 & 7 \\ 2 & 3 & 4 & 5 & 6 & 7 \\ 2 & 3 & 4 & 5 & 6 & 7 \\ 2 & 3 & 4 & 5 & 6 & 7 \\ 2 & 3 & 4 & 5 & 6 & 7\end{array}$




\section{MFQ (continued)}

\section{REMEMBERING PAST EVENTS SCALE}

6. How well you remember things which occurred...

\begin{tabular}{|c|c|c|c|c|c|c|c|}
\hline \multirow[b]{2}{*}{ last month is } & \multicolumn{2}{|c|}{ very bad } & \multicolumn{3}{|c|}{ fair } & \multicolumn{2}{|c|}{ very goo } \\
\hline & 1 & 2 & 3 & 4 & 5 & 6 & 7 \\
\hline $\begin{array}{l}\text { b. between six months and one year } \\
\text { ago is } \ldots \ldots \ldots \ldots \ldots \ldots \ldots\end{array}$ & 1 & 2 & 3 & 4 & 5 & 6 & 7 \\
\hline c. between one and five years ago is & 1 & 2 & 3 & 4 & 5 & 6 & 7 \\
\hline d. between six and ten years ago is & 1 & 2 & 3 & 4 & 5 & 6 & 7 \\
\hline
\end{tabular}

\section{SERIOUSNESS SCALE}

7. When you actually forget in these situations, how serious of a problem do you consider the memory failure to be?...

\begin{tabular}{|c|c|c|c|c|c|c|c|c|c|}
\hline \multirow[b]{2}{*}{ a. } & \multirow[b]{2}{*}{ names } & \multicolumn{3}{|c|}{ very serious } & \multicolumn{3}{|c|}{ somewhat serious } & \multicolumn{2}{|c|}{ not serious } \\
\hline & & $\cdots$ & 1 & 2 & 3 & 4 & 5 & 6 & 7 \\
\hline b. & faces $\ldots . .$. & & 1 & 2 & 3 & 4 & 5 & 6 & 7 \\
\hline c. & appointments $\ldots \ldots$. & & 1 & 2 & 3 & 4 & 5 & 6 & 7 \\
\hline d. & where you put things (e.g. keys) & & 1 & 2 & 3 & 4 & 5 & 6 & 7 \\
\hline e. & performing household chores. & & 1 & 2 & 3 & 4 & 5 & 6 & 7 \\
\hline f. & directions to places $\ldots \ldots \ldots \ldots \ldots$ & & 1 & 2 & 3 & 4 & 5 & 6 & 7 \\
\hline g. & phone numbers you've just checked & & 1 & 2 & 3 & 4 & 5 & 6 & 7 \\
\hline h. & phone numbers used frequently & & 1 & 2 & 3 & 4 & 5 & 6 & 7 \\
\hline i. & things people tell you $\ldots$... & & 1 & 2 & 3 & 4 & 5 & 6 & 7 \\
\hline j. & keeping up correspondence & & 1 & 2 & 3 & 4 & 5 & 6 & 7 \\
\hline k. & personal dates (e.g. birthdays) & & 1 & 2 & 3 & 4 & 5 & 6 & 7 \\
\hline 1. & words $\ldots \ldots \ldots \ldots \ldots \ldots$ & & 1 & 2 & 3 & 4 & 5 & 6 & 7 \\
\hline $\mathrm{m}$. & $\begin{array}{l}\text { going to the store and forgetting } \\
\text { what you wanted to buy } \ldots . . .\end{array}$ & & 1 & 2 & 3 & 4 & 5 & 6 & 7 \\
\hline n. & taking a test $\ldots$. & & 1 & 2 & 3 & 4 & 5 & 6 & 7 \\
\hline 0. & $\begin{array}{l}\text { beginning to do something and } \\
\text { forgetting what you were doing }\end{array}$ & & 1 & 2 & 3 & 4 & 5 & 6 & 7 \\
\hline p. & $\begin{array}{l}\text { losing the thread of thought in } \\
\text { conversation } \ldots \ldots \ldots \ldots \ldots \ldots\end{array}$ & & 1 & 2 & 3 & 4 & 5 & 6 & 7 \\
\hline q. & $\begin{array}{l}\text { losing the thread of thought in } \\
\text { public speaking } \ldots \ldots \ldots \ldots \ldots\end{array}$ & & 1 & 2 & 3 & 4 & 5 & 6 & 7 \\
\hline & $\begin{array}{l}\text { knowing whether you've already } \\
\text { told someone something } \ldots . . .\end{array}$ & & 1 & 2 & 3 & 4 & 5 & 6 & 7 \\
\hline
\end{tabular}


Page 4

\section{MFQ (continued)}

\section{MNEMONICS USAGE SCALE}

8. How often do you use these techniques to remind yourself about things? ...

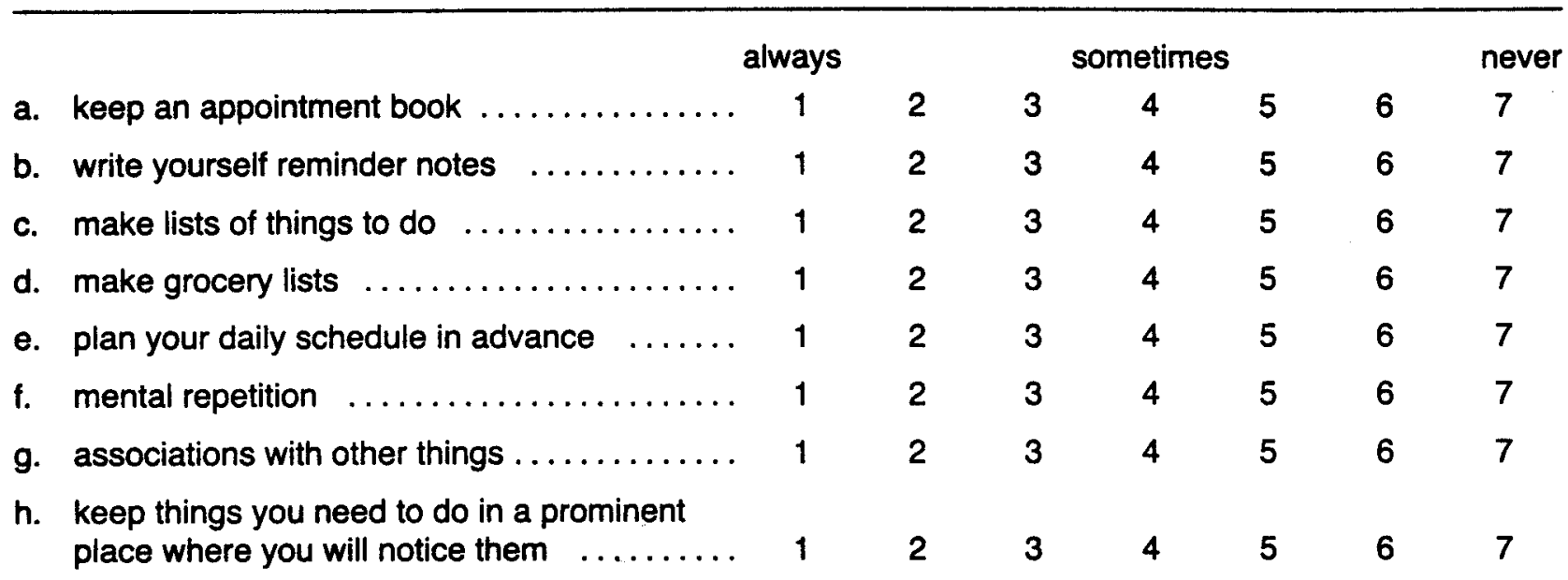




\section{PATIENT HEALTH QUESTIONNAIRE- 9 (PHQ - 9)}

Over the last 2 weeks, how often have you been bothered by any of the following problems?

(Use " $\boldsymbol{~ " ~ t o ~ i n d i c a t e ~ y o u r ~ a n s w e r ) ~}$

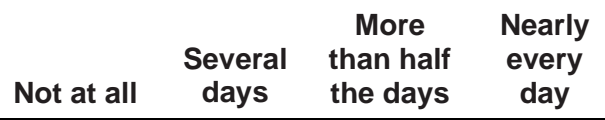

1. Little interest or pleasure in doing things

0

1

2

3

2. Feeling down, depressed, or hopeless

0

1

2

3

3. Trouble falling or staying asleep, or sleeping too much

0

1

2

3

4. Feeling tired or having little energy

0

1

2

3

5. Poor appetite or overeating

0

1

2

3

6. Feeling bad about yourself — or that you are a failure or have let yourself or your family down

0

1

2

3

7. Trouble concentrating on things, such as reading the newspaper or watching television

0

1

2

3

8. Moving or speaking so slowly that other people could have noticed? Or the opposite - being so fidgety or restless that you have been moving around a lot more than usual

9. Thoughts that you would be better off dead or of hurting yourself in some way

$\begin{array}{llll}0 & 1 & 2 & 3\end{array}$

$\begin{array}{llll}0 & 1 & 2 & 3\end{array}$

FOR OFFICE CODING

If you checked off any problems, how difficult have these problems made it for you to do your work, take care of things at home, or get along with other people?

\begin{tabular}{cccc} 
Not difficult & Somewhat & Very & Extremely \\
at all & difficult & difficult & difficult \\
$\square$ & $\square$ & $\square$ & $\square$ \\
\hline
\end{tabular}




\section{Generalized Anxiety Disorder 7-item (GAD-7) scale}

$\begin{array}{|lcccc|}\begin{array}{l}\text { Over the last 2 weeks, how often have you been } \\ \text { bothered by the following problems? }\end{array} & \begin{array}{c}\text { Not at } \\ \text { all sure }\end{array} & \begin{array}{c}\text { Several } \\ \text { days }\end{array} & \begin{array}{c}\text { Over half } \\ \text { the days }\end{array} & \begin{array}{c}\text { Nearly } \\ \text { every day }\end{array} \\ \text { 1. Feeling nervous, anxious, or on edge } & 0 & 1 & 2 & 3 \\ \text { 2. Not being able to stop or control worrying } & 0 & 1 & 2 & 3 \\ \text { 3. Worrying too much about different things } & 0 & 1 & 2 & 3 \\ \text { 4. Trouble relaxing } & 0 & 1 & 2 & 3 \\ \text { 5. Being so restless that it's hard to sit still } & 0 & 1 & 2 \\ \text { 6. Becoming easily annoyed or irritable } & 0 & 1 & 2 \\ \text { 7. Feeling afraid as if something awful might } \\ \text { happen }\end{array}$

If you checked off any problems, how difficult have these made it for you to do your work, take care of things at home, or get along with other people?

Not difficult at all

Somewhat difficult

Very difficult

Extremely difficult

Source: Spitzer RL, Kroenke K, Williams JBW, Lowe B. A brief measure for assessing generalized anxiety disorder. Arch Inern Med. 2006;166:1092-1097. 


\section{Trail Making Test (TMT) Parts A \& B}

\section{Instructions:}

Both parts of the Trail Making Test consist of 25 circles distributed over a sheet of paper. In Part $A$, the circles are numbered $1-25$, and the patient should draw lines to connect the numbers in ascending order. In Part $B$, the circles include both numbers $(1-13)$ and letters $(A-L)$; as in Part $A$, the patient draws lines to connect the circles in an ascending pattern, but with the added task of alternating between the numbers and letters (i.e., 1-A-2-B-3-C, etc.). The patient should be instructed to connect the circles as quickly as possible, without lifting the pen or pencil from the paper. Time the patient as he or she connects the "trail." If the patient makes an error, point it out immediately and allow the patient to correct it. Errors affect the patient's score only in that the correction of errors is included in the completion time for the task. It is unnecessary to continue the test if the patient has not completed both parts after five minutes have elapsed.

Step 1: $\quad$ Give the patient a copy of the Trail Making Test Part A worksheet and a pen or pencil.

Step 2: $\quad$ Demonstrate the test to the patient using the sample sheet (Trail Making Part A SAMPLE).

Step 3: $\quad$ Time the patient as he or she follows the "trail" made by the numbers on the test.

Step 4: $\quad$ Record the time.

Step 5: $\quad$ Repeat the procedure for Trail Making Test Part B.

\section{Scoring:}

Results for both TMT A and B are reported as the number of seconds required to complete the task; therefore, higher scores reveal greater impairment.

$\begin{array}{llll} & \text { Average } & \text { Deficient } & \text { Rule of Thumb } \\ \text { Trail A } & 29 \text { seconds } & >78 \text { seconds } & \text { Most in } 90 \text { seconds } \\ \text { Trail B } & 75 \text { seconds } & >273 \text { seconds } & \text { Most in } 3 \text { minutes }\end{array}$

\section{Sources:}

- Corrigan JD, Hinkeldey MS. Relationships between parts A and B of the Trail Making Test. J Clin Psychol. 1987;43(4):402-409.

- Gaudino EA, Geisler MW, Squires NK. Construct validity in the Trail Making Test: what makes Part B harder? J Clin Exp Neuropsychol. 1995;17(4):529-535.

- Lezak MD, Howieson DB, Loring DW. Neuropsychological Assessment. 4th ed. New York: Oxford University Press; 2004.

- Reitan RM. Validity of the Trail Making test as an indicator of organic brain damage. Percept Mot Skills. 1958;8:271-276. 


\section{Trail Making Test Part A}

Patient's Name:

Date:

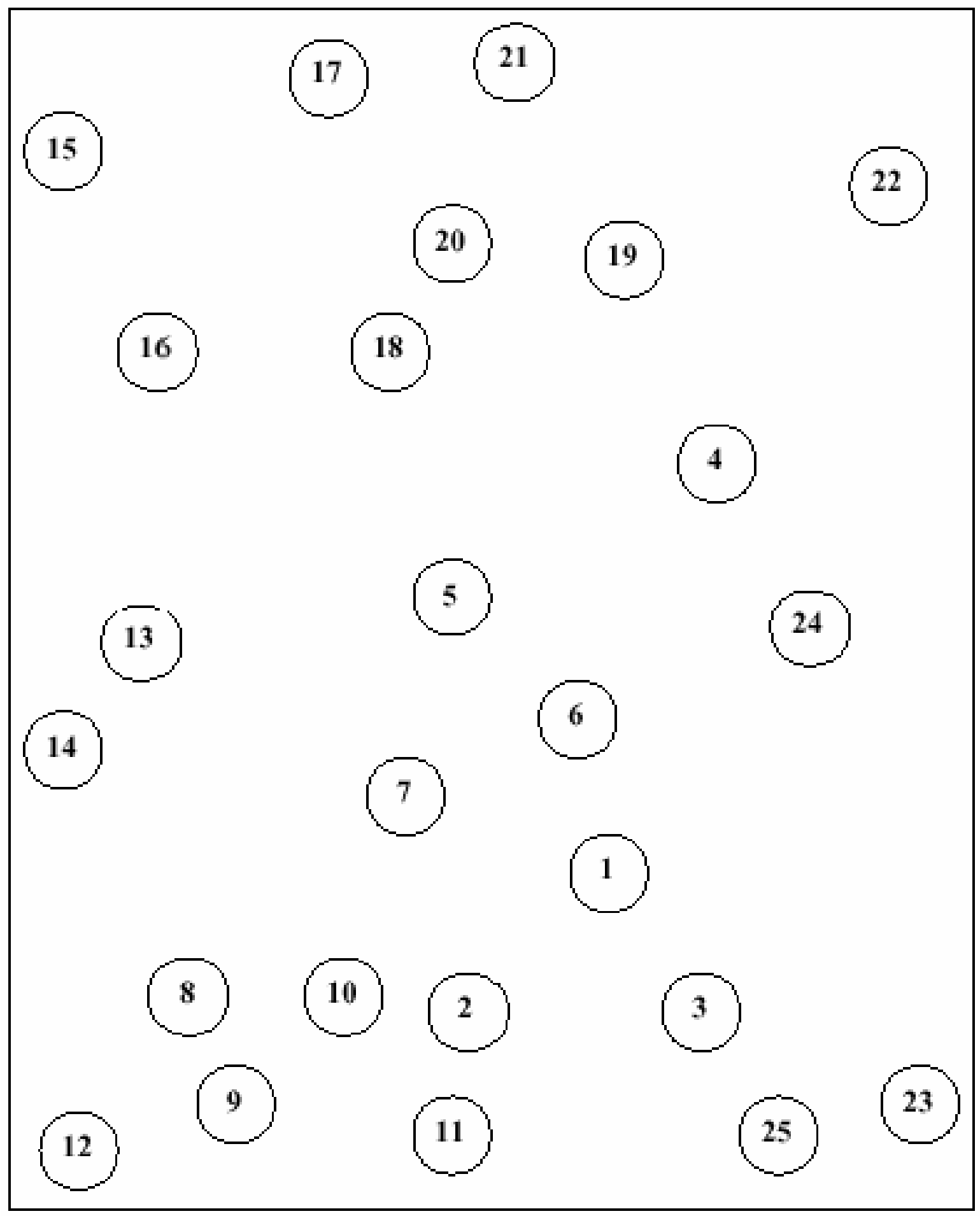




\section{Trail Making Test Part A - SAMPLE}

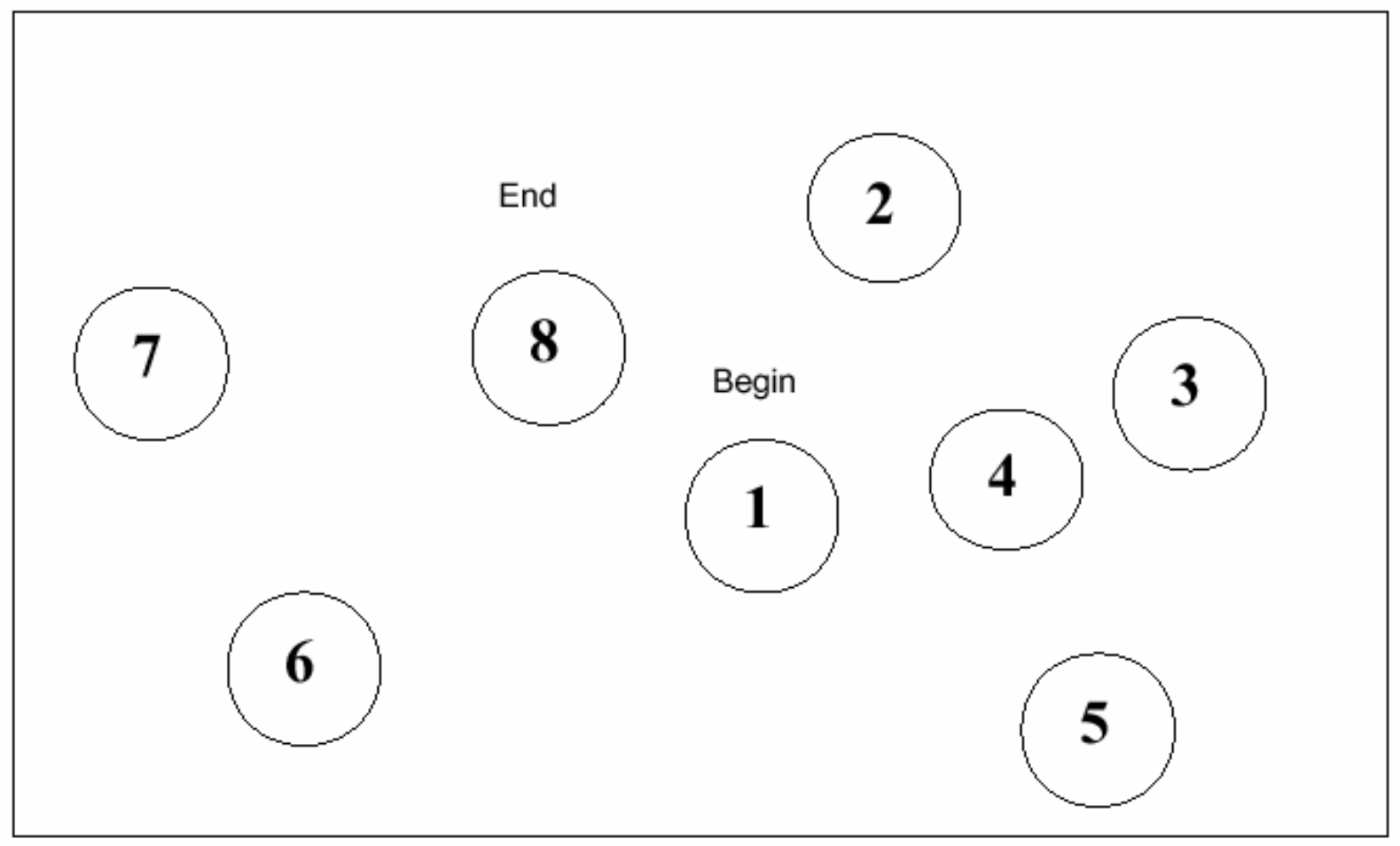




\section{Trail Making Test Part B}

Patient's Name:

Date:

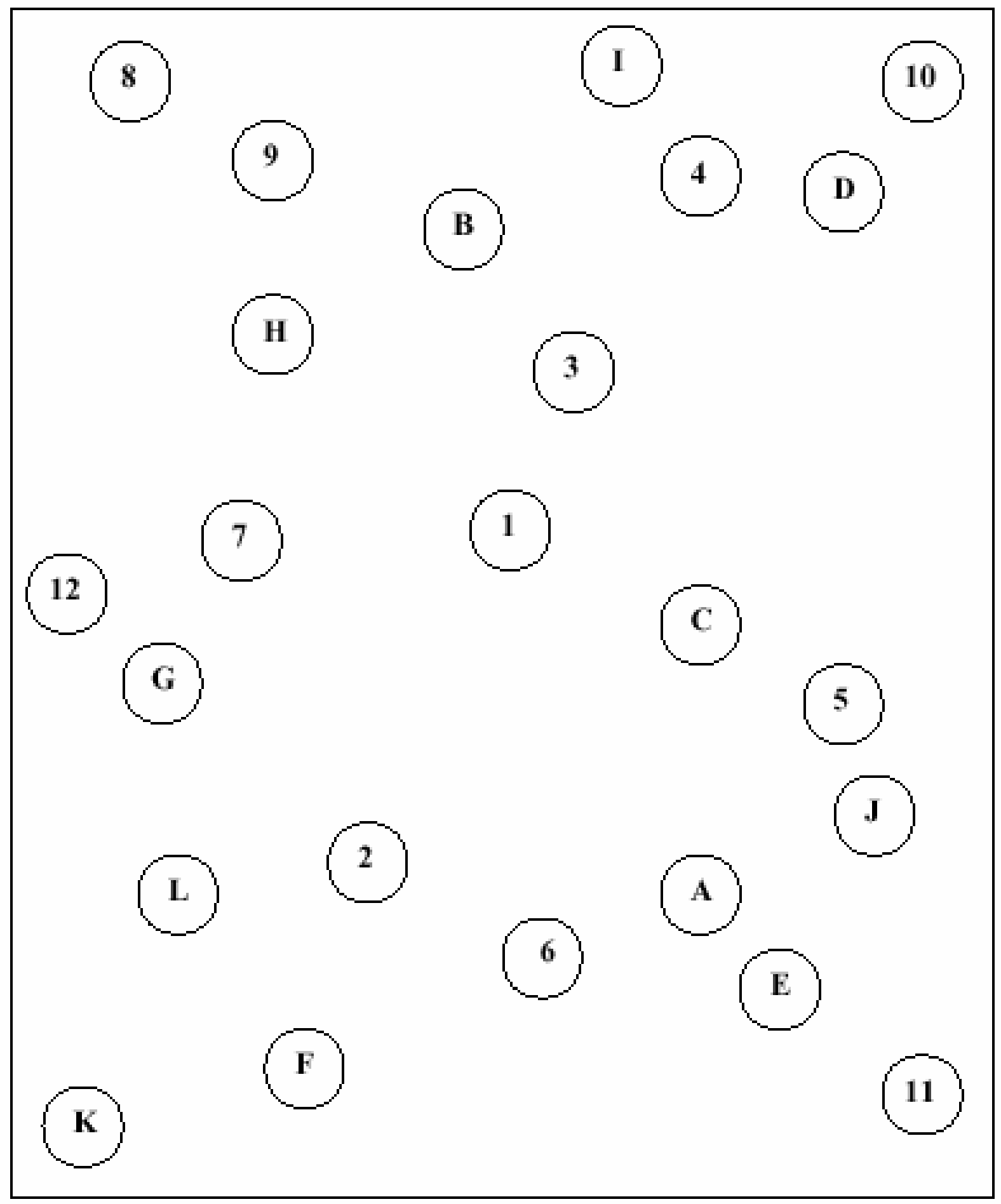




\section{Trail Making Test Part B - SAMPLE}

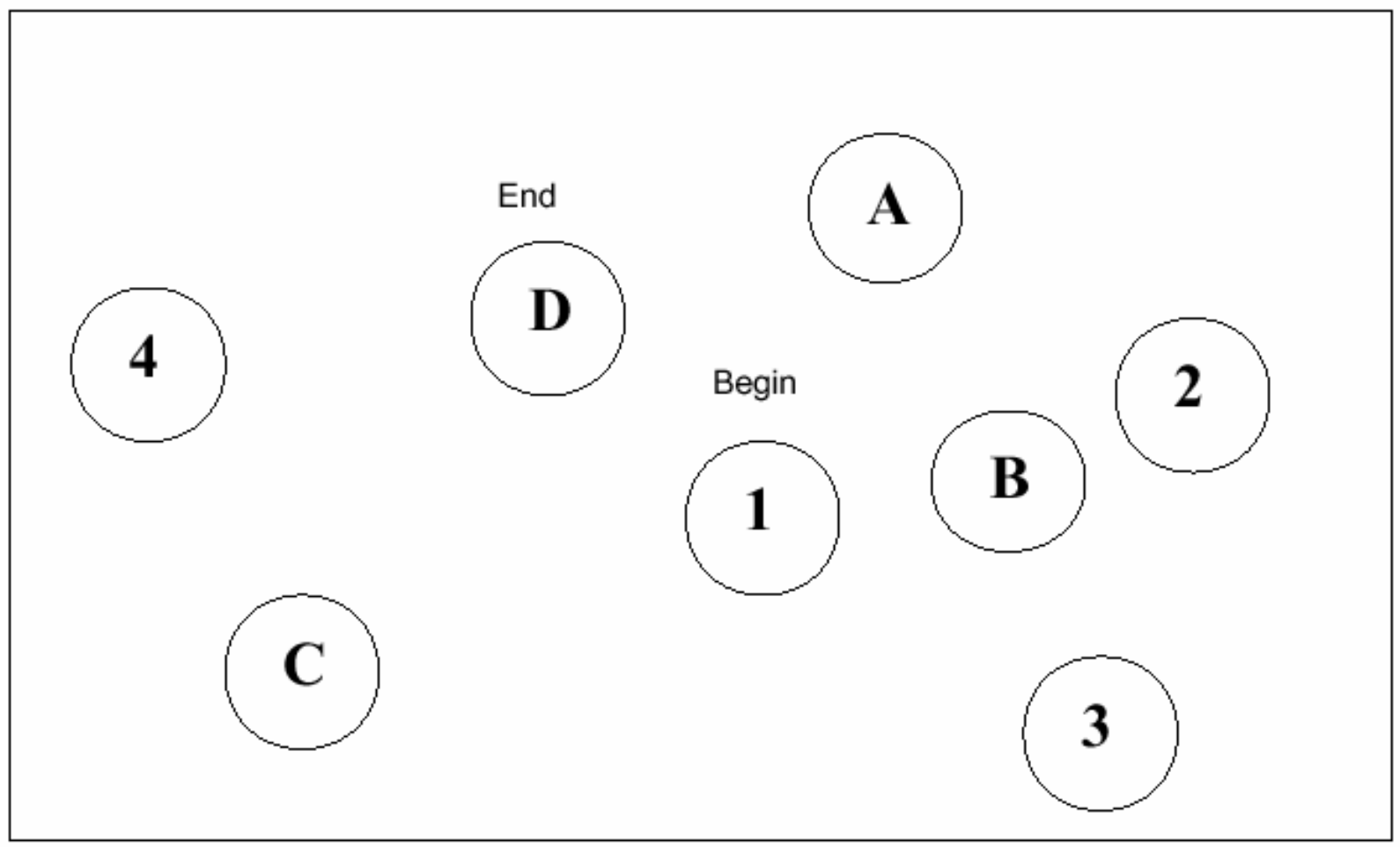


On a scale of 1 to 7 ( 1 being very easy to complete, 7 being very difficult to complete and 4 being neural) please rate how easy or difficult you found each test to complete. (circle the box)

Simple reaction time: press a button when a green circle with GO! appears.

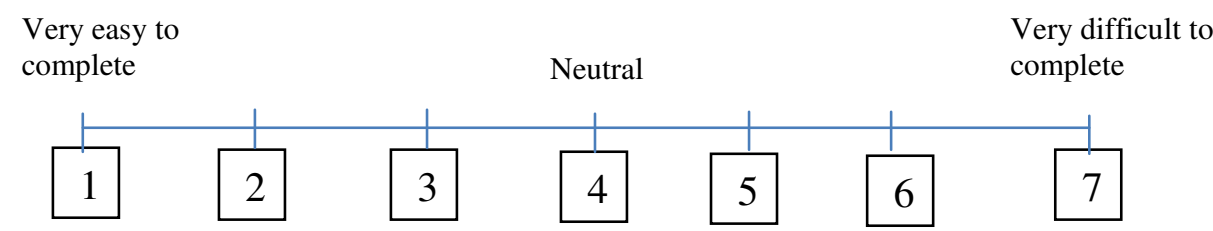

Choice reaction task: Press ' $\mathrm{z}$ ' if $\mathrm{X}$ appears and ' $\mathrm{m}$ ' if $\mathrm{O}$ appears.

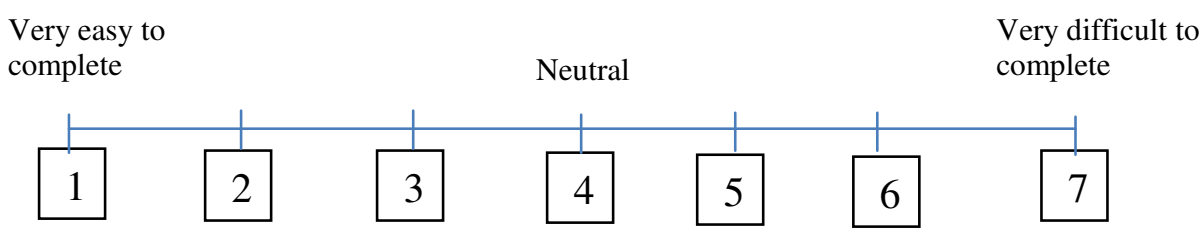

Visual search: responding to the direction of the target arrow

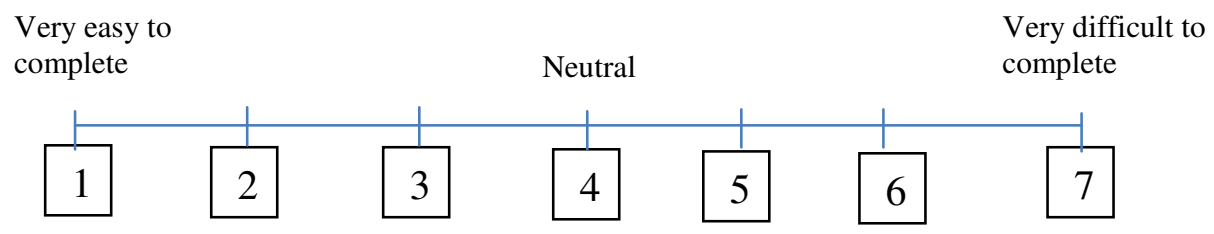


College of Human and Health Sciences

Coleg y Gwyddorau Dynol ac lechyd

Trails A: pen a paper test matching the numbers in order.

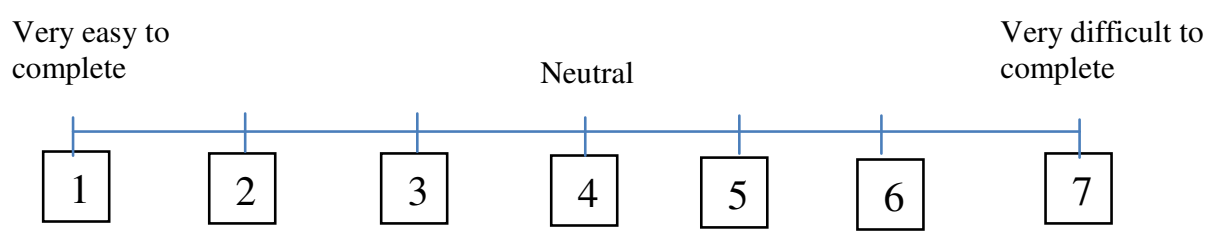

Trails B : pen a paper test matching the numbers and letters in order.

\begin{tabular}{|l|l|l|l|l|l|l|}
$\begin{array}{l}\text { Very easy to } \\
\text { complete }\end{array}$ & Neutral & \multicolumn{1}{l}{$\begin{array}{l}\text { Very diffi } \\
\text { complete }\end{array}$} \\
\hline 1 & 2 & 3 & 4 & 5 & 6 & 7 \\
\hline
\end{tabular}

Milo: IPad test tapping the numbers in order.

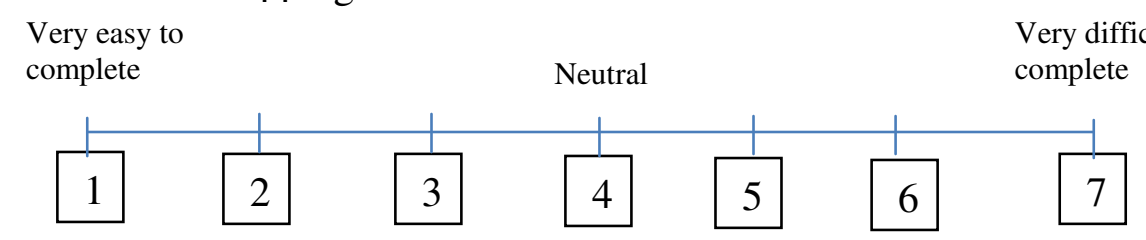




\title{
Measuring Information Processing Speed in Mild Cognitive Impairment: Clinical Versus Research Dichotomy
}

\author{
Judy Haworth ${ }^{\mathrm{a}}$, Michelle Phillips ${ }^{\mathrm{b}}$, Margaret Newson ${ }^{\mathrm{c}}$, Peter J. Rogers ${ }^{\mathrm{d}}$, \\ Anna Torrens-Burton ${ }^{\mathrm{e}}$ and Andrea Tales ${ }^{\mathrm{e}, *}$ \\ a Southmead Hospital, North Bristol Trust, Bristol, UK \\ ${ }^{\mathrm{b}}$ University of Bristol, Centre of Academic Mental Health, Oakfield House, Oakfield Grove, Bristol, UK \\ ${ }^{\mathrm{c}}$ Brain Centre, Elgar House, Southmead Hospital, North Bristol Trust, Bristol, UK \\ ${ }^{\mathrm{d}}$ School of Experimental Psychology, University of Bristol, Bristol, UK \\ ${ }^{\mathrm{e}}$ Department of Psychology, Swansea University, Swansea, Wales, UK
}

Accepted 1 December 2015

\begin{abstract}
A substantial body of research evidence is indicative of disproportionately slowed information processing speed in a wide range of multi-trial, computer-based, neuroimaging- and electroencephalography-based reaction time (RT) tests in Alzheimer's disease and mild cognitive impairment (MCI). However, in what is arguably a dichotomy between research evidence and clinical practice, RT associated with different brain functions is rarely assessed as part of their diagnosis. Indeed, often only the time taken to perform a single, specific task, commonly the Trail making test (TMT), is measured. In clinical practice therefore, there can be a failure to assess adequately the integrity of the rapid, serial information processing and response, necessary for efficient, appropriate, and safe interaction with the environment. We examined whether a typical research-based RT task could at least match the TMT in differentiating amnestic MCI (aMCI) from cognitively healthy aging at group level. As aMCI is a heterogeneous group, typically containing only a proportion of individuals for whom aMCI represents the early stages of dementia, we examined the ability of each test to capture intra-individual variation in performance. The results indicate that as well as significant slowing in performance of the operations involved in TMT part B (but not part A), individuals with aMCI also experience significant slowing in RT compared to controls. The results also suggest that research-typical RT tests may be superior to the TMT in differentiating between cognitively healthy aging and aMCI at group level and in revealing the performance variability one would expect from an etiologically heterogeneous disorder such as aMCI.
\end{abstract}

Keywords: Dementia, information processing speed, mild cognitive impairment, reaction time

\section{INTRODUCTION}

Although information processing speed tends to slow with age [1], disproportionate slowing appears related to cognitive limitations [2-5] and a wide range of brain disorders [6], including degenerative brain

\footnotetext{
*Correspondence to: Prof. Andrea Tales, Department of Psychology, Swansea University, Swansea SA2 8PP, Wales, UK. Tel.: +44 01792 602567; E-mail: a.tales@swansea.ac.uk.
}

changes such as Alzheimer's disease (AD) [6-10], vascular dementia [11], vascular cognitive impairment [12, 13], cerebral small vessel disease [14], amnestic mild cognitive impairment (aMCI) and its conversion to dementia [8] and faster decline in AD progression [15, 16].

Behaviorally, as reaction time (RT) is an important factor in relation to the integrity and efficiency of brain functions such as those involved in attention, cognition, and perception, it may provide a 
'real-life' indicator of changes to everyday functions and explain, at least in part, some of the signs, symptoms, and changes in behavior related to aMCI and $\mathrm{AD}$. Indeed, disproportionate slowing is associated with the cessation of driving [17, 18], mortality among community-dwelling older adults [19], functional dependence in activities of daily living [19, 20], walking speed [21], and outcome after stroke [22].

Of particular relevance to potential clinical impact of such research is the substantial body of evidence indicative of the relationship between RT and some aspects of the brain's structural integrity. Information processing speed as indicated by RT (i.e., the time elapsed between the presentation of a stimulus and the behavioral response), measured over a number of trials of computer-based stimulus response tests, can represent a behavioral 'marker' of neurophysiological integrity. For example, disruption to white matter and cerebral integrity and change in neurotransmitters is associated with disproportionate slowing of and raised intra-individual variability in RT $[1,8$, 23-30]. Arguably therefore this link between structural and functional integrity and behavioral RT indicates the potential for simple RT tests as valid adjuncts to the assessment of diagnosis, status, stage, progression, and interventional success in dementia and related disorders [31].

Furthermore, with appropriate methodological design, RT can be used to assess the integrity of specific aspects of brain function including attention, perception, visual processing, and cognition, at various levels of processing and in response to different processing and resource demands [8]. Indeed, the majority of dementia-related research studies examining RT in relation to brain structure and function (e.g., using techniques such as DTI, EEG, MRI, fMRI) have tended to utilize multi-trial computerbased tasks. These have not only allowed measures of the relationship between behavioral RT and structure and function but generated additional information related to performance variability, fatigue, stimuli and threshold responses, processing load, resource availability and utilization, patterns of functional decline and integrity, and response to intervention. RT is arguably therefore an indicator of an individual's ability to respond rapidly, efficiently, appropriately, and repeatedly to ever-changing stimuli and thus represents a valid, yet easily obtained, indicator of the efficiency by which a person can successfully interact with their surroundings. An example being the constant need for vigilance and response to change required by driving.
Clinically, despite this research evidence, information processing speed tends not to be assessed using a variety of function-specific, computer-based, multitrial RT tests. Although a variety of RT-based tests are available and in use clinically, in some cases information processing speed is assessed by measuring the time taken to perform a given task, namely using a stop-watch to measure the single trial performance of the pen and paper Trail Making Test (TMT) [32-38]. The TMT is a test administered in two parts. In Trails A, individuals are required to connect a series of consecutively numbered circles that are presented in a random pattern on the paper: a task typically described as probing functions such as speed of processing in relation to attention, visual scanning and search, number recognition, numeric sequencing, and motor speed. In Trails B, individuals are required to connect a series of numbered and lettered circles alternating between the two sequences; a task typically described as probing the efficiency of set shifting, mental flexibility, executive function, divided attention, attention switching, visual search set shifting, simultaneous maintenance of two sequences, working memory and cognitive flexibility; arguably a measure of information processing speed in relation to multiple high level, non-specific functions [33, 34, 39]. TMT performance, in both parts $\mathrm{A}$ and $\mathrm{B}$, is evaluated by scoring the time for completion in seconds, using one trial only.

Although research indicates that TMT performance, as in RT, is slower in older compared to younger adults [33, 40], with additional slowing related to pathological aging such as MCI, AD [41, 42], and vascular dementia [43] (but see also [10, 15, $21,41,44-54])$, there are potential limitations associated with the clinical use of the TMT. Although Trails $\mathrm{B}$ is more difficult than Trails A, involving greater information processing load, range and depth of operations, and arguably of greatest clinical usefulness, not all individuals can complete it [55]. Any visual or motor impairment due to extraneous factors such as stroke, injury, blindness, or arthritis for example, will affect test performance. In those circumstances the test is not administered, or it is interpreted with caution. Furthermore, the fact that TMT performance is examined over one trial only precludes the complex analysis of speed/error trade-off, the motor and nonmotor components of reaction time, thresholds for change, the influence of environmental factors and stimulus properties, practice or fatigue effects, the intra-individual variability of an individual's performance at any one time, and the components of rapid, 
successive information processing and response, possible with the use of RT tests. RT tests arguably therefore can provide more 'data rich' results than the TMT. Furthermore the fact that the TMT is pen and paper-based also means that it is difficult to use in its exact clinical format in conjunction with neuroimaging, EEG, and many other imaging studies relating behavioral response with underlying physiology (although see Müller et al. [36] and Hagen et al. [34]).

Arguably, in the time it takes to administer the TMT, a highly sophisticated and function-specific RT and RT variability test (and one typically used in neuroimaging studies) could be administered using the user-friendly interfaces of computers, laptops, or mobile technology devices (e.g., tablets). Potentially, the use of such technology can permit the automatic and immediate availability and electronic transfer of results for interpretation and comparison with previous results. Taking this concept one step further, mobile device technology means that clinically relevant and research-based RT testing does not have to be performed within a clinical setting and does not necessarily require someone to administer it [56].

Although a major research focus in aMCI relates to the potential for disproportionate RT slowing as a 'marker' of the increased risk of developing dementia, the generally ignored fact remains that irrespective of cause, substantially slowed information processing and response may have significant detrimental impact upon daily living. Arguably, in terms of ecological validity and clinical relevance, measuring processing speed with respect to TMT performance only, means that degradation in the integrity of the operations involved in rapid responses to constantly changing or repeatedly presented information is not considered when evaluating task competences and behaviors with inherent RT components. The operations involved in processing and responding to rapid and repeated stimuli in a RT test will be different to those related to the performance of the TMT, and we suggest that measuring RT may be more akin to, ecologically valid or relevant with respect to the investigation of the integrity of information processing speed related to every day tasks which typically require repeated rapid processing, decision making, and response. Taking into account all these considerations, should information about processing speed included in the clinical diagnosis and follow-up of aMCI and dementia be based solely on TMT performance?
We address this question by investigating two measures commonly used as indicators of processing speed in aMCI and AD, namely the TMT, sometimes used in clinical assessment, and the RT component of a form of visual search test commonly employed in research and specifically that is used in a series of previous studies by Tales and colleagues (e.g., [8]), in which RT to a target appearing in isolation is compared to the time taken to respond to the same target when it is surrounded by distracting information. These tasks are similar to Trails B and Trails A, in that they are generally assumed to involve complex and higher processing levels and varying loads, attention shifting, eye movements, sequencing, suppression and inhibition of irrelevant or previously attended locations and stimuli, but unlike the TMT, numerous trails are presented in quick succession (as described in the methods section to follow). We examine in the first instance whether the visual search based RT tests can at least match the ability of the TMT to differentiate aMCI from cognitively healthy aging at group level. Secondly, as aMCI is a clinically heterogeneous group typically containing a proportion of individuals for whom aMCI represents the early stages of a dementing process, a proportion for whom it remains of unknown etiology and others for whom it is a temporary condition, we examine the ability of each test to provide intra-group variation in performance. As both disproportionately slower task-completion time and RT are related to the presence of dementia, one would expect to see some performance variability within the aMCI but not the cognitively healthy control group in both tests.

Research has also indicated that in older adults both age and educational level can influence TMT performance, although outcome appears to vary with respect to TMT scoring methods, type of analysis, and the interpretation of errors [33-35, 41, 57-62]. There is some evidence also that gender can influence RT although this effect appears to be task dependent [33, 58, 63-66]. In the present study therefore, as is common practice, education, age, and gender were matched at group level to the best of our abilities. Furthermore, as earlier research on TMT has been criticized because of the lack of inclusion of a measure of IQ [41, 67], we employed the National adult reading test (NART) $[68,69]$ to provide an estimate of general/pre-morbid intelligence or indeed a proxy of cognitive reserve. As previous evidence highlights the potential for outcome variability between studies of processing speed in such populations $[8,70,71]$, 
the same individuals are included in all tests so direct comparisons of performance can be made.

\section{METHODS}

This study was conducted according to the principles in the Declaration of Helsinki. It was approved by Frenchay Research Ethics Committee and all participants gave written informed consent to participate. Only individuals with the capacity to consent were included. Capacity to consent was assessed by the clinician ( $\mathrm{JH})$ with specialist expertise in this field and consistent with the requirements of the Mental Capacity Act.

\section{Participants}

Community-dwelling cognitively healthy older adults and patients with $\mathrm{aMCI}^{+}$(multi-domain amnestic MCI) were recruited via the Bristol Memory Disorders Clinic. All had normal or corrected-tonormal vision. Medication could not be controlled but none of the participants were receiving medication or intervention deemed likely to affect cognitive or reaction-time-related function and those with $\mathrm{aMCI}^{+}$were receiving no drug treatment or intervention for this condition, none were classed as anxious or depressed. All participants performed a typical Bristol Memory Disorders clinic battery of neuropsychological tests including Mini-Mental State Examination (MMSE) [72], WAIS-III (Wechsler Adult Intelligence Scale) subtests (digit span, similarities, picture completion) [73], Hopkins Verbal Learning Test-Revised [74], CLOX (executive clock drawing task) [75], Visual Form Discrimination Task [76], NART [68], S-word fluency and animal fluency [45], Story Recall (Adult Memory Information Processing Battery) [77], Bristol Activities of Daily Living scale (BADLS) [78], and Brief Assessment Schedule, Depression Cards (BASDEC) (screen for depression) [79]. By definition, all the cognitively healthy older adults performed at ageappropriate levels on all tests (i.e., z-score above -1.5). Individuals with a diagnosis of $\mathrm{aMCI}^{+}$had self-reported and informant corroborated change in memory and objective decline (z scores equal or less than -1.5 in memory and at least one other area of function), in the absence of dementia and intact ability to perform activities of daily living. Exclusion criteria included past history of serious head injury, evidence of physical slowing (e.g., related to Parkinson's disease or arthritis), stroke, and other significant neurological or psychiatric condition (see Phillips et al. [8] and [80, 81]). In total, 87 individuals took part in this research, 48 older adults with mild cognitive impairment and 39 cognitively healthy older adult controls ${ }^{1}$. The demographic details are shown in Table 1.

The cognitively healthy older adult and the aMCI ${ }^{+}$ groups did not differ significantly with respect to mean Age $[Z=-1.65, p=0.098]$ or mean Educational level $[Z=-0.53, p=0.6]$, indicating that attempts at matching these demographics between groups was successful. However, the NART score (level of pre-morbid intelligence) was significantly poorer for the $\mathrm{aMCI}^{+}$compared to the cognitively healthy older adult group $[Z=-3.3, p=0.001]$. As expected, MMSE score was significantly lower for the $\mathrm{aMCI}^{+}$ compared to the cognitively healthy older adult group $[Z=-2.98, p=0.003]$.

\section{Experimental task and procedure}

In a counter balanced procedure, the TMT (both Trails A and Trails B) and the visual search task (both target alone and target plus distracter conditions) were administered to all participants by a trained psychometrist. Testing took place within the Bristol Memory Disorders Clinic.

\section{The pen and paper TMT}

When administering Trails A, the psychologist provided the participants with a practice sheet as a way of visually explaining the task. Once the participants completed the practice sheet, they completed the full Trails A. For this task the participants were instructed to draw one continuous line joining a series of circled numbers in ascending order on a sheet a paper as fast as they could. They were given a maximum of 2 minutes to complete this task. Similarly for Trails B, participants were given a practice sheet and when happy with the instructions were asked to complete the full Trails B. The test required them to draw one continuous line joining a series of circled numbers and letters in ascending and alphabetical order on a sheet of paper as fast as they could. A time limit of 5 minutes is stipulated for this task. Scores are based on the number of seconds until completion and the participant's age. If errors were made on either

\footnotetext{
${ }^{1}$ Please note that some of the participants included in this research were part of a larger cohort Phillips et al. [8] for whom both Trails A and Trails B and visual search RT1 and RT8 conditions were completed in full.
} 
Table 1

Demographic details

\begin{tabular}{lccrrr}
\hline & & Education & Age & NART & MMSE \\
\hline Cognitively healthy older adults $(n=39)$ & Mean & 14.56 & 70.5 & 118.3 & 27.1 \\
& SD & 3.1 & 8.3 & 8.1 & 1.5 \\
& SEM & 0.49 & 1.3 & 1.3 & 0.2 \\
${ }^{*} \mathrm{MCI}^{+}(n=48)$ & Mean & 14.4 & 67.6 & 111.8 & 25.9 \\
& SD & 3.72 & 8.6 & 16.6 & 1.8 \\
& SEM & 0.54 & 1.2 & 2.4 & 0.25 \\
\hline
\end{tabular}

tasks participants are immediately informed and they are allowed to correct them. Errors like this were accounted for in the time to complete the task or if they failed to complete the task in the allotted time.

\section{The computer-based Visual Search Task}

The visual search task used was one employed in several previous studies by Tales and colleagues [8, 80, 81, see also 7], in which the time taken to respond to a target (namely to discriminate whether an arrow-head was pointing to the left or right) when it appeared in isolation upon the computer screen and the time taken to respond to the same target when it was surrounded by similar but irrelevant and distracting stimuli was determined. This paradigm was presented on a Toshiba Satellite-Pro lap top computer viewed at a distance of $57 \mathrm{~cm}$. Superlab software (Cedrus Corporastion San Pedro, CA) was used to deliver stimulus presentation and response capture. This choice RT task included a black target, either a right or left-pointing arrow head; with participants required to indicate whether the arrowhead was pointing to the left or right. The distracting stimuli consisted of seven black arrow-heads pointing either up or down. A 'clock-face' configuration (see Fig. 1) was used to position the target, both when it appeared alone and when surrounded by seven distracters, in a counterbalanced arrangement in order to eliminate any visual field position-related differences in processing. The target appeared eight times

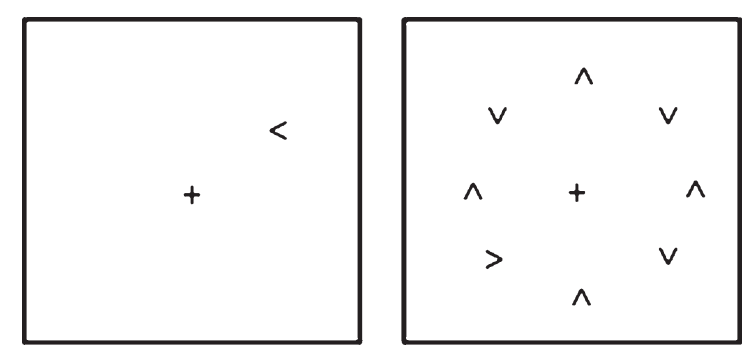

Fig. 1. Schematic of the visual search task: target alone and target plus distracter conditions. at each of the possible 'clock-face' locations giving a total of sixty-four trials. Distracters were presented for half the trials. On each trial the central fixation cross appeared on screen for $1000 \mathrm{~ms}$ prior to the appearance of the target and remained on screen for the duration of the trial. The stimuli remained on screen until a response was made. Participants were instructed to fixate on the center cross at the beginning of each trial and to respond as quickly but as accurately as possible as to whether the target was pointing to the right or left by pressing one of two computer keyboard keys. After instruction, all participants were asked to explain the task to the researcher in order to demonstrate that they fully understood the task and then performed approximately 10 practice trials. The ability of the participants to fixate upon the central cross was checked at the beginning of each trial by researcher observation. The researcher was also in a position to record any lack of trial response and to prompt re-engagement of the task. Participants received no performance-feedback during testing.

\section{DATA ANALYSIS AND RESULTS}

Group mean analysis for RT speed was based on the mean values (of correct trials only) for each individual within the group. The mean response times for TMT, Trails A and B, and the mean RTs (ms) for the target alone and the target plus distracter search are displayed in Table 3, together with the corresponding standard deviation and standard error of the mean. Note that in the TMT, no participants exceeded the five-minute time limit. For the visual search tasks, only correct trials were included in the statistical analysis. Accuracy was high; the mean percentage of errors overall was low for both the cognitively healthy $(3.2 \%)$ and $\mathrm{aMCI}^{+}(4.6 \%)$ groups with no evidence of speed accuracy trade off effects. No participants failed to respond to a trial and none required prompting.

In response to the generally non-normal distribution of our RT data (see Table 2, Figs. 2 and 3), SPSS 
Table 2

Normality of distribution (Shapiro Wilkes test)

\begin{tabular}{|c|c|c|c|c|c|c|}
\hline & \multicolumn{3}{|c|}{ OLD } & \multicolumn{3}{|c|}{$\mathrm{aMCI}^{+}$} \\
\hline & statistic & df & Sig. & statistic & df & Sig \\
\hline Age & 0.939 & 39 & 0.036 & 0.941 & 48 & 0.017 \\
\hline Education & 0.953 & 39 & 0.104 & 0.911 & 48 & 0.002 \\
\hline NART & 0.924 & 39 & 0.012 & 0.764 & 48 & 0.000 \\
\hline MMSE & 0.950 & 39 & 0.085 & 0.932 & 48 & 0.008 \\
\hline Search: target alone & 0.911 & 39 & 0.005 & 0.944 & 48 & 0.022 \\
\hline Search: target \& distracters & 0.949 & 39 & 0.077 & 0.911 & 48 & 0.001 \\
\hline Trails A & 0.973 & 39 & 0.459 & 0.942 & 48 & 0.019 \\
\hline Trails B & 0.833 & 39 & 0.000 & 0.822 & 48 & 0.000 \\
\hline
\end{tabular}

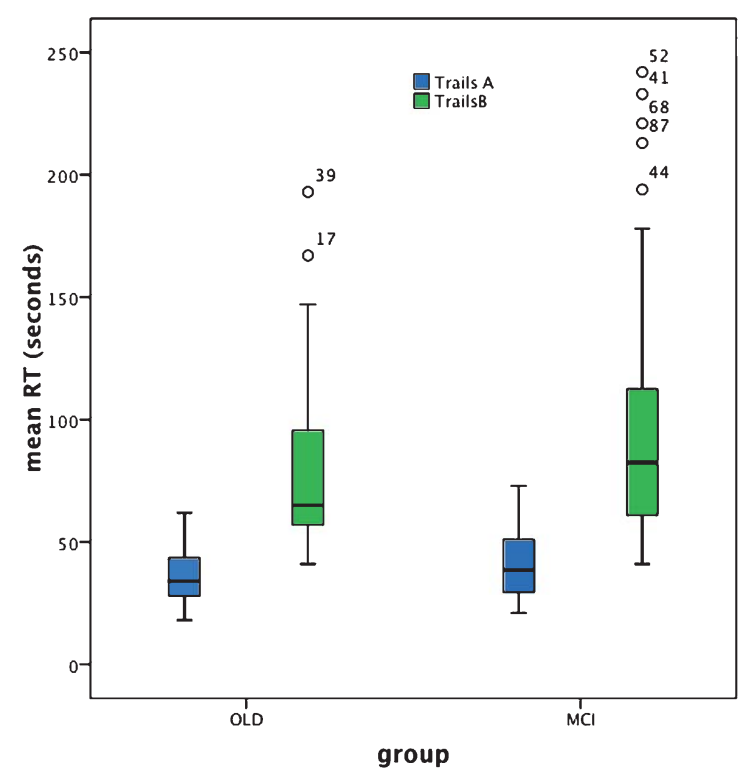

Fig. 2. BOX plot of Trails A and B performance based on individual response speed (seconds).

non-parametric statistical analysis was employed in line with common practice.

\section{Trails $A \& B$ response time analysis}

For Trails A, analysis revealed no significant difference in group-mean response time (in seconds) between cognitively healthy aging and $\mathrm{aMCI}^{+}$ $[Z=-1.4, p=0.16]$. Note, however, that whereas Trails A performance was normally distributed for the cognitively healthy older adult group, this was not the case for the $\mathrm{aMCI}^{+}$group. Note also that multiple correlational analysis (with Bonferroni correction) failed to reveal any correlation of response time with age, education, NART score, or MMSE score in either group [all $p$-values $>0.05$ ].

For Trails B, mean response time was significantly slower in $\mathrm{aMCI}^{+}$compared to cognitively healthy

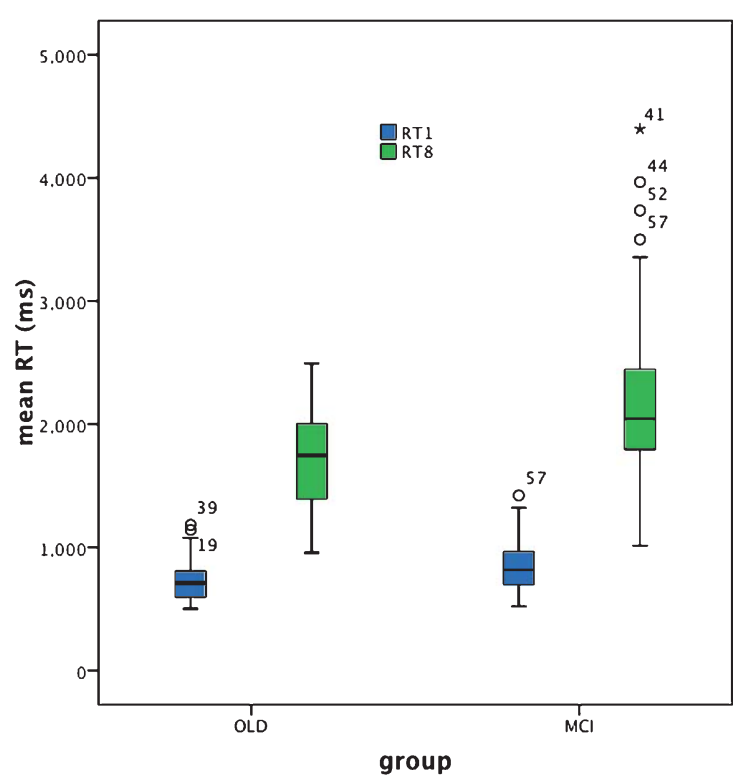

Fig. 3. Box plot for target alone (RT1) and target plus distracters search (RT8) performance based on individual reaction time (RT) (ms).

aging $[Z=-1.96, p=0.05$; effect size $(r)=0.21]$. For both groups, performance of Trails B was not normally distributed and multiple correlational analysis (with Bonferroni correction) failed to reveal any correlation of response time with age, education, NART score, or MMSE score in the older adult group [all $p$-values $>0.05$ ] and education, NART score, or MMSE score in the $\mathrm{aMCI}^{+}$group [ $p$-values $>0.05]$, although performance of Trails $\mathrm{B}$ in the $\mathrm{aMCI}^{+}$group was significantly correlated with age $[r=0.522, p<0.001$ which survives Bonferroni correction; $p=0.004]$.

\section{Visual search target alone and target plus} distracter reaction time analysis

Mean RT in response to the target alone Visual Search task was significantly slower for the $\mathrm{aMCI}^{+}$ 
Table 3

Mean response times (s) for the TMT (Trails A and B) and the mean RTs (ms) for the target alone and the target plus distracter visual search tasks, together with the corresponding standard deviation (SD) and standard error of the mean (SEM)

\begin{tabular}{lcccrr}
\hline & & $\begin{array}{c}\text { Trails A Mean } \\
\text { response time (s) }\end{array}$ & $\begin{array}{c}\text { Trails B Mean } \\
\text { response time (s) }\end{array}$ & $\begin{array}{c}\text { Search Target alone } \\
\text { Mean RT (ms) }\end{array}$ & $\begin{array}{c}\text { Search Target \& distracters } \\
\text { Mean RT (ms) }\end{array}$ \\
\hline $\begin{array}{l}\text { Healthy older } \\
\text { adult controls }\end{array}$ & & 35.9 & 78.7 & 744.2 & 1730.6 \\
& SD & 10.4 & 34.7 & & \\
& SEM & 1.7 & 5.6 & 27.9 & 64.5 \\
aMCI $^{+}$ & & 40.6 & 98.2 & 861.1 & 2230.4 \\
& SD & 13.8 & 51.6 & 209.2 & 709.9 \\
& SEM & 2.0 & 7.5 & 30.2 & 102.5 \\
\hline
\end{tabular}

compared to the cognitively healthy older adult group $[Z=-2.8, p=0.006$; effect size $(\mathrm{r})=0.3]$. For both groups RT was not normally distributed and was not significantly correlated with age, education, NART or MMSE scores [all $p$-values $>0.05$ ].

In response to the target plus distracters Visual Search task, mean RT was significantly slower for the $\mathrm{aMCI}^{+}$compared to the cognitively healthy older adult group $[Z=-3.5, p<0.001$; effect size $=0.38]$. Although performance of this task was normally distributed for the cognitively healthy older adult group this was not the case for the $\mathrm{aMCI}^{+}$group. RT was not significantly correlated with age, education, NART or MMSE scores [all $p$-values $>0.05$ ] in either group.

\section{Gender}

Males and females in the cognitively healthy older adult group did not differ significantly in mean response time for Trails A [ $Z=-0.745, p=0.45]$ or $\mathrm{B}[Z=-1.32, p=0.186]$, or in RT for the target alone $[Z=-0.056, p=0.96]$ or target plus distracters Visual Search tasks $[Z=-0.787, p=0.43]$.

In the $\mathrm{aMCI}^{+}$group, there was no gender difference in target plus distracters Visual Search task $[Z=-1.041, p=0.298]$, Trails A $[Z=-0.433$, $p=0.665]$ or $\mathrm{B}[Z=-0.928, p=0.353]$ performance. In response to the target alone Visual Search task, however, males were significantly faster than females $[Z=-2.073, p=0.038$, effect size $r=0.3]$. It is possible that this gender difference may be related to the greater educational level of males than females in this group $[Z=-3.061, p=0.002$, effect size (r) $=0.44]$, further analysis revealed, however, that RT was not correlated with educational level $[p>0.05]$. Note that in both groups there was no significant difference between males and females with respect to mean age, NART score, or MMSE score and no difference in educational level within the cognitively healthy older adult group [all $p$-values $>0.05$ ].

\section{DISCUSSION}

The TMT is sometimes used clinically to assess the speed of information processing in dementia, MCI, and related disorders, by measuring the time taken to complete the task of consecutively joining a series of numbers and or letters on a sheet of paper. However, in research terms, speed of information processing is generally described with respect to reaction time, i.e., the time elapsed between the relatively rapid presentation of a stimulus and the behavioral response, measured over a number of trials at relatively short intervals. Arguably, the processing involved in processing and responding to such rapid and repeated stimuli is different from that involved in performing the TMT, and we suggest that measuring RT may indeed be more akin to, ecologically valid or relevant with respect to the investigation of the integrity of information processing speed related to every day tasks which typically require rapid processing, decision making, and response.

However, although RT tests may, in theory, represent a clinically valid replacement of the TMT, we have not been able to identify any evidence investigating both measures in clinical populations. Here, therefore, we examined the performance of the TMT (both A and B versions) and two versions of a computer based multi-trial visual search-based RT task (a target alone and a target plus distracters condition) in the same groups of cognitively healthy older adults and patients with $\mathrm{aMCI}^{+}$, in an attempt to determine whether the visual search based RT tests can at least match the ability of the TMT to differentiate $\mathrm{aMCI}^{+}$ from cognitively healthy aging at group level. Secondly, as aMCI ${ }^{+}$is a clinically heterogeneous group typically containing a proportion of individuals for whom $\mathrm{aMCI}^{+}$represents the early stages of a dementing process, a proportion for whom it remains of unknown etiology and others for whom it is a tempo- 
rary condition, we examined the ability of each test to provide intra-group variation in performance. As both disproportionately slower task-completion time and RT are related to the presence of dementia, one would expect to see some performance variability within the $\mathrm{aMCI}^{+}$but not the cognitively healthy control group in both tests.

\section{Trails $A$ and $B$ performance}

Trails A performance failed to significantly differentiate $\mathrm{aMCI}^{+}$from cognitively healthy aging at group level. Arguably this result is indicative of a lack of sensitivity of the Trails A test to the presence of MCI. Indeed the box plot (Fig. 2) reveals just how similar the results are for the patient and control groups. Furthermore, response time was normally distributed for the cognitively healthy older adult but not for the $\mathrm{aMCI}^{+}$group (see Table 2). Given the etiological heterogeneity of $\mathrm{aMCI}^{+}$, in that at least a proportion of such individuals would be expected to be in the prodromal stages of dementia with an generally acknowledged accompanying slowing of response time, the relative lack of individual variability in response time within this group is surprising.

In contrast, Trails B performance significantly differentiated $\mathrm{aMCI}^{+}$from cognitively healthy aging. As shown in Fig. 2, response time is clearly slowed for the $\mathrm{aMCI}^{+}$compared to the control group and there are a number of individuals within the $\mathrm{aMCI}^{+}$group whose performance is represented by clear outliers. This potentially represents the within-group heterogeneity one might expect from such an etiologically varied group. However, Trails B also reveals heterogeneity of performance within the cognitively healthy older adult group; indeed response time is not normally distributed in either group (see Table 2), with both characterized by some disproportionately slower reaction times and therefore some degree of performance overlap.

\section{Visual search reaction time performance}

The RT in response to the simple target alone visual search test was significantly slower in $\mathrm{aMCI}^{+}$compared to cognitively healthy aging. However, as is clear from Fig. 3, RT was abnormally distributed for both groups, with a number of disproportionately slower responses, i.e., outliers characterizing both groups and thus indicative of some degree of overlap between cognitively healthy aging and $\mathrm{aMCI}^{+}$. Nev- ertheless, the effect size of the significant difference in performance between the two groups was greater than that for the Trails B test (effect size ' $r$ ' $=0.21$ and 0.3 ), respectively.

For the target plus distracter visual search task, mean RT was significantly slower in $\mathrm{aMCI}^{+}$compared to cognitively healthy aging. The effect size of this outcome $(r=0.38)$ was greater than that exhibited for the target alone search $(r=0.3)$ and the Trails $\mathrm{B}(r=0.21)$ tasks, indicating that the target plus distracter visual search task is the one most sensitive to $\mathrm{aMCI}^{+}$. Furthermore, whereas the distribution of RT performance was normal within the cognitively healthy older adult group for the target plus distracter visual search task this was not the case for the $\mathrm{aMCI}^{+}$group, revealing instead a number of considerably slower responses, i.e., outliers (see the Box plot in Figs. 2 and 3, and Table 2). There is, of course, once again some degree of overlap between performance in the control and the patient group and therefore not everyone with $\mathrm{aMCI}^{+}$reveals slower mean RT compared to cognitively healthy aging. It appears rather that the $\mathrm{aMCI}^{+}$group contains a greater proportion of individuals with disproportionately slower, responses. However, unlike Trails A, Trails B, and the simple target alone visual search RT task, the target plus distracter visual search RT task promotes outliers, i.e., disproportionately slowed responses only within the $\mathrm{aMCI}^{+}$group. It may be the case that this RT task does not produce as many 'false positives' i.e., disproportionately poor performance within the control group, as does the target alone search RT task and the Trails B test. Note also that three individuals in the $\mathrm{aMCI}^{+}$group are outliers in both the Trails B and the target plus distracter visual search RT test. This may indicate that for some people with $\mathrm{aMCI}^{+}$, both aspects of information processing speed are abnormal whereas for others only specific aspects of such processing are affected. If this is the case, changes over time in the profile of performance of an individual may be more useful than just testing one function. As suggested by Johnstone et al. [50], profile analysis is likely to assist in making differential diagnoses between cognitive disorders, and it may be the case that the identification of distinct deficit profiles of processing speed integrity and changes over time can better identify individuals' cognitive strengths and weaknesses.

Although such disproportionate slowing within the $\mathrm{aMCI}^{+}$group may be related to an increased risk of developing dementia (e.g., Phillips et al. [8]; Kochan 
et al. [10]), or represent a decline to dementia if such tests are administered to an individual over time, lack of follow up of all the participants in this study precludes us from making such an analysis. Such heterogeneity within the $\mathrm{aMCI}^{+}$may, however, explain, at least in part, some of the disparity in RT outcome between previous studies, as slowing may be related to specific etiologies of $\mathrm{aMCI}^{+}$, only some of which have a neurodegenerative basis. Importantly, however, that a proportion of individuals with $\mathrm{aMCI}^{+}$have very slow RTs, much beyond those associated with cognitively healthy aging, indicates that for some individuals processing related to continuous changes in the environment and situations in everyday life that require serial, rapid, and repeated detection, processing, and response can be significantly impaired. These effects are of potential importance irrespective of whether an individual with $\mathrm{aMCI}^{+}$has the early stages of dementia or not.

\section{Gender, age, education, IQ (NART), and MMSE}

A gender-related difference in performance was evident only within the $\mathrm{aMCI}^{+}$group. RT was significantly faster for males than females for the target alone visual search group. Although there was a significantly greater educational level for males than females in this group, which may have potentially contributed to this result, there was no significant correlation between educational level in either males or females and RT and it remains to be seen why a gender-related effect should be evident in the target alone visual search task RT only. Nevertheless this pattern of results indicates that within the same research groups, processing speed outcome and its relation to gender may be influenced by the test used, although one does have to consider that it may be a spurious effect.

In previous studies, TMT outcome has reportedly varied considerably with respect to whether or not performance is correlated with age and education (e.g., [41, 57]). In the present study we found that for the cognitively healthy older adult group, although initial analysis suggested some degree of correlation between Trails A performance speed and age, this did not survive Bonferroni correction for multiple correlational analysis; and performance was not significantly correlated with age per se for the aMCI ${ }^{+}$ group. It appears that over the relatively narrow range of ages within our data set this was a weak effect and one that only appears to occur in cognitively healthy aging. It may be the case that such a rela- tionship occurs only when a wider range of ages is included. In contrast, although performance of Trails B was not significantly correlated with age in the control group, it was significantly correlated with age (surviving Bonferroni correction) in the $\mathrm{aMCI}^{+}$ group: a finding which if further research finds to be robust, may have implications for the interpretation of results over this age range. The finding that age is not similarly correlated with performance in both cognitively healthy aging and $\mathrm{aMCI}^{+}$also breaks an assumption necessary for covariate analysis (if parametric analysis of RT data is attempted; another reason why we used non-parametric testing for our results). In contrast to Trails $A$ and $\mathrm{B}$, RT of both versions of the visual search task was not significantly correlated with age for either the cognitively healthy older adult or the $\mathrm{aMCI}^{+}$ groups.

Performance of Trails A, B, and both versions of the visual search RT task was not significantly correlated with educational level for either the cognitively healthy older adult or the $\mathrm{aMCI}^{+}$groups (although there was some evidence of a correlation for Trails A performance and education for the $\mathrm{aMCI}^{+}$group it did not survive Bonferroni correction). Although the $\mathrm{aMCI}^{+}$group had a significantly lower IQ (NART score), explained by the lower score for females compared to males in this group, performance of none of the four tasks was significantly correlated with IQ. Performance of TMT and both visual search tasks was not significantly correlated with MMSE (note however that for the $\mathrm{aMCI}^{+}$group, Trails B was significantly correlated with MMSE score but again this did not survive Bonferroni correction).

There is therefore some room for debate about whether some relatively small effects of age and education and MMSE occur in relation to TMT and whether they differ with respect to group. However, such effects may be contingent upon the ranges of these factors within the groups (e.g., in wider or different ranges than those used in the present study) and dependent upon the numbers tested over each age range (i.e., related to the determination of normative data in which smaller numbers tend be tested for each age range and research studies in which a narrower range of measures are usually employed).

What is clear from the present study, however, is the lack of relationship between age, education, MMSE, IQ, and visual search based RT performance. This may indicate that such tests are less susceptible to, i.e., relatively independent of, the influence of age, education, and IQ upon performance [57, 82]. 


\section{Potential study limitations}

Unfortunately we were unable to follow up both groups in order to determine clinical outcome thus precluding analysis of whether those individuals with outlying response times and $\mathrm{RTs}$ in the $\mathrm{aMCI}^{+}$group were most likely to develop dementia. Furthermore, interpretation of the results and thus their potential for impact is limited by the fact that we did not test performance repeatedly and over various time periods. Ideally we would have combined our behavioral tasks with an imaging study in order to determine the relationship between our RT and response speed results and structural and functional integrity both at group and individual level. We would have also explored the impact of methodological manipulations of RT tests such as time pressure, processing load, and various types of distraction in order to explore deficits in a wider range of information processing operations.

Furthermore, we only measured response speed and $\mathrm{RT}$ in $\mathrm{aMCI}^{+}$; it would have been useful to determine the sensitivity of such tests to aMCI and to subjective cognitive decline, conditions that may represent lower stages on a continuum between health and dementia. Repeating this study with a larger sample size and wider range of demographic factors would allow further investigation in to the potential relationship between processing speed and RT and factors such as age, IQ, educational level, and level of cognitive impairment. Further research would also investigate a wider range of processing speed and RT tests typically used in research and clinically and in relation to factors such as practice effects.

Nevertheless, the very fact that the search tests seem more sensitive to $\mathrm{aMCI}^{+}$than the Trails B tests and the fact that the search tests appear less affected by demographic factors than the TMT indicates that such tests have a place in clinical assessment.

\section{Conclusions}

These results indicate that as well as potential slowing in performance of the operations involved in the TMT used in clinical assessment, individuals with $\mathrm{aMCI}^{+}$may also experience a substantial slowing of the rapid RT responses necessary for the safe, appropriate, and efficient environmental interaction required in real life; the importance of which is great irrespective of etiology. The results also suggest that RT tests typically used in research may be superior to the TMT in their ability to significantly differenti- ate between cognitively healthy aging and $\mathrm{aMCI}^{+}$ at group level and in revealing a heterogeneity of performance one would expect from an etiologically heterogeneous disorder such as $\mathrm{aMCI}^{+}$. These findings, together with evidence from previous studies regarding the relationship between RT and neurological status, indicates that RT tests should at least be included in the diagnosis and follow-up of cognitive impairment.

\section{ACKNOWLEDGMENTS}

BRACE-Alzheimer's Research [registered charity number 297965] and Maggie Agg for assistance in participant recruitment.

Authors' disclosures available online (http://j-alz. com/manuscript-disclosures/15-0791r2).

\section{REFERENCES}

[1] Ferreira D, Molina Y, Machado A, Westman E, Wahlund LO, Nieto A, Carreia R, Junqué C, Díaz-Flores L, Barroso J (2014) Cognitive decline is mediated by gray matter changes during middle age. Neurobiol Aging 35, 1086-1094.

[2] Salthouse TA (1996) The processing-speed theory of adult age differences in cognition. Psychol Rev 103 403-428.

[3] Zimprich DM (2002) Can longitudinal changes in processing speed explain longitudinal changes in fluid intelligence? Psychol Aging 17, 690-695.

[4] Wahl HW, Schmitt M, Danner D, Coppin A (2010) Is the emergence of functional ability decline in early old age related to change in speed of cognitive processing and also to change in personality? J Aging Health 22, 691-712.

[5] Salthouse TA (1994) The nature of the influence of speed on adult age-differences in cognition. Develop Psychol 30, 240-259.

[6] Warkentin S, Erikson C, Janciauskiene S (2008) rCBF pathology in Alzheimer's disease is associated with slow processing speed. Neuropsychologia 46, 1193-1200.

[7] Amieva H, Rouch-Leroyer I, Fabrigoule C, Daetigues JF (2000) Deterioration of controlled processes in the preclinical phase of dementia: A confirmatory analysis. Dement Geriatr Cogn Disord 11, 46-52.

[8] Phillips M, Rogers P, Haworth J, Bayer A, Tales A (2013) Intra-individual reaction time variability in mild cognitive impairment and Alzheimer's disease: Gender, processing load and speed factors. PLOS One 8, e65712.

[9] Van Deursen JA, Vuurman EFPM, Smits LL, Verhey FRJ, Riedel WJ (2009) Response speed, contingent negative variation and P300 in Alzheimer's disease and MCI. Brain Cogn 69, 592-599.

[10] Kochan N, Bunce D, Pont S, Slavin M, Reppermund S, Brodaty H, Crawford J, Mather K, Kang K, Sachdev P (2013) Reaction-time measures predict incident dementia over 4 years: The Sydney Memory and Ageing Study. Alzheimers Dement 9(Suppl) 536.

[11] Rosler A, Billino J, Muller NG, Weidauer S, Steinmetz H, Kleinschmidt A (2005) Visual search in patients with sub- 
cortical vascular dementia: Short fixations but long reaction times. Dement Geriatr Cogn Disord 20, 375-380.

[12] Duering M, Gesierich B, Seiler S, Pirpamer L, Gonik M, Hofer E, Jouvent E, Duchesnay E, Chabriat H, Ropele S, Schmidt R, Dichgans M (2014) Strategic white matter tracts for processing speed deficits in age-related small vessel disease. Neurology 82, 1946-1950.

[13] Jacobs HIL, Leritz EC, Williams VJ, Van Boxtel MPJ, van der Elst W, Jolles J, Verhey FRJ, McGlinchey RE, Milberg WP, Salat DH (2013) Association between white matter microstructure, executive functions, and processing speed in older adults: The impact of vascular health. Hum Brain Mapp 34, 77-95.

[14] Righart R, Duering M, Gonik M, Jouvent E, Reyes S, Hervé D, Chabriat H, Dichgans M (2013) Impact of regional cortical and subcortical changes on processing speed in cerebral small vessel disease. Neuroimage Clin 2, 854-861.

[15] Parikh M, Hynan LS, Weiner MF, Lacritz L, Ringe W, Cullum CM (2014) Single neuropsychological test scores associated with rate of cognitive decline in early Alzheimer's disease. Clin Neuropsychol 28, 926-940.

[16] Caramelli P, Chaves ML, Engelhardt E, Machado JC, Schultz RR, Vale FA, Charchat-Fichman H (2004) Effects of galantamine on attention and memory in Alzheimer's disease measured by computerised neuropsychological tests: Results of the Brazilian multi-center galantamine study [GAL-BRA-01]. Arq Neuropsiquiatr 62, 379-384.

[17] Edwards JD, Delahunt PB, Mahncke HW (2009) Cognitive speed of processing delays driving cessation. J Gerontol A Sci Med Sci 64, 1262-1267.

[18] Edwards JD, Bart E, O'Connor ML, Cissell G (2010) Ten years down the road: Predictors of driving cessation. Gerontologist 50, 393-399.

[19] Iwasa H, Kai I, Yoshida Y, Suzuki T, Kim H, Yoshida H (2014) Information processing speed and 8-year mortality among community-dwelling elderly Japanese. J Epidemiol 24, 52-59.

[20] Iwasa H, Gondo T, Yoshida Y, Kwon J, Inagaki H, Kawaai C, Masui Y, Kim H, Yoshida H, Suzuki T (2008) Cognitive performance as a predictor of functional decline among the non-disabled elderly dwelling in a Japanese community: A 4-year population-based prospective cohort study. Arch Gerontol Geriatr 47, 139-149.

[21] Welmer A-K, Rizzuto D, Qui C, Caracciolo B, Laukka EJ (2014) Walking speed, processing speed, and dementia: A population-based longitudinal study. J Gerontol A Biol Sci Med Sci 69, 1503-1510.

[22] Cumming TB, Brodtmann A, Darby D, Bernhardt J (2012) Cutting a long story short: Reaction times in acute stroke are associated with longer term cognitive outcomes. J Neurol Sci 322, 102-106.

[23] Reuter-Lorenz P, Park DC (2010) Human neuroscience and the aging mind: A new look at old problems. J Gerontol B Psychol Sci Soc Sci 64B 405-415.

[24] Lu PH, Lee GJ, Tishler TA, Meghpara M, Thompson PM, Bartzokis G (2013) Myelin breakdown mediates age-related slowing in cognitive processing speed in healthy elderly men. Brain Cogn 81, 131-138.

[25] Ritchie SJ, Bates TC, Der G, Starr JM, Deary IJ (2013) Education is associated with higher later life IQ scores but not with faster cognitive processing speed. Psychol Aging 28, 515-521.
[26] Kerchner GA, Racine CA, Hale S, Wilheim R, Laluz V, Miller BL, Kramer JH (2012) Cognitive processing speed in older adults: Relationship with white matter integrity. PLOS One 7, e50425.

[27] Kochunov P, Coyle T, Lancaster J, Robin DA, Hardies J, Kochunov V, Bartzokis J, Royall D, Schlosser AE, Null M, Fox PT (2010) Processing speed is correlated with cerebral health markers in the frontal lobes as quantified by neuroimaging. Neuroimage 49, 1190-1199.

[28] Schneider KK, Schote AB, Meyer J, Markett S, Reuter M, Frings C (2015) Individual response speed is modulated by variants of the gene encoding the alpha 4 sub-unit of the nicotinic acetylcholine receptor (CHRNA4). Behav Brain Res 284, 11-18.

[29] Bartzokis G, Lu PH, Geschwind DH, Tingus K, Huang D, Mendez M, Edwards N, Mintz J (2007) Apolipoprotein E affects both myelin breakdown and cognition: Implications for age-related trajectories of decline into dementia. Biol Psychiatr 63, 1380-1387.

[30] Bartzokis G, Lu PH, Tingus K, Mendez MF, Richard A, Peters DG, Oluwadara B, Barrall KA, Finn JP, Villablanca P, Thompson PM, Mintz J (2010) Lifespan transition of myelin integrity and maximum motor speed. Neurobiol Aging 31, 1554-1562.

[31] Wolinsky FD, Vander Weg MW, Bryant Howren M, Jones MP, Dotson MM (2013) A randomized controlled trial of cognitive training using a visual speed of processing intervention in middle aged and older adults. PLOS One $\mathbf{8}$, e61624.

[32] Reitan RM, Wolfson D (1993) The Halstead-Reitan neuropsychological test battery: Theory and clinical interpretations. Neuropsychology Press, Tuscon, AZ.

[33] Tombaugh TN (2004) Trail Making Test A and B: Normative data stratified by age and education. Arch Clin Neuropsychol 19, 203-214.

[34] Hagen K, Ehlis AC, Haeussinger FB, Heinzel S, Dresler T, Mueller LD, Herrmann MJ, Fallgatter AJ, Metzger FG (2014) Activation during the Trail Making Test measured with functional near-infra-red spectroscopy in healthy elderly subjects. Neuroimage $\mathbf{8 5}, 583-591$.

[35] Salthouse TA (2011) What cognitive abilities are involved in trail-making performance? Intelligence 39, 222-232.

[36] Müller LD, Guhn A, Zeller JBM, Biehl SC, Dresler T, Hahn T, Fallgatter AJ, Polak T, Deckert J, Herrmann MJ (2014) Neural correlates of a standardized version of the trail making test in young and elderly adults: A functional near-infra-red spectroscopy study. Neuropsychologia 56, 271-279.

[37] Rabin LA, Barr WB, Burton LA (2005) Assessment practices of clinical neuropsychologists in the united states and Canada: A survey of INS, NAN, and APA Division 40 members. Arch Clin Neuropsychol 1, 33-65.

[38] Schmid NS, Ehrensperger MM, Berres M, Beck IR, Monsch AU (2014) The extension of the German CERAD Neuropsychological assessment battery with tests assessing subcortical, executive and frontal functions improves accuracy in dementia diagnosis. Dement Geriatr Cogn Dis Extra 4, 322-334.

[39] Lezak MD (1995) Neuropsychological Assessment. Oxford University Press, New York Oxford.

[40] Sofko C, Boettcher A, Hoadley R, Hil B (2014) Differential rates of age-related decline on Trail Making tests. Arch Clin Neuropsychol 29, 511. 
[41] Bezdicek O, Motak L, Axelrod BN, Preiss M, Nikolai T, Vyhnalek M, Poreh A, Ruzicka E (2012) Czech version of the trail making test: Normative data and clinical utility. Arch Clin Neuropsychol 27, 906-914.

[42] Baudic S, Dalla Barba G, Thibaudet MC, Smagghe A, Remy P, Traykov L (2006) Executive function deficits in early Alzheimer's disease and their relations with episodic memory. Arch Clin Neuropsychol 21, 15-21.

[43] Boyle PA, Cohen RA, Paul R, Moser D, Gordon N (2002) Cognitive and motor impairments predict functional declines in patients with vascular dementia. Int J Geriatr Psychiatry 17, 164-169.

[44] Barr A, Benedict R, Tune L, Brandt J (1992) Neuropsychological differentiation of Alzheimer's disease from vascular dementia. Int J Geriatr Psychiatry 7, 621-627.

[45] Spreen O, Strauss E (1998) A Compendium of Neuropsychological Tests, Oxford University Press, Oxford.

[46] Mendez MF, Cherrier MM, Perryman KM (1997) Differences between Alzheimer's disease and vascular dementia on information processing measures. Brain Cogn 34, 301310.

[47] Yuspeh RL, Vanderploeg RD, Crowel TA, Mullan M (2002) Differences in executive functioning between Alzheimer's disease and subcortical ischemic vascular dementia. J Clin Exp Neuropsychol 24, 745-754.

[48] Greenlief CL, Margolis RB, Erker GJ (1985) Application of the Trail Making Test in differentiating neuropsychological impairment of elderly persons. Percept Mot Skills 61, 12831289.

[49] Paul R, Moser D, Cohen R, Browndyke J, Zawacki T, Gordon N (2001) Dementia severity and pattern of cognitive performance in vascular dementia. Appl Neuropsychol 8, 211-217.

[50] Johnstone B, Hogg JR, Schopp LH, Kapila C, Edwards S (2002) Neuropsychological deficit profiles in senile dementia of the Alzheimer's type. Arch Clin Neuropsychol 17, 273-281.

[51] Nestor PG, Parasuraman R, Haxby JV (1991) Speed of information processing and attention in early Alzheimer's dementia. Dev Neuropsychol 7, 243-356.

[52] Rasmusson XD, Zonderman AB, Kawas C, Resnick SM (1998) Effects of age \& dementia on the Trail Making Test. Clin Neuropsychol 12, 169-178.

[53] Classen S, Wang Y, Winter SM, Velozo CA, Lanford DN, Bédard M (2013) Concurrent criterion validity of the safe driving behavior measure: A predictor of on-road driving outcomes. Am J Occup Ther 67, 108-116.

[54] Pillon B, Gouider-Khouja N, Deweer B, Vidailhet M, Malapani C, Dubois B, Agid Y (1995) Neuropsychological pattern of striatonigral degeneration: Comparison with Parkinson's disease and progressive supranuclear palsy. $J$ Neurol Neurosurg Psychiatry 58, 174-179.

[55] Schmid N (2013) Neuropsychological signs of Alzheimer's disease 8 years prior to diagnosis. J Alzheimers Dis $\mathbf{3 4 , 5 3 7 -}$ 546.

[56] Lee H, Baniqued PL, Cosman J, Mullen S, McAuley E, Severson, Kramer AF (2012) Examining cognitive function across the lifespan using a mobile application. Comp Hum Behav 28, 1934-1946.

[57] Ptotek W, tyskawa W, Kluzik A, Grześkowiak M, Podlewski R, Żaba Z, Drobnik L (2014) Evaluation of the trail making test and interval timing as a measures of cognition in healthy adults: Comparisons by age, education, and gender. $\mathrm{Med}$ Sci Monit 20, 173-181.
[58] Cangoz B, Karakoc E, Selekler K (2009) Trail making test: Normative data for Turkish elderly population by age, sex and education. J Neurol Sci 283, 73-78.

[59] Ashendorf L, Jefferson AL, O'Connor MK, Chaisson C, Green RC, Stern RA (2008) Trial making test errors in normal aging, mild cognitive impairment, and dementia. Arch Clin Neuropsychol 23, 129-137.

[60] Seo EH, Lee DY, Kim KW, Lee JH, Jhoo JH, Youn JC, Choo IH, Ha J, Woo JI (2006) A normative study of the Trail Making Test in Korean elders. Int J Geriatr Psychiatry 21, 844-852.

[61] Liu Y, Julkunen V, Paajanen T, Westman E, Wahlund L-O, Aitken A (2012) Education increases reserve against Alzheimer's disease-evidence from structural MRI analysis. Neurorad 54, 929-938.

[62] Corrigan JD, Hinkeldey NS (1987) Relationships between parts A and B of the Trail Making Test. J Clin Psychol 43, 402-409.

[63] Lu P, Lee G, Raven E, Tingus K, Khoo T, Thompson PM, Bartzokis G (2011) Age-related slowing in cognitive processing speed is associated with myelin integrity in a very healthy elderly sample. J Clin Exp Neuropsychol 33, 10591068.

[64] Der G, Deary IJ (2006) Age and sex differences in reaction time in adulthood: Results from the United Kingdom Health and Lifestyle Survey. Psychol Ageing 21, 62-73.

[65] Dykiert D, Der G, Starr JM, Deary IJ (2012) Sex differences in reaction time mean and intraindividual variability across the lifespan. Dev Psychol 48, 1262-1276.

[66] Roivainen E (2011) Gender differences in processing speed: A review of recent research. Learn Individ Differ 21, 145-149.

[67] Mitrushina M, Boone K (2005) Handbook of normative data for neuropsychological assessment, Oxford University Press, New York.

[68] Nelson HE, Willison JR (1991) National Adult Reading Test (NART). Test manual including new data supplement, NFER-NELSON, Windsor.

[69] Dykiert D, Deary IJ (2013) Retrospective validation of WTAR and NART scores as estimators of prior cognitive ability using the Lothian Birth Cohort 1936. Psychol Assess 25, 1361-1366.

[70] van Deursen JA, Vuurman EFPM, Smits LL, Verhey FRJ, Riedel WJ (2009) Response speed, contingent negative variation and P300 in Alzheimer's disease and MCI. Brain Cogn 69, 592-599.

[71] Levinoff E, Saumier D, Chertkow H (2005) Focused attention deficits in patients with Alzheimer's disease and mild cognitive impairment. Brain Cogn 57, 127-130.

[72] Folstein MF, Folstein SE, McHugh PR (1975) Mini-mental state: A practical method for grading the cognitive state of patients for the clinician. J Psychiatr Res 12, 189-198.

[73] Wechsler D (1998) Adult Intelligence Scale-III. The Psychological Corporation, London, UK.

[74] Brandt J (1991) The Hopkins Verbal Learning Test: Development of a new memory test with six equivalent forms. Clin Neuropsychol 5, 125-142.

[75] Royall DR, Cordes JA, Polk M (1988) An executive clock drawing task. J Neurol Neurosurg Psychiatry 64, 588-594.

[76] Benton AL, Sivan AB, Hamshere KD, Varney NR, Spreen O (1994) Visual Form Discrimination Test. Contributions to Neuropsychological Assessment, 2nd ed, Oxford University Press, Oxford. 
[77] Coughlan AK, Hollows S (1995) The adult memory and information processing battery. St. James University Hospital, Leeds, UK.

[78] Bucks RS, Ashworth DL, Wilcock GK, Siegfried K (1996) Assessment of activities of daily living in dementia: Development of the Bristol Activities of Daily Living Scale. Age Ageing 25, 113-120.

[79] Adshead F, Cody DD, Pitt B (1992) BASDEC: A novel screening instrument for depression in elderly medical inpatients. BMJ 305, 397
[80] Tales A, Bayer AJ, Haworth J, Snowden RJ, Philips M, Wilcock G (2011) Visual search in mild cognitive impairment: A longitudinal study. J Alzheimers Dis 24, 151-160.

[81] Tales A, Haworth J, Nelson S, Snowden R, Wilcock G (2005) Abnormal visual search in mild cognitive impairment in Alzheimer's disease. NeuroCase 11, 80-84.

[82] Rabbitt P, Osman P, Moore B (2001) There are stable individual differences in performance variability, both from moment to moment and from day to day. Q J Exp Psychol A 54A, 981-1003 


\title{
Perception and Reality of Cognitive Function: Information Processing Speed, Perceived Memory Function, and Perceived Task Difficulty in Older Adults
}

\author{
Anna Torrens-Burton ${ }^{\mathrm{a}}$, Nasreen Basoudan ${ }^{\mathrm{a}}$, Antony J. Bayer ${ }^{\mathrm{b}}$ and Andrea Tales ${ }^{\mathrm{a}, *}$ \\ ${ }^{a}$ Department of Psychology, Swansea University, Singleton Park, Swansea, Wales, UK \\ ${ }^{\mathrm{b}}$ Division of Population Medicine, Cardiff University, University Hospital Llandough, Penarth, Wales, UK
}

Accepted 21 August 2017

\begin{abstract}
This study examines the relationships between two measures of information processing speed associated with executive function (Trail Making Test and a computer-based visual search test), the perceived difficulty of the tasks, and perceived memory function (measured by the Memory Functioning Questionnaire) in older adults (aged 50+ y) with normal general health, cognition (Montreal Cognitive Assessment score of 26+), and mood. The participants were recruited from the community rather than through clinical services, and none had ever sought or received help from a health professional for a memory complaint or mental health problem. For both the trail making and the visual search tests, mean information processing speed was not correlated significantly with perceived memory function. Some individuals did, however, reveal substantially slower information processing speeds (outliers) that may have clinical significance and indicate those who may benefit most from further assessment and follow up. For the trail making, but not the visual search task, higher levels of subjective memory dysfunction were associated with a greater perception of task difficulty. The relationship between actual information processing speed and perceived task difficulty also varied with respect to the task used. These findings highlight the importance of taking into account the type of task and metacognition factors when examining the integrity of information processing speed in older adults, particularly as this measure is now specifically cited as a key cognitive subdomain within the diagnostic framework for neurocognitive disorders.
\end{abstract}

Keywords: Aging, information processing speed, metacognition, reaction time, subjective cognitive impairment

\section{INTRODUCTION}

Although debate continues with respect to the theoretical and applied relationship between slowing and cognition, information processing speed is a measure commonly used in research as a behavioral indicator, or proxy, of the integrity of cognitive function. The relationship is underpinned by a substantial body

*Correspondence to: Andrea Tales, Department of Psychology, Swansea University, Singleton Park, Swansea, SA2 8PP, Wales, UK. E-mail: A.Tales@swansea.ac.uk. of evidence linking behaviorally measured change in information processing speed to brain structure (e.g., to deterioration in white and grey matter) and function in aging, mild cognitive impairment (MCI), and dementia [1-14]. The potential relevance of information processing speed in research and clinical practice is highlighted by evidence indicating that it can predict ability to perform aspects of daily activity and quality of life [15-20], and it is now specifically cited as a key cognitive subdomain within the diagnostic framework for neurocognitive disorders in DSM-5 [21]. 
Concerns about self-perceived cognitive decline, especially of memory, commonly occur in older adults [22], and it is increasingly apparent that both MCI and dementia can be characterized by an earlier stage, variously termed subjective cognitive impairment (SCI) or decline (SCD), without any objective evidence of deficit from neuropsychological assessment [22-26]. However, subjective cognitive complaints do not always represent a prodromal stage of dementia, with some causes (e.g., anxiety, depression, and sleep disorder) potentially responsive to intervention [22, 27, 28]. Irrespective of causality, concerns about memory function can impact negatively upon everyday life and mental health, with worry about developing dementia and withdrawal from positive health and social behavior [20, 29].

SCI is characterized by objectively defined normal neuropsychological test performance. It is of course possible that objective change is absent in functions such as memory because the tests used are insensitive or do not measure the specific aspect of memory that an individual perceives as having changed. Detrimental change in brain functions other than memory may occur in what we term SCI, but this may be difficult for the general public to describe and, if not tested, may manifest only as vague perceptions of change. Indeed, emerging evidence indicates that fundamental brain operations may be disrupted in individuals with SCI [30]. Therefore, it is possible that a reduction in integrity of fundamental brain processes may impair memory function to a level that may be perceived by the individual, but not evident from current neuropsychological testing protocols.

Despite evidence of inter-relationships between information processing speed, cognition, white and grey matter integrity and behavior, in aging, MCI and dementia [23, 30, 31], there is a lack of research into information processing speed in relation to SCI, and this is particularly so in those individuals who experience subjective changes in memory function in the absence of formal clinical investigation. It is possible that a slowing of information processing speed in individuals experiencing subjective changes in memory might be indicative of structural change (in white matter for example) to which routine neuropsychological tests are not sensitive, but which affects general brain function, cognition, and the perception of functional integrity. Preservation of information processing speed in individuals reporting impaired memory may indicate structural normality.
In this study, therefore, we examine perceived memory function in relation to information processing speed in community-living older adults who have not approached health care services with concerns about their memory or cognitive function $[23,27]$ and with normal levels of general cognitive function and no significant anxiety or depression. In addition, as metacognition can be a factor in the self-perception of the integrity of memory and cognition [31], we also ask whether there is any relationship between reported memory performance and the perception of task difficulty (i.e., is high level of perceived memory dysfunction associated with greater perception of task difficulty?) and whether perceived task difficulty is related to actual (objectively measured) speed of information processing.

There is evidence (e.g., [5]) to suggest that the speed of information processing, and thus study outcome, can differ significantly with respect to the test used, because of the different brain networks and processes recruited by specific task demands. We therefore report studies using two different measures, the pen-and-paper-based trail making test (TMT) and a computer-administered visual search task.

The TMT is commonly used in clinical settings and in aging, MCI, and dementia research to examine information processing speed and executive function [32]. Trails A is a one-trial task typically described as probing functions such as speed of processing in relation to attention, visual scanning and search, number recognition, numeric sequencing and motor speed; giving a baseline measure of perceptual processing and motor speed. Trails B is again a one-trial task typically described as probing the efficiency of set-shifting, mental flexibility, executive function, divided attention, attention switching and shifting, visual search set shifting, simultaneous maintenance of two sequences, working memory and cognitive flexibility; and, thus, is arguably a measure of information processing speed in relation to multiple high level, non-specific functions. The computer-based visual search task requires rapid serial information processing and response over numerous trials, with measurement of the time taken to respond to an isolated target (whether an arrow is pointing right or left) and the time taken to respond to the same target when it is surrounded by similar but irrelevant distracters. This allows us to determine information processing speed per se, but also to measure the effect of irrelevant but distracting stimuli upon such processing. 
Table 1

Mean demographic scores and Trails A and Trails B information processing speeds for older adults. Standard deviation in parenthesis. Note that range refers to observed range within the data

\begin{tabular}{lcccccc}
\hline $\begin{array}{c}\text { Age } \\
(\mathrm{y})\end{array}$ & $\begin{array}{c}\text { Education } \\
(\mathrm{y})\end{array}$ & $\begin{array}{c}\text { MFQ-total } \\
\text { score }\end{array}$ & $\begin{array}{c}\text { Trails A } \\
(\mathrm{s})\end{array}$ & $\begin{array}{c}\text { Perceived } \\
\text { difficulty scale } \\
\text { for Trails A }\end{array}$ & $\begin{array}{c}\text { Trails B } \\
(\mathrm{s})\end{array}$ & $\begin{array}{c}\text { Perceived } \\
\text { difficulty scale } \\
\text { for Trails B }\end{array}$ \\
\hline $\begin{array}{l}\text { Older adults } \\
(n=81)\end{array}$ & $\begin{array}{c}65(5.5) \\
\text { Range 50-79 }\end{array}$ & $16(4.8)$ & $295(49.1)$ & $29.05(9.3)$ & $\begin{array}{c}2(1.2) \\
\text { Range 1-6 }\end{array}$ & $\begin{array}{c}43.43(9.4) \\
\text { Range 1-6 }\end{array}$ \\
\hline
\end{tabular}

Information processing speed for both Trails A and Trails B is represented by the box plot in Fig. 1. Note the presence of outliers in the performance of this task.

\section{STUDY 1. TRAIL MAKING TEST: METHODS}

This study was approved by the ethics committee of Swansea University, Department of Psychology. All participants gave written informed consent to participate.

\section{Participants}

Community-dwelling older adults $(n=100)$ were recruited through adverts placed in local newspapers and social clubs throughout the Swansea area and by word of mouth. Exclusion criteria included poor selfreported general health, any past history of significant medical, neurological, or mental health problems, evidence of physical slowing (e.g., related to Parkinson's disease or arthritis), or previous visit to a health care professional with memory complaints, anxiety, or depression. From those recruited, 19 individuals were excluded. Of these, 8 had Montreal Cognitive Assessment (MoCA) scores of 24 or 25, 3 had MoCA scores below this; 8 had current or past history of significant medical problems, anxiety, or depression. Of those included in the study $\left(n^{1}=81\right.$; age $50+y$; 31 male, 50 female), all had normal or correctedto-normal vision and hearing. Although medication could not be controlled, none of the participants reported receiving medication likely to affect information processing speed or cognitive function. All had normal overall cognition (score of 26 or above) using the MoCA [33] and no significant anxiety or depression, as determined by the Generalized Anxiety Disorder 7-item (GAD-7) score less than 5 $[34,35]$ and the Patient Health Questionnaire (PHQ9) score less than 4 [36], respectively. Participants' age, sex, and years of full time education were recorded. A trained researcher administered the tests.

\footnotetext{
${ }^{1}$ Note that participant estimate number was based on a twotailed analysis with an effect size 0.4 , an alpha of 0.05 , and a power of 0.8: giving an estimate of 46 individuals.
}

Testing took place within the Psychology Department at the University of Swansea.

\section{Subjective memory assessment}

Subjective memory function was measured using the Memory Functioning Questionnaire (MFQ) [37]. This 64-item questionnaire assesses the perception of everyday memory functioning with seven sections on general rating of memory, retrospective functioning (compares current memory with past ability), frequency of forgetting, frequency of forgetting while reading, remembering past events, seriousness of forgetting (how memory impairment impacts daily life), and mnemonics usage. Each item is scored on a 1 to 7 Likert scale ( $1=$ severe memory problems; $7=$ no problems). Scores range between 64 and 448 with high scores reflecting less severe memory complaints.

Table 1 shows the demographics and TMT data.

\section{Trail Making Test}

Practice trails were provided for both Trails A and B. For Trails A, the participants were instructed to draw one continuous line joining a series of circled numbers in ascending order on a sheet of paper as fast but as accurately as possible. For Trails B, the participants were instructed to draw one continuous line joining a series of circled numbers and letters alternately in ascending and alphabetical order on a sheet of paper as fast but as accurately as they could. Test outcome was the time taken in seconds to complete the test (with the time required to rectify any error forming part of the information processing speed score). No performance feedback was provided. Immediately after completing each of trails A and $\mathrm{B}$, participants were asked to rate, using a scale of 1 to 7 , how easy or difficult they found each test to complete, with 1 very easy to complete and 7 very difficult. Study debrief was performed at the end of the experimental session. 
Table 2

Mean baseline demographics, information processing speed and errors. Standard deviation in parenthesis. Note that range refers to observed range within the data

\begin{tabular}{|c|c|c|c|c|c|c|c|c|}
\hline & \multirow[t]{2}{*}{$\begin{array}{l}\text { Age } \\
(y)\end{array}$} & \multirow[t]{2}{*}{$\begin{array}{l}\text { Education } \\
\text { (y) }\end{array}$} & \multirow[t]{2}{*}{$\begin{array}{l}\text { MFQ Total } \\
\text { score }\end{array}$} & \multicolumn{2}{|c|}{$\begin{array}{c}\text { Information } \\
\text { processing speed }\end{array}$} & \multicolumn{2}{|c|}{ Mean group errors } & \multirow{2}{*}{$\begin{array}{c}\text { Perceived } \\
\text { performance } \\
\text { Likert scale }\end{array}$} \\
\hline & & & & $\begin{array}{l}\text { Target } \\
\text { alone }\end{array}$ & $\begin{array}{l}\text { Target plus } \\
\text { distracters }\end{array}$ & $\begin{array}{l}\text { Target } \\
\text { alone }\end{array}$ & $\begin{array}{l}\text { Target plus } \\
\text { distracters }\end{array}$ & \\
\hline $\begin{array}{l}\text { Older adults } \\
(n=54)\end{array}$ & $\begin{array}{c}66 \\
\text { Range 55-79 } \\
(5.2)\end{array}$ & $15(3.7)$ & $290(46.5)$ & $743.02(164.91)$ & $1685.55(314.23)$ & 0.37 & 0.33 & $\begin{array}{c}3(1.4) \\
\text { Range 1-6 }\end{array}$ \\
\hline
\end{tabular}

Information processing speed for both the Target alone and Target plus distracter conditions is represented by the box plot in Fig. 3 . Note the presence of outliers in the performance of this task.

\section{RESULTS}

TMT: Information processing speed and subjective memory function

Spearman's correlational analysis showed no association between MFQ total score and information processing speed for either Trails A or B (all $p$ values $>0.05$ ). Of the seven subscales of the MFQ, none showed an association with either Trails A or Trails B (all $p$ values $>0.05$ ) except for a significant negative correlation of Trails A with the Mnemonics subscale ( $\mathrm{r}=-0.295, p=0.007$, which survives Bonferroni correction, $p=0.042$ ).

\section{TMT: Information processing speed and perceived task difficulty}

The mean (sd) perceived task difficulty score was 2 (1.2) with a range from 1 to 6 for Trails A and 3 (1.6) with a range of 1 to 6 for Trails B. This was not significantly correlated with performance on Trails A $(p>0.05)$ but was significantly positively correlated with performance on Trails B $(r=0.293$, $p=0.008$ ), with slower actual information processing speed associated with a greater perceived task difficulty. Post hoc tests revealed that these results did not vary with respect to educational level or whether the participant was male or female.

\section{Subjective memory function and perceived task difficulty}

MFQ total score was significantly negatively correlated with perceived task difficulty for Trails A $(\mathrm{r}=-0.275, p=0.013)$ and Trails $\mathrm{B}(\mathrm{r}=-0.334$, $p=0.002$ ), with higher levels of subjective memory complaint related to greater perception of task difficulty. Post hoc tests revealed that these results did not vary with respect to educational level or whether the participant was male or female.

\section{STUDY 2: VISUAL SEARCH TASK: METHODS}

\section{Participants}

In the second study, another (separate) group of older adults $(n=62)$ were recruited. The protocol (i.e., inclusion and exclusion factors) was exactly the same as in study 1 , as was the recruitment procedure. From those recruited, 6 individuals were excluded due to MOCA scores of 25 or less, with 2 further individuals excluded as a result of current poor medical health. Demographic details for the participants $\left(n^{2}=54\right.$; age $50+y ; 24$ male, 30 female $)$ of this second study are shown in Table 2. All participants completed all 64 items on the MFQ.

\section{Visual Search Task: Experimental task and procedure}

For the computer-based visual search task, the time taken to respond to a target (target discrimination) when it appeared in isolation upon the screen and the time taken to respond to the same target when it was surrounded by similar but irrelevant and distracting stimuli were determined. This paradigm was presented on a Dell Precision PC running on Windows XP X86 CPU, viewed at a distance of $57 \mathrm{~cm}$. All trials included a black target that was either a left- or right-pointing arrow, the task being to indicate whether the arrow was pointing to the right or left. The distracting stimuli consisted of seven black arrows that pointed up or down. A clock-face configuration (see Fig. 2) was used to position the target, both when it appeared alone and when surrounded by 7 distracters, in a counterbalanced arrangement

\footnotetext{
${ }^{2}$ Note that participant estimate number was based on a twotailed analysis with an effect size 0.4 , an alpha of 0.05 , and a power of 0.8: giving an estimate of 46 individuals.
} 
in order to eliminate any differences in processing between right and left and upper and lower visual fields. A total of 64 trials were presented; the target appearing 8 times at each of the possible 'clock-face' locations. For half of the trials distracters were presented at the other locations and for the other half no distracters were presented. For each trial the central fixation cross appeared on screen for $1000 \mathrm{~ms}$ prior to the appearance of the target (with or without distracters) and remained on screen for the duration of the trial. The stimuli remained on screen until the participant responded, after which the fixation point re-appeared. The participants were instructed to fixate on the center cross at the beginning of each trial and to respond as quickly but as accurately as possible to whether the target was pointing to the right or left by pressing one of two computer keyboard keys. After instruction, all participants were asked to reiterate the instructions to ensure understanding and then performed a practice block of no more than 10 trials. The ability of the participants to fixate on the cross at the beginning of each trial continued to be checked throughout the procedure by researcher observation. No performance feedback was provided.

Group mean errors were calculated. Responses were eliminated if they were incorrect or obviously due to a disturbance/lapse of concentration or below $150 \mathrm{~ms}$ (faster than 'natural' reaction time therefore representing the pre-empting of the stimulus). No participants failed to respond to a trial. For each participant, the median time (information processing speed) taken to respond for the target alone and the target plus distracter trials was determined and group mean data produced (see Table 2).

\section{Perception of task difficulty}

Immediately after completing the test participants were asked to rate, using a Likert scale of 1 to 7 , how easy or difficult they found each test to complete, with 1 very easy to complete and 7 very difficult. Study debrief was performed at the end of the experimental session.

\section{RESULTS}

Visual search: information processing speed and subjective memory function

MFQ total score and subscales scores were not significantly correlated with information processing speed for either the target alone or the target plus distracters conditions ( $p$ values $>0.05$ ). Overall errors on the visual search tasks were very small (mean group errors 0.37 for target alone and 0.33 for target plus distracters) and the number of errors was not significantly correlated with MFQ scores ( $p$ values $>0.05$ ).

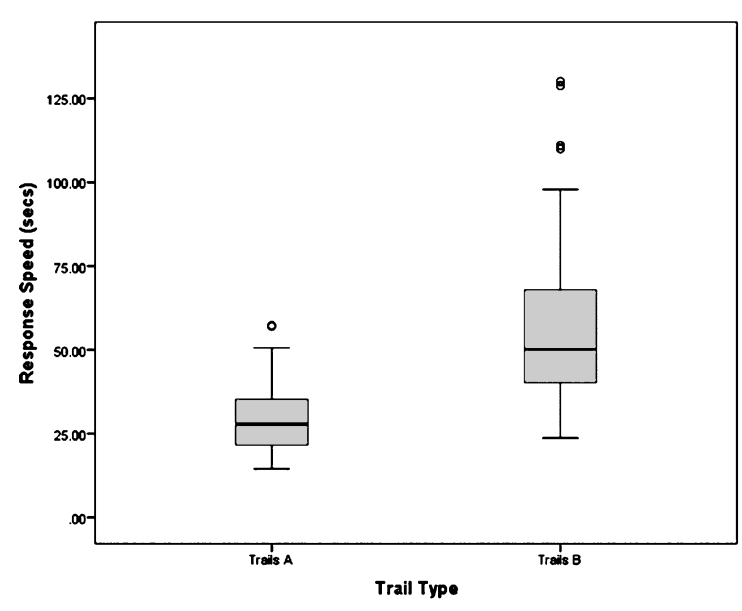

Fig. 1. Box plot of mean information processing speed (s) for Trails A and B performance in older adults.

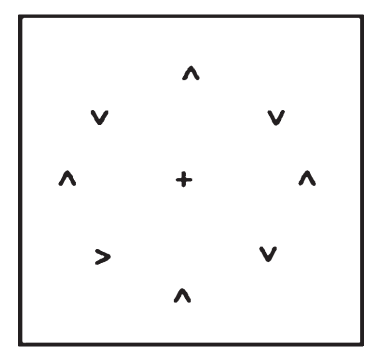

Fig. 2. Search stimulus.

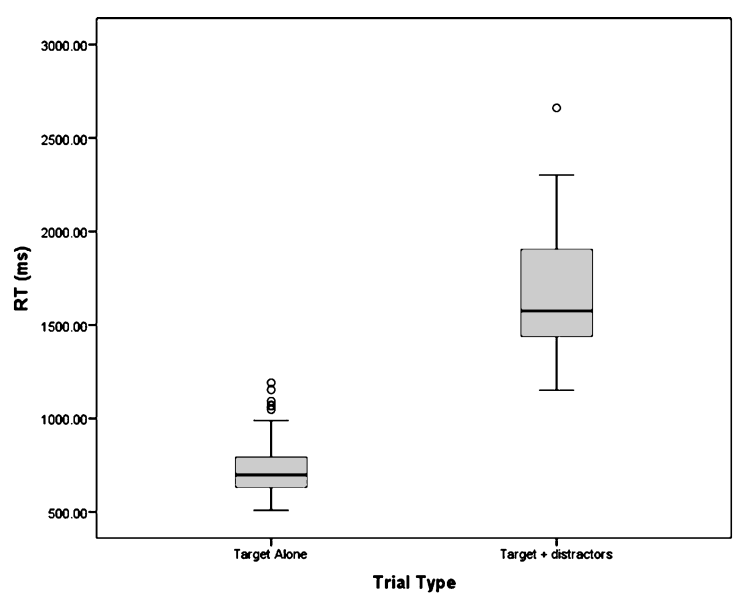

Fig. 3. Box plot of mean information processing speed (ms) for target alone and target plus distracters trials. 


\section{Visual search: information processing speed and perceived task difficulty}

The mean (sd) perceived task difficulty score for the visual search task was 3 (1.4) with a range from 1 to 6 . This was significantly negatively correlated with the target alone condition $(\mathrm{r}=-0.294, p=0.031)$, with slower actual information processing speed associated with less perceived task difficulty, but not for the target plus distracters conditions $(p>0.05)$. The number of errors was not significantly correlated with perceived task difficulty ( $p$ values $>0.05$ ).

\section{Subjective memory function and perceived task difficulty}

MFQ total score was not significantly correlated with perceived task difficulty for either the target alone or the target plus distracters condition ( $p$ values $>0.05$ ). Post hoc tests revealed that all the above results did not vary with respect to whether the participant was male or female.

\section{Educational level and information processing speed}

Further post hoc analysis revealed that although educational level was not significantly related to subjective memory performance it was significantly positively correlated with perceived test difficulty $(\mathrm{r}=0.440, p=0.01)$ and it was significantly negatively correlated with information processing speed for target plus distracter $(\mathrm{r}=-0.398, p=0.003)$ condition, but not for the target alone condition $(p>0.05)$, i.e., a higher level of education is related to faster information processing speed when distractors are present.

\section{DISCUSSION}

The aim of these studies was to determine if a significant relationship exists between information processing speed, subjective memory function, and perceived task performance difficulty in older adults. In a difference of approach from some previous studies, we looked at people recruited from the community rather than from clinical settings, with normal general cognition and without significant depression or anxiety.

\section{Information processing speed and subjective memory}

Greater levels of overall subjective memory complaint (MFQ total score) were not significantly associated with slower information processing speed as measured by the TMT or Visual Search Task. For Trails A there was a significant negative correlation between information processing speed and the mnemonics subset of the MFQ and although this survived Bonferroni correction, the significance level of this effect was low $(p=0.042)$. For Trails B and both conditions of the visual search test, information processing speed was not significantly correlated with any of the MFQ subsets.

Given the close relationship between information processing speed and white and grey matter structure [6-12], the general absence of an association with perceived memory dysfunction in otherwise cognitively healthy, euthymic older adults suggests perceived memory dysfunction is less likely to be related to structural abnormality or possible neurodegenerative change. Similarly, given the association between Alzheimer's disease, vascular dementia and MCI and slowed information processing speed the lack of a relationship between perceived memory function and information processing speed may be indicative of a non-neurodegenerative basis for perceived impairment in individuals with 'normal' levels of general cognition (e.g., MoCA score within the normal range).

Note, however, that these ideas are speculative in nature given the absence of neuroimaging, a full range of objective and subjective measures of memory, cognitive and information processing speed performance, and longitudinal analysis examining the risk of developing MCI and/or dementia. The possibility that changes in brain structure and function and in memory may occur in the absence of changes in information processing speed must also be considered. Individuals also may perceive problems with memory but still perform at normal information processing speed as there may be, for some tasks, factors which influence memory and perceived memory but not information processing speed.

Nevertheless, if such findings were found to be robust after further research and development, the measurement of behavioral information processing speed might be of use in helping to determine for whom priority should be given with respect to further investigation and follow up of subjective memory complaints. For example, disproportionately slower 
responses (such as the outliers evident in our results, see Figs. 1 and 3) may be representative of detrimental change in underlying structure and function and thus a greater possibility of underlying neurodegeneration. Clinical follow-up and medical intervention with these patients could be prioritized, whereas others may benefit more from focus on psychological support, adjusting expectations regarding normal changes in cognitive performance, and providing metacognitive strategies to reduce the required effort for everyday tasks.

\section{Perception of task difficulty and subjective memory}

Greater subjective memory complaint was associated with a perception of greater task difficulty for both Trails A and Trails B of the TMT, especially for the more difficult Trails B test, with higher levels of subjective memory complaint related to greater perceived task difficulty. With respect to the TMT therefore, individuals who reported higher levels of perceived memory dysfunction (total MFQ score) are those who also reported the greater levels of task difficulty, despite actual (objectively measured) information processing speed not being associated with perceived memory function. To speculate, the relationship between perceived memory and perceived task difficulty in individuals with MOCA scores within the normal range in the absence of objective change in information processing speed suggests that perceived memory dysfunction may be related more to metacognitive factors than underlying structural change.

In contrast, for the visual search task, greater subjective memory complaint was not associated with a perception of greater task difficulty for both the target alone and the target plus distracter conditions. Individuals who reported higher levels of memory dysfunction did not report greater levels of task difficulty.

Such outcome variability indicates that the metacognition relationship between perceived memory and difficulty of task may not be generalizable to all tests, but mediated by factors such as difficulty, resource requirements and brain functions and areas recruited. However, different participants were used in the two studies and so the outcome effects may not be directly comparable. Nevertheless, our results raise the possibility that examining patterns of perceived memory function and the perception of task difficulty may help to determine whether perceived memory impairment (in the absence of objective change in memory function) is related to structural change or metacognition (which is more likely to be responsive to intervention and treatment than structural change), or indeed whether a much more complex relationship exists between metacognition, structural change and actual and perceived functional integrity.

\section{Information processing speed and perceived task difficulty}

For Trails A, perceived task difficulty was not significantly correlated with objectively measured information processing speed. In contrast, perceived task difficulty was positively correlated with information processing speed for Trails B, i.e., slower information processing speed was associated with greater perceived task difficulty. For the target plus distracters condition of the visual search task, perceived task difficulty was not significantly correlated with objectively measured information processing speed. For the target alone condition however, perceived task difficulty was significantly negatively correlated with information processing speed, i.e., slower information speed was associated with a lower level of perceived task difficulty. This pattern of results indicates that, irrespective of perceived memory function, the judgement of task difficulty is related to the nature of the task and is not always related to actual performance.

\section{Educational level}

For both Trails A and B, educational level was not significantly associated with information processing speed, subjective memory function or perceived task difficulty. For the visual search task, educational level was also not significantly related to subjective memory performance but it was significantly positively correlated with perceived test difficulty, i.e., a higher level of education was associated with higher levels of perceived task difficulty. Furthermore, educational level was significantly negatively correlated with information processing speed for the target plus distracter condition, i.e., a higher level of education was associated with faster information processing. In contrast, educational level was not significantly correlated with information processing speed for the target alone condition. Although educational level was not significantly related to perceived memory function, this pattern of results indicates the potential 
influence of education upon perceived task performance and actual task performance per se and a failure to take such factors into account in research may influence the interpretation of study outcome.

\section{Study limitations}

The participants in each study cohort were relatively young (mean age $65 \mathrm{y}$ ). However, this is the age group when subjective memory changes are typically reported and when pathological changes of neurodegenerative disease start to become more common. Future work, including neuroimaging, needs to explore the structural and functional relationships between information processing speed, actual and perceived memory function and metacognition factors in older age groups and in those seeking clinical diagnosis and with respect to mood and personality. Further research is also required to investigate why some individuals do not approach health care services about their perceived memory function. Some individuals may be anxious about a formal diagnosis, fearful that they may be developing dementia and unaware of other possible reversible causes of impairment or potential interventions to improve quality of life.

We tested participants on only one occasion and could not take into account the possibility that perceived memory may fluctuate, as it can be due to temporary conditions such as fatigue, or everyday stressful life events. Perceived memory was measured using only the MFQ. Related to this issue is the fact that we were not able to determine the accuracy of self-report on this measure. Furthermore, we did not include a battery of tests objectively measuring memory and cognition, while information processing speed was measured by only two tests, both of which had large executive function components. Nor did we measure separate specific components of behavioral information processing speed, such as motor function. The relationship between actual and perceived memory function, information processing speed and indeed metacognitive processes such as the perception of task difficulty, remains to be determined in relation to a variety of different tests, and ones that recruit different brain structure and functional domains and specific metacognitive factors. Finally, although it may not be possible to control medication, future studies should record and report medication in order to facilitate a greater understanding the generalizability of results to the population in general.

\section{ACKNOWLEDGMENTS}

The authors would like to thank all our participants, Dr. Jade Norris for participant recruitment, and BRACE-Alzheimer's Research for funding part of this study.

Authors' disclosures available online (http://j-alz. com/manuscript-disclosures/17-0599r1).

\section{REFERENCES}

[1] Salthouse TA (1996) The processing-speed theory of adult age differences in cognition. Psychol Rev 103, 403-428.

[2] Woods DL, Wyma JM, Yund EW, Herron TJ, Reed B (2015) Age-related slowing of response selection and production in a visual choice reaction time task. Front Hum Neurosci 9 , 193.

[3] Troyer AK, Vandermorris S, Murphy KJ (2016) Intraindividual variability in performance on associative memory tasks is elevated in amnestic mild cognitive impairment. Neuropsychologia 90, 110-116.

[4] Gorus E, De Raedt R, Lambert M, Lemper JC, Mets T (2008) Reaction times and performance variability in normal aging, mild cognitive impairment and Alzheimer's disease. J Geriatr Psychiatry Neurol 21, 204-218.

[5] Phillips M, Rogers P, Haworth J, Bayer A, Tales A (2013) Intra-individual reaction time variability in mild cognitive impairment and Alzheimer's disease: Gender, processing load and speed factors. PLoS One 8, e65712.

[6] Lu PH, Lee GL, Tishler TA, Meghpara M, Thompson PM, Bartzokis G (2013) Myelin breakdown mediates age-related slowing in cognitive processing speed in healthy elderly men. Brain Cogn 81, 131-138.

[7] Kerchner GA, Racine CA, Hale S, Wilheim R, Laluz V, Miller BL, Kramer JH (2012) Cognitive processing speed in older adults: Relationship with white matter integrity. PLoS One 7, e50425.

[8] Tamnes CK, Fjell AM, Westlye LT, Østby Y, Walhovd KB (2012) Becoming consistent: Developmental reductions in intraindividual variability in reaction time are related to white matter integrity. J Neurosci 32, 972-982.

[9] Nilsson J, Thomas AJ, O'Brien JT, Gallagher P (2014) White matter and cognitive decline in aging: A focus on processing speed and variability. J Int Neuropsychol Soc 20, 262-267.

[10] Hong Z, Ng KK, Sim SKY, Ngeow MY, Zheng H, Lo JC (2015) Differential age-dependent associations of gray matter volume and white matter integrity with processing speed in healthy older adults. Neuroimage 123, 42-50.

[11] Jacobs HIL, Leritz EC, Williams VJ, van Boxtel MPJ, van der Elst W, Jolles J (2013) Association between white matter microstructure, executive functions, and processing speed in older adults: The impact of vascular health. Hum Brain Mapp 34, 77-95.

[12] Yang Y, Bender AR, Raz N (2015) Age related differences in reaction time components and diffusion properties of normal-appearing white matter in healthy adults. Neuropsychologia 66, 246-258.

[13] Strauss E, Bielak AA, Bunce D, Hunter MA, Hultsch DF (2007) Within-person variability in response speed as an indicator of cognitive impairment in older adults. Neuropsychol Dev Cogn B Aging Neuropsychol Cogn 14, 608-630. 
[14] Fjell AM, Walhovd KB (2010) Structural brain changes in aging: Courses, causes and cognitive consequences. Rev Neurosci 21, 187-221.

[15] Lin F, Chen D-G, Vance D, Mapstone M (2013) Trajectories of combined laboratory- and real world-based speed of processing in community-dwelling older adults. J Gerontol Series B: Psychol Sci Soc Sci 68, 364-373.

[16] Batterham PJ, Bunce D, Mackinnon AJ, Christensen H (2014) Intra-individual reaction time variability and allcause mortality over 17 years: A community-based cohort study. Age Ageing 43, 84-90.

[17] Welmer AK, Rizzuto D, Qiu C, Caracciolo B, Laukka EJ (2014) Walking speed, processing speed, and dementia: A population-based longitudinal study. J Gerontol A Biol Sci Med Sci 69, 1503-1510.

[18] Anstey KJ, Dear K, Christensen H, Jorm AF (2005) Biomarkers, health, lifestyle, and demographic variables as correlates of reaction time performance in early, middle, and late adulthood. QJEP Section A: Hum Exp Psychol 58, 5-21.

[19] Eckert MA (2011) Slowing down: Age-related neurobiological predictors of processing speed. Front Neurosci 5,25 .

[20] Hagger-Johnson GE, Shickle DA, Roberts BA, Deary IJ (2012) Neuroticism combined with slower and more variable reaction time: Synergistic risk factors for 7-year cognitive decline in females. J Gerontol B Psychol Sci Soc Sci 67, 572-581.

[21] American Psychiatric Association (2013) Diagnostic and Statistical Manual of Mental Disorders: DSM-5. 5th ed. American Psychiatric Association, Washington, DC.

[22] Jessen F, Amariglio RE, van Boxtel M, Breteler M, Ceccaldi M, Chételat G, Dubois B, Dufouil C, Ellis KA, van der Flier WM, Glodzik L, van Harten AC, de Leon MJ, McHugh P, Mielke MM, Molinuevo JL, Mosconi L, Osorio RS, Perrotin A, Petersen RC, Rabin LA, Rami L, Reisberg B, Rentz DM, Sachdev PS, de la Sayette V, Saykin AJ, Scheltens P, Shulman MB, Slavin MJ, Sperling RA, Stewart R, Uspenskaya O, Vellas B, Visser PJ, Wagner M, Subjective Cognitive Decline Initiative (SCD-I) Working Group (2014) A conceptual framework for research on subjective cognitive decline in preclinical Alzheimer's disease. Alzheimers Dement 10, 844-852.

[23] Mark RE, Sitskoorn MM (2013) Are subjective cognitive complaints relevant in preclinical Alzheimer's disease? A review and guidelines for healthcare professionals. Rev Clin Gerontol 23, 61-74.

[24] Mitchell AJ, Beaumont H, Fergusen D, Yadegarfar M, Stubbs B (2014) Risk of dementia and mild cognitive impairment in older people with subjective memory complaints: Meta-analysis. Acta Psychiat Scand 130, 439-451.
[25] Mendonça MD, Alves L, Bugalho P (2015) From subjective cognitive complaints to dementia: Who is at risk? A systematic review. Am J Alzheimers Dis Other Demen 31, 105-114.

[26] Roehr S, Villringer A, Angermeyer MC, Luck T, RiedelHeller SG (2016) Outcomes of stable and unstable patterns of subjective cognitive decline - results from the Leipzig longitudinal study of the aged (LEILA75+). BMC Geriatrics 16, 180.

[27] João AA, Maroco J, Ginó S, Mendes T, de Mendonça A, Martins IP (2016) Education modifies the type of subjective memory complaints in older people. Int J Geriatr Psychiatry 31, 153-160.

[28] Barba GD, La Corte V, Dubois B (2015) For a cognitive model of subjective memory awareness. J Alzheimers Dis 48, S57-S61.

[29] Hill NL, Mogle J, Wion R, Munoz E, DePasquale N, Yevchak AM, Parisi JM (2016) Subjective cognitive impairment and affective symptoms: A systematic review. Gerontologist 56, e109-e127.

[30] Haworth J, Phillips M, Newson M, Rogers P, TorrensBurton A, Tales A (2016) Measuring information processing speed in mild cognitive impairment: Clinical versus research dichotomy. J Alzheimers Dis 51, 263-275.

[31] Fleming SM, Dolan RJ (2012) The neural basis of metacognitive ability. Philos Trans $R$ Soc Lond B Biol Sci $\mathbf{3 6 7}$ 1338-1349.

[32] Reitan RM (1958) Validity of the trail making test as an indicator of organic brain damage. Percept Mot Skills $\mathbf{8}$, 271-276.

[33] Nasreddine ZS, Phillips NA, Bédirian V, Charbonneau S, Whitehead V, Collin I, Cummings JL, Chertkow H (2005) The Montreal Cognitive Assessment (MoCA): A brief screening tool for mild cognitive impairment. J Am Geriatr Soc 53, 695-699.

[34] Spitzer R, Kroenke K, Williams JB, Löwe B (2006) A brief measure for assessing generalized anxiety disorder: The GAD-7-7. Arch Int Med 166, 1092-1097.

[35] Löwe B, Decker O, Müller S, Brähler E, Schellberg D, Herzog W, Herzberg PY (2008) Validation and standardization of the Generalized Anxiety Disorder Screener (GAD-7-7) in the general population. Med Care 46, 266-274.

[36] Kroenke K, Spitzer RL, Williams JB (2001) The PHQ-9: Validity of a brief depression severity measure. J Gen Int Med 16, 606-613.

[37] Gilewski MJ, Zelinski EM, Schaie KW (1990) The Memory Functioning Questionnaire for assessment of memory complaints in adulthood and old age. Psychol Aging 5, 482-490. 


\section{Visual search parametric testing}

\section{Information processing speed: age and trial type}

Analysis of variance (ANOVA) comparing reaction time between Trial Type (Target plus distractors and Target alone conditions) and age (older and young adults) revealed a significant main effect of Trial Type; reaction time was significantly slower in Target plus distractors condition compared to Target alone condition $\left[\mathrm{F}(1,200)=480.73, \mathrm{p}<.001 ; \eta_{\mathrm{p}}{ }^{2}=.706\right]$ and a significant main effect of age; older adults reaction time was significantly slower compared to young adults $[\mathrm{F}(1,200)=165.14, \mathrm{p}$ $\left.<.001, \eta_{\mathrm{p}}{ }^{2}=.452\right]$. There was a significant interaction of Trial Type and Age; reaction time in Target plus distractors and Target alone conditions were significantly slower in older adults compared to both trial types in young adults $\left[\mathrm{F}(1,200)=38.54, \mathrm{p}<0.001, \eta_{\mathrm{p}}{ }^{2}=.162\right]$.

\section{Correlations: comparing older RT with demographics}

In older adults, there was a significant negative correlation between RT and education for both Target plus distracters $[\mathrm{r}=-.398, \mathrm{p}=.003$ does survive bonferroni correction $\mathrm{p}=.012]$ and for Target alone $[\mathrm{r}=-.316, \mathrm{p}=.020$ doesn't survive bonferroni correction $\mathrm{p}=.08]$. There was no significant correlation between RT and MoCA, MFQ, depression or anxiety for both Target alone and Target plus distracters conditions [all $\mathrm{p}$ values $>.05]$.

\section{Correlations: comparing young RT with demographics}

In young adults Pearson's correlation coefficient revealed a significant negative correlation between RT and depression (PHQ-9) in Target plus distractors $[r=-.342, p=.017$ did not survive Bonferroni correction] and in the Target alone condition $[r=-.313, p=.03$ did not survive Bonferroni correction]. There was no significant correlation between RT and education, MOCA, or anxiety (GAD-7) for both Target plus distractors [all $p$ values $>0.05$ ] and Target alone [all $p$ values $>0.05$ ] conditions.

\section{Intraindividual variability: age and trial type}

ANOVA comparing RT variability (IIV) between trial type and age revealed a significant main effect of trial type; the variability of RT was significantly greater for Target plus distractors compared to Target alone $\left[\mathrm{F}(1,200)=507.31, \mathrm{p}<.001, \eta_{\mathrm{p}}{ }^{2}=.717\right]$ and a significant main effect of age; the variability of RT was significantly greater in older adults compared to young adults [F (1, 200) $\left.=73.0, \mathrm{p}<.001, \eta_{\mathrm{p}}{ }^{2}=.267\right]$. 
There was a significant interaction of trial type and age; reaction time variability in Target plus distractors and Target alone condition were significantly greater in older adults compared to both trial types in young adults $\left[\mathrm{F}(1,200)=30.99, \mathrm{p}<.001, \eta_{\mathrm{p}}{ }^{2}=.134\right]$.

\section{Correlations: comparing young IIV with demographics}

In young adults, RT variability (IIV) for Target plus distractors significantly negatively correlated with anxiety level $[\mathrm{r}=-.329, \mathrm{p}=.022]$ and depression level $[\mathrm{r}=-.393, \mathrm{p}=.006]$ but in Target alone trials, RT variability significantly negatively correlated with depression level only $[r=-.30, p=$ .038]. For both Target alone and target plus distracters conditions IIV was not significantly correlated with MOCA or education [all $\mathrm{p}$ values >.05].

\section{Correlations: Comparing older IIV with demographics}

In older adults there was a significant negative correlation between RT variability and education for Target plus distractors $[\mathrm{r}=-.339, \mathrm{p}=.012]$ and for Target alone $[\mathrm{r}=-.411, \mathrm{p}=.002]$. RT variability significantly positively correlated with anxiety for Target plus distractors $[\mathrm{r}=.297, \mathrm{p}=.029]$ but not for Target alone [ $p>0.05]$. There was no significant correlation between RT variability and MoCA, MFQ or depression level for Target plus distractors [all $p$ values $>.05$ ] or for target alone [all $p$ values $>.05]$.

\section{Information processing speed: age, trial and sex}

ANOVA comparing reaction time (RT) between age (older and young adults), sex (male and female) and trial type (Target plus distractors and Target alone) revealed a significant main effect of age; RT was significantly slower in older adults compared to the younger adults $[\mathrm{F}(1,196)=171.17, \mathrm{p}<.001$, $\left.\eta p^{2}=.466\right]$, a significant main effect of trial type; RT was significantly slower for the Target plus distractors compared to Target alone $\left[\mathrm{F}(1,196)=462.14, \mathrm{p}<.001, \eta \mathrm{p}^{2}=.702\right]$ and a significant main effect of sex; females performed significantly slower overall compared to males $[\mathrm{F}(1,196)=$ $\left.4.05, \mathrm{p}=.045, \eta \mathrm{p}^{2}=.020\right]$. There was a significant interaction between age and trial type $[\mathrm{F}(1,196)$ $\left.=40.18, \mathrm{p}<.001, \eta \mathrm{p}^{2}=.170\right]$ but no significant interaction between age and sex $[\mathrm{F}(1,196)=2.01, \mathrm{p}$ $\left.=.158, \eta \mathrm{p}^{2}=.010\right]$ nor a significant interaction between sex and trial type $[\mathrm{F}(1,196)=2.29, \mathrm{p}=$ $\left..132, \eta \mathrm{p}^{2}=.012\right]$ and no significant age, sex and trial type interaction $[\mathrm{F}(1,196)=.484, \mathrm{p}=.487$, $\left.\eta \mathrm{p}^{2}=.002\right]$.

\section{Intraindividual variability: age, trial and sex}

ANOVA comparing RT variability (IIV) between age (older and young adults), sex (male and female) and trial type (Target plus distractors and Target alone) revealed a significant main effect of age; RT variability was significantly greater in older adults compared to the younger adults [F $\left.(1,196)=75.57, \mathrm{p}<.001, \eta \mathrm{p}^{2}=.278\right]$, a significant main effect of trial type; RT variability was significantly greater for Target plus distractors compared to Target alone $[\mathrm{F}(1,196)=484.78, \mathrm{p}<$ 
$\left..001, \eta p^{2}=.712\right]$ but no significant main effect of sex; RT variability was not significantly different between males and females $\left[\mathrm{F}(1,196)=3.24, \mathrm{p}=.073, \eta \mathrm{p}^{2}=.016\right]$.

There was a significant interaction between age and trial type $\left[\mathrm{F}(1,196)=30.64, \mathrm{p}<.001, \eta \mathrm{p}^{2}=\right.$ $.135]$ but no significant interaction between age and $\operatorname{sex}\left[F(1,196)=1.22, p=.270, \eta p^{2}=.006\right]$ nor a significant interaction between sex and trial type $\left[\mathrm{F}(1,196)=1.77, \mathrm{p}=.185, \eta \mathrm{p}^{2}=.009\right]$ and no significant age, sex and trial type interaction $\left[\mathrm{F}(1,196)=.001, \mathrm{p}=.977, \eta \mathrm{p}^{2}=.00\right]$.

\section{Distractor effect of RT between age and sex}

ANOVA comparing the difference in RT between trial types [Target plus distractors RT - target alone RT] between age (older and young adults) and sex (male and female) revealed a significant main effect of age; the difference in RT between trial types was significantly greater for older adults compared to young adults $\left[\mathrm{F}(1,98)=99.28, \mathrm{p}<.001, \eta_{\mathrm{p}}{ }^{2}=.501\right]$ and a significant main effect of sex; the difference in RT between trial types was significantly greater in females compared to males [F $\left.(1,98)=5.60, \mathrm{p}=.020, \eta_{\mathrm{p}}{ }^{2}=.054\right]$. There was no significant interaction between age and sex; the difference in RT between trial types was not significantly different between males and females in older adults compared to young adults $\left[\mathrm{F}(1,98)=1.18, \mathrm{p}=.279, \eta_{\mathrm{p}}{ }^{2}=.012\right]$.

\section{Distractor effect of IIV between age and sex}

ANOVA comparing the difference in RT variability [target + distractor IIV - target alone IIV] between age and sex revealed a significant main effect of age; the difference in RT variability was significantly greater for older adults compared to young adults $\left[\mathrm{F}(1,98)=35.81, \mathrm{p}<.001, \eta_{\mathrm{p}}{ }^{2}=.268\right]$ but no significant main effect of sex; the difference in RT variability was not significantly different between males and females $\left[\mathrm{F}(1,98)=2.07, \mathrm{p}=.153, \eta_{\mathrm{p}}{ }^{2}=.021\right]$. There was no significant interaction between age and $\operatorname{sex}\left[\mathrm{F}(1,98)=.001, \mathrm{p}=.975, \eta_{\mathrm{p}}{ }^{2}=.00\right]$.

\section{Young adult correlations: Distractor effect (RT), MOCA, anxiety and depression}

In young adults, Pearson's correlation coefficient revealed no significant correlation between the mean difference in RT and education, MOCA, anxiety or depression [all p values >0.05].

\section{Older adult correlations: Distractor effect (RT), MOCA, MFQ, anxiety and depression}

In older adults the mean difference in RT significantly negatively correlated with education [ $\mathrm{r}=-.334$, $\mathrm{p}=.014$ ] but did not significantly correlate with MOCA, MFQ, anxiety or depression [all $\mathrm{p}$ values $>0.05]$. 


\section{Difference in RT variability, MoCa, anxiety and depression in young adults}

In young adults the mean difference in RT variability significantly negatively correlated with depression level $[\mathrm{p}=-.313, \mathrm{p}=.030]$ but did not significantly correlate with education, anxiety or MOCA [all p values $>0.05]$.

\section{Difference in RT variability, MOCA, MFQ, anxiety and depression in older adults}

In older adults the mean difference in RT variability significantly positively correlated with anxiety [r=-.293, $\mathrm{p}=.031]$ but did not significantly correlate with education, depression, MOCA score or MFQ score [all $\mathrm{p}$ values $>0.05$ ].

\section{Correlations between factors in young adults}

In young adults there was a significant positive correlation between depression and anxiety $[r=.743$, $p<.001]$. There was no significant correlation between MOCA, education, depression or anxiety respectively [all $\mathrm{p}$ values $>0.05$ ]. Spearman's rho revealed no significant correlation between perceived test difficulty and education, depression or anxiety [all $\mathrm{p}$ values $>0.05$ ].

\section{Correlations between factors in older adults}

In older adults there was a significant negative correlation between anxiety and depression $[\mathrm{r}=.483$, $\mathrm{p}<.001]$ and depression significantly positively correlated with education [ $\mathrm{r}=.294, \mathrm{p}=.031]$. MOCA score did not correlate with level of education, MFQ, anxiety or depression [all $p$ values $>0.05$ ]. All factors had no influence on objective cognitive performance. MFQ score did not correlate with level of education, anxiety or depression [p > 0.05]. All factors had no influence on how older adults perceived their memory. Spearman's rho revealed perceived test difficulty significantly positively correlated with education $[\mathrm{r}=.440, \mathrm{p}=.001]$ and depression $[\mathrm{r}=.273, \mathrm{p}=.046]$ and significantly negatively correlated with MOCA score $[\mathrm{r}=-.279, \mathrm{p}=.041]$ but did not significantly correlate with anxiety, and MFQ score [all $\mathrm{p}$ values $>0.05$ ]. 


\section{TRAILS PARAMETRIC TESTING}

\section{Age and Trial Comparison}

Analysis of variance ANOVA comparing age (older and young adults) and tests (Trails A and Trails B) revealed a significant main effect of test, with significantly slower overall RT for the Trails B compared to Trails A $[\mathrm{F}(1,300)=186.32, \mathrm{p}<.001$, partial eta squared $=.383]$ : a significant main effect of age; with significantly slower overall RT in the older compared to the younger adults [F $\left.(1,300)=24.21, \mathrm{p}<0.001, \eta \mathrm{p}^{2}=.075\right]$, but no significant age by test interaction, i.e., the difference in RT between Trails A and Trails B was not significantly different in the older group compared to young group $\left[\mathrm{F}(1,300)=3.06, \mathrm{p}=.081, \eta \mathrm{p}^{2}=.010\right]$.

\section{Reaction time, MOCA, education, anxiety \& depression in young adults}

In young adults Pearson's correlation coefficient revealed no significant correlation between RT and depression score (PHQ-9), anxiety score (GAD-7), MOCA score or education level or for both Trails A [all $p$ values $>.05$ ] and Trails B [all $p$ values >.05]. Spearman's rho (for Likert scale) revealed no significant correlation between RT and perceived test difficulty for both Trails A [p >.05] and Trails B [p >.05].

\section{Reaction time, MOCA, education, anxiety \& depression in older adults}

In older adults there was no significant correlation between RT and education level, depression, anxiety, MOCA score, MFQ score for both Trails A[all p values >.05] and Trails B [all $\mathrm{p}$ values $>$.05]. Spearman's rho revealed a significant positive correlation between RT in Trails B and perceived difficulty of Trails $\mathrm{B}[\mathrm{r}=.293, \mathrm{p}=.008]$ but no significant correlation between RT in Trails A and perceived test difficulty of Trails A [p > .05].

\section{$\operatorname{Sex}$}

Analysis of variance ANOVA comparing age (older and young adults), sex (male and female) and test (Trails A and Trails B) revealed a significant main effect of age; with significantly slower overall $\mathrm{RT}$ in the older compared to the younger adults $\left[\mathrm{F}(1,296)=26.3, \mathrm{p}<0.001, \eta \mathrm{p}^{2}=.082\right]$ and of test, with significantly slower overall RT for the Trails B compared to Trails A $[\mathrm{F}(1,296)=172.48$, p < $\left..001, \eta p^{2}=.368\right]$ :but no significant main effect of sex with no significant difference in RT between males and females $\left[\mathrm{F}(1,296)=.004, \mathrm{p}=.950, \eta \mathrm{p}^{2}=.00\right]$. There was no significant interaction of test and age $\left[F(1,296)=3.18, p=.076, \eta p^{2}=.011\right]$, of test and gender $\left[F(1,296)=.016, p=898, \eta p^{2}=\right.$ $.00]$ or of age and gender $\left[\mathrm{F}(1,296)=2.28, \mathrm{p}=.132, \eta \mathrm{p}^{2}=.008\right]$ nor between age, sex and test $[\mathrm{F}$ $\left.(1,296)=.160, \mathrm{p}=.689, \eta \mathrm{p}^{2}=.001\right]$. 


\section{Correlations between factors}

In young adults, Pearson's correlation coefficient revealed that MOCA score significantly positively correlated with depression $[\mathrm{r}=.285, \mathrm{p}=.025]$. Depression levels significantly positively correlated with anxiety levels $[r=.794, p<.001]$. Spearman's rho revealed that MOCA score significantly negatively correlated with the perceived test difficulty of Trails $\mathrm{A}[\mathrm{r}=-.314, \mathrm{p}=.013]$ but not with the perceived test difficulty of Trails B [p > .05]. Perceived test difficulty of Trails A significantly positively correlated with the perceived test difficulty of Trails B $[r=.432, p<.001]$ but the perceived difficulty of both Trails A and Trails B did not significantly correlate with education, , anxiety or depression [all $\mathrm{p}$ values $>.05$ ].

In older adults, MFQ score significantly negatively correlated with education $[\mathrm{r}=-.242, \mathrm{p}=.030]$ and depression $[r=-.229, p=.040]$. Depression levels significantly positively correlated with anxiety levels $[\mathrm{r}=.537, \mathrm{p}<.001]$. Spearman's rho revealed a significant negative correlation between MFQ score and perceived test difficulty of Trails $\mathrm{A}[\mathrm{r}=-.275, \mathrm{p}=.013]$ and Trails $\mathrm{B}[\mathrm{r}=-$ $.334, \mathrm{p}=.002]$. MOCA score significantly negatively correlated with perceived test difficulty of Trails A [ $\mathrm{r}=-.245, \mathrm{p}=.027]$ but not Trails B [ $\mathrm{p}>.05]$. Education significantly positively correlated with perceived test difficulty of Trails $\mathrm{A}[\mathrm{r}=.238, \mathrm{p}=.033]$ but not Trails $\mathrm{B}[\mathrm{p}>.05]$.

\section{SIMPLE RT PARAMETRIC TESTING}

\section{Demographics}

An independent samples $t$ test revealed no significant difference in mean education or MOCA score between young and old adult groups [ $\mathrm{p}>$.05]. PHQ score was significantly greater for the young group compared to the older group $[\mathrm{t}(\mathrm{df} 163)=-5.26, \mathrm{p}<.001,95 \%$ confidence interval $[\mathrm{CI}]$ lower $=$ 4.24; Upper $=-1.94$; effect size (Cohen's $\mathrm{d})=-0.83$ ] and GAD score was significantly greater for the young group compared to the older group $[\mathrm{t}(\mathrm{df} 163)=-4.34, \mathrm{p}<.001,95 \%$ confidence interval $[\mathrm{CI}]$ lower $=-3.54$; Upper $=-1.35$; effect size $($ Cohen's $d)=-0.68]$.

\section{Information processing speed: age comparison}

A one-way ANOVA comparing mean reaction time (RT) for age group (older adults and young adults) revealed a significant main effect of age; older adults produced slower reaction time compared to young adults $\left[\mathrm{F}(1,170)=10.53, \mathrm{p}=.001, \eta \mathrm{p}^{2}=.058\right]$. 


\section{Correlations: RT, MOCA, education, anxiety \& depression}

In young adults, Pearson's correlation coefficient revealed a significant negative correlation between $\mathrm{RT}$ and level of education $[\mathrm{r}=-.257, \mathrm{p}=.020]$ but no significant correlation between RT and MOCA score, depression (PHQ-9) or anxiety (GAD-7) [all p values > .05]. RT significantly positively correlated with IIV $[\mathrm{r}=.739, \mathrm{p}<.001]$. Spearman's rho (for Likert scale) revealed no significant correlation between RT and perceived test difficulty [p $>.05]$.

In older adults, Pearson's correlation coefficient revealed no significant correlation between RT and education level, MOCA score, MFQ, depression or anxiety [all p values > .05]. RT significantly positively correlated with IIV $[\mathrm{r}=.632, \mathrm{p}<.001]$. Spearman's rho (for Likert scale) revealed no significant correlation between RT and perceived test difficulty [p $>.05]$.

\section{Intraindividual variability: age comparison}

A one-way ANOVA comparing variability of reaction time (IIV) and age group (older adults and young adults) revealed a significant main effect of age; older adults produced greater variability of reaction time compared to young adults $\left[\mathrm{F}(1,170)=13.63, \mathrm{p}<.001, \eta \mathrm{p}^{2}=.074\right]$.

\section{Intraindividual variability , MOCA, education, anxiety \& depression}

In young adults, Pearson's correlation coefficient revealed no significant correlation between variability and education level, MOCA score, depression levels or anxiety levels [all p values > .05]. Spearman's rho (for Likert scale) revealed no significant correlation between variability and perceived test difficulty $[\mathrm{p}>.05]$.

In older adults, Pearson's correlation coefficient revealed no significant correlation between IIV and MOCA, MFQ, depression levels or anxiety levels [all $p$ values > .05]. Spearman's rho (for Likert scale) revealed no significant correlation between variability and perceived test difficulty [p $>.05]$.

\section{Correlations between factors}

In young adults Pearson's correlation coefficient revealed that depression level significantly positively correlated with anxiety level; as depression levels increased, anxiety levels increased $[\mathrm{r}=$ $.755, \mathrm{p}<.001]$. There was no significant correlation between MOCA and depression, anxiety or education level respectively [all $\mathrm{p}$ values $>.05$ ]. Spearman's rho revealed no significant correlation between perceived test difficulty and education, MOCA, anxiety or depression [all $\mathrm{p}$ values $>.05$ ].

In old adults depression level significantly positively correlated with anxiety level $[\mathrm{r}=.547, \mathrm{p}<$ .001]. There was no significant correlation between MFQ score and MOCA, depression, anxiety or education level [all $\mathrm{p}$ values $>.05]$. 
Spearman's Rho revealed a significant negative correlation between perceived test difficulty and MOCA score $[\mathrm{r}=-.208, \mathrm{p}=.049]$ and MFQ score $[\mathrm{r}=-.241, \mathrm{p}=.022]$ but not with education, depression or anxiety [all $\mathrm{p}$ values $>.05$ ].

\section{Information processing speed: age and sex comparison}

A two-way between-subjects ANOVA comparing RT for age (older and young) and sex (females and males) revealed a significant main effect of age; older adults produced significantly slower RT in compared to young adults $\left[\mathrm{F}(1,168)=9.55, \mathrm{p}=.002, \eta \mathrm{p}^{2}=.054\right]$ and a significant main effect of gender; females produced slower reaction times compared to males $\left[\mathrm{F}(1,168)=4.71, \mathrm{p}=.031, \eta \mathrm{p}^{2}=\right.$ .027]. There was no significant age by gender interaction; reaction time for females and males in older adults was not significantly different to reaction time for females and males in young adults $\left[\mathrm{F}\left(1,168=1.17, \mathrm{p}=.281, \eta \mathrm{p}^{2}=.007\right]\right.$.

\section{Intraindividual Variability: age and sex comparison}

A two-way between-subjects ANOVA comparing IIV for age (older and young) and sex (females and males) revealed a significant main effect of age; older adults produced significantly greater IIV in compared to young adults $\left[\mathrm{F}(1,168)=13.35, \mathrm{p}<.001, \eta \mathrm{p}^{2}=.074\right]$ but there was no significant main effect of sex; the difference in IIV between females and males was not significant $\left[\mathrm{F}(1,168)=.546, \mathrm{p}=.461, \eta \mathrm{p}^{2}=.003\right]$ nor was there an age by sex interaction; variability of reaction time for females and males in older adults was not significantly different to reaction time for females and males in young adults $\left[\mathrm{F}(1,168)=.014, \mathrm{p}=.907, \eta \mathrm{p}^{2}=.00\right]$.

\section{CHOICE PARAMETRIC TESTING}

\section{Demographics}

An independent samples t test revealed no significant difference in mean education or MOCA score between young and old adult groups [p > .05]. PHQ score was significantly greater for the young group compared to the older group $[\mathrm{t}(\mathrm{df} 151)=-4.98, \mathrm{p}<.001,95 \%$ confidence interval $[\mathrm{CI}]$ lower $=-$ 4.16; Upper $=-1.80$; effect size $($ Cohen's $d)=-0.81]$ and GAD score was significantly greater for the young group compared to the older group $[\mathrm{t}(151)=-4.87, \mathrm{p}<.001,95 \%$ confidence interval $[\mathrm{CI}]$ lower $=-3.87$; Upper $=-1.64$; effect size $($ Cohen's $d)=-0.79]$.

\section{Information processing speed: age comparrison}

A one-way ANOVA comparing mean RT between age (young adults and older adults) revealed a significant main effect of age; older adults produced significantly slower mean RT compared to the younger adults $\left[\mathrm{F}(1,159)=59.39, \mathrm{p}<.001, \eta \mathrm{p}^{2}=.272\right]$. 


\section{Correlations: Reaction time, MOCA, education, MFQ, anxiety \& depression}

In young adults Pearson's correlation coefficient revealed a significant positive correlation between $\mathrm{RT}$ and IIV; as reaction time increased, variability also increased $[\mathrm{r}=.706, \mathrm{p}<.001]$. There was no significant correlation between RT and education, MOCA, depression level (PHQ-9) or anxiety level (GAD-7) [all $\mathrm{p}$ values $>$.05]. Spearman's rho revealed no significant correlation between perceived test difficulty and reaction time $[\mathrm{p}>.05]$.

In older adults Pearson's correlation coefficient revealed a significant positive correlation between $\mathrm{RT}$ and IIV; as reaction time increased, variability also increased [ $\mathrm{r}=.698, \mathrm{p}<.001]$. There was no significant correlation between RT and education, MOCA, MFQ, depression level (PHQ-9) or anxiety level (GAD-7) [all $\mathrm{p}$ values $>$.05]. Spearman's rho revealed no significant correlation between perceived test difficulty and reaction time $[p>.05]$.

\section{Intraindividual Variability: age comparison}

Analysis of variance ANOVA comparing variability of reaction time (IIV) between age (young adults and older adults) revealed a significant main effect of age; older adults produced greater variability of reaction time compared to young adults $\left[\mathrm{F}(1,159)=27.51, \mathrm{p}<.001, \eta \mathrm{p}^{2}=.148\right]$.

\section{Intraindividual Variability, MOCA, education, anxiety \& depression}

In young adults Pearson's correlation coefficient revealed that there was a significant positive correlation between IIV and MOCA score $[\mathrm{r}=-.283, \mathrm{p}=.017]$. There was no significant correlation between IIV and education, depression or anxiety [all $\mathrm{p}$ values $>.05$ ]. Spearman's rho revealed no significant correlation between perceived test difficulty and variability [ $p>05]$.

In older adults Pearson's correlation coefficient revealed that there was no significant correlation between IIV and education, MOCA, MFQ, depression level or anxiety level [all $p$ values > .05]. Spearman's rho revealed no significant correlation between perceived test difficulty and reaction time $[p>.05]$.

\section{Comparing factors: MOCA, MFQ, education, anxiety \& depression}

In young adults Pearson's correlation coefficient revealed a significant positive correlation between MOCA score and depression $[\mathrm{r}=.241, \mathrm{p}=.043$, a significant positive correlation between MOCA score and anxiety $[\mathrm{r}=.253, \mathrm{p}=.034]$ and a significant positive correlation between depression and anxiety $[\mathrm{r}=.716, \mathrm{p}<.001]$. Spearman's rho revealed no significant correlation between perceived test difficulty and education, MOCA score, anxiety or depression [all $\mathrm{p}$ values $>.05$ ]. 
In older adults Pearson's correlation coefficient revealed a significant negative correlation between MFQ score and education $[\mathrm{r}=-.242, \mathrm{p}=.032]$ and depression $[\mathrm{r}=-.301, \mathrm{p}=.007]$ There was a significant positive correlation between depression level and anxiety level $[\mathrm{r}=.531, \mathrm{p}<.001]$. Spearman's rho revealed a significant negative correlation between perceived test difficulty and MFQ [ $\mathrm{r}=-.389, \mathrm{p}<.001]$ but there was no significant correlation between perceived test difficulty and education, MOCA, anxiety or depression [all $\mathrm{p}$ values $>.05$ ].

\section{Comparison of age between blocks}

A two way mixed ANOVA comparing age group (older and young) and block number (block 1, 2, 3 and 4) revealed no significant main effect of block number on reaction time overall $[\mathrm{F}(2.69,477)=$ $\left.1.26, \mathrm{p}=.289, \eta \mathrm{p}^{2}=.008\right]$ but a significant main effect of age group; older adults were significantly slower compared to young adults $\left[\mathrm{F}(1,159)=85.47, \mathrm{p}<.001, \eta \mathrm{p}^{2}=.350\right]$ and a significant interaction of block number and age group; older adults were significantly slower across all four blocks compared to young adults $\left[\mathrm{F}(2.69,477)=9.40, \mathrm{p}<.001, \eta \mathrm{p}^{2}=.056\right]$.

A two way mixed ANOVA revealed no significant main effect of block number on reaction time variability overall $\left[\mathrm{F}(3,477)=2.14, \mathrm{p}=.095, \eta \mathrm{p}^{2}=.013\right]$ but a significant main effect of age group; older adults were significantly more variable compared to young adults $[\mathrm{F}(1,159)=29.58, \mathrm{p}<.001$, $\left.\eta \mathrm{p}^{2}=.157\right]$ and a significant interaction of block number and age group; older adults were significantly more variable across all four blocks compared to young adults $[\mathrm{F}(3,477)=5.77, \mathrm{p}=$ $\left..001, \eta p^{2}=.035\right]$.

\section{Information processing speed: age and sex comparison}

A two-way between-subjects ANOVA comparing RT for age (older and young) and sex (females and males) revealed a significant main effect of age; older adults produced significantly slower RT in compared to young adults $\left[\mathrm{F}(1,157)=55.0, \mathrm{p}<.001, \eta \mathrm{p}^{2}=.259\right]$ but there was no significant main effect of sex; the difference in RT between females and males was not significant $[\mathrm{F}(1,157)=.442$, $\mathrm{p}$ $\left.=.507, \eta \mathrm{p}^{2}=.003\right]$ nor was there a significant age and gender interaction; reaction time for females and males in older adults was not significantly different to reaction time for females and males in young adults $\left[F(1,157)=.122, p=.727, \eta p^{2}=.001\right]$.

\section{Intraindividual Variability: age and sex comparison}

A two-way between-subjects ANOVA comparing IIV for age (older and young) and sex(females and males) revealed a significant main effect of age; older adults produced significantly greater IIV in compared to young adults $\left[\mathrm{F}(1,157)=27.64, \mathrm{p}<.001, \eta \mathrm{p}^{2}=.150\right]$ but there was no significant main effect of gender; the difference in IIV between females and males was not significant $\left[\mathrm{F}(1,157)=.163, \mathrm{p}=.687, \eta \mathrm{p}^{2}=.001\right]$. There was no significant age and gender interaction; variability of reaction time for females and males in older adults was not significantly different to reaction time for females and males in young adults $\left[\mathrm{F}(1,157)=.408, \mathrm{p}=.524, \eta \mathrm{p}^{2}=.003\right]$. 


\section{Comparing Age and Sex across blocks: information processing speed}

A two way mixed ANOVA comparing age group (older and young adults), sex (male and female) and block number (block 1, 2, 3 and 4) revealed a significant main effect of age $[F(1,157)=80.08, p$ $\left.<.001, \eta p^{2}=.338\right]$ but no significant main effect of block number $\left[F(1,157)=2.06, p=.110, \eta p^{2}=\right.$ $.013]$ or sex $\left[F(1,157)=.746, p=.389, \eta p^{2}=.005\right]$ and no significant interaction of age and sex $\left[\mathrm{F}(1,157)=.028, \mathrm{p}=.868, \eta \mathrm{p}^{2}=.00\right]$. There was a significant main effect of block number and age $\left[\mathrm{F}(2.76,471)=9.8, \mathrm{p}<.001, \eta \mathrm{p}^{2}=.059\right]$ and a significant interaction of block number and sex $[\mathrm{F}$ $\left.(2.76,471)=6.79, \mathrm{p}<.001, \eta \mathrm{p}^{2}=.041\right]$ but no significant interaction of block number, sex and age $\left[\mathrm{F}(2.76,471)=.943, \mathrm{p}=.414, \eta \mathrm{p}^{2}=.006\right]$.

\section{Comparing Age and Sex across blocks: Intraindividual Variability}

A two way mixed ANOVA revealed a significant main effect of block number $[\mathrm{F}(3,471)=2.74, \mathrm{p}=$ $\left..043, \eta p^{2}=.017\right]$ and a significant main effect of age $\left[\mathrm{F}(1,157)=28.1, \mathrm{p}<.001, \eta \mathrm{p}^{2}=.152\right]$ but no significant main effect of $\operatorname{sex}\left[\mathrm{F}(1,157)=.00, \mathrm{p}=.989, \eta \mathrm{p}^{2}=.00\right]$. There was no significant interaction of age and sex $\left[\mathrm{F}(1,157)=.001, \mathrm{p}=.989, \eta \mathrm{p}^{2}=.00\right]$ or of block number and sex $[\mathrm{F}(3$, $\left.471)=2.17, \mathrm{p}=.09, \eta \mathrm{p}^{2}=.014\right]$ but there was a significant interaction of block number and age $[\mathrm{F}$ $\left.(3,471)=5.81, \mathrm{p}=.001, \eta \mathrm{p}^{2}=.036\right]$. There was no significant interaction of block number, sex and age $\left[\mathrm{F}(3,471)=.985, \mathrm{p}=.40, \eta \mathrm{p}^{2}=.006\right]$.

\section{MILO PARAMETRIC TESTING}

\section{Demographics}

Independent $\mathrm{t}$ test analysis revealed no significant difference in mean education or MOCA score between young and old adult groups [p > .05]. PHQ-9 depression score was significantly greater for the young group compared to the older group $[\mathrm{t}(\mathrm{df} 136)=-4.98, \mathrm{p}<.001,95 \%$ confidence interval $[\mathrm{CI}]$ lower $=-4.47$; Upper $=-1.93$; effect size $($ Cohen's $\mathrm{d})=-0.86]$ and GAD-7 anxiety score was significantly greater for the young group compared to the older group $[\mathrm{t}(\mathrm{df} 136)=-4.43, \mathrm{p}<.001$, $95 \%$ confidence interval $[\mathrm{CI}]$ lower $=-3.70$; Upper $=-1.42$; effect size $($ Cohen's $\mathrm{d})=-0.77]$. 


\section{Comparison of age and sex}

\section{RT 1}

ANOVA comparing reaction time of the first ball between age (older and young adults) and sex(male and female) revealed a significant main effect of age; older adults were significantly slower compared to young adults $\left[\mathrm{F}(1,134)=17.39, \mathrm{p}<.001, \eta \mathrm{p}^{2}=.115\right]$ but no significant main effect of sex; no significant difference in RT 1 between males and females $\left[F(1,134)=.877, p=.351, \eta p^{2}=\right.$ $.007]$ nor a significant interaction of age and $\operatorname{sex}\left[\mathrm{F}(1,134)=.956, \mathrm{p}=.330, \eta \mathrm{p}^{2}=.007\right]$.

\section{RT 8 -1}

ANOVA comparing overall reaction time between age (older and young adults) and sex(male and female) revealed a significant main effect of age; older adults were significantly slower compared to young adults $\left[\mathrm{F}(1,134)=68.35, \mathrm{p}<.001, \eta \mathrm{p}^{2}=.338\right]$ but no significant main effect of sex; no significant difference between males and females $\left[\mathrm{F}(1,134)=3.21, \mathrm{p}=.075, \eta \mathrm{p}^{2}=.023\right]$ nor a significant interaction of age and $\operatorname{sex}\left[\mathrm{F}(1,134)=1.62, \mathrm{p}=.205, \eta \mathrm{p}^{2}=.012\right]$.

\section{Reaction time, MOCA, MFQ, perceived test difficulty, education, anxiety and depression}

In young adults, Pearson's correlation coefficient revealed no significant correlation between MOCA and RT 1 or RT 8 minus RT1 [all p values > .05]. Spearman's rho revealed no significant correlation between perceived test difficulty and RT 1 or RT 8 minus RT1 [all p values $>.05$ ]. There was no significant correlation between education and RT 1 or RT 8minus 1 [all p values > .05]. there was no significant correlation between anxiety and RT 1 or RT 8minus 1 [all p values > .05]. No significant correlation between depression and RT 1 or RT 8minus 1 [all p values > .05].

In older adults, there was no significant correlation between MOCA and RT1 but revealed a significant negative correlation between RT8 minus RT1 and MOCA score [r $=-.277, \mathrm{p}=.013$, didn't survive bonferroni correction $\mathrm{p}=.078]$. There was no significant correlation between MFQ score and RT 1 or RT 8minus 1 [all p values > .05]. Spearman's rho revealed no significant correlation between perceived test difficulty and RT 1 or RT 8minus 1 [all p values $>.05$ ]. There was no significant correlation between education and RT 1 or RT 8minus 1 [all p values $>.05$ ]. In older adults there was no significant correlation between anxiety and RT 1 or RT 8minus 1 [all p values > .05]. There was no significant correlation between depression and RT 1 or RT 8minus $1 \mathrm{v}$ [all p values $>.05]$. 


\section{Intraindividual Variability}

\section{IIV 1}

ANOVA comparing reaction time variability of the first ball between age (older and young adults) and sex(male and female) revealed a significant main effect of age; older adults were significantly more variable compared to young adults $\left[\mathrm{F}(1,134)=21.90, \mathrm{p}<.001, \eta \mathrm{p}^{2}=.140\right]$ but no significant main effect of sex; no significant difference in RT 1 variability between males and females [F (1, $\left.134)=1.11, \mathrm{p}=.293, \eta \mathrm{p}^{2}=.008\right]$ nor a significant interaction of age and $\operatorname{sex}[\mathrm{F}(1,134)=.022, \mathrm{p}=$ $\left..883, \eta p^{2}=.00\right]$.

\section{IIV8 -1}

ANOVA comparing overall reaction time variability between age (older and young adults) and sex(male and female) revealed no significant main effect of age; older adults no more variable compared to young adults $\left[\mathrm{F}(1,134)=2.45, \mathrm{p}=.120 \eta \mathrm{p}^{2}=.018\right]$ and no significant main effect of sex; no significant difference between males and females $\left[\mathrm{F}(1,134)=1.84, \mathrm{p}=.178, \eta \mathrm{p}^{2}=.014\right]$ nor a significant interaction of age and $\operatorname{sex}\left[\mathrm{F}(1,134)=.012, \mathrm{p}=.912, \eta \mathrm{p}^{2}=.00\right]$.

\section{Correlations: Variability, MOCA, MFQ, perceived test difficulty}

In young adults, Pearson's correlation coefficient revealed no significant correlation between MOCA and IIV1, or IIV 8 minus IIV1 [all p values > .05]. Spearman's rho revealed no significant correlation between perceived test difficulty and IIV1, or IIV 8 minus IIV1 [all p values $>.05$ ]. There was no significant correlation between education and IIV1, or IIV 8 minus IIV1 [all p values > .05]. There was no significant correlation between anxiety and IIV1, or IIV 8 minus IIV1 [all $\mathrm{p}$ values $>.05]$. There was no significant correlation between depression and IIV1 or IIV 8minus IIV1 [all $\mathrm{p}$ values $>.05]$. 
In older adults, Pearson's correlation coefficient revealed no significant correlation between MOCA and IIV1, or IIV 8 minus IIV1 [all p values > .05] but a significant negative correlation between MOCA and IIV8 minus $1[\mathrm{r}=-.245, \mathrm{p}=.029$, didn't survive bonferroni correction $\mathrm{p}=.174]$. There was a significant negative correlation between MFQ and IIV1 $[r=-.224, p=.029$, didn't survive bonferroni correction $\mathrm{p}=.186$ ] IIV8 minus IIV1 [p > .05]. Spearman's rho revealed no significant correlation between perceived test difficulty and IIV1, or IIV 8 minus IIV1 [all p values $>.05$ ].

There was no significant correlation between education and IIV1, or IIV 8 minus IIV1 [all p values > .05]. There was a significant positive correlation between anxiety and IIV1[ $\mathrm{r}=.224, \mathrm{p}=.046$, didn't survive bonferroni correction $p=.276$ ] but not with IIV8 minus IIV1 [ $p>.05]$. There no significant correlation between depression and IIV1, or IIV 8 minus IIV1 [all p values > .05]. 
APPENDIX R

\section{$\underline{\text { Correlations between tests }}$}

In older adults, mean RT for TMT Trails B significantly positively correlated with mean RT of Trails A [r =.682, $p<.001]$ and with Simple RT $[\mathrm{r}=.228, p=.041]$. The TMT and the Simple RT were considered poorer attention tests i.e. less sensitive to RT differences between young and older adults as both tests produced small effect sizes. In the MILO the first response (RT1) significantly positively correlated with overall performance excluding the first response (RT8-RT1) $[\mathrm{r}=.391, p<.001]$. The MILO was considered more sensitive to RT differences. There were no significant correlations between tests for IIV.

In young adults, TMT Trails A significantly positively correlated with Simple RT [r $=.309, p=.009]$ and MILO RT8-RT1 [r $=.259, p=.05]$. TMT Trails B significantly positively correlated with Trails A [r $=.363, p=.002]$, Simple RT $[\mathrm{r}=.321, p=$ $.006]$ and MILO RT8-RT1 [r $=.406, p=.002]$. Simple RT significantly positively correlated with MILO RT8-RT1 [r=.277, $p=.035]$. MILO RT1 significantly positively correlated with Choice RT [r $=.447, p<.001]$. TMT and Simple RT were considered less sensitive tests (small effect sizes) and MILO was considered more sensitive (large effect sizes). As with older adults the poorer tests correlated together and the 'best' tests correlated together but also poorer tests correlated with 'best' tests. For IIV only MILO IIV1 positively correlated with Choice RT [r $=.368, p=$ .004]. Both tests produced high effect sizes so considered more sensitive to ageing effects.

Finding correlations between tests support research that there is likely to be some correlation between any test of RT although this will depend on what aspect of brain function the RT is related to. 